\title{
Universität Szeged
}

Philosophische Fakultät

Promotionskolleg Sprachwissenschaft - Germanistische Linguistik

\section{Integrationsgrad vorangestellter Adverbialsätze im Neuhochdeutschen (1650-2000)}

\author{
Dissertation \\ zur Erlangung des akademischen Grades $\mathrm{PhD}$ in germanistischer Linguistik \\ an der Universität Szeged
}

Vorgelegt von:

Péter Kappel

Wissenschaftlicher Betreuer:

Prof. Dr. Vilmos Ágel

Szeged 


\section{Danksagung}

Mein größter Dank richtet sich an meinen Doktorvater Prof. Dr. Vilmos Ágel (Kassel) für die Möglichkeit, die Grammatik des Neuhochdeutschen bereits als Student wissenschaftlich in den Blick zu nehmen, für seine wegweisenden Ideen und Anregungen und für seine geduldige Unterstützung in den vergangenen Jahren, ohne die diese Arbeit nicht möglich gewesen wäre.

Bedanken möchte ich mich auch bei den Leiterinnen und Leitern des Instituts für Germanistik in Szeged, die meine wissenschaftliche Arbeit stets unterstützt haben: Prof. dr. Árpád Bernáth, Dr. habil. Géza Horváth, Dr. habil. Endre Hárs, Dr. Tünde Katona und v.a. Prof. dr. Péter Bassola sowie Dr. habil. Ewa Drewnowska-Vargáné vom Lehrstuhl für Germanistische Linguistik.

Für anregende Diskussionen bin ich, ohne sie einzeln aufzuzählen, mehreren Kolleginnen und Kollegen im In- und Ausland dankbar. Ein besonderer Dank geht an Prof. Dr. Mathilde Hennig (Gießen) und Dr. habil. Attila Péteri (Budapest), die die erste Fassung der Dissertation begutachtet und mit kritischen Kommentaren versehen haben. Für die sorgfältige Korrektur der Arbeit möchte ich Christina Schrödl (Szeged) meinen herzlichen Dank aussprechen.

Nicht zuletzt möchte ich meiner Familie, insbesondere meinen Eltern, meiner Frau Ildikó und meinen Kindern Kamilla und Dániel danken, die mir den Rückhalt gaben und mit denen ich die schönsten Momente der langen Dissertationsjahre erleben durfte.

Szeged, August 2017

Péter Kappel 


\section{Inhaltsverzeichnis}

1. Einleitung ..................................................................................................................... 1

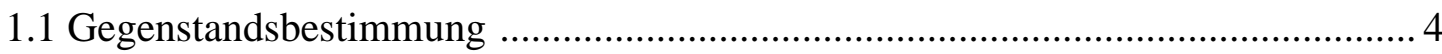

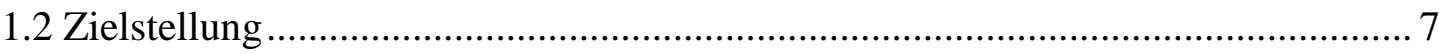

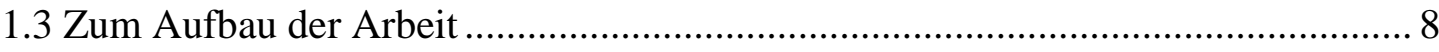

2. Forschungsstand .................................................................................................................. 10

2.1 Integration vorangestellter Adverbialsätze in der Gegenwartssprache .................. 10

2.1.1 Zur syntaktischen Variation im Allgemeinen ............................................... 11

2.1.2 Ebenenbezug: propositionale, Sprechakt- und epistemische Ebene ............. 13

2.1.3 Bedingungen für die Wahl bzw. die Bevorzugung einer Variante ................ 16

2.1.4 Integration im Spannungsfeld von Mündlichkeit/Schriftlichkeit ................. 22

2.2 Zur Erforschung der historischen Entwicklung von Adverbialsätzen .................... 24

2.2.1 Zur formalen Markierung von Nebensätzen in der Sprachgeschichte .......... 25

2.2.1.1 Wortstellung im Nebensatz ........................................................... 26

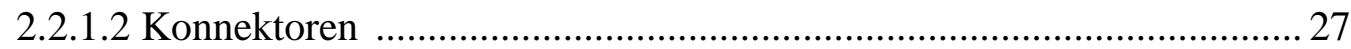

2.2.2 Zur topologischen Integration von Adverbialsätzen bis zum

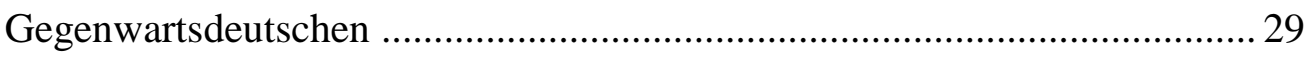

2.2.2.1 Althochdeutsch und Mittelhochdeutsch .............................................. 30

2.2.2.2 Frühneuhochdeutsch und frühes Neuhochdeutsch ............................. 34

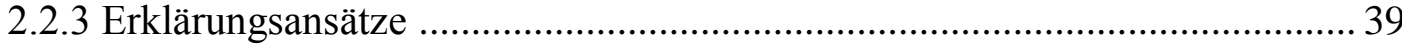

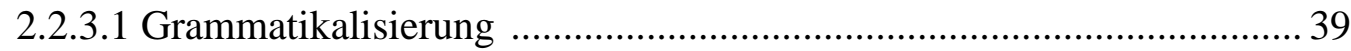

2.2.3.2 Rekategorisierung und Reanalyse ................................................... 41

2.2.3.3 Soziopragmatische Erklärungen ................................................... 44

2.3 Zur Notwendigkeit eines Neuansatzes ......................................................... 48

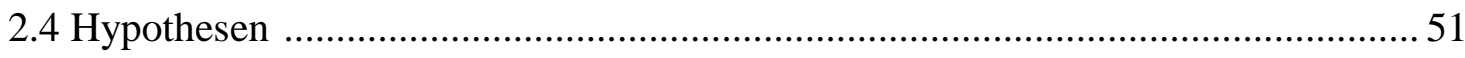

3. Theoretische Grundlegung .......................................................................................................5 53

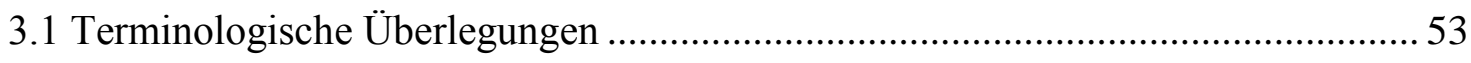

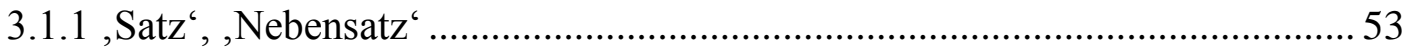

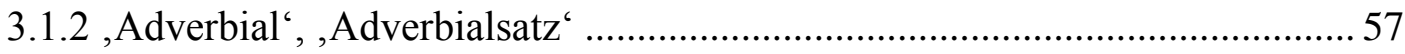




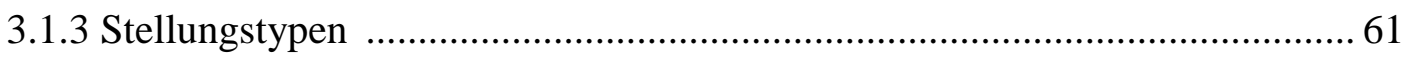

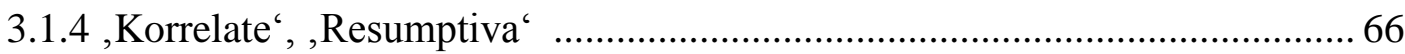

3.2 Zur Erfassung der Linearstruktur des komplexen Satzes ........................................ 69

3.2.1 Das Vorfeld und das linke Außenfeld ......................................................... 70

3.2.2 Vorangestellte Nebensätze im Rahmen des Stellungsfeldermodells ............. 72

3.2.3 Das Stellungsfeldermodell in historischen Untersuchungen ........................ 73

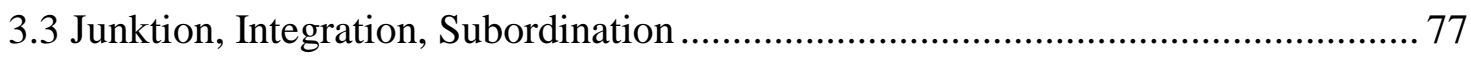

3.2.1 Subordination im Rahmen der Junktionstheorie ......................................... 79

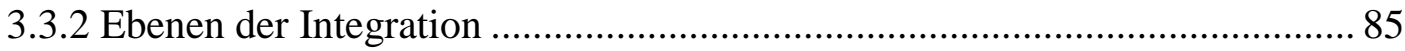

3.4 Semantische Typen von Adverbialsätzen ............................................................. 93

3.4.1 Klassifizierungen von Adverbialsätzen...................................................... 94

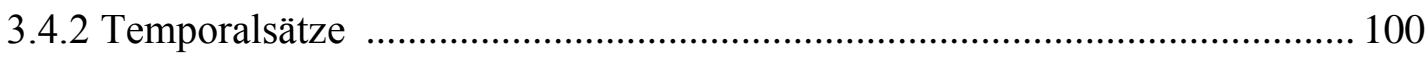

3.4.3 Konditionalsätze und Irrelevanzkonditionale............................................. 102

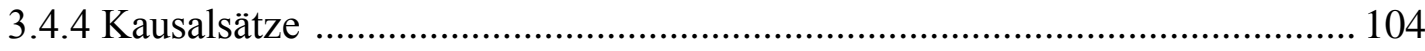

3.4.5 Weitere Typen von Adverbialsätzen im engeren Sinne ................................ 105

3.4.5.1 Final- und Konsekutivsätze ............................................................... 106

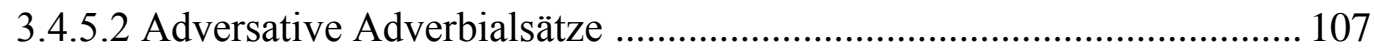

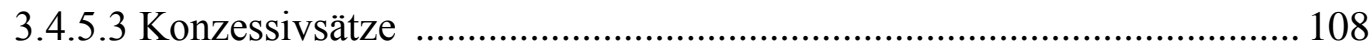

3.4.5.4 Spezifizierende Adverbialsätze (Explikativ- und Restriktivsätze)........ 110

3.4.5.5 Vergleichssätze (Komparativ- und Proportionalsätze) ........................ 112

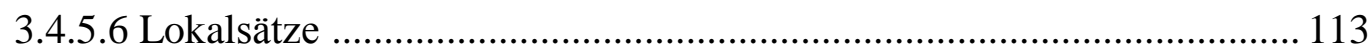

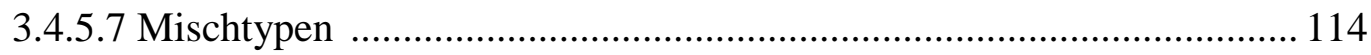

3.4.6 Periphere bzw. pragmatische Adverbialsätze.............................................. 116

3.5 Mündlichkeit/Schriftlichkeit bzw. Nähe/Distanz ................................................... 121

3.5.1 Grundlagen und Operationalisierung .................................................. 121

3.5.2 Mündlichkeit/Schriftlichkeit aus sprachgeschichtlicher Sicht .................... 123

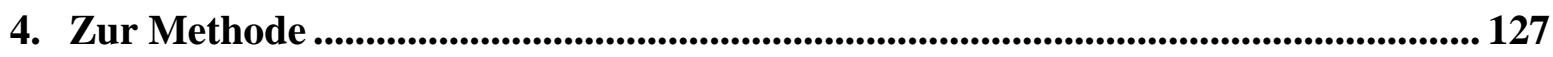

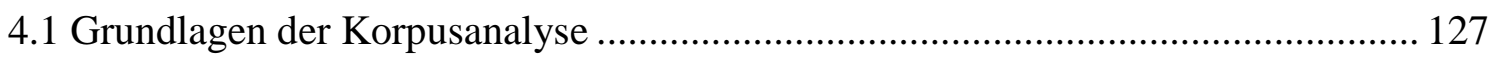

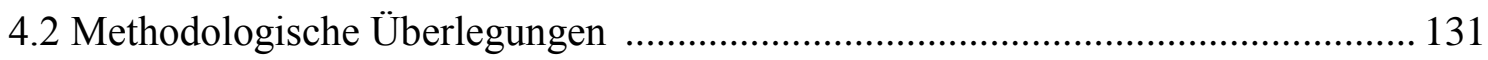

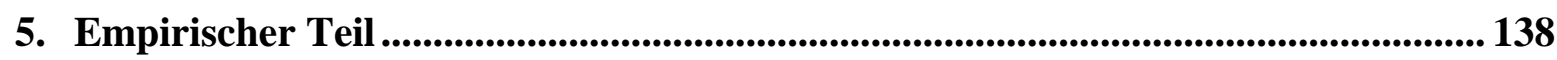

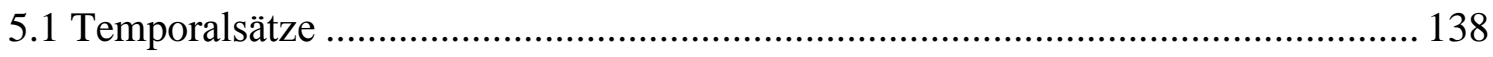

5.1.1 Temporalsätze in den Korpustexten aus dem 17. Jh. ................................... 139

5.1.2 Temporalsätze in den Korpustexten aus dem 18. Jh. ................................. 148 
5.1.3 Temporalsätze in den Korpustexten aus dem 19. Jh. ................................... 153

5.1.4 Temporalsätze in den Korpustexten aus dem 20. Jh. .................................. 158

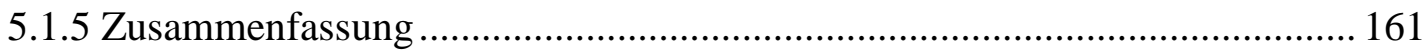

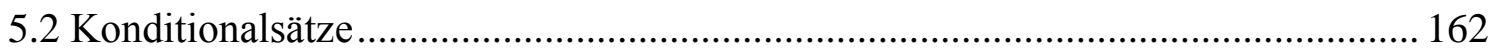

5.2.1 Konditionalsätze in den Korpustexten aus dem 17. Jh................................ 163

5.2.2 Konditionalsätze in den Korpustexten aus dem 18. Jh................................ 169

5.2.3 Konditionalsätze in den Korpustexten aus dem 19. Jh................................ 171

5.2.4 Konditionalsätze in den Korpustexten aus dem 20. Jh............................... 174

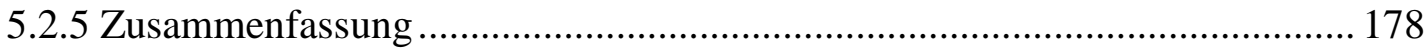

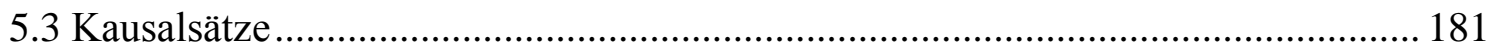

5.3.1 Kausalsätze in den Korpustexten aus dem 17. Jh. ...................................... 182

5.3.2 Kausalsätze in den Korpustexten aus dem 18. Jh. ...................................... 185

5.3.3 Kausalsätze in den Korpustexten aus dem 19. Jh. ....................................... 190

5.3.4 Kausalsätze in den Korpustexten aus dem 20. Jh. ....................................... 192

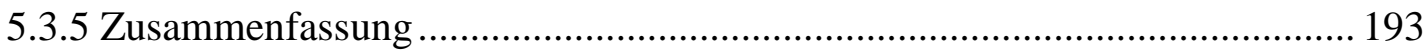

5.4 Weitere Typen von Adverbialsätzen im engeren Sinne ........................................ 195

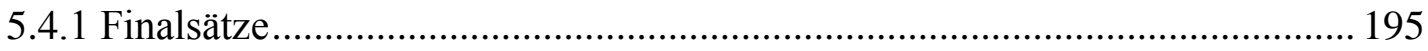

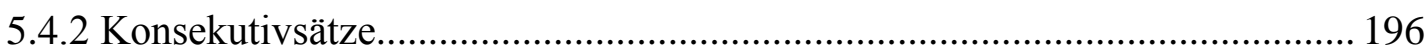

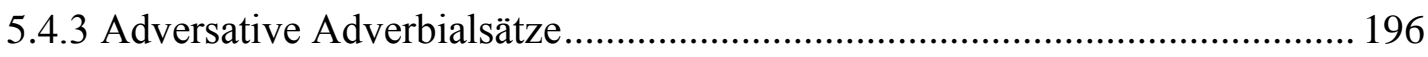

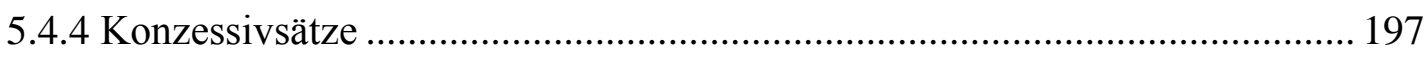

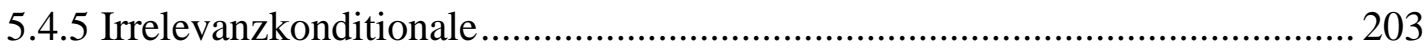

5.4.6 Spezifizierende Adverbialsätze (Explikativ- und Restriktivsätze)................ 208

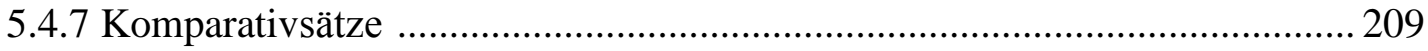

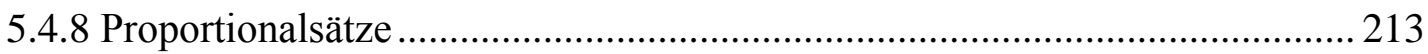

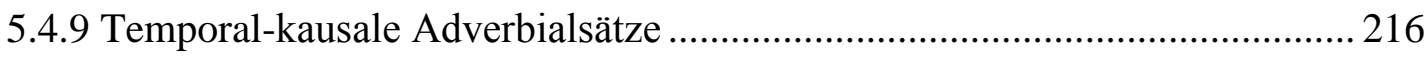

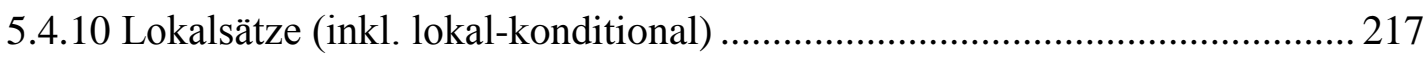

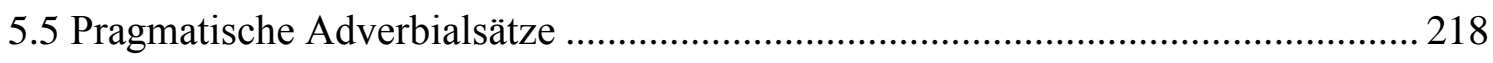

5.5.1 Moduskommentierende Adverbialsätze .................................................... 220

5.5.2 Modusmodifizierende Adverbialsätze ....................................................... 223

5.5.3 Diktums- bzw. einstellungskommentierende Adverbialsätze ...................... 224

5.5.4 Formulierungskommentierende Adverbialsätze......................................... 225

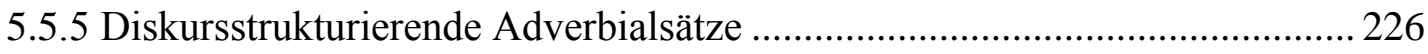

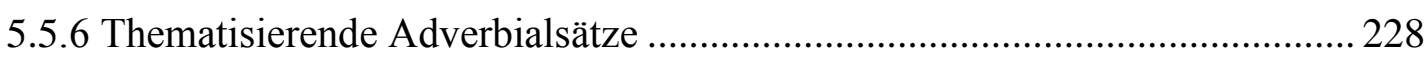

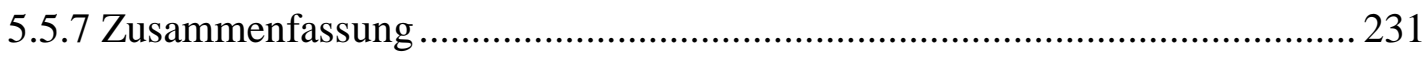




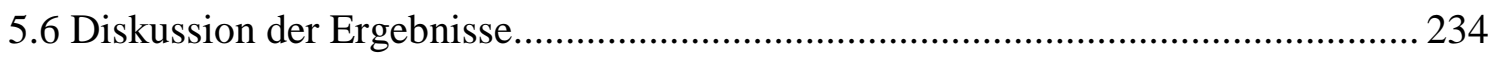

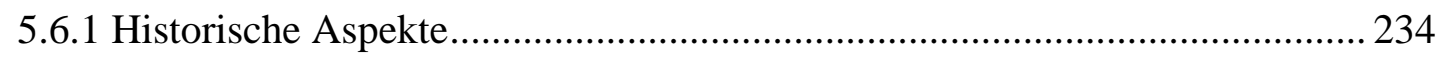

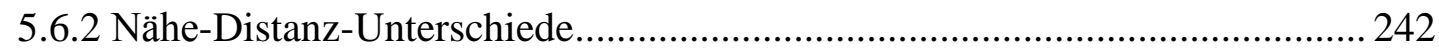

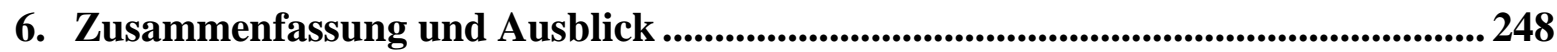

7. Literaturverzeichnis.................................................................................................. 251

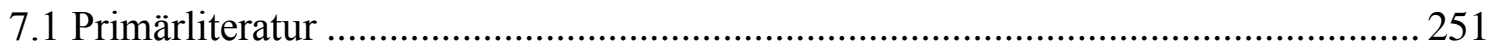

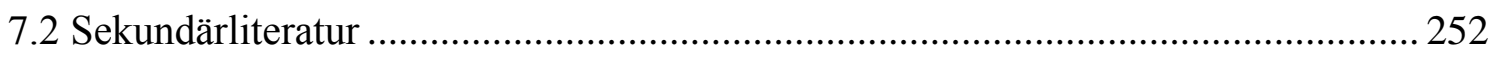

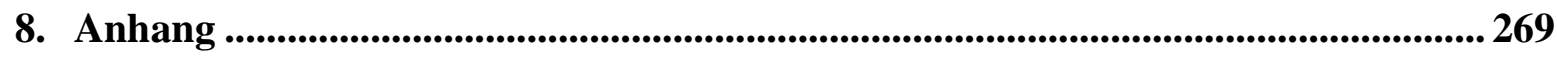

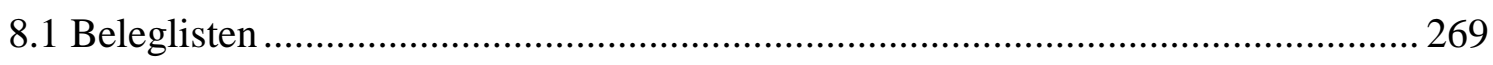

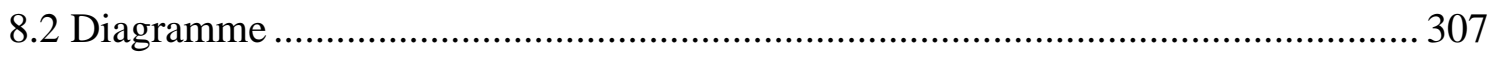




\section{Einleitung}

Die vorliegende Dissertation setzt sich zum Ziel, die Integration vorangestellter Adverbialsätze im Neuhochdeutschen anhand eines selbst zusammengestellten Korpus zu untersuchen und dadurch einen Beitrag zur historischen Syntax zu liefern. Die zentrale Fragestellung der Arbeit wurde im Anschluss an die Hypothese von König/van der Auwera (1988: 107f.) entwickelt. Sie skizzieren in ihrem viel beachteten Aufsatz zur Integration von Konditional- und Konzessivsätzen im Deutschen und im Niederländischen einen Grammatikalisierungspfad: Die beiden Sprachen haben demnach einen dreistufigen Prozess der Satzintegration durchlaufen, indem die vorangestellten Adverbialsätze zunehmend in den nachfolgenden Hauptsatz integriert wurden. Das zu untersuchende Phänomen wurde jedoch nicht nur rein grammatisch (vgl. König/van der Auwera 1988, Axel 2002, Axel-Tober 2012), sondern auch unter Heranziehung weiterer (u.a. pragmatischer bzw. stilistischer) Faktoren erklärt (vgl. Lötscher 2005, 2010). Daher soll der Frage nachgegangen werden, ob die weit verbreitete, aber heutzutage kontrovers beurteilte Auffassung von König/van der Auwera (1988: 107f.) einen angemessenen Erklärungsansatz für die Beschreibung der Integration vorangestellter Adverbialsätze darstellt. Im Folgenden wird die eigene Arbeit in einem breiteren Forschungskontext verortet.

Die vorliegende Arbeit ist angelehnt an die ,neue deutsche Sprachgeschichtsforschung. In der Sprachgeschichtsforschung ist in den letzten Jahrzehnten eine Verlagerung des Interesses auf die jüngere Geschichte des Deutschen zu verzeichnen (vgl. Gardt/Mattheier/Reichmann 1995, darin v. a. Mattheier 1995, bzw. Elspaß 2007a: 2ff.). Das wissenschaftliche Denken über die deutsche Sprache in der Neuzeit wird immer mehr durch verschiedene Fragestellungen der äußeren Sprachgeschichte geprägt, während die innere Sprachgeschichte zu den weniger favorisierten Themenbereichen gehört (vgl. die Bestandsaufnahme zur neuhochdeutschen Syntax von Ágel 2000). Auch im Bereich der inneren Sprachgeschichte, d. h. in der historischen Grammatik, werden jedoch Untersuchungen durchgeführt, die eine gewisse Sensibilität gegenüber der äußeren Sprachgeschichte haben. Seit den 80er Jahren entwickelt sich eine soziopragmatisch orientierte Sprachgeschichtsforschung, die das Verhältnis von syntaktischen Strukturen und der Vertikalisierung des Varietätenspektrums (im Sinne von Reichmann 1988, 2003), der Herausbildung von Sprachwertsystemen 
(Mattheier 1981: 298ff.), dem Einfluss des Sprachbewusstseins u.v.m. zu erschließen hat. In diese Tendenz sind etwa die Arbeiten von Ebert (1998), Elspaß (2005 und 2015), von Polenz (1995) und Takada (1998) einzuordnen. Die vorliegende Arbeit möchte diesem letzten, jüngeren Ansatz der Sprachgeschichtsforschung gerecht werden: Es wird eine Untersuchung zur historischen Syntax des Neuhochdeutschen durchgeführt, die aber durch Verknüpfungen zur äußeren Sprachgeschichte über den Rahmen der reinen Systembeschreibungen hinausgreift. Das Vorhaben steht unter dem Zeichen der inneren Sprachgeschichte, indem der Integrationsgrad der vorangestellten Adverbialsätze und die Tendenz zur zunehmenden Integration in der neuhochdeutschen Periode untersucht werden. Es ist davon auszugehen, dass sich im untersuchten Zeitraum wichtige Veränderungen im Bereich der vorangestellten Adverbialsätze abspielen, bzw. dass diese nicht einfach systemhistorisch aufgedeckt werden können. Es wird deshalb auch nach außersprachlichen Faktoren des Wandels und der syntaktischen Variation gesucht. Eine dem untersuchten Gegenstand angepasste Vorgehensweise sollte bestimmte Bereiche der äußeren Sprachgeschichte berücksichtigen und systematisch analysierbar machen. In der vorliegenden Arbeit wird die Einbeziehung der Unterscheidung zwischen konzeptioneller Mündlichkeit und Schriftlichkeit (= Sprache der Nähe und Sprache der Distanz, vgl. Koch/Oesterreicher 1985 bzw. 1994, Ágel/Hennig 2006a) bei der Beschreibung der Satzverknüpfung im Neuhochdeutschen geleistet. Dabei reiht sich diese Arbeit in die sozio- bzw. varietätenlinguistisch inspirierte historische Syntax des Deutschen ein, die nach Fleischer (2015: 381) bis heute bedeutend ist.

In der vorliegenden Dissertation wird mithilfe einer empirischen Analyse versucht, eine Forschungslücke der historischen Syntax komplexer Sätze zu schließen. ${ }^{1}$ Während frühere Zeitstufen relativ gut erforscht sind, liegen zum Integrationsprozess vorangestellter Adverbialsätze in der neuhochdeutschen Epoche nur Einzelstudien vor. Die ungünstige Lage der Erforschung des Neuhochdeutschen ist u.a. wohl darauf zurückzuführen, dass man oft stillschweigend davon ausgeht, dass in dieser Periode keine relevanten syntaktischen Veränderungen mehr zu beobachten sind (zur Kritik dieser Auffassung s. Mattheier 1995 bzw. Ágel 2000: 1855ff.). Einige Untersuchungen (z.B. Axel-Tober 2012) legen nahe, dass die Durchsetzung des integrativen Typs zu Beginn des Neuhochdeutschen bereits abgeschlossen ist. Es bleibt jedoch zu klären, wann und ob überhaupt der Integrationsprozess zum Abschluss kommt.

Zu weiteren Desideraten der Syntax des Neuhochdeutschen s. Ágel (2000). 
Die Frage nach dem Verhältnis von Mündlichkeit/Schriftlichkeit und Satzintegration wurde bisher fast völlig ausgeblendet, obwohl - wie im Kapitel 2 gezeigt wird - angenommen werden kann, dass diese Ebene der sprachlichen Variation einen Einfluss auf das zu untersuchende Phänomen ausüben kann. Daher wird eine empirische Untersuchung durchgeführt, die neben dem historischen Aspekt auch dem Unterschied zwischen konzeptioneller Mündlichkeit und konzeptioneller Schriftlichkeit Rechnung trägt. In den letzten Jahren fanden grammatische Gemeinsamkeiten und Unterschiede zwischen konzeptionell mündlichen und schriftlichen Texten in mehreren Projekten und Untersuchungen besondere Berücksichtigung. Im Rahmen eines OTKA-Projekts zur Neuhochdeutschen Grammatik 1650-2000 (Laufzeit 2001-2004) wurden wichtige theoretische und methodologische Vorarbeiten zur „Grammatik aus Nähe und Distanz“ durchgeführt, die im Sammelband von Ágel/Hennig (2006) dokumentiert sind. Das Nähe/Distanz-Modell von Ágel/Hennig (2006a) wurde auch im DFG-Projekt „Explizite und elliptische Junktion in der Syntax des Neuhochdeutschen. Pilotprojekt zu einer Sprachstufengrammatik des Neuhochdeutschen“ (Laufzeit 2007-2009) auf historische Quellentexte angewendet. Es wurde u.a. untersucht, in welchem Verhältnis die Verteilung der einzelnen Junktionstechniken ${ }^{2}$ mit der Nähe- bzw. Distanzsprachlichkeit steht (vgl. Ágel/Hennig 2007). Im Junktionsmodell von Ágel/Hennig, das auf Raible (1992) aufbaut, wurden im Phänomenbereich, explizite Junktion‘ u.a. Grundtechniken der Subordination erfasst und operationalisiert (vgl. Ágel 2010). Ihre Vorkommenshäufigkeit wurde auch zu anderen Junktionstechniken u.a. bei der Koordination in Beziehung gesetzt. Es konnten dabei Unterschiede im Junktionsprofil von konzeptionell mündlichen bzw. schriftlichen Texten nachgewiesen werden (Ágel 2012). Zudem wurden mehrere Einzelphänomene anhand historischer Quellentexte behandelt (u.a. Czicza 2014, Hennig 2010a, Kappel 2004). Die aufgeführten Arbeiten sind eingebunden in das größere Langfristprojekt „Neuhochdeutsche Grammatik“, das zum Ziel hat, eine Sprachstufengrammatik für die neuhochdeutsche Epoche (1650-2000) zu erstellen. ${ }^{3}$ Diese Grammatik, die als die letzte noch fehlende Sprachstufengrammatik des Hochdeutschen in der Reihe „Sammlung kurzer Grammatiken germanischer Dialekte“ erscheinen wird, soll die Nähe/Distanz-Dimension, also grammatische Gemeinsamkeiten und Unterschiede zwischen konzeptionell mündlichen und schriftlichen Texten systematisch berücksichtigen.

Zu den Begriffen ,Junktion` bzw. ,Junktionstechniken`s. Kapitel 3.3.

Zur Beschreibung des von Vilmos Ágel (Universität Kassel) geleiteten Projekts s. Ágel (o.J.). 


\subsection{Gegenstandsbestimmung}

Zur Präzisierung der Gegenstandsbestimmung sollen zunächst einige begriffliche Vorerklärungen angeführt werden. Eine detailliertere Erläuterung der Begriffe ist im Kapitel 3 zu finden. Der Gegenstand der Untersuchung ist der Grad der Integration vorangestellter Adverbialsätze in den nachfolgenden Hauptsatz. Adverbialsätze können mit dem übergeordneten Satz unterschiedlich verknüpft werden. Im Folgenden wird der Schwerpunkt auf die topologische Integration gelegt, die mit einer dreistufigen Skala zwischen Integration und Desintegration erfasst werden kann.

Bezüglich der vorangestellten Adverbialsätze im Deutschen werden von König/van der Auwera (1988: 102f., 107) drei Stellungstypen unterschieden und durch folgende Beispielsätze illustriert:

(1) Weil er krank ist, kann Fritz, nicht mitkommen.

Dieser Satz veranschaulicht die am stärksten integrierte Form, sie wird als ,integrativ“ bezeichnet. Der vorangestellte Nebensatz steht direkt vor dem Hauptsatzfinitum, er ist in die Satzstruktur (ins Vorfeld, s. Kapitel 3.2.2) des übergeordneten Satzes integriert.

(2) Wenn er krank ist, dann bleibt er zu Hause.

Beim zweiten Typ, der als ,resumptiv‘ bezeichnet wird, ist die Integration des Nebensatzes im Hauptsatz grammatisch nicht so offensichtlich markiert wie im vorigen Fall. Der vorangestellte Nebensatz wird durch ein Korrelat (dann) wieder aufgenommen, erst danach folgt das Hauptsatzfinitum. Da bei diesem Typ keine topologische Einbettung vorliegt, ist er schwächer integriert als der erste, der integrative, Typ. Das Korrelat stellt aber eine Entsprechung des Adverbialsatzes im Hauptsatz dar und bildet mit ihm eine semantische Einheit, daher ist dieser Typ stärker integriert als der dritte, nicht-integrative, Typ.

(3) Selbst wenn sie nicht alle gekommen sind, wir können mit dem Besuch zufrieden sein.

Nach dem vorangestellten Nebensatz steht hier kein Korrelat, sondern ein anderes, selbständiges Stellungsglied (das pronominale Subjekt wir), erst diesem folgt das Hauptsatzfinitum. Der vorangestellte Nebensatz ist zwar formal durch die Nebensatzgestalt integriert (s. dazu Kapitel 3.3.2), eine topologische Integration liegt jedoch nicht vor. Die Wortstellung des Nachsatzes wird vom vorangestellten Nebensatz nicht beeinflusst. In Anlehnung an Faucher (2000: 6) könnte man auch so formulieren, dass die Verb-Zweit-Regel den vorangestellten Nebensatz ausgrenzt. Dieser Typ ist am schwächsten integriert, er wird 
,nicht-integrativ‘ genannt. Diese Unterscheidung hat sich sowohl in der gegenwartsbezogenen als auch in der sprachhistorischen Forschungsliteratur eingebürgert (vgl. Kapitel 3.1.3), und wird auch der vorliegenden Untersuchung zugrunde gelegt. Die Wahl der Terminologie und die kritische Betrachtung von alternativen Begriffsbestimmungen ist im theoretischen Teil nachzulesen (s. Kapitel 3.1.3).

Kausalität, Konditionalität, Konzessivität bzw. die weiteren Inhaltsrelationen können nicht nur durch die Junktionstechniken der Subordination ausgedrückt werden. Im Sinne der Junktionstheorie von Raible (1992) gibt es sowohl integrativere als auch weniger integrative Techniken. Im Falle der Kausalität wäre ein Satz mit der Inkorporation einer Präpositionalgruppe wie in (4) integrativer, die koordinative Verknüpfung wie in (5) jedoch weniger integrativ als die Subordination im Beispielsatz (1):

(4) Wegen seiner Krankheit kann Fritz nicht mitkommen.

(5) Fritz kann nicht mitkommen, er ist nämlich krank.

Dabei können bestimmte inhaltliche Relationen durchaus präferierte Ausdrucksformen besitzen: Zum Beispiel die Konzessivität wird nur selten mit einer koordinativen Verknüpfung ausgedrückt. Zwischen konzeptionell mündlichen bzw. konzeptionell schriftlichen Texten bestehen wichtige Unterschiede, so wird die Kausalität in einem konzeptionell mündlichen Text aus dem 17. Jh. „fast nur konjunktional“, im konzeptionell schriftlichen Vergleichstext aus dem 17. Jh. ,,überwiegend subjunktional ausgedrückt“ (Ágel 2012: 200). Es wäre interessant der Frage nachzugehen, inwiefern eine Tendenz zur Verwendung integrativerer Junktionstechniken im Neuhochdeutschen zu beobachten ist. Einschlägige Ergebnisse zu dieser Frage liegen bereits vor (vgl. Ágel 2012: 203). In der vorliegenden Arbeit finden jedoch koordinative Aussagenverknüpfungen, auch wenn sie mit dem gleichen Inhalt in Form eines Adverbialsatzes und eines übergeordneten Hauptsatzes ausgedrückt werden könnten, keine Berücksichtigung. Sie sind eben verschiedene Junktionstechniken (im Sinne von Raible 1992 und Ágel 2010). Eine alle Junktionstechniken berücksichtigende empirische Untersuchung ist im Rahmen der vorliegenden Dissertation nicht zu leisten.

Auch die Integration vorangestellter Subjekt-, Objekt- bzw. Relativsätze bildet nicht den Gegenstand der vorliegenden Arbeit. Die Stellungscharakteristika der Ergänzungssätze und Relativsätze wurden in mehreren Arbeiten untersucht (Oppenrieder 2006: 909ff., Boszák 2009, bzw. Lehmann 1984 und 1995, Zitterbart 2013). Dabei wurden wichtige Unterschiede 
zwischen einzelnen Nebensatztypen nachgewiesen. ${ }^{4}$ Ich beschränke mich auf die Analyse der Adverbialsätze, denn der Integrationsgrad vorangestellter Adverbialsätze ist ein komplexes Phänomen, dessen Untersuchung größte theoretische und methodische Sorgfalt erfordert.

Ebenfalls denkbar, allerdings schwer realisierbar wäre eine Ausdehnung des Forschungsgegenstandes auf frühere Zeitepochen des Deutschen (z.B. auf das Frühneuhochdeutsche oder gar auf das Althochdeutsche). ${ }^{5}$ In der vorliegenden Arbeit werden jedoch andere Schwerpunkte gesetzt, die linguistisch genauso, oder eventuell noch relevanter sind. Wenn man vom Problem der Übertragbarkeit einschlägiger Termini und Konzepte auf frühere historische Epochen und weiteren methodologischen Schwierigkeiten absieht (vgl. Kapitel 2.2), dann ist der wichtigste Grund für die Einschränkung des Themenbereiches darin $\mathrm{zu}$ suchen, dass die einschlägigen Entwicklungen im Neuhochdeutschen bisher nicht systematisch analysiert wurden, obwohl gerade diese Epoche wichtige Erkenntnisse über die Satzintegration verspricht.

Ausgeklammert bleibt auch der kontrastive Aspekt, die Integration und ihre Veränderungen in anderen Sprachen. Einschlägige Untersuchungen liegen bereits zu mehreren, v.a. germanischen Sprachen vor. ${ }^{6}$ Laut König/van der Auwera (1988: 128) prägt der Integrationsprozess mehrere germanische Sprachen, aber in unterschiedlichem Maße, skandinavische Sprachen mehr als das Deutsche, das Deutsche aber mehr als das Niederländische.

4 Ein wichtiger Unterschied besteht darin, dass satzwertige Attribute nicht alleine im Vorfeld stehen können (Dürscheid 2012: 95). In sog. substantivierten Relativsätzen wie (i) analysiere ich den Nebensatz im Gegensatz zu Sätzen mit einem Bezugsnomen und anaphorischer Wiederaufnahme wie (ii) nicht als Attributsatz, sondern als einen echten Gliedsatz (hier: Subjektsatz):

(i) Der das getan hat, wird es büßen.

(ii) Wir kennen einen Arzt, dem Anna vertraut (Lehmann 1983: 1200, 1205)

Relativsätze mit einem anaphorischen Element am Anfang des übergeordneten Satzes ohne Bezugsnomen wie (iii) können jedoch durchaus als Attributsätze analysiert werden:

(iii) Wem Anna vertraut, dem kannst auch du trauen.

5 Im Kapitel 2.2 wird - soweit dies für die Zielsetzung der vorliegenden Arbeit sinnvoll erscheint - auf die historische Entwicklung der Adverbialsätze in früheren Zeitepochen eingegangen. Eine systematische Untersuchung, die die Dimension Mündlichkeit/Schriftlichkeit im obigen Sinne berücksichtigt, ist aufgrund der Quellenlage ,für die vorherige Sprachgeschichte nicht möglich" (Elspaß 2015: 395) bzw. auch aus methodischer Sicht problematisch (vgl. die Diskussion der Anwendbarkeit des Nähe-Distanz-Modells auf frühere Zeitstufen bei Lötscher 2010).

6 Zum Deutschen und Niederländischen s. König/van der Auwera (1988), zum Deutschen und Französischen s. Wegener (2001), bezüglich des Teilbereichs der V1-Konditionale s. auch Hilpert (2010) zum Deutschen und Schwedischen, Van den Nest (2010) zum Deutschen und Englischen. Des Weiteren s. auch Donaldson (2012) zum Altfranzösischen, Vandenberghe (2003) zum Mittelniederländischen. Zur typologischen Untersuchung der linken Peripherie im Allgemeinen aufgrund sieben europäischer Sprachen (Deutsch, Englisch, Schwedisch, Französisch, Russisch, Finnisch, Ungarisch) s. auch Molnár (2012). 


\subsection{Zielstellung}

Zur Erfassung der Integration vorangestellter Adverbialsätze in die nachfolgenden Hauptsätze im Neuhochdeutschen werden folgende Ziele gesetzt. ${ }^{7}$ Da das Phänomen ,Integration vorangestellter Adverbialsätze‘ bisher einerseits zeitlich einseitig behandelt wurde, indem frühere Epochen in den Vordergrund gerückt wurden, andererseits methodisch vom möglichen Einfluss des Varietätenspektrums losgerissen wurde, soll in der vorliegenden Arbeit der Forschungshorizont erweitert und überprüft werden, welche neuen Erkenntnisse durch die Untersuchung neuhochdeutscher Texte aus den Bereichen der konzeptionellen Mündlichkeit und Schriftlichkeit gewonnen werden können. Zentrales Anliegen der Arbeit ist die Analyse der Veränderungen der Satzintegration im Neuhochdeutschen. Es soll in diesem Sinne überprüft werden, ob im Neuhochdeutschen relevante Häufigkeitsunterschiede beim Vorkommen der im Kapitel 1.1 angeführten Stellungstypen auftreten. Dabei wird überprüft, ob die Durchsetzung des integrativen Typs zu Beginn des Neuhochdeutschen bereits abgeschlossen ist bzw. ob er die dominierende Variante im Neuhochdeutschen ist. Des Weiteren bleibt zu klären, wie der Integrationsgrad verschiedener Typen von Adverbialsätzen ist. $\mathrm{Zu}$ unterscheiden sind dabei semantische Typen wie etwa Kausalsätze oder Konzessivsätze, aber auch strukturelle Typen wie uneingeleitete Verberst-Sätze, die überwiegend konditional verwendet werden (vgl. Freywald 2013: 321). Es wird der Frage nachgegangen, ob es überhaupt sinnvoll ist, verschiedene Adverbialsatztypen zusammenzufassen und im Allgemeinen deren Integrationsgrad zu bestimmen.

Letztlich soll der Einfluss außersprachlicher Faktoren beim Integrationsprozess nachgewiesen werden. Dies wäre ein Argument gegen die Auffassung des Integrationsprozesses als ein rein grammatisches Phänomen. Die operationalisierbare Erfassung der Unterschiede zwischen konzeptioneller Mündlichkeit und Schriftlichkeit nach dem Nähe-Distanz-Modell von Ágel/Hennig (2006a und 2006b) bietet m.E. einen methodologisch nachvollziehbaren Zugang zu diesem Problembereich. Es soll also überprüft werden, ob die Variable Mündlichkeit/Schriftlichkeit einen Einfluss auf die syntaktische Variation im Bereich der Satzintegration hat.

Die vorliegende Arbeit, die zu den obigen zentralen Bereichen einen Beitrag liefern möchte, soll auf empirischen Daten basieren. Es kann kein Ziel der vorliegenden Arbeit sein, ein repräsentatives Korpus zum Neuhochdeutschen zusammenzustellen (vgl. Kapitel 4.1). Daher wird bewusst in Kauf genommen, dass die zu ermittelnden Daten über die untersuchte

\footnotetext{
7 Zur Ermittlung der Forschungsdesiderate und zur ausführlichen Begründung der Zielstellung s. Kapitel 2.
} 
Datenmenge hinaus nicht ohne Weiteres generalisierbar sind. Die Leitprinzipien der Korpuszusammenstellung sind die Unterscheidung mehrerer synchroner Schritte und die Unterscheidung zwischen konzeptionell mündlichen und konzeptionell schriftlichen Texten. Weitere Dimensionen der sprachlichen Variation sollen möglichst konstant gehalten werden, um die Möglichkeit ihrer Einflüsse auf die syntaktische Variation zu reduzieren.

\subsection{Zum Aufbau}

Im Einleitungsteil (1.1 und 1.2) wurden u.a. der Gegenstand der Arbeit kurz umrissen und in einem größeren Forschungskontext verortet sowie die Zielstellung der Arbeit erläutert. Im zweiten Teil wird ein Überblick über den Stand der Forschung zur Integration vorangestellter Adverbialsätze in Gegenwart und Geschichte geboten. Zudem soll auch gezeigt werden, welche Bereiche des angenommenen Integrationsprozesses bisher wenig erforscht wurden und somit ein Desiderat darstellen. Der Forschungsstand wird in zwei Teilen - getrennt nach gegenwartsbezogenen (2.1) und sprachhistorischen Untersuchungen (2.2) - skizziert. Davon werden dann wissenschaftlich relevante Fragestellungen abgeleitet und die Hypothesen für die eigene Untersuchung festgelegt (2.3 bzw. 2.4). Dadurch wird die eigene Arbeit im Kontext der einschlägigen Forschungsliteratur eingeordnet.

Im dritten, umfassenderen Teil werden die wichtigsten Termini und Theoriebausteine der Untersuchung behandelt. Das Kapitel beginnt mit der Diskussion der einschlägigen Begriffe ,Satz‘, bzw. ,Nebensatz‘, und Adverbial' bzw. ,Adverbial` sowie den Möglichkeiten zur Erfassung der Stellungstypen (Kapitel 3.1). Danach erfolgt - v. a. aufgrund der Fachliteratur zur Gegenwartssprache - die Behandlung vorangestellter Nebensätze im Rahmen des Stellungsfeldermodells (Kapitel 3.2) bzw. der syntaktischen Bereiche der Satzverknüpfung (Kapitel 3.3). Veränderungen im Bereich der Integration vorangestellter Adverbialsätze sollen in einem theoretischen Rahmen erfasst werden, der in der Lage ist, neben dem intensiv erforschten topologischen Aspekt auch weitere Aspekte der Subordination zu berücksichtigen und Unterschiede zwischen weniger integrativen und integrativeren Techniken adäquat erfassen zu können. Im Kapitel 3.3 soll daher v.a. in Anlehnung an die Arbeiten von Raible (1992), Fabricius-Hansen (1992), Zifonun et al. (1997: 2250-2253) sowie Ágel (2010) ein komplexes Mehr-Ebenen-Modell zur Erfassung von Satzverknüpfungstechniken skizziert werden.

Im nächsten Unterkapitel (3.4) werden die für die Analyse notwendigen semantischen Klassen aufgearbeitet. Bezüglich der semantischen Klassen wird zunächst die Klassifizierung 
von Adverbialsätzen problematisiert, um sich im zweiten Schritt auf die Analysekategorien und die Integration in den einzelnen semantischen Bereichen konzentrieren zu können. Der theoretische Teil endet mit einem Überblick über die Erfassung der Mündlichkeit/Schriftlichkeit im Rahmen des Nähe-Distanz-Modells von Ágel/Hennig (2006a und 2006b) (Kapitel 3.5).

Anschließend wird auf die Datengrundlage (Kapitel 4.1) und auf methodologische Fragen (Kapitel 4.2) eingegangen. Im fünften Teil sind die empirischen Analysen zu finden. Dabei werden verschiedene Typen von Adverbialsätzen getrennt behandelt und neben der topologischen Integration der Adverbialsätze, die im Vordergrund der vorliegenden Arbeit steht, auch weitere Aspekte der Integration thematisiert.

Nach der Zusammenfassung (Kapitel 6) und dem Literaturverzeichnis (Kapitel 7) ist ein Anhang zu finden, in dem die Beleglisten (Kapitel 8.1) und Diagramme (Kapitel 8.2) aufgeführt werden, die zur angemessenen Dokumentation des Forschungsprozesses beitragen sollen. 


\section{Forschungsstand}

In diesem Kapitel soll ein Überblick über die gegenwartsbezogene und historische Syntaxforschung im Bereich der Satzverknüpfungstechniken, insb. der Integration vorangestellter Adverbialsätze gegeben werden. ${ }^{8}$ Die Bestandsaufnahme der gegenwärtigen Forschungssituation erfolgt in drei Teilen: Da die vorliegende Arbeit darauf abzielt, die Kluft zwischen Beschreibungen der älteren Sprachstufen und der Gegenwartssprache mithilfe einer empirischen Analyse zum Neuhochdeutschen zu überbrücken, wird in den Kapiteln 2.1 und 2.2 der Forschungsstand bezüglich der Gegenwartssprache und der historischen Entwicklung getrennt umrissen. Demnach werde ich vom skizzierten Forschungsstand Schlüsse ziehen und die Notwendigkeit eines Neuansatzes, einer empirischen Untersuchung des Integrationsgrades vorangestellter Adverbialsätze im Neuhochdeutschen begründen und die Hypothesen für die vorliegende Untersuchung festlegen (Kapitel 2.3 bzw. 2.4).

Gegenwartsbezogene und sprachhistorische Arbeiten sind natürlich nicht immer klar zu trennen, denn in mehreren historischen Arbeiten wird ein synchroner Schnitt der Gegenwartssprache mit einem älteren synchronen Schnitt verglichen, um eine diachrone Analyse zu ermöglichen (vgl. z.B. Baschewa 1983). Deshalb dient die verwendete Unterscheidung an dieser Stelle nur zur besseren Überschaubarkeit des Materials.

\subsection{Integration vorangestellter Adverbialsätze in der Gegenwartssprache}

Was die Breite des bearbeiteten Materials betrifft, so lässt sich feststellen, dass sich die einschlägigen Arbeiten i.d.R. nicht mit allen Typen von Adverbialsätzen befassen. Baschewa (1983) und König/Eisenberg (1984) wenden sich z.B. den Konzessivsätzen zu, Flämig (1964) behandelt Finalsätze, Breindl/Walter (2009) untersuchen Kausalsätze, Volodina (2011a und 2011b) Konditional- und Kausalsätze. In anderen Fällen werden nur jeweils kleinere, spezifische Teilbereiche behandelt. So liegen mehrere Arbeiten zu V1-Konditionalsätzen (Reis/Wöllstein 2010, Pittner 2011) und Irrelevanzkonditionalen (Waßner 2006, Leuschner 2005) vor. Oft ist eine formale Einschränkung des Gegenstandes in Form einer Festlegung auf

\footnotetext{
Die Spezialliteratur zu den einzelnen Unterarten von Adverbialsätzen wird in diesem Kapitel nur dann herangezogen, wenn dies für einen Gesamtüberblick über die Entwicklungstendenzen bei Adverbialsätzen als sinnvoll erscheint. Sie wird im Kapitel 3.4 ausführlicher behandelt.
} 
einen Subjunktor zu beobachten, vgl. etwa die einschlägigen Arbeiten zu wenn-Sätzen (z.B. Auer 2000, Günthner 1999a, Volodina 2006) bzw. zu weil-Sätzen (Konopka 2006). Zudem ist nicht von vornherein eindeutig, welche Nebensatztypen für eine Untersuchung der Adverbialsätze einschlägig sein sollten. Behaghel (1929: 414) behandelt z.B. auch den Typus was das betrifft, ${ }^{9}$ der bei der Behandlung von Adverbialsätzen i.d.R. keine Berücksichtigung findet. Des Weiteren ist auch die Berücksichtigung von sprechaktbezogenen Adverbialsätzen (vgl. Kapitel 3.1.2 und 3.4.6) fraglich. In der umfangreichen Literatur werden oft andere Schwerpunkte gesetzt, die topologische Integration von Adverbialsätzen wird dann nur am Rande erwähnt (vgl. z.B. König/Eisenberg 1984, Wolf 1998). Empirische Untersuchungen, die die Integration vorangestellter Adverbialsätze in ihrer Ganzheit erfassen würden, stellen also ein Desiderat der Forschung dar.

Im Folgenden werden Ergebnisse der gegenwartsbezogenen Forschung referiert. In den einschlägigen Arbeiten wird jedoch nicht immer expliziert, was unter ,(Gegenwarts)deutsch“ genau verstanden wird bzw. auf welchen zeitlichen Abschnitt sich die Aussagen beziehen. Das Deutsche wird oft stillschweigend mit dem Gegenwartsdeutschen, noch genauer mit der Standardsprache im journalistischen Bereich gleichgesetzt. ${ }^{10}$ Des Weiteren wird die zeitliche Erstreckung des Gegenwartsdeutschen unterschiedlich bestimmt. Auffallend ist etwa Baschewas (1983: 77) weit gefasste Periode ab 1900 als die „Zeit der deutschen Gegenwartssprache“" (so auch Flämig 1964: 6). Wir wenden uns im Folgenden der Variation unter den Stellungstypen zu. Dabei stütze ich mich v.a. auf König/van der Auwera (1988) und Kappel (2008).

\subsubsection{Zur syntaktischen Variation im Allgemeinen}

Zum Stand der Forschung vor ca. 30 Jahren liefern König/van der Auwera (1988) einen kompakten Überblick über die einschlägigen Arbeiten - allerdings nur zu einem sehr eingeschränkten semantischen Bereich der Konditionalität und Konzessivität. ${ }^{11}$ Bezüglich der Wortstellungseigenschaften im Nachsatz (dem Nebensatz nachgestellten Hauptsatz) unterscheiden König/van der Auwera (1988: 102f.) drei Stellungstypen im

\footnotetext{
Wir folgen dieser wissenschaftlichen Tradition bei der Bestimmung des Gegenstandes und werden für die Einbeziehung dieses Typs in die Untersuchung von Adverbialsätzen plädieren (vgl. Kapitel 3.4.6).

10 Vgl. z.B. die Formulierung „im Deutschen“ im Titel von Cortès (2003) Arbeit: Bis auf eine Ausnahme wurden alle Belege dem Spiegel aus den Jahrgängen zwischen 1997 und 2000 entnommen. Ein weiteres Beispiel für die Untersuchung eines Zeitungskorpus der Gegenwartssprache ist Carlsen (1998).

11 König/van der Auwera (1988: 106f.) unterscheiden eigentlich neben Konditionalität und Konzessivität auch einen Mischtyp der konzessiven Konditionalität mit drei Unterklassen. Sprechaktbezogene Verwendungsweisen werden jedoch ausgeklammert (1998: 111). Dieses Problem wird erst im Kapitel 3.1.2 bei der Diskussion des Adverbialbegriffs und im Kapitel 3.4 bei der Behandlung der semantischen Klassen aufgegriffen.
} 
Gegenwartsdeutschen, zwischen denen eine Variation besteht: integrativ, resumptiv und nicht-integrativ (s. Kapitel 1.1, vgl. auch die unten stehenden Beispielsätze (6) bis (8)). Im Bereich der von König/van der Auwera untersuchten Konditionalität und Konzessivität sind unter Umständen alle drei Varianten möglich (1988: 103f.). Dies soll an den folgenden Beispielen illustriert werden: ${ }^{12}$

(6) Wenn ich an deiner Stelle wäre, würde ich es tun.

(7) Wenn ich an deiner Stelle wäre, dann würde ich es tun.

(8) Wenn ich an deiner Stelle wäre, ich würde es tun.

Dies bedeutet allenfalls nicht, dass die Variation willkürlich wäre. Laut König/van der Auwera (1988: 129f.) könnten bei Konditionalsätzen im Konjunktiv wie in den obigen Belegen die Faktoren Emphase und Kontrastfokus bzw. Sprechaktbezug bei der Wahl des integrativen bzw. nicht-integrativen Musters eine Rolle spielen. ${ }^{13}$

König/van der Auwera (1988) behandeln mehrere Aspekte der syntaktischen Variation.

Bei der Wählbarkeit der einzelnen Stellungstypen handelt es sich darum, ob bestimmte Sätze auf eine Variante festgelegt sind. In diesen Fällen besteht eigentlich keine Möglichkeit zur Wahl unter den Varianten. Eine bestimmte Stellung wird jedoch nur selten zwingend vorgeschrieben. Dies ist etwa der Fall beim ersten Typ der konzessiven Konditionalsätze, dessen Antezedens (d.h. die Annahme bzw. die Voraussetzung des Urteils, die links vom Hauptsatz steht) durch Disjunktion spezifiziert wird (König/van der Auwera 1988: 118):

12 Eine Google-Recherche am 2.8.2015. lieferte für alle drei Sätze einschlägige Treffer (integrativ: sieben, resumptiv: einen, nicht-integrativ: vier authentische Beispiele, vgl. z.B. die Belege aus den folgenden Forenbeiträgen: http://www.gutefrage.net/frage/was-wuerdet-ihr-tunweiter-wartenneue-stelle-suchen\#answers, https://www.allmystery.de/themen/rs47798, https://pflegeboard.de/thema/ab-nach-holland-div-fragen-zur-pflegedort.31434/). Für ähnliche Sätze lassen sich in gegenwartssprachlichen Texten problemlos Belege finden.

13 König/van der Auwera (1988: 129f.) führen die Wahl zwischen den beiden Stellungstypen auf die argumentative Orientierung der anschließenden Äußerung zurück. Es handele sich bei der nicht-integrativen Variante (8) nach König/van der Auwera (1988: 129f.) um einen Ratschlag in einer Situation, in der der Adressat in Erwägung zieht, etwas zu tun. Demgegenüber sei es bei der integrierten Variante (6) der Sprecher, der erwägt, etwas zu tun. Die syntaktische Variation ist jedoch in den folgenden Belegen (i) und (ii) aus dem Deutschen Referenzkorpus (DeReKo, Institut für Deutsche Sprache 2016) nicht auf dieser Weise zu erklären, da hier seiner in der dritten Person steht und sich somit nicht auf den Rezipienten beziehen kann:

(i) Wenn ich an seiner Stelle wäre, würde ich mich mehr für die Jugendlichen einsetzen, d. h. mehr Geld für die Schulen und Kindergärten zur Verfügung stellen, damit sie ihre Einrichtungen moderner gestalten können. (NUZ03/JUN.00512 Nürnberger Zeitung, 06.06.2003; Jedem Menschen helfen, der in Not ist)

(ii) „Es wäre eine gute Chance für ihn, sein Gewissen zu erleichtern“", meint der niederländische Nebenkläger Paul Hellmann, dessen Vater in Sobibor starb. „Wenn ich an seiner Stelle wäre, ich würde diese letzte Chance nutzen." (NUN09/DEZ.00133 Nürnberger Nachrichten, 02.12.2009, S. 2; Demjanjuk setzt auf Schweigen - Zur Anklage sagt der 89-Jährige nichts - Anwälte wollen den Prozess kippen).

Ein weiterer Kritikpunkt ist, dass nicht danach gefragt wird, wie die Wahl des resumptiven Typs (wie in (7)) in diesem Rahmen zu erklären ist. Nach König/van der Auwera (1988: 129f.) ist ein weiterer Faktor mit im Spiel: Bei der nichtintegrierten Variante (8) ist ein stärkerer Akzent auf ich zu erwarten. Im anderen Fall ist das Konsequens weniger emphatisch oder assertiv als in (8). 
(9) (Ganz gleich) ob wir ihm helfen oder nicht, er schafft es nie.

(10) * (Ganz gleich) ob wir ihm helfen oder nicht, schafft er es nie.

(11) * (Ganz gleich) ob wir ihm helfen oder nicht, so/dann schafft er es nie.

Mit dem Asterisk * wird markiert, dass die entsprechenden Sätze grammatisch nicht wohlgeformt sind, dass also die beiden anderen Stellungstypen im Gegenwartsdeutschen gar nicht möglich sind. Die Wahl eines bestimmten Stellungstyps sei auch bei durch so+Adjektiv eingeleiteten Proportionalsätzen (s. Kapitel 3.4.5.5) zwingend vorgeschrieben. In diesen Fällen ist die integrative oder die nicht-integrative Stellung nicht möglich (Zifonun et al. 1997: 2351f.): ${ }^{14}$

(12) So freundlich sie war, so abweisend konnte sie plötzlich werden.

Des Weiteren ist die Wahl der integrativen Variante bei bestimmten nebensatzförmigen Sprechhandlungskommentaren nicht möglich (vgl. aber (19) weiter unten). Dieser Typ wurde bei den Überlegungen von König/van der Auwera (1988: 111ff.) nicht berücksichtigt. Sprechaktbezogene Verwendungsweisen wurden jedoch intensiv untersucht (vgl. z.B. Pittner 1999; Wolf 1998, Zifonun et al. 1997: 2326ff.). Es wird davon ausgegangen, dass sie mit Desintegration korrelieren bzw. dass der lockeren inhaltlichen Relation eine losere syntaktische Verknüpfung entspricht. (Des-)Integration ist also in diesem Sinne funktionalisiert. Im Folgenden wird auf den Ebenenbezug der Adverbialsätze ausführlicher eingegangen.

2.1.2 Ebenenbezug: propositionale, Sprechakt- und epistemische Ebene

Die Unterscheidung der propositionalen, der epistemischen und der Sprechaktebene geht auf Sweetser (1990) zurück. Im Folgenden sollen die kognitiven Ebenen in Anlehnung an das HDK-2 (Breindl et al. 2014: 109) am Beispiel der weil-Sätze erläutert werden:

(13) Die Kinder schlafen schon, weil sie einen anstrengenden Tag hatten.

(14) Die Kinder schlafen schon, weil man gar nichts mehr von ihnen hört.

(15) Weil die Kinder schon schlafen, wie wäre es mit einem Spaziergang um den Block?

14 Bei Proportionalsätzen sind die ,Korrelate‘ nach Zitterbart (2013: 621) integrative Bestandteile der Korrelatverbindung und sind somit nicht weglassbar (zur Anwendbarkeit des Korrelatbegriffs auf Proportionalsätze s. Kapitel 3.1.4.). 
Im Beispiel (13) ist der im weil-Satz ausgedrückte Sachverhalt die Realursache dafür, dass die Kinder schon schlafen. In (14) ist dagegen der im weil-Satz ausgedrückte Sachverhalt nicht die Ursache für das Schlafen der Kinder. Vielmehr wird aus der Stille (d.h., aus dem Inhalt des weil-Satzes) der Schluss gezogen, dass die Kinder schon schlafen. Mit dem weil-Satz wird also die Begründung der Annahme des Sprechers zum propositionalen Gehalt des Obersatzes ausgedrückt. Im letzten Beispiel (15) wird durch den weil-Satz der Grund für den im Obersatz realisierten Sprechakt angegeben.

Beim propositionalen Bezug wird eine Relation zwischen zwei Sachverhalten in einer realen oder möglichen Welt hergestellt, die semantische Beziehung ist also auf der Sachverhaltsebene angesiedelt (vgl. (13)). Bei der nicht-propositionalen Bezugsgröße besteht die Relation nicht zwischen den Sachverhalten. Dabei lassen sich folgende, metaphorische (vgl. Breindl et al. 2014: 188) Ebenen unterscheiden: Auf der epistemischen Ebene wird eine Annahme oder Einstellung des Sprechers zum propositionalen Gehalt des Obersatzes ausgedrückt (s. (14)). Bei der Sprechaktebene besteht die Relation zwischen der im Nebensatz ausgedrückten Proposition und dem im Obersatz realisierten Sprechakt (wie Bitte, Befehl oder Frage, vgl. (15)).

Auch weitere Konnektoren können neben der Sachverhaltsebene auf den metaphorischen Ebenen verknüpfen, vgl. z.B. konditionale wenn-Sätze. Welche Lesart im konkreten Fall gemeint ist, ergibt sich u.a. aus dem Weltwissen oder aus dem Wissen über die Situation (vgl. Breindl et al. 2014: 188). Unter der Einbeziehung kommunikativ-pragmatischer Faktoren kann der Konditionalsatz im folgenden Beleg so gedeutet werden, dass er keine Bedingung für die Proposition des Hauptsatzes enthält (die traditionelle, grammatisch-semantisch fundierte Interpretation als Standardkonditional wäre irreführend, denn was die Politiker alles wissen, kann nicht vom Wissenstand des Sprechers abhängen), sondern durch ihn die Einstellung zum Vermuteten ausgedrückt wird.

(16) Wenn ich mich nicht ganz täusche, dann haben wohl alle führenden Politiker davon gewußt.

Der Kommunikationspartner muss die epistemische Einstellung des Sprechers zur im Hauptsatz ausgedrückten Vermutung unter einer kommunikativen Akzeptanzbedingung erkennen können. Diese kommunikativ-pragmatischen Funktionen, „[können] mit grammatisch-semantisch fundierten Beschreibungsmodellen nicht erfaßt werden [...]“ (Wolf 2001: 161). Das folgende Beispiel illustriert einen sprechaktbezogenen Konditionalsatz 
(,Relevanzkonditional'), der angibt, unter welchen Bedingungen die Äußerung des Hauptsatzes relevant ist (vgl. Pittner 1999: 345):

(17) Wenn du Hunger hast, der Kühlschrank ist voll.

Es handelt sich um einen metakommunikativen Kommentar (vgl. auch Wolf 1998). ${ }^{15}$ Das Konsequens, also die Proposition des Nachsatzes, ist separat assertierbar und hängt nicht von der Gültigkeit des Antezedens im wenn-Satz ab. Mit anderen Worten: Ob der im Antezedens ausgedrückte Sachverhalt wahr ist oder nicht, modifiziert die Proposition des Hauptsatzes nicht: Eine propositionsbezogene Interpretation im Sinne des Märchens ,Tischlein-DeckDich` wäre zwar auch möglich, stellt jedoch gewiss nicht den Normalfall dar.

Bezüglich der Integration von Adverbialsätzen stellt Pittner (2013a: 521) den folgenden Zusammenhang fest: „Der Grad der syntaktischen Integration in den Bezugssatz korreliert mit dem Ebenenbezug der Adverbialsätze.“ So konnte z.B. Konopka (2006: 122f.) in seiner Untersuchung von weil-Verbletztsätzen feststellen, dass sie bei nicht-propositionaler Verwendung topologisch bzw. prosodisch desintegriert sind, während propositionsbezogene weil-Sätze im Vorfeld erscheinen können. ${ }^{16}$

Die nicht-integrative Stellung markiert oft eine nicht-propositionale Verwendung auf der Sprechaktebene. Die syntaktische Desintegration von metakommunikativen Beiträgen zu einer nachgestellten Äußerung (wie Weil du es unbedingt wissen willst; Obwohl es dich eigentlich nichts angeht; Damit du es weißt; Falls dich jemand fragen sollte) folgt nach Küper (1993: 42f.) aus dem ikonischen Prinzip:

Syntaktische Integration (die von intonatorischer Integration begleitet wird) signalisiert semantische bzw. konzeptuelle Nähe, und syntaktische Nicht-Integration (die von intonatorischer Diskontinuität begleitet wird) signalisiert semantische bzw. konzeptuelle Distanz zwischen den betreffenden Sätzen. (Küper 1993: 43)

Bei metakommunikativen Nebensätzen ist nach Küper (1993: 43) eine maximale semantische bzw. konzeptuelle Distanz anzunehmen, ihre nicht-integrative Stellung ist pragmatisch motiviert.

Es besteht allerdings keine Eins-zu-eins-Entsprechung von Form und Funktion bezüglich des Parameters (Des-)Integration und der Verknüpfungsebenen. Einerseits erscheint die Integration des vorangestellten Nebensatzes nicht nur bei der propositionalen Verknüpfung.

15 Thim-Mabrey untersucht die metakommunikative Funktion von Vorvorfeld-Ausdrücken und unterscheidet dabei sechs Gruppen, aus denen - von einer Gruppe abgesehen - auch Nebensätze im Vorvorfeld einschlägig sind (1988: 54ff.).

16 Blühdorn (2006: 280) geht davon aus, dass im Bereich der Kausalität nicht-propositionale (= modale) Lesearten durch vier Faktoren begünstigt werden: Neben der syntaktischen Desintegration werden getrennte Akzentuierung der Teilsätze, Definitheit der Ursache und Modalitätsmarker in der Satzumgebung genannt. ,Jeder einzelne dieser Faktoren macht für sich genommen modale Konnektor-Lesarten wahrscheinlicher." Umgekehrt wird die propositionale Lesart unwahrscheinlich, wenn mehrere Faktoren zusammenwirken. 
Im folgenden Satz liegt eine epistemische Verwendung vor (Beispiel zit. n. Breindl et al. 2014: 199, vgl. auch Zifonun et al. 1997: 2296, Pasch et al. 2003: 395):

(18) Weil die Straße nass ist / muss es geregnet haben.

Auch epistemische Konditionale können integrativ angebunden werden (vgl. Breindl et al. 2014: 199). Bei Konditionalsätzen mit Sprechaktbezug ist die Vorfeldstellung ebenfalls möglich:

(19) Wenn ich das so sagen darf, ist er nicht besonders höflich. (zit. n. Pittner 1999: 360)

Andererseits ist die nicht-propositionale Lesart bei der Desintegration nicht zwingend, denn z.B. bei konzessiven Konditionalsätzen, deren Antezedens durch Disjunktion spezifiziert wird (vgl. oben Beleg (9)), ist sogar die nicht-integrative Stellung zwingend. Eine konsequente funktionale Trennung des integrativen bzw. nicht-integrativen Typs für die Markierung der propositionalen bzw. nicht-propositionalen Lesarten liegt also im Gegenwartsdeutschen (noch?) nicht vor. Bei der Beziehung von Stellungstypen und Bezugsebenen könnte höchstens von einer Tendenz die Rede sein, wie aus der folgenden Formulierung hervorgeht:

Die syntaktische Integration ist bei einem Bezug auf die Inhaltsebene am größten, während ein Bezug auf die epistemische oder die Sprechaktebene tendenziell mit einer geringeren syntaktischen und prosodischen Integration einhergeht. (Pittner 2013a: 521)

Bei der Analyse der Desintegration sollte man u.a. auch semantisch-konzeptuelle Aspekte berücksichtigen. Im Folgenden wird auf die Bedingungen, die die Wahl einer Variante ermöglichen bzw. zur Bevorzugung einer Variante führen, eingegangen.

\subsubsection{Bedingungen für die Wahl bzw. die Bevorzugung einer Variante}

Neben der Wählbarkeit der einzelnen Stellungstypen behandeln König/van der Auwera (1988: 109f.) zwei weitere Aspekte der syntaktischen Variation: Die Bedingungen, unter denen die Realisierung einer Stellungsvariante möglich ist und die Präferenz/Häufigkeit der Stellungstypen. Diese Aspekte werden im Folgenden ausführlicher diskutiert und mit Beispielen veranschaulicht. Wenn eine Variante neben einer anderen oder neben den anderen beiden Alternativen möglich ist, wird unterschieden zwischen markierten und unmarkierten Fällen. Es handelt sich nach König/van der Auwera (1988: 109f.) um markierte Strukturen, wenn sie nur unter bestimmten Bedingungen gewählt werden können. Unmarkierte 
Strukturen, die unter diesen Bedingungen ebenfalls möglich sind, können jedoch auch dann vorkommen, wenn die Bedingungen nicht erfüllt sind.

Bei eingeleiteten (kanonischen) Konditionalsätzen, bei denen im Prinzip alle drei Stellungstypen möglich sind, sind nach König/van der Auwera (1988: 117), integrativ` und ,resumptiv` die unmarkierten Stellungen, wobei die integrative Stellung bevorzugt wird. ${ }^{17}$ Die nicht-integrative Stellung ist dagegen bei eingeleiteten Konditionalsätzen markiert und kann nur dann realisiert werden, wenn bestimmte Bedingungen erfüllt sind (s. weiter unten).

Uneingeleitete Verberstsätze wie der folgende Beleg (20) sind König/van der Auwera (1988: 116) zufolge nicht-kanonisch. In diesem Bereich scheint die resumptive Stellung unmarkiert und bevorzugt zu werden, die integrative Stellung ist ebenfalls unmarkiert, aber nicht die bevorzugte Variante. Laut König/van der Auwera (1988: 116f.) ist die nichtintegrative Stellung bei uneingeleiteten (nicht-kanonischen) Konditionalsätzen wie im folgenden Beispiel (vereinfacht zit. n. König/van der Auwera 1988: 118) - im Gegensatz zu eingeleiteten (kanonischen) Konditionalsätzen - unmarkiert:

(20) Wäre ich in Paris, ich würde zum Louvre gehen.

Anhand der obigen Kriterien kann die Wählbarkeit der Stellungstypen bei Konditionalsätzen wie folgt zusammengefasst werden:

\begin{tabular}{|l|l|l|}
\hline & \multicolumn{1}{|c|}{ kanonisch } & \multicolumn{1}{c|}{ nicht-kanonisch } \\
\hline nicht-integrativ & markiert & unmarkiert, nicht bevorzugt \\
\hline resumptiv & unmarkiert, nicht bevorzugt & unmarkiert, bevorzugt \\
\hline integrativ & unmarkiert, bevorzugt & unmarkiert, nicht bevorzugt \\
\hline
\end{tabular}

Abb. 1: Stellungstypen bei Konditionalsätzen nach König/van der Auwera (1988: 117)

So logisch diese Unterscheidung auch ist, zeigt sich bei der Behandlung einzelner Typen, dass die Einordnung in die obigen Kategorien nicht immer eindeutig ist, es ist z.B. nicht klar, ob in eingeleiteten Konditionalsätzen die separate Assertierbarkeit des Konsequens die nichtintegrative Stellung bevorzugt oder einfach ermöglicht. ${ }^{18}$ Zur Beantwortung dieser Frage wären weitere empirische Untersuchungen erforderlich. Auch bei der nicht-integrativen Variante in asyndetischen Verknüpfungen wird die Charakterisierung von König/van der

17 Lötscher (2005: 347) stellt fest, dass ,nach König/van der Auwera (1988: 117) der resumptive Typ die bevorzugte Variante bildet". Sie unterscheiden jedoch kanonische und nicht-kanonische Strukturen. An der angegebenen Stelle behandeln König/van der Auwera (1988: 117) nicht-kanonische Strukturen im konditionalen Bereich, bei denen sich tatsächlich der resumptive Typ als die bevorzugte Variante erweist. Dies darf jedoch nicht verallgemeinert werden, denn bei kanonischen Strukturen im konditionalen Bereich wird der integrative Typ bevorzugt.

18 Vgl. dazu auch den Widerspruch in der Beschreibung von König/van der Auwera (1988: 110) zu einem Satztyp: Ein sprechaktbezogener Konditionalsatz „requires or, at least, strongly favors“ die nicht-integrative Stellung. 
Auwera (1988: 117) kritisiert: Sie ist nach Van den Nest (2010: 105) nicht als „unmarked, non-favoured“, sondern als markiert einzustufen. Nach Van den Nest (2010: 105) gibt es weder bei der Markiertheit noch bei der Präferenz (s. dazu weiter unten) der einzelnen Stellungstypen relevante Unterschiede zwischen kanonischen und nicht-kanonischen Konditionalen.

Ein wichtiges Ergebnis der Forschung ist, dass gezeigt werden konnte, unter welchen Bedingungen bestimmte Konstruktionen bei Konditionalen (König/van der Auwera 1988: 111-117) bzw. bei konzessiven Konditionalen und Konzessiven (König/van der Auwera 1988: 117-127) möglich sind. Der integrative und der resumptive Typ sind grundsätzlich unmarkierte Strukturen im Gegenwartsdeutschen, die nicht-integrative Stellung kann jedoch nur unter besonderen Bedingungen verwendet werden (vgl. auch Lötscher 2005: 347ff., Zifonun 1997: 2350f.).

Eine pragmatische Motiviertheit bei der Wahl des nicht-integrativen Stellungstyps liegt nicht nur bei nicht-propositionsbezogenen Adverbialsätzen (s. 2.1.2), sondern auch bei Konditionalsätzen, konzessiven Konditionalsätzen und Konzessivsätzen vor, die einen Sachverhalt ausdrücken, „der für die Geltung des im Hauptsatz ausgedrückten Sachverhalts weitgehend irrelevant ist“ (Küper 1993: 42). Die nicht-integrative Stellung wird auf die illokutive Selbständigkeit bzw. selbständige Assertierbarkeit des Hauptsatzes zurückgeführt (Küper 1993: 42, König/van der Auwera 1988: 111, 118, 126). Das Prinzip der Ikonizität erklärt auch in diesem Fall die Funktion der Nicht-Integration. Sie sollte den geringen semantischen Zusammenhang zwischen den Teilsätzen widerspiegeln bzw. einer schwächeren syntaktisch-semantischen Integration entsprechen (vgl. auch Zifonun et al. 1997: 2351).

Die sonst restringierte nicht-integrative Stellung des Nebensatzes ist im folgenden Beleg möglich, weil das Antezedens durch einen sog. ,free-choice'-Ausdruck eingeleitet wird (König/van der Auwera 1988: 118). Der Hauptsatz ist - wie auch in sprechaktbezogenen Verwendungen, s. 2.1.2 - selbstständig assertierbar, der Inhalt des Nebensatzes ändert nämlich nichts an der Gültigkeit der Proposition des Hauptsatzes. In diesen, sowie auch in den unten $\mathrm{zu}$ behandelnden Fällen konkurriert er mit den anderen beiden Stellungstypen (König/van der Auwera 1988: 115):

(21) Wie viel er auch verdient, er ist nie zufrieden.

(22) Wie viel er auch verdient, (so) ist er nie zufrieden. 
Auch bei weiteren Typen ist die Gültigkeit der Proposition im Hauptsatz nicht durch das Antezedens eingeschränkt. $\mathrm{Zu}$ diesem Typ gehören die mit $o b$ eingeleiteten Disjunktivkonditionale und Irrelevanzkonditionale mit auch/selbst wenn (vgl. Axel 2002: 3):

(23) Ob du es einsiehst oder nicht, du mußt ins Krankenhaus.

(24) Selbst wenn ich Millionär wäre, einen Porsche würde ich nicht fahren wollen.

Die formalen Spezifika und die Funktionen von Irrelevanzkonditionalen wurden von Leuschner (2005: 286ff.) eingehend untersucht (vgl. auch Waßner 2006, Leuschner 2007).

Der nicht-integrative Typ kommt auch in Konditionalsätzen vor, „bei denen der Hauptsatz anaphorisch auf den Nebensatz Bezug nimmt“" (Zifonun et al. 1997: 2351). Auch in solchen Fällen ist der Hauptsatz selbständig assertierbar (vgl. König/van der Auwera 1988: 113f.).

(25) Wenn du mitkommen willst, ich habe nichts dagegen. (zit. n. König/van der Auwera 1988: 114)

Es stellt sich jedoch die Frage, ob in solchen Fällen überhaupt von Adverbialsätzen die Rede sein kann. Nach Zifonun et al. (1997: 2351) handelt es sich um wenn-Sätze in Komplementfunktion.

Ein weiterer Typ, bei dem Desintegration vorkommt, stellen nicht-kanonische Konzessivsätze mit V1, mit diskontinuierlichem wenn ... auch bzw. so-Konzessiva dar (König/van der Auwera 1988: 125ff.).

(26) Wenn/So sehr die Texte auch Geschmacksache sind, die Melodien sind wunderbar.

Nach König/van der Auwera (1988: 112) kommt der nicht-integrative Typ auch bei faktischen Konditionalen vor. Der nicht-integrative Typ ist schließlich auch bei konjunktivisch formulierten Konditionalgefügen bzw. bei kontrafaktiven Konditionalsätzen (nach Axel 2002: 3) möglich (vgl. Beispiel (8) weiter oben).

Zusammenfassend kann festgestellt werden, dass bei den Bedingungen für die Wahl der nicht-integrativen Stellung der „Grad der semantisch-konzeptuellen Abhängigkeit zwischen den beiden Konjunkten“ (Wegener 2000: 43) relevant ist. Der hohen Abhängigkeit bei echten Bedingungssätzen entspricht ihre Integration, der Nicht-Abhängigkeit bei Konzessiva oder Irrelevanzkonditionalen ihre Desintegration. ${ }^{19}$ Der gemeinsame Nenner für die obigen Verwendungsweisen mit dem nicht-integrativen Stellungstyp scheint die selbständige

19 Bei echten Konditionalen ist der im Nebensatz ausgedrückte Sachverhalt die Voraussetzung für den Sachverhalt im Obersatz, bei Konzessiva ist er aber irrelevant bzw. kann auch im Widerspruch zum Sachverhalt des Obersatzes stehen. In semantisch-konzeptueller Hinsicht besteht also nur bei echten Konditionalen eine semantisch-konzeptuelle Abhängigkeit. (vgl. Wegener 2000: 40ff.) 
Assertierbarkeit des Hauptsatzes zu sein (vgl. König/van der Auwera 1988: 128 bzw. Zifonun et al. 1997: 2351).

Ein weiterer, relevanter Faktor dürfte die Emphasis sein, die unter besonderen Umständen sowohl integrative als auch nicht-integrative Stellung bevorzugen kann (vgl. König/van der Auwera 1988: 124ff., 129, vgl. auch die Beispiele (6)-(8)). In diesen Fällen der Hervorhebung hängen Semantik und Prosodie zusammen. Als Beispiel sei auf vorangestellte obwohl-Sätze verwiesen, die nur dann nicht-integrativ verwendet werden, wenn bestimmte Teile des Obersatzes mit ,assertiver Emphase“ realisiert werden, d.h. sie „Träger eines Gewichungsakzentes werden“"(Zifonun et al. 1997: 2351). Im folgenden Beispielsatz steht im Vorfeld des Obersatzes ein Vollverb mit besonderer Akzentuierung. Die beiden anderen Stellungstypen sind damit blockiert (vgl. auch Lötscher 2005: 348):

(27) Obwohl ich deinen Ärger verstehe, anfahren hättest du mich nicht gleich müssen. (zit. n. Zifonun et al. 1997: 2351, Hervorhebung leicht modifiziert, P.K.)

Abbildung 2 fasst die oben referierten Bedingungen für die Wahl des nicht-integrativen Variante mit einigen Beispielen stichpunktartig zusammen.

\begin{tabular}{|l|l|}
\hline sprechaktbezogener Adverbialsatz & $\begin{array}{l}- \text { metakommunikative Verwendung von Konditionalen } \\
\text { (z.B. Relevanzkonditional), von Konzessiv-, Kausal- } \\
\text { und Finalsätzen }\end{array}$ \\
\hline $\begin{array}{l}\text { selbständig assertierbarer, vom Nebensatz semantisch- } \\
\text { konzeptuell unabhängiger Hauptsatz }\end{array}$ & $\begin{array}{l}\text { - Irrelevanzkonditionale (,free-choice‘-Ausdrücke, mit } \\
\text { ob eingeleitete Disjunktivkonditionale und } \\
\text { Irrelevanzkonditionale mit } \text { auch/selbst wenn) } \\
\text { - Konditionale (konjunktivisch formulierte, } \\
\text { kontrafaktive sowie faktische Konditionalsätze) } \\
\text { - Konzessiva (nicht-kanonische Konzessivsätze mit } \\
\text { V1, mit diskontinuierlichem wenn ... auch bzw. so- } \\
\text { Konzessiva) }\end{array}$ \\
\hline $\begin{array}{l}\text { Loslösung des Nebensatzes durch die Hervorhebung } \\
\text { eines stark akzentuierten Elements im Vorfeld des } \\
\text { Hauptsatzes }\end{array}$ & $\begin{array}{l}\text { - Konditionale und Konzessiva (sowohl kanonische } \\
\text { mit } \text { obwohl } \text { als auch nicht-kanonische), die durch } \\
\text {,assertive Emphase“ vom ihrem Obersatz losgelöst } \\
\text { werden }\end{array}$ \\
\hline
\end{tabular}

Abb. 2: Bedingungen für die Wahl der nicht-integrativen Stellung

Im Folgenden wenden wir uns der Frage zu, welche Variante im Gegenwartsdeutschen bevorzugt wird. Beim Gebrauch der Stellungstypen wurden Häufigkeitsunterschiede nachgewiesen. Im Allgemeinen geht man heute davon aus, dass die integrative Stellung ,im heutigen Deutsch gegenüber den beiden anderen Möglichkeiten als der Normalfall“" gilt (Zifonun et al. 1997: 2349). Viele Korpusauszählungen stärken dieses Bild. In einem Gegenwartskorpus (s. dazu König/van der Auwera 1988: 115) ist etwa bei Konditionalsätzen 
die integrative Stellung gerade dreimal so oft belegt als die resumptive Stellung. ${ }^{20}$ In manchen Korpora ist ein noch größerer Unterschied zu beobachten. Nach der Auszählung von Axel (2002: 9) ist im Bereich der vorangestellten Adverbialsätze der integrative Typ sogar mit 85\% die dominierende Variante. ${ }^{21}$

An dieser Stelle sollte allerdings darauf verwiesen werden, dass solche Häufigkeitsunterschiede von mehreren Faktoren abhängen können. ${ }^{22}$ Kanonische (eingeleitete) Nebensätze verhalten sich anders als nicht-kanonische (uneingeleitete): Der resumptive Typ wird bei uneingeleiteten Konditionalsätzen häufiger realisiert als bei eingeleiteten Konditionalsätzen (König/van der Auwera 1988: 117, Van den Nest 2005: 35, Zifonun et al. 1997: 2350), er ist sogar nach mehreren Korpusauszählungen die bevorzugte Struktur bei V1-Konditionalen im Gegenwartsdeutschen (König/van der Auwera 1988: 115, 117). Im Lichte neuerer empirischer Daten aus geschriebenen Korpora ist allerdings die Charakterisierung der asyndetischen Verknüpfung durch König/van der Auwera (s. Abb. 1) in Frage zu stellen (Van den Nest 2010: 105): ${ }^{23}$ Im Gegensatz zu König/van der Auwera (1988: 117) scheint auch in diesem Bereich die integrative Stellung bevorzugt zu sein (mit 71\%), die resumptive Stellung (28\%) ist ebenfalls unmarkiert, aber nicht die bevorzugte Variante.

Auch bei konzessiven wenn-auch-Sätzen, die ebenfalls als nicht-kanonisch aufgefasst werden (König/van der Auwera 1988: 123), kommen Resumptiva häufiger vor, als bei kanonischen Konzessivsätzen mit obwohl. König/van der Auwera (1988: 117) nehmen daher an, dass kanonische Strukturen auf dem Integrationsprozess weiter fortgeschritten sind als andere Strukturen. Auch strukturelle Faktoren könnten also einen Einfluss auf die Wahl der Stellungstypen haben.

Bei der Wahl der Stellungstypen können des Weiteren auch die ausgedrückten semantischen Bereiche von Bedeutung sein. Bei kausalen Verknüpfungen werden integrativere Strukturen verwendet als etwa bei konditionalen Verknüpfungen - nach dem Motto "Je stärker die semantische Relation, desto enger die syntaktische Zusammengehörigkeit“" (vgl. König/van der Auwera 1988: 128).

20 Es wurden 471 Konditionalsätze untersucht, davon weisen 353 (75\%) die integrative Stellung auf, der resumptive Anschluss ist mit 118 Belegen (25\%) die nicht bevorzugte Variante. Der Stellungstyp ,nicht-integrativ“ ist nicht belegt.

21 Das gegenwartsdeutsche Korpus aus dem Ende des 20. Jhs. umfasst insg. 300 Belege. Die Hälfte entstammt dem Spiegel und der Süddeutschen Zeitung, die andere Hälfte setzt sich aus einem Roman von Christoph Hein und einer Textsammlung von R. Goetz zusammen.

22 Auf den eventuellen Einfluss der Mündlichkeit/Schriftlichkeit soll weiter unten eingegangen werden (s. Kapitel 2.1.4).

23 Von 4800 Belegen aus dem DeReKo konnte die integrative Stellung in 3415 Belegen (71\%) nachgewiesen werden, der Anteil der resumptiven Stellung ist viel niedriger (1338 Belege, 28\%). Das nicht-integrative Muster wird nur in sehr wenigen Fällen verwendet (47 Belege, 1\%). 


\subsubsection{Integration im Spannungsfeld von Mündlichkeit/Schriftlichkeit}

Wie im Kapitel 2.1.1 erwähnt, weisen viele Korpusauszählungen darauf hin, dass im Gegenwartsdeutschen die integrative Stellung den Normalfall darstellt. Wenn es nicht um die standardisierte Schriftsprache geht, scheint aber die Vormachtstellung des integrativen Typs nicht mehr so eindeutig zu sein. Es ist bezeichnend für die einschlägige Literatur, dass fast ohne Ausnahme nur die geschriebene Sprache untersucht wird. ${ }^{24}$ In letzter Zeit wird jedoch bei Korpusuntersuchungen auch der gesprochenen Sprache ein besonderes Interesse geschenkt. Es liegen auch zur Integration der Adverbialsätze Untersuchungen vor, die einen Vergleich von gesprochensprachlichen und schriftsprachlichen Daten ermöglichen. Obwohl diese Untersuchungen nicht alle Adverbialsatztypen systematisch erfassen, können sie uns zu einer relevanten Erkenntnis verhelfen.

Auer (2000) analysiert vorangestellte wenn-Sätze im gesprochenen und geschriebenen Deutsch. Während in seinem schriftsprachlichen Korpus (Die Zeit, Frankfurter Allgemeine Zeitung) in etwa $65 \%$ der Fälle der integrative Typ verwendet wird, kommt im gesprochensprachlichen Korpus aus spontanen Gesprächen der resumptive Typ am häufigsten vor - der integrative Typ weist dabei nur einen Anteil von ca. 25\% auf (Auer 2000: 180). ${ }^{25}$ Die Ergebnisse dieser Analyse deuten also darauf hin, dass die Wahl zwischen den obigen Stellungstypen sehr wohl mit Unterschieden zwischen Mündlichkeit und Schriftlichkeit zu tun haben könnte. Daher wäre das einseitige Bild der bisherigen Forschung durch gezielte Analysen der medialen sowie konzeptionellen Mündlichkeit (im Sinne von Koch/Oesterreicher 1985 bzw. Ágel/Hennig 2006a) zu ergänzen.

Im gesprochenen Deutsch können neben den Graden der syntaktischen Integration auch die Grade der prosodischen Integration bestimmt werden (vgl. Kapitel 3.3.2). Unter hohem Grad der prosodischen Integration versteht Volodina (2011a: 146) „Konnekte[...] mit integrierendem Grenzton, die in einer einzigen Intonationseinheit realisiert sind“. Wenn

24 Eine Berücksichtigung gesprochensprachlicher Daten ist sicherlich für viele Forschende wünschenswert. „,[D]er aktuelle Stand der verfügbaren Korpora der gesprochenen Sprache im Deutschen erlaubt aber zum gegenwärtigen Zeitpunkt keine vergleichbare Untersuchung“" (Breindl/Walter 2009: 37), wie sie für den Bereich der geschriebenen Sprache in mehreren Arbeiten vorliegen. Auch Redder (1987: 324) erfordert in ihrer empirischen Untersuchung weitere Analysen vom gesprochen- sowie schriftsprachlichen Material, daneben u.a. auch sprachhistorische Untersuchungen, die für die systematische Erfassung der Variation zwischen den Korrelaten so und dann bei wenn-Sätzen notwendig wären.

25 Von 237 Belegen für vorangestellte wenn-Sätze aus dem schriftsprachlichen Korpus tritt die integrative Stellung (64,56\%, d.h. 153 Belege) am häufigsten auf, beim resumptiven bzw. nicht-integrativen Typ finden sich deutlich weniger Belege (62 Belege, 26,16\% bzw. 22 Belege, 9,28\%). Bei 280 Belegen für vorangestellte wenn-Sätze aus dem gesprochensprachlichen Korpus erscheint jedoch die resumptive Stellung mit der höchsten Frequenz (47,8\%, d.h. 134 Belege), die integrative Stellung kann nur in 72 Belegen (25,72\%) nachgewiesen werden. Das nicht-integrative Muster wird in 18,48\% der einschlägigen Fälle realisiert. Es soll angemerkt werden, dass bei 9,42\% der Belege der Stellungstyp nicht bestimmt werden konnte (,others“). 
jedoch Konnekte (d.h. die durch den Konnektor verknüpften Teilsätze ${ }^{26}$ mit separierendem Grenzton in zwei oder mehreren Intonationseinheiten realisiert sind, dann handelt es sich um niedrigen Grad der prosodischen Integration. ${ }^{27}$ Die Beziehungen zwischen syntaktischer und prosodischer Integration werden von Volodina (2011a) am Beispiel von kausalen und konditionalen Gefügen untersucht. Aufgrund eines Korpus aus authentischen spontanen Gesprächen (Gerichtsverhandlungen, Studiengespräche, Talkshows, Alltagskommunikation und Gespräche am Kiosk) konnte festgestellt werden, dass bei Konditionalsätzen mit wenn (n=328) der integrative Stellungstyp 19\% und der nicht-integrative Typ 13\% ausmacht, und dass die (Des-)Integration mit den Graden der prosodischen Integration korreliert. ${ }^{28}$ Dem hohem Grad syntaktischer Integration entspricht ein hoher Grad prosodischer Integration und umgekehrt, der niedrige Grad syntaktischer Integration korreliert mit einem niedrigen Grad prosodischer Integration. ${ }^{29}$ Der Zusammenhang zwischen niedrigem Grad syntaktischer sowie prosodischer Integration konnte auch im kausalen Bereich nachgewiesen werden. ${ }^{30}$ Zur Kodierung von Kausalität und Konditionalität in der gesprochenen Sprache liegen weitere Untersuchungen vor, die auch die prosodische Realisierung einbeziehen (vgl. Gohl 2002a, 2006 bzw. Günthner 1999a).

Volodina (2011a: 147ff.) untersucht auch die Frage, inwiefern der Grad der (syntaktischen und prosodischen) Integration mit den kognitiven Ebenen, die von Sweetser (1990) unterschieden werden (Sachverhaltsebene, Sprechakteben, epistemische Ebene, vgl. Kapitel 2.1.2), zusammenhängt. Es lässt sich feststellen, dass sich Strukturen auf der

26 Konnektoren sind ,alle [1] unflektierbaren, [2] nicht kasusregierenden Einheiten, deren [3] Bedeutung eine spezifische zweistellige Relation [4] mit propositionalen Argumenten ist, welche [5] die Form von finiten Sätzen haben können müssen“ (Breindl/Waßner 2006: 48, vgl. auch Pasch et al. 2003: 1-6). Subjunktoren leiten Verbletztsätze ein. Die beiden finiten Sätze, die mit dem Subjunktor verknüpft werden, nennen Breindl/Waßner (2006) in Anlehnung an Pasch et al. (2003) Konnekte: das interne Konnekt ist der vom Subjunktor eingeleitete Satz, das externe Konnekt ist der syntaktisch übergeordnete Matrixsatz, vgl. z.B. (i):

(i) Weil $\quad$ es regnen soll $\}$, \{bleiben wir zu Hause $\}$.

Subjunktor internes Konnekt externes Konnekt

27 Prosodische Desintegration kann in der geschriebenen Sprache über eine Interpunktion mit Satzschlusszeichen transportiert werden (Breindl/Walter 2009: 56). Bei Texten, die vor der Normierung der Rechtschreibung entstanden sind, ist jedoch dieses Kriterium nicht ohne Weiteres anwendbar. Daher können Grade der prosodischen Integration in der Untersuchung neuhochdeutscher Texte nicht berücksichtigt werden.

28 Der Anteil des resumptiven Typs lässt sich nicht genau ermitteln, da Volodina (2011a: 146) unter dem mittleren Grad syntaktischer Integration auch parenthetisch eingeschobene und nachgestellte wenn-Sätze erfasst. Der folgende Verweis lässt jedoch vermuten, dass der Anteil der parenthetisch eingeschobener und nachgestellter wenn-Sätze verhältnismäßig gering sein könnte: „Am häufigsten werden Strukturen nach dem für die gesprochene Sprache typischen wenn-dannMuster gebildet.“(Volodina 2011a: 146)

29 Beim integrativen Typ liegen demnach 62 Belege vor, alle mit hohem Grad prosodischer Integration. Beim nichtintegrativen Typ weisen 40 von 43 Belegen einen niedrigen Grad prosodischer Integration auf. Die absoluten Zahlen wurden aufgrund der Prozentzahlen bei Volodina (2011a: 146) errechnet.

30 Da im kausalen Bereich nicht nur Subordination mit den Konnektoren weil und $d a$, sondern auch koordinierende Verknüpfungen mit denn, nämlich bzw. weil mit Verbzweit erfasst werden, soll auf die weitere Analyse der Daten verzichtet werden. Es soll jedoch angemerkt werden, dass Volodina (2011a: 146) eine Korrelation ,zwischen den Graden syntaktischer und prosodischer Integration und dem Ausdruck von Kausalität und Konditionalität“ feststellt: Bei konditionalen Relationen ist der Grad syntaktischer und prosodischer Integration insgesamt höher als bei den kausalen Relationen. 
Sachverhaltsebene anders verhalten als Strukturen auf der Sprechaktebene. Am häufigsten werden Strukturen mit einem hohen und mittleren Grad syntaktischer Integration verwendet, wenn auf der Sachverhaltsebene argumentiert wird. In den meisten Fällen liegt dann ein hoher Grad prosodischer Integration vor. Auf der Sprechaktebene finden sich jedoch keine Strukturen mit einem hohen Grad syntaktischer oder prosodischer Integration. Sie werden typischerweise mit einem niedrigen oder mittleren Grad syntaktischer Integration (und mit einem niedrigen Grad prosodischer Integration) realisiert. Bei der dritten kognitiven Ebene erlauben die Daten keine eindeutige Interpretation solcher Zusammenhänge: „Während sich im Falle kausaler Relationen die epistemische Ebene genau so verhält wie die Sprechaktebene, verhält sie sich im Falle konditionaler Relationen ähnlich wie die Sachverhaltsebene.“ (Volodina 2011a: 149) Volodina (2006) geht auch der Frage nach, ob sich semantische Untertypen von wenn-Sätzen bezüglich der Integriertheit voneinander unterscheiden. Das Vorhandensein des nicht-integrativen Typs lässt sich zwar nicht auf die Oppositionspaare ,faktisch“ vs. ,nicht-faktisch ${ }^{\star}$ bzw. ,generisch“ vs. ,spezifisch“ zurückführen, das wenn-Konnekt in temporaler Lesart (faktisch, generisch) und in temporalkonditionaler Lesart (nicht-faktisch, spezifisch) kommen jedoch im Korpus nicht desintegriert vor, während der nicht-integrative Stellungstyp bei den anderen semantischen Unterarten mit einem Anteil von 10 bis 19\% der einschlägigen wenn-Konnekte (inkl. Postposition und Parenthese) belegt ist. Das relativ häufige Vorkommen (19\%) von desintegrierten wennKonnekten in rein-konditionaler Lesart (nicht-faktisch, spezifisch) führt Volodina (2006: 371) darauf zurück, dass „ein enger Zusammenhang zwischen der Spezifizität des vom wennKonnekt bezeichneten Sachverhalts [...] und der kommunikativen Situation [besteht], die eine - syntaktisch gesehen - ,lockerere' Konstruktion erlaubt.“

\subsection{Zur Erforschung der historischen Entwicklung von Adverbialsätzen}

Die Integration der Nebensätze in den nachfolgenden Hauptsatz ist ein sich über mehrere Sprachepochen hinziehender Prozess. Wie es zu zeigen sein wird, betrifft sie mehrere Bereiche, von denen in letzter Zeit die topologische Einbettung der Nebensätze im Mittelpunkt stand. Da sich jedoch aus weiteren Bereichen der historischen Syntax komplexer Sätze wichtige Erkenntnisse für die Untersuchung der Integration von Adverbialsätzen ergeben, soll zunächst auf die Entwicklung der formalen Markierung von Nebensätzen kurz eingegangen werden (Kapitel 2.2.1). Anschließend soll ein Überblick über die historischen Veränderungen bezüglich der topologischen Integration der Nebensätze gegeben werden. Da 
der syntaktische Wandel i.d.R. langfristig ist, empfiehlt es sich, einen Blick auf ältere Zeitstufen zu werfen bzw. die jüngeren Veränderungen in einem größeren Rahmen zu verorten. Es dürfte hilfreich sein, den Bogen der einschlägigen Entwicklungen zumindest ansatzweise anzudeuten.

\subsubsection{Zur formalen Markierung von Nebensätzen in der Sprachgeschichte}

Nach der geläufigen Lehrmeinung entwickelt sich die Unterordnung aus der Nebenordnung (s. dazu bereits Biener 1920/22: 165). ${ }^{31}$ Das bedeutet nicht nur, dass unterordnende Techniken ursprünglich jünger sind, sondern auch, dass verschiedene Satzverknüpfungstechniken ineinander übergehen können. Dies wiederum deutet darauf hin, dass bei der Untersuchung der Satzverknüpfungstechniken nur ein theoretischer Rahmen angemessen sein kann, der im Gegensatz zu einer starren Dichotomie auch mit fließenden Übergängen umzugehen weiß. Die Frage, seit wann es überhaupt subordinierte Sätze gibt, wird zwar in der Fachliteratur unterschiedlich beantwortet (vgl. Axel 2002: 16), die untergeordneten Sätze in früheren Stadien der Sprachgeschichte waren aber bestimmt noch keine Nebensätze im heutigen Sinne, weder was ihre (Un-)Selbständigkeit (vgl. Fleischmann 1973: 316), noch was ihre formale Markierung betrifft. ${ }^{32}$ Erst seit dem Mittelhochdeutschen ist eine Tendenz zur strukturellen Trennung von Haupt- und Nebensatz zu beobachten. ${ }^{33}$ Als kanonische Marker der Subordination im heutigen Deutsch gelten die Verbendstellung im Nebensatz bzw. subordinierende Konnektoren. In der einschlägigen Forschung wurde der Entwicklung der Nebensatzwortstellung und den Veränderungen im Bestand der Konnektoren besonderes Interesse gewidmet (vgl. z.B. Eichinger 1995 bzw. Fleischmann 1973). Auf diese beiden Bereiche soll im Folgenden kurz eingegangen werden.

31 Das bekannteste Beispiel hierfür ist wohl die Entwicklung der Hypotaxe nach dass: Die unterordnende Konjunktion war nach der geläufigen Lehrmeinung ursprünglich ein ,auf den folgenden satz hinweisendes demonstrativum“ im ersten Satz (Biener 1920/22: 173), erst später wurde es durch Reanalyse der Struktur zum Bestandteil des zweiten Satzes. Der hypotaktische Satz entwickelte sich demnach aus dem parataktischen Satz, z.B. ich sagte das: er kommt > ich sagte, dass er kommt (vgl. Paul 1920: 241, Dorchenas 2005: 9; s. auch Axel 2009). Axel-Tober (2012: 366) kommt jedoch zum Schluss, dass die Hypothese, dass sich hypotaktische Konstruktionen aus parataktischen entwickelt haben, nicht haltbar ist.

32 Im Althochdeutschen gilt die Späterstellung des Finitums als formales Merkmal von eingeleiteten Nebensätzen. Die Verbendstellung hängt von der Zahl der Satzglieder im Nebensatz ab. Das System der Konnektoren war noch nicht zur Unterscheidung der Neben- bzw. Unterordnung funktionalisiert. Zur Verbstellung im Nebensatz in althochdeutscher Zeit sowie zu den Konnektoren s. Schrodt (2004: 206ff. bzw. 143ff.).

33 Nach Admoni (1980: 348),,wird der Nebensatz in der Zeit um 1700 im Ganzen sehr konsequent dem Nicht-Nebensatz gegenübergestellt“. Ágel (2000: 1880f.) argumentiert jedoch dafür, dass diese Tendenz bis heute nicht abgeschlossen ist und verweist sogar auf gegenläufige Tendenzen. 


\subsubsection{Wortstellung im Nebensatz}

Die formale Markierung der subordinierten Sätze durch besondere Wortstellung gehört zu den intensiv erforschten Bereichen der neueren Sprachgeschichte. Die formale Trennung von Hauptsatz und Nebensatz setzt die Festigung von unterschiedlichen Klammerkonstruktionen (Hauptsatz- und Nebensatzklammer) voraus. Bereits im Frühneuhochdeutschen ist eine Zunahme der Klammerbildung zu beobachten (vgl. Erben 2000: 1588, mit weiterführender Literatur), doch der Höhepunkt wurde erst im 17./18. Jh. erreicht (von Polenz 1994: 268; Ágel 2000: 1874). ${ }^{34}$ Demnach dürfte eigentlich der Terminus ,Satzklammer' nicht auf frühere Sprachperioden angewandt werden (vgl. auch Ágel 2000: 1874, Elspaß 2005: 206, s. auch Kapitel 3.2.3). ${ }^{35}$ In der Forschung wurde auch nach den Gründen bzw. den Quellen dieser syntaktischen Entwicklungen gesucht. Nach dem heutigen Forschungsstand lässt sich etwa feststellen, dass das Klammerprinzip zwar ihren Ursprung in der Mündlichkeit hat, die konsequente Dehnung der Satzklammer und der Ausbau des Mittelfeldes jedoch auf die Schriftlichkeit zurückzuführen sind (vgl. Nübling 2013: 100). Es wurden auch Schlüsse auf regionale und Textsortenunterschiede gezogen: Bei der Stärkung des Klammerprinzips spielten das Ostmitteldeutsche und das Ostoberdeutsche bzw. die Fachprosa eine Vorreiterrolle (Schildt 1976; Ágel 2000: 1874). In einer Untersuchung von Briefen aus Nürnberg aus dem Zeitraum 1300-1600 konnte Ebert (1980) nachweisen, dass die vollständige Rahmenbildung mit dem Bildungsgrad der Schreiber zusammenhängt. Diese Ergebnisse zeigen m.E. eindrucksvoll, wie wichtig die soziopragmatisch sensible (d.h. außersprachliche Faktoren berücksichtigende) Erfassung von syntaktischen Entwicklungen ist.

Die Entstehung der Endstellung im Nebensatz wurde in der Forschungsliteratur unterschiedlich interpretiert. Einige Auffassungen, wie etwa die These vom lateinischen Einfluss (so noch Maurer 1926 bzw. Behaghel 1932: XIII), gelten heute als überholt (vgl. z.B.

34 Im 17. Jh. ist die Distanzstellung der grammatisch zusammengehörenden Prädikatsteile im Hauptsatz bereits grammatikalisiert, zu dieser Zeit ist jedoch noch nicht von einer ,Klammerung“ im Sinne von Eichinger (1995: 304) zu sprechen. Dies würde einen „spezifisch strukturierten ,Inhalt““, ein Mittelfeld mit geregelter Abfolge seiner Elemente voraussetzen. Die Grammatikalisierung der Satzklammer setzt nach Eichinger (1995) erst zu Beginn des 18. Jhs. ein. Zu dieser Zeit sind neben voll ausgebildeten Klammern auch partiell ausgebildete Klammern zu finden. In einer Untersuchung von Prosatexten aus dem Zeitraum 1670-1730 stellt Schildt ein Verhältnis von ca. 4:1 zugunsten der vollständigen Klammerung fest, fehlende Klammerung stellt jedoch mit $0,8 \%$ bereits die Ausnahme dar (vgl. Schildt 1976: 271). Zum Forschungsstand und zu methodologischen Überlegungen der Erforschung der Satzklammer s. Ágel (2000: 1873-1876).

35 Ob der Begriff, Satzklammer` auf das frühe Neuhochdeutsche angewendet werden kann, hängt davon ab, ob wir vom Begriffsverständnis von Eichinger (1995: 304) ausgehen und somit ein Mittelfeld mit geregelter Abfolge seiner Elemente als Kriterium voraussetzen. In Bezug auf sprachhistorische Untersuchungen im Allgemeinen zieht Elspaß (2005: 206) den Schluss, dass die Anwendung des Stellungsfeldermodells auf Sprachstufen vor der Ausbildung der Satzklammer problematisch ist. 
Betten 1987: 131ff.). Andere werden z.T. kontrovers diskutiert. Die Endstellung im Nebensatz diente nach der Relieftheorie von Fleischmann (1973) zunächst zur Kennzeichnung eines Hintergrundgeschehens (im Gegensatz zu einem Vordergrundsachverhalt). Diese Fähigkeit wurde später auf einzelne Partikeln (= Konnektoren) übertragen, sodass am Ende eine feste Fügung von satzeinleitender Partikel und Endstellung entstand: Die beiden Marker sind heute gekoppelt, d.h., Endstellung ist nur noch bei bestimmten Einleitewörtern möglich. ${ }^{36}$ Die Endstellung des Finitums wurde auch zum Signal der logischen Unterordnung. In einer Übergangsphase vom Mittelhochdeutschen zum Neuhochdeutschen vollzieht sich „die Eingliederung des Endstellungssatzes in den Zweitstellungssatz: Der Endstellungssatz wird von nun an im allgemeinen als Glied des folgenden Zweitstellungssatzes betrachtet“ (Fleischmann 1973: 318). Damit wird m.E. ein wesentlicher Bereich der Integration vorangestellter Nebensätze erfasst. Es sollte also überlegt werden, ob vor dieser Stufe überhaupt von ,Nebensätzen' zu sprechen ist.

Die Nebensatzklammer (bzw. ,Adjunktklammer' nach der Terminologie von Weinrich 2005: 56f.) scheint seit dem 18. Jh. fest zu sein (Nübling 2013: 100). In der Wortstellung sind jedoch bis heute noch weitere syntaktische Veränderungen $\mathrm{zu}$ beobachten. Diesbezüglich wurde u.a. die Stellung des Finitums im mehrgliedrigen Nebensatzprädikat untersucht (Härd 1981): Dabei ist ein Vordringen seiner Nachstellung zu beobachten, bei zweigliedrigen Nebensatzprädikaten bereits im 16., bei dreigliedrigen Nebensatzprädikaten seit dem 17. Jh. Die letzte Position des Finitums im mehrgliedrigen Nebensatzprädikat konnte allerdings bis heute keine feste Regel werden, vgl. Nebensätze mit vorangestelltem Finitum (wie dass sie es hätte machen müssen) im Gegenwartsdeutschen. Gleichzeitig ist bei der Stellung der infiniten Teile im Nebensatzprädikat das neuhochdeutsche Vordringen des Prinzips, rechtsdeterminiert-links' zu beobachten.

\subsubsection{Konnektoren}

Im Bereich der Satzkonnektoren sieht Betten (1987: 99f.) wichtige Veränderungen vom Mittelhochdeutschen bis zum Neuhochdeutschen und stellt diese Veränderungen mit der Verschriftlichung in Zusammenhang:

Die Tendenz zu einer eindeutigen, mehr logisch-kausal als räumlich- bzw. zeitlich-deiktisch ausgerichteten Ausdrucksweise läuft parallel mit der Entfaltung differenzierter schriftsprachlicher Kommunikationsformen gegenüber nur oder vorwiegend mündlichen: und zwar als notwendige Konsequenz dieser grundlegend veränderten medialen Verwendung von Sprache. (Betten 1987: 100)

\footnotetext{
36 Zur „Verbendstellung im Hauptsatz in der deutschen Prosa des 15. und 16. Jahrhunderts“ s. Lötscher (2000).
} 
Es ist eine Reduktion der Polyfunktionalität der einzelnen Subjunktionen zu verzeichnen, die zu einer klaren Trennung der $d / s$ - bzw. w-Konnektoren führt (vgl. auch Fleischmann 1973: 319). Die $d / s$-Konnektoren werden auf die adverbiale Funktion festgelegt. Dies bedeutet, dass mit ihnen nur ausnahmsweise Nebensätze eingeleitet werden (vgl. $d a$ ). Im modernen Deutsch treten aber kaum noch Partikeln auf, die sowohl subjunktional als auch adverbial verwendet werden. ${ }^{37}$ Der subjunktionale Bereich wird mit $w$-Konnektoren abgedeckt. Eine weitere, wichtige Entwicklung, die natürlich mit dem obigen Phänomen zusammenhängt, ist die Entwicklung neuer Konnektoren (wie etwa die Entstehung von obwohl durch Univerbierung, vgl. Nübling 2013: 283f.). In der Forschungsliteratur werden auch Veränderungen der Konnektoren im Bereich der Adverbialsätze beschrieben (vgl. z.B. Ágel 2000 mit weiterführender Literatur). Diese betreffen zum einen den Konkurrenzkampf einzelner Konnektoren (wie etwa das Vordringen von damit im Finalsatz bzw. das Vordringen von obwohl im Konzessivsatz), zum anderen auch strukturelle Entwicklungen (das Vordringen von Infinitivkonstruktionen im Finalsatz sowie die Zunahme der eingeleiteten Nebensätze im konzessiven Bereich). Die oben angedeuteten Veränderungen führen dazu, dass die Arten der Verknüpfungen zwischen Teilsätzen präziser ausgedrückt werden. Dies steht offenbar wiederum mit der Ausbreitung der Schriftlichkeit bzw. mit den Bedingungen schriftlicher Kommunikation im Zusammenhang. Nach der Vertikalisierungstheorie von Reichmann (2003: 47, s. dazu ausführlicher Kapitel 3.5.2) kann davon ausgegangen werden, dass die zunehmende Orientierung an einer Leitvarietät ab dem 16. Jh. eine Umschichtung des Varietätenspektrums nach sich zieht. Wie Ágel (2012: 201ff.) durch einen Vergleich der Junktionsprofile von neuhochdeutschen Nähe- und Distanztexten zeigen konnte, ist die Vertikalisierung auch im Bereich der Satzverknüpfungstechniken festzumachen: Die tendenziell aggregativen Nähetexte passen sich strukturell den tendenziell integrativen Distanztexten an.

Soweit der knappe Überblick über die wichtigsten Aspekte für die formale Markierung von untergeordneten Sätzen. Eine weitere, wichtige Entwicklung in der Geschichte der Nebensätze betrifft die Stellungsmöglichkeiten der Nebensätze, auf die ich mich im folgenden Kapitel (2.2.2) konzentrieren möchte.

37 Bei $d a$ ist ein semantischer Unterschied zu registrieren: Im adverbialen Bereich hat es eine lokale, im subjunktionalen Bereich eine kausale Bedeutung. 
2.2.2 Zur topologischen Integration von Adverbialsätzen bis zum Gegenwartsdeutschen

Was die Breite des bisher bearbeiteten Materials betrifft, so lässt sich - wie auch in der gegenwartsbezogenen Forschung, vgl. 2.1 - feststellen, dass die einschlägigen Arbeiten oft nur auf jeweils kleinere Bereiche beschränkt sind. Baschewa (1983) und Lühr (2004) wenden sich z.B. den Konzessivsätzen zu, Flämig (1964) und Babenko (1988) untersuchen Finalsätze, Lühr (2010) und Kotin (2013) Konditionalsätze. Des Weiteren soll auch festgehalten werden, dass nicht alle Arbeiten über Adverbialsätze bzw. einzelne Adverbialsatztypen auf die topologische Integration eingehen. Diese Frage wird z.B. bei Babenko (1988) gar nicht thematisiert. $^{38}$

Das Mittel- und Frühneuhochdeutsche wurden bezüglich der historischen Entwicklung der Adverbialsätze gründlich untersucht und auch zum Althochdeutschen liegen eine Reihe einschlägiger Arbeiten vor, ein Überblick findet sich bei Axel (2002). Sie untersucht die frühere Entwicklung der Adverbialsätze seit dem Althochdeutschen und liefert dazu quantitative Daten aus der Sekundärliteratur, die in drei Fällen (bei dem mittelhochdeutschen >Prosa Lancelot I<, dem frühneuhochdeutschen >Prosa Lancelot II und einem Gegenwartsdeutsch-Korpus) mit stichprobenartigen Untersuchungen ergänzt wurden. Zum Integrationsgrad in der neuhochdeutschen Epoche liegen im Gegensatz zu den früheren Sprachstufen nur vereinzelte diachrone Untersuchungen vor. Ein Beispiel dafür stellt die Untersuchung von Baschewa (1983) dar, die einerseits zeitlich auf zwei synchrone Schnitte aus der Zeit zwischen 1700-1830 bzw. dem Gegenwartsdeutschen ab 1900, andererseits thematisch auf den konzessiven bzw. konzessiv-konditionalen Bereich beschränkt ist. Die quantitative Untersuchung der $d a$-Sätze von Axel-Tober (2012, s. weiter unten) erfasst nur das frühe Neuhochdeutsch zwischen 1650-1700. Wiktorowitz (2013) untersucht ebenfalls Adverbialsätze aus dem 17. Jh. Aus den Mosaiksteinen, die zur Verfügung stehen, lässt sich der Integrationsprozess in der neuhochdeutschen Epoche nicht rekonstruieren.

Die topologische Einbettung von Nebensätzen, die zunehmend ihren Hauptsätzen vorausgehen können (vgl. Behaghel 1932: 263f.), läuft nach der diachronen Hypothese von König/van der Auwera (1988: 127) in drei Stufen ab. Die oben (Kapitel 1.1) eingeführten drei Typen (,integrativ‘, ,resumptiv ${ }^{\star}$ und ,nicht-integrativ') werden auf die diachrone Achse projiziert und als Stufen der Entwicklung betrachtet: „subordinate clauses have tended to develop from non-integration to integration via resumption" (König/van der Auwera 1988:

\footnotetext{
38 Dass Babenko (1988) in ihrer Arbeit über Finalsätze im 16. und 17. Jh. nicht auf die topologische Integration von vorangestellten Finalsätzen, die ja nur selten vorangestellt vorkommen (1988: 108), eingeht, ist nicht als Kritik zu verstehen.
} 
127). Diese These von einem Prozess der Satzintegration im Deutschen, der vom ,nichtintegrativ‘ über ,resumptiv‘, bis hin zum ,integrativ' führt, wurde übrigens auch in einigen älteren Arbeiten (z.B. Hammarström 1923: 49-55) explizit genannt.

\subsubsection{Althochdeutsch und Mittelhochdeutsch}

Die Beschäftigung mit der Geschichte der Integration vorangestellter Adverbialsätze begann bereits am Ende des 19. Jhs. Starker (1883) untersuchte „Die Wortstellung der Nachsätze in den ahd. Übersetzungen“. Dabei wurden Sätze, die vom lateinischen Original abweichen, hervorgehoben. Nach Starkers Auffassung wurde die eher noch „parataktische Anfügung“ (d.h. kein Einfluss des Vordersatzes auf die Wortstellung des Nachsatzes, nach der Terminologie von König/van der Auwera (1988) ,nicht-integrativ`) durch das Auftreten von „anaphorischen“ Pronomen oder Partikeln im Nachsatz (= ,resumptiv“) verdrängt. Die sog. Anfangstellung des Verbs im Nachsatz (=, integrativ') entwickelte sich wiederum über ein Übergangsstadium der resumptiven Wiederaufnahme des Vordersatzes. ${ }^{39}$ Diese Annahme von der Abfolge der Stellungstypen ,nicht-integrativ ${ }^{\star}>$,resumptiv ${ }^{\star}>$, integrativ ${ }^{\star}$, die nach gut 100 Jahren bei König/van der Auwera (1988: 127) auftritt, wurde von Behaghel (1929: 404) heftig kritisiert. ${ }^{40}$

Nach Behaghel (1929: 402ff.) existieren die drei Stellungstypen bereits im Germanischen nebeneinander: Er führt entsprechende altnordische, angelsächsische, althochdeutsche Belege für den resumptiven Anschluss bzw. gotische, altnordische, altenglische und althochdeutsche Belege für die Spitzenstellung des Verbs im Nachsatz (=,integrativ $\left.{ }^{\natural}\right)$ auf und zieht den Schluss, dass es keinen Anlass gibt, ,eine der drei fügeweisen im germ. für älter zu halten als die andern.“ Nach seinen Auszählungen kommt in den meisten gesichteten althochdeutschen Texten sogar der resumptive Typ am häufigsten vor. Die einzige Ausnahme bildet >Isidor (um 790/800), mit einem ebenfalls bedeutenden Anteil des resumptiven Typs (29\%) ${ }^{41}$.

39 Der Entwicklungsprozess mit den drei Stufen wird wie folgt beschrieben: „Allmählich machte sich das Streben geltend, den Nachsatz in engere Beziehung zu setzen zum hypot.[aktischen, P.K.] Vordersatze; dasselbe äußerte sich zunächst in der Einschiebung eines anaphorischen Pronomens oder solcher Partikel in den Nachsatz (dieselbe entspricht meistens derjenigen, welche den Vordersatz einleitet). [...] War der Gebrauch der anaph. Partikel am Anfange des Nachsatzes erst einmal Gesetz geworden, so mußte, da jene den Vordersatz in einem einzigen Worte zusammenfaßte, unwillkürlich das Gefühl immer lebendiger werden, daß der Vordersatz gleichstehe einem an die Spitze gestellten Satzteile; einem solchen aber hatte sich nach dem bereits erwähnten Gesetze das Verbum unmittelbar anzuschließen. Die zunächst regelmäßig angewendete anaph. Partikel konnte nunmehr entbehrlich erscheinen [...] erst allmählich wurde sie $[=$ die Anfangsstellung des Verbs im Nachsatz, P.K.] häufiger, bis sie schließlich als gleichberechtigt neben die Anfügung vermittelst anaph. Partikel trat. Je mehr aber diese Stellung des Verbs dem Nachsatze eigentümlich wurde, um so mehr mußte dieselbe in den gewöhnlichen Aussagesätzen außer Gebrauch kommen“" (Starker 1883: 15f.).

40 „Der versuch von Starker, die hauptsatzstellung als älter zu erweisen, [...] ist, schon vom ahd. aus betrachtet gänzlich mißlungen." (Behaghel 1929: 404).

41 Die Prozentzahl wurde nach den absoluten Zahlen bei Behaghel (1929: 405) errechnet. 
Die Stellungstypen im Althochdeutschen wurden auch in jüngerer Zeit gründlich untersucht (Axel 2002: 16-27, Axel 2004: 26-37, Lötscher 2005: 358-361, zu thō-Sätzen s. auch Axel-Tober 2012: 273-301). Sowohl die Auswertung früherer Untersuchungen ${ }^{42}$ (Axel 2002: 24, 27f., Lötschers 2005: 364) als auch Lötschers (2005: 359) eigene Auszählungen zeigen eindeutig, dass der resumptive Typ - im Gegensatz zu einer Annahme seines relativ späten Vorkommens (s. oben) - bereits mit einem verhältnismäßig hohen, den nichtintegrativen Typ sogar weit übertreffenden Anteil belegt ist. ${ }^{43}$ In Notkers >Martianus Capella und Notkers >De Consolatione` wird der resumptive Typ bei über $85 \%$ der , linksperipheren Adverbialsätze' realisiert (vgl. auch ähnliche quantitative Daten bei Behaghel 1929: 405). Der nicht-integrative Typ überwiegt nur im `Isidor`. Darin ist jedoch die Anzahl der einschlägigen vorangestellten Adverbialsätze im Vergleich zu den anderen Quellentexten sehr niedrig, außerdem ist - im Einklang zu der oben erwähnten Auszählung Behaghels - auch ein bedeutender Anteil des resumptiven Typs zu finden (vgl. Axel 2002: 24 sowie Lötscher 2005: 359). Axel stimmt dementsprechend Behaghel zu: Es gibt keinen Anlass zur Annahme, dass ,nicht-integrativ` historisch gesehen der originelle Stellungstyp ist (vgl. auch Van den Nest 2010: 122). „Es gibt keine Evidenz dafür, dass es ein Stadium gab, in dem nur die nichtintegrative Stellung [...] möglich war oder zumindest deutlich dominierte“. (Axel-Tober 2012: 357) Die Auffassung des Resumptivums als Übergangsstufe scheint daher fragwürdig zu sein.

Axel führt auch althochdeutsche Adverbialsätze mit dem Muster AS- $\mathrm{V}_{\text {fin }}$ (also mit unmittelbar anschließendem Hauptsatzfinitum, nach der Terminologie von König/van der Auwera (1988: 127) ,integrativ‘, vgl. Kapitel 3.1.3) auf, sie analysiert diese Belege jedoch nicht als eingebettete Nebensätze (2002: 22ff.). Sie argumentiert dafür, dass in solchen Fällen eine sog. V1-Apodosis vorliegt, sodass der nachfolgende Hauptsatz als V1-Deklarativsatz zu interpretieren ist. Nach dieser Auffassung wären vorangestellte Adverbialsätze im Althochdeutschen noch nicht vorfeldfähig. ${ }^{44}$ Schrodt (2004: 212) weist jedoch darauf hin, dass auch Beispiele $\mathrm{zu}$ finden sind, in denen die V1-Apodosis-Interpretation nicht

42 Zur Sekundärliteratur und den dort herangezogenen Quellen vgl. die Angaben in den Arbeiten von Axel (2002) und Lötscher (2005). Axel (2002: 10, Fußnote 8) bemerkt, dass einigen Untersuchungen die entsprechenden quantitativen Daten nicht problemlos zu entnehmen sind. Da Entwicklungen bis zum Neuhochdeutschen nicht den Gegenstand meiner empirischen Untersuchung bilden, können wir eventuelle Ungenauigkeiten - soweit sie die skizzierten Tendenzen nicht betreffen - in Kauf nehmen.

43 Bei der Auszählung von Axel (2002) wurden drei althochdeutsche Korpora mit insg. 148 Belegen herangezogen: >Isidor (ca. 790/800), >Tatian` (ca. 830) und >Martianus Capella< (ca. 1000). Bei Lötscher wurden insg. 285 Belege den drei einschlägigen Stellungstypen zugeordnet und zwar aus den folgenden Texten: aus der Isidorübersetzung, aus Otfrids >Evangelienharmonie und Notkers 〉De Consolatione〈. Lötscher (2005: 259) unterscheidet dabei auch insg. 11 Belege für die Hauptsätze mit Verbletztstellung, die dem Vordersatz folgen. Diese Belege werden hier nicht mitgerechnet (vgl. auch Axel 2002: 9, Fußnote 9).

44 Die Frage, auf welche Zeitepochen das Vorfeldkonzept übertragen werden kann, wird im Kapitel 3.2.3 aufgegriffen. 
nachvollziehbar ist. Lötscher (2005: 360f.) stellt die Anwendbarkeit der oben (Kapitel 1.1) eingeführten drei Stellungstypen grundsätzlich in Frage, da sie „die V2-Felderstruktur mit Vorfeld voraussetzen, die sich erst relativ spät herausgebildet hat". Demnach dürfe die Voranstellung des Nebensatzes direkt vor dem Hauptsatzfinitum im früheren Althochdeutschen nicht als integrative Stellung analysiert werden. Lötscher stimmt Axel zu, indem er in solchen Fällen von einer syntaktisch lockeren Anfügung mit Verberststellung im Nachsatz ausgeht (2005: 360f.). ${ }^{45}$ Nach dieser Überlegung bzw. auch im Lichte der empirischen Daten zum Althochdeutschen ist die Auffassung der Abfolge Nicht-Integration > Resumption > Integration zu verwerfen.

Die Stellungstypen im Mittelhochdeutschen wurden ebenfalls ausführlich untersucht (zum Überblick vgl. Axel 2004: 37-39). Die folgende Abbildung, der die Daten in Axel (2002: 9) und Lötscher (2005: 364) zugrunde liegen, soll zu einem groben Bild über die Verteilung der Stellungstypen in mittelhochdeutschen Prosatexten verhelfen. ${ }^{46}$

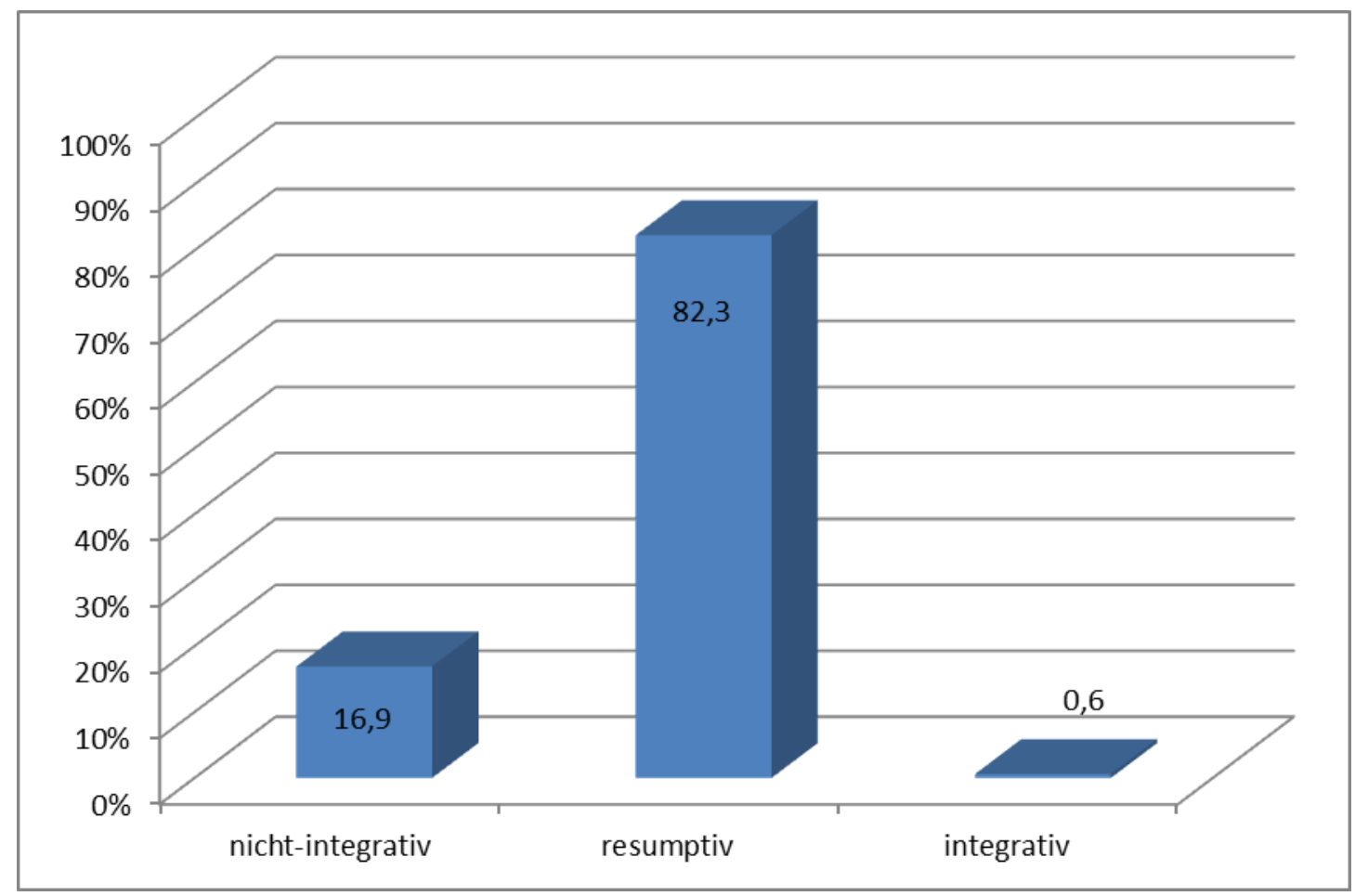

Abb. 3: Integrationsgrad vorangestellter Adverbialsätze in mittelhochdeutschen Prosatexten (n=1247) (vgl. Axel 2002: 9, Lötscher 2005: 364)

45 Das Fehlen des AS-V $\mathrm{V}_{\text {fin }}$-Musters im Mittelhochdeutschen erklärt sich mit dem Rückgang der V1-Deklarativsätze während der mittelhochdeutschen Periode.

46 In Axel (2002) wurden sechs mittelhochdeutsche Korpora mit insg. 1520 Belegen untersucht, aus denen allerdings in der Abbildung 3 nur die Prosatexte >Bertholds Predigten $(1240-1272)$ und der ältere Teil des Prosaromans $>$ Lancelot $>$ (um 1250) berücksichtigt werden (237 bzw. 279 Belege). Bei Lötscher wurden 15 Prosatexte mit insg. 731 Belegen herangezogen. 
Im Mittelhochdeutschen ist - bis auf sehr wenige Ausnahmen - keine integrative Anbindung vorangestellter Adverbialsätze zu finden (Behaghel 1929: 405, Axel 2002: 5): In den herangezogenen Prosatexten kommen lediglich acht von ca. 1250 Belegen mit integrativer Stellung vor (0,6\%). Lötscher (2005: 363f.) geht davon aus, dass bei der Verteilung der beiden anderen Stellungstypen Textsortenunterschiede und stilistische Aspekte eine Rolle spielen. Dies kann durch den Vergleich der Stichproben aus Versdichtung und Prosatexten bestätigt werden: In der Versdichtung kommt der nicht-integrative Typ viel häufiger vor als der resumptive Typ (786 vs. 412 Belege). ${ }^{47}$ Dies führt er auf den Umstand zurück, „dass in der Versdichtung der Satzbau stark vom Versbau beeinflusst ist"“ (2005: 365f.). Behaghel (1929: 406) vertritt die Auffassung, dass die Verteilung von resumptiver und nichtintegrativer Spitzenstellung regional verschieden ist: Während in der niederdeutschen Region deutlich die nicht-integrative Stellung bevorzugt werde, tendieren hochdeutsche Regionen eher zum resumptiven Typ. ${ }^{48}$ Lötschers stichprobenweise untersuchte Texte zeigen zwar keine relevanten regionalen Unterschiede bei der Verteilung der Stellungstypen (2005: 363, Anm. 16), aber die syntaktischen Untersuchungen zum älteren Deutsch (vgl. Kapitel 2.2.1) legen nahe, dass bei der Zusammenstellung des Korpus der vorliegenden Arbeit der mögliche Einfluss der diatopischen Variation unbedingt beachtet werden soll (vgl. Kapitel 4.1).

Der resumptive Typ ist mit der höchsten Frequenz die bevorzugte Variante im Mittelhochdeutschen, obwohl nicht-satzwertige Herausstellungen nach links zu dieser Zeit nicht verbreitet sind (Lötscher 1995: 47, Axel 2002: 10). Was den nicht-integrativen Typ betrifft, so lässt sich feststellen, dass sein Auftreten im Mittelhochdeutschen (und auch im Frühneuhochdeutschen) im Gegensatz zum heutigen Gebrauch nicht von bestimmten semantischen-pragmatischen Bedingungen abhängt (Lötscher 2005: 349, Axel 2002: 6). Er kann auch bei gewöhnlichen Konditional-, Kausal- und Temporalsätzen frei verwendet

47 In der mittelhochdeutschen Versdichtung stammen die meisten Belege (786 von 1199) aus >Parzival< (1200). Der integrative Typ hat übrigens auch in der Versdichtung eine marginale Rolle: ein Einzelbeleg von 1199 (Lötscher 2005: 363, Voranstellung mit Verbletztstellung im nachfolgenden Hauptsatz nicht mitgezählt, vgl. auch Axel 2002: 9, Fußnote 9).

48 Behaghel (1929: 413) weist auch darauf hin, dass die Stellungsmöglichkeiten in den Mundarten wenig erforscht sind. Falls sie behandelt werden, findet man oft pauschale Behauptungen. Die Stellungsmöglichkeiten werden z.T. kontrovers beurteilt, vgl. z.B. die widersprüchlichen Aussagen im Schwäbischen Wörterbuch: Einmal heißt es, korrelatives so „sei nicht populär“, bzw. an einer anderen Stelle liest man, es sei „,die gewöhnlichste einleitung des hauptsatzes nach adv. Nebensatz“ (zit. n. Behaghel 1929: 413). Diese Verwirrung könnte eventuell auf Normkonflikte zurückgeführt werden und zeigen, dass Sprachbewusstsein durchaus eine Rolle bei der Wahl zwischen den Stellungstypen gespielt haben konnte. Eine Untersuchung metasprachlicher Äußerungen zu den Stellungstypen könnte zu interessanten Ergebnissen führen. Die Unterscheidung der drei Stellungstypen ist (noch ohne die oben genannte Terminologie) bereits in den Grammatiken des 19. Jh. zu finden, s. z.B. die dritte Auflage der Grammatik von Blatz (1896: 127-130). In der zweiten Auflage wird jedoch nur die integrative Stellung beschrieben bzw. eher vorgeschrieben (1880: 500). Eine ausführliche Analyse der grammatikografischen Werke der neuhochdeutschen Zeit würde den Rahmen der vorliegenden Arbeit sprengen. 
werden. ${ }^{49}$ Beim gegenwärtigen Forschungsstand bleibt die Frage nach den Einzelheiten der Festlegung des nicht-integrativen Typs auf bestimmte Kontexte unbeantwortet. Dass sein Anteil im Vergleich zu Notkers spätalthochdeutschen Übersetzungen >Martianus Capella und >De Consolatione` sogar zuzunehmen scheint, steht jedoch im Widerspruch zur Annahme einer unidirektionalen Entwicklung von Desintegration zur Integration (s. König/van der Auwera 1988).

Bereits Paul (1920: 315) weist darauf hin, dass nach einem vorangestellten Nebensatz die Wortstellung des Nachsatzes eine wichtige Veränderung vom Mittelhochdeutschen zum Neuhochdeutschen zeigt. Im Mittelhochdeutschen beeinflusste der vorangestellte Nebensatz nicht die Wortstellung des Nachsatzes, das Verb des Nachsatzes musste nicht an erster Stelle stehen. Für das Neuhochdeutsche aber nimmt Paul (1920: 315) die selbe Wortstellung nach dem vorangestellten Nebensatz an als beim nicht-satzwertigen Satzglied, „das Verb. muß ihm also unmittelbar folgen.“ Es muss betont werden, dass Paul im direkten Anschluss des Verbs an den Vordersatz im Neuhochdeutschen eine „Regel“ vermutet, von der es allerdings Ausnahmen gibt. ${ }^{50}$ Die Ausführungen von Paul (1920) legen also eine Verdrängung des nichtintegrativen Typs nahe. Nun wenden wir uns dem Frühneuhochdeutschen zu, um die Frage zu überprüfen, inwiefern der nicht-integrative Typ verdrängt bzw. der integrative Typ verbreitet war.

\subsubsection{Frühneuhochdeutsch und frühes Neuhochdeutsch}

Zur Integration vorangestellter Adverbialsätze im Frühneuhochdeutschen liegen mehrere Auszählungen vor (u.a. Hammarström 1923, Axel 2002, Axel 2004: 40ff., Lötscher 2005). Die Auswertung der Daten aus Axel (2002: 9) und Lötscher (2005: 367f.) ergibt den folgenden groben Überblick über die Verteilung der Stellungstypen: ${ }^{51}$

$49 \mathrm{Zu}$ einschlägigen Belegen für den nicht-integrativen Typ im älteren Deutsch s. Lötscher (2005: 349).

50 Die integrative Stellung werde v.a. bei Konzessivsätzen aufgegeben. Paul (1920: 315f.) führt nicht-integrative Beispiele für mehrere Typen der Konzessivsätze auf. Sehr modern ist Paul in der Hinsicht, dass er sogar pragmatische Verwendungen wie daß ich mich kurz fasse bzw. daß ich dirs gestehe erwähnt. Auch diese Sätze beeinflussen die Wortstellung des Nachsatzes nicht, werden also nicht-integrativ verwendet. Nur in wenigen Fällen geht Paul (1920: 316) von einem Schwanken in der Wortstellung aus, so z.B. bei den „aus Bedingungssätzen entwickelten Konzessivsätzen“, vgl. (i):

(i) Wenn du auch noch so sehr klagtest, er würde / würde er sich doch nicht rühren lassen;

Alles andere ist für Paul eine „seltene Ausnahme“ (1920: 316). Dabei sollte jedoch angemerkt werden, dass Pauls Auffassung den in der neueren Fachliteratur ,resumptiv' genannten Typ durchaus erlaubt und er ihn mit dem integrativen Typ auf gleicher Höhe sieht (vgl. Kap. 3.1.3). Dagegen nimmt Axel an, dass der resumptive Typ im Mittelhochdeutschen eine „Realisierungsvariante von V3“ ist (2002: 12).

51 In Axels Durchsicht wurden insg. 1021 Belege aus fünf Quellentexten berücksichtigt (2002: 9). Dies wird von Lötscher (2005: 367f.) stichprobenweise um weitere 1157 Belege aus 23 Quellentexten (in den meisten Fällen ca. 50 Belege pro Quellentext) ergänzt. 


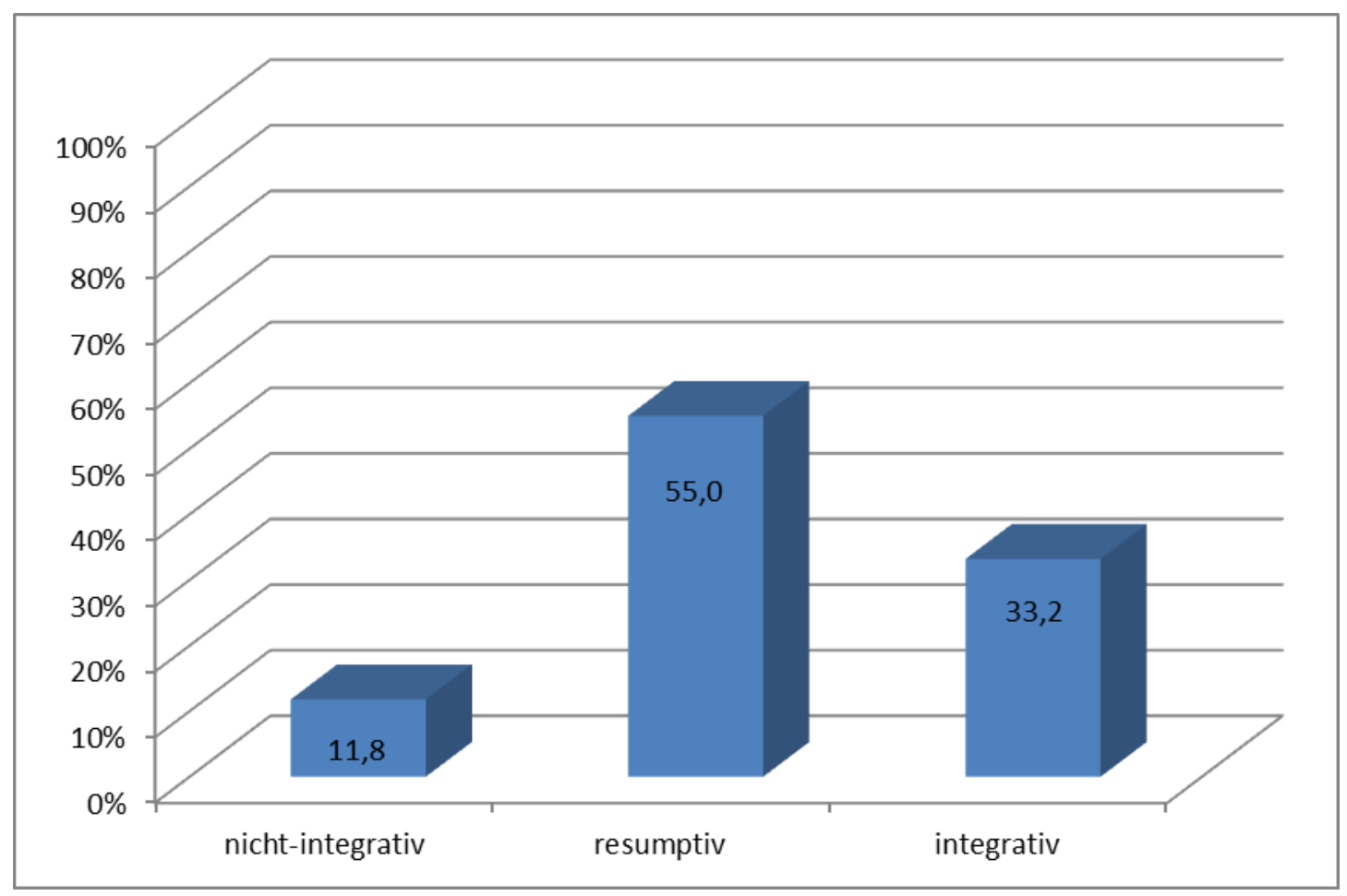

Abb. 4: Integrationsgrad vorangestellter Adverbialsätze im Frühneuhochdeutschen $(n=2178)$

(vgl. Axel 2002: 9 bzw. Lötscher 2005: 367f.)

Der integrative Typ verbreitet sich seit dem Frühneuhochdeutschen, wobei in dieser Epoche insgesamt noch das Resumptivum überwiegt (vgl. Axel 2002: 9). Auch der nicht-integrative Typ bleibt jedoch v.a. in literarischen Texten ein gebräuchliches Muster (Lötscher 2005: 370). Laut Axel-Tober (2012: 365f.) behalten nur spezielle Adverbialsatztypen den nichtintegrierten Status. Der nicht-integrative Typ wird bei einzelnen Unterarten von Adverbialsätzen komplett verdrängt (z.B. bei da-Sätzen, vgl. Axel-Tober 2012: 350f.). Diese ,Marginalisierung‘ des nicht-integrativen Typs vollzog sich nach Axel-Tober (2012: 365f.) bereits in der frühneuhochdeutschen Periode.

Die Etablierung des integrativen Typs als allgemein akzeptiertes Stellungsmuster erfolgt im letzten Drittel des 15. Jhs., vor Mitte des 15. Jhs. kommt er kaum vor (Lötscher 2005: 369f.). Zwischen den einzelnen Texten zeigen sich jedoch ab dieser Zeit große Unterschiede: Z.B. wird im >Pilger`(Geiler von Kaisersberg, 1494) und in der >Jerusalemfahrt` (Füessli, 1523) bis auf einen Einzelbeleg immer der resumptive Typ realisiert (100\% bzw. 98\%), während in der >Bayer Chronik` (Aventinus, 1530/31) der integrative Typ mit 92\% überwiegt. Nach den Ergebnissen einer anderen quantitativen Untersuchung aufgrund des Bonner Frühneuhochdeutsch-Korpus (vgl. http://www.korpora.org/Fnhd/) scheint sich der neue Typ bereits in der ersten Hälfte des 16. Jhs. durchgesetzt zu haben: 


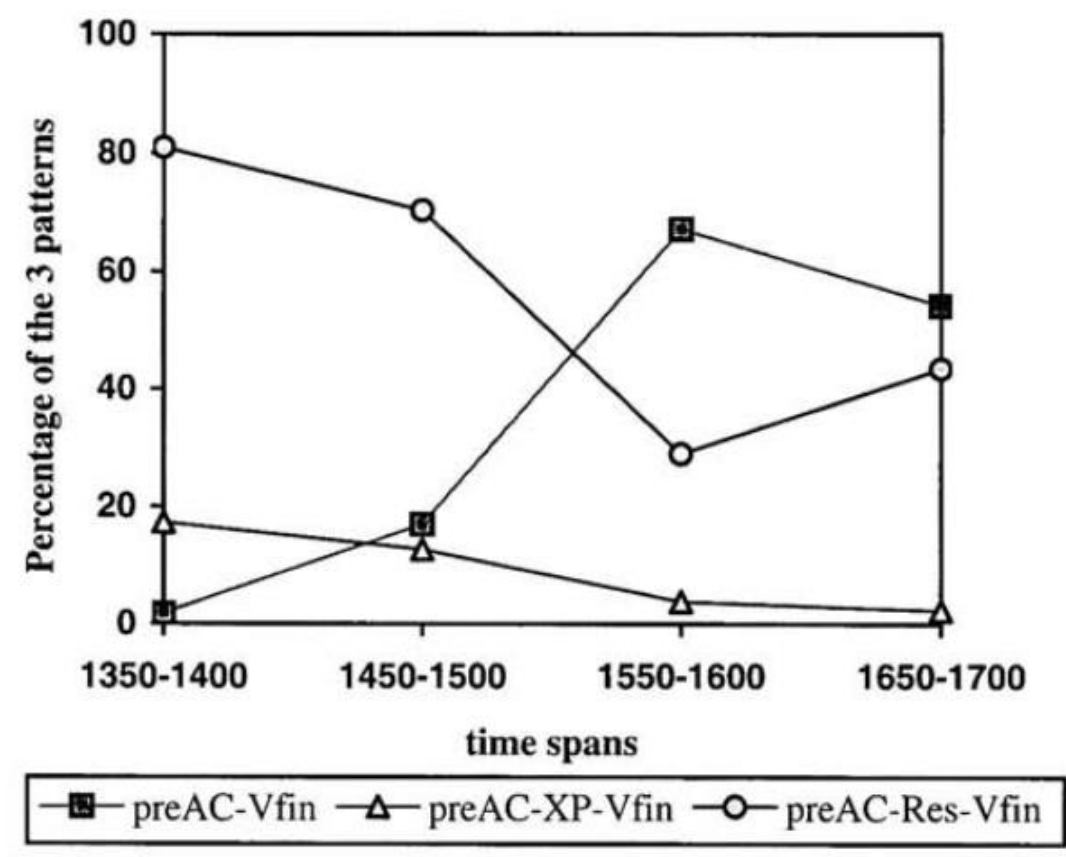

Abb. 5: Integrationsgrad vorangestellter Adverbialsätze im Bonner

Frühneuhochdeutsch-Korpus ( $\mathrm{n}=$ ca. 2700) (Axel 2004: 40)

In den Korpustexten aus dem Zeitraum zwischen 1550 und 1600 erreicht der integrative Typ (das Muster , preAC- $\mathrm{V}_{\text {fin }}{ }^{\text {) }}$ ) eine Häufigkeit von fast $70 \%$. Man könnte sich fast schon der Versuchung hingeben und feststellen, dass sich der integrative Typ innerhalb von 200 Jahren etablierte. Dies ist aber eher unwahrscheinlich - und nicht nur deshalb, weil beim syntaktischen Wandel eher ein längerer Ablauf erwartet wird. Problematisch sind die z.T. sehr großen Unterschiede zwischen den einzelnen Texten aus dem selben Jh., die eine rein grammatische Erklärung des Phänomens in Frage stellen. Lötscher (2005: 369) geht davon aus, dass der Integrationsprozess nicht alle Textarten in der gleichen Weise erfasst (s. 2.2.3).

Die folgenden Ergebnisse zur Verbreitung des resumptiven Typs (,preAC-Res- $V_{\text {fin }}{ }^{6}$ ) legen wiederum keine lineare, unidirektionale Entwicklung im Sinne von König/van der Auwera (1988) nahe. Zwischen 1350 und 1600 geht seine Häufigkeit allmählich zurück. Während er zwischen 1450 und 1500 noch der am meisten benutzte Stellungstyp ist (ca. 70\%), beträgt sein Anteil am Ende des 16. Jhs. knapp 30\%. Beim Übergang zum Neuhochdeutschen wird der resumptive Typ jedoch wieder etwas häufiger (über 40\%). Zur Frequenz des resumptiven Stellungstyps bemerkt Paul, dass er im Neuhochdeutschen „eine große Ausdehnung erlangt, die aber in der neuesten Zeit wieder eingeschränkt ist“" (1920: 317). ${ }^{52}$

\footnotetext{
52 Auf den Vordersatz kann v.a. so zurückweisen, das sich allgemein erhalten habe nach ,Bedingungsatz', nach Konzessivsatz und nach Sätzen wie was das betrifft. Es wurde jedoch eher ungebräuchlich nach Kausalsatz, ,Zeitsatz', nach ehe, das eine Präferenz ausdrückt, bzw. nach ,Absichtsatz‘ (Paul 1920: 317ff.).
} 
Durch die Auswertung eines frühneuhochdeutschen Volksbuches wurde gezeigt, dass die Stellungstypen ,integrativ‘, ,resumptiv ${ }^{\star}$ sowie ,nicht-integrativ ${ }^{\star}$ bei den einzelnen semantischen Adverbialsatzklassen nicht im gleichen Maße möglich sind, dass Final- und Konzessivsätze nur mit resumptiver Stellung gebraucht werden, während bei Konditionalsätzen alle drei Stellungstypen auftreten (vgl. Axel 2002, Hammarström 1923). Auf Unterschiede zwischen semantischen Klassen wird in der Forschungsliteratur mehrmals hingewiesen. Z.B. sind Kausalsätze im Frühneuhochdeutschen integrativer als Temporalsätze, die aber selbst integrativer sind als Konditionalsätze. Bereits Behaghel (1929: 408) weist in seinem Aufsatz, in dem „Der Nachsatz“ und dessen Anschluss an den Vordersatz behandelt werden, darauf hin, dass „seit Luther“ bereits eine Regel der Verteilung nach semantischen Typen zu erkennen ist. Bezüglich neuerer historischer Untersuchungen kann jedoch festgestellt werden, dass mehrfach undifferenzierte Analysen im Hinblick auf die semantischen Klassen von Adverbialsätzen zu finden sind, vgl. z.B. Axel (2002: 9), Axel (2004: 40), Lötscher (2005: 367f.). Dass die Tendenz zur zunehmenden Integration bei den semantischen Subtypen von Adverbialsätzen unterschiedlich ausgeprägt ist und zwischen ihnen Unterschiede hinsichtlich des Integrationsgrades beobachtet werden können, wird für das 17. Jh. auch von Wiktorowitz (2013) angenommen, bei der Auswertung seines Korpus werden jedoch keine quantitativen Daten genannt. Im Kapitel 4.2 wird dafür argumentiert, semantische Untertypen bei der quantitativen Untersuchung systematisch zu berücksichtigen.

Die Bevorzugung einzelner Stellungstypen ist auch auf strukturelle Faktoren zurückzuführen. Axel/Wöllstein (2009: 18ff.) untersuchen die Häufigkeit der Wortstellungstypen bei vorangestellten Adverbialsätzen mit anschließenden deklarativen Hauptsätzen im Bonner Korpus. Kanonische Adverbialsätze wurden von V1-Gefügen getrennt analysiert. Bei vorangestellten kanonischen Adverbialsätzen zeigt sich eine Verteilung der Stellungstypen, die im Vergleich mit dem Gegenwartsdeutschen v.a. durch den niedrigen Anteil des Typs ,integrativ' im ersten Zeitabschnitt (1350-1400, nur 2\% aus ca. 600 Belegen) auffällt. Bereits im 16.-17. Jh. überwiegt jedoch gerade dieser Stellungstyp, der im Gegenwartsdeutschen am häufigsten vorkommt. Beim Blick auf die vorangestellten V1Konditionale zeigt sich, dass sie an der rasanten Verbreitung der integrativen Stellung zwischen 1350-1650 nicht teilgenommen haben: Der integrative Stellungstyp erscheint im Korpus erst ab 1650, d.h., ihre Verbreitung in V1-Konditionalen beginnt 300 Jahre nach der entsprechenden Entwicklung bei kanonischen Adverbialsätzen (Axel/Wöllstein 2009: 19, 22). 
Behaghel (1929: 410) bemerkt am Material von Thomasius, dass die (Nicht-)Setzung des Korrelats mit der Länge des Vordersatzes zusammenhängen kann. ${ }^{53}$ Wenn der Raum zwischen Nebensatzanfang und Nachsatz klein ist, wird kein Korrelat gesetzt. Wenn der Zwischenraum aber mehrere Zeilen erfüllt, wird auf das Korrelat zurückgegriffen, das in diesem Fall den Anfang des übergeordneten Satzes deutlich markieren soll. ${ }^{54}$ Auch rhythmische Gründe könnten eine Rolle spielen: Mit dem Korrelat kann der Nachsatz mit einer unbetonten Silbe beginnen (Behaghel 1929: 406). Dies könnte zur Verbreitung des resumptiven Typs im Mittelhochdeutschen beigetragen haben.

Die Distribution der resumptiven Elemente ist im Mittelhochdeutschen und noch im Frühneuhochdeutschen teilweise anders als im Gegenwartsdeutschen (Axel 2002: 11). Die einleitenden Ausdrücke von Adverbialsatz und Bezugssatz bilden oft Paare wie z.B. da-da, so-so, ee-ee usw. ${ }^{55}$ Zudem wird so auch bei Kausal- und Temporalsätzen verwendet.

Zur Geschichte der Integration im Neuhochdeutschen liegen nur wenige Auszählungen, die alle Unterarten von Adverbialsätzen berücksichtigen, vor. Der obigen Abbildung 5 sind Daten zum frühen Neuhochdeutsch (1650-1700) zu entnehmen, die Axel (2004: 40) aufgrund des Bonner Frühneuhochdeutsch-Korpus ermittelt hat. Der Anteil des nicht-integrativen Typs ist im Gegensatz zu früheren Teilkorpora etwas niedriger, dieser Typ ist jedoch nicht komplett verdrängt. Dominant sind der resumptive und der integrative Typ, wobei der letztere um ca. $10 \%$ häufiger anzutreffen ist. Diese Daten legen nahe, dass beim nicht-integrativen Typ die Verdrängung und Festlegung auf bestimmte Verwendungen vor neuhochdeutscher Zeit noch nicht abgeschlossen ist und der Konkurrenzkampf zwischen dem resumptiven und integrativen Muster erst im Neuhochdeutschen ausgefochten wird.

Es finden sich auch Daten, die die diachrone Hypothese von König/van der Auwera (1988: 127) zu unterstützen scheinen. Baschewa (1983) beobachtet an ihrem Korpus aus dem 18.-20. Jh. eine abnehmende Frequenz vom Typ ,resumptiv‘ im konzessiven bzw. konzessivkonditionalen Bereich. Auch zur früheren Geschichte der Integration wurden entsprechende

53 Dieser Faktor wird bereits in der Mitte des 17. Jh. in einem Korpusvolltext genannt. Er sollte jedoch nicht überbewertet werden, wie es im folgenden Beleg der Fall ist:

(i) Die übrigen Conjunctionen, als caussales, conditionales, consecutiuae, wenn sie im voerdern Satze stehen, erfordern im Anfang des Nachsatzes so: welches so aber, wenn der vordere Satz kurz ist, auch wegbleiben kann, z.B. weil du von Kindheit auf die H. Schrifft weißt: (so) kann dich dieselbe unterweisen; woferne du mich zahlest, (so) muß ich dich verklagen; nachdem er ausstudiert hat, (so) wartet er nunmehr auf Dienste. (Aichinger III: 517)

54 Wenn im älteren Deutsch dem Hauptsatz ein komplexes syntaktisches Gebilde (z.B. ein komplexes Nebensatzgefüge) vorangestellt wird, kann eine Loslösung des Hauptsatzes vom Nebensatz erfolgen. Die nicht-integrative Stellung markiert einen Neuanfang. Lötscher (2005: 362) nennt dafür zwei Beispiele aus dem 13. und 15. Jh.

55 Bereits Behaghel (1929: 403) verweist darauf, dass tho und so als Einleitung des Bezugsatzes auf parataktische Sätze mit tho-tho bzw. so-so zurückgehen. Das Inventar von Paaren bei Adverbialsubordinatoren (thanne-thanne, so-so, tho-tho, er-er usw.) war im Althochdeutschen und Mittelhochdeutschen viel größer als heute. Sie waren bei mehreren semantischen Untertypen von Adverbialsätzen möglich (Axel 2004: 29). 
Daten genannt (s. weiter oben, vgl. auch Paul 1920: 317ff., Axel 2002: 16 usw.). Es könnte angenommen werden, dass der nicht-integrative Typ seit dem Frühneuhochdeutschen verdrängt und auf bestimmte spezielle Verwendungen festgelegt wird, während die Erscheinung des integrativen Typs eine neuere Entwicklungsstufe darstellt. Es wurden jedoch m.E. genügende, der Hypothese von König/van der Auwera (1988) widersprechende Ergebnisse genannt. Daher erscheint es an dieser Stelle sinnvoll, alternative Erklärungsansätze zu diskutieren (s. dazu auch Kappel 2014).

\subsubsection{Erklärungsansätze}

\subsubsection{Grammatikalisierung}

König/van der Auwera (1988) erklären den Integrationsprozess im Rahmen der Grammatikalisierungstheorie: Das eher parataktische Nebeneinander zweier selbständiger Sätze wird durch eine syntaktische Struktur mit einem untergeordneten, in den übergeordneten Satz eingebetteten Nebensatz abgelöst (1988: 107f., 127). In diesem Sinne geht es um Grammatikalisierung, ,a process in which something becomes or is made more grammatical“ (Lehmann 2015: 11) bzw. um die Grammatikalisierungsstufe ,Syntaktisierung (= die Entstehung syntaktischer Strukturen aus freien Diskursstrukturen, s. Lehmann 2015: 15). König/van der Auwera (1988) liefern auch historische Daten für die Verbreitung des integrativen Typs bzw. für den Rückgang des nicht-integrativen Typs in mehreren germanischen Sprachen, u.a. im Deutschen. Ihre These von einer Tendenz zur zunehmenden Integration, die in den drei Stadien Nicht-Integration > Resumption > Integration ablaufe, dürfte auch in der heutigen Forschung als die geläufige Lehrmeinung angesehen werden (vgl. z.B. Wegener 2000: 42), die auch Eingang in die IDS-Grammatik gefunden hat (Zifonun et al. 1997: 2349).

Um diese Hypothese bestätigen zu können, müsste nachgewiesen werden, dass die drei Stufen in der Tat in der angenommenen zeitlichen Abfolge (nicht-integrativ > resumptiv > integrativ) nacheinander erscheinen. Die Verbreitung bzw. Abnahme der jeweiligen Konstruktionen sollte dementsprechend unidirektional, d.h nur in einer, nicht aber in gegenläufiger Richtung ablaufen. Diese These erwies sich jedoch im Lichte der empirischen Daten zum älteren Deutsch als nicht haltbar. Der nicht-integrative Typ kann nämlich nicht als die älteste Variante nachgewiesen werden. Zudem ist der resumptive Typ vom Althochdeutschen bis zum Frühneuhochdeutschen die bevorzugte Variante, in den meisten 
Korpora mit einem Anteil zwischen 30-75\% (vgl. Axel 2002: 9ff.). Es ist fraglich, ob er wirklich vom integrativen Typ abgelöst wird, seine Auffassung als Übergangsstufe ist also nicht unproblematisch (vgl. Kapitel 2.2.2). Axel (2002: 27f.) führt noch folgende Argumente gegen die Auffassung von König/van der Auwera (1988) auf, die sie als „Grammatikalisierungsanalyse“ interpretiert: Im Gegenwartsdeutschen können nicht nur einbettbare, sondern auch nicht-einbettbare Adverbialsätze resumptiv angeschlossen werden (vgl. auch Köpcke/Panther 1989 und Vandergriff 2009: 198), sodass es nicht klar sei, „ob und inwiefern, diachron betrachtet, die resumptive Struktur einen Fortschritt in Richtung syntaktische Integration darstellt.“ (Axel 2002: 28) Ein Grammatikalisierungsszenario mit einem Kontinuum zwischen Parataxe und Einbettung, wie es in Anlehnung Lehmann (1988: 189) angenommen wird (König/van der Auwera 1988: 127), setze ein rein parataktisches Nebeneinander von selbständigen Sätzen am Anfang voraus. Dies lässt sich jedoch nicht rekonstruieren, im Althochdeutschen sei der Vordersatz bereits ein subordinierter Satz gewesen (Axel 2002: 28f., Axel-Tober 2012: 357). Der Anteil der Konstruktionen, die im Gegenwartsdeutschen nur noch Entwicklungsrückstände darstellen (Zifonun et al. 1997: 2349), nimmt zwischenzeitlich sogar zu, daher ist es fraglich, ob der Entwicklungsprozess linear, unidirektional und in den angenommenen drei Stufen (erst ,nicht-integrativ ${ }^{6}$, dann abgelöst durch ,resumptiv', am Ende der Entwicklung, integrativ') abläuft. Des Weiteren können die Stellungstypen von König/van der Auwera (1988) vor dem (Spät-)Althochdeutschen nicht angewandt werden, sodass für eine Annahme einer potenziell noch früheren Zeitstufe mit Dominanz des nicht-integrativen Typs auch die theoretische Grundlage fehlt (s. auch Lötscher 2005: 361).

Laut Behaghel (1929: 403f.) entwickelte sich der resumptive Typ nicht aus dem nichtintegrativen Typ (wie von König/van der Auwera 1988 angenommen). Er hat seinen Ursprung in temporalen Verknüpfungen mit $d o-d o$. Die Einleitung des Nachsatzes gehe auf parataktische Verbindungen zurück, in denen Hauptsätze aneinandergereiht und mit da verbunden werden:

(28) sie wünschten mit Christus zu gehn; da kamen sie weiter: da fanden sie (zit. n. Behaghel 1929: 403)

Behaghel (1929: 403) erklärt auch den Ursprung des einleitenden so ähnlich: Es sei „aus zwei parataktischen sätzen mit so - so entstanden [...], wo jedes so auf zurückliegendes hinwies““. Auch der nicht-integrative Typ sei aus Parataxe entstanden, wobei die „wortstellung des allein 
stehenden hauptsatzes“ die Folge davon ist, dass der Vordersatz ein selbständiger Fragesatz ${ }^{56}$ oder ein Wunschsatz war:

(29) ist er der tiuvel oder sin wip, er kann mir einec niht geschaden (zit. n. Behaghel 1929: 402)

Behaghel (1929) bietet also einen Erklärungsansatz für den Integrationsgrad der vorangestellten Nebensätze, der nicht von einem stufenweisen Übergang (,nicht integrativ` > ,resumptiv ‘ , integrativ') ausgeht, sondern beim Ursprung und Wandel der drei Stellungstypen zwischen einzelnen Untertypen unterscheidet.

Eine ähnliche Erklärung findet sich bei Rieck (1977: 164, vgl. Axel 2002: 15). Das resumptive Element habe demnach die „formal syntaktische Funktion“, nachgestellte Hauptsätze und Nebensätze zu unterscheiden. Dies habe sich zunächst bei V1-Konditionalen entwickelt und dann auf weitere Adverbialsatztypen übertragen. Da jedoch V1-Konditionale im frühneuhochdeutschen Korpus von Thim-Mabrey (1987: 199) nicht häufiger resumptiv angeschlossen werden als andere Unterarten von Adverbialsätzen und die resumptive Stellung bereits vor der Entwicklung der V1-Konditionale verbreitet war, ist diese Erklärung nicht angemessen (Axel 2002: 15f.). Bei Rieck (1977: 165) ist auch ein Erklärungsversuch für die Verdrängung des Resumptivums zu finden: Die resumptive Verwendung von so wird bei der „Einführung einer geregelten Interpunktion“ redundant (zur Relevanz der Interpunktion beim Integrationsgrad vorangestellter Adverbialsätze s. auch Kapitel 3.2.2. sowie 3.3.2).

\subsubsection{Rekategorisierung bzw. Reanalyse}

Axel (2002) argumentiert in ihrer generativ ausgerichteten Untersuchung für einen Wandel im kategorialen Status von Adverbialsätzen. Nach dieser Auffassung waren die Adverbialsätze vom Althochdeutschen bis zum Frühneuhochdeutschen nicht vorfeldfähig (Axel 2002: 16). Im Althochdeutschen und im Mittelhochdeutschen waren demnach Adverbialsätze keine echten Gliedsätze. D.h., sie waren nur subordinierte, nicht aber syntaktisch integrierte Sätze (vgl. auch Axel 2004: 40ff. und Axel 2007: 233). Die Entwicklung der Vorfeldfähigkeit bei vorangestellten Adverbialsätzen wird auf spätmittelhochdeutsche/frühneuhochdeutsche Zeit

56 Die Entstehung von asyndetischen (uneingeleiteten) Konditionalsätzen aus parataktischen Diskurssequenzen und ihre Grammatikalisierung kann - parallel zum Deutschen - auch im Englischen nachgezeichnet werden (vgl. Van den Nest 2005). Dabei erweist sich jedoch - entgegen der Hypothese von König/van der Auwera (1988) - der nicht-integrative Typ nicht als die unmarkierte Variante des Althochdeutschen. Van den Nest (2005: 35f.) stellt die Auffassung, der resumptive Typ sei die später hinzugekommene, sekundäre Variante, in Frage. 
datiert und rein grammatisch erfasst. ${ }^{57}$ Den Grund für die Einbettung seit dem Frühneuhochdeutschen sieht Axel (2002: 35f.) darin, dass Adverbialsätze syntaktisch (dem früheren CP-Status gegenüber) als PP rekategorisiert wurden und somit Gliedsatzstatus erlangt haben. Die Adverbialsätze wurden ,in zunehmenden Maße in satzinternen Modifizierer-Positionen lizenziert“ (Axel 2002: 30). ${ }^{58}$

Lötscher (2005: 350f.) kritisiert jedoch diese Auffassung. Er verweist u.a. darauf, dass mit einer rein syntaktischen Rekategorisierungsthese nicht erklärt werden kann, warum im Neuhochdeutschen neben dem integrativen Typ auch weitere Stellungen möglich sind, wobei nach dieser These im Neuhochdeutschen „nur noch integrative Spitzenstellung möglich sein“ dürfte. Für einen zweiten Mangel hält Lötscher den Umstand, dass Axels These einen klaren Übergang von einem Konstruktionstyp zu einem anderen impliziert, während höchstens nur ein allmähliches Ausbreiten des integrativen Typs nachzuweisen ist. Ein weiteres Problem ist, dass die verschiedenen Stellungsvarianten sogar im selben Text parallel verwendet werden. Lötscher weist stilistisch-funktionale Unterschiede beim Gebrauch der Stellungstypen nach (s. weiter unten), die ,mit einer rein syntaktischen Erklärung über Rekategorisierung nicht erklärbar" seien. Schließlich wird auch eingewendet, dass nicht danach gefragt wird, warum der angenommene syntaktische Wandel bei Adverbialsätzen überhaupt vorgegangen ist. Die Ursache des syntaktischen Wandels könne im Rahmen der Rekategorisierungshypothese nicht sinnvoll thematisiert werden.

Nach einer theoretischen Weiterentwicklung anhand der temporalen $d a$-Sätze ${ }^{59}$ in AxelTober (2012: 270ff.) ist die syntaktische Kategorie der Adverbialsätze nicht mehr wesentlich. Nach dem Reanalyseszenario von Axel-Tober (2012: 320) entwickelt sich das Adverb da (mhd. dô) über das Zwischenstadium eines Relativadverbs zu einer Konjunktion, was mit dem Wandel von der unintegrierten korrelativen Satzfügung zu einer Einbettungsstruktur mit einem Gliedsatz einhergeht. Die Ausgangskonstruktion war nach dieser Auffassung eine korrelative Relativkonstruktion (2012: 344) ${ }^{60}$ Die dô-Sätze haben eine periphere Position, sie

57 Diese Datierung ist insofern etwas überraschend, dass V2-Stellung bereits im Spätalthochdeutschen sehr verbreitet ist, vgl. Lötscher (2005: 355).

58 Ob und wie einschlägige Veränderungen bei einzelnen Korpusbelegen analysiert und kategorial erfasst werden können, bzw. wie an konkreten Sätzen gezeigt werden könnte, dass sie „in zunehmenden Maße in satzinternen ModifiziererPositionen lizenziert" werden (Axel 2002: 30), bleibt jedoch fraglich.

59 Da-Sätze seien im Gegenwartsdeutschen nicht-kanonische Konstruktionen, da sie nur formal-syntaktisch subordiniert und eingebettet sind, während sie sich informationsstrukturell, semantisch und pragmatisch eher wie Hauptsätze verhalten, also keine informationsstrukturelle-semantische Integriertheit und illokutive Unterordnung zeigen (Axel-Tober 2012: 10ff.).

60 Diese Strategie wird das korrelative Diptychon genannt. Es handelt sich um eine Konstruktion mit einem Teilsatz, der im Hauptsatz nicht eingebettet ist und mit einem anaphorischen Pronomen oder Adverb wiederaufgenommen wird, vgl. auch Viti (2015: 460 bzw. 317ff.). Diese aus dem Indogermanischen ererbte Relativkonstruktion war noch im älteren Deutsch verbreitet. 
stehen - anders als im Gegenwartsdeutschen - nicht im Vorfeld des Bezugsatzes (2012: 37). Den dô-Sätzen vor der Reanalyse weist Axel-Tober (2012: 346) keine Position im Restsatz $\mathrm{zu}$, ,sie waren lediglich an die Matrix-CP adjungiert“. Dies änderte sich erst im Spätmittelhochdeutschen oder $\mathrm{zu}$ Beginn der frühneuhochdeutschen Periode, als diese Konstruktion als Linksversetzung reanalysiert wurde. Erst danach waren $d \hat{o}$-Sätze (und die anderen Kernadverbialsatztypen) syntaktisch in den Bezugssatz integriert und vorfeldfähig bzw. auch mittelfeldfähig (2012: 344, 353).

Axel-Tober (2012: 350ff.) schlägt ein Reanalyseszenario mit lediglich zwei Stadien vor. Beim sog. korrelativen Stadium (Althochdeutsch/Mittelhochdeutsch) seien die beiden Oberflächenabfolgen ,AdvS-XP- $\mathrm{V}_{\text {fin }}{ }^{6}$ und ,AdvS-Pro-Adv- $\mathrm{V}_{\text {fin }}{ }^{\text {' }}$ strukturell identisch, beide stellen eine Art ,hängende Topikkonstruktion' dar (Axel-Tober 2012: 343). Das korrelative Pro-Adverb ist zwar nur beim zweiten Muster overt realisiert, aber auch hinter dem anderen Muster verbirgt sich die korrelative Konstruktion: Diese Variante der korrelativen Konstruktion sei also eine nur scheinbar nicht-integrierte Struktur (Axel-Tober 2012: 350, 361). Für das ,Gliedsatzstadium' (Frühneuhochdeutsch) seien ebenfalls zwei Abfolgen, ,AdvS- $\mathrm{V}_{\text {fin }}{ }^{6}$ und ,AdvS-Pro-Adv- $\mathrm{V}_{\text {fin }}{ }^{\text {}}$ charakteristisch, denn das nicht-integrative Muster wird bei $d a$-Sätzen nicht mehr verwendet. ${ }^{61}$ Sowohl beim ersten Muster mit einem Adverbialsatz im Vorfeld, als auch beim zweiten Muster, bei dem ein linksversetzter Adverbialsatz vorliegt, handelt es sich um syntaktisch integrierte Konstruktionen. Dies führt Axel-Tober (2012: 358) darauf zurück, dass nur solche Phrasen linksversetzt werden können, die syntaktisch in den Restsatz eingebettet sind (vgl. aber Beleg (30) weiter unten). Der korrelative Typ (in Axel-Tobers Terminologie die Abfolge ,AdvS-Pro-Adv- $\mathrm{V}_{\text {fin }}{ }^{6}$ ) stellt also nach dieser Auffassung kein Übergangsstadium dar, er entwickelt sich - wie auch im Sinne der Erklärung von Behaghel (1929), s. weiter oben - nicht aus dem nicht-integrativen Typ. Er ist eigentlich kein dritter Typ im Sinne von König/van der Auwera (1988), denn Axel-Tober argumentiert dafür, „dass die Konstruktionen mit und ohne Korrelat strukturell identisch sind.“ (2012: 38). Zu Beginn der frühneuhochdeutschen Periode ist der resumptive Typ eher eine ambige Konstruktion: Er konnte sowohl als korrelative als auch als Linksversetzungskonstruktion analysiert werden, was die Voraussetzung für seine Reanalyse war:

Die Oberflächensyntax der beiden Konstruktionen ist sehr ähnlich. Was die Distribution der linksperipheren Phrase (bzw. des linksperipheren Satzes angeht) [sic!], unterscheiden sie sich jedoch in Bezug auf die Frage,

\footnotetext{
61 Bei vorangestellten $d a$-Sätzen im Gegenwartsdeutschen ist das nicht-integrative Muster ungrammatisch. Zwischen 1650 und 1700 fand Axel-Tober (2012: 349) ebenfalls keine Belege in den neun Quellentexten aus dem Bonner Frühneuhochdeutsch-Korpus.
} 
ob diese auch in anderen Positionen auftreten kann. Nur bei der Linksversetzung kann die betroffene XP alternativ auch im Innern des Restsatzes stehen. (Axel-Tober 2012: 360f.)

Axel-Tober untersucht nur einen Untertyp von Adverbialsätzen, es wird jedoch nahegelegt, dass auch in anderen Fällen eine ähnliche Entwicklung von Adverbialsatzkonstruktionen aus Relativkonstruktionen anzunehmen ist (2012: 272). Da bei anderen Unterarten von Adverbialsätzen der nicht-integrative Stellungstyp auch im Gegenwartsdeutschen verwendet wird (vgl. Kapitel 2.1.3), kann jedoch Axel-Tobers Reanalyseszenario nicht auf alle Adverbialsätze angewandt werden. Der Status des nicht-integrativen Typs im ,Gliedsatzstadium' (seit dem Frühneuhochdeutschen) und sein Verhältnis zum korrelativen Typ bei nicht-eingebetteten Adverbialsätzen sind nicht geklärt. Weiter oben (Kapitel 2.2.3.1) wurde schon angedeutet, dass im Gegenwartsdeutschen nicht nur einbettbare, sondern auch nicht-einbettbare Adverbialsätze resumptiv angeschlossen werden können (Axel 2002: 27f.):

(30) Wenn du meine Meinung hören willst, (dann) fallen die Aktien bald. (Beispielsatz nach Köpcke/Panther 1989, vgl. auch Vandergriff 2009: 198)

Viel problematischer ist jedoch, dass im Rahmen des Reanalyseszenarios von Axel-Tober (2012) die klar sichtbare syntaktische Variation $\mathrm{zu}$ frühneuhochdeutscher bzw. neuhochdeutscher Zeit nicht erfassbar ist. Im Rahmen des generativen Ansatzes von AxelTober ist die Frage nach möglichen außersprachlichen Faktoren der Variation nicht zu beantworten bzw. nicht relevant. ${ }^{62}$

\subsubsection{Soziopragmatische Erklärungen}

Die Frage nach möglichen außersprachlichen Faktoren der Variation wurde bereits in der ersten Hälfte des 20. Jhs. aufgeworfen. Die Daten Hammarströms (1923) zu den beiden frühneuhochdeutschen Volksbüchern >Till Eulenspiegel` (aus der Zeit um 1515) bzw. >Doctor Faust (1587) legen nahe, dass hinter der scheinbar diachronen Entwicklungstendenz ein anderes Prinzip steckt. Dies soll mit Hilfe der folgenden Abbildung gezeigt werden:

62 Die folgenden Gegenargumente Lötschers (2005: 350f.), die gegen die Rekategorisierungshypothese von Axel (2002) aufgeführt wurden, gelten auch für Axel-Tobers (2012) Reanalyseszenario. Die stilistisch-funktionalen Unterschiede beim Gebrauch der Stellungstypen können mit einem rein grammatischen Ansatz nicht erklärt werden. Des Weiteren werden die verschiedenen Stellungsvarianten sogar im selben Text parallel verwendet. 


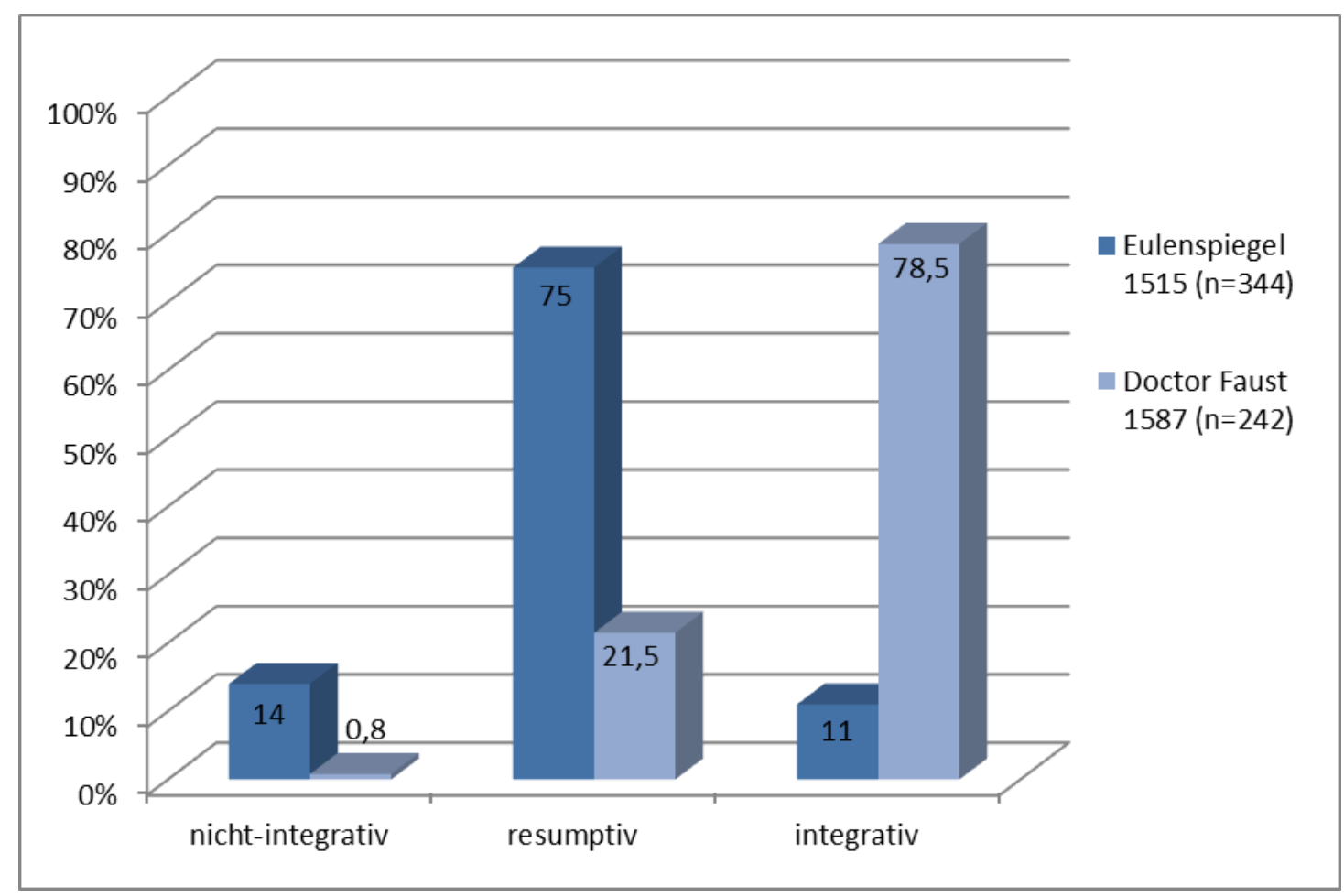

Abb. 6: Integrationsgrad vorangestellter Adverbialsätze in frühneuhochdeutschen Volksbüchern

(vgl. Hammarström 1923: 1 bzw. Axel 2002: 9)

Die Abbildung zeigt, dass der integrative Typ nur im zweiten Text dominiert (und zwar mit fast $80 \%$ ). Wahrscheinlich nicht (oder nicht nur) deshalb, weil er später entstanden ist. Diese ausgesprochen große Variation innerhalb des selben Jhs., die übrigens nicht nur für das bekanntlich variationsreiche Frühneuhochdeutsche zutrifft, wird in der Fachliteratur (bis auf Lötscher 2005, s. weiter unten) überraschenderweise nicht hervorgehoben, sie ist m.E. jedoch der Schlüssel zur Erklärung der Entwicklung. ${ }^{63}$

Was könnte nun im Hintergrund stehen, wenn der rein syntaktische Erklärungsansatz nicht mehr ausreichend ist? Die Anmerkungen Hammarströms können uns m.E. einen Schritt näher zur Lösung bringen. Das Volksbuch 〉Till Eulenspiegelく weist eine „durch schriftsprachliche und fremde einflüsse weniger bedingte [...] im volke übliche Sprache” auf (1923: 3), während das Faustbuch mit den folgenden Anmerkungen charakterisiert wird: „einfluss des herrschenden kanzleistils”, „lateinisierende Prosa" und „weitschweifige Ausdrücke” (Hammarström 1923: 4). Die Verweise auf volkstümliche Literatur und Kanzleistil der Urkundensprache sprechen für einen konzeptionellen Unterschied zwischen den beiden Texten, wobei sie natürlich nicht eindeutig als nähesprachlicher bzw. distanzsprachlicher Text bezeichnet werden können. Dazu wäre eine detaillierte Nähenalyse (vgl. Ágel/Hennig 2006b)

${ }^{63}$ Zum engen Verhältnis von Sprachvariation und Sprachwandel s. von Polenz (2000: 58-71). 
nötig. ${ }^{64}$ Die erwähnten quantitativen Unterschiede und Schwankungen deuten m.E. auf den größten Mangel der bisherigen Forschung hin: Sie konzentriert sich allzu sehr auf die diachrone Dimension und verliert die Sprache als Varietätensystem (bzw. Veränderungen im Varietätenspektrum, s. Reichmann 1988, 2003 bzw. Kapitel 3.5.2) aus den Augen. Die Texte werden nur nach zeitlichen Aspekten ausgewählt und Unterschiede zwischen ihnen werden nicht (oder nicht genügend) reflektiert. M.E. können aber nicht alle Texte in den gleichen Topf geworfen werden, da mediale bzw. konzeptionelle Unterschiede auf die syntaktische Variation einwirken könnten. Auch die Einwirkung weiterer Dimensionen des Varietätensystems ist nicht auszuschließen (s. dazu Kapitel 2.2.2.1, vgl. auch Kappel 2007).

Lötscher (2005: 353ff.) stellt dem syntaktischen Beschreibungsansatz einen pragmatischen gegenüber. Er sieht eine Korrelation zwischen Integriertheit der Konstituenten und dem Aufwand ihrer Verarbeitung: Der integrative Typ könne im Vergleich mit dem nichtintegrativen Typ nur mit einem größeren Verarbeitungsaufwand produziert bzw. rezipiert werden, weil in diesem Fall die gesamte, komplexe Satzkonstruktion im Gedächtnis behalten werden müsse. ${ }^{65}$ Integrierte Strukturen haben dagegen den Vorteil, dass sie als grammatisch kohärent bewertet werden. Nach Lötscher (2005: 354f.) können beim Integrationsgrad der Adverbialsätze konkurrierende Bewertungskriterien angesetzt werden, deren Verhältnis sich zeitlich ändern kann. Dem sprachpsychologischen Kriterium des Verarbeitungsaufwandes wird das konkurrierende Kriterium der grammatischen Kohärenz entgegengesetzt, das im älteren Deutsch noch keine größere Rolle spielt, aber mit der Zeit immer mehr zur Geltung kommt:

Je besser integriert in eine syntaktische Struktur ein Element mit einer relevanten Funktion in der semantischen Struktur ist, desto kohärenter bildet die syntaktische Struktur die semantische Struktur ab, und desto höher wird eine Satzstruktur auf einer Kohärenzskala gegenüber anderen, weniger kohärenten und/oder integrierten Strukturen bewertet. [...] Je nachdem, in welchem Grad grammatische Kohärenz als Bewertungskriterium eine Rolle spielt, werden kohärente Strukturen gegenüber anderen Strukturen bevorzugt (oder eher nicht). (Lötscher 2005: 354f.)

Die jeweiligen Bewertungsmaßstäbe beeinflussen die Wahl zwischen den Varianten. Die Vermeidung des integrativen Typs bis zum Frühneuhochdeutschen wird ,auf eine generell beschränkte Toleranz gegenüber Satzkomplexität zurückgeführt“ (2005: 356). Zunehmende Integration sei deshalb unerwünscht, weil bei der Wahl der Stellungstypen zu dieser Zeit das sprachpsychologische Kriterium des geringen Verarbeitungsaufwandes entscheidend ist: „Je

\footnotetext{
64 Lötscher (2010) sieht methodische Probleme bei der Anwendung des Nähe-Distanz-Modells auf frühere Zeitstufen. Demnach sind z.B. die Kandidaten für nähesprachliche Signale in frühneuhochdeutschen Texten z.T. anders zu beurteilen als in gegenwartsdeutschen Texten, vgl. weiter Kapitel 2.2.2.

65 Die These von der Gedächtnisbelastung syntaktischer Konstruktionen wurde bisher v.a. in Bezug auf die Satzklammer diskutiert (s. Thurmair 1991 bzw. Marschall 1994).
} 
geringer integriert ein Teilsatz als Nebensatz ist, desto geringer ist der Verarbeitungsaufwand bei der (mündlichen) Sprachproduktion und -rezeption - und umgekehrt.“ (Lötscher 2005: 354) Der Wandel besteht nach Lötscher (2005: 358) darin, dass das Kriterium der grammatischen Kohärenz zunehmend höher gewichtet wird als das Kriterium des geringen Verarbeitungsaufwandes, bzw. somit formal mögliche syntaktische Varianten pragmatisch anders gewertet werden. Daher wird mit der Zeit ein größeres Maß an Nebensatzintegration toleriert und es verbreiten sich kohärentere Konstruktionen ${ }^{66}$, was Lötscher (2005: 358) auf die zunehmende Verschriftlichung des Deutschen zurückführt. Diese Kohärenz wird allerdings bei semantisch-pragmatisch lockeren Verbindungen im Sinne der Ikonizität nicht verlangt.

Die Veränderungen im Integrationsgrad vorangestellter Adverbialsätze werden also von Lötscher (2005: 358) nicht rein syntaktisch erklärt, sondern auf die Bewertung von den Sprechern und auf die Verschriftlichung der deutschen Sprache zurückgeführt. Die Wahl zwischen den Stellungstypen hänge auch von der jeweiligen Textsorte ${ }^{67}$ bzw. von stilistischen Faktoren ab (2005: 363). Bei Prosatexten werden methodisch nur schwer erfassbare, stilistische Kriterien behandelt. Die nicht-integrative Stellung kommt eher in Texten vor, die „der Rhetorik gesprochener Sprache“ nahe stehen, bzw. die ungezwungen, lebendig und adressatenorientiert formuliert werden (2005: 365). Fachtexte und Texte mit einem einfachen, nüchternen Satzbau weisen dagegen die nicht-integrative Stellung seltener auf. Auch die Ausbreitung des integrativen Typs seit dem Ende des 15. Jhs. ist nach Lötscher (2005: 369f.) ähnlich zu deuten: Dieser Stellungstyp sei ,zumindest zu Beginn Signal einer gewissen stilistischen Modernität in Texten elaborierter Sprache“ gewesen. Er war bei Texten von humanistisch gebildeten Autoren und gebildeten Stadtbürgern am wahrscheinlichsten. Der zu Beginn pragmatische, dann stilistische Wandel wird durch Konventionalisierung der (Nicht-)Verwendung einzelner Stellungstypen in bestimmten Kontexten $\mathrm{zu}$ einem grammatischen Wandel (Lötscher 2005: 372). Diese stilistischen Faktoren, deren Operationalisierung wohl nicht problemlos ist, werfen die Frage auf, ob sie nicht im Rahmen des Nähe-Distanz-Modells zu deuten sind.

Lötscher geht in einem jüngeren Beitrag der Frage nach, ob bestimmte Konstruktionen, „die gegenwartssprachlich potenziell als typisch mündlich und mithin nähesprachlich gelten“ (2010: 114) - u.a. die resumptive und nicht-integrative Anbindung der Adverbialsätze - auch

66 Auch weitere, stark integrative syntaktische Phänomene werden bis zum 15.-16. Jh. vermieden und danach häufiger verwendet, vgl. z.B. die Einschachtelung von Nebensätzen (Lötscher 2005: 356f., 370f.)

67 Vgl. dazu Kapitel 2.2.2.1. Syntaktische Unterschiede zwischen Prosa und Versdichtung sind in der Forschung auch für frühere Sprachstufen bekannt, zum Althochdeutschen s. Fleischer (2006: 27) mit weiterführender Literatur. 
in frühneuhochdeutschen Texten als Nähesignale zu identifizieren sind. Er räumt ein, dass die Kanditaten für nähesprachliche Signale in frühneuhochdeutschen Texten aus Zeitlichkeitsbedingungen $\mathrm{zu}$ erklären sind (2010: 118), betont aber, dass aggregative Formulierungsmuster im älteren Deutsch nicht unbedingt als nähesprachlich aufzufassen sind. Weniger integrative Satzanbindungen tauchen nämlich nicht nur in konzeptionell nähesprachlich(er)en Texten auf:

Im Gegenteil, sie kommen praktisch in allen Arten von Texten, auch solche ohne erkennbare Tendenz zu einer konzeptuellen Mündlichkeit vor. Anders gesagt, man kann die genannten Phänomene mindestens der damals generell gültigen Sprachnorm für geschriebene Sprache, wenn nicht sogar dem generellen Sprachsystem zuordnen. Beispielsweise ist der resumptive Anschluss von vorangestellten Nebensätzen im Mittelhochdeutschen praktisch obligatorisch [...]. (Lötscher 2010: 119)

In der Tendenz zu stärkerer Integrativität im 15. und 16. Jh. sieht Lötscher (2010: 127f.) ein prestigesprachliches Phänomen und deutet die integrative Anbindung der Adverbialsätze im Frühneuhochdeutschen als Distanzsignal.

Schrittweise werden in Texten von anspruchsvolleren Autoren [Hervorhebung von mir, P.K.] Formen verwendet, die weniger aggregativ oder grammatisch kohärenter sind als die herkömmlichen, dies aber offensichtlich in Kontexten, die als distanzsprachlich anzusehen sind. So verwenden etwa humanistische Autoren wie die Übersetzer Steinhöwel und Niclas von Wyle zunehmend die integrative Anbindung von vorangestellten Nebensätzen ohne resumptive Partikeln oder Pronomen, dies entgegen den Gebräuchen in den sonstigen zeitgenössischen Texten (Lötscher 2010: 128).

Im Frühneuhochdeutschen ist der integrative Typ eine markierte Form der konzeptionellen Schriftlichkeit, die resumptive Form ist dagegen unmarkiert. Der resumptive Typ kommt zwar im Neuhochdeutschen auch weiterhin vor, er wird jedoch mit dem nicht-integrativen Typ (bis auf spezielle Kontexte wie die Verwendung als Irrelevanzkonditionale) allmählich zurückgedrängt und wird zur markierten Form der konzeptionellen Mündlichkeit. An dieser Stelle sei anzumerken, dass semantische Unterschiede in den Untersuchungen von Lötscher (2005) und (2010) nicht systematisch berücksichtigt wurden. Die oben angeführten Überlegungen sprechen jedoch m.E. eindeutig für die systematische Berücksichtigung der Dimension Mündlichkeit/Schriftlichkeit in empirischen Untersuchungen. ${ }^{68}$

\subsection{Zur Notwendigkeit eines Neuansatzes}

Im Folgenden soll nun der Frage nachgegangen werden, welche Schlüsse sich aus dem oben skizzierten Forschungsstand ziehen lassen. Davon ausgehend werde ich die Notwendigkeit

68 Vgl. auch Mattheier (1995). In diesem Aufsatz wird scharfe Kritik an den zugrundeliegenden Theorien, an den Forschungsmethoden und an dem Gegenstandsbereich historischer Untersuchungen geübt. U.a. verweist Mattheier auf das Verhältnis von Mündlichkeit und Schriftlichkeit, das trotz einschlägiger Studien in letzter Zeit m.E. weiterhin zu den Desideraten der Sprachgeschichte gehört. 
eines Neuansatzes, einer empirischen Untersuchung des Integrationsgrades vorangestellter Adverbialsätze im Neuhochdeutschen, begründen.

Trotz der hohen Anzahl einschlägiger Arbeiten wissen wir bei weitem nicht alles über den Prozess der Integration der vorangestellten Adverbialsätze in den nachfolgenden Hauptsatz. Es mangelt an empirischen Untersuchungen der Adverbialsätze im Neuhochdeutschen, die auf alle Adverbialsatztypen eingehen. Bei den vorliegenden Korpusuntersuchungen sind größere Unterschiede $\mathrm{zu}$ beobachten, deren adäquate Erklärung bisher noch aussteht. Die quantitativen Unterschiede bei einzelnen Korpusauszählungen und die Schwankungen bei den Stellungstypen können m.E. nur dann adäquat erklärt werden, wenn auf die Auswahl der Korpustexte größerer Wert gelegt wird und die zugrunde liegende Methode stärker reflektiert wird.

Die Gemeinsamkeit der oben diskutierten grammatischen Erklärungen (2.2.3.1 und 2.2.3.2) sehe ich darin, dass sie sich auf die Entstehung einer Innovation konzentrieren. Bezüglich der Neuzeit dürften jedoch - im Sinne von Peter von Polenz (1995) - Fragen zur Verbreitung einer (zu ihrer Entstehungszeit) innovativen Form wichtiger sein als die Fragen zur Entstehung selbst. ${ }^{69}$ Die syntaktische Variation und die Verbreitung einer syntaktischen Form auf Kosten einer anderen syntaktischen Form sind in einem sozio-pragmatischen Kontext zu erfassen. Gegen eine rein grammatische Erklärung der Entwicklungen im Bereich der Satzintegration spricht auch die erhebliche Variation der Stellungstypen in Texten aus demselben Jahrhundert (vgl. Kappel 2008: 40f.). Außerdem zeigen viele Ergebnisse der oben referierten Forschungsliteratur (vgl. z.B. Kapitel 2.2.1.1) m.E. eindrucksvoll, wie wichtig die soziopragmatisch sensible, d.h. außersprachliche Faktoren berücksichtigende, Erfassung von syntaktischen Entwicklungen ist. Die Möglichkeiten des soziopragmatischen Ansatzes wurden bisher bei der Untersuchung der topologischen Integration nicht völlig ausgeschöpft. Einige Ergebnisse der einschlägigen Forschung (v.a. Auer 2000, Hammarström 1923, Lötscher 2010) deuten darauf hin, dass die Wahl zwischen den obigen Stellungstypen sehr wohl mit Unterschieden zwischen Mündlichkeit und Schriftlichkeit zu tun haben könnte. In mehreren Fällen wurden einschlägige Veränderungen in der Integration von Nebensätzen mit der Verschriftlichung in Zusammenhang gebracht (v.a. Lötscher 2005 und Ágel/Hennig 2006), dieser Aspekt wurde jedoch bisher nicht systematisch untersucht. Da die Untersuchung

69 Peter von Polenz (1998: 46) wirft die Frage zur Abgrenzung von Innovation und Sprachwandel auf: „Viele erst in der etablierten dt. Schriftsprache hochbewertete bzw. allgemein übliche Sprachmittel sind bereits Jahrzehnte oder Jahrhunderte früher als Varianten in bestimmten Quellen oder Textsorten nachzuweisen, waren also längst Teile des dt. Sprachsystems, ehe sie sprachgeschichtlich relevant (z. B. sprachkritisch auffällig) wurden. Wo genau ist die Grenze zwischen Innovation und Sprachwandel?““ 
des Verhältnisses der Stellungstypen und der Nähe-Distanz-Dimension besonders vielversprechend ist, wären gezielte Analysen der konzeptionellen Mündlichkeit (im Sinne von Koch/Oesterreicher 1985 bzw. Ágel/Hennig 2006a und 2006b) wünschenswert. Da dieser Aspekt in den Mittelpunkt der vorliegenden Untersuchung gestellt wird, sollen weitere Faktoren, deren Einfluss auf die Integration in der Forschung angenommen wurde (u.a. diatopische und Textsortenunterschiede), möglichst konstant gehalten werden (s. Kapitel 4.1).

In Bezug auf die Verteilung der einzelnen Stellungstypen im Neuhochdeutschen sollen noch zentrale Fragen beantwortet werden. Es sollte überprüft werden, ob die Faktoren, die die nicht-integrative Stellung im Gegenwartsdeutschen ermöglichen, auch im älteren Neuhochdeutsch gelten. Beim ,nicht-integrativen“ Typ sind zudem große Schwankungen in den einzelnen Korpora zu registrieren, deren Erklärung noch aussteht. Es sollte auch überprüft werden, ob alle nicht-integrativen Sätze wirklich in den gleichen Topf zu werfen sind, zumal sie nach der Festigung des integrativen Typs eine vom Normalfall abweichende, pragmatische Funktion übernehmen könnten. $\mathrm{Zu}$ prüfen ist u.a., ob dieser Wandel sich wirklich im Frühneuhochdeutschen vollzieht, oder auch im Neuhochdeutschen noch „Reste“ des älteren Gebrauchs zu finden sind (vgl. Axel-Tober 2012: 323).

Die Fragen, wie und wann sich der integrative Typ, dessen Ausbreitung seit dem Ende des 15. Jhs. andauert, etablierte bzw. wie und wann sich die von Lötscher angenommene konzeptionell mündliche/schriftliche Markiertheit der einzelnen Stellungstypen veränderte, wurden m.E. noch nicht befriedigend geklärt. Außerdem wurden bisher viele Detailfragen nicht systematisch untersucht. Man denke nur an die Faktoren, die zur Bevorzugung des resumptiven Typs führen können, wie etwa die Herstellung der syntaktischen Ruhelage (Behaghel 1903) bzw. der Raum zwischen Nebensatzanfang und Nachsatz. Eine eingehende Analyse verdient auch die Variation zwischen den verwendeten Korrelaten bei resumptiver Stellung. Es sollte auch überprüft werden, ob in den einzelnen Texten Zusammenhänge zwischen dem Grad der formalen Integration im Allgemeinen (vgl. Kapitel 3.3) und der topologischen Integration im Besonderen nachzuweisen sind.

Die in diesem Kapitel bezüglich der Syntax komplexer Sätze gewonnenen Erkenntnisse können resümierend wie folgt zusammengefasst werden: Bei der Untersuchung syntaktischer Phänomene im Neuhochdeutschen sind außersprachliche Faktoren zu berücksichtigen. Polenz (1995: 44) spricht sogar von der „Untrennbarkeit von Sprachsystemwandel und soziopragmatischer Sprachgeschichte in der Neuzeit“", da beim Sprachwandel die „Durchsetzung bestimmter Varianten in der Variantenselektion, also in der außersprachlich bedingten Entwicklung der Sprachnorm (in Coserius Sinne)“ eine wichtige Rolle hat (von 
Polenz 1995: 45). Mehrere syntaktische Veränderungen vom Mittelhochdeutschen bis zum Neuhochdeutschen könnten mit der Verschriftlichung und weiteren außersprachlichen Faktoren (u.a. kognitiv-kulturgeschichtlichen Bedingungen wie der Vertikalisierung im Sinne von Reichmann 1988, 2003, vgl. dazu Kapitel 3.5.2) in Zusammenhang stehen. Eine weitere Erkenntnis ist, dass die starre Dichotomie bei und die unreflektierte Übertragung von einschlägigen Termini vermieden werden sollte (,Adverbial', ,(Neben-)Satz‘, ,Vorfeld“ usw.). Diese Erkenntnisse sind wohl auch bei der Analyse der Integration von Adverbialsätzen im Neuhochdeutschen bedeutsam und prägen deshalb das Untersuchungsdesign der vorliegenden Arbeit (vgl. Kapitel 4.1).

\subsection{Hypothesen}

Aus dem Forschungsstand werden folgende Hypothese für die eigene Untersuchung abgeleitet. Es soll geklärt werden, wann (bzw. ob überhaupt) der Integrationsprozess zum Abschluss kommt. Daher soll überprüft werden, ob die Durchsetzung des integrativen Typs $\mathrm{zu}$ Beginn des Neuhochdeutschen bereits abgeschlossen ist und ob damit zu Beginn des Neuhochdeutschen eine Art Stillstand in der syntaktischen Entwicklung eintritt.

Hypothese 1: In den Korpustexten aus dem 17. Jahrhundert dominiert bereits der integrative Typ. Die Durchsetzung des integrativen Typs ist vor dem Beginn der neuhochdeutschen Periode bereits abgeschlossen.

Der integrative Typ stellt im heutigen Deutsch nach Zifonun et al. (1997: 2349) den Normalfall dar. Es soll überprüft werden, ob eine Dominanz des integrativen Typs auch in der neuhochdeutschen Periode (1650-2000) nachzuweisen ist.

Hypothese 2: Der integrative Typ ist die bevorzugte Variante im Neuhochdeutschen.

Des Weiteren bleibt zu klären, wie der Integrationsgrad verschiedener Typen von Adverbialsätzen ist. Auf einschlägige Unterschiede zwischen semantischen (wie etwa Konditionalen oder Konzessiva) und strukturellen Typen (eingeleitete vs. uneingeleitete Verberst-Sätze) wurde in der Forschungsliteratur mehrmals hingewiesen. Das begründet eine entsprechende Gliederung bei der Beschreibung empirischer Daten zum Neuhochdeutschen: Die einzelnen Typen werden getrennt behandelt und erst dann zueinander in Beziehung gesetzt.

Hypothese 3: Der Integrationsgrad ist bei den einzelnen semantischen Adverbialsatztypen z.T. unterschiedlich bzw. bei ihnen werden jeweils verschiedene Stellungstypen bevorzugt. 
Dadurch kann auch untersucht werden, ob es sinnvoll ist, die verschiedenen Typen gemeinsam unter dem Begriff Adverbialsätze zu subsumieren bzw. zu behandeln.

Zentrales Anliegen der Arbeit ist die Analyse der Veränderungen der Satzintegration im Neuhochdeutschen. Es soll in diesem Sinne überprüft werden, ob im Neuhochdeutschen relevante Häufigkeitsunterschiede beim Vorkommen der im Kapitel 1.1 angeführten Stellungstypen auftreten.

Hypothese 4: Im Neuhochdeutschen sind relevante syntaktische Veränderungen im Bereich der Satzintegration nachzuweisen, die sich in deutlichen Häufigkeitsunterschieden bei den Stellungstypen ,nicht-integrativ', ,resumptiv` und ,integrativ` erfassen lassen.

Letztlich soll der Einfluss außersprachlicher Faktoren beim Integrationsprozess nachgewiesen werden. Es soll überprüft werden, ob die Variable Mündlichkeit/Schriftlichkeit einen Einfluss auf die syntaktische Variation im Bereich der Satzintegration hat.

Hypothese 5: Bei der Verteilung der Stellungstypen ,nicht-integrativ', ,resumptiv ${ }^{\text {‘ }}$ und ,integrativ ${ }^{6}$ sind relevante Unterschiede zwischen konzeptionell mündlichen bzw. konzeptionell schriftlichen Texten nachzuweisen.

Wenn diese Hypothese bestätigt werden kann, dann wäre das ein Argument gegen die Auffassung des Integrationsprozesses als ein rein grammatisches Phänomen. 


\section{Theoretische Grundlegung}

\subsection{Terminologische Überlegungen}

Im Folgenden werden grundlegende Begriffe erläutert und die uneinheitliche Terminologie der Stellungstypen diskutiert. Zunächst sollte geklärt werden, was in der vorliegenden Arbeit unter ,Adverbialsatz' zu verstehen ist. Eine Definition des Begriffs ,Adverbialsatz sollte zwei Komponenten enthalten: Die erste Komponente bestimmt untergeordnete Satzstrukturen und sollte auf den Satz-Status Bezug nehmen (s. Kap. 3.1.1), die zweite Komponente ist rein funktional (s. Kap. 3.1.2).

\subsection{1 ,Satz ${ }^{6}$, Nebensatz}

In der historischen Syntaxforschung ist ein formaler, syntaktischer Satzbegriff verbreitet. Die vorliegende Arbeit ist angelehnt an diese Tradition. Die Teilsätze eines komplexen Satzes werden von Admoni (1990: 4f.) ,Elementarsätze` genannt. Dieser Begriff bezeichnet eine syntaktische Struktur, die im Grunde genommen

mit der Struktur des selbständigen Satzes übereinstimmt, ohne Rücksicht darauf, ob er eine abgeschlossene Einheit darstellt, und unabhängig davon, welche Rolle er im Redestrom spielt. Selbständiger Satz, Hauptsatz, Nebensatz, beigeordneter Satz - alle diese Sätze sind Elementarsätze.

Bei einem strukturellen Satzbegriff kann der Begriffsinhalt von ,Satz‘ generell eingeschränkt werden auf syntaktische Einheiten, die ein Verb (ein Finitum) als wichtigstes strukturbestimmendes Element aufweisen.

Die Satzwertigkeit wird auch in modernen Grammatiken des Gegenwartsdeutschen (vgl. z.B. die Duden-Grammatik 2016: 775f.) an das Vorhandensein eines Finitums gebunden. In der IDS-Grammatik findet sich folgende Definition: „Sätze sind übergreifende Konstruktionsformen, die mindestens aus einem finiten Verb und dessen - unter strukturellen und kontextuellen Gesichtspunkten - notwendigen Komplementen bestehen.“70 (Zifonun et al. 1997: 91) Das Vorhandensein des Subjekts wird jedoch nicht gefordert, sodass auch

70 Dieser formbezogenen Begriffsbestimmung steht die Definition der ,kommunikativen Minimaleinheit‘ als funktionale Einheit gegenüber. Nicht alle Sätze sind zugleich kommunikative Minimaleinheiten. Dies gilt v.a. für komplexe Sätze mit Ober- und Untersatz, die nur zusammen eine kommunikative Minimaleinheit bilden (Zifonun et al. 1997: 66ff.). 
subjektlose Sätze mit dem unpersönlichen Passiv (wie in (31)) bzw. Aufforderungssätze dem obigen Kriterium entsprechen (Zifonun 2015: 159).

(31) Heute wird mal wieder in die Hände gespuckt. (zit. n. Zifonun 2015: 159)

Die Verbzentriertheit in der Definition ist nachvollziehbar, denn „Finitheit ist notwendig für die Existenz eines Subjekts, die Existenz eines Subjekts jedoch nur hinreichend für ein finites Verb.“ (Zifonun 2015: 159)

Die Verbzentriertheit als Kriterium wird auch in der vorliegenden Arbeit angenommen. Die Anwendung der obigen Definition auf das ältere Deutsch ist jedoch problematisch. Beim epochentypischen Phänomen der sog. afiniten Konstruktionen, die v.a. im 17. und in der ersten Hälfte des 18. Jhs. verbreitet waren, wird das Finitum (i.d.R. das Hilfsverb sein, seltener haben) nicht realisiert, wobei das Vollverb und all seine Komplemente (inkl. Subjekt) im Satz stehen (vgl. Ágel 2000: 1888). In diesen Fällen ist also Finitheit anscheinend nicht notwendig für das Vorhandensein des Subjekts. Solche eingeleiteten Nebensätze ohne Finitum sind auch in den untersuchten neuhochdeutschen Korpustexten belegt:

(32) waß für Trauben so nicht gar zeitig gewesen, hat man müssen stehen lassen (Schuhmacher Chronik II: 443)

Der finite Prädikatsteil des vorangestellten Objektsatzes wird zwar eingespart, er ist jedoch eindeutig rekonstruierbar in Form des temporalen Hilfsverbs sein. Diese und ähnliche Fälle (wie etwa bei der integrativen Koordinationsellipse, vgl. Hennig 2010a: 85f.) werden in der vorliegenden Arbeit als ,Sätze“ betrachtet. $^{71}$ Im Gegensatz dazu sind „satzwertig[e] Ausdrücke mit einer infiniten Verbform (Partizip, Gerundium, Infinitiv), denen ein eigenes Subjekt fehlt oder fehlen kann“ (Blühdorn 2012a: 301), keine ,Sätze‘, sondern nur ,Satzäquivalente‘ (vgl. auch die Duden-Grammatik 2016: 905). Der folgende Beispielsatz von Blühdorn (2012a: 309f.) enthält eine Partizipialgruppe, die als Adverbial zu deuten ist:

(33) Angekommen in Las Vegas, stand für die Miss-Universe-Kandidatinnen ein volles Programm an.

Sie erfüllt jedoch - im Gegensatz zum Adverbialsatz Als sie in Las Vegas angekommen waren - das Satzkriterium nicht.

71 Einschlägig sind auch elliptische Strukturen des Typs ,Verdichtung der konditionalen Hypotaxe‘ (Auer 2002: 171ff.) wie in (i), in denen das fehlende Subjekt und Verb aufgrund des Kontextes rekonstruiert werden können:

(i) Wenn sie kommt, machen wir einen Spaziergang, wenn sie nicht kmt, bleiben wir zu Hause. (zit. n. Zifonun 2013: 308)

Solche kondensierten hypotaktischen Konstruktionen stellen bezüglich des Parameters ,Verlust an Satzwertigkeit‘ (im Sinne des Konzepts von ,clause linkage‘, Lehmann 1988, vgl. dazu Kapitel 3.3.1) eine integrativere Technik als die entsprechenden Satzstrukturen dar. 
Es kann Folgendes festgehalten werden: Alle satzförmigen Ausdrücke, die mindestens ein (an der Oberfläche realisiertes oder eindeutig rekonstruierbares) Finitum und dessen - unter strukturellen und kontextuellen Gesichtspunkten - notwendige Komplemente enthalten, werden als ,Sätze' bezeichnet. Als Oberbegriff für die übergeordneten oder untergeordneten Sätze in einem komplexen Satz (,Obersatz bzw. ,Untersatz') wird auch der Terminus ,Teilsatz‘ verwendet. Nun soll auf die übergeordneten bzw. untergeordneten Sätze näher eingegangen werden.

Die Bestimmung der Begriffe ,Hauptsatz‘ und ,Nebensatz‘ ist für eine historische Arbeit alles andere als trivial, daher sollen sie an dieser Stelle diskutiert werden. Traditionelle, dichotomisch geprägte Beschreibungen von Satzverknüpfungstechniken wurden bekanntlich (mit Recht) bereits mehrfach kritisiert (vgl. z.B. Hentschel/Weydt 2013: 373; Auer 1998). In Beschreibungen der genannten Art werden die beiden Begriffe ,Haupt-“ und ,Nebensatz“ scharf getrennt. Des Weiteren sind mehrere (oft synonym gebrauchte) dichotomische Termini auf die Art der syntaktischen Verknüpfung von Satzkonstruktionen zu beziehen, wie Hypotaxe vs. Parataxe, Subordination vs. Koordination, Unterordnung vs. Beiordnung/Nebenordnung (vgl. z.B. Bußmann 2008: 272, 507). Welche Kriterien zur Unterscheidung dieser Begriffe in den Gegenwartsgrammatiken hergezogen werden, hängt eng mit deren Konzeption zusammen. Während z.B. die IDS-Grammatik von einer kommunikativ-funktionalen Sichtweise geprägt ist und demnach eher die kommunikative (Un-)Selbstständigkeit als Kriterium nennt (Zifonun et al. 1997: 2235f.), zieht sich durch die Grammatik von Engel (1988 bzw. 2004) das Dependenzprinzip durch, sodass demnach das Kriterium der Abhängigkeit dominiert. Jede Grammatik ist jedoch gezwungen, bei der Unterscheidung der obigen Begriffe Kompromisse zu schließen. So kann zumindest die Einführung der Begriffe ,Hauptsatzfragment ${ }^{\star}$ in der IDS-Grammatik ${ }^{72}$ bzw. ,Hauptsatzkomplex`/,Hauptsatzgefüge‘ bei Engel (1988: 241 sowie 2004: 137) ${ }^{73}$ gedeutet werden. Man braucht wohl nicht besonders hervorzuheben, dass die Bestimmung der einschlägigen Begriffe für sprachhistorische Arbeiten noch viel problematischer ist. Dass der Begriff ,Nebensatz' nicht unreflektiert auf historische Sprachstufen angewandt werden kann,

72 Im Satz Niemand verstand, was mit dieser Behauptung gemeint war. ist der erste Teilsatz (der ,Obersatz'), dem der nachfolgende Nebensatz (,Untersatz') untergeordnet wird, bei der Ablösung aus dem Satzkomplex nicht selbständig. In solchen Fällen wird statt ,Hauptsatz' der Begriff ,Hauptsatzfragment' verwendet, da der Obersatz nur zusammen mit dem Untersatz eine (komplexe) kommunikative Minimaleinheit darstellt (Zifonun et al. 1997: 2238).

73 Im Satz Ich fürchte, er wird es bemerken. ist der abhängige Satz nach Engel (1988: 241) ein potentiell selbständiger Hauptsatz. In diesem Fall handelt es sich nicht um ein Satzgefüge, sondern um einen ,Hauptsatzkomplex ' (Engel 1988: 241) oder ein ,Hauptsatzgefüge‘ (Engel 2004: 137). Bei den konditionalen Angabesätzen (Engel 1988: 269 bzw. 2004: 148) ist jedoch unabhängig von der Form der abhängigen Sätze von ,Nebensätzen` die Rede, was die Unsicherheit bei der Einordnung von subjunktorlosen Satzkonstruktionen zeigt. 
wird von Ágel (2000: 1881) so auf den Punkt gebracht, dass er den Integrationsprozess vorangestellter Adverbialsätze als „Eingliederung des Noch-nicht-Nebensatzes in den Nochnicht-Hauptsatz in Nachstellung durch die Erstplazierung des Hauptsatzfinitums“ bezeichnet. Eine Bestimmung der Kategorie ,Nebensatz' nach dem formalen Kriterium - nach Verbstellung, wie dies etwa bei Fabricius-Hansen (1992: 460) geschieht - wäre deshalb problematisch, weil die formale Kennzeichnung der Nebensätze zeitlich veränderlich ist und ein epochenübergreifendes formales Merkmal schwer zu bestimmen wäre (zur formalen Markierung von Nebensätzen aus historischer Perspektive vgl. Kap. 2.2.1). Das Kriterium der Selbständigkeit wirft die Frage auf, auf welche Weise die einschlägigen Sätze eines historischen Quellentextes getestet werden könnten. Wie sollten nun die beiden Begriffe ,Haupt-` bzw. ,Nebensatz ${ }^{6}$ im Hinblick auf die Erfordernisse der historischen Syntax bestimmt werden?

M.E. sollte man in einer historischen Arbeit von einer relationalen Bestimmung der untergeordneten Sätze ausgehen. Statt der Form oder Selbständigkeit der einzelnen Teilsätze wird also nach der Beziehung zwischen den Teilsätzen gefragt. Eine entsprechende Unterscheidung findet sich auch in mehreren Gegenwartsgrammatiken. Nach Zifonun et al. (1997: 2236f.) werden die Begriffe ,Obersatz' und ,Untersatz' relational definiert: Die ,Untersätze‘ können als Teile der übergeordneten Sätze (,Obersätze`) aufgefasst werden. Auch für die Grammatik von Eisenberg (2013: 50, 318) ist ein relationales Begriffsverständnis charakteristisch, demnach sind ,Nebensätze“ als Teile der übergeordneten Sätze (meist ,Hauptsätze`) aufzufassen und haben eine syntaktische Funktion im Hauptsatz. Die Beziehung zwischen den Teilsätzen kann durch formale Merkmale gekennzeichnet werden, muss aber nicht. Mit diesen Begriffsbestimmungen ist gesichert, dass das zu untersuchende Phänomen in ein skalares Modell der Satzverknüpfungsmöglichkeiten integrierbar ist, das uns zu einem detaillierteren Bild über den Wandel der verschiedenen Techniken verhilft und dessen Grundzüge weiter unten erläutert werden (vgl. Kap. 3.3). Im Folgenden sei auf die Frage eingegangen, ob untergeordnete Sätze mit Verbendstellung (,Nebensätze‘ im Sinne von Fabricius-Hansen 1992: 460) bzw. weitere Untersätze mit V1 oder V2 immer eine Satzgliedfunktion im übergeordneten Satz haben müssen bzw. wie diese Satzgliedfunktion erfasst werden kann. 
Die Einsicht, dass die begriffliche Erfassung des ,Adverbials ' nicht unproblematisch ist, da es „die heterogenste unter den gebräuchlichen syntaktischen Relationen, ein typischer Restbegriff“" ist (Eisenberg 2013: 45), dürfte wohl verbreitet sein. ${ }^{74}$ In der vorliegenden Arbeit wird ein Adverbialbegriff verwendet, der neben der Satzgliedfunktion des Adverbials und seinem typischen propositionalen Bezug auch den sog. Sprechaktbezug ohne Satzgliedfunktion im engeren Sinne erlaubt. Diese Entscheidung kann auf folgende Gründe zurückgeführt werden: Erstens wird die Frage nach der Satzintegration in Bezug auf das Gegenwartsdeutsche insbesondere bei den sog. pragmatischen Nebensätzen, die keinen propositionalen Bezug zum übergeordneten Satz haben, eingehend behandelt (vgl. z.B. Pittner 1999; Wolf 1998; Zifonun et al. 1997: 2326ff.). Zweitens stellt die Grammatikalisierung der sog. nicht-integrativen Adverbialsätze im Neuhochdeutschen ein wichtiges Forschungsdesiderat dar. Ein zu eingegrenzter Adverbialbegriff hätte somit die Konsequenz, dass wesentliche Züge der Satzintegration nicht erfasst würden. Drittens gilt die Proposition nach Ágel (2007: 53) ,als digitales und modulares Organisationsformat für sprachliche Inhalte“, der Propositionsbegriff sollte daher nur zur „Beschreibung von kontextgrammatisch organisierten Inhalten“ verwendet werden, d.h. seine Übertragung etwa auf die althochdeutsche Zeit in sprachgeschichtlichen Untersuchungen wäre ,gegenstandsinadäquat““. Viertens ist in den letzten Jahrzehnten eine Loslösung vom traditionellen Verständnis des Terminus ,Adverbial` zu bemerken. Das Adverbial bezeichnet zwar nach Pittner ,,primär eine Satzgliedfunktion“ (1999: 47, vgl. auch 1999: 202), aber neben Adverbialsätzen, die Teil der Proposition eines Satzes sind (V-Adverbiale oder VP-Adverbiale) oder Sprechereinstellungen zur Proposition bezeichnen (Satzadverbiale, vgl. Pittner 1999: 46), werden u.a. auch ,sprechaktbezogene Adverbialsätze ${ }^{75}$ (Pittner 1999: 361) als besondere Unterklasse von Adverbialsätzen behandelt.

74 Zur Zeit der 4. Auflage des Grundrisses ist diese Formulierung von Eisenberg, die seit gut 20 Jahren in den neueren Auflagen unverändert beibehalten wurde, m.E. nach wie vor gültig, vgl. die folgende Formulierung von Pittner (2013b: o.S.): „Adverbiale stellen eine sehr heterogene Satzgliedfunktion dar, die ex negativo als die Funktion derjenigen Satzglieder bestimmt werden kann, die weder als Subjekt, Objekt noch als Prädikativ fungieren." Vgl. auch Pittner (1999: 46).

75 Die Terminologie ist dabei recht uneinheitlich, vgl. auch ,parenthetische Adverbiale‘ (Bartsch 1972: 66f.), ,Adverbialsätze mit Äußerungsbezug', ,illokutive oder Sprechakt-Adverbiale‘ (Pittner 1999: 46, 200, 320), ,pragmatische Adverbiale‘ bzw. ,pragmatische Konditionalsätze‘ (Pittner 2000), ,Metakommunikative Nebensätze im Vorvorfeld“ (Wolf 1998). Auch bezüglich des Englischen wird bei dieser Unterklasse von Adverbialsätzen der Sprechaktbezug hervorgehoben, vgl. z.B. Breul (1998: 137): „the position that one type of adverbial clauses are constituents of the VP of their superordinate clauses (and thus embedded) and that the other type of adverbial clauses are no constituents of other clauses at all (and thus 'tactic'), may have speech act theoretical implications". 
Der Begriff ,Adverbial` wird traditionell mit dem folgenden, allgemein formulierten semantischen Kriterium erfasst: „Adverbiale drücken die näheren Umstände des Geschehens aus: den Ort (Lokaladverbial), die Zeit (Temporaladverbial), die Art und Weise (Modaladverbial), den Grund (Kausaladverbial) u. a.“ (Dürscheid 2012: 38, vgl. dieselben Grundtypen bei Musan 2013: 5f.). Diese Grundtypen von Adverbialen sind Situativadverbiale, die „sich direkt auf eine Situation [beziehen] und an[geben], wo, wann, wie und warum sie stattfindet.“ (Musan 2013: 63) Es stellt sich die Frage, ob der Bezugsbereich des Adverbials bzw. des Adverbialsatzes am Verb festzumachen ist. „Adverbialsätze stehen in den meisten Ansätzen in direkter Abhängigkeit zum Verb des Matrixsatzes“" (Pasch/Zifonun 2006: 926). Mir scheint es sinnvoll, beim syntaktischen Kriterium eine Art Flexibilität zu erlauben: Adverbiale können sich sowohl auf das Verb als auch auf den ganzen Satz beziehen. Für Letzteres bringt Dürscheid (2012: 38) folgendes Beispiel:

(34) Wahrscheinlich kommt er heute nicht.

Innerhalb der Adverbialsätze unterscheidet die IDS-Grammatik (Zifonun et al. 1997: 2275) ,verbgruppenbezogene Adverbialsätze‘ und ,Satzadverbialsätze ‘ ${ }^{76}$ In diesem Sinne lässt sich der Begriff des Adverbials mit den Worten von Dürscheid (2012: 229) definieren: „Satzglied, das die näheren Umstände des im Verb ausgedrückten Geschehens bezeichnet oder sich auf die Aussage des ganzen Satzes bezieht.“ Diese Bestimmung erlaubt m.E. neben dem typischen propositionalen Bezug auch den Sprechaktbezug bzw. den epistemischen Bezug (d.h. Bezug auf den Wissenstand des Sprechers, vgl. Pittner 2013a: 501f.). Im Sinne der Unterscheidung von Sweetser (1990) kann der Begriff ,Adverbial' nicht nur auf die Sachverhaltsebene, sondern auch auf die Sprechaktebene und die epistemische Ebene bezogen werden (zum Konzept des Drei-Ebenen-Modells s. auch Breindl et al. 2014: 186-193, vgl. dazu 2.1.2). In diesem Sinne unterscheidet Pittner in ihrem Lexikonartikel zu ,Adverbial ‘ drei Möglichkeiten: ,Adverbial“ ist ein „Satzglied, das die Umstände eines Sachverhalts näher spezifiziert, eine Sprechereinstellung zum Sachverhalt angibt oder sich auf die Umstände der Äußerung bezieht.“ (2013b: o.S.) Bei Adverbialen, die Einstellungen des Sprechers zum bezeichneten Sachverhalt ausdrücken (emotionale Bewertungen wie z.B. leider, Bewertungen der Wahrscheinlichkeit wie etwa sicher oder Bewertungen hinsichtlich der Beteiligung des

76 Wenn sich der Subjunktorsatz auf eine nominale bzw. präpositionale Konstituente oder auf ein Adverb bezieht, wie im folgenden Beleg aus der Berliner Zeitung mit dem temporalen Subjunktor als, dann hat er die syntaktische Funktion eines Attributs (Zifonun 2013: 310):

(i) Im Jahr 2003, als das Wetter außergewöhnlich war, wurden in den Sommer- und Freibädern 2,4 Millionen Gäste gezählt. 
Subjekts am Geschehen wie z.B. intelligenterweise), bzw. bei Adverbialen mit Sprechaktbezug handelt es sich nicht um die Umstände eines im Verb ausgedrückten Sachverhalts. Ihr Satzgliedstatus ist auch deshalb fraglich, weil sie bestimmten Satzgliedeigenschaften nicht entsprechen (z.B. Erfragbarkeit und Ersetzbarkeit durch Pronomen, vgl. Pittner 2013b).

Die Frage, ob der Satzgliedstatus im herkömmlichen Sinne ein notwendiges Kriterium der Adverbiale ist, wird kontrovers beantwortet. Nach Pittner (2013a: 501) sind

Adverbialsätze [...] subordinierte Sätze, die in Bezug auf ihren Matrixsatz die syntaktische Funktion eines Adverbials ausüben. Adverbialsätze übernehmen eine Satzgliedfunktion in ihrem Bezugsatz und sind somit Gliedsätze.

Nach dieser Definition handelt es sich in dem Fall, wenn ,einige oder sogar alle Satzgliedeigenschaften“ fehlen (2013a: 521), ,strenggenommen“ nicht um Adverbialsätze. An anderer Stelle (Pittner 2013b) werden jedoch die Satzgliedeigenschaften nicht als notwendiges Kriterium des Adverbialbegriffs angesehen, was die Unsicherheit beim Umgang mit den traditionellen Termini eindrücklich belegt.

Das Dilemma ist auch im Satzgliedkonzept selbst begründet. Neef (2014: insbes. 448ff.) kritisiert die traditionelle Definition des ,Satzglieds‘ und stellt den Satzgliedbegriff in seinem radikalen Vorschlag im Grunde genommen mit ,Ergänzung ${ }^{67}$ gleich, was jedoch dazu führt, „dass das Adverbial aus der Menge der Satzgliedfunktionen ausscheidet; Adverbiale sind Elemente von Sätzen, die nicht unter die Kategorie Satzglied fallen“ (2014: 451). Neef räumt jedoch ein, dass eine sehr heterogene Gruppe entsteht, wenn man den Adverbialbegriff in diesem Sinne als eine Art Restklasse bestimmt (2014: 453).

Bei einem strengen Begriffsverständnis müsste auch traditionellen Adverbialen wie etwa Konsekutivsätzen mit so dass/sodass der Adverbialstatus bzw. der Satzgliedcharakter aberkannt werden, weil sie „weder erfragbar, verschiebbar, noch pronominalisierbar sind“ (Pittner 2013a: 516, s. auch Demske 2009). Der Ausweg aus dieser terminologischen Sackgasse könnte ein Prototypenansatz sein, den Pittner nahelegt. Der Kernbereich der Adverbialsätze lässt sich wohl mit ,prototypische[n] Gliedsätze[n]“(2013a: 521) erfassen. An

77 Eine Ergänzung ist nach Dürscheid (2012: 231) ein „Satzglied, das valenzgebunden ist“. Adverbiale sind nach der traditionellen Auffassung meist Supplemente (d.h. freie Angaben), sie können jedoch auch Komplemente (Ergänzungen) sein (vgl. z.B. Pasch/Zifonun 2006: 921, 932). Von einigen Verben wird z.B. ein bestimmter Adverbialtyp mit dem Merkmal [+dir] (Richtungsadverbial) selegiert (Pittner 1999: 51). Bei Verben wie sich freuen, sich ärgern registriert Eisenberg (2013: 339) einen „Kollaps von adverbialer und Ergänzungsfunktion der wenn-Sätze“ wie in Wir freuen uns, wenn du kommst. In solchen Fällen bleibt die Rekonstruktion der Adverbialfunktion notgedrungen eine Frage der Interpretation. In der vorliegenden Untersuchung werden valenzgebundene Adverbialsätze nicht berücksichtigt. Beim Stellungsverhalten bzw. bei den anaphorischen Elementen im nachgestellten Obersatz treten nämlich wichtige Unterschiede auf, vgl. den folgenden Beleg:

(i) Als ich in der Badewanne lag und mich alle verwöhnten, war das, wie ein kleines Wunder, wie ein schöner Traum, wie ein Happy End einer melancholischen Geschichte. (Jugendtagebuch VII: 15) 
der Peripherie finden sich Adverbialsätze im weiteren Sinne, bei denen die Satzgliedeigenschaft fehlt und die daher auch begrifflich von den prototypischen Adverbialsätzen, die sich auf die Proposition ihres Bezugssatzes beziehen, unterschieden werden müssen (so bereits beim Terminus ,Sprechakt-Adverbiale' bei Pittner 1999: 320). Diese könnte man in Anlehnung an Zifonun et al. (1997: 2275) als ,periphere Adverbialsätze“ bezeichnen. $^{78}$ M.E. ist dieser Schritt, den Pittner selbst nicht explizit durchführt, unentbehrlich, selbst wenn dadurch der Bruch mit traditionellen Annahmen der Schulgrammatik in Kauf genommen werden muss.

Auch der Blick in neuere Syntaxdarstellungen untermauert die Plausibilität der Berücksichtigung der nicht-prototypischen Adverbiale. So können etwa nach Musan (2013: 63) auch folgende, nicht-prototypische Adverbiale unterschieden werden, die ,im weitesten Sinne inhaltliche Verhältnisse zwischen Situationen angeben“: Substitutiv-, Explikativ-, Restriktiv- und Irrelevanzkonditionaladverbiale. Außerdem gibt es weitere Adverbiale, die „sich gewissermaßen ,von außen“ auf die Satzaussage [beziehen]“ (Musan 2013: 63): Sie unterscheidet zwei Unterarten von Satzadverbialen: Kommentaradverbial und Textadverbial. Auch bei Boettcher (2009: 194) werden neben Satzgliedern, die auf der propositionalen Ebene angesiedelt sind, auch solche behandelt, die eher zum pragmatischen Gehalt des Satzes beitragen.

In der vorliegenden Arbeit werden im Sinne der obigen Ausführungen auch periphere, d.h. nicht-prototypische Adverbiale mitberücksichtigt. Sprechaktadverbiale, bei denen die Frage nach der Integration der vorangestellten Nebensätze eingehend behandelt wird, werden dadurch ebenfalls erfasst. Für die vorliegende Arbeit wird aufgrund der oben referierten Begriffsbestimmungen folgende übergreifende Definition vorgeschlagen: Adverbiale beziehen sich entweder auf ein Verb bzw. eine Verbgruppe (,Verbgruppenadverbiale') oder auf einen ganzen Satz (,Satzadverbiale') und geben in Bezug auf diese inhaltliche Verhältnisse im weitesten Sinne an. Entweder drücken sie die näheren Umstände des im Verb

78 In der vorliegenden Arbeit werden mit dem Terminus ,periphere Adverbialsätze - im Gegensatz zu Zifonun et al. (1997: 2323 ) - nicht nur Komitativsätze, Konfrontativsätze und periphere Vergleichssätze erfasst, sondern auch alle nicht propositionsbezogenen Satzadverbialsätze, inkl. modusmodifizierende, moduskommentierende, diktumskommentierende und weiterführende Adverbialsätze, die inhaltlich ebenfalls locker angefügt sind (vgl. Zifonun et al. 1997: 2242ff.). Weitere Beispiele für die Unterscheidung von Adverbialsätzen im Rahmen der Prototypentheorie liegen mit Haegeman (2006), Frey (2011) und Blühdorn (2013) vor. Haegeman (2006: 1653) unterscheidet für das Englische ,central adverbial clauses' bzw. ,peripheral adverbial clauses': Während ,central adverbial clauses“ „to events/states of affairs, and lack manifestations of speaker anchoring" referieren, sind ,peripheral adverbial clauses' diskursbezogen. Nach Haegeman (2006: 1654) können ,peripheral adverbial clauses' nicht oder nur schwach in ihren Matrixsatz eingebettet werden. Blühdorn (2013) verwendet zur Unterscheidung von zentralen und peripheren Subjunktorsätzen die syntaktischen Testverfahren Negation (das Kriterium, ob sie im Skopus der Negation liegen können) und Abspaltung mit Es ist/war... . Nach Frey (2011: 75) können jedoch auch ,peripheral adverbial clauses' eingebettet, d.h. in ihren Matrixsatz syntaktisch integriert, auftreten. 
ausgedrückten Geschehens aus (und sind somit Teil der Proposition, ,propositionsbezogene Adverbiale') oder sie tragen zum pragmatischen Gehalt des Satzes bei, indem sie die Umstände der Äußerung über einen Sachverhalt bzw. eine Sprechereinstellung zum Sachverhalt näher spezifizieren. Adverbiale haben typischerweise eine Satzgliedfunktion und sind nicht valenzgebunden. Nicht-propositionsbezogene Adverbiale sind jedoch keine Satzglieder im engeren Sinne (,periphere oder pragmatische Adverbiale“).

Der Begriff ,Adverbialsatz‘ kann als Einschränkung der obigen Definition des ,Adverbials' mit Hilfe des Satzkriteriums (vgl. Kap. 3.1.1) bestimmt werden, nämlich als ein Adverbial in Form eines untergeordneten Teilsatzes in einem komplexen Satzgefüge. Aufgrund der oben zitierten Begriffsbestimmungen von Pittner (2013a: 501 und 2013b) ließe sich folgende Definition ableiten: Adverbialsätze sind subordinierte Sätze, die typischerweise eine Satzgliedfunktion in ihrem Bezugsatz übernehmen (und somit Gliedsätze sind). Sie können nicht nur die Umstände eines Sachverhalts näher spezifizieren, sondern auch eine Sprechereinstellung zum Sachverhalt angeben oder sich auf die Umstände der Äußerung beziehen.

\subsubsection{Stellungstypen}

Es wurde bereits in Anlehnung an König/van der Auwera (1988) angedeutet, dass bei vorangestellten Adverbialsätzen im Gegenwartsdeutschen eine synchrone Variation zwischen den drei Stellungstypen existiert (s. Kapitel 1.1). Vorangestellte Adverbialsätze können in die Satzstruktur des übergeordneten Satzes eingefügt werden (,integrativ', vgl. (35)), oder mit einem Korrelat vor dem Hauptsatzfinitum wiederaufgenommen werden (,resumptiv`, vgl. (36)) bzw. von der Satzstruktur des Hauptsatzes unabhängig bleiben (,nicht-integrativ`, vgl. (37)):

(35) Weil er krank ist, kann Fritz nicht mitkommen.

(36) Wenn er krank ist, dann bleibt er zu Hause.

(37) Wenn ich die Bilanz der letzten Jahre zog, es blieb ein Plus.

In der Fachliteratur wurden auch andere Termini für die obigen Stellungstypen verwendet. Diese sollen im Folgenden knapp diskutiert werden.

Paul (1920) unterscheidet grundsätzlich nur zwei Stellungstypen und sieht bei resumptiver Stellung keinen eigenen Typ, sondern eher eine Alternative zur topologischen Integration, mit der Begründung, dass in diesem Fall auch kein selbständiges Satzglied vor dem Verb des 
Nachsatzes gebildet wird (vgl. 1920: 316). Das entscheidende Kriterium für seine Beschreibung der Wortstellung im Nachsatz ist also, ob vor dem Verb des Nachsatzes ein selbständiges Satzglied steht.

Hammarström (1923: 50f.) unterscheidet drei Stellungstypen im Nachsatz, die mit den folgenden Termini bezeichnet werden: ,gerade Stellung', ,ungerade Stellung ' und ,Anfangsstellung des Verbs'. Problematisch dabei ist m.E. die Unterscheidung zwischen gerader und ungerader Stellung, die ja von der Position des Subjekts im Verhältnis zum Verb abhängt. Während bei der geraden Stellung das Subjekt vor dem Verb des Nachsatzes steht, folgt es bei der ungeraden Stellung dem Verb, d.h. vor dem Verb steht ein anderes Element (z.B. ein resumptives so). Die gerade Stellung entspricht nicht völlig dem nicht-integrativen Typ, sondern nur einem Untertyp des nicht-integrativen Typs, bei dem vor dem Verb das Subjekt - und nicht ein anderes Satzglied steht. Daher ist auch die ungerade Stellung nicht einfach mit dem resumptiven Typ gleichzusetzen, denn zu dieser Möglichkeit gehören zudem auch alle Verwendungsweisen, bei denen nicht das Subjekt, sondern ein anderes Satzglied vor dem Verb des Nachsatzes steht, etwa auch der folgende Beleg:

\section{(38) Damit ichs nun aussage; heut Mittag kam Wallroth. (Wagner III: 56)}

Behaghels Aufsatz „Der Nachsatz“ (1929) war richtungsweisend für die Untersuchung der Integration vorangestellter Nebensätze. Er erklärt den Unterschied in der Stellung des Subjekts als irrelevant (1929: 401f.). In neueren Arbeiten wird die Stellung eines selbständigen Satzglieds vor dem Verb als eigenständiger Typ behandelt, egal ob es ein Subjekt oder ein anderes Satzglied ist. Behaghel (1929: 401f.) unterscheidet drei Möglichkeiten (,hauptsatzstellung', ,conjunctionelle einleitung', , anfangsstellung'), die zwar nicht mit der später gängig gewordenen Terminologie bezeichnet werden, aber im Grunde genommen den Typen von König/van der Auwera (1988) entsprechen. Obwohl die Bezeichnungen Behaghels aus mehreren Gründen problematisch sind, ${ }^{79}$ soll festgehalten

79 Die Bezeichnung, conjunctionelle einleitung‘ ist m.E. missverständlich, weil sie bei Behaghel (1929: 409) nicht nur auf Hauptsätze, sondern auch auf eingeleitete Nebensätze (Vordersätze) bezogen wird, die somit ebenfalls ,,mit conjunction eingeleitet" werden können. Auch die beiden anderen Bezeichnungen sind nicht befriedigend, denn sie berücksichtigen nur die Verbstellung im nachfolgenden Hauptsatz. Damit kann das dreistufige Kontinuum zwischen Integration und Desintegration nicht präzise erfasst werden. Unpräzise ist dabei die Bezeichnung ,hauptsatzstellung', denn neben dem durchaus gängigen Typ der Aussage-V2-Sätze existieren auch Aussage-V1-Sätze. Bei direktem Anschluss des Hauptsatzverbs wäre demnach Anfangsstellung und Hauptsatzstellung nicht klar zu trennen. Eine ,anfangsstellung' des Hauptsatzverbs ist auch bei Entscheidungsfragesätzen oder Imperativsätzen zu beobachten, denen ein Adverbialsatz vorangestellt wird. In diesen Fällen zeigt jedoch die Anfangsstellung des Verbs - wie weiter unten gezeigt wird - keine topologische Integration des Vordersatzes an. Außerdem wurde auch die umstrittene Wortartzugehörigkeit der Elemente wie so angesprochen: Sie seien von den reinen Konjunktionen, die keinen Satzgliedcharakter aufweisen, auch begrifflich zu trennen (s. Horacek 1957: 423ff.). Auf diese letzte Frage wird weiter unten eingegangen. 
werden, dass in jüngerer Zeit im Grunde genommen Behaghels Klassifizierung verwendet wird. Die Terminologie und der theoretische Rahmen wurden jedoch weiter verfeinert.

Horacek (1957: 428) erfasst mit ihrer Gruppierung m.E. ebenfalls das Wesentliche der Wortstellungseigenschaften. Als Ausgangspunkt nimmt sie die obige Gruppierung von Behaghel und modifiziert sie v.a. beim zweiten Typ. Sie unterscheidet folgende Typen: Erstens kann der Nachsatz unabhängig vom Vordersatz sein; zweitens kann der Vordersatz mit einem eröffnenden Stellungsglied des Nachsatzes aufgenommen werden; drittens kann der Nachsatz durch das Verb eröffnet werden. Diese Gruppierung entspricht weitgehend den drei Typen von König/van der Auwera (1988). Der Leser vermisst nur noch kurze, aus einem Wort bestehende Bezeichnungen für die jeweiligen Typen. Was Horaceks Klassifizierung besonders auszeichnet, ist die Charakterisierung der aufnehmenden Elemente beim zweiten Typ als Satzglieder. Diese Auffassung ist zwar nicht unproblematisch, es soll jedoch eingeräumt werden, dass es Horaceks Verdienst ist, die Frage nach dem Satzgliedcharakter der aufnehmenden Elemente überhaupt aufgeworfen zu haben. Neuerdings wird eher die Auffassung akzeptiert, nach der die aufnehmenden Elemente auf Satzgliedebene mit den Vordersätzen zusammengehören (s. dazu ausführlicher Kapitel 3.3.2).

Axel (2002: 2ff.) verwendet in ihrem Aufsatz zur Integration von Adverbialsätzen im Althochdeutschen auf der Grundlage der Unterscheidung von König/van der Auwera (1988: 102f.) eine andere Terminologie, in der (ähnlich wie bei Behaghel, s.o.) auf die Verbstellung zurückgegriffen wird. Sätze wie (1) und (3) werden von Axel (2002: 3) als V2 bzw. V3 bezeichnet. Obwohl die Bezeichnung V2 beim integrativen Typ noch sinnvoll wäre, da in Aussagesätzen wie in (1) das Verb nicht am Satzanfang (vgl. ,,anfangsstellung“ bei Behaghel, 1929: 401f.), sondern an der zweiten Position hinter dem integrierten Nebensatz steht, ist der Terminus V3 problematisch. Er impliziert nämlich, dass der vorangestellte Nebensatz in Sätzen wie (3) an der ersten Position des Hauptsatzes steht und wirklich zu seiner Satzstruktur gehört. In solchen Fällen scheint dagegen eine der tatsächlichen Satzstruktur vorangestellte und damit nur lose verknüpfte Konstruktion vorzuliegen. Nach Axel (2002: 19) können vorangestellte Adverbialsätze ,auch in der V3-Struktur unintegriert links ihres deklarativen Bezugssatzes auf[treten]”. Bei anderen unintegrierten, lose verknüpften „linksperipheren“ Adverbialsätzen, die mit direkt nachgestelltem Hauptsatzfinitum angebunden werden, wird jedoch das Prinzip der Verbstellung aufgegeben. Axel (2002: 22) deutet sie nicht als V2, sondern als V1-Apodosis (Adverbialsatz links von einem V1-Deklarativsatz). Der terminologische Gebrauch in Axel (2002: 2ff.) ist daher m.E. problematisch. 
Im Folgenden soll die Frage geklärt werden, ob die Stellungstypen von König/van der Auwera (1988: 102f.) auf komplexe Sätze mit V1-Obersätzen angewandt werden können. Bei Imperativsätzen oder Entscheidungsfragesätzen ist die Anfangsstellung des Verbs obligatorisch und zeigt keine topologische Integration des vorangestellten Adverbialsatzes an. Pittner (1999: 303) nimmt daher beim folgenden Beispielsatz völlig zu Recht eine nichtintegrierte Stellung des Vordersatzes an: ${ }^{80}$

(39) Wenn Ihnen Ihr Alltag arm scheint, klagen Sie ihn nicht an.

Wenn nach einem vorangestellten Adverbialsatz direkt das Finitum eines Imperativsatzes bzw. Entscheidungsfragessatzes realisiert wird, darf keine Vorfeldstellung des vorangestellten Adverbialsatzes (=, integrativ') angenommen werden, da in diesen Fällen das Vorfeld i.d.R. nicht besetzbar ist. ${ }^{81}$ Anhand der folgenden Beispielsätze soll überprüft werden, ob bei diesen Satztypen die nicht-integrative Stellung die einzige Wahl ist. Der Obersatz im folgenden Beleg ist ein Entscheidungsfragesatz mit Verberststellung: ${ }^{82}$

(40) Und selbst hätte die Schichauwerft ihn geklaut, wäre dir dann geholfen gewesen? (G. Grass, zit. n. Pittner 1999: 302)

Der Obersatz weist jedoch ein Korrelat (dann) im Mittelfeld auf, was Pittner (1999: 301f.) zur Annahme einer linksversetzten Konstruktion veranlasst. Die Mittelfeldstellung des Korrelats unterscheidet sich jedoch vom klassischen Typ ,resumptiv ${ }^{6}$ mit Stellung des Korrelats im Vorfeld des übergeordneten Aussagesatzes. Da beim Entscheidungsfragesatz kein Vorfeld vorhanden ist (vgl. auch Zifonun et al. 1997: 641), kann diese Stellungsvariante nur innerhalb des nicht-integrativen Typs behandelt werden. ${ }^{83}$ Daher ist anzunehmen, dass selbst beim topologisch am schwächsten integrierten Typ relativ schwächer und stärker integrative Techniken unterschieden werden können, was in einer Analyse der Integriertheit mitberücksichtigt werden sollte (vgl. Kapitel 3.3.1).

80 Auer (1997: 70) und Günthner (1999a) analysieren entsprechende Belege aus der gesprochenen Sprache ebenfalls nicht als Vorfeldstellung, sondern als Vor-Vorfeldstellung des wenn-Satzes vor einem Imperativ- oder Fragesatz.

$81 \mathrm{Zu}$ (scheinbaren) Ausnahmen für Aufforderungssätze und Fragesätze mit Verbzweitstellung vgl. Zifonun et al. (1997: 654f. bzw. 643f.).

82 Nach Günthner (1999a: Fußnote 32) ist bei Entscheidungsfragesätzen in der gesprochenen Sprache „schwieriger zu beurteilen“, ob der vorangestellte Adverbialsatz im Vorfeld oder im Vor-Vorfeld seines Obersatzes steht. Sie schließt eine Vorfeldbesetzung bei Entscheidungsfragesätzen nicht aus und nimmt das Kriterium der prosodischen (Des-)Integration für die Unterscheidung zwischen Vorfeld- bzw. Vor-Vorfeldkonstruktionen an.

83 Integrativ angeschlossene Adverbialsätze mit einem Korrelat im Mittelfeld des Obersatzes wie im folgenden Beleg (i) aus dem Deutschen Referenzkorpus (DeReKo, Institut für Deutsche Sprache 2016) sind analog: Da sie nicht im linken Außenfeld stehen, ist keine ,resumptive" Stellung, sondern eine Variante der ,integrativen' Stellung anzunehmen.

(i) Sollte Volkswagen zur Finanzierung von Rückholaktionen und Gerichtsfällen frisches Geld vom Kapitalmarkt brauchen, sind dann die Voraussetzungen dafür schlecht. (P15/NOV.03612 Die Presse, 28.11.2015, S. 18; Wie Aktionäre das Management torpedieren)

Das Vorhandensein des Korrelats im Mittelfeld des Obersatzes ist also sowohl beim integrativen als auch beim nichtintegrativen Stellungstyp möglich. 
Welcher Stellungstyp ist anzunehmen, wenn das Korrelat bei Imperativsätzen nicht im Mittelfeld des Obersatzes erscheint? Im folgenden Internetbeleg aus dem Deutschen Referenzkorpus (DeReKo, Institut für Deutsche Sprache 2016) steht nach dem vorangestellten wenn-Satz ein resumptives so, erst darauf folgt das Finitum des Aufforderungssatzes:

(41) Wenn das so ist, so nenne mir bitte Quellen, sonst glaube ich das nicht. (WDD11/B01.01563: URL: http://de.wikipedia.org/wiki/Diskussion:Bandbreite: Wikipedia, 2011)

Bei diesem Typ kann - aus demselben Grund wie bei (39) - weder der vorangestellte Adverbialsatz noch das Resumptivum topologisch in den Aufforderungssatz integriert werden. Für so kann in diesen Fällen eine Position angenommen werden, „die auch Konjunktoren einnehmen, nämlich zwischen den Konnekten“" (Pasch et al. 2003: 487), also eine Position im linken Außenfeld. Diese Annahme machen auch Sätze wie (42) plausibel, in denen das Vorfeld des Obersatzes durch das Fragewort wie besetzt ist. Schließt man die Möglichkeit der mehrfachen Vorfeldbesetzung durch dann und wie aus, so ist auch in diesem Fall aus einer Stellung des resumptiven Elements im linken Außenfeld auszugehen:

(42) Wenn Liebe Unsterblich ist dann wie sollte Scheidung betrachtet werden? (http://www.proghiphop.com/wenn-liebe-unsterblich-ist-dann-wie-sollte-scheidungbetrachtet-werden/)

Die Unterscheidung zwischen ,resumptivem“ und ,nicht-integrativem Typ ist bei solchen Sätzen aus terminologischer Sicht nicht unproblematisch. Vom ,resumptiven Typ` im Sinne von König/van der Auwera (1988: 102f.) kann nur dann die Rede sein, wenn eine klassische Herausstellungsstruktur mit linksangebundenem Adverbialsatz und resumptive Wiederaufnahme im Vorfeld des V2-Satzes vorliegt. Die oben diskutierten Aufforderungsund Fragesätze haben jedoch die Eigenschaft gemeinsam, dass das resumptive Element nicht im Vorfeld des Obersatzes steht bzw. stehen kann. Diese sind daher als ,nicht-integrativ` zu analysieren. Im Bereich der topologischen Desintegration können aber neben der korrelatlosen Technik (wie im Beleg (39)) die Technik mit nicht-resumptiven Korrelat im Mittelfeld des Obersatzes (wie im Beleg (40)) und das Auftreten eines Korrelats im linken Außenfeld des Obersatzes (wie im Beleg (41)) unterschieden werden.

Zusammenfassend lässt sich feststellen, dass das Dreiermodell von König/van der Auwera (1988: 102f.) nur für die Erfassung der wohl sehr frequenten Aussagesätze verwendbar ist. Bei Aufforderungs- und Fragesätzen ist die Wählbarkeit der Stellungstypen anders als bei komplexen Sätzen mit V2-Obersätzen, daher sollen diese Typen bei der Gesamtbewertung des Integrationsgrades getrennt behandelt werden. Obwohl die Terminologie von König/van 
der Auwera (1988: 102f.) nicht widerspruchsfrei ist, hat sie sich sowohl in der gegenwartsbezogenen als auch in der sprachhistorischen Forschungsliteratur eingebürgert (vgl. z.B. Karhiaho 2003: 95, Lötscher 2005: 347f., Lötscher 2010: 129, Pittner 1999: 295ff., Van den Nest 2005: 34f., Zifonun et al. 1997: 2349), und wird auch der vorliegenden Untersuchung zugrunde gelegt. Die Integration vorangestellter Adverbialsätze wird - im Anschluss an König/van der Auwera (1988: 102f.) - i.d.R. auf der Grundlage des Konzepts des Stellungsfeldermodells erfasst (vgl. Zifonun et al. 1997: 2349, Lötscher 2005: 347f., Pittner 1999, Van den Nest 2005: 34f.). ${ }^{84}$ Bevor auf diesen Theoriebaustein eingegangen wird, sollen jedoch die Termini ,Korrelate‘ bzw. ,Resumptiva‘ diskutiert werden.

\subsection{4, Korrelate“, ,Resumptiva“}

Unter ,Korrelat“ versteht man ein „Element, das als Bezugseinheit für einen Nebensatz oder eine Infinitivgruppe fungiert“ (Glück/Rödel 2016: 376). Korrelate haben eine Verweisfunktion, sie können auf untergeordnete Sätze voraus- oder zurückverweisen (vgl. Zifonun et al. 1997: 1475). „Im Standardfall verbleiben sie im Matrixsatz, während der Teilsatz, den sie vertreten, extraponiert wird.“ (Zitterbart 2013: 603). Sie werden auch ,Platzhalter' genannt (zu einer ausführlicheren Begriffsbestimmung s. Zitterbart 2013: 602607).

Wenn der korrelierte Nebensatz dem Korrelat vorausgeht, erscheint er im linken Außenfeld, das darauf folgende Korrelat besetzt das Vorfeld des übergeordneten Satzes (vgl. z.B. Zifonun et al. 1997: 1492 sowie Kapitel 3.2.2, für Beispielsätze s. (2) oder (36)). Das Korrelat fungiert in solchen Fällen als Resumptivum, ,indem es den ersten Teil der Aussage wiederaufnimmt“ (Zitterbart 2013: 621). Im Kapitel 3.3.1 werden auch nichtresumptive Korrelate behandelt.

Was die semantische Leistung der Korrelate angeht, so werden mehrere Verwendungen unterschieden (Zifonun et al. 1997: 1492f., Zitterbart 2002: 164). Neben Korrelaten mit semantisch eindeutiger Funktion (z.B. deshalb, darum, die selbst als Kausaladverbien verwendet werden) gibt es auch Korrelate, die semantisch weniger spezifisch (dann, $d a$, die nicht auf eine semantische Klasse festgelegt sind) oder sogar unspezifisch sind $(s o) .{ }^{85}$ Bei der

\footnotetext{
84 Auch bei Axel wird öfter auf die Bedeutung des Vorfelds verwiesen (z.B. 2002: 3-7), obwohl ihrem Aufsatz der theoretische Rahmen der Generativen Grammatik zugrunde liegt. Bezüglich ihrer Terminologie kritisiert jedoch Lötscher (2005: 351) m.E. zu Recht, dass es unklar ist, „,wie die V3-Stellung in Bezug auf das Konzept der Felderstruktur des deutschen Satzes zu interpretieren ist".

85 Baschewa (1983: 80) definiert Korrelate als Wörter im übergeordneten Satz, die „als Indikatoren der semantischen Relation zwischen den verknüpften Satzbedeutungen [gelten]“. Bei Konzessivsätzen sind danach die Korrelate doch,
} 
Wahl der Korrelate sind die jeweiligen semantischen Klassen relevant, so wird etwa $d a$ eher bei durch als eingeleiteten Temporalsätzen oder Lokalsätzen, so dagegen bei durch wenn auch eingeleiteten Konzessivsätzen und bei Irrelevanzkonditionalen bevorzugt (vgl. Zifonun et al. 1997: 1493f.). In manchen Fällen ist jedoch eine Variation bei der Wahl der Korrelate nachzuweisen und diese Variation scheint im Lichte der modernen Forschung im Gegenwartsdeutschen nicht beliebig zu sein. Redder (1987: 315) untersucht „die spezifische Leistung von so im Unterschied zu dann" in resumptiv angebundenen wenn-Sätzen. Auf die Propositionen in diesen wenn-Sätzen wird anders verwiesen: Bei dann handelt es sich um eine „lineare Fortentwicklung von Gedanken“ (1987: 324), wie sie etwa bei Temporalität oder beim Nacheinander von Voraussetzung und Konsequenz (Konditionalität) zu beobachten ist. Mit so können aber im Nebensatz thematisierte begriffliche Aspekte der Nebensatzproposition aufgegriffen und unter einem bestimmten Vergleichsaspekt anders entfaltet werden. Bei solchen nicht-linearen Ausführungen spricht Redder auch von „Umformungen des Hörerwissens“ (1987: 318). Laut Redder (1987: 322f.) scheint so auch mit dem faktischen Gebrauch von wenn-Sätzen zu korrelieren. In diesem Fall drücken wennSätze eine verwirklichte Bedingung aus. Mir sind jedoch keine Korpusauszählungen bekannt, die auf solche Unterschiede Bezug nehmen würden.

Im Zusammenhang mit den Korrelaten wurde in erster Linie ihrer Setzung/Nicht-Setzung besonderes Interesse gewidmet. Dabei scheint u.a. die Polyfunktionalität der Konjunktionen eine Rolle zu spielen. Nach weil-Sätzen ist das Korrelat weglassbar, da die kausale Relation durch die Konjunktion explizit gekennzeichnet ist (Gohl 2002a: 11). Nach vorangestellten weil-Sätzen tritt dementsprechend nur selten ein Korrelat auf (vgl. Konopka 2006: 109). Nach dem polyfunktionalen wenn wird dagegen das Korrelat häufiger gesetzt, da z.B. mit der Korrelatverbindung ${ }^{86}$ wenn-Satz+dann der semantische Bezug zwischen den Teilsätzen genauer gekennzeichnet werden kann. Die Setzung des Korrelats kann auch bei sog. uneingeleiteten Verberstsätzen, „die ohnehin nicht über ein eindeutiges Subordinationszeichen verfügen, für syntaktische Klarheit sorgen“ (Zitterbart 2013: 621). In neueren Arbeiten wird die korrelative Anbindung auch in einem textlinguistischen Rahmen betrachtet. Korrelate nach vorangestellten Gliedsätzen können den Gliedsatz thematisieren und somit zur thematischen Progression der Textgestaltung beitragen (vgl. Cortès 2003: 156).

dennoch von der Partikel so zu unterscheiden, die Baschewa (1983: 80) nicht als Korrelat, sondern ,als einen strukturellen Anschluß“" ansieht.

86 Nach der IDS-Grammatik (Zifonun et al. 1997: 1487-1490) bilden der Gliedsatz und das Korrelat gemeinsam eine ,Korrelatverbindung‘. In Pasch et al. (2003: 248) wird dafür der Terminus ,Korrelatkonstruktion` verwendet. 
Zitterbart (2002: 48ff.) behandelt Sonderfälle im adverbialen Bereich. Die einschlägigen „Satztypen heben sich aufgrund des Zusammenspiels zwischen obligatorischem Korrelat und Subjunktor von anderen Adverbialsätzen hervor.“ (2002: 48) Als Beispiel werden u.a. Proportionalsätze wie (43) genannt, in denen je als Subjunktor und desto als Korrelat aufgefasst werden.

(43) Je mehr sich dann im Laufe der Verhandlungen und Kämpfe die Situation zuspitzt, desto angespannter wird die Lage nicht nur zwischen den bangenden Eltern, sondern auch unter den Entführern. (zit. n. Zitterbart 2002: 49)

Bei Zifonun et al. (1997: 2337) ist eine analoge Analyse von Proportionalsätzen zu finden: „Bei Proportionen der Steigerungsstufe Positiv wird der Untersatz durch so eingeleitet, der Obersatzrest enthält als Korrelat ebenfalls $s o$ ":

(44) So gut er sich für andere einsetzen kann, so schlecht kann er sich selbst verkaufen. (zit. nach Thurmair 2001: 34)

Es ist allerdings umstritten, ob desto bzw. so in den beiden obigen Beispielsätzen als Korrelate aufzufassen sind. Von typischen Korrelaten unterscheiden sie sich nicht nur darin, dass sie als konstitutiver Bestandteil der Korrelatverbindung anzusehen und nicht weglassbar sind (Zitterbart 2002: 165). In (44) liegt nicht das typische, resumptive so vor, vgl. die folgende semantische Beschreibung der Struktur von Thurmair (2001: 34):

So verweist [...] auf etwas, was zum Vergleich heranzuziehen ist und aus dem der Ausprägungsgrad der mit
so verbundenen adjektivischen Eigenschaft abzuleiten ist. [...] Beide mit den Adjektiven bezeichneten
Eigenschaften weisen somit den gleichen Ausprägungsgrad auf - dies wird durch den Verweis des zweiten so
geleistet. Bedingung dafür, daß dieser Vergleich nicht vage bleibt, ist, daß aus dem Kontext der
Ausprägungsgrad für eine der beiden adjektivischen Eigenschaften abzuleiten ist, also für Sprecher wie Hörer
bereits bekannt ist. Dies wird durch das erste so geleistet, das auf einen aus dem Kontext bekannten oder
abzuleitenden Vergleichspunkt verweist. Sowohl der Obersatz als auch der Untersatz enthalten Adjektive, die bei der Vergleichsstruktur konstitutiv und nicht weglassbar sind. Die Notation „so[ADJEKTIV]“ von Thurmair (2001: 34) legt nahe, dass das Adjektiv Teil der Konstruktion ist und seine Position mit einem beliebigen Adjektiv gefüllt werden kann. Es ist zu betonen, dass im Vorfeld des Obersatzes nicht das Wort so steht, sondern der Ausdruck so+Adjektiv. Da aber der kategoriale Status der Korrelate bei Adverbialssätzen als Wort (Adverb oder Präpositionaladverb) bestimmt wird (vgl. z.B. Zifonun et al. 1997: 1490), ist es fraglich, ob die Ausdrücke so+Adjektiv bzw. desto+Adjektiv als Korrelate anzusehen sind. Zu überlegen wäre die folgende alternative Analyse: Nach Hennig/Buchwald-Wargenau (2010: 14) liegt im Satz (44) kein konjunktional eingeleiteter und mit einem Korrelat angeschlossener Vergleichssatz vor, sondern ein komplexer, Junktionsausdruck‘. In Anlehnung an Thurmair 
könnte diese Struktur mit der Formel so+Adjektiv ... (Verbletztstellung) - so+Adjektiv ... (Verbzweitstellung) erfasst werden. Die formale Parallelisierung spricht dafür, neben ,paarigen Junktoren“ wie weder ... noch im Bereich der Koordination (Ágel 2010: 909f.) auch den Begriff ,paariger Junktionsausdruck' im Bereich der Subordination einzuführen. In der Duden-Grammatik (2016: 1119) werden weitere Parallelisierungen mit je+Adjektiv ... (Verbletztstellung) - je/desto/umso+Adjektiv ... (Verbzweitstellung) bzw. oder wie+Adjektiv ... (Verbletztstellung) - so+Adjektiv ... (Verbzweitstellung) aufgeführt. Bei diesen paarigen Konstruktionen ist die Anwendbarkeit des Dreitypenmodells von König/van der Auwera (1988) strittig. Aus diesem Grund dürfen die obigen Typen bei der Gesamtbewertung des Integrationsgrades nicht als ,resumptiv‘ mitgerechnet werden.

\subsection{Zur Erfassung der Linearstruktur des komplexen Satzes}

Betrachtet man die theoretische Grundlage der einschlägigen Arbeiten, erkennt man zwei Ansätze zur Beschreibung der Linearstruktur. Für die moderne Forschung (sowohl für Grammatiken als auch für die Spezialliteratur) ist kennzeichnend, dass mit großer Vorliebe auf das Stellungsfeldermodell als Grundlage für die Beschreibung von Wortstellungsregularitäten im komplexen Satz zurückgegriffen wird (vgl. etwa das einschlägige Kapitel von Ursula Hoberg in Zifonun et al. 1997: 2251f., Thim-Mabrey 1988, Günthner 1999a, Pasch et al. 2003: 67ff., Peyer 1997: 84ff., Wegener 2000: 38f., Wolf 1998: 95 usw.). Die theoretische Grundlage zur Beschreibung der Linearstruktur wird jedoch in mehreren Arbeiten nicht expliziert bzw. nicht befriedigend erläutert. In diesen Fällen wird statt des Stellungsfeldermodells oft auf eine Beschreibung nach der Verbstellung des nachfolgenden Hauptsatzes zurückgegriffen, wobei Strukturen mit V2 bzw. V3 unterschieden werden. Dieser Ansatz ist jedoch aus mehreren Gründen problematisch (vgl. Kapitel 3.1.3). ${ }^{87}$ Die Beschreibung der Linearstruktur der hier zu untersuchenden komplexen Sätze basiert auf dem Stellungsfeldermodell.

Im Folgenden soll zunächst den Fragen nachgegangen werden, wie vorangestellte Adverbialsätze im Rahmen des Stellungsfeldermodells zu verorten sind (Kapitel 3.2.1 und 3.2.2). Da in letzter Zeit der Anwendbarkeit des Stellungsfeldermodells für sprachhistorische Untersuchungen hinterfragt wurde, soll anschließend auf die Diskussion dieser Frage eingegangen werden (Kapitel 3.2.3).

87 In generativen Modellen wird, wie Zifonun (2013: 313) bemerkt, die Topologie „integraler Bestandteil eines syntaktischen Strukturmodells, bei dem lineare Positionen direkt mit strukturellen Positionen assoziiert sind.“ 


\subsubsection{Das Vorfeld und das linke Außenfeld}

Die Grundlagen des Konzepts der Stellungsfelder im deutschen Satz, das sich bei Beschreibung der Linearstruktur des Satzes bewährt hat, ${ }^{88}$ werden u.a. von Dürscheid (2012: 87-105), Altmann/Hofmann (2004), Weinrich (2005: 33-80) bzw. Zifonun et al. (1997: insbes. die Abschnitte E4 1. und 3., C6 2.1, H1 9.3) ausführlich erläutert. Der Ausgangspunkt für die Erfassung der Linearstruktur in diesem Rahmen ist die Klammerstruktur, auf deren Basis die Aufteilung des Satzes in Felder erfolgt (vgl. z.B. Weinrich 2005: 60). Nach der Position der Klammerteile (linke Satzklammer und rechte Satzklammer) werden etwa Sätze mit V2 wie in (45) auf folgende Stellungsfelder aufgeteilt (Dürscheid 2012: 88ff., 94): ${ }^{89}$

(45) Paul hat sich an der Uni eingeschrieben für Mathematik, Physik und Philosophie. (zit. n. Dürscheid 2012: 102)

\begin{tabular}{|l|l|l|l|l|}
\hline Vorfeld & linke SK & Mittelfeld & rechte SK & Nachfeld \\
\hline Paul & hat & sich an der Uni & eingeschrieben & für Mathematik, Physik und Philosophie. \\
\hline
\end{tabular}

Abb. 7: Beispiel für die Gliederung von V2-Sätzen in Vor-, Mittel und Nachfeld anhand der Satzklammer

Das Vorfeld wird in der IDS-Grammatik im Grunde genommen als „,der Satzabschnitt vor dem linken Satzklammerteil in Verbzweitsatzstrukturen“ definiert (Zifonun et al. 1997: 1576). Die linke Satzklammer wird in Verbzweitsatzstrukturen immer durch ein Finitum besetzt. Es gehört jedoch nicht alles, was vor dem Finitum steht, zum Vorfeld. Der Vorfeldbegriff wird syntaktisch eingegrenzt: Syntaktisch nicht integrierte Einheiten vor der linken Satzklammer gehören nicht zum Satzabschnitt Vorfeld. ${ }^{90}$

Das Vorfeld wird vom linken Außenfeld unterschieden (Zifonun et al. 1997: 1577). ${ }^{91}$ Das linke Außenfeld enthält Einheiten, die sich aus der Satzstruktur in unterschiedlichem Grad lösen. Wichtig ist dabei die Einsicht, dass die lineare Abfolge der Elemente im linken

88 In der IDS-Grammatik wird darauf verwiesen, dass das Konzept des Stellungsfeldermodells „Eingang in die meisten Grammatiken gefunden hat.“ (Zifonun et al. 1997: 1498)

89 Für V1-Sätze gilt nur der Unterschied, dass sie kein besetzbares Vorfeld haben (Dürscheid 2012: 91, Weinrich 2005: 78ff.). Auf die interne Topologie der formal durch subordinierende Elemente und Verbendstellung (= linke und rechte Satzklammer nach Dürscheid 2012: 92 und Zifonun et al. 1997: 1501) markierten Untersätze wird hier nicht näher eingegangen (s. dazu Dürscheid 2012: 92ff.). Im Fokus der vorliegenden Untersuchung steht nämlich die externe Topologie der Nebensätze, also die Frage, inwieweit diese in den nachfolgenden Hauptsatz integriert werden. (Zur Unterscheidung von interner und externer Topologie s. Zifonun et al. 1997: 2341f.)

90 Diese Auffassung ist in der einschlägigen Literatur weit verbreitet, vgl. z.B. auch Eroms (2000: 353): Syntaktisch nicht integrierte Einheiten vor der linken Satzklammer besetzen keine Satzposition, sie werden ,aus der engeren Satzstruktur herausgenommen“. Vgl. auch Dürscheid (2012: 97f.).

91 Bei anderen Autoren erscheint hierfür der weitgehend synonyme Terminus, Vorvorfeld‘ bzw. ,Vor-Vorfeld‘ (vgl. z.B. Auer (1997: 67), Thim-Mabrey (1988) bzw. Dürscheid (2012: 97), Günthner (1999a: 212)) oder ,Nullstelle‘ (vgl. Pasch et al. 2003: 487, Breindl et al. 2014: 177). 
Außenfeld den „Grad ihrer Bindung an den nachfolgenden Satz“ (1997: 1580) abbildet. Im linken Außenfeld können (in der angegebenen Reihenfolge) folgende Einheiten erscheinen (nach Zifonun et al. 1997: 1577ff.): ${ }^{92}$ (i) Interaktive Einheiten wie Interjektionen (z.B. $n a$ ), Responsive (z.B. ja) oder Anredeformen (z.B. Mensch); (ii) Koordinierende Ausdrücke wie z.B. und, oder; (iii) Thematisierungsausdrücke, ,die vorwegnehmend das Thema der KM nennen, das dann im Satz selbst (im Vor- oder Mittelfeld) anaphorisch oder anadeiktisch fortgeführt wird“" (Zifonun et al. 1997: 1579).

Der Typ (iii) kann nach dem Grad der Bindung der Ausdrücke an den nachfolgenden Satz weiter klassifiziert werden in links angebundene und freie Thematisierungsausdrücke (vgl. dazu näher Zifonun et al. 1997: 518-522). ${ }^{93}$ Links angebundene Thematisierungsausdrücke enthalten eine Nominal-, Pronominal-, Präpositional- oder Adverbphrase, die durch anaphorische Pronomina oder anadeiktische Elemente i.d.R. im Vorfeld des Satzes thematisch wieder aufgenommen wird. Vgl. dazu den folgenden Beispielsatz, in dem auf die vorangestellte Präpositionalphrase mit Hilfe des anadeiktischen Ausdrucks $d a$ verwiesen wird:

(46) Im Wald, da sind die Räuber. (zit. n. Zifonun et al. 1997: 1579)

Ist der linksangebundene Thematisierungsausdruck eine Nominalphrase, so kann mit dem wiederaufnehmenden Element Kongruenz bezüglich der nominalen Kategorisierungen (Genus, Numerus, Kasus) bestehen (1997: 518). Intonatorisch erfolgt die Anbindung im mündlichen Medium nach einem progredienten Tonmuster ohne längere Pause. Freie Thematisierungsausdrücke sind aber intonatorisch sowie syntaktisch vom nachfolgenden Satz unabhängig und weisen einen geringeren Grad an Bindung auf. Der Thematisierungsausdruck, der intonatorisch durch eine Pause - oder in der Schrift z.B. durch einen Doppelpunkt - abgetrennt ist, wird nicht unmittelbar im Vorfeld aufgenommen, sondern erst im Mittelfeld mit Anaphern, Anadeixis oder einer Nominalphrase thematisch fortgeführt. Vgl. dazu den folgenden Beispielsatz:

(47) Hollywood: In mehr als sieben Jahrzehnten hat diese Traumfabrik nicht nur Stars, immer auch Rebellinnen hervorgebracht und das ist gut so. (zit. n. Zifonun et al. 1997: 1579)

\footnotetext{
92 Eroms (2000: 352) gibt in Anlehnung an Schanen (1993) eine noch detailliertere Liste von möglichen Elementen vor dem eigentlichen Vorfeld an. Da aber nicht-satzwertige Voranstellungen nicht den Gegenstand der vorliegenden Arbeit bilden, soll nach der Klassifizierung der IDS-Grammatik (Zifonun et al. 1997) vorgegangen werden.

93 Diese Unterscheidung geht auf Altmann (1981) zurück. Er bezeichnet die beiden ,Formen der Herausstellung ${ }^{`}$ als ,Linksversetzung' bzw. ,freies Thema'.
} 
Die Unterscheidung von links angebundenen und freien Thematisierungsausdrücken erfolgt zwar im Einzelnen nach z.T. verschiedenen Kriterien, in jedem Fall wird jedoch der unterschiedliche Grad an Bindung unterstrichen. ${ }^{94}$

Zifonun et al. (1997: 518, 520) weisen darauf hin, dass sowohl linksangebundene als auch freie Thematisierungsausdrücke durch Nebensätze realisiert werden können. Als dritter Untertyp von Thematisierungsausdrücken werden formelhafte Thematisierungsausdrücke genannt (1997: 522-526), die ebenfalls die Form von Nebensätzen haben können, z.B.:

(48) Was den Weg zu ihm betreffe, so sei er ohne besondere Beschwerden und Anstände (...) (zit. n. Zifonun et al. 1997: 522)

\subsubsection{Vorangestellte Nebensätze im Rahmen des Stellungsfeldermodells}

Das Stellungsfeldermodell kann auch auf vorangestellte Nebensätze angewandt werden. Die Vorfeldbesetzung von satzwertigen Stellungsgliedern im Rahmen eines komplexen Satzes gleicht der „,normalen“ Vorfeldbesetzung der einfachen Sätze: Sowohl Ergänzungs- als auch Adverbialsätze können im heutigen Deutsch im Vorfeld ihres Obersatzes stehen (vgl. z.B. Dürscheid 2012: 95 bzw. Zifonun et al. 1997: 2345, 2348). Die Position der vorangestellten Nebensätze im Vorfeld zeigt ihre starke Integration in den Obersatz.

Lötscher (2005: 352) argumentiert dafür, das linke Außenfeld „für die Charakterisierung der Voranstellung von Nebensätzen aller Art (also neben Adverbialsätzen auch Ergänzungssätzen und Relativsätzen) zugrunde zu legen“. Auf eine funktionale Ähnlichkeit der Linksanbindung von Termsätzen und nicht satzwertigen (z.B. nominalen) Einheiten wird von Zifonun et al. (1997: 2349) explizit verwiesen. Mit dem linken Außenfeld erscheint eine Position für intonatorisch und syntaktisch schwächer integrierte Einheiten, deren Integrationsgrad jedoch unterschiedlich sein kann. Denn bei der am schwächsten integrierten Variante fehlt die korrelative Verbindung, daher ist die Anfügung des Nebensatzes lockerer. Die Stellungsmöglichkeiten von vorangestellten Adverbialsätzen werden von Zifonun et al. (1997: 2349, in Anlehnung an König/van der Auwera 1988) wie folgt zusammengefasst:

Bei integrativer Spitzenstellung ist der Nebensatz einziges Stellungsglied des Vorfeldes; bei resumptiver Spitzenstellung befindet sich der Nebensatz im linken Außenfeld, das eigentliche Vorfeld wird durch ein

\footnotetext{
94 Selting (1993: 315) betont, dass die beiden Typen primär durch prosodische Merkmale unterschieden werden können. Ágel/Hennig (2006c: 393) führen dagegen die Unterscheidung nach syntaktischen Kriterien durch: ,Linksversetzungen“ können als doppelte Realisierungen eines Elements der Projektionsstruktur aufgefasst werden, während dies beim ,freien Thema' nicht möglich ist. Vor allem bei ,Linksversetzungen', bei denen der Linksversetzungsausdruck und das Vorfeldelement im Folgesatz morphologisch übereinstimmen, könnte auch überlegt werden, sie gegebenenfalls als doppelte Vorfeldbesetzung zu analysieren (vgl. Altmann/Hofmann 2004: 146f.). Der Grad an Integration in den Folgesatz ist auf jeden Fall höher als beim anderen Typ.
} 
,resumptives' Korrelat in Form von so, dann, da eingenommen. Bei nicht-integrativer Spitzenstellung findet sich der Nebensatz ebenfalls im linken Außenfeld, ein Korrelat fehlt jedoch.

Lötscher (2005: 352) sieht Parallelen zwischen den beiden oben behandelten Typen von Thematisierungsausdrücken und den Stellungstypen ,resumptiv` und ,nicht-integrativ`. Der links angebundene Thematisierungsausdruck entspreche nach Lötscher (2005: 352ff.) dem resumptiven Typ (49), der freie Thematisierungsausdruck dem nicht-integrativen Typ (50).

(49) Dass es heute regnet, das macht die Ferien nicht unterhaltsamer.

(50) Dass es heute regnet, unterhaltsamer macht das die Ferien auch nicht.

In der Tat ist die Anbindung von nicht-satzwertigen, links angebundenen Thematisierungsausdrücken und resumptiv aufgenommenen Nebensätzen ähnlich. Vgl. die folgenden Beispielsätze:

(46) Im Wald, da sind die Räuber. (zit. n. Zifonun et al. 1997: 1579)

(51) Als die Entscheidung fiel, daß Freys DVU zur Bundestagswahl, also auch hier in Berlin antritt, berichtet einer der Organisatoren der Initiative, da haben wir gedacht: wir müssen ein deutliches Zeichen gegen Intoleranz setzen und Zivilcourage zeigen. [Die Rheinpfalz 2.7.1998, S. 3] (zit. n. Pasch et al. 2003: 390)

$\mathrm{Ob}$ auch der nicht-integrative Typ dem freien Thematisierungsausdruck entspricht, sei hier dahingestellt. Es lassen sich leicht Zusammenhänge finden, wie etwa die Abhebung der vorangestellten Elemente durch Interpunktionszeichen (v.a. Gedankenstrich) oder entsprechende intonatorische Charakteristika (steigendes bzw. fallendes Tonmuster). Es trifft jedoch nicht $\mathrm{zu}$, dass nicht-integrative Adverbialsätze etwas thematisieren, was im nachfolgenden Satz mit Anaphern, Nominalphrasen usw. thematisch fortgeführt werde (vgl. Zifonun et al. 1997: 521). Auf jeden Fall zeichnet sich der nicht-integrativ angeschlossene Vordersatz durch einen hohen Grad an intonatorischer und syntaktischer Unabhängigkeit von der nachfolgenden Satzstruktur aus.

\subsubsection{Das Stellungsfeldermodell in historischen Untersuchungen}

Das Stellungsfeldermodell wurde auch auf frühere Sprachstufen des Deutschen angewandt (für das Althochdeutsche vgl. z.B. Dittmer/Dittmer 1998, Robinson 1997). In den letzten Jahren entzündete sich jedoch eine Debatte über die Anwendbarkeit des Stellungsfeldermodells, insbesondere für die Beschreibung des linken Satzrands in älteren Sprachstufen. Diese Diskussion soll knapp nachgezeichnet werden. 
Lötscher spricht sich ausdrücklich dafür aus, dass sich das Stellungsfeldermodell auch für die Beschreibung älterer Sprachstufen eignet und bezeichnet es als eine „Tatsache [...], dass [...] die Felderstruktur formal über Sprachvarianten und Sprachepochen gleich bleibt" (2005: 353). ${ }^{95}$ Diese Auffassung teilen auch Schrodt (2004) bzw. Ramers (2005: 89), die sogar behaupten, dass bezüglich der Stellungsregularitäten keine tiefgreifenden Entwicklungen vom Althochdeutschen bis zum Neuhochdeutschen anzusetzen seien. ${ }^{96}$

Auf der anderen Seite gibt es zu diesem Thema jedoch kritische Äußerungen: Der Gedankengang von Wich-Reif (2008) lässt sich etwa so zusammenfassen: Das Stellungsfeldermodell ist bei einer festen Verbstellung durchaus brauchbar, wenn aber diese Voraussetzung nicht erfüllt ist, eignet es sich für die syntaktische Beschreibung nicht. Sie bezweifelt somit die Tauglichkeit des Stellungsfeldermodells für die Erfassung des Althochdeutschen, das über keine festen Verbpositionen verfüge. Nach Wich-Reif (2008) deutet die mehrfache Besetzung des Vorfeldes im Gegenwartsdeutschen, die v.a. in der Mündlichkeit verbreitet ist, sogar darauf hin, dass das Finitum im Aussagesatz nicht fest an der zweiten Stelle steht, sondern möglicherweise auch an der dritten Stelle stehen kann: ${ }^{97}$

(52) [Gestern] [im Kino] haben wir uns den Film: „Sophie Scholl - die letzten Tage“ angesehen. (Beispielsatz zit. n. Wich-Reif 2008: 189)

Das Stellungsfeldermodell sei deshalb auch bezüglich der Gegenwartssprache nicht brauchbar, wenn nicht nur die standardisierte Schriftsprache, sondern auch der Bereich der Mündlichkeit beschrieben wird.

Wie ist nun mit der Frage umzugehen, ob die Verbstellung im älteren Deutsch fest war bzw. ob dies die Prämisse der Anwendung des Stellungsfeldermodells sei? Bei der Beurteilung der Anwendbarkeit des Feldermodells für die vorliegende Untersuchung ist wohl die Tauglichkeit eines Vorfeldkonzepts in älteren Sprachstufen der entscheidende Punkt.

Lötscher (2005: 355) argumentiert für die Anwendbarkeit des Stellungsfeldermodells in historischen Untersuchungen, die nach dem Spätalthochdeutschen ansetzen. Er räumt zwar ein, dass für das frühe Althochdeutsche die heutigen Satzgliedstellungs- und Felderregeln noch nicht gültig sind. Die Felderstruktur mit einem Vorfeld und einem linken Außenfeld war

95 Dies schließt jedoch die synchrone bzw. diachrone Variation bei der Besetzung der einzelnen Felder nicht aus. „Aus der Nichtverwendung des linken Vorfelds in gewissen Fällen kann [...] nicht geschlossen werden, dass es in diesen Fällen nicht doch als >Slot< potenziell existiert" (Lötscher 2005: 353).

96 Allerdings wird von Ramers (2005: 89) auch auf die Notwendigkeit der Erweiterung des Modells verwiesen, damit die Besetzung und die Funktion der einzelnen Felder adäquat beschrieben werden können.

97 Die sog. mehrfache Vorfeldbesetzung kann oft so interpretiert werden, dass die zweite Konstituente in die erste eingebettet ist und diese spezifiziert. Auch im obigen Beispiel handelt es sich um ein einziges Satzglied mit komplexer Konstituentenstruktur. Wich-Reif (2008: 201) weist darauf hin, dass die Zuordnung ähnlicher Sätze zum V2- oder V3Satzmuster bzw. zur einfachen oder doppelten Vorfeldbesetzung eine Interpretationssache ist. 
noch nicht entwickelt (2005: 360). ${ }^{98}$ Er vertritt jedoch die Auffassung, dass bereits seit dem Spätalthochdeutschen „am Satzanfang grundsätzlich die gleichen Felder-Muster gelten“. Im Falle des Spätalthochdeutschen ist nach Lötscher (2005: 355) bereits eine „Felderstruktur mit einem Vorfeld, das von genau einem, beliebigen vollen Satzglied besetzt werden kann“, anzusetzen. ${ }^{99}$ Nach Lötscher bleibt also die Felderstruktur vom Spätalthochdeutschen bis zum Gegenwartsdeutschen formal gleich, was aber nicht ausschließt, dass die Besetzbarkeit der einzelnen Felder „sowohl synchron zwischen verschiedenen Stilebenen wie diachron [...] differieren kann.“ (2005: 353) Lötscher (1995: 42) geht davon aus, dass im älteren Deutsch die gleichen Strukturen am linken Satzrand verwendet werden wie im Gegenwartsdeutschen. Er zieht bezüglich der Analyse der Integration von Adverbialsätzen vom Spätalthochdeutschen bis zum Neuhochdeutschen den folgenden Schluss:

Sobald die V2-Stellung mit Vorfeld und gleichzeitig die Stellung im linken Außenfeld als Positionsmöglichkeiten definiert sind, besteht für beliebige Satzglieder und somit auch für Adverbialsätze wenigstens formal immer die Wahlmöglichkeit zwischen den verschiedenen Formen der Spitzenstellung, die wir für das Gegenwartsdeutsche unterschieden haben. (Lötscher 2005: 356)

Die V2-Stellung im Aussagesatz ist nicht so zu verstehen, dass vor dem Verb immer genau eine Konstituente stehen muss (vgl. auch Zifonun et al. 1997: 1639). Unter grammatisch beschreibbaren Bedingungen kann und konnte das Vorfeld auch unbesetzt bleiben (,leeres Vorfeld‘, vgl. etwa Weinrich 2005: 78ff.). Des Weiteren war es auch im älteren Deutsch möglich, dass der Vorfeldkonstituente weitere Elemente vorangestellt wurden. In diesen Fällen handelt es sich jedoch um eine Position des linken Außenfelds. Dazu gehören Elemente, die nur locker zur Satzstruktur gehören bzw. - wie in Zifonun et al. (1997: 1577) formuliert wird - „nicht [...] dem Satz zuzurechnen sind“. Sie können jedoch mit einer plausiblen Typisierung dem Verbstellungstyp V2 zugeordnet werden, um im zweiten Schritt ihre Besonderheiten auf der Folie der Normalfälle erfassen zu können. Diesen Weg geht z.B. auch die IDS-Grammatik (vgl. Zifonun et al. 1997: 1500). Selbst Wich-Reif (2008: 188), die gegen die Tauglichkeit des Stellungsfeldermodells argumentiert, räumt ein: „Alle relevanten Vor- beziehungsweise Vorvorfeldphänomene sind bereits in der althochdeutschen Sprachepoche nachweisbar“.

98 Die Sprachdenkmäler aus dem 8. und 9. Jh. zeichnen sich durch eine größere Stellungsfreiheit des Verbs aus, „die möglicherweise noch Reste von germanischen Stellungsregeln widerspiegelt" (Lötscher 2005: 359). Die Satzgliedstellung unterlag primär pragmatischen Faktoren. Ágel (2015: 140f.) argumentiert für eine verbzentrierte Modellierung der althochdeutschen Wortstellung, in der die nichtverbalen Elemente um das Verb herum nach informationsstrukturellen Kriterien, relativ frei angeordnet werden.

99 Lötscher untermauert seine Position mit den Verhältnissen in Notkers >Consolatio<-Bearbeitung, in der ,mit geringen Ausnahmen, die V2-Stellung mit beliebiger Besetzung der Stelle vor dem Verb als Grundregel“" gelte (Lötscher 2005: $355)$. 
Wich-Reif (2008) verweist auf mehrere Argumente gegen die Tauglichkeit des Stellungsfeldermodells. Sie bemerkt, dass im Stellungsfeldermodell ursprünglich kein Vorvorfeld vorgesehen wurde (2008: 202). Trotz dieser Tatsache spielt m.E. bei der Beurteilung der Anwendbarkeit des Stellungsfeldermodells keine Rolle, welchen Stand die Theorie etwa bei Erich Drachs Grundgedanken der deutschen Satzlehre (Erstausgabe aus dem Jahre 1937) hatte. ${ }^{100}$ Heute sollte vielmehr nur sein aktueller Stand diskutiert werden. Die Kritik von Wich-Reif (2008: 203) bezieht sich auch auf die Terminologie, u.a. auf Ausdrücke wie ,Linksherausstellung‘. Der Begriff ,Linksversetzung ${ }^{6}$ entstand als Übersetzung zum englischen Terminus ,left dislocation“ und wurde in Anlehnung an Altmann (1981) in der einschlägigen Forschung verbreitet (vgl. Lötscher 1995: 34). Beide Bezeichnungen suggerieren nach Wich-Reif (2008) eine nicht haltbare Vorstellung von Sprachproduktion und -rezeption: Konstruktionen wie Seinen Bruder, den kenne ich nicht. können mit einer Bewegung des Elements seinen Bruder aus einer zugrunde liegenden Struktur Seinen Bruder kenne ich nicht. nicht sinnvoll beschrieben werden. Neben ,Linksversetzung' sind jedoch

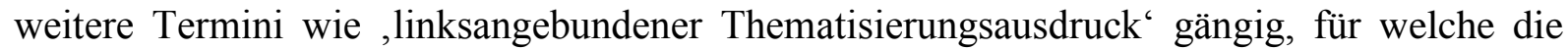
Kritik von Wich-Reifs (2008) nicht zutrifft. Auch die Kritik an der Terminologie ,links' und ,rechts‘ als Begriffe der Räumlichkeit, die nicht (oder nur metaphorisch) auf die mündliche Sprachproduktion angewendet werden können, ist nicht neu (s. z.B. Fiehler 2000). Sie betrifft jedoch m.E. gar nicht die Tauglichkeit des Stellungsfeldermodells an sich. Der Ausdruck ,Linksversetzung ${ }^{6}$ ist nämlich kein fester Bestandteil der Theorie, er entstammt vielmehr der Generativen Grammatik und wurde nur zur Spezifizierung der Vorvorfeldbesetzungen rein deskriptiv verwendet. ${ }^{101}$

Die Anwendbarkeit des Feldermodells für die Analyse älterer Zeitstufen setzt im engeren Sinne nicht nur die Tauglichkeit des Vorfeldkonzepts, sondern auch die Tauglichkeit des Klammerprinzips voraus, weil die Klammerteile als Kriterien für die Bestimmung der Stellungsfelder dienen (vgl. Ágel 2015: 141). Würde man dieses strengere Kriterium anwenden, so wäre das Klammerprinzip für ältere Zeitstufen gar nicht anzusetzen, da die Grammatikalisierung der Klammerstrukturen erst zu Beginn der neuhochdeutschen Periode erfolgt (vgl. Kapitel 2.2.1.1). Nach Ágel (2015: 144), der als Kriterium nicht die Grammatikalisierung der ,Distanzstellung', sondern die Grammatikalisierung der ,Klammer

\footnotetext{
100 Das Konzept des Stellungsfeldermodells wurde in einer Reihe von Arbeiten ausgebaut (zur weiterführenden Literatur s. Zifonun et al. 1997: 1498,1502). Man beachte auch, dass moderne topologische Modelle ihre Vorläufer bereits im 19. Jh. haben (vgl. Ramers 2005: 79).

101 Auf diese, theoretisch nicht auf Transformationen oder Bewegungen bezogene, sondern rein deskriptive Verwendung der einschlägigen Termini verweist bereits Altmann (1981: 47).
} 
anwendet (s. dazu Kapitel 2.2.1.1), ist sogar „davon auszugehen, dass es vor dem 18. Jh. keine Klammerstrukturen und Stellungsfelder im heutigen Sinne gab“. M.E. könnte aber schon bei einer Grammatikalisierung der ,Distanzstellung“ (d.h. Fernstellung von zwei grammatisch zusammengehörenden Teilen, also der linken und rechten Satzklammer) von ,Vorfeld“ die Rede sein. In diesem Sinne wäre das Feldermodell seit dem frühen Neuhochdeutschen anwendbar.

Die Übertragung des Klammerprinzips und des Vorfeldkonzepts auf die Anfänge der deutschen Sprache ist ein Ergebnis synchronizistischen Denkens (im Sinne von Ágel 2015: 122). Vor dem Spätalthochdeutschen waren Wortstellungsregularitäten ganz anders angelegt, daher ist die Anwendung der Felderregeln auf das frühe Althochdeutsche nicht sinnvoll. Auch die unreflektierte Übertragung des Klammerprinzips und des Vorfeldkonzepts auf die Zeit zwischen dem Spätalthochdeutschen und dem frühen Neuhochdeutschen ist nicht unproblematisch. Die im Entstehen begriffenen Stellungsfelder am Anfang des Neuhochdeutschen sind nicht mit den voll grammatikalisierten Stellungsfeldern der gegenwärtigen (konzeptionellen) Schriftlichkeit gleichzusetzen.

Die im Laufe der Zeit im Entstehen begriffenen Stellungsfelder gehören nach Ágel (2015: 144) zur sog. Offlinesyntax, d.h. sie ,stellen ein Produkt der entzeitlichten Linearität der konzeptionellen Schriftlichkeit dar“. „Historisch ist von einem dynamischen Verhältnis von Online- und Offlinesyntax auszugehen.“ (Ágel 2015: 149) Die Grammatikforschung steht vor der Herausforderung, die Überlagerung zeitlicher Strukturen (Onlinestrukturen) von entzeitlichten (linearisierten) Strukturen in einem theoretischen Modell zu erfassen. Beim aktuellen Forschungsstand müssen wir uns in der vorliegenden Untersuchung damit begnügen, die unreflektierte Verwendung einschlägiger Termini zu vermeiden. Beim ,Vorfeld' sollte berücksichtigt werden, dass die Grammatikalisierung dieses Stellungsfelds erst am Anfang bzw. während des Untersuchungszeitraums der vorliegenden Analyse erfolgt.

\subsection{Junktion, Integration, Subordination}

Es dürfte heutzutage bereits unumstritten sein, dass die vielfältigen Möglichkeiten der Satzverknüpfung in der Gegenwartssprache mit der traditionellen Unterscheidung zwischen Hauptsatz und Nebensatz bzw. Koordination und Subordination nicht präzise genug erfasst werden können (vgl. z.B. Auer 1998; Fabricius-Hansen 1992; Helbig 2003). Diese Feststellung gilt erst recht bei der Analyse von Quellentexten früherer Zeiten. Zunächst sollte also geklärt werden, in welchem Rahmen die Adverbialsätze des Neuhochdeutschen zu 
erfassen sind. Dabei kann auf erfolgversprechende Ansätze der jüngeren Zeit zurückgegriffen werden. Nach der knappen Darstellung und Kritik der traditionellen Auffassung wird ,Subordination“ im Sinne der modernen Fachliteratur als skalarer Begriff erfasst. Weiter unten (Kapitel 3.3.2) werden auch die Arten der Integration ausführlicher beschrieben.

Nach einer traditionellen Definition versteht man unter ,Subordination“ die „Art der Verknüpfung zweier Sätze, wobei der eine syntakt. in den anderen integriert wird“ (Glück/Rödel 2016: 683). Ein Teilsatz des Satzkomplexes wird einem anderen Teilsatz des Satzkomplexes untergeordnet (vgl. auch Bußmann 2008: 272, 467, 697). Wenn man jedoch die Frage stellt, welche Merkmale mit Integration bzw. Unterordnung eines Teilsatzes im Zusammenhang stehen, ist eine dichotomische Unterscheidung zwischen Koordination und Subordination nicht mehr unproblematisch. Die Merkmale, die bei der Unterordnung vorliegen, sind bekanntlich unterschiedlich und können sich diachron verändern (zur sprachgeschichtlichen Tragfähigkeit des formalen Kriteriums vgl. auch Kapitel 3.1.1).

Der ,Nebensatz' wird traditionell aufgrund der folgenden Kriterien erfasst: Stellung des finiten Verbs, Satzgliedwert und fehlende Selbständigkeit. ,Nebensätze‘ weisen demnach typischerweise Verbletztstellung auf, sie können als Satzglieder des Hauptsatzes bestimmt werden und sind kommunikativ nicht selbständig. Bei genauerem Hinsehen stellt sich jedoch heraus, dass die einzelnen Kriterien an sich keine eindeutige Unterscheidung ermöglichen bzw. mehrere Typen von Nebensätzen nicht alle Eigenschaften teilen. Quantitativ gesehen weisen zwar die meisten Nebensätze tatsächlich Verbletztstellung auf. Mit diesem formalen Kriterium können jedoch V1-Adverbialsätze bzw. ,uneingeleitete Nebensätze‘ in Ergänzungsfunktion (vgl. Auer 1998) sowie hypothetische Vergleichssätze, in denen auf das einleitende als direkt das Finitum folgt (vgl. Demske 2011, s. auch die Beispiel (61) weiter unten) nicht als Nebensätze erfasst werden - obwohl sie nach dem funktionalen Kriterium eindeutig Nebensätze sind. Aber auch das Satzgliedkriterium führt zu Schwierigkeiten. Typische Nebensätze sind Gliedsätze oder Gliedteilsätze, es gibt jedoch auch Verbletztsätze, deren Status dem Satzgliedkriterium nicht entspricht, wie etwa die sog. weiterführenden Nebensätze (s. dazu Brandt 1990). Das Kriterium der Selbständigkeit ist schwer zu operationalisieren, zudem gibt es einerseits relativ selbständige, unabhängige Verbletztsätze (z.B. Wenn er doch endlich kommen würde!, vgl. Blühdorn 2013: 150f.) bzw. ,abhängige Hauptsätze، (im Sinne von Auer 1998), andererseits auch Hauptsätze, die nicht ganz selbständig bzw. unvollständig sind (z.B. bei der Redeeinleitung von Sie sagt, sie ist müde., vgl. den Terminus ,Hauptsatzfragment‘ weiter oben im Kapitel 3.1.1). 
Aus den obigen Gründen nimmt Fabricius-Hansen (1992) für die Subordination im Deutschen eine Prototypenstruktur an: Es werden prototypische und marginale Fälle der Subordination unterschieden. Neben den klassischen Subordinationskriterien der Verbletztstellung und Satzgliedwert (,semantische Integration“) müssen prototypische Nebensätze auch prosodische und pragmatische Kriterien erfüllen. Nach dem prosodischen Kriterium wird prototypischen Nebensätzen keine eigene Intonationseinheit zugewiesen (,phonologische Integration“). Nach dem pragmatischen Kriterium haben prototypische Nebensätze keine eigene Illokution (,pragmatische Integration“). Nach dem fünften, topologischen Kriterium stehen prototypische Nebensätze im Vorfeld des Hauptsatzes. Aufgrund dieser Subordinationsmerkmale werden Nebensatzklassen unterschieden vom prototypischen Fall A (alle Kriterien erfüllt) bis Fall E (Abweichung in formaler Hinsicht, d.h. semantisch-pragmatisch integrierte Hauptsätze), der Subordination im marginalen Sinne darstellt. Im Anschluss an Fabricius-Hansen (1992) wird Subordination auch in weiteren Arbeiten als ein vieldimensionales Verfahren dargestellt (vgl. Peyer 1997, Zifonun et al. 1997: 2250-2253, Helbig 2003). Im Kapitel 3.3.2 werden die Ebenen der Integration erörtert. Zunächst soll jedoch auf die Frage eingegangen werden, wie Subordination in einem umfassenden Modell der Satzverknüpfungstechniken erfasst werden könnte.

\subsubsection{Subordination im Rahmen der Junktionstheorie}

Die Satzkomplexität bzw. die Techniken der Satzverknüpfung werden in der Junktionstheorie von Raible (1992) in einem größeren theoretischen Rahmen behandelt. Er modelliert unterschiedliche Möglichkeiten der sprachlichen Darstellung von Inhaltsrelationen zwischen zwei Sachverhalten. Die verschiedenen Techniken können acht Ebenen zugeordnet werden, wobei allerdings nur die Ebenen I-IV zum Ausdruck von Relationen zwischen satzwertigen Sachverhaltsdarstellungen gehören. Bei den Ebenen V-VIII handelt es sich um nicht-finite Formen bzw. um nicht-verbale (d.h. nominale) Fügungen, die in einen Satz integriert werden. Bei der Junktion werden ,linear aufeinanderfolgende (Satz-)Einheiten [...] zueinander in Relation gesetzt" (Raible 1992: 30). Die dazu gehörenden Techniken werden in einem Kontinuum zwischen ,Aggregation“ und ,Integration“ dargestellt: ${ }^{102}$ „Am Extrem der

\footnotetext{
102 Das Begriffspaar ,Aggregation \%/,Integration“ erfasst grundlegende Unterschiede bei der Perspektivität von Bildern und in der Grammatik. In Anlehnung an Panofsky (1927) unterscheidet Köller (1993: 21, 2004: 55) den ,Aggregatraum` der frühen Kunst, in dem einzelne Elemente additiv nebeneinandergestellt werden, bzw. den ,Systemraum" der zentralperspektivischen Kunst der Renaissance, dessen Elemente systemhaft angeordnet und von einer Perspektive aus (monozentrisch) organisiert sind. Diese Unterscheidung wird auch auf die grammatische Organisation übertragen (vgl.
} 
Aggregation stehen zwei Sätze unverbunden nebeneinander. Am anderen Extrem bleibt ein einziger, völlig integrierter Satz übrig“ (Raible 1992: 27). Dies soll nun anhand zweier Beispiele knapp erläutert werden (vgl. Raible 1992: 14-18).

Die Sätze werden im folgenden Beispiel unverbunden nebeneinander gestellt, die inhaltliche Relation zwischen den Sachverhaltsdarstellungen wird nicht expliziert. Diese Technik, die am aggregativsten ist, wird ,Juxtaposition“ genannt (Ebene I).

(53) Peter liegt mit Grippe im Bett. Er geht nicht zur Schule.

Zwischen den beiden gleichrangigen Sätzen ist keine syntaktische Kohäsionsmarkierung zu finden. Ihr Zusammenhang ist nur aufgrund des Nacheinanderstehens der beiden Sätze bzw. mithilfe pragmatischer Kriterien (wie etwa Weltwissen) zu erschließen. Die dem folgenden Satz zugrunde liegende Technik ist integrativer (Ebene VII). Die Kausalrelation wird explizit markiert und eine der Sachverhaltsdarstellungen in Form einer Präpositionalphrase in die andere eingebettet. Die Nominalisierung erlaubt eine starke Kondensierung der Informationen. $^{103}$

(54) Wegen einer Grippeerkrankung geht Peter nicht zur Schule.

Zwischen den beiden obigen Techniken, auf Ebene IV, wird die Technik der Subordination eingeordnet. Unter den satzwertigen Sachverhaltsdarstellungen stellt sie die integrativste Form dar.

Die Junktionstheorie von Raible erlaubt im Gegensatz zu traditionellen Darstellungen, weniger integrative und integrativere Techniken in einem Kontinuum anzuordnen. Sie ist somit flexibler als die starre, dichotomische Auffassung der traditionellen Grammatiken. ${ }^{104}$ Die Junktionstheorie wurde jedoch v.a. im Hinblick auf romanische Sprachen ausgearbeitet, zudem nahm Raible weniger die Subordination, als vielmehr die integrativeren Techniken

Raible 1992: 27-31, Köller 1993 bzw. 2004, Koch/Oesterreicher 1990: 11, 96, Ágel 2003, Ágel 2007: 45, Ágel/Hennig 2006a: 26f.).

103 Auch in Lehmanns (1988: 182) Konzept von „clause linkage“ sind neben satzwertigen Strukturen auch Nominalisierungen miteinbezogen. Mit dem Merkmalbündel ,Desententialisierung ' wird der Verlust an Satzwertigkeit des zugeordneten Satzes und zugleich die Zunahme an nominalen Eigenschaften erfasst. Lehmann (1988: 200) nennt diesen Parameter „,desententialisation of the subordinate clause“ und behandelt ihn unter dem Stichwort ,Expansion vs. Reduktion“. ,Desententialisierung' liegt z.B. vor, wenn flektierte Verben durch infinite Konstruktionen ersetzt werden. Der Beispielsatz (i) ist integrativer, als (ii) oder (iii) (vgl. auch Peyer 1997: 36):

(i) Sie hörte das Pfeifen ihrer Schwester.

(ii) Sie hörte, dass ihre Schwester pfeift.

(iii) Sie hörte ihre Schwester laut pfeifen

Der Verlust an Satzwertigkeit zeigt also einen höheren Grad der Integration an. Umgekehrt kann auch der (ursprünglich) übergeordnete Teilsatz reduziert werden. Dies geschieht etwa bei der Grammatikalisierung von Konstruktionen wie ich mein(e), die vom selbständigen Matrixsatz eines Satzgefüges zum Diskursmarker reduziert werden und in Kombination mit einem ,abhängigen Hauptsatz' auftreten können (vgl. Lehmann 1988: 204, Auer 1988, Imo 2007). Ich beschränke mich jedoch im Weiteren auf die Kombination von Teilsätzen.

104 Auch weitere, sprachtypologisch orientierte oder auf das Deutsche bezogene Darstellungen bieten im Vergleich mit der traditionellen Auffassung ein erweitertes Modell (vgl. z.B. Lehmann 1988 bzw. Fabricius-Hansen 1992). 
unter die Lupe. Dies hat zur Folge, dass die Subordination - aus der Perspektive meines Untersuchungsgegenstandes - viel zu undifferenziert beschrieben wird, denn auch innerhalb der Subordination können weniger integrative und integrativere Techniken unterschieden werden. Daher kann Raibles theoretischer Rahmen nicht ohne Weiteres auf das Neuhochdeutsche übertragen werden. ${ }^{105}$ Eine Weiterentwicklung des Junktionsmodells bieten Ágel (2010) zur expliziten Junktion und Hennig (2010a) zur elliptischen Junktion.

Auch in der Theorie zur expliziten Junktion (Ágel 2010) werden Junktionstechniken auf der Skala zwischen Aggregation und Integration modelliert. Aufgrund der Anzahl und der Art der regierten Konnekte (Ágel 2010: 907) werden innerhalb der expliziten Junktion vier Junktionsklassen unterschieden. Diese sind nach zunehmender Integrativität geordnet: Koordination, Subordination, Inkorporation und Unifikation. ${ }^{106}$ Die beiden Extreme, Koordination (maximale Aggregation) und Unifikation (maximale Integration), unterscheiden sich in der Anzahl der regierten Konnekte: „Während koordinierende Junktoren kein Konnekt regieren, regieren unifizierende Junktoren, die selber Prädikatsausdrücke sind“, zwei verbale/nominale Konnekte (2010: 907). Diese Junktionsklassen werden mit folgenden Beispielsätzen veranschaulicht (2010: 906):

(55) Peter geht nicht zur Schule. Er ist nämlich krank.

(56) Seine Erkrankung ist der Grund dafür, dass Peter nicht zur Schule gehen kann.

Im Beispielsatz (55) werden zwei verbale Konnekte mit dem koordinierenden Junktor nämlich explizit verknüpft, wobei weder das erste noch das zweite Konnekt regiert ist (,Koordination‘). Im Beispielsatz (56) werden ebenfalls zwei Konnekte (ein nominales und ein verbales) mit dem Junktor verknüpft, der unifizierende Junktor (= Prädikatsausdruck ist der Grund dafür) regiert aber beide Konnekte. Ágel (2010: 916) begründet mit der „Beidseitigkeit der Integration (= zwei regierte Konnekte)“, dass die Junktionsklasse ,Unifikation` am integrativsten ist.

Zwischen den beiden Extrempolen werden die Junktionsklassen ,Subordination“ und ,Inkorporation“ verortet, bei denen jeweils ein Konnekt durch den Junktor regiert ist. Der Unterschied lässt sich an der Art des regierten Konnektes (bzw. an der Art des Junktors)

\footnotetext{
$105 \mathrm{Zu}$ weiteren Gründen dafür, warum die Junktionstheorie von Raible (1992) bei der Anwendung auf das Neuhochdeutsche anpassungsbedürftig ist, s. Ágel (2010: 900f.).

106 In der Theorie der expliziten Junktion ist - im Gegensatz zu Raible (1992) - nicht die Juxtaposition die aggregativste Junktionstechnik, sie ist nämlich keine explizite Junktionstechnik. Es findet sich da per definitionem kein Junktor, der Sachverhaltsdarstellungen verbinden könnte.
} 
festmachen. Dies soll mithilfe der folgenden Beispielsätze veranschaulicht werden (Ágel 2010: 906):

(57) Weil Peter krank ist, geht er nicht zur Schule.

(58) Wegen seiner Erkrankung kann Peter nicht zur Schule gehen.

Das vom subordinierenden Junktor regierte Konnekt im Satz (57) ist „,verbal und damit noch relativ selbstständig“ (Ágel 2010: 907). Das von der Präposition wegen regierte Konnekt der Inkorporation (58) ist dagegen nominal: Es wird durch die Nominalgruppe seiner Erkrankung realisiert, die eine Sachverhaltsdarstellung komprimiert ausdrückt und „daher syntaktisch voll in das andere Konnekt eingegliedert ist“"(2010: 907f.).

Innerhalb der oben erläuterten Junktionsklassen können aufgrund des Kriteriums Aggregation/Integration mehrere Junktionstechniken unterschieden werden, die „einzelsprachliche syntaktische Jungierungsoptionen“ darstellen (2010: 908). Die Junktionsklasse der ,Subordination“ wird im Gegensatz zu Raible (1992) sehr differenziert erfasst. Im ersten Schritt ist zwischen finiten und infiniten (ohne $z u$ bzw. mit $z u$ ) Techniken zu unterscheiden. Bei den finiten Techniken der Subordination, die für die vorliegende Untersuchung einschlägig sind, werden in Anlehnung an die syntaktische Subklassifizierung der Konnektoren im HDK-1 (Pasch et al. 2003: 40) drei Grundtechniken bestimmt. Diese sind: Subordination durch Verbzweitsatzeinbetter, Subordination durch Subjunktorersatz ${ }^{107}$, Subordination durch Subjunktoren ${ }^{108}$. Das Rektum des internen Konnekts ist im ersten Fall ein V2-Satz (s. Beispiel (59), daher ist diese Grundtechnik aggregativer als die Subordination durch Subjunktor, bei der das Rektum des internen Konnekts als Verbletztsatz realisiert ist (Beispiel (60)):

(59) Vorausgesetzt es geht ihr gut, kann sie dazu noch viel beitragen. (zit. n. Pasch et al. 2003: 351)

(60) Mir kam es vor, als ob ich ewig gewartet hätte. (2010: 912)

Subordination durch Subjunktorersatz ist aggregativer als Subordination durch Subjunktor. Sie zeichnet sich durch „Subjunktor-Reduktion und deren Indizierung durch Nicht-Verbletzt“ (2010: 912) aus. Im Gegensatz zu ihren Entsprechungen mit Subjunktoren (als ob s. (60) bzw.

107 Bei Subordination durch Subjunktorersatz (Ágel 2010: 912) handelt es sich im Sinne des HDK-1 um eingebettete, aber nicht um subordinierte Sätze (vgl. Pasch et al. 2003: 241).

108 Sog. Postponierer wie z.B. sodass, wobei, bei denen im Gegensatz zu den Subjunktoren die Reihenfolge der Konnekte nicht frei ist (Pasch et al. 2003: 40), werden hier im Rahmen der Grundtechnik ,Subordination durch Subjunktoren“ erfasst, da sie ebenfalls Verbletztsätze einbetten (vgl. Ágel 2010: 912). 
wenn s. (62) weiter unten) bleibt in den folgenden Beispielen nur „ein Restsubjunktor“ als (61) mit besonderer Wortstellung bzw. nur die markierte Verberststellung (63) übrig.

(61) Mir kam es vor, als hätte ich ewig gewartet. (2010: 912)

(62) Wenn es hier ungemütlich ist, können wir auch woanders hingehen. (2010: 912)

(63) Ist es hier ungemütlich, können wir auch wo anders hingehen. (2010: 912)

Mit ,Subordination durch Subjunktorersatz ' werden also sog. uneingeleitete Konditionalsätze und auch weitere uneingeleitete Adverbialsätze (vgl. Kapitel 2.1.1) erfasst.

Die obigen Grundtechniken der Subordination werden weiter ausdifferenziert. Aufgrund der Position des internen Konnekts können mehrere Junktionstechniken unterschieden werden. Besonders aggregativ ist die Junktionstechnik, wenn das interne Konnekt in der sog. Nullstelle (Ágel 2010: 914 im Anschluss an Pasch et al. 2003: 487) bzw. im sog. linken Außenfeld (vgl. Kapitel 3.2.1) auftritt. Etwas integrativer ist die Junktionstechnik, wenn sich das interne Konnekt im Nachfeld, noch integrativer, wenn es sich im Mittelfeld (= Einschub) befindet. Am integrativsten ist die Technik mit der Stellung des internen Konnektes im Vorfeld (= Einbettung).

Aufgrund des Kriteriums des Korrelats (Vorhandensein und Position) wird innerhalb dieser Techniken weiter differenziert (2010: 913ff.). Bezüglich der Voranstellung ${ }^{109}$ des internen Konnektes lassen sich folgende drei Möglichkeiten bestimmen. Am aggregativsten ist die Technik, wenn am Anfang des nachgestellten Hauptsatzes (d.h. direkt nach dem vorangestellten Nebensatz, der im linken Außenfeld des übergeordneten Satzes steht), ein resumptives Korrelat auftritt (Typ ,resumptiv‘, vgl. König/van der Auwera (1988), s. dazu Beispiel (2) weiter oben). Da das resumptive Korrelat die Integration des Nebensatzes im Vorfeld des nachgestellten Hauptsatzes verhindert, ist es aggregativer als die korrelatlose Technik (= ,-KORR`) mit Vorfeldstellung des Nebensatzes (Typ ,integrativ‘ nach König/van der Auwera (1988), s. dazu Beispiel (1) weiter oben). Ágel (2010: 914f.) unterscheidet jedoch im Gegensatz zu König/van der Auwera (1988) resumptive und nichtresumptive Korrelate. Nichtresumptive Korrelate (=,+KORR`, wie dazu im folgenden Beispiel für eine infinite Technik mit $u m$... zu) befinden sich im Mittelfeld des Hauptsatzes und ermöglichen eine Vorfeldstellung des Nebensatzes (wie bei ,-KORR'):

(64) Um meine Familie zu sehen, fahre ich dazu nach Hause. (2010: 915)

109 Die an dieser Stelle erläuterte Unterscheidung ist übrigens auch bei der Nachfeldstellung, z.T. auch bei der Mittelfeldstellung des internen Konnektes möglich, vgl. Ágel (2010: 914). 
Die Technik mit nichtresumptivem Korrelat $(=,+$ KORR`) ist integrativer als die Technik mit resumptivem Korrelat, sie ist sogar nach Ágel (2010: 914f.) am integrativsten. ${ }^{110}$

Der Typ ,nicht-integrativ‘ (König/van der Auwera 1988) erscheint in diesem Modell nicht als eine selbständige Subordinationstechnik. Formal gesehen sind vorangestellte Adverbialsätze mit nicht-integrativer Stellung aber offensichtlich aggregativer als die obigen Typen (vgl. auch Ágel 2010: 920f.). Bei ,ikonische[r] Aggregation“ von SprechaktKonditionalen ist zwar die nicht-integrative Stellung von Adverbialsätzen motiviert, eine integrative Stellung würde sogar Interpretationsschwierigkeiten verursachen. ${ }^{111}$ In seltenen Fällen ist jedoch die prosodische (Des-)Integration für die Interpretation entscheidend, wenn prosodisch integrierte, propositional $\mathrm{zu}$ deutende wenn-Strukturen mit nicht-integrativer Stellung realisiert werden (v.a. wenn sie kontrafaktisch sind, vgl. Breindl et al. 2014: 745f.). In diesen Fällen ist die syntaktische Kodierung nicht ikonisch, die nicht-integrative Stellung korreliert nicht mit der Interpretation als Sprechakt-Konditional, sondern mit der Interpretation als Standardkonditional. Aus diesem Grund folge ich hier nicht dem Modell von Ágel (2010), sondern betrachte den Typ ,nicht-integrativ` als eine selbständige (und bezüglich der Form die aggregativste) Subordinationstechnik bei Voranstellung des Adverbialsatzes. Bei Imperativ- und Fragesätzen, in denen eine integrative Stellung des vorangestellten Adverbialsatzes ausgeschlossen ist, wurde eine nicht-integrative Stellung im linken Außenfeld angenommen (vgl. Kapitel 3.1.3). Im Bereich der nicht-integrierten Adverbialsätze dieser Art sind drei Techniken zu unterscheiden (nach zunehmendem Integrationsgrad geordnet): Die korrelatlose Technik, die Technik mit nichtresumptiven Korrelat im Mittelfeld des Obersatzes und das Auftreten eines Korrelats im linken Außenfeld des Obersatzes (vgl. Kapitel 3.1.3).

Mithilfe des hier erläuterten Junktionsmodells kann die Subordination differenziert erfasst werden. Zudem kann aufgrund der Junktionstechniken auf der Skala der AggregationIntegration auch der Junktionswert bzw. die Junktionsintensität eines Textes im Vergleich mit anderen Texten (als tertia comparationis) bestimmt werden. Somit ist die „Quantifizierung des Grades an Aggregativität bzw. Integrativität von Texten“ bzw. der Anteil jungierter Konnekte an der Gesamtzahl der Konnekte operationalisierbar (2010: 903).

110 Wenn das interne Konnekt im Nachfeld steht, lässt das Korrelat im Mittelfeld des Hauptsatzes „den Anschluss des internen Konnekts [...] antizipieren“ (Ágel 2010: 914f.). Die Antizipation ist einem kataphorischen, nicht-resumptiven Korrelat (,+KORR`) zu verdanken, die im vorangestellten Hauptsatz eine Projektion aufbaut. Diese zusätzliche Leistung von ,+KORR' im Vergleich zu ,-KORR` ist bei nachgestellten Hauptsätzen (und internen Konnekten im Vorfeld) nicht vorhanden, da in solchen Fällen ein rückverweisendes Korrelat auftritt.

111 Bei der Operationalisierung des Junktionsmodells wird berücksichtigt, dass die nicht-integrative Stellung z.B. von Sprechakt-Konditionalen ikonisch sein kann: „Der syntaktischen Nichtintegration entspricht eine semantische.“ (Ágel 2010: 920) 


\subsubsection{Ebenen der Integration}

Bezüglich des Begriffs ,Integration“ herrscht in der Fachliteratur große Uneinheitlichkeit. Er bezeichnet einerseits einen Extrempol auf der Skala zwischen ,Aggregativität/Aggregation“ und ,Integrativität/Integration“ (s. Kapitel 3.3.1). Im Falle der Integration in diesem Sinne werden kohärente syntaktische Einheiten gebildet und dadurch eine monozentrische Organisationsweise ermöglicht (Ágel 2007: 53). König/van der Auwera (1988) nehmen eine Tendenz zur zunehmenden (syntaktischen), Integration“ der vorangestellten Adverbialsätze an, die in drei Stufen abläuft (s. Kapitel 2.2). Ebenfalls mit dem Begriff ,Integration“ werden verschiedene Aspekte der Verknüpfung eines untergeordneten Satzes mit einem übergeordneten Satz im Rahmen der Subordination erfasst. Auf diese Aspekte soll im Folgenden kurz eingegangen werden. ${ }^{112}$

Wie bereits erwähnt (s. Kapitel 3.3.1), wird Subordination in jüngeren Arbeiten als ein vieldimensionales Verfahren dargestellt (vgl. Fabricius-Hansen 1992; Zifonun et al. 1997: 2250-2253). Es handelt sich dabei um die Fragen, wie die Unterordnung formal markiert wird bzw. welche Relationen zwischen den verknüpften Teilsätzen bestehen. Dementsprechend werde ich die Forschungsliteratur getrennt in ,formale' und ,funktionale Unterordnung behandeln. Die Möglichkeit der Trennung von formalen und funktionalen Aspekten der Subordination deuten bereits Fabricius-Hansen (1992: 481) und Helbig (2003: 1f.) an, wobei sie allerdings zum Teil unterschiedliche Kriterien aufführen. Bei der ,formalen Unterordnung‘ werde ich folgende Ebenen der Integration ins Auge fassen: ,Nebensatzgestalt` (Zifonun et al. 1997: 2250), ,topologische Integration“ (Zifonun et al. 1997: 2250) und ,prosodische Integration“ (Volodina 2011a: 146, vgl. Kapitel 2.1.2). Bezüglich der dritten Ebene sei erwähnt, dass hier mit Eisenberg (2006: 27) die Auffassung vertreten wird, dass die Intonation - ebenso wie die Wortstellung - zu den syntaktischen Mitteln gehört. Die obigen Kriterien beziehen sich also alle auf die äußere Form der verknüpften Sätze, daher scheint es berechtigt zu sein, sie unter dem Begriff ,formale Markierung von Unterordnung ${ }^{`}$ zu behandeln.

Mit ,Nebensatzgestalt‘ bzw. ,gestaltsbezogener Integration“ (Zifonun et al. 1997: 2250) wird die Stellung des Finitums im Nebensatz erfasst. Die Verbletztstellung ist im Gegenwartsdeutschen mit dem Auftreten eines Subjunktors, Relativpronomens bzw. wAusdrucks am Anfang des Nebensatzes gekoppelt: „Im Gegensatz zu Konjunktionen leiten Subjunktionen Verbletztsätze ein: Das finite Verb nimmt die letzte Position im Nebensatz

112 Auf die Diskussion weiterer Verwendungsweisen des Begriffs ,Integration', wie etwa bei der Integration von Fremdwörtern ins Deutsche (vgl. Duden-Grammatik 2016: 371), wird aus Platzgründen verzichtet. 
ein“ (Duden-Grammatik 2016: 637). Sätze mit Endstellung des Finitums sind in der Regel Nebensätze. Umgekehrt gilt auch, dass die Unterordnung eines Teilsatzes im prototypischen Fall durch die Verbletztstellung markiert wird. Auf beiden Seiten gibt es jedoch Ausnahmen für die Korrelation Nebensatz-Verbletztstellung. Auf selbständige, unabhängige Verbletztsätze, die keinen Obersatz haben, wurde im Kapitel 3.3 bereits verwiesen. Des Weiteren wurden auch untergeordnete Sätze beschrieben, die keine Verbletztstellung aufweisen. Die gestaltsbezogene Desintegration von uneingeleiteten Konditionalsätzen mit Verberststellung (vgl. Beleg (63) weiter oben) kann nur auf anderen Ebenen, u.a. mit prosodischer und pragmatischer Integration kompensiert werden. ${ }^{113}$ Es stellt sich dabei auch die Frage, ob sie auch nach anderen Kriterien weniger integriert sind als prototypische Nebensätze, d.h., ob zwischen den einzelnen Ebenen der Integration Zusammenhänge nachzuweisen sind. Helbig (2003: 5) bringt diesen Gedanken wie folgt auf den Punkt: „Die beiden Kriterien (der ,Form“ und der ,Funktion') decken sich [...] durchaus nicht immer.“

Bei der ,topologischen Integration“ (Zifonun et al. 1997: 2250) geht es um die Frage, ob der Nebensatz die gewöhnliche Stelle eine Satzglieds innerhalb des übergeordneten Satzes einnimmt oder ob eine Art „Sonderstellung“ auftritt (vgl. Fabricius-Hansen 1992: 474f.). ${ }^{114}$ Durch die Vorfeldstellung von untergeordneten Sätzen ist im Gegenwartsdeutschen eine Möglichkeit gegeben, auch bei Sätzen, die keine ,Nebensatzgestalt' haben, die Unterordnung formal durch Einbettung zu markieren (vgl. Beleg (63) mit ,integrativer' Stellung im Sinne von König/van der Auwera (1988)). Subjunktional eingeleitete Adverbialsätze mit Verbletztstellung, die eine ,integrative' Stellung aufweisen, sind formal gesehen mehrfach integriert. Bei ,nicht-integrativer' Stellung wiederum kann auch im Falle von Verbletztsätzen auf Besonderheiten in der Relation von Haupt- und Nebensatz geschlossen werden. Die nichtintegrative Stellung zeigt eine „nur schwach ausgebildete syntaktisch-semantische Integration“ (Zifonun et al. 1997: 2351). ${ }^{115}$ Die dritte Möglichkeit nach König/van der Auwera (1988) ist die Wiederaufnahme des vorangestellten Adverbialsatzes mit einem resumptiven Element im Vorfeld des Nachsatzes (= Typ ,resumptiv'). Dabei würde eine

113 Reis/Wöllstein (2010: 113) vertreten die Ansicht, dass uneingeleitete V1-Konditionalsätze nicht integriert sind. Auch unselbständige V2-Sätze mit argumentrealisierender Funktion sind nach Reis (1997: 121, 142), ,syntaktisch ,relativ unintegrierte Nebensätze““. Pittner (2011: 102f.) kritisiert jedoch diese Auffassung und verweist darauf, dass die angenommene Unintegriertheit der V1-Konditionalsätze nur auf den einzelnen Ebenen gezeigt werden kann, bzw. „dass Integration auf den einzelnen Ebenen auch prinzipiell unabhängig stattfinden kann“. D.h., aus der „Tatsache, dass sie prosodisch nicht integriert sind und eine eigene FHG aufweisen", lässt sich nicht darauf folgern, dass sie syntaktisch nicht integriert wären. Pittner (2011: 103) argumentiert also für die Trennung der informationsstrukturellen und der syntaktischen Ebene.

114 Es handelt sich hier also um die ,externe Topologie‘ der Nebensätze, vgl. Kapitel 3.2.1.

115 Peyer (1997: 80, Fn. 48) verweist jedoch darauf, dass diese Korrelation umgekehrt nicht gültig ist, denn „topologisch vollständig integrierte Nebensätze bedeuten nicht immer einen engen inhaltlichen Zusammenhang." Vgl. dazu die Beispielsätze im Kapitel 3.4.6 weiter unten. 
,topologische Integration“ des vorangestellten Adverbialsatzes nur dann vorliegen, wenn man annehmen würde, dass sowohl der Vordersatz als auch das wiederaufnehmende Korrelat im Vorfeld des Nachsatzes stehen. Obwohl dies sicherlich nicht die herrschende Meinung ist, kann diese Analysse bei Blühdorn (2013: 155) und Günthner (1999a) gefunden werden. In der Tat könnte die Korrelatverbindung aus vorangestelltem Adverbialsatz und wiederaufnehmendem Element als eine Konstituente aufgefasst werden (vgl. auch Zitterbart 2013: 621), im Rahmen des Stellungsfeldermodells wird aber in diesem Fall die Stellung des Adverbialsatzes im sog. linken Außenfeld angenommen. Der vorangestellte Adverbialsatz ist syntaktisch nicht mehr voll integriert, da der Platz, den er im Obersatz einnehmen könnte, durch das Korrelat besetzt ist (vgl. Fabricius-Hansen 1992: 471). Das Korrelat stellt also syntaktisch gesehen eine Entsprechung des Adverbialsatzes im Obersatz dar (Zitterbart 2013: 602-606). Syntaktische und semantische Aspekte sind dabei schwer zu trennen. Die semantische Funktion von Korrelaten als Anschlusselement zwischen den beiden Teilsätzen wird weiter unten beschrieben. Wir unterscheiden also den hohen, mittleren und niedrigen Grad der topologischen Integration (vgl. z.B. Pasch et al. 2003, Volodina 2011: 18). Beim nicht-integrativen Stellungstyp liegt demnach keine Einbettung vor, aber auch in diesem Fall handelt es sich um Subordination. Subordiniert und auch eingebettet sind sowohl der integrative als auch der resumptive Typ, wobei jedoch der erste einen hohen Grad, der zweite - wegen Herausstellung - nur einen mittleren Grad topologischer Integration darstellt.

Die dritte, formale Ebene bezeichne ich in Anlehnung an Volodina (2011a: 146) und Pittner (2011: 96) als ,prosodische Integration“ (= ,phonologische Integration“ nach FabriciusHansen 1992: 467, ,intonatorische Integration“ nach Zifonun et al. 1997: 2250, vgl. auch Kapitel 2.1.2). ${ }^{116}$ Hierbei handelt es sich um die Frage, ob den verknüpften Teilsätzen eine eigene Intonationseinheit zugewiesen werden kann. Prosodisch eigenständige Satzeinheiten, deren Autonomie auch durch finale Grenztonmuster, deutliche Pausen und „,zurückgenommene[...] Artikulation“ gezeigt werden kann, sind nicht voll in den Matrixsatz integriert (Zifonun et al. 1997: 2252).

Die prosodischen Unterschiede können auch in der Schriftlichkeit zum Ausdruck kommen. Peyer (1997: 56) nimmt daher die Subordinationskomponente ,Intonation und Interpunktion im Gefüge‘ an. Die Markierung der geringen bzw. fehlenden Integration durch Doppelpunkt -

\footnotetext{
116 Der Begriff ,prosodische Integration“ scheint aus mehreren Gründen angemessener als seine Alternativen. Es soll hervorgehoben werden, dass etwa die progrediente Intonation nicht unbedingt mit dem Fehlen/Vorhandensein eigenständiger Intonationskonturen korreliert (Reis/Wöllstein 2010: 147). Dabei spielen auch weitere prosodische Faktoren eine Rolle, wie etwa die eventuelle Abtrennung der Teilsätze durch eine Pause.
} 
wie im nächsten Beispielsatz aus einem Spiegel-Interview aus dem Jahre 1999 - ist jedoch m.E. sekundär, sie imitiert bzw. bildet die prosodische Eigenständigkeit des Vordersatzes ab:

(65) Wenn Ihnen das Manager-Gewerbe schon suspekt ist: Können Sie sich denn vorstellen, nach dem Karriere-Ende eine Clubmannschaft zu trainieren? (zit. n. Karhiaho 2003: 94)

In ihrem Korpus aus Pressetexten und literarischen Texten der Gegenwart bzw. in der einschlägigen Literatur fand Karhiaho (2003: 95) in allen einschlägigen Beispielen mit dem Doppelpunkt eine nicht-integrative Stellung. Gerade bei Spitzenstellung des Hauptsatzfinitums kann der Doppelpunkt „die Stellung des Adverbialsatzes als nichtintegrativ fest[legen]“ (2003: 95), wie dies auch im obigen Beispiel zu beobachten ist. Die nicht-integrative Stellung von Adverbialsätzen vor Frage- bzw. Aufforderungssätzen mit Verberststellung wird jedoch nicht immer durch Interpunktionszeichen markiert. ${ }^{117}$

Freywald (2013: 323) sieht einen Zusammenhang zwischen Prosodie und FokusHintergrund-Gliederung bei Verberstsätzen. Wie eng die prosodische und die informationsstrukturelle Ebene miteinander verknüpft sind, zeigt das folgende Zitat, in dem die beiden Ebenen gleichgesetzt werden: „Prosodisch haben uNS [= uneingeleitete Nebensätze, P.K.] und Bezugssatz notwendig separate Fokus-Hintergrund-Gliederungen [...], mit (optionaler) Pausenmarkierung; sie bilden also separate Informationseinheiten“ (Reich/Reis 2013: 551). M.E. sollte jedoch die formale und die funktionale Ebene auch in diesem Fall getrennt werden. Die informationsstrukturelle Ebene wird daher weiter unten erläutert.

Mit den oben beschriebenen drei Möglichkeiten stehen unterschiedliche Verfahren zur formalen Markierung von Integration/Desintegration zur Verfügung, die im Grunde genommen „,die selbe Funktion [haben] und [...] sie in ähnlicher Weise [leisten]“ (Wegener 2000: 39). Sie bilden die enge/losere semantische und pragmatische Relation zwischen den verknüpften Sätzen ikonisch ab. Wie Wegener (2000) am Beispiel der weil-Sätze zeigt, können dabei dank des Zusammenspiels der drei Verfahren mehrere Stufen unterschieden werden, vgl. weil-Verbletztsätze, die trotz Nebensatzgestalt nur schwach integriert sind. ${ }^{118}$

\footnotetext{
117 Zur Erläuterung der Gründe, warum in diesen Fällen eine nicht-integrative Stellung angenommen wird, bzw. zu einschlägigen Beispielsätzen ohne besondere grafische Markierung der nicht-integrativen Stellung vgl. Kapitel 3.2.2.

118 In Beispielen wie Weil du es sowieso erfährst, - ich lasse mich scheiden. bzw. Möchten Sie noch ein Viertel Wein? Weil ich jetzt die letzte Runde abfrage. (zit. $\mathrm{n}$. Wegener 2000: 37f.) sind die weil-Sätze prosodisch und - wegen der nichtintegrativen Voranstellung bzw. wegen der Nachstellung - auch topologisch nicht integriert. Zwischen den Teilsätzen besteht nur eine losere inhaltliche Beziehung (epistemische Rechtfertigung oder Äußerungsbegründung). Durch die Nebensatzgestalt kann jedoch angezeigt werden, dass der Inhalt des weiterführenden weil-Satzes nur eine Nebeninformation darstellt. Auch die parataktische Verknüpfung mit weil-Verbzweit kann eine losere inhaltliche Beziehung zwischen den Teilsätzen anzeigen.
} 
Unter ,formaler Markierung von Unterordnung' können neben den oben genannten Ebenen der Integration auch weitere Merkmale der Subordination erfasst werden. Auf den Subjunktorersatz bzw. markierte Wortstellung von uneingeleiteten Adverbialsätzen war im Kapitel 3.3.1 bereits eingegangen. Auch die Konjunktivverwendung wird als Nebensatzmerkmal zur Bestimmung der Grade der Abhängigkeit herangezogen, vgl. z.B. Auer (1988), der bei ,uneingeleiteten Nebensätzen“ - wie im Beispiel (66) - den Konjunktiv im nachgestellten Inhaltssatz als nebensatzindizierendes Phänomen ansieht:

(66) Josef fürchtete, er habe/hätte einen Fehler gemacht.

Es ist jedoch fraglich, ob der Konjunktiv auch bei Adverbialsätzen als Subordinationsmerkmal angesehen werden kann. Bei Finalsätzen kann er einfach stilistisch markiert sein: Der Konjunktiv I kommt in damit-Sätzen eher im gehobenen Register vor (Zifonun 2013: 308). Schließlich könnte überlegt werden, auch die ,korrelative Subordination“ (Zitterbart 2002: 33) als eigenständige Ebene bei der formalen Unterordnung anzunehmen. Aber Korrelate ,erfüllen Funktionen im Satzgefüge, die oft vielschichtig sind und mehr als nur grammatische Informationen enthalten können“ (Zitterbart 2013: 602), sodass hier die formale und die funktionale Ebene bei Korrelaten getrennt behandelt wird. Wie gezeigt wurde, gestaltet sich ,formale Markierung von Unterordnung' als graduales Konzept. Neben formal voll integrierten Strukturen gibt es auch solche, die nur schwach bzw. nur auf einer Ebene integriert sind.

Als nächster Schritt wird die ,funktionale Unterordnung` erschlossen. Dabei soll zunächst die Frage nach Unterscheidungskriterien der ,funktionalen Unterordnung ${ }^{6}$ gestellt werden. Dazu gehören m.E. die ,semantische Integration“ (Fabricius-Hansen 1992: 467f.) und die ,pragmatische Integration“ (Fabricius-Hansen 1992: 467, 472f., Helbig 2003: 8). Die beiden Kriterien werden bereits von Fabricius-Hansen (1992: 481) als zusammengehörend beschrieben, wenn die Subordination in funktionaler Hinsicht erfasst wird.

Bei der semantischen Ebene geht es in erster Linie um das Kriterium ,Satzgliedwert`,Satzgliedschaft im übergeordneten Satz‘(Peyer 1997: 56 bzw. Helbig 2003: 2), d.h. um eine funktionale Abhängigkeit des untergeordneten Satzes vom übergeordneten Satz. Dieses Kriterium ist erfüllt, wenn der untergeordnete Satz eindeutig als ein Satzglied (oder Satzgliedteil) in den übergeordneten Satz eingebettet ist. ${ }^{119}$ Lehmann (1988: 189)

119 Fabricius-Hansen (1992: 467f.) unterscheidet drei semantische Funktionen des Untersatzes bei der Integration in den Obersatz. Bei ,Argument-Subordination“ ist der untergeordnete Satz ein Argument (oder eine Ergänzung) des Obersatzprädikats. Bei der ,Restriktor-Subordination' geht es um Verknüpfungen, die traditionell als attributiv bezeichnet werden könnten. Adverbialsätze werden mit dem Terminus ,Operator-Subordination` erfasst (vgl. z.B. Zitterbart 2002: 
spricht in seinem sprachtypologischen Modell zur ,clause linkage“ von Degradierung eines Satzes zum Satzglied eines anderen Satzes (vgl. auch Peyer 1997: 30ff.). Dies ist jedoch nicht der Fall bei weiterführenden Nebensätzen, des Weiteren wird der Satzgliedstatus der eingeleiteten Nebensätze auch bei Postponierern wie so dass in Frage gestellt (FabriciusHansen 1992: 463, vgl. auch weiter oben). In der IDS-Grammatik (Zifonun et al. 1997: 2251), in der der Terminus ,kompositionale Integration ${ }^{، 120}$ verwendet wird, werden mehrere Fälle der Desintegration aufgeführt: U.a. werden diktumskommentierende, modusmodifizierende und moduskommentierende Satzadverbialsätze (s. dazu ausführlicher Kapitel 3.4.6) erwähnt, die kompositional nicht voll in den Nachsatz integriert sind. Der ,kompositionalen“ bzw. ,semantischen' Desintegration entspricht jedoch nicht immer eine topologische Desintegration. In diesen Fällen ist die Behandlung des Satzgliedkriteriums besonders problematisch: Trotz Vorfeldstellung, also topologisch vollständiger Integration, liegt kein enger inhaltlicher Zusammenhang zwischen den Teilsätzen vor.

Zur semantischen Ebene der Integration gehören m.E. auch weitere Kriterien. Wie oben erwähnt, könnte das ,(Nicht-)Auftreten von Korrelaten und Proformen` evtl. als eigenständige Komponente von Subordination aufgefasst werden (Fabricius-Hansen 1992: 470-473, vgl. auch Peyer 1997: 56, 66-77). Die syntaktische Fügung ist zwar in solchen Fällen lockerer, aus funktionaler Sicht kann jedoch eine zusätzliche Leistung von Korrelaten beobachtet werden. Obwohl der vorangestellte Adverbialsatz semantisch nur indirekt, durch das Korrelat eingebettet ist (Fabricius-Hansen 1992: 471), ${ }^{121}$ stärkt das Korrelat die semantische Verknüpfung der Teilsätze: „Unter semantisch/inhaltlichem Aspekt [...] verweist das Korrelat auf ein zu ergänzendes Informationselement, kennzeichnet damit den Hauptsatz als nicht selbständig und bewirkt, daß die Teilsätze stark aufeinander bezogen werden.“ (Peyer 1997: 71). Des Weiteren kann das Korrelat als Teil der Korrelatverbindung auch „die semantischlogische [...] Beziehung“ zwischen den Teilsätzen verdeutlichen und somit zur Eindeutigkeit bei der Interpretation beitragen (Zitterbart 2002: 35).

142). Im Falle der ,Operator-Subordination“ sind wohl die Teilsätze semantisch weniger eng verknüpft als bei der ,Argument-Subordination“, denn die Untersätze können im Normalfall ,nicht als primärer Aktant des Verbums eingestuft werden“ (Zitterbart 2002: 35), es sei denn, der Adverbialsatz ist valenzgebunden (vgl. Kapitel 3.1.2).

120 Diesem Kriterium entsprechen nach Zifonun et al. (1997: 2250) „Nebensätze, die die Wahrheitsbedingungen des Obersatzrestes tangieren, also Nebensätze, die als Teile von Propositionsausdrücken oder Teile von Teilen von Propositionsausdrücken zu verstehen sind“. Helbig (2003: 8) verwendet den Begriff ,semantisch-propositionale Integration' wohl in dem hier erläuterten Sinne (eigene Proposition/Prädikation oder nicht), er nimmt aber hierfür eine eigenständige Ebene der Integration an.

121 Beim resumptiven Typ wie in (2) ist nach dem HDK-1 (Pasch et al. 2003: 74) keine syntaktische Funktion des vorangestellten Adverbialsatzes im Obersatz anzunehmen: „Der linksversetzte Ausdruck bezeichnet hier einen ,Diskursgegenstand', von dem der nachfolgende Satz etwas prädiziert. Er steht zu diesem nur in einer textuellen, nicht aber in einer syntaktischen Funktion.“ 
Bei der Explizitheit der Verknüpfung unterscheidet Lehmann (1988: 210-213) mehrere Möglichkeiten auf dem Kontinuum zwischen Syndese und Asyndese. Bei sehr expliziten Verknüpfungsmitteln sind die beteiligten Sätze miteinander stärker verbunden als bei unspezifisch(er)en Verknüpfungsmitteln (wie z.B. dass) oder asyndetischen Verknüpfungen (vgl. auch Peyer 1997: 40ff.). Bei asyndetischen V1-Konditionalen, in denen keine Subjunktoren die logisch-semantische Beziehung zwischen den Teilsätzen kennzeichnen können, kann die semantische Verknüpfung der Teilsätze durch ein Korrelat markiert und der V1-Satz dadurch desambiguiert werden. Zitterbart (2002: 149) geht sogar soweit, dass sie das Korrelat bei V1-Konditionalen als Subordinationszeichen ansieht, „das die Verhältnisse (Verberstsatz als Nebensatz!) im Gesamtsatz verdeutlicht und stabilisiert“. ${ }^{122}$

Hierfür ist jedoch nicht nur zwischen semantisch eindeutigen und polyfunktionalen Subjunktoren, sondern auch zwischen semantisch eindeutigen und polyfunktionalen Korrelaten zu unterscheiden (vgl. Kapitel 3.1.4), deren Bestand und Merkmale sich historisch ändern. $^{123}$ Bei semantisch eindeutigen Korrelaten wie deswegen (kausal) und Korrelatverknüpfungen wie wenn-dann (konditional) ist der Grad der semantischen Integration höher. Im Gegensatz dazu ist so ein „semantisch unspezifisches Korrelat“ (Zitterbart 2002: 152).

Schließlich könnte auch überlegt werden, bei der semantischen Integration auch den „Grad der semantisch-konzeptuellen Abhängigkeit zwischen den beiden Konjunkten“ (Wegener 2000: 43) zu berücksichtigen. So wäre die hohe Abhängigkeit bei echten Bedingungssätzen und Nicht-Abhängigkeit bei Konzessiva zu unterscheiden. ${ }^{124}$

\footnotetext{
${ }^{122}$ Im frühen Neuhochdeutschen war die Polyfunktionalität der Verberststellung noch stärker als im Gegenwartsdeutschen, vgl. die exemplarische Analyse eines nähesprachlichen Textes in Kappel (2004). Im Neuhochdeutschen gab es eine Tendenz zur Reduktion der uneingeleiteten Adverbialsätze zugunsten der eingeleiteten Adverbialsätze, wobei jedoch uneingeleitete Konditionalsätze eine Ausnahme bildeten (vgl. Ágel 2000: 1881). Im Gegenwartsdeutschen sind dementsprechend v.a. uneingeleitete Konditionalsätze häufig.

123 Zur Übergangszeit vom Frühneuhochdeutschen zum Neuhochdeutschen wird der Gebrauch der semantisch mehrdeutigeren Konnektoren, deren Bedeutungen sich erst mit Einbeziehung des Kontextes ergeben und die somit mehrere Gebrauchsmöglichkeiten zulassen, vom Gebrauch der semantisch eindeutigeren Konnektoren, die kontextentbundene Bedeutungen aufweisen, verdrängt. Die Tendenz zur Reduktion von Bedeutungsvielfalt bzw. zur zunehmenden Wohlbestimmtheit im Bereich des Wortschatzes wird von Reichmann (1988) als ,Monosemierung ‘ bezeichnet. Zur Monosemierungstendenz im Bereich der Satzkonnektoren s. z.B. Betten 1987: 87ff., Kappel/Németh 2003 sowie Kapitel 2.2.1 weiter oben. Für die letzten Jahrzehnte wird öfters auch eine gegensätzliche Entwicklung angenommen, z.B. bei den viel diskutierten, angeblich neueren Verwendungsmöglichkeiten von weil (zum Überblick und weiterer Literatur s. Selting (1999); zu obwohl vgl. Günthner (1999b), zu wobei vgl. Günthner (2000), zu wo vgl. Günthner (2002)). Dies dürfte aber mit der Entwicklung einer neuen, tertiären Mündlichkeit (vgl. Ágel 2003: 15), also mit dem wachsenden Einfluss der konzeptionell gesprochenen Sprache auf die Lockerung der Normen - und möglicherweise auch mit der Verlagerung des Beschreibungsgegenstandes der Sprachwissenschaftler auf die gesprochene Sprache zusammenhängen.

124 Bei echten Konditionalen ist der Sachverhalt A die Voraussetzung für B, bei Konzessiva ist er aber irrelevant bzw. kann auch im Widerspruch zu B stehen. In semantisch-konzeptueller Hinsicht besteht also nur bei echten Konditionalen eine Abhängigkeit zwischen A und B. (vgl. Wegener 2000: 40ff.)
} 
Beim zweiten, pragmatischen Kriterium geht es um die Frage, ob die verknüpften Teilsätze eine eigene Illokution haben oder der untergeordnete Satz „kein eigenes illokutives Potential besitzt und der komplexe Satz nur die dem entsprechenden einfachen Satz zukommende illokutive Kraft aufweisen kann.“ (Fabricius-Hansen 1992: 468) Diese Ebene wird von Zifonun et al. (1997: 2252) - der Terminologie der IDS-Grammatik entsprechend - als ,KMModus-bezogene Integration“ bezeichnet. Dass dabei im Grunde genommen das selbe Kriterium gemeint ist, zeigt auch die Verwendung des Synonyms ,illokutive Integration“ (Zifonun et al. 1997: 2253). Bei Helbig (2003: 2) ist von kommunikativer Selbständigkeit die Rede.

In der Fachliteratur werden gelegentlich weitere Ebenen der Integration angenommen. So unterscheidet Pittner (2011: 101f.) die ,informationsstrukturelle Integration“. „Komplexe Sätze können eine gemeinsame Fokus-Hintergrund-Gliederung (im folgenden FHG) oder separate FHGs für die einzelnen Teilsätze aufweisen“ (Pittner 2011: 86). Auch Helbig (2003: 8) nimmt hier eine eigene Ebene der Integration an und bezeichnet sie als ,thematische Integration'. Bei thematischer Integration in der Informationsstruktur geht es darum, dass im komplexen Satz nur ein gemeinsamer Fokus bzw. nur eine Thema-Rhema-Struktur zu finden ist. Auch wenn keine informationsstrukturelle Integration vorliegt, also wenn der untergeordnete Satz und der Matrixsatz jeweils eine eigene Fokus-Hintergrund-Gliederung aufweisen, ist die Informationseinheit des untergeordneten Satzes kommunikativ weniger gewichtig als die des Matrixsatzes, sie stellt nur eine Hintergrundinformation dar (Brandt 1989, Pittner 2011: 86). Umgekehrt ist es jedoch auch möglich, dass der Subjunktorsatz die Vordergrund- und der Obersatz die Hintergrundinformation liefert (Zifonun 2013: 305). Bei Wegener (2000: 36f.) werden die Parameter illokutive Selbständigkeit bzw. selbständige Informationseinheiten/eine Informationseinheit unter dem pragmatischen Aspekt subsumiert. Dazu gehört nach Wegener (2000: 36) auch die Frage, ob der eine Satz unter den Skopus eines Operators des anderen Satzes fällt.

Die Ebenen der Integration werden durch die folgende Abbildung 8 zusammenfassend dargestellt: 


\begin{tabular}{|l|c|}
\hline \multicolumn{1}{|c|}{ formale Unterordnung } & funktionale Unterordnung \\
\hline - Nebensatzgestalt & - semantische Integration \\
\hline - topologische Integration & - Satzgliedschaft im übergeordneten Satz \\
& - Explizitheit der Verknüpfung \\
\hline - prosodische Integration & - semantisch-konzeptuelle Abhängigkeit \\
(bzw. Markierung der Desintegration durch & - illokutive Integration \\
Interpunktionszeichen) & - informationsstrukturelle Integration \\
\hline - Konjunktiv als Abhängigkeitssignal & \\
\hline
\end{tabular}

Abb. 8: Ebenen der Integration

Diese Ebenen der Integration sind nicht nur bei der Subordination anwendbar. Der Beleg (58) weiter oben, der die Inkorporation eines von der Präposition wegen regierten Konnekts veranschaulicht, zeigt auf mehreren Ebenen einen hohen Grad der Integration. Aus formaler Sicht ist die Präpositionalgruppe Wegen seiner Erkrankung topologisch und prosodisch integriert. Es liegt zwar keine Nebensatzgestalt vor, die Nominalisierung stellt jedoch im Sinne des Gesagten einen höheren Grad der Integration dar, formal ist diese Struktur also noch stärker integriert als ein prototypischer Nebensatz. Aus funktionaler Sicht liegt eine pragmatische und informationsstrukturelle Integration vor. Das semantische Kriterium der ,Satzgliedschaft‘ wird ebenfalls erfüllt, zudem ist die logisch-semantische Beziehung zwischen den Konnekten explizit markiert. Im Gegensatz dazu weisen die intensiv erforschten weil-Sätze mit Verbzweitstellung einen niedrigen Grad der Integration auf und werden zu Recht zur Parataxe gerechnet (s. Beleg (69) weiter unten, vgl. Küper 1991, Selting 1999, Wegener 2000 und Ágel 2016).

\subsection{Semantische Typen von Adverbialsätzen}

Die bisherigen Forschungen haben gezeigt, dass es beim Integrationsgrad von Adverbialsätzen relevante Unterschiede hinsichtlich der semantischen Subtypen gibt. So sind etwa im Gegenwartsdeutschen Temporalsätze stärker integriert als Konzessivsätze (König/van der Auwera 1988, vgl. Kapitel 2.1.1). Auch im früheren Deutsch wurden relevante Unterschiede nachgewiesen (vgl. Kapitel 2.2.2.2). Aus diesen Forschungsergebnissen konnte die Hypothese abgeleitet werden, dass die Tendenz zur zunehmenden Integration vorangestellter Adverbialsätze in der neuhochdeutschen Epoche 
(1650-2000) bei den semantischen Subtypen von Adverbialsätzen unterschiedlich ausgeprägt ist und zwischen ihnen Unterschiede hinsichtlich des Integrationsgrades beobachtet werden können (vgl. Kapitel 2.4). Um die Gültigkeit dieser Hypothese überprüfen zu können, werden die Korpusbelege getrennt nach semantischen Typen ausgewertet und in eigenen Kapiteln diskutiert. Falls statistisch relevante Unterschiede nach den semantischen Subtypen nachgewiesen werden können und gezeigt werden kann, dass die Integration bei den verschiedenen semantischen Klassen anders abläuft, dann soll die Frage gestellt werden, ob es sinnvoll ist, die verschiedenen Typen gemeinsam unter dem Begriff Adverbialsätze zu subsumieren bzw. zu behandeln. Eine alternative Auffassung wäre, dass nicht ein Prozess der Integration bei den Adverbialsätzen, sondern mehrere Integrationsprozesse bei den verschiedenen semantischen Typen ablaufen. Im Folgenden soll der theoretische Rahmen für die Analyse nach semantischen Typen dargelegt werden.

\subsubsection{Klassifizierungen von Adverbialsätzen}

Traditionell werden bei den Konjunktionen, Adverbialbestimmungen und zum Teil auch bei Nebensätzen semantische Typen wie etwa temporal, kausal, konditional unterschieden (vgl. Bußmann 2008: 467). Im HDK-2 (Breindl et al. 2014: 244) wird jedoch zu Recht konstatiert, dass es in den grammatischen Darstellungen des Deutschen keinen Konsens gibt bei der Frage, welche Klassen von Adverbialsätzen unterschieden werden sollten. Dies wird in der folgenden Abbildung 9 anschaulich gezeigt. Es finden sich insg. nur 6 Nebensatzklassen, die in allen herangezogenen Darstellungen auftreten. Zu diesem „Kernkanon“ (2014: 242) gehören folgende Typen: Temporal-, Konditional-, Kausal-, Konsekutiv-, Final-, Konzessivsatz. Zudem wird der Restriktivsatz bis auf eine Darstellung meist mitbehandelt. ${ }^{125}$

125 Bei der Ausnahme handelt es sich um die Syntax von Dürscheid, die diesen Typ auch in der neuesten Auflage (2012) nicht aufführt. Der Restriktivsatz kann ebenfalls zum „Kernkanon“ gerechnet werden. Die entsprechenden Typen sind durch Schattierung hervorgehoben. 


\begin{tabular}{|c|c|c|c|c|c|c|c|}
\hline & $\begin{array}{l}\text { Dürscheid } \\
2010\end{array}$ & $\begin{array}{l}\text { Duden- } \\
\text { Grammatik } \\
2009\end{array}$ & $\begin{array}{l}\text { Engel } \\
2004\end{array}$ & $\begin{array}{l}\text { Weinrich } \\
2003\end{array}$ & GDS 1997 & $\begin{array}{l}\text { Helbig/ } \\
\text { Buscha } \\
1999\end{array}$ & $\begin{array}{l}\text { Hoffmann } \\
2013\end{array}$ \\
\hline Lokalsatz & & & + & & & + & + \\
\hline Temporalsatz & + & + & + & + & + & + & + \\
\hline Modalsatz & + & + & & & & + & \\
\hline Konditionalsatz & + & + & + & + & + & + & + \\
\hline Kausalsatz & + & + & + & + & + & + & + \\
\hline Konsekutivsatz & + & + & + & + & + & + & + \\
\hline Finalsatz & + & + & + & + & + & + & + \\
\hline Instrumentalsatz & & & + & & & + & \\
\hline Konzessivsatz & + & + & + & + & + & + & + \\
\hline Adversativsatz & + & + & & & + & + & \\
\hline $\begin{array}{l}\text { Irrelevanz- } \\
\text { konditionalsatz }\end{array}$ & & + & & & + & & \\
\hline Restriktivsatz & & + & + & + & + & + & + \\
\hline Komitativsatz & & & + & & + & & + \\
\hline Konfrontativsatz & & & & & + & & + \\
\hline Proportionalsatz & & & & & + & + & \\
\hline Negationssatz & & & & & + & & \\
\hline Komparativsatz & & & & & & + & \\
\hline $\begin{array}{l}\text { Ereignis- } \\
\text { präzisierender } \\
\text { Adverbialsatz }\end{array}$ & & & & & & & + \\
\hline Vergleichssatz & & & & & & & + \\
\hline Explikativsatz & & + & & & & & \\
\hline
\end{tabular}

Abb. 9: Nebensatzklassen in Grammatiken des Deutschen (Breindl et al. 2014: 243)

In der vorliegenden Untersuchung werden z.T. die traditionellen Typen aus dem obigen Kernkanon übernommen (wie etwa Temporal-, Konditional-, Kausal- und Konzessivsätze). Im Folgenden soll auf die Problematik der semantischen Relationen detaillierter eingegangen werden.

Die semantischen Typen sollen in einem Modell erfasst werden, das satzsemantische Verhältnisse nicht nur bei der Subordination, sondern auch bei anderen Junktionstechniken beschreiben kann. In der germanistischen Linguistik ist v.a. die Klassifizierung von Peter von 
Polenz (1985: 269-287) verbreitet. ${ }^{126}$ Er unterscheidet 15 Klassen (1985: 268ff.) für semantische Relationen bei Aussagen-Verknüpfungen.

Die semantischen Klassen von Peter von Polenz sind als Weiterführung und Präzisierung der traditionellen Gliederungen der Konjunktionen, Adverbialbestimmungen und Nebensätze nach inhaltlichen Kriterien interpretierbar. Diese traditionellen Kategorien werden bei von Polenz (1985: 268ff.) - unter Beibehaltung der lateinischen Termini - aufgegriffen. ${ }^{127} \mathrm{Im}$ nächsten Beispiel werden zwei Prädikationen/Aussagen (P1 und P2) bzw. „zwei Satzinhalte samt pragmatischem Gehalt“" (von Polenz 1995: 287) durch die subordinative Konjunktion (weil) mit kausaler Relation (R) verknüpft. Der durch die subordinative Konjunktion eingeleitete, vorangestellte Nebensatz (P1) fungiert als Angabesatz als Teil des folgenden Hauptsatzes (P2). Das folgende Beispiel enthält die einschlägige Notation nach von Polenz (1985: 267):

(67) Weil $_{R}$ er es getan hat gl , musste er gehen $_{P 2}$.

Die 15 Klassen, die nach der verknüpfenden Relation unterschieden werden, sollen nun aufgezählt und (falls möglich) mit einem Beispiel für Nebensätze veranschaulicht werden. Für die ersten beiden Typen (kopulativ und disjunktiv) können keine subordinativen Verknüpfungen als Beispiele herangezogen werden. Da diese beiden Relationen jedoch bei der koordinativen Verknüpfung von Nebensätzen vorkommen, sollen sie mit aufgeführt werden. Peter von Polenz unterscheidet folgende Verknüpfungen (1985: 268-286): ${ }^{128}$

${ }^{126}$ In seiner Junktionstheorie (vgl. Kapitel 3.3) unterscheidet Raible (1992) 18 semantische Relationen. Da seine Beschreibung auf die romanischen Sprachen zugeschnitten ist, und da in der germanistischen Linguistik andere Klassifizierungen etabliert sind, wird auf die Klassifizierung von Raible nicht weiter eingegangen.

127 Die semantischen Klassen sind nicht nur auf Nebensätze, Konjunktionen und Adverbialbestimmungen beschränkt, sondern erfassen auch weitere syntaktische Ausdrucksformen wie etwa mit einem Relationsverb (vgl. 1985: 266f.):

(i) Seinen Abgang ${ }_{P 2}$ verdankt $_{R}$ er seinem Verhalten ${ }_{P 1}$.

Für unsere Zielsetzung einschlägig ist v.a. die Satzbaumöglichkeit, bei der zwischen satzwertigen Sachverhaltsdarstellungen satzsemantische Relationen ausgedrückt werden.

128 Die Beispielsätze wurden ebenfalls von Polenz (1985) entnommen, bzw. leicht modifiziert. An den Stellen, wo von Polenz zum jeweiligen Typ kein einschlägiges Beispiel aufführt, habe ich aus seinen erläuternden Textstellen die Beispiele entnommen. In Klammern werden auch die Handlungstypen angegeben, die die verknüpfende Relation (R) näher erläutern. Durch Unterstreichung werden die Konnektoren markiert, die die jeweilige Relation ausdrücken. 
1. Kopulativ (HINZUFÜGEN, ERGÄNZEN...)

Er wollte plaudern $n_{\mathrm{P} 1}$ und $_{\mathrm{R}}$ rauchen $_{\mathrm{P} 2}$.

2. Disjunktiv (ZUR WAHL STELLEN, ENTSCHEIDUNG OFFEN LASSEN)

Niemand darf wegen ...seiner religiösen $\mathrm{P}_{\mathrm{P} 1} \underline{\text { oder }}_{\mathrm{R}}$ politischen Anschauungen $\mathrm{P}_{\mathrm{P} 2}$ benachteiligt ...werden.

3. Adversativ (ENTGEGENSETZEN, KONTRASTIEREN...)

Inge liest gern $n_{\mathrm{P} 1}$, während ${ }_{\mathrm{R}}$ Hans lieber Klavier spielt ${ }_{\mathrm{P} 2}$.

4. Konzessiv (ZUGESTEHEN UND ENTGEGENSETZEN)

... dass die Opposition nicht mit nach Moskau gefahren sei $i_{\mathrm{P} 2}, \underline{o b w o h l_{R}}$ er sie eingeladen habe $e_{\mathrm{P} 1}$.

5. Explikativ, spezifizierend und exemplifizierend (ERKLÄREN, PRÄZISIEREN...)

... betreibt de facto Kunstförderung ${ }_{\mathrm{P} 1}, \underline{\text { was }}_{\mathrm{R}}$ ein Bundesgesetz nicht tun dürfte $e_{\mathrm{P} 2}$.

6. Restriktiv (EINSCHRÄNKEN)

Jeder hat das Recht auf ... 1 1, soweit ${ }_{\mathrm{R}}$ er nicht die Rechte anderer verletzt ... $\mathrm{P} 2$

7. Komparativ/vergleichend (VERGLEICHEN)

$\underline{S}_{\mathrm{R}}$ entschieden ich mich ... wehre $e_{\mathrm{P1}}, \underline{\boldsymbol{s}_{\mathrm{R}}}$ entschieden bin ich gegen $\ldots \mathrm{P} 2$

8. Temporal (ORIENTIEREN ÜBER ZEITBEZIEHUNGEN)

Nachdem $_{\mathrm{R}}$ alle da waren $_{\mathrm{P} 2}$, fingen wir an $n_{\mathrm{P} 1}$.

9. Komitativ (MITEINANDER-GESCHEHEN ZWEIER SACHVERHALTE)

... zu unterrichten $n_{\mathrm{P} 1}, \underline{\text { ohne dass }} \underline{\mathrm{R}}_{\mathrm{R}}$ er von jemandem daran gehindert wird ${ }_{\mathrm{P} 2}$.

10. Instrumental (ZUM ZWECK ANWANDTE MITTEL NENNEN)

...seine Meinung frei zu äußern $\mathrm{P}_{\mathrm{P}}$, wofür $\underline{\mathrm{R}}_{\mathrm{R}}$ er Wort, Schrift und Bild benutzt $t_{\mathrm{P} 2}$.

11. Final (ZWECK ANGEBEN)

Die Warnschilder sind rot $_{\mathrm{P} 1}$, damit ${ }_{\mathrm{R}}$ sie auffällig $\operatorname{sind}_{\mathrm{P} 2}$.

12. Konsekutiv (FOLGERN, BEHAUPTETE/ERWARTETE FOLGE NENNEN)

Er sprach langweilig ${ }_{\mathrm{P} 1}, \underline{\text { so das }}_{\mathrm{R}}$ man einschlief $f_{\mathrm{P} 2}$. (1998: 268)

13. Kausal (BEGRÜNDEN, AUS EINER URSACHE AUF IHRE FOLGE SCHLIESSEN)

Weil $_{\mathrm{R}}$ er von der Richtigkeit seiner These überzeugt war ${ }_{\mathrm{P} 1}$, setzte er alles durch $\mathrm{P}_{\mathrm{P}}$.

14. Konditional (BEDINGUNG SETZEN, BEDINGTEN SACHVERHALT VORAUSSAGEN) Typ A-B Wenn $_{\mathrm{R}}$ das Wetter schlecht ist $t_{\mathrm{P} 1}$, geht es nicht $t_{\mathrm{P} 2}$.

15. Metakommunikativ (BEDEUTUNG/AUSDRUCKSWEISE KOMMENTIEREN)

$\ldots \mathrm{P} 1, \underline{\text { worunter }}_{\mathrm{R}} z$ uverstehen ist, $\ldots \mathrm{P} 2$

Abb. 10: Semantische Relationen nach Peter von Polenz (1985: 268-286) 
Die bei von Polenz aufgeführten semantischen Klassen sind nur zum Teil mit den traditionellen Kategorien identisch. Einerseits fehlt z.B. die lokale Verknüpfung, andererseits enthält die Klassifizierung auch Typen, die in traditionellen Darstellungen unberücksichtigt bleiben (z.B. metakommunikative Verknüpfungen). An mehreren Textstellen weist von Polenz (s. z.B. 1985: 265) darauf hin, dass neben der Verknüpfung von Satzinhalten auch die Möglichkeit besteht, Sätze „samt Handlungsgehalt“ in eine semantische Relation zu setzen. Auf diesen Punkt soll im Folgenden näher eingegangen werden.

Der Unterschied zwischen der rein satzsemantischen Verknüpfung von Satzinhalten und der eher pragmatischen Verknüpfung von Sätzen bzw. Äußerungen kann am konditionalen Bereich erklärt werden (1985: 283ff.). Den ersten Typ konditionaler Verknüpfungen erläutert von Polenz (1985: 283) wie folgt:

Aus einem als ,möglich' VORAUSGESETZTEN, als HYPOTHESE GESETZTEN bzw. als REGEL GEWUSSTEN Sachverhalt P1, der als BEDINGUNG R gekennzeichnet wird, einen von P1 BEDINGTEN Sachverhalt P2 VORAUSSAGEN, auf ihn HINWEISEN usw. (Versalien nach dem Originaltext, P.K.)

Diese Art von Verknüpfung kann so gedeutet werden, dass die Gültigkeit einer Aussage P1 die Gültigkeit der Aussage P2 nach sich zieht (d.h. etwa ,wenn P1 - dann P2‘). Daneben wird auch ein zweiter, pragmatischer Typ konditionaler Verknüpfungen behandelt, bei dem die konditionale Relation mit Sprecherhandlungen/Illokutionen verbunden ist. Betrachten wir dazu den folgenden Beispielsatz (von Polenz 1985: 285):

(68) Fall $_{\mathrm{R}}$ Sie inzwischen den angeforderten Betrag entrichtet haben sollten $\mathrm{P}_{\mathrm{P} 1}$, betrachten Sie bitte diese Mahnung als gegenstandslos $\mathrm{P} 2$.

Hier handelt es sich also darum, dass ein Sachverhalt P1 als Bedingung für die eigene Handlung des Sprechers (hier: Aufforderung) gesetzt wird. Die Aufforderung im mit P2 indizierten Satz ist nur unter der Bedingung gültig, dass der Inhalt des vorangestellten Nebensatzes zutrifft.

Ebenfalls pragmatisch/sprechaktbezogen ist der ,Typ E` der kausalen Verknüpfungen bei von Polenz (1985: 281ff.): „Eine Sprechhandlung P1 BEGRÜNDEN durch eine als GRUND gekennzeichnete Sprecherhandlung P2“. Beispiele für diese Relation werden insb. in der Fachliteratur zu weil-Sätzen mit Verbzweitstellung diskutiert. Mit weil-Sätzen wie in (69) wird nicht die Proposition des Hauptsatzes, sondern der Sprechakt selbst begründet (vgl. z.B. Küper 1991: 137):

(69) Ich habe das Geld genommen, weil - Sie hätten's ja sowieso rausgekriegt. (Küper 1991: 137) 
Für die Erfassung der nicht rein satzsemantischen Verknüpfungen ${ }^{129}$ dieser Art bietet sich v.a. der Typ „metakommunikative Verknüpfungen“ bei von Polenz (1985: 285f.) an. Da dieser Terminus aber auf die Kommentierung der Ausdrucksweise bzw. Bedeutung beschränkt ist und somit nicht alle Typen von sprechaktbezogenen Adverbialsätzen erfasst, werden sie im Folgenden unter dem Terminus ,periphere Adverbialsätze‘ (vgl. Kapitel 3.1.2) erfasst und im Kapitel 3.4.6 behandelt.

In der Duden-Grammatik (2016: 1091) werden sieben Bedeutungsgruppen mit Untertypen unterschieden. Der erste Typ, kopulativ (mit den Unterarten additiv und alternativ) wird nicht durch Subjunktoren realisiert. Die weiteren Typen sind (in Klammern die Unterarten): temporal (vorzeitig, nachzeitig, gleichzeitig), konditional, „im weiteren Sinne kausalgleichläufig“ (i.e.S. kausal, konsekutiv, modal-instrumental, final), „im weiteren Sinne kausal-gegenläufig“ (adversativ und konzessiv), „spezifizierend“ (explikativ und restriktiv) sowie ,vergleichend“ (komparativ und proportional). ${ }^{130}$ Die semantischen Typen komitativ und metakommunikativ werden zwar an dieser Stelle nicht aufgeführt, ansonsten sind die Typen - mit einer teils anderen Gruppierung - mit denen aus der Satzsemantik von Peter von Polenz (1985) identisch.

Auf der Basis der obigen Klassifizierungen erscheint für die vorliegende Arbeit folgende Gliederung für sinnvoll. Zunächst werden die traditionellen Nebensatzklassen temporal (3.4.2), konditional (3.4.3), und im engeren Sinne kausal (3.4.4) behandelt. Weitere Typen aus der Gruppe „im weiteren Sinne kausal-gleichläufig“ (wie etwa Finalsätze), werden mit den Untertypen „im weiteren Sinne kausal-gegenläufig“ (adversativ, konzessiv) und weiteren, nicht-traditionellen Nebensatzklassen (wie etwa ,komparativ' und ,proportional') sowohl im theoretischen als auch im empirischen Teil getrennt behandelt (3.4.5 bzw. 5.4), weil für diese Nebensatzklassen in den untersuchten Korpustexten nur relativ wenige Belege zu finden sind. Schließlich werden nicht-propositionsbezogene bzw. nicht-satzgliedwertige Adverbialsätze erfasst (3.4.6). Dabei werden u.a. sprechaktbezogene Verwendungen bzw.

129 Vgl. dazu Kapitel 3.1.2. Carlsen (1998) untersucht redewiedergebende Satzgefüge mit redeeinleitendem wie-Satz anhand eines Zeitungskorpus. In syntaktischer Hinsicht ist dabei zu bemerken, dass hier (anders als in der indirekten Rede) die Redeeinleitung in Nebensatzform erscheint und die (eingeleitete) Rede selbst im Vordergrund steht (1998: 64). Es lassen sich mehrere Positionen der wie-Sätze im Satzgefüge unterscheiden, u.a. auch ihre Voranstellung, die uns weiter interessiert. Die IDS-Grammatik (Zifonun et al. 1997: 2242ff.) behandelt ,modusmodifizierende‘ und ,-kommentierende Nebensätze', vgl. dazu Kapitel 3.3.2 bzw. 3.4.6.

${ }^{130} \mathrm{Im}$ Vergleich mit dem HDK-2 (Breindl et al. 2014) sind viele Gemeinsamkeiten zu finden. Auch da werden der Oberbegriff ,im weiteren Sinne kausal-gegenläufig“ mit den beiden Untertypen , adversativ“ und ,konzessiv“ bzw. der Oberbegriff „,spezifizierend“ mit den beiden Untertypen ,explikativ“ und ,restriktiv“ verwendet. Es gibt jedoch auch Unterschiede: Restriktive Adverbialsätze wie im folgenden Beispiel werden als ,negativ-konditional` bezeichnet (vgl. Breindl et al. 2014: 1074f.):

(i) Außer wenn das Wetter plötzlich umschlägt habe ich nie Schmerzen. (zit. n. Breindl et al. 2014: 1079).

Die negativ-konditionale Relation ist ,auf eine Konditionalrelation plus eine Negation zurückzuführen [...], wobei die Negation vom negativ-konditionalen Konnektor beigetragen wird“ (Breindl et al. 2014: 1032). 
modusmodifizierende, moduskommentierende und diktumskommentierende Adverbialsätze behandelt, die inhaltlich nur locker angefügt sind (vgl. Zifonun et al. 1997: 2242ff.).

\title{
3.4.2 Temporalsätze
}

Im De-Gruyter-Lexikon Deutsche Grammatik (Hentschel 2010: 359) stehen für ,temporal bzw. ,Temporalsatz‘ folgende knappe Definitionen:

\begin{abstract}
Als temporal bezeichnet man Präpositionen (nach einer Stunde), Adverbien (heute) und Adverbialbestimmungen einschließlich ganzer Adverbialsätze (jeden Freitag; als ich noch zur Schule ging), die zum Ausdruck zeitlicher Verhältnisse dienen.

Ein Temporalsatz ist ein Satz, der ein in einem anderen Satz ausgedrücktes Ereignis zeitlich verankert: Als die Polizei kam, waren die Einbrecher längst über alle Berge. Temporalsätze werden im Deutschen meist durch Konjunktionen wie als, wenn oder nachdem eingeleitet.
\end{abstract}

Bei der Temporalität werden i.d.R. bezüglich des Inhalts der verknüpften Sätze (d.h., in welcher Relation der Inhalt des Hauptsatzes zu dem des Nebensatzes steht) Vorzeitigkeit, Gleichzeitigkeit und Nachzeitigkeit unterschieden. Die temporalen Beziehungen zwischen den Teilsätzen werden u.a. durch die Konjunktionen der Temporalsätze ausgedrückt, so drücken etwa die temporalen Subjunktoren als, während, solange, seit(dem) usw. Gleichzeitigkeit aus (vgl. z.B. von Polenz 1985: 275, Zitterbart 2002: 147, zu einer ausführlichen Liste der Temporalkonnektoren - darunter auch der Subjunktoren - s. Blühdorn 2004: 126, Breindl et al. 2014: 274ff.). Bei der Unterscheidung von Vor- und Nachzeitigkeit wird das Kriterium genannt, wann das durch den Konstituentensatz Bezeichnete im Verhältnis zu dem durch den Matrixsatz Bezeichneten stattfindet (vgl. z.B. Glück/Rödel 2016: 704, Zifonun et al. 1997, Zitterbart 2002: 146). Dementsprechend bezeichnet z.B. nachdem Vorzeitigkeit und bevor bzw. ehe Nachzeitigkeit. ${ }^{131}$

Neben den Subjunktoren tragen auch weitere Elemente zum Ausdruck der Zeitverhältnisse bei. Die Konjunktion als wird z.B. bei von Polenz (1985: 275) sowohl bei der Vorzeitigkeit als auch bei der Gleichzeitigkeit angeführt, ${ }^{132}$ bei der Vorzeitigkeit steht jedoch auch die Bemerkung, dass als in diesen Fällen „mit Vergangenheit II“ steht. Die Tempora der Prädikatsausdrücke spielen dementsprechend bei temporalen Relationen eine wichtige Rolle. Das Plusquamperfekt wird bei von Polenz (1985: 253) als sog. ,Aspekt-Zusatz‘ bezeichnet, der ausdrücken soll, dass die Handlung zum Zeitpunkt des Sprechens bereits abgeschlossen ist

\footnotetext{
131 Bei von Polenz (1985: 275) ist jedoch die Bestimmung der Vor- und Nachzeitigkeit umgekehrt, vgl. die folgenden Beispiele für vorzeitig: bevor, ehe, als bzw. nachzeitig: nachdem, sobald, sowie. Zur uneinheitlichen Terminologie s. auch Breindl et al. (2014: 289).

132 Auch weitere Subjunktoren werden in mehreren Grammatiken sowohl bei der Gleichzeitigkeit als auch bei der Vorzeitigkeit angeführt, vgl. Breindl et al. (2014: 289).
} 
(,perfektiv'). Aufgrund des Gebrauchs bestimmter Tempora kann auch zwischen Vorzeitigkeit und Gleichzeitigkeit von wenn-Sätzen unterschieden werden (Glück/Rödel 2016: 704). Wörter wie oft werden neben ,Aspekt-Zusätzen` auch bei ,Aktionsart-Zusätzen“ aufgeführt (von Polenz 1985: 253). Sie werden traditionell als temporale Adverbien bezeichnet und sind beim Ausdruck zeitlicher Faktoren (z.B. Frequenz, vgl. Glück/Rödel 2016: 703) ebenfalls einschlägig. Breindl et al. (2014: 290) unterscheiden Zeitpunkt, Zeitdauer und Frequenz spezifizierende Temporalkonnektoren.

Die semantischen Beziehungen zwischen den Teilsätzen werden nicht in jedem Fall eindeutig ausgedrückt. In der Konnektorensemantik werden formgleiche Konnektoren des Gegenwartsdeutschen untersucht, die unterschiedliche Bedeutungen bzw. Verwendungen haben können, wie es etwa beim temporalen und konditionalen wenn zu beobachten ist (s. Blühdorn 2004: 125, Zifonun u.a. 1997: 2282ff.). Des Weiteren ist zu berücksichtigen, dass einige Subjunktionen wie wenn nicht nur im Gegenwartsdeutschen ambig sind (zur Tendenz der Monosemierung der Konnektoren s. weiter oben 2.2.1, 3.3.2). Dabei ist auch mit Übergängen (bei wenn zwischen Temporalität und Konditionalität) zu rechnen, die die Einordnung der Belege erschweren. Dies sei mit dem folgenden Beispielsatz illustriert:

(70) Wenn die Sonne scheint, gehen wir spazieren. (zit. n. Eroms 2000: 233)

Eroms (2000: 233f.) verweist darauf, dass dieser Satz - neben der wohl konditionalen Grundbedeutung - latent auch eine temporale Interpretation ermöglicht, da die Proposition des Obersatzes auch ,,in einem allgemein zeitlichen Sinne“ situiert wird. Diese Ambiguität von wenn-Sätzen wird aufgelöst, wenn Konjunktiv II vorliegt (konditionale Interpretation, vgl. Glück/Rödel 2016: 351f.). Eine andere, wohlbekannte Schwierigkeit bei der Interpretation besteht darin, dass temporale Subjunktoren im Sinne einer „Ursache-WirkungInterpretation auf Ereignisebene überlagert werden“ können, sodass etwa beim Subjunktor nachdem eine handlungsbegründende Verwendung entstehen kann, vgl. das folgende Beispiel (zit. n. Fabricius-Hansen 2007: 770):

(71) Nachdem der Patient noch nicht eingetroffen ist, nehmen wir jetzt Frau Müller dran.

Bei der Analyse der vorangestellten Adverbialsätze ist also mit Übergangstypen von temporal-kausalen bzw. temporal-konditionalen Adverbialsätzen zu rechnen (vgl. auch Ágel 2000: 1887). Zur Abgrenzung der semantischen Relationen und zu Übergangstypen s. Kapitel 4.2. bzw. 3.4.5.7.

Bei der Behandlung der Korrelate für Temporalsätze ist auf Zitterbart (2002: 147) zu verweisen. Folgende Zeitadverbien werden als Korrelate für temporale Adverbialsätze 
angeführt: da, damals, dann, darauf, jetzt, seither, soweit, so oft, so lange. ${ }^{133}$ Was die Stellung der Korrelate für Temporalsätze anbelangt, so ist die resumptive Spitzenstellung am Anfang des Matrixsatzes ihre unmarkierte Stellung (Zifonun et al. 1997: 142f., Zitterbart 2002: 147), sie können aber auch im Mittelfeld des vorangestellten Matrixsatzes auftreten. Bezüglich der (Nicht-)Setzung der Korrelate bemerkt Zitterbart (2002: 147), dass bei temporalen Angabesätzen die Korrelate sehr häufig fehlen, dies aber bei wenn-Sätzen nicht der Fall ist. Das Setzen des Korrelats dann als wiederaufnehmender Ausdruck wäre in bestimmten Fällen ungewöhnlich, vgl. z.B. den folgenden Beleg (zit. n. Zitterbart 2002: 149).

(72) Immer wenn er Alkohol trinkt, bekommt er Probleme mit der Polizei.

Bei vorangestellten temporalen wenn-Sätzen dürfte die (Nicht-)Setzung des resumptiven Elements dann nicht nur mit der Gefahr der Verwechslung mit konditionalen Relationen, sondern auch weiteren Besonderheiten zusammenhängen, „deren Konsequenzen sich bis zum Bereich der Stilistik verfolgen lassen.“ (Zitterbart 2002: 150) Die integrative Stellung von Temporalsätzen scheint bereits im 17. Jh. weit verbreitet zu sein, der resumptive Typ tritt nur „,in Ausnahmefällen“ auf (Wiktorowicz 2013: 395).

\subsubsection{Konditionalsätze und Irrelevanzkonditionale}

Im De-Gruyter-Lexikon Deutsche Grammatik (Hentschel 2010: 152) findet sich folgende Definition des Begriffs ,Konditionalsatz':

\footnotetext{
133 Im HDK-1 (Pasch et al. 2003: 263f.) werden folgende temporale Korrelatkonstruktionen (Verknüpfung von Subjunktoren und Korrelaten) unterschieden:

Subjunktor Korrelat(e)

als $\quad d a($ und - selten - dann $)$

bevor $\quad d a$ und dann

bis (dass) da

da da; so

ehe $\quad d a$

nachdem da und dann

seit(dem) $\quad d a$

sobald da; dann; sobald

solange da und solange

sooft so oft

während $\quad d a$

wie $\quad d a$

Es ist auffallend, dass der Subjunktor wenn auf dieser Liste fehlt. Die Korrelate da/dann/so in Linksversetzungskonstruktionen nach wenn-Sätzen werden jedoch an anderer Stelle (Pasch et al. 2003: 403) aufgeführt. Wenn der Subjunktor mehrere semantische Varianten zulässt, kann das Korrelat zur Unterscheidung beitragen. Der Subjunktor $d a$ ist auch, bzw. vor allem in kausaler Verwendung möglich (mit den Korrelaten daher, darum, so, vgl. Pasch et al. 2003: 263, 402). Es ist jedoch umstritten, ob vorangestellte $d a$-Sätze im Gegenwartsdeutschen mit Korrelaten wiederaufgenommen werden können, s. Axel-Tober (2012: 271). Zu temporalen $d a$-Sätzen im älteren Deutsch s. AxelTober (2012). Des Weiteren ist während auch als adversativer Subjunktor (mit dem Korrelat so) verwendbar.
} 
Ein Konditionalsatz ist ein Nebensatz, in dem die Bedingung für ein Ereignis ausgedrückt wird, das in einem anderen Satz beschrieben wird: Wenn es am Wochenende schön ist, machen wir einen Ausflug. Konditionalsätze werden im Deutschen meist durch Konjunktionen wie wenn, falls, sofern eingeleitet.

Der Konjunktiv II kann als ein „Marker von Konditionalität“ (Breindl et al. 2014: 700) aufgefasst werden. ${ }^{134}$ Durch die Verwendung des Konjunktiv Präteritum wird Konditionalis ausgedrückt, durch Konjunktiv Plusquamperfekt im Konditionalsatz Irrealis (vgl. Glück/Rödel 2016: 352).

Neben den im obigen Zitat genannten konditionalen Konjunktionen werden im HDK-2 auch der Subjunktor so (im Gegenwartsdeutschen allerdings stilistisch eingeschränkt, nur in einem höheren Register gebräuchlich) und u.a. die aus Verbzweitsatz-Einbettern abgeleiteten komplexen Subjunktoren angenommen, dass bzw. vorausgesetzt, dass aufgeführt (Breindl et al. 2014: 692, 772). Im Gegenwartsdeutschen ist wenn der häufigste Konditionalkonnektor (Breindl et al. 2014: 756). Uneingeleitete Konditionalsätze stehen in Verberststellung und werden ihrem Obersatz meist vorangestellt (Glück/Rödel 2016: 352). Bei indikativischen V1Konditionalen ist die Nachstellung nach Breindl et al. (2014: 702) nicht möglich. Als Korrelate fungieren dann und so. In den Prosatexten aus dem 17. Jh. tritt nach Wiktorowicz (2013: 395f.) ausschließlich das Korrelat so auf, außerdem ist die resumptive Stellung vorangestellter Konditionalsätze der dominierende Stellungstyp.

Einen Sondertyp stellen Irrelevanzkonditionale dar (Pittner 1999: 271, Leuschner 2005: 284, Duden-Grammatik 2016: 639), deren semantische Besonderheit im Vergleich zu den Standardkonditionalen darin besteht, „dass der Inhalt des Antezedens keinerlei Einfluss auf die Wahrheitsbedingungen des Konsequens hat“" (Breindl et al. 2014: 967), es drückt eine irrelevante Bedingung aus. Irrelevanzkonditionale werden in der vorliegenden Arbeit in Anlehnung an die IDS-Grammatik (Zifonun et al. 1997: 2319-2322) nicht als Subklasse der Konditionale, sondern als eigenständiger Typ behandelt. Das ,Irrelevanzkonditional` wird wie folgt definiert (Zifonun et al. 1997: 2319):

Es spezifiziert eine konditionale Beziehung zwischen einem Konsequens und einer Menge von Antezedensbedingungen, die ein ganzes Spektrum von Möglichkeiten mehr oder weniger erschöpfend erfassen. Dabei wird ausgedrückt, daß keine dieser Möglichkeiten die Gültigkeit des Konsequens beeinflußt.

Irrelevanzkonditionale stehen zwischen Konditionalität und Konzessivität in dem Sinne, dass das Antezedens nicht als gültig dargestellt wird (und stehen somit der Konditionalität

134 Im Bereich der Konditionalität können die Untertypen faktisch, hypothetisch und kontrafaktisch unterschieden werden (Breindl et al. 2014: 733-737). Als Abgrenzungskriterium von hypothetischen sowie kontrafaktischen Konditionalen fungiert die Kompatibilität mit dem Sprecher-Wissen. Wenn sowohl die Proposition des Konditionalsatzes als auch die des Obersatzes falsch sind, liegen kontrafaktische Konditionale vor (Breindl et al. 2014: 726). Die kontrafaktische Relation kann durch Konjunktiv Plusquamperfekt eindeutig ausgedrückt werden. Beim Konjunktiv Präteritum und Indikativ finden sich jedoch Übergänge zwischen kontrafaktischen und hypothetischen bzw. faktischen und hypothetischen Konditionalen. 
nahe) aber auf die Gültigkeit des Konsequens geschlossen werden kann (wie bei der Konzessivität). Sie werden von König/van der Auwera (1988) als ,concessive conditionals“ bezeichnet, während Baschewa (1983) sie als Konzessivsätze behandelt. Bei Irrelevanzkonditionalen drückt die Protasis im Gegensatz $\mathrm{zu}$ prototypischen Konditionalsätzen mehrere Antezedenswerte aus (Zifonun et al. 1997: 2319, Leuschner 2005: 284). Diese müssen jedoch nicht explizit als Alternativen formuliert werden, wie dies beim Subtyp ,alternative Irrelevanzkonditionale' der Fall ist (z.B. ob (...) oder (ob), sei es (...) sei es). Sie können auch einer offenen Menge entsprechen (,universale Irrelevanzkonditionale‘, z.B. bei wer auch immer, so ADJ auch immer). Des Weiteren ist es auch möglich, dass im Antezedens ein unerwarteter Extremwert genannt und dadurch impliziert wird, „dass dies auch für niedrigere Werte, also für weniger auffällige und weniger unwahrscheinliche Bedingungen gilt.“ (Breindl et al. 2014: 983) Standardkonditionale und Irrelevanzkonditionale weisen andere Stellungseigenschaften auf. Irrelevanzkonditionale werden häufig nicht-integrativ angeschlossen, beim letztgenannten Subtyp (,skalare Irrelevanzkonditionale') kann jedoch auch der integrative und (seltener) der resumptive Stellungstyp vorkommen (Breindl et al. 2014: 983). Sprechaktbezogene Konditionalsätze, u.a. Relevanzkonditionale (Breindl et al. 2014: 737) bzw. moduskommentierende Adverbialsätze (Zifonun et al. 1997: 2242ff.) werden im Kapitel 3.4.6 behandelt.

\title{
3.4.4 Kausalsätze
}

An dieser Stelle werden nur Kausalsätze im engeren Sinne behandelt (vgl. die Erläuterungen im Kapitel 3.4.1). ${ }^{135}$ Der Begriff ,kausal‘ wird im De-Gruyter-Lexikon Deutsche Grammatik in diesem Sinn gedeutet:

\begin{abstract}
Zwei Elemente stehen dann in einem kausalen Verhältnis zueinander, wenn das eine Element die Ursache oder der Grund für das andere Element ist. In der Grammatik werden z.B. Konjunktionen als kausal bezeichnet, wenn sie einen Teilsatz einleiten können, in dem die Ursache für ein Ereignis angegeben wird,

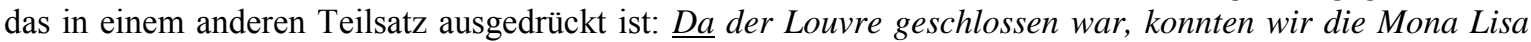
leider nicht besichtigen. (Hentschel 2010: 147, Hervorhebung im Original, P.K.)
\end{abstract}

Bei der kausalen Beziehung lassen sich mehrere Untertypen unterscheiden. Im De-GruyterLexikon Deutsche Grammatik wird die traditionelle Auffassung vertreten, dass im Kausalsatz „die Ursache für ein Ereignis ausgedrückt wird, das in einem anderen Satz beschrieben wird: Er kam zu spät, weil die Bahnbediensteten streikten.“ (Hentschel 2010: 147) Diese Definition

\footnotetext{
135 In der Duden-Grammatik (2016: 1091) werden bei den Bedeutungsgruppen „im weiteren Sinne kausal-gleichläufig“ und „im weiteren Sinne kausal-gegenläufig“ mehrere Untertypen unterschieden und u.a. Konzessiv-, Konsekutiv- und Finalbestimmungen als kausal im weiteren Sinne kategorisiert.
} 
erfasst nur den sog. propositionalen Bezug. Bei einem erweiterten Kausalitätsbegriff können auch weil-Sätze mit epistemischem und Sprechaktbezug als Kausalsätze kategorisiert werden (vgl. Breindl et al. 2014: 835ff., s. auch Kapitel 2.1.1).

Kausalsätze werden im Metzler-Lexikon-Sprache (Glück-Rödel 2016: 328) als Adverbialsätze definiert, die durch die kausalen Subjunktoren da und weil eingeleitet werden. Die beiden Subjunktoren sind im Gegenwartsdeutschen die mit Abstand frequentesten (Breindl et al. 2014: 818), im HDK-2 werden allerdings auch weitere kausale Subjunktoren aufgeführt: alldieweil, dieweil, nachdem, sintemal(en) (Breindl et al. 2014: 790). ${ }^{136}$ Bei diesen Subjunktoren kann die kausale Bedeutung im Neuhochdeutschen nicht immer eindeutig von ihrer ursprünglichen temporalen Bedeutung getrennt werden (vgl. Kapitel 3.4.1). Daher können nicht alle $d a$ - bzw. nachdem-Sätze zwangsläufig als Kausalsätze eingestuft werden (s. dazu Kapitel 4.2). Auch bei weil ist die temporale Verwendung „bis ins 20. Jahrh.“ (DWb, Bd. 28, Sp. 764) zu beobachten. Es gibt auch uneingeleitete Kausalsätze mit Verberststellung und obligatorischer Modalpartikel doch. Diese werden ihrem Bezugssatz immer nachgestellt (Pittner 2013a: 505) und sind für die vorliegende Arbeit nicht einschlägig.

Bei vorangestellten Kausalsätzen wird im 17. Jh. die resumptive Stellung mit dem Korrelat als bevorzugt (Wiktorowicz 2013: 396). Im Gegenwartsdeutschen ist die resumptive Stellung nicht mehr so frequent, als Korrelat können deshalb bzw. deswegen auftreten (Breindl et al. 2014: 819).

Kausalität ist mit der konditionalen Relation verwandt, als wichtigstes Abgrenzungsmerkmal wird die Faktizität bzw. Nonfaktizität des Sachverhalts des Antezedens genannt (Breindl et al 2014: 721).

\subsubsection{Weitere Typen von Adverbialsätzen im engeren Sinne}

In diesem Kapitel werden aufgrund ihrer geringen Frequenz einige Untertypen aus den Gruppen „im weiteren Sinne kausal-gleichläufig“ bzw. „im weiteren Sinne kausalgegenläufig“" gemeinsam und weniger ausführlich behandelt: Final- und Konsekutivsätze bzw. adversative und Konzessivsätze (3.4.5.1 bis 3.4.5.3). Dazu kommen weitere Typen aus den Gruppen „spezifizierend“ (explikativ und restriktiv) sowie „vergleichend“ (komparativ und

\footnotetext{
136 Nach dem De-Gruyter-Lexikon Deutsche Grammatik (Hentschel 2010: 147) können Kausalsätze auch durch die Konjunktion denn eingeleitet werden. Wir gehen dagegen davon aus, dass Kausalsätze Adverbialsätze sind (vgl. GlückRödel 2016: 328). Der Konnektor denn ist durchaus als kausal zu bestimmen, er kann jedoch keine subordinierten Sätze einleiten.
} 
proportional) (3.4.5.4 sowie 3.4.5.5). Abschließend werden Lokalsätze und Mischtypen behandelt (3.4.5.6 sowie 3.4.5.7).

\subsubsection{Final- und Konsekutivsätze}

Finalsätze stellen einen Untertyp aus der Gruppe „kausal-gleichläufig“ dar. Sie „geben einen Zweck oder ein Ziel des im übergeordneten Satz ausgedrückten Geschehens an“" (Pittner 2013a: 513). Die Finalitätsrelation wird im Deutschen häufiger durch Infinitivkonstruktionen mit $z u$ ausgedrückt, finite Finalsätze werden eher selten verwendet (Breindl et al. 2014: 1014). Typische Subjunktoren im Gegenwartsdeutschen sind damit und (auf) dass. ${ }^{137}$ Als Korrelate treten deshalb bzw. deswegen auf. Vorangestellte Finalsätze sind nach den Stichproben von Breindl et al. (2014: 1043f.) selten. Aus je 200 Belegen treten bei auf dass nur 7 vorangestellt auf, bei damit sind es 34 Belege, während dass - mit Ausnahme der Verwendungen mit Sprechaktbezug - nur nachgestellte Finalsätze einleitet (Breindl et al. 2014: 1039f., 1043f.). Die Bevorzugung der Nachstellung von Finalsätzen kann nach Flämig (1964: 8) auf die logische Denkfolge zurückgeführt werden, ,wobei Zweck und Ziel des Hauptsatzgeschehens den eigentlichen Aussageschwerpunkt bilden“. Bei Voranstellung werden Finalsätze nach Flämig (1964: 9) „ins Vorfeld des Gesamtsatzes“ integriert.

Im De-Gruyter-Lexikon Deutsche Grammatik (Hentschel 2010: 68) wird ,Konsekutivsatz folgenderweise definiert:

Ein Konsekutivsatz ist ein Satz, in dem die Folgen eines Ereignisses ausgedrückt werden, das in einem anderen Satz beschrieben wird: Sie war so wütend, dass sie kaum noch an sich halten konnte. Konsekutivsätze werden im Deutschen durch Konjunktionen wie (so) dass eingeleitet.

Die Subjunktoren so dass bzw. sodass werden von Pasch et al. (2003: 418) als Postponierer behandelt, denn der durch sie eingeleitete Verbletztsatz (,das interne Konnekt) „darf nicht vor dem externen Konnekt auftreten.“ (2003: 419). In den eingesehenen Gegenwartsgrammatiken wird die Möglichkeit der Voranstellung von Konsekutivsätzen nicht behandelt, vielmehr wird von einer ikonischen Kodierung mit umgekehrter Reihenfolge ausgegangen (vgl. z.B. Breindl et al. 2014: 265). ${ }^{138}$

\footnotetext{
137 Der frequenteste finale Subjunktor ist damit (Flämig 1964: 24, Breindl et al. 2014: 1014f.), v.a. umgangssprachlich wird auch dass verwendet (Breindl et al. 2014: 1014f.), das nach Flämig (1964: 24) in der Goethezeit (1760-1820) noch häufiger als damit verwendet wurde. „Das eher veraltete auf daß findet sich heute überwiegend, wo eine spezielle, z. B. ironisierende Stilwirkung erreicht werden soll.“ (Zifonun et al. 1997: 2317f.)

138 Die - das Prinzip der Ikonizität verletzende - Strategie der Vorwegnahme der Wirkung ist jedoch bei konsekutiven Präpositionalphrasen (wie in Zu unserer Enttäuschung war das Versteck leer) durchaus möglich (Blühdorn 2012b: 210f.).
} 


\subsubsection{Adversative Adverbialsätze}

Adversative Adverbialsätze, von Zifonun et al. (1997: 2324) ,Konfrontativsätze“ genannt, drücken einen Gegensatz zwischen den Sachverhalten aus, die in dem Ober- und dem Untersatz bezeichnet werden. Sie gehören nach der Angabe der Duden-Grammatik (2016: 1091) mit dem konzessiven Typ zur Kategorie „im weiteren Sinne kausal-gegenläufig“. Adversative Adverbialsätze werden v.a. durch den adversativen Subjunktor während eingeleitet, dessen primäre Bedeutung temporal ist (vgl. Kapitel 3.4.1). Die temporale Beziehung der Gleichzeitigkeit ist bei einigen während-Sätzen schon aufgrund des Tempusgebrauchs auszuschließen, die adversative Lesart ist also auf grammatische Faktoren zurückführbar. Die Gegensätzlichkeit der beiden Sachverhalte ,wird in der Regel an bestimmten lexikalischen Oppositionen erkennbar“" (Zifonun et al. 1997: 2324). Bei der Unterscheidung von temporalen und adversativen Lesarten ist nach Breindl et al. (2014: 50) der Grad der Parallelität der beiden Konnekte als Abgrenzungsparameter heranzuziehen: „Die Existenz paarweise kontrastierender Abschnitte [...] erzeugt [...] eine adversative Lesart des Subjunktors während“،. Im folgenden Beleg aus dem DeReKo (Institut für Deutsche Sprache 2016) ist die adversative Lesart auf die Kontraste Mutter/Vater bzw. sich auf die Straße retten/im Wohnzimmer sterben zurückzuführen.

(73) Während sich die Mutter auf die Straße rettete, wurde der Vater nach den Löscharbeiten tot im Wohnzimmer gefunden. (M91TG/104.02935 Mannheimer Morgen, 08.04.1991, Regionales; Hausbesitzer starb in den Flammen)

Die temporale Lesart ist hier auch durch den Kontext blockiert: Eine Gleichzeitigkeit der Ereignisse ist nicht anzunehmen, da das Ereignis im internen Konnekt während des Brandes, das Ereignis im externen Konnekt nach dem Löschen des Brandes stattfindet.

Gelegentlich werden sog. Substitutivadverbiale von Adversativadverbialen unterschieden: „Substitutivadverbiale geben an, dass eine Situation an Stelle einer anderen Situation stattfindet.“ (Musan 2013: 63) Breindl et al. (2014: 488, 500) argumentieren für die Trennung der adversativen und substitutiven Relation. Sie ordnen die Subjunktoren (an)statt bzw. (an)statt dass nicht als adversative, sondern als substitutive (negationsinduzierende additive) Konnektoren ein, während sie in der Duden Grammatik (2016: 640) nur als ein Untertyp der adversativen Subjunktionen angesehen werden. Da auch in Zifonun et al. (1997: 2325) Untersätze mit adversativem während bzw. mit (an)statt daß gemeinsam behandelt werden, wird von einer Unterscheidung in der vorliegenden Arbeit abgesehen. Der Oberbegriff für die 
beiden Verwendungen wird jedoch im Gegensatz zu Zifonun et al. (1997: 2324) nicht ,Konfrontativsätze‘, sondern ,adversative Adverbialsätze‘ genannt.

\subsubsection{Konzessivsätze}

Bei faktischen Konzessivsätzen werden sowohl die Wahrheit des Antezedens als auch die des Konsequens behauptet, wobei auch ausgedrückt wird, dass nicht zu erwarten ist, dass beide zutreffen. ${ }^{139}$ Es handelt sich um eine „blockierte Bedingung“ (Breindl et al. 2014: 263), die nicht zur erwartbaren Folge führt, bzw. um einen „unwirksamen Gegengrund“ (Engel 1988: 723). Konzessivsätze sind sowohl mit Konditionalsätzen (Glück/Rödel 2016: 370) als auch mit Kausalsätzen (Pittner 2013a: 509) verwandt.

Konzessivsätze werden u.a. durch die Subjunktoren obwohl, obgleich, obschon, obzwar, wiewohl, wenngleich, trotzdem bzw. durch wenn ...auch eingeleitet oder als Verberstsatz ${ }^{140}$ realisiert (Glück/Rödel 2016: 370, Pittner 2013a: 509f., zu weiteren konzessiven Subjunktoren s. Breindl et al. 2014: 903f. sowie Di Meola 1997: 169f.). Mehrere Konnektoren wie etwa trotzdem werden neben ihrer subordinierenden Verwendung auch als Adverbkonnektor gebraucht (Breindl et al. 2014: 905). Die Entstehung konzessiver Subjunktoren der $o b$ - und wenn-Gruppe wird als ein Prozess der Univerbierung nachgezeichnet (vgl. Nübling 2013: 283f.). Aus der Verknüpfung von konditionalen Subjunktoren und „einer ursprünglich affirmativen, geltungsbekräftigenden Partikel“ (Breindl et al. 2014: 909) wie wohl, schon, gleich enstehen neue Subjunktoren wie wenngleich, wennschon bzw. obwohl, obgleich, obschon. Bei uneingeleiteten Konzessivsätzen tragen Partikeln wie auch zum Ausdruck der konzessiven Relation bei (Pittner 2013a: 509):

(74) Regnet es auch, geht er doch spazieren.

Das Auftreten einer Partikel im Verberstsatz ist im Gegenwartsdeutschen nach Breindl et al. (2014: 910) obligatorisch.

Baschewa (1983) wertet in ihrer Untersuchung der Konzessivsätze im Neuhochdeutschen ${ }^{141}$ u.a. die Entwicklungen in der Form der Einleitemittel aus. Während noch im 18. Jh. sowohl die Distanzstellung ( $o b$... gleich) als auch die Zusammenrückung der

139 Dieses Kriterium gilt nicht bei konzessiven Konditionalen (,Irrelevanzkonditionalen', vgl. Kapitel 3.4.3), die des Öfteren dem Bereich der Konzessivität zugeordnet werden (vgl. Di Meola 1997). Bei ihnen wird nämlich die Wahrheit des Antezedens nicht behauptet.

140 Die Verbzweitstellung in uneingeleiteten Konzessivsätzen tritt sehr selten auf(Baschewa 1983, Di Meola 1997: 210f.).

141 Sowohl das erste (1700-1830) als auch das zweite Teilkorpus (ab 1900) sollen die Literatursprache repräsentieren, beide stützen sich auf die Textsorten der Belletristik, Wissenschaft und Presse. Mit dem ersten Korpus erfasst Baschewa (1983: 77) die „Zeit der endgültigen Festigung des Systems der deutschen Literatursprache“, die zweite - zwar relativ weit gefasste - Periode soll die „Zeit der deutschen Gegenwartssprache“ abdecken. 
Konjunktionsteile zu beobachten sind, sind im 20. Jh. die Partikeln zu einer festen Einheit mit $o b$ verschmolzen. Die Distanzstellung kommt nur noch bei wenn mit nachgestellten Partikeln vor, wobei sie heute nur bei wenn ... schon häufiger als die Kontaktstellung ist (vgl. Di Meola 1997: 172). Vom 18. Jh. zum 20. Jh. nimmt auch der Anteil eingeleiteter Konzessivsätze auf Kosten der uneingeleiteten Konzessivsätze mit Verberststellung zu (Baschewa 1983: 86). Des Weiteren verbreiten sich die subordinativen Konzessivverknüpfungen mit Subjunktoren der $o b$ - und wenn-Gruppe auf Kosten anderer konzessiver Konnektoren. Dabei ist insbesondere das Vordringen von obwohl zu verzeichnen (Baschewa 1983: 91).

Im Gegenwartsdeutschen ist obwohl bei weitem der frequenteste konzessive Subjunktor (Breindl et al. 2014: 912, Di Meola 1997: 169f.). Im Bestand der Subjunktoren lassen sich regionale und stilistische Unterschiede feststellen. So ist z.B. obschon im Schweizer Hochdeutsch und im Österreichischen Deutsch frequenter als obwohl, bzw. obgleich gilt als „dichterisch“ (vgl. Breindl et al. 2014: 943f., für regionale, stilistische und auch idiolektale Unterschiede im Neuhochdeutschen s. auch Baschewa 1983: 91f.).

Vorangestellte Konzessivsätze können mit dem Resumptivum so wieder aufgenommen werden. Im Mittelfeld des Obersatzes können zudem adversative Adverbkonnektoren doch bzw. dennoch stehen, sodass eine Art „Doppelmarkierungkonstruktionen“ entstehen (Breindl et al. 2014: 923). Breindl et al. (2014: 923f.) argumentieren dafür, dass diese Adverbkonnektoren nicht in Korrelatkonstruktionen eingehen, u.a. weil sie nicht als wiederaufnehmendes Element im Vorfeld des Obersatzes verwendet werden, „vielmehr kann in diesen Konstruktionen zusätzlich ein wiederaufnehmendes Element im Vorfeld auftreten“. Im folgenden Beispiel steht das Korrelat so im Vorfeld des Obersatzes, der Adverbkonnektor doch im Mittelfeld:

(75) Obschon im Zusammenhang mit der Erstellung des Richtplanes konkrete Zielsetzungen formuliert worden sind, so ist damit doch nur ein Teilbereich abgesteckt. (St. Galler Tagblatt, 11.07.1997, o.S., zit. n. Breindl et al. 2014: 923)

Di Meola (1997: 200ff.) betrachtet doch in dieser Verwendung als ein Korrelat, das der Verstärkung des konzessiven Gegensatzes dient, während das polyseme so die Funktion habe, „die Verbindung der beiden Teilsätze im hypotaktischen Gefüge hervorzuheben.“ Das Auftreten von Adverbkonnektoren im Mittelfeld scheint nach der Auszählung von Baschewa (1983: 93f.) im 18. Jh. noch typisch zu sein. ${ }^{142}$ Der Anschluss des Obersatzes mit so nimmt im Neuhochdeutschen stark ab (vgl. Baschewa 1983: 93f.). Während im 18. Jh. so mit

\footnotetext{
142 Baschewa (1983: 93) unterscheidet folgende drei Strukturtypen bei „Vorderstellung“ der Konzessivsätze: a) ohne Korrelat, b) so +/- Korrelat /adversativ/ (z.B. so ... doch), c) Korrelat /adversativ/. Hier wird so nicht als Korrelat analysiert, bzw. wird alleinstehendes doch auch bei Stellung im Mittelfeld als Korrelat aufgefasst.
} 
$(74,7 \%)$ oder ohne Partikel im Mittelfeld (15,1\%) noch weit verbreitet und bei Konjunktionen der $o b$-Gruppe sogar obligatorisch ist (nur zwei Gegenbelege von 82), tritt so mit $(13,6 \%$ ) oder ohne Partikel im Mittelfeld (4,2\%) im 20. Jh. viel seltener nach vorangestellten Konzessivsätzen auf. ${ }^{143}$

An dieser Stelle sei angemerkt, dass konzessive Subjunktoren wie obwohl im Gegenwartsdeutschen auch parataktisch verwendet werden können (vgl. zuletzt Ágel 2016). Günthner (1999b) erklärt diese Besonderheit damit, dass sich der Konzessivkonnektor obwohl zum Diskursmarker entwickelt. ${ }^{144}$

Auch Konzessivsätze haben nicht-propositionsbezogene Verwendungen (vgl. Zifonun et al. 1997: 2313f.). Vorangestellte Konzessivsätze können auch beim epistemischen Bezug integrativ angebunden werden (vgl. Breindl et al. 2014: 935), sie weisen jedoch häufig den nicht-integrativen Stellungstyp auf, der gerade bei konzessiven Verberstsätzen den Normalfall darstellt. Im Korpus von Di Meola (1997: 208f.) überwiegt die integrative Stellung (553 Belege von 671 vorangestellten Konzessivsätzen), die nicht-integrative Stellung ist v.a. bei Konzessivsätzen zu finden, die durch einen Subjunktor aus der wenn-Gruppe eingeleitet werden. ${ }^{145}$ Wiktorowicz (2013: 396) findet in Prosatexten des 17. Jhs. ausschließlich Konzessivsätze, die resumptiv angebunden sind.

\subsubsection{Spezifizierende Adverbialsätze (Explikativ- und Restriktivsätze)}

Während Explikativ- und Restriktivsätze bei von Polenz (1985: 272f.) als zwei verschiedene Verknüpfungen behandelt werden, sind sie in der Duden-Grammatik (2016: 1114f.) unter dem Typ ,spezifizierend“ zusammengeführt. Die Gemeinsamkeit wird bei der Definition ,spezifizierender Konnektoren“ wie folgt bestimmt: Sie „binden erläuternde Informationen an Aussagen an.“ (Duden-Grammatik (2016: 1114) Bei der semantischen Beziehung und beim Bezugsbereich unterscheiden sich jedoch die beiden Untertypen voneinander: Die

143 Resumptives so wird im schriftsprachlichen Korpus für den Zeitraum 1990-1995 von Di Meola (1997: 202f.) v.a. bei Konzessivsätzen verwendet, die durch einen Subjunktor aus der wenn-Gruppe eingeleitet werden. So und doch als Verstärkungselemente im Hauptsatz sind „in Fachtexten überproportional vertreten“ (1997: 204), dabei kommt so in Kombination mit einem zweiten Element (meist doch) etwas häufiger vor als alleinstehendes so (1997: 200f.).

144 Beim epistemischen Bezug können nachgestellte obwohl-Sätze sowohl mit Verbendstellung als auch mit NichtVerbendstellung auftreten. Bei Nachstellung wie im folgenden Beleg hat der durch obgleich eingeleitete, syntaktisch desintegrierte Adverbialsatz ,die Funktion einer nachgetragenen Einschränkung der Gültigkeit oder Relevanz des externen Konnekts, insofern sie einen nicht recht zum Inhalt des externen Konnekts passenden Sachverhalt nennt. Solche Verwendungen werden mitunter als ,restriktive" oder ,indirekte Konzessivität" bezeichnet", sie stellen jedoch einen Übergang zur adversativen Relation dar (Breindl et al. 2014: 925):

(i) ,,Ich will niemanden sehen!", sagt sie. Obgleich da zwei Nachbarn sind, die sich um sie kümmern, mal einkaufen oder für sie etwas mitkochen. (Frankfurter Rundschau, 21.12.1998, S. 15, zit. n. Breindl et al. 2014: 929).

145 Nach Di Meola (1997: 209) treten ,[h]ochgradig integrierte und grammatikalisierte Konnektive wie obwohl, obgleich oder wiewohl" kaum mit nicht-integrativer Stellung auf. Dieser Stellungstyp ist v.a. bei semantisch marginalen Typen wie „konditional“ oder „skalar“ zu finden, die in der vorliegenden Arbeit unter ,Irrelevanzkonditionale“ erfasst werden. 
erläuternden Informationen ,,beziehen sich explikativ auf den Sachverhalt oder restriktiv auf die Äußerung.“ (Duden-Grammatik (2016: 1114) Der Untertyp ,explikativ“ wird in der Duden-Grammatik (2016: 1114) wie folgt erklärt:

Eine explikative (erklärende) Anknüpfung liegt vor, wenn der Sachverhalt der vorangehenden Aussage durch weitere Einzelheiten näher erläutert wird. Explikative Konnektoren setzen voraus, dass ein Zusammenhang zwischen dem zugrunde liegenden Sachverhalt und der Erläuterung besteht. Die tatsächlichen Bezüge muss der Leser rekonstruieren. Meist erfolgt die Explikation textfolgefest, auch wenn die Subjunktion insofern (als) verwendet wird.

Im Folgenden soll ein Beispielsatz für nachgestellte Explikativsätze aus der DudenGrammatik (2016: 1063) aufgeführt werden:

(76) Die Lage war kritisch, insofern als das Lämpchen noch immer blinkte.

In der Satzsemantik von Peter von Polenz (1985: 272) werden mehrere Untertypen der explikativen Verknüpfung angenommen:

Explikative, spezifizierende und exemplifizierende Verknüpfungen (nach lat. explicare ,erklären', species ,Art, einzelner Fall', exemplum ,Beispiel'): Eine Aussage P1 durch eine Aussage P2 näher ERKLÄREN ${ }_{\mathrm{R}}$, P1 durch P2 GENAUERSAGEN ${ }_{R} /$ PRÄZISIEREN $_{R}$ bzw. EXEMPLIFIZIEREN E. $_{R}$.

Bei restriktiven Verknüpfungen wird die Gültigkeit einer Aussage durch eine andere Aussage eingeschränkt (von Polenz 1985: 237, Zifonun et al. 1997: 2338). Bezüglich der Reihenfolge der Konnekte wird darauf verwiesen, dass die vorangehende Aussage im folgenden Nebensatz eingeschränkt wird, d.h., dass Restriktivsätze i.d.R. nachgestellt sind (vgl. von Polenz 1985: 237), in der Duden-Grammatik (2016: 640) ist jedoch auch die Voranstellung eines Restriktivsatzes belegt. Restriktivsätze, die im Gegenwartsdeutschen mit insofern (...) als, soweit, außer daß/wenn usw. eingeleitet werden, sind in der IDS-Grammatik als ,kategorienvariable Nebensätze“ eingestuft, da „verschiedene Gebrauchsweisen mit jeweils unterschiedlichen Bezugsbereichen erkennbar [sind]“. (Zifonun et al. 1997: 2338) Sie sind von peripheren Adverbialsätzen (3.4.6) nicht scharf zu trennen, die äußerungsbezogene Gebrauchsweise wird in der IDS-Grammatik unter dem Stichpunkt ,modusmodifizierende Nebensätze‘ (s. dazu 3.4.6) behandelt: Modusmodifizierende Nebensätze „schränken den Gültigkeitsanspruch, der für das Gesagte unter dem Aspekt ,so ist es` angemeldet wird, in unterschiedlicher Weise ein.“ (Zifonun et al. 1997: 2326). In der IDS-Grammatik (Zifonun et al. 1997: 2326f.) werden insg. fünf einschlägige Gebrauchsweisen aufgeführt, auf die an dieser Stelle nicht näher eingegangen wird. Es soll nur festgehalten werden, dass Restriktivsätze im Gegensatz zu der oben referierten Aussage von Gallmann in der DudenGrammatik (2016: 1114) nicht nur äußerungsbezogen sein können. Im folgenden Beispielsatz aus der Duden-Grammatik (2016: 640) ist m.E. eine Verknüpfung auf der propositionalen 
Ebene zu beobachten. Er enthält, wie aus dem Kommentar von Nübling hevorgeht, eine „Einschränkung des Sachverhalts, der im übergeordneten Satz ausgedrückt wird“.

(77) Die Diskussion war sehr interessant, insofern (als) sie Aufschluss über das Tatmotiv lieferte.

\subsubsection{Vergleichssätze (Komparativ- und Proportionalsätze)}

Bei Vergleichssätzen werden zwei ähnliche bzw. parallele Sachverhalte miteinander in Beziehung gesetzt. Diese Nebensatzklasse wird nur in der Grammatik von Hoffmann (2013: 360f.) und Zifonun et al. (1997: 2333) explizit behandelt (vgl. Kapitel 3.4.1). ${ }^{146}$ Gelegentlich werden komparative und vergleichende Verknüpfungen gleichgesetzt (von Polenz 1985: 273f., Pittner 2013a: 517f.). In der vorliegenden Arbeit werden in Anlehnung an die DudenGrammatik (2016: 1117) die beiden Untertypen ,komparativ ${ }^{6}$ und ,proportional unterschieden: Während bei ,proportionalen` Verknüpfungen die Ähnlichkeit zwischen den im Bezugssatz bzw. im Vergleichssatz ausgedrückten Sachverhalten parallel graduierend ist, ist sie in ,komparativen“ Verknüpfungen ,allgemeiner Art“ ${ }^{6147}$ Bei Proportionalsätzen ist der Anschuss des vorangestellten Adverbialsatzes - im Gegensatz zu Komparativsätzen - nicht variabel. Sie werden in der vorliegenden Arbeit als formale Parallelisierungen im Rahmen eines paarigen Junktionsausdrucks ,so+Adjektiv ..., so+Adjektiv ...“ analysiert (s. dazu Kapitel 3.1.4). Komparativsätze der realen Gleichheit werden durch wie eingeleitet, im Hauptsatz können die Korrelate so, genau, genauso stehen (Bergerová 1998: 149, von Polenz 1985: 273).

Auf der semantischen Ebene können Vergleichssätze der realen (Un-)Gleichheit sowie hypothetische (oder irreale) Vergleichssätze unterschieden werden (Bergerová 1998: 149). Hypothetische Vergleichssätze haben nach Eggs (2006: 176) die folgende Funktion: „Sie erlauben dem Sprecher, eine als Hypothese formulierte Erklärung für den im Hauptsatz genannten Sachverhalt zum Ausdruck zu bringen." Sie werden durch die zusammengesetzten Subjunktoren wie wenn, als ob/als wenn eingeleitet, bei Subjunktorersatz tritt ein durch als (,Restsubjunktor') eingeleiteter, konjunktivischer Verberstsatz auf (vgl. Pittner 2013a: 518, Ágel 2010: 912, Pasch et al. 2003: 356, 619f., Eggs 2006: 172f., von Polenz 1985: 273). Im

\footnotetext{
146 Die im Kapitel 3.4.1 angegebene Abbildung 9 aus dem HDK-2 (Breindl et al. 2014: 243) ist nicht präzise genug, denn bei der „GDS“ (Zifonun et al. 1997) fehlt der entsprechende Eintrag „,+“ beim ,Vergleichssatz“.

147 In der IDS-Grammatik werden ,reine Vergleichssätze“ und ,Vergleichssätze als Teile von Gradphasen“ unterschieden (Zifonun et al. 1997: 2333). Die Bezeichnung ,Komparativsätze‘ wird nicht benutzt. ,Proportionalsätze` bilden dort keinen Untertyp von Vergleichssätzen, sondern stellen eine eigene Kategorie dar.
} 
folgenden Beispiel wird der irreale Vergleichssatz seinem Bezugssatz vorangestellt und integrativ angschlossen:

(78) Als hätten sie nur auf ein Zeichen von ihm gewartet, stürzten alle auf die Tanzfläche. (zit. n. Pasch et al. 2003: 697)

Einige pragmatische Verwendungen von Vergleichssätzen (z.B. Verwendungen als Modusmodifikator, vgl. Zifonun et al. 1997: 2334), die nicht als propositionsbezogene Adverbialsätze aufzufassen sind, werden im Kapitel 3.4.6 behandelt.

\subsubsection{Lokalsätze}

Die Nebensatzklasse der Lokalsätze findet sich nicht in jeder Grammatik (s. aber Engel 2004: 146, Hoffmann 2013: 361, Zifonun et al. 2280). Obwohl Lokaladverbialia meist als Untertyp der situierenden bzw. kontextspezifizierenden Adverbialia behandelt werden (vgl. z.B. Bußmann 2008: 415, Duden-Grammatik 2016: 795, Dürscheid 2012: 38, Hentschel 2010: 18, Zifonun et al. 1997: 1150f.), ist der Status von Sätzen wie in (81) nicht geklärt:

(79) Wo gehobelt wird, fallen Späne. (zit. n. Holler 2013: 287)

Der durch wo eingeleitete Nebensatz kann als satzförmige Realisation eines Lokaladverbials gedeutet und nach Pasch/Zifonun (2006: 921f.) „der traditionellen Kategorie des Adverbialsatzes zugeordnet werden“ (vgl. auch Pittner 2004: 361, Zitterbart 2002: 146f.). Obwohl in diesen Fällen kein Bezugselement im übergeordneten Satz vorliegt, ,ist ein Adverb da oder dort hinzudenken“ (Duden-Grammatik 2016: 1050), daher wird der obige Satz meist als ,freier Relativsatz، analysiert (vgl. z.B. Pittner 1999: 225, 317). Es besteht jedoch keine Einigkeit darüber, ob freie Relativsätze nach ihrer Funktion attributiven Relativsätzen gleichen oder eher Adverbialen zuzuordnen sind (vgl. z.B. Holler 2013: 282f., Pittner 2004: 361).

Lokalsätze können in Anlehnung an Pittner (1999: 225) als ein Sonderfall von Adverbialsätzen betrachtet werden: „Sie unterscheiden sich von anderen Adverbialsätzen dadurch, daß sie nicht Sachverhalte, sondern Entitäten bezeichnen. Das bedeutet, daß mittels des im Lokalsatz bezeichneten Sachverhalts ein Ort bestimmt wird.“" ${ }^{148}$ Lokalsätze werden

\footnotetext{
148 Nach von Polenz (1985: 252) sind Lokaladverbiale Zusätze zum „Aussagengehalt des Satzinhalts“. Die lokale Relation wird weder bei den semantischen Klassen von Aussagen-Verknüpfungen (von Polenz 1985: 268-287) noch bei den semantischen Klassen von Konnektoren (Breindl et al. 2014: 253) berücksichtigt. In seiner lokalen Verwendung ist wo nicht als Konnektor zu betrachten, denn es charakterisiert nicht ein semantisches Verhältnis zwischen zwei Sachverhalten bzw. Satzinhalten, seine Bedeutung ist keine spezifische zweistellige Relation mit propositionalen Argumenten (vgl. Pasch et al. 2003: 1f., von Polenz 1985: 265f.).
} 
zudem nicht durch Subjunktoren eingeleitet, sondern meist durch das ,W-Adverb“ wo (vgl. Zifonun et al. 1997: 2280). ${ }^{149}$ Es soll jedoch betont werden, dass wo nicht nur die Funktion hat, Lokalsätze einzuleiten. Im Folgenden soll auf das Verhältnis der lokalen Relation zu anderen semantischen Relationen eingegangen werden.

Nach Eisenberg (2013: 277) ist beim Gebrauch von wo als Relativadverb (wie in der Moment, wo das passiert) die Tendenz zu beobachten, „nicht nur bei lokalen, sondern auch bei anderen Inhaltsbeziehungen aufzutreten.“ Diese Tendenz führt er darauf zurück, „dass das Lokale besonders häufig metaphorisiert wird und Grundstrukturen für viele andere Inhaltsbereiche abgibt.“ (2013: 277) In der Erforschung der gesprochenen Sprache wurden auch andere Gebrauchsweisen von wo untersucht, in denen es keine Relativsätze, sondern als Subjunktor Adverbialsätze einleitet. Dabei wurden temporale, kausale bzw. konzessive Verwendungen beschrieben (vgl. Günthner 2002: 1, Gohl 2006: 44ff., Zifonun et al. 1997: 2299f., 2312f.). Ein weiteres Beispiel für Übergänge stellen die durch wo eingeleiteten Konditionalsätze dar. Zu ihrer Entstehung bemerkt Behaghel (1928: 350): „Aus der greifbaren Örtlichkeit kann [...] die geistige hervorgehen“. ${ }^{150}$ Daher ist im Korpus sowohl mit rein lokalen Verwendungen als auch mit Übergängen zwischen Lokalität und weiteren Inhaltsbeziehungen zu rechnen, was als Argument für die Berücksichtigung der Lokalsätze verstanden werden kann. Aus dieser knappen Erläuterung wird zwar deutlich, dass Lokalsätze aus mehreren Gründen einen Sonderfall darstellen. Da das Ziel der vorliegenden Arbeit aber darin besteht, möglichst alle Adverbialsatztypen systematisch $\mathrm{zu}$ untersuchen, sollte im Zweifelsfall für die Berücksichtigung des Satztyps entschieden werden. Mit anderen Worten, in der vorliegenden Untersuchung werden - trotz der obigen Bedenken - Lokalsätze bzw. die davon schwer abgrenzbaren Mischtypen mitberücksichtigt.

\subsubsection{Mischtypen}

Im Folgenden soll erläutert werden, aus welchem Grund Mischtypen von den reinen temporalen bzw. kausalen oder konditionalen Relationen getrennt behandelt werden. Die Etablierung einer besonderen Gruppe der Mischtypen ist m.E. deshalb sinnvoll, weil „Übergänge von einer Bedeutungsgruppe in andere stattgefunden haben, indem die

\footnotetext{
${ }^{149}$ Es gibt jedoch auch Lokalsätze, die „das Geschehen im Bezugssatz im Hinblick auf (...) eine Richtung oder einen Ausgangspunkt [situieren]“ (Pittner 2013a: 502). Sie werden durch wohin bzw. woher eingeleitet. Da diese Untertypen in den untersuchten Korpustexten nicht belegt sind, wird darauf nicht ausführlicher eingegangen.

150 Der wo-Satz kommt in diesen Fällen „,der allgemeinen Bedingung nahe“, vgl. das folgende Beispiel (Behaghel 1928: 350):

(i) er demüthigte sinen Gegner, wo es nötig war.
} 
Einleitungswörter im Laufe der Entwicklung neue Bedeutungsinhalte annehmen.“ (Dal/Eroms 2016: 245) Diese Veränderungen betreffen z.B. im Falle von da, nachdem oder während die neuhochdeutsche Zeit, sodass eine eindeutige Zuordnung der Belege nicht immer möglich ist.

Die zeitliche Relation wird bekanntlich in mehreren Sprachen weiter interpretiert, wie es auch bei da oder nachdem im Deutschen der Fall war: Mit der Zeit entwickelte sich eine kausale Lesart im Sinne von post hoc ergo propter hoc (d.h. „danach, also aus diesem Grunde“, vgl. Blühdorn 2004: 131f., Breindl et al. 2014: 101f.). Die Veränderung der Bedeutung von $d a$ ist nach Axel-Tober (2012: 272) „eine sprachgeschichtlich junge Erscheinung, die sich erst in nhd. Zeit systematisch vollzogen hat“. Die kausale Bedeutung von $d a$ entwickelt sich erst am Ende des 16. Jhs., wohl aus der temporalen Bedeutung (AxelTober 2012: 355). Die neue Bedeutung verbreitet sich erst im 17. Jh. und ist in der Übergangszeit schwer von der alten Bedeutung zu trennen. D.h., es ist schwer exakt zu bestimmen, „ab wann die lediglich implikatierte kausale Bedeutung Teil der wörtlichen Bedeutung geworden ist“" (Axel-Tober 2012: 352). ${ }^{151}$ Heute wird $d a$ als Subjunktor im kausalen Bereich verwendet, temporale $d a$-Sätze werden im Gegenwartsdeutschen v.a. nur literarisch verwendet und gelten als gehoben (Fabricius-Hansen 2007: 775f., Breindl et al. 2014: 312). Der Subjunktor nachdem leitet nach den Angaben des DWb (1889, Sp. 34, Z. 3) bereits im 17. Jh. vorangestellte Kausalsätze ein. Auch bei diesem Subjunktor ist schwer zu bestimmen, ab wann (bzw. ob überhaupt) die kausale Bedeutung Teil der wörtlichen Bedeutung geworden ist. ${ }^{152}$

Der Subjunktor während ist relativ jung, in der ersten Hälfte des 18. Jhs. wird er noch in zusammengesetzter Form verwendet (z.B. während dessen (dasz), vgl. DWb 1922: Sp. 807, Z. 23). Seine Bedeutung ist primär temporal, das einfache während mit adversativer Bedeutung tritt aber schon am Ende des 18. Jhs. auf. Während der Bedeutungswandel des

151 Die temporale Bedeutung von $d a$ (im Sinne von nachdem und während) tritt noch bis zum 19. Jh. auf (vgl. Breindl et al. 2014: 802 in Anlehnung an Arndt 1956:129f.). Es hat ,heute ausschließlich kausale[...] Bedeutung“ (Betten 1987: 86). Während die temporale Variante des Subjunktors $d a$ im Gegenwartsdeutschen bereits ,veraltet klingt“ (Blühdorn 2004: 132, vgl. auch Duden-Grammatik 2016: 638), kann beim semantisch unspezifisch(er)en Adverbkonnektor $d a$ auch im Gegenwartsdeutschen eine temporale Funktion angenommen werden (vgl. Breindl et al. 2014: 314f.).

152 Nach Brooks (2006: 168) überwiegt in oberdeutschen Drucken bereits um 1530 die kausale Funktion von nachdem. In der historischen Syntax von Dal/Eroms (2016: 233) wird jedoch bezüglich des Alters der kausalen Bedeutung erst ein Beleg aus dem Jahre 1772 angegeben. Welke (2007: 159f.) nimmt sogar bei dem gegenwartsdeutschen nachdem nur eine temporale Bedeutung an und unterscheidet diese von einer möglichen Implikatur aus der temporalen Bedeutung: „Eine mögliche Implikatur des Hörers (und auch eine mögliche Absicht des Sprechers) besteht darin, dass aus der temporalen Beziehung auf eine kausale gefolgert werden soll. Damit wird die Temporalbestimmung jedoch nicht zu einer Kausalbestimmung. Denn die Konjunktion nachdem hat (noch) keine kausale Bedeutung." Im Gegenwartsdeutschen finden sich jedoch Belege wie (i), die keine temporale Interpretation zulassen und eine rein kausale Bedeutung zeigen. Bei nachdem ist also die Kausalität im Gegenwartsdeutschen bereits konventionalisiert.

(i) Nachdem die Ausstellung einen sehr repräsentativen und qualitativ sehr hochstehenden Querschnitt der zeitgenössischen Kunst darstellt, würde sie sich natürlich als Einstieg für ein Tiroler Museum der Moderne geradezu anbieten. (Neue Kronen Zeitung, 14.05.1995, S. 27, zit. n. Breindl et al. 2014: 226) 
Subjunktors $d a$ bereits abgeschlossen ist, sind polyseme Konnektoren, bei denen sowohl die ursprüngliche als auch die neue Bedeutung vorhanden sind, auch im Gegenwartsdeutschen keine seltene Erscheinung (für weitere Beispiele der regulären Polysemie von Subjunktoren s. Breindl et al. 2014: 103). Bei der Analyse der vorangestellten Adverbialsätze ist also mit Übergangstypen zu rechnen (vgl. auch Ágel 2000: 1887). ${ }^{153}$

\subsubsection{Periphere bzw. pragmatische Adverbialsätze}

Wie im Kapitel 3.1.2 bereits erwähnt, werden in der vorliegenden Arbeit mit dem Terminus ,periphere Adverbialsätze‘ - im Gegensatz zur etwas eingeschränkteren Verwendung in Zifonun et al. (1997: 2323) - alle nicht-prototypischen, nicht-propositionsbezogenen Satzadverbialsätze erfasst, u.a. moduskommentierende und modusmodifizierende Adverbialsätze, die inhaltlich nur locker angefügt sind (vgl. Zifonun et al. 1997: 2242ff.). Da bei der Klassifizierung kein allgemeiner Konsens besteht, soll eine Typologie v.a. auf der Grundlage von Pittner (2000) und Zifonun et al. (1997: 2251) erstellt werden, die durch eine weitere Kategorie ergänzt wird. Im Folgenden wird auf die Untertypen, peripherer Adverbialsätze‘ knapp eingegangen. Die Frage nach der Desintegration der vorangestellten Nebensätze ist insbesondere bei diesen pragmatischen Verknüpfungen relevant.

In der IDS-Grammatik werden moduskommentierende, modusmodifizierende und diktumskommentierende Nebensätze erwähnt, die kompositional nicht voll in den Nachsatz integriert sind (Beispiele nach Pittner 1999: 337 bzw. Zifonun et al. 1997: 2251):

(80) Wenn es dich interessiert: wir sind völlig pleite.

(81) Wie ich vermute, hat niemand sich an die Vorschläge gehalten.

(82) Wie ich leider feststellen mußte, hat niemand sich an die Vorschläge gehalten.

Diese Nebensatztypen werden in der IDS-Grammatik im Kapitel ,Adverbialsätze“ erfasst, obwohl sie keine Adverbiale im engeren Sinne darstellen (vgl. Kapitel 3.1.2). Im Beispiel (80) stellt der Nebensatz einen Kommentar auf der Modusebene dar. Für diesen Typ ist eine nichtintegrative Anbindung des Adverbialsatzes charakteristisch. Es handelt sich um ein Sprechakt-Adverbial im Sinne von Pittner (1999: 320). Adverbialsätze mit Sprechaktbezug können auch bei Kausal-, Konzessiv- und Finalsätzen auftreten (s. Pittner 1999: 337f.), diese sind als Untertypen moduskommentierender Adverbialsätze aufzufassen. Im Beispiel (81)

\footnotetext{
${ }^{153}$ Vgl. die methodologischen Überlegungen zur Erfassung der semantischen Relationen im Kapitel 4.2.
} 
modifiziert der Nebensatz den KM-Modus, d.h., er schränkt den Gültigkeitsanspruch der Aussage hinsichtlich ihrer Verlässlichkeit ein (vgl. Zifonun et al. 1997: 2326f.). Dazu trägt auch der modale Prädikatsausdruck (vermute) im Untersatz bei.

Mit dem Untersatz wird diejenige Hinsicht genannt, unter der überhaupt ein Geltungsanspruch für die Aussage erhoben werden kann. [...] Mit dem Untersatz wird eine Einschränkung hinsichtlich der Verläßlichkeit der Aussage gemacht. (Zifonun et al. 1997: 2327)

In diesem Satz, wie auch im nächsten Beispiel, wird kein typischer „VerhältnissatzSubjunktor wie weil, wenn“" (Zifonun et al. 1997: 2275) verwendet. Der ,kompositionalen“ bzw. ,semantischen“ Desintegration entspricht in (81) sowie (82) keine topologische Desintegration. Im Kapitel 3.3.2 wurde bereits erwähnt, dass in diesen Fällen der Satzgliedstatus der Untersätze nicht eindeutig ist: Trotz Vorfeldstellung, also topologisch vollständiger Integration, liegt kein enger inhaltlicher Zusammenhang zwischen den Teilsätzen vor. Im Beispiel (82) ist der kommentierende Nebensatz „auf das gesamte Diktum bezogen" und drückt eine emotionale Bewertung des Sprechers aus.

Pittner (2000) behandelt mehrere Typen von Konditionalkonstruktionen, die auf der diskurspragmatischen Ebene anzusiedeln sind. Sie verweist darauf, dass die pragmatischen Charakterisierungen in der Fachliteratur zu kurz greifen. Aufgrund der Fachliteratur wird eine Typologie erstellt, in der fünf Funktionen von Konditionalsätzen mit Sprechaktbezug unterschieden werden. Relevanzkonditionalsätze wurden weiter oben (Kapitel 2.1.2) bereits behandelt, in der Typologie von Zifonun et al. (1997) sind sie dem Typ ,moduskommentierend` zuzurechnen. Illokutionskraftmodifizierende Konditionalsätze beziehen sich auf die Illokution im Bezugssatz.

(83) Wenn ich das so sagen darf, liebe Frau Sager: Das ist schon sehr halbherzig. (PBT/W15.00066 Protokoll der Sitzung des Parlaments Deutscher Bundestag am 16.10.2003. 66. Sitzung der 15. Wahlperiode 2002-2005. Plenarprotokoll, Berlin, 2003)

In diesem Beispielsatz aus dem DeReKo-Korpus (Institut für Deutsche Sprache 2016) wird die Illokutionskraft abgeschwächt: „Der Sprecher holt sich quasi die Erlaubnis des Hörers für seine Äußerung oder suggeriert dies zumindest.“ (Pittner 2000: o.S.) Dieser Typ entspricht der Kategorie ,modusmodifizierend‘ von Zifonun et al. (1997). Bei diesem Typ nimmt Pittner (2000: o.S.) an, dass seine integrative Stellung ,nicht völlig akzeptabel“ scheint. Es konnten in der Tat keine einschlägigen Belege aus dem DeReKo (Institut für Deutsche Sprache 2016) ermittelt werden. Eine Google-Recherche lieferte jedoch mehrere Belege für die integrative Stellung, vgl. den nächsten Beleg aus einem Internetforum: 
(84) Bei der PK 380 sind Stahlteile verbaut und wenn ich das so sagen darf ist die PK 380 qualitativ besser $(-)$ ).

(www.co2air.de/wbb3/index.php?page=Thread\&threadID=78039)

Einstellungskommentierende Konditionalsätze wie Wenn ich es offen sagen darf „drücken eine Sprechereinstellung aus, teilweise kombiniert mit dem Einholen einer Erlaubnis“ (Pittner 1999: 348, 2000: o.S.). Als Oberbegriff wird in der vorliegenden Untersuchung ,diktumsbzw. einstellungskommentierend“ verwendet, da er nicht nur Diktumskommentare im obigen Sinne erfasst. ${ }^{154}$ Einschlägige Adverbialsätze können u.a. eine Sprechereinstellung im obigen Sinne, die emotionale Bewertung des Sprechers zur Proposition des Obersatzes bzw. das Verhalten des Sprechers zur Gültigkeit der nachfolgenden Aussage ausdrücken.

Der nächste Typ von pragmatischen Konditionalsätzen dient dazu, die gewählte Formulierung $\mathrm{zu}$ kommentieren. Dieser Typ entspricht den „metakommunikativen Verknüpfungen“ bei von Polenz (1985: 285f.) und wurde im Kapitel 3.4.1 bereits behandelt. Um metakommunikative Verknüpfungen, die auf die Kommentierung der Ausdrucksweise bzw. Bedeutung beschränkt sind, vom Oberbegriff der pragmatischen, sprechaktbezogenen Adverbialsätzen $\mathrm{zu}$ unterscheiden, wird in der vorliegenden Arbeit eher der Terminus ,formulierungskommentierend“ bevorzugt. Da dieser Typ aber nicht auf Konditionalsätze beschränkt ist, bezeichne ich ihn nicht als ,formulierungskommentierende Konditionalsätze“ (Pittner 2000), sondern als ,formulierungskommentierende Adverbialsätze‘.

Der ,diskursstrukturierende“ Typ „hat vor allem die Funktion, die Aufmerksamkeit des Hörers in eine bestimmte Richtung zu lenken“ (Pittner 2000: o.S.). Dass im folgenden Beispielsatz kein ,rein“ konditionaler Typ vorliegt, ist auch daran zu erkennen, dass der Wahrheitswert des Konsequens nicht von dem des Antezedens abhängt. Mit Hilfe des wennSatzes wird die Beziehung der folgenden Äußerung zum Gesprächskontext verdeutlicht:

(85) Wenn Sie sich daran erinnern, so haben wir diesen Terminus anfangs wie folgt definiert: ... . (zit. n. Pittner 2000: o.S.)

Günthner (1999) nimmt eine etwas andere Typologie vor. Während sie sowohl „Relevanzkonditionale‘ als auch ,diskursstrukturierende‘ Konditionalkonstruktionen behandelt, wird als dritter Typ ,metakommunikative Konditionalkonstruktionen` genannt, der

\footnotetext{
154 Bei pragmatischen Adverbialsätzen sind neben der Kategorie ,diktumskommentierend` noch weitere Typen zu beachten, wie etwa propositionskommentierend, daher soll eine andere Terminologie verwendet werden. Zur Unterscheidung zwischen Diktumsbezug und Propositionsbezug s. Zifonun et al. (1997: 2328f.) Im Beleg (i) liegt ein Propositionsverweis vor:

(i) Sollte Neuberg einem Mord zum Opfer gefallen sein - wofür bis jetzt nicht der geringste Beweis vorlag -, dann war dieser Anschlag durch eine schadhafte Nachttischlampe erfolgt und nicht durch Gift, das stand felsenfest. (zit. n. Zifonun et al. 1997: 2328f., Hervorhebung im Original)
} 
den Pittner'schen Typen ,illokutionskraftmodifizierend', ,einstellungskommentierend ‘ und ,formulierungskommentierend' Konditionalsatz entspricht.

Nach diesem Überblick soll die in der vorliegenden Arbeit angewandte Klassifikation vorgestellt werden. Es werden die folgenden Typen unterschieden: ,moduskommentierend“ (5.5.1), ,modusmodifizierend“ (5.5.2), ,diktums- bzw. einstellungskommentierend‘ (5.5.3), ,formulierungskommentierend' (5.5.4) sowie ,diskursstrukturierend' (5.5.5). Zudem wird im Folgenden für die Annahme eines weiteren Typs argumentiert, der in der Regel nicht als Adverbialsatz behandelt wird. Diesen Typ bezeichne ich als ,thematisierend' (5.5.6).

Sog. ,Sperrsätze‘ oder ,unechte Spaltsätze‘ (auch ,Pseudo-Clefts‘ genannt) sind, obwohl sie auch eine diskurspragmatische bzw. informationsstrukturierende Funktion erfüllen, keine Adverbialsätze. ${ }^{155}$ Die Annahme einer adverbialen Funktion wäre jedoch zu überlegen bei Sätzen wie Was ich nur schade finde oder Was ich eigentlich damit sagen wollte ohne Kopulaverb im übergeordneten Satz, das als konstitutiver Teil von Pseudo-Clefts angesehen wird, vgl. den folgenden Beleg aus dem DeReKo (Institut für Deutsche Sprache 2016):

(86) Tolle Sache, die Antenne-MV-Partys. Was ich schade finde, viele stöhnen, sie haben kein Geld, alles zu teuer .... Trotzdem sind Diskos brechend voll, aber die Antenne$M V$-Partys z. B. beim Lichterfest am Kummerower See oder in Brunn waren nur sehr mäßig besucht. (NKU03/AUG.08814 Nordkurier, 29.08.2003; LESERBRIEF)

Diese Sätze drücken „,eine Bewertung, eine affektive Einstellung bzw. eine Positionierung des Sprechers zur noch ausstehenden Information“ aus oder fungieren als metakommunikativer Rahmen einer Sprechhandlung (Günthner 2006: 83). Hinsichtlich ihrer Funktion steht der obige Beleg den diktumskommentierenden Adverbialsätzen nahe. Das folgende Beispiel veranschaulicht ebenfalls diesen Typ, mit dem die folgende Aussage kommentiert wird (Duden-Grammatik 2016: 1056):

(87) Was mich am meisten erstaunte: Wenn ein Kollege wirklich mal mitbekam, dass ich ein Hörgerät trug, fiel seine Reaktion ganz anders aus, als ich erwartet hatte. (zit. $\mathrm{n}$. Duden-Grammatik 2016: 1056f.)

$155 \mathrm{Zu}$ echten und unechten Spaltsätzen s. ausführlicher Altmann (2009). Der sog. ,echte Spaltsatz b bzw. ,Cleft-Satz ' hat das Muster Es ist $X+$ Relativsatz und dient ebenfalls zur Hervorhebung von Informationen (s. Duden-Grammatik 2016: 1056). Den Cleft-Satz (i) führt Altmann (2009: 13) auf einen einfachen Satz zurück, dessen Subjekt (die Frauen) als Cleft-Ausdruck in Form einer Kopulakonstruktion „abgespalten“ wird, der ein nachgestellter freier Relativsatz untergeordnet wird:

(i) Die Frauen sind es, die Verwirrung stiften.

Altmann (2009: 16) schlägt bei dieser Konstruktion die Analyse als Prädikativsatz vor. Im Pseudocleftsatz (ii) bildet der Cleft-Ausdruck (die Frauen) mit einem Kopulaverb den übergeordneten Satz, dem ein vorangestellter Pronominalsatz untergeordnet wird.

(ii) ?Wer/Was Verwirrung stiftet, (das) sind die Frauen.

Nach Altmann (2009: 29) ist der vorangestellte Gliedsatz entweder als Subjektsatz oder als Prädikativsatz zu analysieren. 
Der Umstand, dass diese Sätze nicht durch einen Subjunktor, sondern durch was eingeleitet werden, dürfte kein Grund dafür sein, sie nicht als Adverbialssätze zu berücksichtigen.

Ferner wäre auch bei Konstruktionen des Typs Was $X$ betrifft/angeht, die als „Einleitungsfloskeln“ (Altmann 1981: 22, 42) oder als „formelhafte Thematisierungsausdrücke“ (Zifonun et al. 1997: 516, 524ff., vgl. dazu auch Kapitel 3.2.1) gedeutet werden, die Annahme der adverbialen Funktion plausibel (so bereits Behaghel 1929: 414). Diese Konstruktionen haben die Funktion, das Thema der Folgeäußerung anzugeben, daher wird an dieser Stelle der Terminus ,thematisierende Satzadverbialsätze` vorgeschlagen. Die Grenze zu anderen Typen (z.B. zu einstellungskommentierenden Sätzen wie Was ich nur schade finde, die auch eine thematisierende Funktion haben) ist fließend. ,Thematisierende Satzadverbialsätze‘ können resumptiv mit so oder da im Vorfeld des nachstehenden Syntagmas wieder aufgenommen werden. Zudem wird im folgenden Beispielsatz der thematische Ausdruck den Weg zu ihm durch das Pronomen er anaphorisch fortgeführt.

(48) Was den Weg zu ihm betreffe, so sei er ohne besondere Beschwerden und Anstände (...) (Thomas Mann, Erzählungen, 950, zit. n. Zifonun et al. 1997: 522)

Dieser Typ von was-X-betrifft/angeht-Konstruktionen ist zwar mit den Pseudoclefts verwandt (Günthner 2006: 84), auch hier geht es um eine Herausstellung eines Satzglieds im Vordersatz. Es gibt jedoch auch was-X-betrifft/angeht-Konstruktionen, die in der IDSGrammatik im Kapitel „Adverbialsätze“ behandelt werden. Diese können einen Aspekt nennen, ,auf den die Gültigkeit der Aussage eingeschränkt werden soll“. Sie werden ,modusmodifizierend' genannt:

(88) Was seine Leistungen angeht, so sind wir ganz zufrieden mit ihm. (zit. n. Zifonun et al. 1997: 2327)

Birkner (2008) untersucht was-X-betrifft-Konstruktionen aufgrund eines Datenkorpus von 300 Belegen und beschreibt sie als zweiteilige Konstruktionen. Der erste Teil ist ein durch Verb-Letzt-Stellung gekennzeichneter, unselbständiger Nebensatz mit der Formel was $\mathrm{X}$ VERB $\{($ an)betrifft/angeht/an(be)langt $\}$, wobei X eine meist mit einer lexikalischen Vollform besetzte NP bezeichnet. Der zweite Teil ist sein nachgestellter Bezugssatz. ${ }^{156}$ In Birkners Aufsatz wird zwar das Problem der (Des-)Integration des was-Syntagmas angesprochen

\footnotetext{
156 Neben der Voranstellung sind allerdings weitere Stellungsvarianten möglich. „Textstrukturierende Verwendungen erscheinen in der Regel in Voranstellung, expansive Verwendungen sind final- oder zwischengestellt.“ (Birkner 2008: 69) Die textstrukturierende Funktion besteht in der Organisation des Textthemas, bei der expansiven Funktion handelt es sich entweder um eine Erweiterung mit zusätzlichen propositionalen Einheiten oder um eine nachträglich einschränkende Spezifizierung eines bereits vorhandenen Referenten (Birkner 2008: 69ff.). Für die vorliegende Arbeit ist v.a. die textstrukturierende Funktion von Bedeutung.
} 
(2008: 64, 68) und die Möglichkeit dreier Varianten beschrieben (Integration, Desintegration, Resumption), quantitative Daten werden jedoch diesbezüglich nicht genannt. ${ }^{157}$

\subsection{Mündlichkeit/Schriftlichkeit bzw. Nähe/Distanz}

Die Berücksichtigung der gesprochenen Sprache in sprachgeschichtlichen Untersuchungen ist stark an das Problem der Überlieferung gebunden. Historische Mündlichkeit kann nur am Material schriftsprachlicher Quellen analysiert werden, indem „,Reflexe` oder ,Spuren` der Mündlichkeit in schriftich überlieferten (...) Texten rekonstruiert“ werden (Betten 2000: 1649). Es soll daher zunächst darauf eingegangen werden, wie die (konzeptionelle) Mündlichkeit im Rahmen einer prototypischen Betrachtungsweise erfasst werden kann. Dabei werden das Modell der ,Sprache der Nähe“ und der ,Sprache der Distanz von Koch/Oesterreicher (1985) und seine Weiterentwickung in Ágel/Hennig (2006a und 2006b) v.a. unter dem Aspekt ihrer praktischen Umsetzbarkeit behandelt (Kapitel 3.5.1). Zur Geschichte, Entwicklung und zu den Anwendungsfeldern des Nähe-Distanz-Ansatzes s. Ágel/Hennig (2010) sowie Feilke/Hennig (2016).

Anschließend wird die Dimension ,Nähe‘ vs. ,Distanz‘ aus sprachgeschichtlicher Sicht erörtert, wobei auf die ,Verschriftung ‘ bzw. ,Verschriftlichung ${ }^{`}$ und deren Zusammenhänge mit Aggregation/Integration eingegangen werden soll (Kapitel 3.5.2).

\subsubsection{Grundlagen und Operationalisierung}

Bei der Beschreibung von gesprochener und geschriebener Sprache fand das Modell der ,Sprache der Nähe‘ und der ,Sprache der Distanz von Koch/Oesterreicher (1985) besondere Beachtung. Sie unterscheiden zwischen medialer und konzeptioneller Ebene (1985: 17): Während das Medium der Realisierung sprachlicher Äußerungen ,phonisch` bzw. ,graphisch“ sein kann, wird auf konzepioneller Ebene (Konzeption im Sinne von Modalität, die die Äußerungen prägt) von einem Kontinuum zwischen den Polen ,Sprache der Nähe` und ,Sprache der Distanz ausgegangen. ,Nähe“ und ,Distanz ${ }^{\star}$ werden auf Kommunikationsbedingungen und Versprachlichungsstrategien zurückgeführt und zu Textsorten in Beziehung gesetzt (1985: 18, 23).

157 Birkner (2008: 68) bemerkt dazu, dass die Beurteilung der syntaktischen Integration ,in vielen Fällen nicht eindeutig möglich“" sei. Diese Feststellung bezieht sich allerdings eher auf die Zwischen- oder Finalstellung des was-Syntagmas. 
Auf diesem Modell aufbauend wird von Ágel/Hennig (2006a) das ,Modell des Nähe- und Distanzsprechens“ ausgearbeitet. Ágel/Hennig (2006a: 13ff.) sehen bezüglich des Modells von Koch/Oesterreicher mehrere theoretische und methodische Probleme. ${ }^{158}$ Ein wichtiger Kritikpunkt ist, dass die Beziehungen zwischen den Kommunikationsbedingungen und den einzelnen sprachlichen Phänomenen nicht modelliert werden (2006a: 16). Bei der Weiterentwicklung des Koch/Oesterreicher'schen Konzepts werden im Rahmen einer hierarchischen Modellierung (Ágel/Hennig 2006a: 17-24) konkrete sprachliche Phänomene auf die Bedingungen der Nähe- bzw. Distanzkommunikation zurückgeführt. Im Folgenden soll mit Hilfe des Beispiels (89) aus einem Radio-Phone-In veranschaulicht werden, wie nähesprachliche Merkmale aus universal-pragmatischen Bedingungen des Nähesprechens abgeleitet werden. Der Beispielsatz enthält eine Ausklammerung (wegen der anschrift).

(89) LEG mal bitte auf wir rufen dich noch mal an wegen der anschrift. (Daniel Domian VII: Transkriptzeile 1293)

In der Hierarchie ist die oberste Ebene I das ,Universale Axiom“: Nähesprechen findet statt, wenn sich Produzent und Rezipient zur gleichen Zeit im gleichen Raum befinden. Aus dieser Grundkonstellation ergeben sich Kommunikationsbedingungen auf Ebene II, z.B. die Zeitgebundenheit der Produktion und der Rezeption. Diese Bedingung führt auf Ebene III zu einer spezifischen, spontanen Diskursgestaltung (d.h. die Zeitgleichheit von Planung und Produktion). Die spezifische Diskursgestaltung wird umgesetzt durch spezifische sprachliche Verfahren wie aggregative Strukturierung (Ebene IV). Diese kann sich einzelsprachlich materialisieren als Diskursmerkmale, in unserem Beispiel als aggregative Strukturen am Satzrand (Ebene V).

Der Vorteil dieses Modells besteht aus der Sicht der vorliegenden Arbeit v.a. darin, dass es die Einordnung von Diskursarten/Textsorten sowie einzelner Texte in das Nähe-DistanzKontinuum ermöglicht (Ágel/Hennig 2006b: 33). Bei der Operationalisierung werden die Ergebnisse der Analyse nähersprachlicher Merkmale des einzuordnenden Textes in Beziehung gesetzt mit denen eines Proto-Nähetextes (des oben erwähnten Radio-Phone-Ins Daniel Domian VII), der einen Nähewert von 100\% hat. Dadurch kann die prozentuale Nähesprachlichkeit des einzuordenden Textes ermittelt werden. Somit kann sichergestellt werden, dass in sprachgeschichtlichen Untersuchungen Texte mit prozentual ähnlichem Nähebzw. Distanzwert verglichen werden.

158 Die Modelle von Koch/Oesterreicher (1985), Ágel/Hennig (2006a) sowie weitere Ansätze zur Erfassung des Spannungsfelds zwischen Mündlichkeit und Schriftlichkeit können an dieser Stelle nicht ausführlich diskutiert werden, Näheres dazu s. Ágel/Hennig (2006a). 
Mit diesem Modell wurden historische Quellentexte zwischen Nähe und Distanz verortet (vgl. die Analysen im Sammelband von Ágel/Hennig 2006). Die Analyse des Quellentextes Güntzer I ergab z.B. einen Wert von 38,6\% Nähesprachlichkeit (vgl. Kappel 2006: 120). Obwohl dieser Text einen weit höheren Grad an Nähesprachlichkeit aufweist als die Mehrheit der medial schriftlichen Texte, ist seine Nähesprachlichkeit nur eine relative (Ágel/Hennig 2006b: 46). ${ }^{159}$ Ein historisches Nähekorpus kann jedoch nur aus solchen relativ nähesprachlichen Quellentexten zusammengestellt werden, daher wird der Begriff ,nähesprachlich` bzw. ,Nähetext` im folgenden Sinne verwendet: „Ein historischer Text kann dann als ,nähesprachlich` bezeichnet werden, wenn er ungefähr die für seine Zeit überlieferte maximale Nähesprachlichkeit aufweist.“ (vgl. Ágel/Hennig 2006b: 46f.) Als ,nähesprachlich“ in obigen Sinne „haben sich insbesondere Privatbriefe, Tagebücher und Lebensberichte einfacher Leute erwiesen“ (Hennig 2009: 102), daher werden bei der Zusammenstellung des Nähekorpus diese Textsorten berücksichtigt (vgl. Kapitel 4.1).

\subsubsection{Mündlichkeit/Schriftlichkeit aus sprachgeschichtlicher Sicht}

Zur kommunikativen und wissenschaftshistorischen Relevanz der Schriftsprache sei auf zwei Aspekte verwiesen. $\mathrm{Zu}$ Beginn der neuhochdeutschen Periode war die Schreib-/Lesekommunikation auf eine kleine Minderheit beschränkt, die Kommunikation vollzog sich größtenteils medial mündlich. In der deutschen Sprachgeschichtsschreibung ist jedoch eine spezifische Orientierung zu beobachten: Sie ist nach Ágel (2000: 1856) „fixiert auf die Herausbildung der Schrift- und Standardsprache“. In den letzten Jahrzehnten ist allerdings eine Neuorientierung in der Sprachgeschichtsschreibung zu beobachten. Nach von Polenz (1995: 44) ist eine „orale Alltags-Sprachgeschichte [...] wünschbar als Ergänzung zur hochkulturellen Sprachgeschichte [...], aber mangels Quellen vorerst kaum realisierbar.“ Auch Elspaß (2007b: 42ff.) verweist darauf, dass bisher v.a. Texte von Literaten, Gelehrten und Politikern den Gegenstand sprachgeschichtlicher Untersuchungen bildeten, obwohl diese hochkulturellen Texte von professionellen Schreibern nur einen kleinen Ausschnitt der Sprachwirklichkeit darstellen. Er plädiert für eine Berücksichtigung von Briefen und Tagebüchern von Menschen aus niedrigeren Bevölkerungsschichten als Quellen der Alltagssprache in der jüngeren Sprachgeschichte. In Elspaß (2005) werden 648 Privatbriefe

159 Bei Güntzer I liegt parallel ein Wert von 61,4\% Distanzsprachlichkeit vor, der Text ist also nur relativ nähesprachlich. Bei der Zusammenstellung des Korpus konnten natürlich keine Texte mit dem gleichen Nähewert ausgewählt werden. Die Prozentzahlen sollen also nicht den Eindruck erwecken, dass eine mathematische Genauigkeit in diesem Sinne angestrebt wurde (vgl. Ágel/Hennig 2006b: 35). 
aus der Auswandererkorrespondenz des 19. Jh. ausgewertet. Diese Texte gehören „zu den ,nähesprachlichsten` Textzeugen, die uns überliefert sind.“ (Elspaß 2007b: 42)

Die Berücksichtigung der medialen sowie konzeptionellen Mündlichkeit/Schriftlichkeit bei der Integration von Adverbialsätzen ist deshalb wichtig, weil diese Faktoren eine wichtige Rolle bei der Entstehung, Verbreitung und Auswahl gewisser syntaktischer Strukturen spielen (vgl. auch Kapitel 2.1.2). Einige der wichtigen grammatischen Veränderungen der Neuzeit dürften eng mit der Literalisierung ${ }^{160}$ der Sprachbevölkerung zusammenhängen (vgl. Ágel 2015). Veränderungen in der Denkweise und im Sprachbewusstsein der Sprachbenutzer dürften auch auf den Bereich der Satzverknüpfung einen Einfluss haben (vgl. Ágel 2007: 51f., Lötscher 1995: 54ff.). Veränderungen der Satzverknüpfung sind folglich vom Aspekt der Mündlichkeit/Schriftlichkeit nicht $\mathrm{zu}$ trennen. Man sollte vielmehr versuchen, die Beschreibung der Systemgeschichte für diesen Aspekt sensibel zu machen. Genau dies, d. h. die Einbeziehung der Unterscheidung zwischen konzeptioneller Mündlichkeit und Schriftlichkeit bei der Beschreibung der Satzverknüpfung im Neuhochdeutschen, wird in der vorliegenden Arbeit geleistet.

Im Neuhochdeutschen finden nicht nur eine Alphabetisierung und Verbreitung der Schriftlichkeit, sondern auch eine mediale Umschichtung statt, also ,eine Entwicklung aus der nicht nur medialen, sondern auch konzeptionellen Mündlichkeit heraus in die konzeptionelle Schriftlichkeit als sprachkulturelles Orientierungszentrum hinein“ (Reichmann 2003: 42). Während die Zeit vor dem 16. Jh. v.a. mit den Stichwörtern ,Polyzentrik‘, ,Horizontalität‘, ,Mündlichkeit des Sprachhandels` zu beschreiben ist, zieht die Entstehung einer Leitvarietät und die zunehmende Orientierung an ihr eine Umschichtung des ganzen Varietätenspektrums nach sich. ${ }^{161}$ Nach der Vertikalisierungstheorie von Reichmann (2003: 30, vgl. auch 1988: 174) kann von einer „fundamentalen Umorientierung des Varietätenspektrums des Deutschen zwischen dem späten 15. und dem 18. Jahrhundert.“ ausgegangen werden. An dieser Stelle kann nicht auf alle Aspekte der Vertikalisierung eingegangen werden (vgl. dazu Reichmann 2000: 38ff.), es sollen jedoch zwei Aspekte hervorgehoben werden. Einerseits wird die Variantenfülle und -toleranz des Frühneuhochdeutschen reduziert. Dies führt zu einer Variantenaussonderung, bei der die folgenden Dichotomien relevant sind: regional vs. überregional, sprech- vs. schreibsprachlich, emotional vs. rational, sprachökonomisch vs.

\footnotetext{
160 Das Neuhochdeutsche zeichnet sich durch eine zunehmende Alphabetisierung breiter Bevölkerungsschichten aus, die ihren Höhepunkt im 19. Jh. erreicht: Während zu Beginn des 19. Jhs. „,noch weniger als die Hälfte der Bevölkerung lesen und schreiben konnte“, kann man zu Beginn des 20. Jhs. bereits von „eine[r] fast vollständige[n] Alphabetisierung“ ausgehen (Hennig 2009: 43, vgl. auch Elspaß 2005: 76ff.).

161 Die entsprechenden Begriffe für die Zeit nach dem 16./17. Jh. sind ,monozentrisch`, ,vertikal‘ und ,Schriftlichkeit‘.
} 
explizit. D.h., dass im neu entstandenen Varietätenspektrum den Varianten, die z.B. raumgebunden sind bzw. der konzeptionellen Mündlichkeit näher stehen, von den Sprechern ein negativer Wert zugesprochen wird, während überregionale Varianten der konzeptionellen Schriftlichkeit als positiv beurteilt werden. Andererseits hat die Vertikalisierung auch sprachstrukturelle (z.B. syntaktische) Konsequenzen (vgl. Reichmann 2003: 46f.), z.B. im Bereich der Regelung der Wortstellung.

An dieser Stelle soll auf die Unterscheidung von ,Verschriftung' und ,Verschriftlichung eingegangen werden: Während ,Verschriftung' für die „mediale Umsetzung vom phonischen ins graphische Medium“ steht, sind unter ,Verschriftlichung“ „konzeptionelle Verschiebungen in Richtung Schriftlichkeit“ (Koch/Oesterreicher 1994: 587) bzw. - wie Hennig (2009: 9) formuliert - „die Anpassung sprachlicher Muster an die Bedingungen des Distanzsprechens“ zu verstehen. Nach Ágel/Hennig (2006a: 29) ändert sich das Verhältnis zwischen Aggregation und Integration im Laufe der Sprachgeschichte wegen der Verschriftlichung: Aggregationsmerkmale werden durch Integrationsmerkmale ersetzt oder die beiden existieren im Sinne einer vertikalen Umschichtung nebeneinander bzw. über-/untereinander (s. auch Ágel 2007: 51). Auch Hennig (2009: 37) vertritt die These, „dass die einschlägige historische Bedingung, die zum Abbau der historischen Nähemerkmale führt, die Verschriftlichung ist.“ In Czicza/Hennig (2013: 8) werden für mehrere grammatische Phänomene eine „Entwicklung $\mathrm{zu}$ mehr Integration und weniger Aggregation“ nachgewiesen und der AggregationIntegration-Parameter als ,übergeordnetes Erklärungsprinzip“ bezeichnet. Sie formulieren folgende These:

Verschriftlichung begünstigt den Aufbau von integrativen syntaktischen Mustern, die Ausdruck von Anpassungsvorgängen an die Distanzbedingungen sind. Aufgrund der soziopragmatischen Voraussetzungen im Zeitraum des Neuhochdeutschen und aufgrund der Vertikalisierungstendenzen kann damit gerechnet werden, dass zunächst auf die Distanzbedingungen reagierende Tendenzen zur Bevorzugung integrativer Muster auch in den Nähebereich vordringen und funktionsäquivalente aggregative Strukturen verdrängen. Wenn es dann im Zuge einer Reorganisation des Nähebereichs zur Aufhebung der Varietätenmarkierung kommt, kann in einem allgemeinen Sinne von einem Wandel von Aggregation zu Integration gesprochen werden. (Czicza/Hennig 2013: 13)

Bezüglich des zu untersuchenden Phänomens ist die Variation der Stellungstypen folgenderweise zu beschreiben. Der resumptive Typ ist als nähesprachliches Merkmal zu deuten und ist aggregativ (s. Ágel/Hennig 2006b: 41, 47f., 2006c: 393), vgl. die folgende Begriffsbestimmung vom „Korrelat als Aggregationsindikator“:

Wenn (bei vorangestelltem Nebensatz) die Integration des Nebensatzes in den Hauptsatz zusätzlich durch ein Korrelat angezeigt wird, obwohl die Integration des Nebensatzes voll grammatikalisiert ist, dann betrachten wir das (...) als ein Zeichen aggregativer Diskursgestaltung. (Ágel/Hennig 2006c: 393) 
Im Sinne der oben angerissenen Konzepte der strukturellen Vertikalisierung und der Verschriftlichung ist das folgende Szenario anzunehmen (vgl. Czicza/Hennig 2013): Die beiden Varianten ,integrativ` und ,resumptiv‘ existieren zu Beginn des Neuhochdeutschen im Sinne einer vertikalen Umschichtung nebeneinander bzw. über-/untereinander, der resumptive Typ wird dann auch in Nähetexten zugunsten des integrativen Typs tendenziell abgebaut. Hennig (2009: 110f.) formuliert folgende Hypothese:

Die Herausbildung des Integrationsprinzips wird durch die Verschriftlichung begünstigt. Von der Verschriftlichung stark betroffene Texte folgen dem Integrationsprinzip in höherem Maße als von der Verschriftlichung kaum betroffene Texte. Deshalb ist zu Beginn des Neuhochdeutschen noch mit einem Nebeneinander von dem Integrationsprinzip folgenden und von diesem Prinzip abweichenden Texten zu rechnen. Im Zuge der Verschriftlichung werden Unterschiede zwischen Nähe und Distanz eingeebnet, das Integrationsprinzip dringt in den Nähebereich vor und führt zu einer Reorganisation des Nähebereichs.

Daher ist eine Analyse der Integration vorangestellter Adverbialsätze, die das Spannungsfeld zwischen Mündlichkeit und Schriftlichkeit berücksichtigt, wünschenswert. 


\section{Zur Methode}

Die Untersuchung des Integrationsgrades vorangestellter Adverbialsätze erstreckt sich auf die neuhochdeutsche Epoche, also auf den Zeitraum zwischen 1650-2000. ${ }^{162}$ Zunächst wird auf einige Überlegungen hinsichtlich der Korpuszusammenstellung bzw. die Wahl und die Charakterisierung der Quellentexte eingegangen (Kapitel 4.1).

\subsection{Grundlagen der Korpusanalyse}

Es kann kein Ziel der vorliegenden Arbeit sein, ein repräsentatives ${ }^{163}$ Korpus zum Neuhochdeutschen zusammenzustellen. ${ }^{164}$ Dabei wird bewusst in Kauf genommen, dass die Daten über die untersuchte Datenmenge hinaus nicht ohne Weiteres generalisierbar sind. Da das Neuhochdeutsche nicht als eine einheitliche Varietät, sondern als eine variationsreiche Sprachperiode aufzufassen ist (vgl. Spiekermann 2010: 345, Sinner 2014: 25), müsste ein repräsentatives Korpus des Neuhochdeutschen möglichst die gesamte (mediale und konzeptionelle) Schriftlichkeit und die überlieferte konzeptionelle Mündlichkeit gewichtet, nach allen Regionen, Textsorten usw. erfassen. Die Zusammenstellung eines ausgewogenen Korpus des Neuhochdeutschen im Sinne von Lemnitzer/Zinsmeister (2010: 52) kann in der vorliegenden Arbeit nicht mal ansatzweise geleistet werden. Damit sieht man sich mit der Schwierigkeit konfrontiert, wie man ein Korpus zusammenstellen könnte, das trotzdem erlaubt, relevante Schlussfolgerungen zu ziehen. Als Ausweg sehe ich ein Korpusdesign, bei dem die Möglichkeit des Einflusses der sprachlichen Variation (so etwa die Diatopik) auf die quantitativen Daten reduziert wird und großer Wert auf die Vergleichbarkeit der Korpustexte

${ }^{162}$ Ich verwende diese zeitliche Bestimmung des Neuhochdeutschen in Anlehnung an Ágel/Hennig (2007: 185f.). Die Frage der Periodisierung der deutschen Sprachgeschichte wurde bis heute nicht ein für alle mal gelöst, es liegen unterschiedliche Periodisierungsversuche vor, die z.T. auch die Abgrenzung des Neuhochdeutschen betreffen (zum Überblick s. Roelcke 1995). In einem jüngeren Beitrag argumentiert Elspaß (2008) z.B. für eine Unterscheidung des Mittelneuhochdeutschen (1650 bis ca. 1950) und des Gegenwartsdeutschen (von 1950 an). Da die vorliegende Arbeit eng ins Projekt ,Sprachstufengrammatik des Neuhochdeutschen' eingebunden ist (vgl. dazu Kapitel 1.2), bleibe ich bei der obigen Periodisierung, in der kein Einschnitt um 1950 angenommen wird.

163 Es handelt sich um die „Frage, inwieweit man Erkenntnisse, die man durch die Analyse von Korpusdaten gewonnen hat, auf den Sprachausschnitt, den das Korpus präsentieren soll, übertragen kann.“" (Lemnitzer/Zinsmeister 2010: 50) Historisch könnte bei einem Korpus einer Sprachperiode „nur in einem eingeschränkten Sinne von Repräsentativität“ die Rede sein, denn die Grundgesamtheit kann nur auf das Überlieferte und Zugängliche bezogen werden, nicht auf die Sprachperiode insgesamt (Wegera/Waldenberger 2012: 15).

164 Die Deutsche Forschungsgemeinschaft (DFG) hat im Dezember 2015 einen Antrag zum Thema „Syntaktische Grundstrukturen des Neuhochdeutschen. Zur grammatischen Fundierung eines Referenzkorpus Neuhochdeutsch“ bewilligt, die Ergebnisse dieses Projekts können jedoch in der vorliegenden Arbeit nicht mehr berücksichtigt werden. 
gelegt wird. Individuelle oder textsortentypische Unterschiede können zwar nicht ausgeschlossen werden, ${ }^{165}$ aber weitere Dimensionen der sprachlichen Variation werden möglichst konstant gehalten (s. weiter unten).

Als empirische Grundlage dienen 8 Quellentexte, die zwischen 1650 und 2000 entstanden sind. Das Korpus wird nach zwei Leitprinzipien zusammengestellt. Das erste Prinzip ist: Es soll dem historischen Aspekt Rechnung tragen in dem Sinne, dass es aus vergleichbaren Texten besteht, deren Entstehungszeit als Variable unterschiedlich ist. Dadurch wird die diachrone Analyse (d.h. der Vergleich von mehreren synchronen Schnitten) ermöglicht. Aus jedem Jahrhundert der neuhochdeutschen Epoche sollen Texte genommen werden. Das zweite Leitprinzip der Korpuszusammenstellung ist die Unterscheidung zwischen konzeptionell mündlichen und konzeptionell schriftlichen Texten. Dadurch sollte überprüft werden, ob konzeptionell bedingte Unterschiede beim Integrationsgrad vorangestellter Adverbialsätze nachgewiesen werden können. Das Korpus besteht aus vier Zeitabschnitten, jedem Jahrhundert ${ }^{166}$ sind zwei Texte (ein Nähe- und ein Distanztext) zugeordnet. Tabelle 1 stellt die Zusammensetzung der Teilkorpora vor. ${ }^{167}$

\begin{tabular}{|l|l|l|}
\hline & Nähe & Distanz \\
\hline $1650-1700$ & Güntzer I & Harsdörffer I \\
\hline $1700-1800$ & Schuhmacher Chronik II & Aichinger III \\
\hline $1850-1900$ & Auswandererbriefe V & Burckhardt V \\
\hline $1950-2000$ & Jugendtagebuch VII & Lorenz VII \\
\hline
\end{tabular}

Tabelle 1: Zusammensetzung der Teilkorpora

165 Als Beispiel für die Relevanz individueller Variation zu Beginn der neuhochdeutschen Zeit sei auf Lefèvres (2012: 491, 505) Untersuchung von Prosaschriften verwiesen, die gezeigt hat, dass es beim Gebrauch der unterschiedlichen Varianten von so durchaus bedeutende Häufigkeitsunterschiede gibt, ,jedes Werk fungiert somit als eigenes kleines System mit einem eigenen kontrastiven Verhältnis zwischen den unterschiedlichen Funktionen von ,so ““

166 In Kappel (2008: 42) hatte ich mir zum Ziel gesetzt, Texte den folgenden Zeitabschnitten zu entnehmen, damit zwischen der Entstehungszeit der jeweiligen Texte jeweils ca. 100 Jahre liegen: 1650-1700, 1750-1800, 1850-1900 bzw. 19502000. Für den Zeitabschnitt 1750-1800 konnte jedoch kein Nähetext gefunden werden, der alle Auswahlkriterien erfüllt hätte. Z.B. wäre der im Kapitel 3.1.3 erwähnte Nähetext Wagner III (= das Sturm-und-Drang-Drama Die Kindermörderinn) von der Herkunftsregion des Autors her geeignet, er ist jedoch kein unveröffentlichter Privattext eines ,unroutinierten' Schreibers (zur exemplarischen Analyse nähesprachlicher Merkmale in Wagner III s. Hennig 2007: 24ff.). Aus diesem Grund ist die Wahl auf den Nähetext Schuhmacher Chronik II gefallen, der bis auf seine Abfassungszeit (1712-1738) auf unsere Anforderungen zugeschnitten ist.

167 Die bibliographischen Daten zu den Quellentexten sind im Literaturverzeichnis zu finden. Die römischen Ziffern beziehen sich auf die Zeitabschnitte á 50 Jahre im Neuhochdeutschen, z.B. I = 1650-1700. Auf eine ausführliche Beschreibung der einzelnen Korpustexte wird verzichtet. Die Distanztexte sind bekannte wissenschaftliche Texte berühmter Autoren, zu den Nähetexten s. Kappel (2006: 104), Kronberger (2008), Hennig (2010b: 947f.), Elspaß (2005) und (2010: 72ff.). An dieser Stelle sei gedankt dem Stadtarchiv Reutlingen für die Bereitstellung des unveröffentlichten Transkripts zu Schuhmacher Chronik II, Stephan Elspaß für die Bereitstellung seines Korpus von Auswandererbriefen und dem Deutschen Tagebucharchiv Emmendingen, in dem ich den letzten, dem Korpusdesign passenden unveröffentlichten Privattext aus dem 20. Jh. finden konnte. 
Der Gesamtumfang beträgt ca. 100.000 Wortformen (ca. 12.000 Wortformen pro Korpustext). Aus dem oben geschilderten Korpusdesign und dem Umfang ergibt sich die Einsicht, dass mit den Ergebnissen der Analyse vorsichtig umgegangen werden muss.

Bei der variationslinguistisch sensiblen Korpuszusammenstellung setzt das zweite Leitprinzip einen entsprechenden theoretischen Rahmen und die Möglichkeit seiner praktischen Umsetzung voraus (vgl. Kapitel 3.5.1). Dabei stütze ich mich v.a. auf das NäheDistanz-Modell von Ágel/Hennig (2006a und 2006b). Die Nähetexte aus dem 17., 18. und 19. Jh. wurden bereits in der Fachliteratur als nähesprachlich ausgewiesen. Die Analyse des Quellentextes Güntzer I ergab einen Wert von 38,6\% Nähesprachlichkeit (vgl. Kappel 2006: 120). Bei Schuhmacher Chronik II wurden die Angaben zur Nähesprachlichkeit auf Mikroebene (Hennig 2010a: 947f.) mit einem selbst ermittelten Wert der Nähesprachlichkeit auf Makroebene ergänzt, die Nähesprachlichkeit des Textes beträgt somit insg. 30\%. Die Analyse von Auswandererbriefen unroutinierter Schreiber ergibt einen Durchschnittswert von 34\% (Elspaß 2010: 72ff.). Auch das Jugendtagebuch VII aus dem 20. Jh. konnte mit Hilfe des Nähe-Distanz-Modells als nähesprachlich verortet werden, er hat sogar den höchsten Nähewert (37,8\%). Die Arbeit mit bekannten wissenschaftlichen Texten berühmter Autoren, die prototypisch für Distanzsprache sind, hat gezeigt, dass auch diese einen geringen Nähewert haben können (z.B. 4,1\% Nähesprachlichkeit, d.h. 95,9\% Distanzsprachlichkeit für Die Geburt der Tragödie von Nietzsche, vgl. dazu Hennig 2010a: 81), dieser Wert jedoch deutlich niedriger ist als bei den herangezogenen Nähetexten. Die genauen Werte meiner Distanztexte sind für die Zielsetzung der Arbeit m.E. nicht relevant, so dass auf eine entsprechende Analyse verzichtet werden kann.

In der vorliegenden Arbeit wird der Integrationsgrad vorangestellter Adverbialsätze nicht auf ihre eventuelle regionale Variation geprüft. Da jedoch bei der Verbreitung syntaktischer Varianten und auch beim Bestand der Subjunktoren regionale Unterschiede nachgewiesen wurden (vgl. Kapitel 2.2.1 bzw. 3.4.5.3) bzw. weil der eventuelle Einfluss der regionalen Dimension beim Integrationsgrad vorangestellter Adverbialsätze kontrovers beurteilt wird (vgl. Kapitel 2.2.2), ist bei der Textauswahl darauf $\mathrm{zu}$ achten, dass der Einfluss der diatopischen Variation bei der Wahl zwischen den Stellungstypen (und somit auch die Gefahr einer falschen Deutung regionaler Variation als historischer Entwicklung) möglichst reduziert werden sollte. Daher wurde die Entscheidung getroffen, bei der Textauswahl nur oberdeutsche Quellentexte zu berücksichtigen. Die nähesprachlichen Quellentexte weisen einen höheren Grad von Dialektalität auf (vgl. Kappel 2007 sowie Elspaß 2010 zum Zusammenhang von Dialektalität und Nähesprachlichkeit). Daher wäre es sicherlich 
wünschenswert gewesen, die Quellentexte aus einer eingeschränkten Region zu wählen. Dies ist jedoch aufgrund der Quellenlage v.a. bei den Nähetexten nicht realisierbar. Obwohl sie alle dem Hauptdialektgebiet des Alemannischen bzw. der Großregion ,westoberdeutsch“ entnommen wurden, sind sie dialektal nicht völlig homogen. ${ }^{168}$ Mit dieser Methode kann also der Einfluss der diatopischen Variation bei der Wahl zwischen den Stellungstypen zwar nicht völlig ausgeschlossen aber deutlich reduziert werden. Die Erfassung der weiteren sechs Großregionen, die nach Elspaß (2010: 81) bei einem Nähekorpus zu berücksichtigen wären, war aufgrund der Quellenlage nicht möglich.

Die untersuchten Text(ausschnitte) gehören unterschiedlichen Textsorten an. Bei den distanzsprachlichen Texten wurde Fachprosa herangezogen. Es handelt sich um gedruckte Texte, die in der zeitgenössischen Rezeption hohe Beachtung fanden. Die Nähetexte stammen aus dem Bereich der privaten Schriftlichkeit (Autobiografie, Chronik, Privatbrief, Tagebuch). Bei den AutorInnen kann aufgrund ihrer Berufe die Beherrschung elaborierter Sprachstile nicht vorausgesetzt werden. Sie sind im Vergleich zu den Autoren der entsprechenden Distanztexte eher als „,ungeübte٪,unroutinierte“ Schreibende“ (Elspaß 2005: 46) zu charakterisieren. Diese textuellen Unterschiede müssen zur Kenntnis genommen werden, zumal ein nach allen möglichen Faktoren einheitliches Korpus von Nähe- und Distanztexten derzeit nicht realisierbar ist. Natürlich unterscheiden sich die Texte auch dahingehend, ob sie eher narrativ oder argumentativ sind, was eine Auswirkung auf die Frequenz der verwendeten Untertypen von Adverbialsätzen haben könnte. Bei eher narrativen Texten ist etwa ein höherer Anteil von Temporalsätzen anzunehmen, bei argumentativen Texten könnte erwartungsgemäß der Anteil von Kausalsätzen höher sein. In der Fachliteratur wurden bereits Affinitäten von Inhaltsrelationen zur Nähe-Distanz-Dimension nachgewiesen. Für die Wissenschaftskommunikation, die prototypisch für Distanzsprachlichkeit ist, ermitteln Czicza et al. (2012: 22ff.) einen hohen Anteil finaler, konsekutiver und weiterer Inhaltsrelationen, die sie ,Relationen der Wissenschaftssprachlichkeit‘ nennen. In den von Ágel (2012: 201) analysierten Nähetexten überwiegen andere Relationen wie temporal. Welche Textsortenunterschiede es diesbezüglich gibt, bildet den Gegenstand weiterer Forschungen. ${ }^{169}$ Czicza et al. (2012: 41) betonen, „dass der Einfluss der jeweiligen Textsorte auf die sprachliche Gestaltung nicht unterschätzt werden darf.“

168 Vgl. die Angaben zur Herkunft der Verfasser der Nähetexte: Reutlingen (Schuhmacher Chronik II) sowie Nürtingen bei Stuttgart (Jugendtagebuch VII) vs. Elsass (Güntzer I). Der Korpustext Auswandererbriefe V enthält 21 Briefe von 10 Schreibenden mit Volksschulausbildung, die westoberdeutsche Herkunft haben.

169 Dass es wohl solche Textsorten- und Texttypenunterschiede gibt, zeigt der nicht-narrative, keinesfalls klar erzählende Nähetext Wagner III: In diesem Drama kommen Konditionalsätze deutlich häufiger als Temporalsätze vor, während dies in den erzählenden Nähetexten gerade umgekehrt ist. 


\subsection{Methodologische Überlegungen}

Die Tendenz zur zunehmenden Integration ist bei den semantischen Subtypen von Adverbialsätzen unterschiedlich ausgeprägt und zwischen ihnen können Unterschiede hinsichtlich des Integrationsgrades beobachtet werden. ${ }^{170}$ Zudem ist die Frequenz der einzelnen semantischen Typen in den Teilkorpora unterschiedlich. Die Unterschiede in der Frequenz einzelner Adverbialsatztypen sind bei der quantitativen Analyse besonders zu berücksichtigen, da es vermieden werden sollte, dass Frequenzunterschiede bei den semantischen Typen fälschlicherweise als Unterschiede im Integrationsgrad oder als NäheDistanz-Unterschiede interpretiert werden. Aus diesen Gründen darf keine undifferenzierte Analyse im Hinblick auf die semantischen Klassen von Adverbialsätzen durchgeführt werden. Semantische Untertypen werden bei der quantitativen Untersuchung zunächst separat behandelt, auch wenn dieses Vorgehen bei einzelnen semantischen Typen zu geringen Belegzahlen führt. Im zweiten Schritt (s. Kapitel 5.6) werden die Nachteile dieses Vorgehens dadurch kompensiert, dass bestimmte Satztypen zusammengezogen ausgewertet werden.

Bei der Frage, welche Schlussfolgerungen meine Untersuchungsergebnisse ermöglichen, wird der sog. Chi-Quadrat-Test angewendet, mit dem die statistische Signifikanz der Untersuchungsergebnisse zu berechnen ist. ${ }^{171} \mathrm{Da}$ bei manchen Untertypen die Anzahl der einschlägigen Belege so gering ist, dass die erfolgreiche Durchführung des Tests gar nicht möglich ist (z.B. bei adversativen Adverbialsätzen), scheint die Zusammenführung von einzelnen Typen bei der Diskussion der Ergebnisse (Kapitel 5.6) sinnvoll. An dieser Stelle wird diskutiert, ob und in welchen Fällen bestimmte Satztypen - getrennt oder zusammengezogen - mit dem Chi-Quadrat-Test auszuwerten sind. Zur Berechnung der $\begin{array}{llll}\text { Signifikanzwerte } \quad \text { wird } & \text { Online-Kalkulatorprogramm }\end{array}$ (http://www.socscistatistics.com/tests/chisquare2/Default2.aspx) verwendet. Bei der Bestimmung der statistischen Signifikanz der Ergebnisse werden nur Stellungsvarianten berücksichtigt, die beim jeweiligen Satztyp wählbar sind (vgl. Kapitel 2.1.1). Z.B. wird im Falle der Temporalsätze, bei denen nur der integrative und der resumptive Typ zur Verfügung

\footnotetext{
${ }^{170}$ Wie weiter oben bereits erwähnt (vgl. Kapitel 2.1.1 bzw. 3.4), gibt es bekanntlich wesentliche Unterschiede bei der Integration von verschiedenen Adverbialsatztypen. Temporalsätze, die nach Axel (2002: 15) früher vorfeldfähig wurden als z.B. Kausal- oder Konditionalsätze, werden bereits in frühneuhochdeutschen Quellentexten mit einiger Häufigkeit integrativ angebunden. In einem Korpus von Prosatexten aus der zweiten Hälfte des 17. Jhs. findet Wiktorowitz (2013: 394f.) „fast ausschließlich“ integrativ angebundene Temporalsätze, während der Anteil dieses Stellungstyps z.B. bei Konditional- oder Konzessivsätzen deutlich niedriger ist.

171 Je niedriger der sog. P-Wert (Signifikanzwert) ist, desto stärker ist der Zusammenhang zwischen den untersuchten Variablen. Unter einem P-Wert von 0,05 (= einer Wahrscheinlichkeit von weniger als 5\%) gilt das Ergebnis als statistisch signifikant. Wenn die Ergebnisse nicht-signifikant (nach dem Chi-Quadrat-Test) sind, bedeutet das jedoch nicht, dass die gewonnenen Daten keine Aussagekraft haben, sondern dass zur Absicherung weitere Analysen nötig wären.
} 
steht, die nicht-integrative Variante, deren Wahl nicht möglich ist, bei der Berechnung nicht berücksichtigt, weil vom Kalkulatorprogramm der Wert 0 nicht akzeptiert wird. ${ }^{172} \mathrm{Im}$ Folgenden sollen methodologische Überlegungen zur Identifizierung und Einordnung der einschlägigen Belege aufgeführt werden.

Im Kapitel 3.1.1 wurde das Kriterium der Rekonstruierbarkeit bei der Satzdefinition erläutert. Dabei gilt, dass Adverbialsätze ohne satzwertigen übergeordneten Satz in der vorliegenden Arbeit nur unter der Bedingung behandelt werden, dass das aus dem Nachsatz fehlende Finitum bzw. dessen unter strukturellen und kontextuellen Gesichtspunkten notwendige Komplemente eindeutig rekonstruierbar sind (vgl. Kapitel 3.1.1). In diesem Sinne liegt im folgenden Beleg trotz des Fehlens des Finitums ein satzwertiger übergeordneter Satz vor.

(90) ich mußte nehmen was ich bekomen konnte, und nicht was ich hätte sollen haben und wie bälder dieselben verkauft wurden desto besser indem sie // täglich ī Werthe fielen, .... (Auswandererbriefe V: 18)

Der Ausdruck desto besser ist als elliptische Struktur aufzufassen. Da in diesem Fall die nicht realisierten Stellungseinheiten (Kopulaverb und Pronomen) rekonstruierbar sind (desto besser war es), wird der obige Beleg in der Untersuchung berücksichtigt. Dieses Kriterium führt jedoch nicht in jedem Fall zu einer eindeutigen Abgrenzung. In solchen Fällen sollten Vergleichsbelege aus dem Korpusvolltext herangezogen werden. Im folgenden Beispiel aus Augustin Güntzers Autobiografie (= Korpustext Güntzer I) wurde das Vollverb kommt erst in der Edition von Fabian Brändle und seinen Mitarbeitern eingetragen. Dieser Eingriff wurde in der Edition durch eckige Klammern markiert. ${ }^{173}$

(91) So mihr einer auß dißem Landt zuhanden [kommt], so wil ich solche Gudtdaten widerunb vergelten und wettmachen. (Güntzer I: 92v-93r)

Da sich in der Autobiografie auch ein vergleichbarer, nicht-rekonstruierter Beleg findet, der die obige Interpretation unterstützt, scheint die Rekonstruktion des Finitums angemessen zu sein:

(92) Will es widerunb wett machen, so mihr ein Bomerr zu Handten kompt. (Güntzer I: Eingeklebt zwischen $89 \mathrm{v}$ und 90r)

\footnotetext{
${ }^{172}$ Wenn ein Stellungstyp wählbar aber in einem Korpustext nicht belegt ist, wird der Wert 0 durch 1 ersetzt.

173 Vgl. die Anmerkungen von Brändle und Sieber zu den Editionsregeln (2002: 72).
} 
Historische Nähetexte enthalten weitere Problemfälle, die nur mit einer Präzisierung der im Kapitel 3.1.1 angeführten Kriterien zu lösen sind. Im Gegensatz zu (91) ist die Interpretation des folgenden Belegs nicht mehr so eindeutig:

(93) Nachdem der große Grieg in Fryol zwisten den Venetige[r]n und Firsten von Gretz [vorbei war?], zog ich durch das Grener Landt in Italiam. (Güntzer I: 54r)

Auch an dieser Stelle wurde der Ausdruck in den eckigen Klammern erst bei der Anfertigung der Edition eingetragen. Der Subjunktor und das Subjekt sind zwar vorhanden, das Subjekt hätte jedoch durchaus auch mit einem anderen Prädikat verknüpft werden können, was durch das Fragezeichen in der Edition angedeutet wird. Des Weiteren scheint es ebenfalls möglich zu sein, dass durch die erweiterte Attribuierung des Substantivs Grieg eine Art Planungsschwierigkeit entstand. Auch der Umstand, dass Nachdem der große am Anfang einer neuen Seite im Original von der deutschen Kurrentschrift abgehoben wurde, ${ }^{174}$ könnte eventuell dazu beigetragen haben, dass der mit dem Subjunktor nachdem eingeleitete Nebensatz nicht mit dem zu erwartenden Prädikat am Ende des Nebensatzes abgeschlossen wird, also die mit nachdem aufgebaute Projektion nicht eingelöst wird. Nach den Präpositionalattributen zu Grieg wird der Satz so weitergeführt, als stünde nicht der Subjunktor nachdem, sondern eine Präpositionalgruppe (vgl. Nach dem großen Krieg) am Satzanfang. Bei solchen Problemfällen wird das zweite Satzkriterium angewendet, das Hennig (2006: 178) für Einheitentypen der gesprochenen Sprache erarbeitet hat: Sie betrachtet Sätze als syntaktische Einheiten, in denen die Realisierung des Verbs und der seiner Valenzpotenz entsprechenden Glieder „in kontinuierlicher Weise erfolgt, d.h., wenn keine Planungsschwierigkeiten an der Satzoberfläche zu erkennen sind.“ Der erste Teil dieses Belegs soll wegen der oben erläuterten Störung in der Projektionsstruktur nicht als ,Satz analysiert werden.

Es werden nur Adverbialsätze berücksichtigt, die ihrem Obersatz vorangestellt sind, Belege mit Zwischenstellung des Adverbialsatzes sind für die vorliegende Arbeit nicht relevant. Im folgenden Beleg wird der sobald-Satz in ihren Obersatz daß sie ... wieder in die sclaverei versetzt würden parenthetisch eingeschoben.

(94) Diese Partei nun wurde von den nördlichen Repuplikanern zu allen ihnen nützlichen Zwecken Mißbraucht würden indem sie ihnen vormahlten daß sie ihre Befreier aus der Sclaverei seien und daß sie sobald die Democraten an die Regierung kämen, wieder in die sclaverei versetzt würden, was allerdings sehr abgeschmackt ist, aber dennoch

${ }^{74}$ Solche Textstellen markierte Güntzer durch lateinische Kursiva, sie sind auch in der Edition kursiv gesetzt. Güntzer mischt jedoch die lateinischen Kursiva häufig mit der deutschen Kurrentschrift, vgl. die Anmerkungen von Brändle und Sieber zu den Editionsregeln. 
seine Wirkung nicht verfehlte indem die meisten serh sehr unwissend sind in und mit recht eine große Angst vor der Sclaverei haben. (Auswandererbriefe V: 2)

Ein weiterer Grund für die Nicht-Berücksichtigung des Belegs ist, dass sich die Frage der topologischen Integration nur in den Fällen stellt, in denen der Adverbialsatz einem Hauptsatz als ,obersten Obersatz' (Zifonun et al. 1997: 2237) vorangestellt wird. Im folgenden Beleg kann die Stellung des weil-Satzes (Vorder- oder Nachstellung) nicht eindeutig bestimmt werden:

(95) So hat Sich der General mit vierzig Tausend Mann auff der Winge gegen Eningen zu gelagert und einen schröck Capitin in die Statt geschickt, den Untergang anzutrohen, So hat Sich der wol Meridierte Herr B. M. Samuel Clewer einen Fußfahl vor Ihro Generalitet gethan, weil Sie tautzbrüder gewessen. So hat Er zu Ihm gesprochen, stehe auff Bruder, dein Hauß und deiner Freunden Hauß sollen verschont werden. (Schuhmacher Chronik II: 444f.)

Der weil-Satz kann sowohl den Kniefall vor dem General als auch die in direkter Rede wiedergegebene Ansprache an ihn begründen, er kann also sowohl als Vorder- als auch als Nachsatz interpretiert werden. Ich werde solche Zweifelsfälle zwar in der qualitativen Analyse ausführlicher beschreiben, aber aus der quantitativen Analyse heraushalten.

Ein weiteres Problem stellen Temporalsätze dar, denen unmittelbar ein temporales Adverb vorangestellt wird. Es stellt sich die Frage, ob in diesen Fällen eine Erweiterung des vorangestellten Adverbials durch einen Attributsatz (und somit eine Zwischenstellung des Nebensatzes) oder ob eine komplexe adverbiale Einheit in Voranstellung anzunehmen ist. In der vorliegenden Arbeit wird die zweite Auffassung vertreten: Bei erst wenn (s. folgenden Beleg), immer wenn bzw. kurz bevor (s. Belege (163) und (164) weiter unten) kann man in Anlehnung an Pasch et al. (2003: 71, 411) davon ausgehen, dass die vorangestellten Adverbiale (z.B. erst, immer, kurz) - wie auch Fokuspartikeln wie sogar - als Modifikator auffassen sind und in Vorerstposition, also in einer dem Vorfeld zugehörigen Position, stehen.

(96) Einen einzelnen Stern, der durch eine kleine Lücke in der Wolkendecke scheint, vermag man nicht zu benennen; erst wenn ein Stück klaren blauen Himmels größer wird und man auf ihm mehrere Sterne in ihrer räumlichen Beziehung zueinander sieht, ist man in der Lage, dieses Muster mit einem bestimmten Teil der bekannten Sternekarte zur Deckung zu bringen, mit ihm zu identifizieren. (Lorenz VII: 41)

Einschlägige Belege sollen daher in der Analyse berücksichtigt werden. Belege wie (97) werden dagegen als restriktive Attributivsätze in Zwischenstellung analyisiert:

(97) In dem Wirtzhau $\beta$, da ich uber Nacht pleib, bezalte ich dem Wirdt den Nachtimes, konde nichts essen und trincken. (Güntzer I: 59v) 
Des Weiteren werden auch valenzgebundene Nebensätze aussortiert. Im folgenden Beleg ist der wie-Satz als Objekt/Ergänzung des Hauptsatzprädikats weiß zu analysieren:

(98) Ich habe im Vertrauen auf Gott wieder eine Anstellung angenomen, wie es mir gehen wird, weiß ich nicht, .... (Auswandererbriefe V: 9)

Der folgende, durch $d a$ eingeleitete Adverbialsatz (resumptiv mit alsbald wiederaufgenommen), stammt aus Sebastian Münsters Cosmographia aus 1544:

(99) ... und schreibet hievon Münsterus I. 5. Gosmograph. C. 21. mit folgenden Worten: Da die Freyer ihren ersten alten Titul (die Edlen) behalten haben/ alsbald haben die Grafen einen hohern Titul wollen haben/ und genennet werden die Wolgebornen: (Harsdörffer I: 26)

Belege, die eindeutig als wörtliche Zitate aus früheren schriftlichen Texten zu rekonstruieren sind, werden in der vorliegenden Untersuchung nicht berücksichtigt. Dies gilt jedoch nicht für Redewiedergaben wie in (95).

Im Folgenden soll geklärt werden, welche weiteren Kriterien zur Erfassung der semantischen Relationen herangezogen werden. Im Kapitel 3.4.2 wurde die Unterscheidung der temporalen und konditionalen Relationen angesprochen. Dabei gilt, dass sich nur bei der konditional-hypothetischen Verwendung die Frage stellt, ob der im Untersatz ausgedrückte Sachverhalt wahr ist bzw. zutreffen kann (vgl. Zifonun et al. 1997: 2284f. und Breindl et al. 2014: 321). Wenn Konjunktiv II vorliegt, ist nur eine konditional-hypothetische Interpretation möglich (vgl. Glück/Rödel 2016: 352). ${ }^{175}$ Beim Subjunktor wenn unterscheiden wir mit der IDS-Grammatik (Zifonun et al. 1997: 2282f.) die konditional-hypothetische Verwendung, die temporale und die faktische ${ }^{176}$ Verwendung. Sowohl die konditional-hypothetische (die Beispiele (100) bzw. (101)) als auch die temporale Verwendung (die Beispiele (102) bzw. (103)) können sowohl generalisierend $(100,102)$ als auch singulär $(101,103)$ sein. Als Beispiele werden dafür u.a. folgende Sätze aufgeführt (zit. n. Zifonun et al. 1997: 2282, Hervorhebungen aus dem Original übernommen, P.K.):

(100) Wenn man Knollenblätterpilze ißt, vergiftet man sich.

(101) Wenn du bis fünf Uhr nicht zurückkommst, eilen wir dir zu Hilfe.

175 Konditionalität ist auch mit weiteren Relationen wie Kausalität bzw. Adversativität/Konfrontativität verwandt, s. dazu Breindl et al. (2014: 718-725).

176 Im folgenden Beispielsatz ist der Inhalt des wenn-Satzes nicht hypothetisch, sondern faktisch bzw. kontextuell gegeben. Aus dem Linkskontext geht hervor, dass ,,das Antezedens [...] der Fall ist“ (Zifonun et al. 1997: 2286):

(i) Ich habe von Ihrem Lottogewinn erfahren. Wenn Sie (schon) soviel Geld gewonnen haben, könnten Sie wenigstens eine Runde spendieren. 
(102) Die Angela glich ihrem Vater so auffällig, daß die Burgl zuweilen erschrak, wenn das Kind sie aus seinen blauen Augen anschaute.

(103) Wenn ich das Buch ausgelesen habe, gebe ich es dir gleich.

In der IDS-Grammatik (Zifonun et al. 1997: 2284f.) wird betont, dass die temporale und die konditional-hypothetische Verwendung nicht immer sauber zu trennen sind. Es werden jedoch Indizien benannt, mit deren Hilfe Problemfälle geklärt werden können:

Temporale wenn-Sätze haben die Funktion, eine Betrachtzeit für den Obersatz zu liefern. Das heißt, sie geben, wie andere temporale Untersätze, z.B. mit als, während, sobald, solange, das Wahrheitsintervall des Untersatzes als maximal mögliches Betrachtzeitintervall an den Obersatz weiter.

Generalisierende Konditionale können von generalisierenden Temporalen auch dadurch unterschieden werden, daß nur bei den Konditionalen die Frage der Wahrheit des Untersatzes überhaupt relevant wird.

Bei der temporalen (und bei der faktischen) Verwendung ,stellt sich die Frage gar nicht, ob der im ANTEZEDENS genannte Sachverhalt auch nicht zutreffen könnte“ (Breindl et al. 2014: 321). Bei (102) ist der im wenn-Satz genannte Sachverhalt zur Äußerungszeit bereits eingetreten, bei (103) ist aus der Sprecherperspektive anzunehmen, dass es auf jeden Fall zum Eintreten des Sachverhaltes kommt. In Konditionalsätzen wird aber nicht behauptet oder vorausgesetzt, dass die bezeichneten Sachverhalte zutreffen, es ist nur „das Zutreffen des Bedingtseins der Konsequenz durch das Antezedens für die Wahrheit des Gesamtsatzes ausschlaggebend“ (Eisenberg 2013: 339). Im empirischen Teil wird die Einordung als Temporal- bzw. Konditionalsatz aufgrund der Kriterien von Zifonun et al. (1997: 2282ff.) durchgeführt. Zur Veranschaulichung sei der folgende Beleg herangezogen:

(104) So ein Waltforster solt zu eich komen, wirdt er eich gefenglich nacher Hagenau fieren. (Güntzer I: 41r)

Bei der Interpretation des vorliegenden Satzes ist zwar die temporale Bedeutung nicht auszuschließen, aber die Verwendung des Modalverbs solt trägt dazu bei, dass hier von einer hypothetischen Verwendung ausgegangen werden kann. Da bei diesem Beleg die Frage nach der Wahrheit des Untersatzes relevant ist, wird dieser Beleg als Konditionalsatz eingestuft und an der entsprechenden Stelle (Kapitel 5.2) behandelt.

In den untersuchten Belegen ließen sich Übergänge zwischen semantischen Relationen registrieren, die die Einordnung der Belege erschwerten. Eine Voraussetzung für die Annahme von Übergangstypen ist, dass die einschlägigen Subjunktoren beide betroffenen Relationen ausdrücken können. Diesem Kriterium entsprechen $d a$-Sätze, wenn sie im jeweiligen Zeitabschnitt sowohl rein temporal als auch rein kausal verwendet werden. Der 
Subjunktor als tritt dagegen nicht in reinen Kausalsätzen auf, ${ }^{177}$ daher ist die Annahme eines kausalen Verhältnisses nur auf der Ebene der bezeichneten Handlungen, aber nicht auf der Ebene ihrer sprachlichen Realisierung möglich. Aus diesem Grund werden die einschlägigen Sätze - bei denen ein kausaler Nebensinn angenommen werden könnte - nicht als Mischtyp temporal-kausal (vgl. Kapitel 5.4.7), sondern als temporal eingestuft, vgl. den folgenden Beleg für einen durch als eingeleiteten Temporalsatz:

(105) Als er vernam, daß ich ein Hochdeischter wahr, so fragt er mich alerhandt, wie es im deischt Landt stunde, aber wußt es beßer, als ich es $i[h] m$ sagen kondt. (Güntzer I: 90r)

177 Der Subjunktor als wird nicht als temporal-kausal, sondern nur als semantisch eindeutig temporal verwendet, wenn auch okkasionell eine kausale Bedeutung des jeweiligen Adverbialsatzes nicht ausgeschlossen werden kann. Der Subjunktor als ist in temporaler Funktion „schon bei Luther [...] nicht selten“ (Dal/Eroms 2014: 246), eine konventionalisierte kausale Bedeutung liegt aber bis heute nicht vor. Für den als-Satz in (i) nehmen Breindl et al. (2014: 134) eine kausale Interpretation an, die darauf zurückzuführen ist, dass „die aspektuelle Forderung nach einer telischen Sachverhaltsbeschreibung verletzt ist":

(i) Als sich die Tür nicht öffnen ließ, rief Hans den Hausmeister.

Wegen der Negation sei der zeitliche Bezugsrahmen der Sachverhaltsbeschreibung des Adverbialsatzes nicht zu bestimmen, daher sei in solchen Fällen „,eine Streichung des Kausalzusammenhangs kaum mehr möglich“. Kausalität ist jedoch kein Teil der lexikalischen Bedeutung des Subjunktors als. Zudem kann als zeitlicher Bezugsrahmen die Zeit angenommen werden, als versucht wurde, die Tür zu öffnen (und sich herausgestellt hat, dass sie sich nicht öffnen lässt). 


\section{Empirischer Teil}

Zunächst werden Temporal-, Konditional-, Kausal-, Konzessiv- und Komparativsätze behandelt. Um den Erkenntniswert der einzelnen Kapitel besser sichtbar zu machen, werden sie jeweils durch kürzere Zusammenfassungen abgeschlossen. Anschließend werden weitere Adverbialsatztypen wie etwa adversative oder Finalsätze, für die im Korpus nur relativ wenige Belege zu finden sind, im Unterkapitel 5.4 behandelt. Pragmatische Adverbialsätze sollen separat, im Kapitel 5.5 analysiert werden. Die im Anhang (Kapitel 8.1) aufgeführten, nach Adverbialsatztypen gegliederten Beleglisten sollen die Nachvollziehbarkeit der gewonnenen Ergebnisse gewährleisten.

Im Folgenden sollen die vorangestellten Temporalsätze aus dem Korpus nach folgenden Kriterien untersucht werden: Die Zusammenhänge zwischen Stellungstypen und Subjunktoren bzw. Korrelaten werden nach Zeitabschnitten geordnet untersucht. In den einzelnen Unterkapiteln wird die Datenmenge auch unter dem Aspekt der Nähe-DistanzDimension und anderen Integrationsebenen analysiert (s. dazu Kappel i.V.).

\subsection{Temporalsätze}

Im Gesamtkorpus finden sich 121 Belege für vorangestellte Temporalsätze. Die folgende Tabelle zeigt anschaulich, dass die Belege in den Korpustexten bzw. in den Teilkorpora nicht einheitlich verteilt sind:

\begin{tabular}{|l|l|l|l|}
\hline & Nähe & Distanz & insg. \\
\hline $17 . \mathrm{Jh}$. & $\begin{array}{l}\text { Güntzer I } \\
\text { 64 Belege }\end{array}$ & $\begin{array}{l}\text { Harsdörffer I } \\
\text { 4 Belege }\end{array}$ & 68 Belege \\
\hline $18 . \mathrm{Jh}$. & $\begin{array}{l}\text { Schuhmacher Chronik II } \\
\text { 9 Belege }\end{array}$ & $\begin{array}{l}\text { Aichinger III } \\
\text { 3 Belege }\end{array}$ & 12 Belege \\
\hline $19 . \mathrm{Jh}$. & $\begin{array}{l}\text { Auswandererbriefe V } \\
11 \text { Belege }\end{array}$ & $\begin{array}{l}\text { Burckhardt V } \\
\text { 7 Belege }\end{array}$ & 18 Belege \\
\hline $20 . \mathrm{Jh}$. & $\begin{array}{l}\text { Jugendtagebuch VII } \\
19 \text { Belege }\end{array}$ & $\begin{array}{l}\text { Lorenz VII } \\
\text { 4 Belege }\end{array}$ & 23 Belege \\
\hline insg. & $\begin{array}{l}\text { 103 Belege } \\
\text { 18 Belege }\end{array}$ & 121 Belege \\
\hline
\end{tabular}

Tabelle 2: Verteilung der Belege für vorangestellte Temporalsätze in den Korpustexten bzw. in den Teilkorpora $(n=121)$ 
Der Anteil an Temporalsätzen ist in den Nähetexten höher (zur Relevanz verschiedener Inhaltsrelationen in Nähe- bzw. Distanztexten s. auch Ágel 2012: 196). Der größte Unterschied ist zwischen den beiden Korpustexten aus dem 17. Jh. zu beobachten: 64 Belegen (mehr als die Hälfte aller Temporalsätze des Gesamtkorpus!) aus Güntzer I stehen lediglich 4 Belege aus Harsdörffer I gegenüber. Dieser deutliche Unterschied bezüglich der Frequenz der Temporalsätze ergibt sich wohl daraus, dass die Texte nicht im gleichen Maße durch die Erzählung früherer Ereignisse geprägt sind. Die Erzählung früherer Ereignisse ist eher für den Korpustext Güntzer I charakteristisch, in dem mehr als die Hälfte der vorangestellten Adverbialsätze als Temporalsätze einzustufen sind. Die relativ höhere Anzahl der Temporalsätze in der Autobiografie ist also nicht überraschend. Der niedrige Anteil von Temporalsätzen im Korpustext Harsdörffer I ist auch nicht verwunderlich, enthält er doch eher Beschreibungen von Regeln u.a. zur Rechtschreibung, die durch konditionale Gefüge ausgedrückt werden können, als Erzählungen von Ereignissen, die zeitlich situiert werden könnten. Die Verteilung der verschiedenen Adverbialsatztypen in den Korpustexten steht also offensichtlich auch mit dem Inhalt der Texte im Zusammenhang (vgl. dazu Kapitel 4.2).

In den untersuchten Korpustexten finden sich keine eindeutigen Belege für uneingeleitete Temporalsätze. Der folgende Beleg enthält einen V1-Satz, der eher als selbständiger Aussagesatz zu interpretieren ist.

(106) Siehet ein grosser Herr ein waidlichs Baüerlein im Hauffen, Männlein, spricht Er, welches Regiment gefelt dir am besten, der Bauren oder der Fürsten. (Schuhmacher Chronik II: 426)

Im Folgenden werden die vorangestellten, eingeleiteten Temporalsätze nach den vier Zeitabschnitten gegliedert untersucht.

\subsubsection{Temporalsätze in den Korpustexten aus dem 17. Jh.}

Im Korpustext Harsdörffer I sind nur vier einschlägige Belege zu finden. Im Sinne des oben Gesagten (vgl. Kapitel 5.1) ist es keinesfalls überraschend, dass im Korpustext Harsdörffer I keine Temporalsätze der Vor- bzw. Nachzeitigkeit belegt sind. Des Weiteren kommt die singuläre Verwendung (im Sinne von Zifonun et al. 1997: 2282f.) der Temporalsätze - mit einer Ausnahme - nicht vor. Der einzige, mit als eingeleitete Temporalsatz in singulärer Verwendung wird trotz syntaktischer Komplexität integrativ angebunden: ${ }^{178}$

\footnotetext{
${ }^{178}$ Im Analyseteil werden die ihrem Obersatz vorangestellten Adverbialsätze (und die mit ihnen - koordinierend, subordinierend oder parenthetisch - verknüpften weiteren Untersätze) der Übersichtlichkeit halber durch farbige Markierung hervorgehoben.
} 
(107) Als aber der Jagermeister geantwortet: Er wisse nicht/ was ein Extract seye? hat der Käiser versetzt: Mein Knoll/ so laß mir auch meine Schreiber und Secretarien zu frieden. (Harsdörffer I: 13)

Der Temporalsatz ist durch zwei abhängige Sätze erweitert, im Vorfeld seines Obersatzes (also vor dem Hauptsatzfinitum hat) steht also eine Einheit aus drei Teilsätzen.

Des Weiteren kommen im Korpustext Harsdörffer I drei Temporalsätze vor, die mit getrennt geschriebenem so offt eingeleitet werden. Auch diese Temporalsätze werden integrativ angebunden, wie dies am folgenden, zu Illustrationszwecken herangezogenen Beispiel zu beobachten ist:

(108) So offt wir Speis und Geträck zu uns nehmen/sollen wir uns erinnern/ daß wir von unsrem Vatterland genehret und erhalten werden: (Harsdörffer I: 1)

Die durch so offt eingeleiteten Temporalsätze enthalten Frequenzangaben (vgl. FabriciusHansen 2007: 776) zum übergeordneten Satz, sie sind Frequenz spezifizierend im Sinne von Breindl et al. (2014: 255). Mit dem vorangestellten Temporalsatz wird die Häufigkeit der im übergeordneten Satz angegebenen Handlung bestimmt. Der Satzkomplex kann wie folgt paraphrasiert werden: „Immer dann, wenn wir Speise und Getränk zu uns nehmen, sollen wir uns erinnern, dass wir von unserem Vaterland ernährt und erhalten werden“. Laut von Polenz (1985: 253) könnte in diesen Fällen von einem iterativen/frequentativen Aktionsart-Zusatz die Rede sein. Die semantischen Beziehungen zwischen den Teilsätzen werden durch den temporalen Subjunktor so offt, der auch im Gegenwartsdeutschen spezifisch für allquantifizierte Sachverhalte ist (Breindl et al. 2014: 125), eindeutig markiert. ${ }^{179}$

Im Korpustext Güntzer I können 64 vorangestellte Adverbialsätze als Temporalsätze klassifiziert werden. Wie auch im anderen Korpustext aus dem 17. Jh., so ist auch in Güntzer I kein vorangestellter Temporalsatz der Nachzeitigkeit belegt. Vorzeitigkeit wird jedoch in mehreren Fällen durch Temporalsätze ausgedrückt. Am offensichtlichsten ist dies in Belegen $\mathrm{zu}$ erkennen, die einen entsprechenden Subjunktor wie etwa nachdem enthalten. ${ }^{180} \mathrm{Im}$ Korpustext sind 3 Belege für nachdem zu finden, neben zwei integrativ angebundenen Sätzen (vgl. z.B. Beleg (110)) tritt ein Einzelbeleg für resumptive Anbindung auf:

(109) Nachdem wihr auß Lickerlandt kemen sindt, so zogen wihr ins Landt zu Gleffen (Güntzer I: 82r)

\footnotetext{
179 Diese Verwendung von so oft bzw. so bald ist auch im Korpus von Lefèvre (2012: 497) belegt, dort werden allerdings ausschließlich Belege aus dem 18. Jh. aufgeführt.

180 Durch nachdem eingeleitete Temporalsätze der Vorzeitigkeit sind im Gegenwartsdeutschen - laut der Ergebnisse einer Stichprobe aus dem DeReKo (vgl. Breindl et al. 2014: 334) - deutlich häufiger vorangestellt als durch bevor bzw. ehe eingeleitete Temporalsätze der Nachzeitigkeit.
} 
Die Vorzeitigkeit des im Obersatz genannten Sachverhalts wird in diesem Beleg nicht nur durch den Subjunktor, sondern auch durch die Verwendung des Perfekts im Temporalsatz angezeigt. ${ }^{181}$ Das Hauptsatzverb steht im Präteritum und markiert somit auch morphologisch, dass das Bezeichnete im Hauptsatz später stattfindet als das Bezeichnete im Temporalsatz. Die Interpretation des folgenden Belegs ist auch deshalb problematisch, weil in den Teilsätzen kein Tempusunterschied festzustellen ist - die Verben außzog und ließ stehen im Präteritum:

(110) Nachdem ich nun im Jahrr ano 1615 auff Barttolimey außzog zu wandtern, ließ mihr mein Vatter ein ney Kleidt machen von Leder, gab mihr zugehöhrige Nodtdurfft wie auch 12 fl. in Gelt (Güntzer I: 40v)

Der Subjunktor nachdem leitet im Gegenwartsdeutschen i.d.R. Temporalsätze der Vorzeitigkeit ein. Die primäre lexikalische Bedeutung des Subjunktors steht hier jedoch im Widerspruch zum Inhalt des komplexen Satzes. Die Vorzeitigkeit des im Nebensatz bezeichneten Ereignisses ist aus logischen Gründen auszuschließen, denn die Reihenfolge der Ereignisse ist wie folgt rekonstruierbar: Erst bekam Güntzer ein neues Kleid und etwas Geld von seinem Vater, dann zog er aus zu wandern. Er muss das Kleid und das Geld vor der Wanderung bekommen haben, sonst hätte er sie nicht mit sich nehmen können. Daher wird an dieser Stelle die Interpretation des obigen Belegs als Temporalsatz mit einer vagen semantischen Beziehung zwischen den Teilsätzen angenommen. Der Subjunktor nachdem scheint bezüglich der zeitlichen Abfolge der Sachverhalte „unspezifisch situierend“ (Breindl et al. 2014: 292) zu sein, da er nur anzeigt, dass sein ,internes Konnekt als eine Art Rahmen die Betrachtzeit liefert für die zeitliche Situierung des externen Konnekts [...] und die konkrete Verortung dieser Betrachtzeit kontextuell festgelegt wird“.

Die temporale Bedeutung von nachdem ist wie auch im Gegenwartsdeutschen primär, es leitet jedoch nach den Angaben des DWb (1889, Sp. 34, Z. 3) bereits im 17. Jh. vorangestellte Kausalsätze ein. Eine kausale Deutung des Belegs ist nicht im Vornhinein auszuschließen, denn auch die folgende Interpretation wäre plausibel: Der Grund dafür, dass Güntzers Vater ihm ein neues Kleid machen ließ, war, dass Güntzer auszog zu wandern. Die kausale Verwendung von nachdem ist im Gegenwartsdeutschen zwar möglich (vgl. Zifonun et al. 1997: 1149), in bestimmten Fällen sogar zwingend (vgl. Breindl et al. 2014: 206f.). ${ }^{182}$ Im

181 Das Vollverb kemen ist als Partizipform zu kommen zu interpretieren. Der Wegfall von ge- ist bei Verben mit Plosiva im Anlaut auch in weiteren nähesprachlichen Quellentexten des Neuhochdeutschen belegt (vgl. z.B. Elspaß 2010: 76) und ist auch in den heutigen süddeutschen Dialekten verbreitet.

182 Im Beleg (i) spricht das Prädikat darstellt, das „,dem Subjektsdenotat permanente Eigenschaften zuschreib[t]“, gegen die Annahme des temporalen Defaultwerts von nachdem. Aufgrund dieses Merkmals muss der folgende Satz kausal interpretiert werden (Breindl et al. 2014: 206f.): 
Korpusvolltext konnten aber keine Belege identifiziert werden, die eine kausale Einordnung des Subjunktors nachdem ermöglichen. Nur der temporale Gebrauch von nachdem ist belegt und im einschlägigen Beleg sind keine Merkmale identifizierbar, die eine eindeutige kausale Interpretation nahelegen würden. ${ }^{183}$ Daher wird dieser Beleg als Temporalsatz eingestuft.

In den meisten Fällen drücken die Temporalsätze des Korpus Gleichzeitigkeit aus, wobei 53mal der Subjunktor als verwendet wird. Gleichzeitigkeit und Vorzeitigkeit sind jedoch nicht immer scharf zu trennen. Im folgenden Beleg handelt es sich - anders als dies bei den temporalen Subjunktoren als und während im Normalfall zu erwarten wäre (vgl. Breindl et al. 2014: 78) - nicht darum, dass ,eines der Relata ein zeitlich ausgedehnteres Ereignis [bezeichnet], das das im anderen Relat bezeichnete Ereignis umschließt“. Es gilt hier vielmehr die zeitliche Abfolge, nach der erst das im Temporalsatz bezeichnete Ereignis und direkt danach das im Matrixsatz bezeichnete Ereignis stattfindet.

(111) Als ich auffstundt, fiel ich widerumb nider, ging auff alen Fieren gleich wie Dierr. (Güntzer I: 59v)

Der Subjunktor als kann auch im Gegenwartsdeutschen bei Vorzeitigkeit verwendet werden (vgl. Breindl et al. 2014: 299). Im angeführten Beleg wird jedoch auf einen Tempusunterschied in den Teilsätzen verzichtet, obwohl dies zur Explizierung der semantischen Relation hätte beitragen können.

In den meisten Temporalsätzen des Korpustextes ist die singuläre Verwendung zu beobachten. Während für den am häufigsten verwendeten Subjunktor als 53 Belege nachzuweisen sind, kommen andere Subjunktoren nur vereinzelt vor. Die generalisierende Verwendung der Temporalsätze ist bloß in vier Belegen zu beobachten. Bezüglich ihres Stellungsverhaltens lässt sich feststellen, dass nur die resumptive Anbindung belegt ist (4mal).

(i) Nachdem die Ausstellung einen sehr repräsentativen und qualitativ sehr hochstehenden Querschnitt der zeitgenössischen Kunst darstellt, würde sie sich natürlich als Einstieg für ein Tiroler Museum der Moderne geradezu anbieten. (Neue Kronen Zeitung, 14.05.1995, S. 27, zit. n. Breindl 2014: 206f.)

183 Lefèvre (2012: 498) sieht die Übertragbarkeit der semantischen Typen auf seine Quellentexte aus dem 17. und dem beginnenden 18. Jh. kritisch: „Beim klassischen periodischen Stil tritt dabei die eigene temporale, kausale, konditionale, konzessive Semantik hinter dem logischen und argumentativen Aufbau der Periode zurück: Es wird vorrangig die Protasis als Thema, als allgemeine Prämisse, als Wiederaufgreifen schon bekannten Sachgehalts, und die Apodosis als Rhema, als kleine Prämisse, als Hinzufügen neuen Sachgehalts gekennzeichnet." Auch bei nachdem kann davon ausgegangen werden, dass die modernen Analysekategorien der Temporalität nicht problemlos anwendbar sind. Bei der Gliederung von Perioden im 17. Jh. hat nachdem „vor allem eine anaphorische Funktion, sie verweist auf den voranstehenden Kotext oder gar implizit auf den Kontext.“ (Lefèvre 2013: 278) In diesem Sinne ist zu vermuten, dass der Subjunktor nachdem beim Verweis auf den - bereits im vorausgehenden Kotext (Güntzer I: 32v) erwähnten - Beginn der „Wandterschafft“ am Bartholomäustag anaphorisch auf die Reisevorbereitung inkl. Gebete zu Gott (u.a. Güntzer I: 38r39r) Bezug nimmt. Lefèvre (2013: 317f.) führt mehrere Belege aus dem 17. Jh. an, in denen die Konjunktionen wie nachdem nicht zur Angabe der Abfolge der bezeichneten Ereignisse im heutigen Sinne beitragen. Er nimmt in diesen Fällen bei nachdem keine temporale, sondern eine diskursive Funktion an und betrachtet es nicht als syntaktisch subordinierend, sondern als parataktisches Verknüpfungsmittel bzw. als Gliederungssignal (2013: 319f.). Da die Wortstellung jedoch Abhängigkeit markiert, liegt möglicherweise ein Kollaps von adverbialer und subjunktionaler Verwendung vor. 
Im folgenden Beispiel wird durch den wan-Satz angegeben, in welchen Zeitintervallen die Aussage des Hauptsatzes gültig ist, er ist daher temporal zu interpretieren.

(112) Walzet mich hin und her, ruffete mihr balt die Augen auß dem Kopff, weinete wie ein Kindt. Wan ich dan deß Abens spadt nacher Hauß kam, so fragt man mich, waß mihr an dem Augen wahr, daz sie also rodt wahren. (Güntzer I: 101v-102 r)

Es liegt eine generalisierende Verwendung vor. Generalisierende wenn-Sätze können im Gegenwartsdeutschen sowohl temporal als auch konditional interpretiert werden, daher stellt sich die Frage nach der semantischen Relation. Die temporale Interpretation ist m.E. angemessener als die Deutung als konditional-hypothetische Verwendung, denn es ist weniger sinnvoll anzunehmen, dass Güntzer überhaupt nicht nach Hause kam, „es stellt sich die Frage gar nicht, ob der im ANTEZEDENS genannte Sachverhalt auch nicht zutreffen könnte“ (Breindl et al. 2014: 321). Ein generalisierender wenn-Satz liegt auch im folgenden Beleg vor:

(113) Wan mihr etwaß wol ist, so beschaue ich die Statt, ihr Gebey und abgottische Kirchen. (Güntzer I: 60v)

Im Korpustext treten zwei durch so eingeleitete Adverbialsätze auf, die als Temporalsatz einzustufen sind. Ihre semantische Interpretation ist jedoch nicht völlig eindeutig:

(114) Lage des Nachts under dem Himel neben einer Vischerhitt. Ich duhe mich mit meinem Gelt sehr genau behelffen. In den gleinen Stetten ging ich deß Nacht im Abent nicht hinein, behalffe mich bey einem Bauren im Stro oder under einem Schopff auff Stein oder Holz oder under dem hellen Himel. Den gantzen Tag ging ich in den kleinen Stetten spatzieren. So man die Dore zuschließt, so ging ich hinauß. (Güntzer I: 84r)

Der obige Beleg wird als Temporalsatz interpretiert: Im Linkskontext schildert Güntzer, dass er die Nächte nicht in den Städten verbrachte. Er blieb den ganzen Tag in den Städten, bis die Tore geschlossen wurden, zu diesem Zeitpunkt verließ er immer die Stadt. Im Sinne dieser Interpretation liegt hier eine generalisierende Verwendung des Temporalsatzes vor. Eine konditionale Deutung des Belegs (nach dem Wortlaut: Nur in dem Falle verließ er die Städte, wenn die Tore geschlossen wurden) ist weniger naheliegend, denn es kann angenommen werden, dass zu dieser Zeit die Stadttore am Abend immer geschlossen wurden.

Im nächsten Beleg liegt eine singuläre Verwendung des durch so eingeleiteten Temporalsatzes vor. Eine Seite vor der im Folgenden angegebenen Textstelle beschreibt Güntzer, welche gesundheitlichen Probleme er bei der Überquerung der eiskühlen Donau erlitten habe. ${ }^{184}$ In der nachfolgenden Textstelle wird eine harte, aber erfolgreiche Therapie beschrieben. Es wurde ihm befohlen, die Füße in eiskaltes Wasser zu stellen. Sie durften erst

\footnotetext{
184 „Da fiel ich hinein mit dem lincken Schenckel. Da frore mihr der Schenckel und Strimpff aneinander.” (Güntzer I: 97r).
} 
dann aus dem Wasser genommen werden, als die Füße schon ,an daß Geschire wolten frieren“. Dann nahm Güntzer einen Hammer und „schlug daz Eiß mit auff“.

(115) Auff die Weinachtfeirtag kam ich in die Wiltau. Es wirdt mihr von Artzen und andern Leidten freyhertzig bevollen, Midel zu prauchen, darmit ich nicht etwan auff Krocken mußt in mein Vatterlandt gon. Da wirdt mihr befollen, die Schenckel in Eiß und alerkeltiste Waßer zu stellen biß an die Kney, welches ich es offt getan. In der grosten Kelten saß ich auff die Zinen deß Hauß, die Schenckel biß an die Kney in daz Waßer gestelt, offt 2 Stund lang. So mihr dan die Schenkel an daß Geschire wolten frieren, so name ich ein Hamer, schlug daz Eiß mit auff. Du[r]ch dißes Mittel wirdt mihr die Kelten und Frost widerum auß den Schencklen gezogen. (Güntzer I: 98v99r)

In diesem Beleg - wie auch im oben zitierten so-Temporalsatz mit generalisierender Verwendung - ist eine Parallelkonstruktion zu beobachten: Der Temporalsatz wird durch den Subjunktor so eingeleitet, der am Anfang des Hauptsatz mit dem Korrelat so wiederaufgenommen wird. Die Möglichkeit der Entstehung einer Parallelkonstruktion in diesem Sinne scheint die Wahl des resumptiven Typs zu begünstigen. ${ }^{185}$

Die weiteren temporalen Subjunktoren mit geringer Frequenz sind indem, so offt als und $d a$. In den folgenden Belegen ist die integrative Stellung zu beobachten. Der nächste Beleg mit indem ist eindeutig als temporal $\mathrm{zu}$ interpretieren, der Adverbialsatz ist mit der Präpositionalgruppe während der Reise im Churlandt paraphrasierbar:

(116) Indem wihr in Churlandt thun reißen, durchzogen wihr vil großer langer Welt. (Güntzer I: 97v)

Die temporale Verwendung des Subjunktors indem ist im heutigen Deutsch veraltet (Breindl et al. 2014: 1197). Eine modal-instrumentale Bedeutungsrelation, die im Gegenwartsdeutschen beim Subjunktor indem ebenfalls möglich oder gar prototypisch ist (vgl. Breindl et al. 2014: 588, Duden-Grammatik 2016: 640, Fabricius-Hansen 2011: 21), kann bei dem obigen Beleg wohl nicht angenommen werden. Die semantische Interpretation einiger $d a$-Sätze ist jedoch problematisch. Der vorangestellte Adverbialsatz im folgenden Beleg situiert das im Obersatz bezeichneten Ereignis temporal.

(117) Da wihr gutten Sudtwindt bekamen und fordtseglet[e]n dem Sundt zu, empfinde ich eine Lifferung meiner schwohrmiedtigen Gedancken und sehe, daß Gott nahe ist, mihr zu helffen in meiner großer Nodt und Traurigkeitt, die mich umpfangen hatt in dem Landt zu Preißen. (Güntzer I: 105v)

\footnotetext{
185 Auch im Gegenwartsdeutschen sind Parallelkonstruktionen bei den Subjunktoren da, sobald, solange, sooft, dazu möglich, vgl. die Liste für temporale Korrelatkonstruktionen aus dem HDK-1 (Pasch et al. 2003: 263f.) im Kapitel 3.4.2 weiter oben. Korrelatkonstruktionen mit einleitendem so sind nach Lefèvre (2012: 497) noch im 18. Jh. häufig, sie treten aber beim Ausdruck temporaler Beziehungen in Verbindung mit bald oder oft auf.
} 
Der Grund für die Erleichterung Güntzers ist nicht im Inhalt des vorangestellten Adverbialsatzes zu suchen, er wird eher in den folgenden Teilsätzen beschrieben. An dieser Stelle liegt also die temporale Variante des Subjunktors $d a$ vor, die im Gegenwartsdeutschen bereits „veraltet klingt“ (Blühdorn 2004: 132, vgl. auch Duden-Grammatik 2016: 638). Im Korpustext sind auch $d a$-Sätze belegt, die eindeutig als kausal zu interpretieren sind und an der entsprechenden Stelle behandelt werden (vgl. Kapitel 5.3). Des Weiteren liegen auch Belege vor, in denen die Temporalität kaum von der Kausalität zu trennen ist. In diesen Fällen wird ein Mischtyp temporal-kausal angenommen, einschlägige Belege werden im Kapitel 5.4 .7 behandelt.

Der nächste Beleg enthält auch einen Adverbialsatz, der eher temporal zu interpretieren ist. Es soll jedoch auch überprüft werden, ob er einen kausalen Nebensinn besitzt.

(118) So offt als er hinder mich ging, spring ich von den gelegten Beimen herunder undt ging widerumb dahinden. (Güntzer I: 42v)

In diesem Temporalsatz, der eine Frequenzangabe (vgl. Fabricius-Hansen 2007: 776, s. auch Beleg (108) weiter oben) enthält, wird ein komplexer Subjunktor verwendet. Im Gegensatz zu den Temporalsätzen mit „so offt“ aus dem Korpustext Harsdörffer I handelt es sich beim untergeordneten Teilsatz im obigen Beleg um vergangene Ereignisse in Bezug auf den Sprechzeitpunkt. Dies ist sowohl an den Tempora als auch am als, das als Subjunktor auf die Vergangenheit Bezug nimmt und diese Bedeutung auch im vorliegenden komplexen Subjunktor mitbringt, ablesbar. Die dargestellten Ereignisse könnten auch in ein kausales Verhältnis gestellt werden, in der sprachlichen Formulierung wird jedoch der Obersatz zeitlich situiert.

Bei Temporalsätzen, die durch den Subjunktor als eingeleitet sind, ist der integrative Typ die bevorzugte Variante (33 zu 20). In einem Korpus von Prosatexten aus der zweiten Hälfte des 17. Jhs. findet Wiktorowitz (2013: 394f.) „fast ausschließlich“ integrativ angebundene Temporalsätze, sodass der relativ hohe Anteil des resumptiven Typs im Nähetext Güntzer I überraschend ist. Im Korpus von Wiktorowitz (2013: 395) ist der resumptive Typ nur selten bei einfachen vorangestellten Temporalsätzen $\mathrm{zu}$ finden, auch bei umfangreichen Temporalsätzen bzw. zwei Temporalsätzen vor dem Obersatz überwiegt der integrative Typ. Im Nähetext Güntzer I dürfte die Komplexität des Vordersatzes bei der Wahl zwischen den Stellungstypen eine größere Rolle spielen. Wenn der Vordersatz durch einen weiteren Teilsatz erweitert wird, kommt von 5 Belegen 4 mal die resumptive Stellung vor (vgl. z.B. die Belege 
(123) und (125) weiter unten). Dies ist auch im folgenden Beleg zu beobachten, in dem der vorangestellte Temporalsatz durch einen Objektsatz erweitert wird:

(119) Als aber der Zingießer von andern seinen Gesellen erfuhr, wehr ich sey, so gab er mihr den andern Tag Arbeidt. (Güntzer I: 94r)

Das einzige Gegenbeispiel mit integrativer Stellung weist bei dem vorangestellten als-Satz und dem Objektsatz als dessen Erweiterung insgesamt 9 Wörter auf, sodass dieser Satz trotz Komplexität der Satzstruktur wohl keine extreme Belastung des Gedächtnisses verursacht:

(120) Als ich i[h]n erkandte, das es ein Man wahr, erschracke ich zim Teil, dieweil er so lang auff mich warttete, er dirfft mich dodt schlagen. (Güntzer I: 91v)

Bei den Belegen für als-Sätze ohne Komplexität des Vordersatzes kommt die integrative Stellung doppelt so häufig vor als die resumptive (32 zu 16). Es lässt sich in diesem Bereich kein Kriterium festmachen, das bei der Wahl der Stellungstypen entscheidend wäre. In den nächsten Belegen sind die vorangestellten Temporalsätze fast buchstabengetreu gleich, sie werden jedoch unterschiedlich in den Obersatz eingebunden:

(121) Als es Tag wirdte, reißete ich mein[e]r Straßen und dancket dem Wirdt um die Herberg. (Güntzer I: 84v)

(122) Als es Tage wirdt, so danckete ich ihren um die Herberg, gab ihr eine Verehr. (Güntzer I: 79v)

Im Korpustext Güntzer I wird bis auf einen Einzelbeleg immer das Resumptivum so verwendet. Im einzigen Gegenbeispiel steht $d a$ im Vorfeld des Obersatzes:

(123) Als ich nun solches hordte auff der italienischer Sprach, daß diße 3 Gesellen Banditen und Merder wahren, da wahr mihr angst und bange. (Güntzer I: 56r)

In diesem Beleg könnten die Ereignisse auch in einem kausalen Verhältnis erscheinen, weil der Umstand, dass Güntzer die Gefahr erkannte, der Grund für seine Angst war. Die sprachliche Realisierung erfasst hier jedoch eher den temporalen Aspekt.

Wie oben erwähnt, finden sich in den analysierten Sätzen auch Belege, die nicht eindeutig der Temporalität zugeordnet werden können. Bei diesen Sätzen könnte zwar evtl. ein kausaler Nebensinn angenommen werden, der Subjunktor als tritt jedoch nicht in reinen Kausalsätzen auf. Aus den Belegen für auch kausal zu interpretierende Temporalsätze weisen ein Satz integrative (vgl. Beleg (118)) und zwei Sätze resumptive Stellung ((124) und (125)) auf:

(124) Als er vernam, daß ich ein Hochdeischter wahr, so fragt er mich alerhandt, wie es im deischt Landt stunde, aber wußt es beßer, als ich es i[h]m sagen kondt. (Güntzer I: 90r) 
(125) Als ich die feindliche Biß am Kopff und Fießen empfinde, so erwachete ich, erschr[e]cke uber dißer Geschicht und wunderlicher Gest, die freßen wolten. (Güntzer I: 84r-v)

Die beiden Belege für die resumptive Stellung sind insofern auffällig, dass der eine einen durch einen Objektsatz erweiterten Temporalsatz vor dem Matrixsatz enthält (124), während der andere (125) den längsten Temporalsatz mit kausalem Nebensinn (10 Wörter) darstellt.

Wie ersichtlich, weisen die obigen Belege aus dem Korpustext Güntzer I entweder die integrative oder die resumptive Stellung auf. Ein eindeutiger Beleg für den nicht-integrativen Typ liegt im Korpustext nicht vor. Dabei sollte allerdings der folgende Beleg angeführt werden, bei dem die Stellung des als-Satzes in Bezug auf den Obersatz relevant ist.

(126) Es wahr um Marttiny Zeitt, als ich von Dantzig zog, die Best fing an zu krasier[e]n. Die Burger und die Handtwercksgesellen zogen sehr vil hin. (Güntzer I: 94r)

Man könnte eine Nachstellung annehmen, aber auch die Interpretation als eine ApokoinuStruktur $^{186}$ ist nicht auszuschließen: Es handelt sich also um einen Spezialfall, sodass dieser Einzelbeleg nicht als sicherer Hinweis für die sonst nicht belegte nicht-integrative Stellung von Temporalsätzen gedeutet werden kann. Er wird daher in der quantitativen Auswertung nicht berücksichtigt.

Zusammenfassend können die Temporalsätze in den beiden Korpustexten aus dem 17. Jh. wie folgt charakterisiert werden: Im Korpustext Harsdörffer I finden sich nur relativ wenige Beispiele für vorangestellte Temporalsätze, diese werden jedoch ausnahmslos integrativ angebunden. Im Korpustext Güntzer I ist die Frequenz der vorangestellten Temporalsätze viel höher. Dabei ist der Anteil des integrativen Typs (38 Belege) deutlich höher als der des resumptiven Typs (26 Belege). In den beiden Korpustexten aus dem 17. Jh. findet sich kein einziger, eindeutiger Beleg für die nicht-integrative Stellung von vorangestellten Temporalsätzen.

Da aber im nähesprachlichen Text auch die resumptive Variante mit 40,6\% vertreten ist, sollte die Frage gestellt werden, welche Faktoren evtl. diese Stellung begünstigen können. Eine Satzkomplexität in der Einheit vor dem Obersatz liegt im Korpustext Güntzer I in fünf Fällen vor, dabei wird bis auf einen Beleg die resumptive Variante verwendet. Die Satzkomplexität in der Einheit vor dem Obersatz führt in den Temporalsätzen aus dem Korpustext Harsdörffer I nicht zur Verwendung des resumptiven Typs, beide Belege sind integrativ angebunden. Bezüglich der Konnektoren ist hervorzuheben, dass die

186 Der Begriff ,Apokoinu“ steht für „eine syntaktische Konstruktion, bei der zwei Sätze ein Satzglied gemeinsam haben“ (Ágel/Hennig 2006c: 39). 
vorangestellten Temporalsätze aus Harsdörffer I durch Subjunktoren eingeleitet werden, die spezifisch für singuläre (als) bzw. allquantifizierte (so offt) Sachverhalte sind (vgl. Breindl et al. 2014: 125), sodass die semantischen Beziehungen zwischen den Teilsätzen eindeutig markiert sind. Im Nähetext sind jedoch die semantischen Beziehungen zwischen den Teilsätzen nicht immer eindeutig. Obwohl die semantischen Untertypen manchmal schwer abzugrenzen sind, ist auffällig, dass bei der generalisierenden Verwendung der resumptive Stellungstyp verwendet wird. Was die Form der Resumptiva betrifft, so wird im Korpustext Güntzer I bis auf einen Einzelbeleg immer das „semantisch unspezifische[...]“ (Zitterbart 2002: 152) Korrelat so verwendet.

\subsubsection{Temporalsätze in den Korpustexten aus dem 18. Jh.}

Im Korpustext Aichinger III kommen, wie auch im Distanztext aus dem 17. Jh., relativ wenige Temporalsätze vor. Im folgenden Beleg wird der Subjunktor ehe zum Ausdruck der Nachzeitigkeit verwendet:

(127) Ehe die lateinische Sprache zu ihrer Schönheit gelangte, hatte sie auch allerley diphthongos, welche in alten römischen Schriften, als, den Gesetzten der Könige, den 12. Tafeln, der Duillischen und Sripionischen Tafel gefunden werden, als: ei für e oder i, (Aichinger III: 26)

Dieser Beleg weist ebenso die integrative Stellung auf, wie der folgende Temporalsatz. Hier kann nicht eindeutig zwischen Vorzeitigkeit und Gleichzeitigkeit unterschieden werden. Der Subjunktor sobald kann im Gegenwartsdeutschen auch Vorzeitigkeit ausdrücken, dies ist jedoch nach der Duden-Grammatik (2016: 1096) nur „eine Variante eines temporalgleichzeitigen Verhältnisses“.

(128) Z.B. wenn ich das Wort Baum aussprechen soll: so muß ich erstlich die Lippen zusammen schliessen, und, so bald ich sie aufthu, muß jener Schall heraus fahren, der die weitere Eröffnung des Mundes erfordert. (Aichinger III: 20)

Der Verzicht auf Tempuswechsel in den einschlägigen Teilsätzen erlaubt eine Interpretation, nach der der betroffene Sprachlaut zur Zeit der Verschlusslösung entsteht. Auch der dritte Beleg im Korpustext Aichinger III, der Gleichzeitigkeit bezeichnet, wird mit integrativer Stellung realisiert, obwohl der Vordersatz durch mehrere Teilsätze erweitert wird und somit eine sehr komplexe Konstruktion darstellt:

(129) Denn als der König in Frankreich dem Kaiser im Jahr 1444. etliche 1000. Mann wider die Schweizer zu Hülffe schickte, deren Oberhaubt war der Marschall Graf von Armagnac, diese Franzosen aber gegen das teutsche Reich selbst nichts gutes im 
Sinne hatten, und daher etliche Mahle geschlagen wurden, wurde ihnen von den Teutschen nach ihrem Feldherrn, und zugleich zu Schimpfe der Name der armen Jäcken beygeleget. (Aichinger III: 44)

Der durch als eingeleitete Temporalsatz wird zunächst durch einen Relativsatz (deren Oberhaubt war der Marschall Graf von Armagnac) erweitert, der aufgrund der Verbstellung auch als Parenthese zu deuten ist. Es folgt ein nicht eingeleiteter Nebensatz (diese Franzosen aber gegen das teutsche Reich selbst nichts gutes im Sinne hatten), dessen syntaktische Relation zu den vorangestellten Teilsätzen nur sehr vage markiert wird. Der Konnektor aber und der Konnektor und vor dem nächsten Teilsatz lassen vermuten, dass diese Teilsätze mit dem Temporalsatz am Anfang des Satzkomplexes koordiniert werden. Die semantische Relation der Temporalität ist jedoch schwer nachvollziehbar. Auch eine formal nicht markierte kausale Relation (Grund für die Entstehung eines Necknamens) ist nicht auszuschließen. Diese logische Beziehung zwischen den Teilsätzen wird jedoch nicht markiert, sondern kann nur vom Rezipienten rekonstruiert werden. Wegen dieser semantischen Vagheit kann der vorangestellte als-Satz nur formal als Temporalsatz aufgefasst werden. Nach vier Teilsätzen, insgesamt 47 Wörtern, wird der ebenfalls relativ lange Obersatz (mit 18 Wörtern) realisiert. Der Satzkomplex besteht also aus 65 Wörtern, seine Rezeption wird durch eine Gedächtnisbelastung als Folge der lang gedehnten Projektionsstruktur und durch vage semantische Relationen erschwert. Die Wahl des integrativen Stellungstyps ist daher überraschend. Ein derart hohes Ausmaß von Aufnahmefähigkeit des Vorfeldes ist bei Temporalsätzen nur für den Distanztext aus dem 20. Jh. belegt.

Im Korpustext Schuhmacher Chronik II kommen 9 vorangestellte Temporalsätze vor. Der weiter oben (Kapitel 5.1) bei den Korpustexten aus dem 17. Jh. festgestellte Frequenzunterschied von Temporalsätzen ist also auch für die Korpustexte aus dem 18. Jh. wenn auch in einem kleineren Maße - gültig.

Im Korpustext ist die generalisierende Verwendung der Temporalsätze nicht belegt. Alle Belege können dem singulären Gebrauch zugeordnet werden. Im Vergleich zum nähesprachlichen Korpustext aus dem 17. Jh., in dem meistens ebenfalls die singuläre Verwendung von Temporalsätzen zu beobachten ist und als mit 83\% (53 Belege von 64 eingeleiteten Temporalsätzen) den frequentesten Subjunktor darstellt, ist im nähesprachlichen Korpustext aus dem 18. Jh. eine deutlich andere Verwendung der Subjunktoren zu beobachten. Es findet sich kein einziger Beleg für vorangestellte, durch als eingeleitete Temporalsätze. Der Subjunktor als ist aber im Korpustext belegt, er leitet z.B. im folgenden 
Satzkomplex (mit recht vagen Relationen zwischen den Teilsätzen) einen nachgestellten Temporalsatz ein:

(130) Betriebt ist es anzusehen gewessen. Daß ein Sohn, der schon wider herau $\beta$ ware, als er seinen umb Hülff schreienden Vatter retten wolte und sich wider in die Tieffe deß wassers begeben, daß sie beyde von der Fluth deß Wassers $u$. Eys ergriffen $u$. beyde elendiglich ertrüncken müssen. (Schuhmacher Chronik II: 331f.)

In zwei Fällen werden Temporalsätze der Nachzeitigkeit durch biß eingeleitet. Sie spezifizieren hier keine Zeitdauer, sondern eher einen Zeitpunkt. Die Satzlänge ist beim resumptiv angeschlossenen Temporalsatz (Beleg (131)) mehr als doppelt so hoch wie beim Temporalsatz mit integrativer Stellung (Beleg (132)):

(131) So hat mann sie Gefänglich eingezogen. (So ist der pacient den 17 Novemb. gestorben) Biß sie von einem Ehrsamen rath durch Zeugen $u$. Kundschafft seindt verhört worden So haben die Medici u. Barbierer in Eröffnung gefunden, daß ein Stich so gefährlich gewessen, der Ihm daß Leben verkürtzt. (Schuhmacher Chronik II: 336)

(132) den 5. Janw. deß Nachts, So daß die Sheur auff der Alten-Burg ist in die Asche gelegt worden und ist deß Nachts umb 1 uhr der Lerm angangen, biß man aber ist hinauff kom [m]en, ist Sie schon fast verbrunnen gewessen, daß keine Errettung mehr zu hoffen und ist durch einen Gott und ruchlossen Menschen angezindt worden \& also sampt Frucht und Futter Jäm [m] erlich verbrunnen. (Schuhmacher Chronik II: 398f.)

Die Satzkomplexität führt im folgenden Beleg zur resumptiven Wiederaufnahme des vorangestellten, mit einem indirekten Fragesatz erweiterten Temporalsatzes: ${ }^{187}$

(133) und ist von den Weh-Müetteren besichtiget worden. So haben Sie bey Ihren gefunden, Daß Sie Milch gegeben, welches schon ein anzeigen eines Kinds gewessen, darauff ist Sie widerumb von der Obrigkeit Examiniert worden, Ob Sie ein Kind gebohren oder nicht, Sie es aber durchauß gelaugnet, wie man Ihren hat vorgehalten, warumb Sie dann Milch geben, So hat Sie Sich mit disen Worten vernem [m]en lassen, Sie habe vor einem Jahr schon Milch gegeben, (Schuhmacher Chronik II: 378)

Die Wahl des resumptiven Typs könnte durch die Länge und die Satzkomplexität der Satzeinheiten vor dem Matrixsatz begünstigt sein. $\mathrm{Da}$ in den anderen, eindeutigen Belegen keine Satzkomplexität in diesem Sinne $\mathrm{zu}$ beobachten ist, können keine weiteren Schlussfolgerungen gezogen werden. Wie auch im Korpustext aus dem 17. Jh., sind Temporalsätze der Vor- bzw. Gleichzeitigkeit auch in Schuhmacher Chronik II nicht immer klar zu trennen. Im obigen Beleg gilt eine zeitliche Abfolge, nach der erst das im

\footnotetext{
${ }^{187}$ Der Temporalsatz wird nicht mit einer Subjunktion, sondern mit einem Frageadverb (wie) eingeleitet.
} 
Temporalsatz bezeichnete Ereignis und direkt danach das im Matrixsatz bezeichnete Ereignis stattfindet. Dies ist auch im folgenden Beleg mit resumptiver Stellung zu beobachten:

(134) hat Sie Es auff dem Weg dem schon gemelten Statt-Diener Elias Klein bestanden, Sie habe Es auff der Büne in einem Wollen-Ständlein in Speier ligen, man solle nur daß Schlisselein bey ihrem Vatter holen, wie mans von Ihm begert, So ist Ihm hart unmächtig worden, daß man vermeint Er werde gleich deß Todes sein. (Schuhmacher Chronik II: 379)

Der folgende, durch wie eingeleitete Temporalsatz, der im Gegensatz zu den beiden obigen Belegen eindeutig der Gleichzeitigkeit zuzuordnen ist, ${ }^{188}$ wird integrativ angeschlossen:

(135) Nachmittag hat man daß Gastmal gehalten, mit einer schönen Auffwartung der zwelff Falckenäten, wie auch dero Schiltwachten, die Herren haben auch gespeiset mit dem Graffen Eben, dem Keyser auch zu Ehren des Keysers Gesundheit truncken, hat man auch Loß gebrunnen 12 Falckeneten Hunden es waren auch besonnen die Herren nun von Stunden, der Keyserin Gesundheit zu trincken mit Bescheidt so hat man auch zu Ehren 10 Falckenäten gehen, wie es an graffen kom[m]en, hat man 2 weniger genom $[\mathrm{m}]$ en und sie so gelöset, wie es zu Ehren gneset. (Schuhmacher Chronik II: 327)

Interessant an diesem mit Reimen bestückten Satzkomplex ist, dass die resumptive Wiederaufnahme des Temporalsatzes zu einer deutliche(re)n rhythmischen Abweichung geführt hätte, wobei der Matrixsatz auch im Original bereits länger ist als die rhythmischen Einheiten vor ihm.

Im folgenden Beleg wird der getrennt geschriebene Subjunktor so bald verwendet. Im nachfolgenden Beleg wird statt so bald als einleitendes Element nur bald verwendet.

(136) Nehmlich vierzig Personen seind auff die Donau Brücken gangen dem Eiß durch zu helffen, so bald sie aber angefangen zu schaffen ist der gewalt des Eyses $u$. Wassers so schleunig angeloffen daß Es die Brücke eingerissen wovon 24 Menschen ertruncken (Schuhmacher Chronik II: 331)

(137) man hat vermeint es werd ein Hunger abgeben weil So 24ig Compagnien eingemaschiert sind, man hat bey Obrigkeitlicher Straff der Becken-Zunfft umgebotten, Sie sollen Brodt bachen, damit kein mangel erschöne, aber Gott sey Danck ist alles genug zubekommen gewessen, den gantzen Winter durch. Bald Sie aber außgerückt sind im Fryh-Jahr Anno 1704 So hat Es auffgeschlagen und ist Theur worden, der Laib Brodt galt der Zeit $10 x$. die Maß Wein $5 x$. auch $4 x$. bey den Bürgern (Schuhmacher Chronik II: 324f.)

Bei Breindl et al. (2014: 1180) wird bald nicht als Subjunktor verzeichnet, sondern nur als „nicht nacherstfähiger Adverbkonnektor“. Es wird jedoch erwähnt, dass die Form ohne so in älteren Sprachstufen belegt ist und im Bairischen auch heute noch mit Verbendstellung

188 Im Gegenwartsdeutschen ist wie als temporaler Subjunktor sehr selten und „funktionsgleich mit dem rahmensetzenden als" (Breindl et al. 2014: 308). 
verwendet werden kann (Breindl et al. 2014: 358). Im DWb (Bd. 1, Sp. 1083) ist der Gebrauch von bald mit Verbendstellung für den Anfang des 17. Jh. auch für andere Regionen belegt. Im Korpustext finden sich jedoch keine Vergleichsbelege. Da es nicht eindeutig ist, ob hier eine Korrelatverbindung von einem unselbständigen Nebensatz und einem wiederaufnehmenden Element so oder zwei selbständige Sätze vorliegen, wird dieser Beleg bei der quantitativen Analyse nicht berücksichtigt.

In den beiden folgenden Belegen mit $d a$ ist die semantische Relation (temporal oder kausal) schwer zu bestimmen. Der Beleg (138) könnte als Apokoinu-Konstruktion analysiert werden, bei dieser Interpretation wäre jedoch der $d a$-Satz rein temporal.

(138) Also hat Lutherus daß Revormations Werck angefangen und hat geweret biß auff $30 i g$ in werenter Zeit hat Sich viel Unheil gezeiget von allerhand Rotten \& Secten und theure Zeiten, auch Anno 1524 der Bauren-Krieg sich erhoben, da Sich die Bauren mit ihren Müstgablen \& Pfleglen und Bauersfedren verfast, pfiff Pfeiffer dem gemeinen Mann zum Tantz und bließ Lerma. in dieser Auffrur sind viel Tausend Bauren umkom[m]en, wie die gefangne Auffrierer erbärmlich zusamen koppet (Schuhmacher Chronik II: 425f.)

Im Beleg (139) wird der $d a$-Satz mit dem resumptiven $d a$ wiederaufgenommen, d.h., es liegt eine parallele Korrelatkonstruktion vor.

(139) Dises war die Ursach weil der Papst Leo der Zehende den Johann Tetzel ein Dominicaner Münch ins Teuschland außgesant Ablaß umbs Gelt zu verkauffen, damit Er Alle Schärtz in Eropa zusamen gebracht welches ist geschehen Anno 1517 Da Tetzeleus seinen verdampten Ablaß-Kram zu Gütterbach ausgeleget. Da hat Lutherus anheben darwider zu prodestieren und mit dem Schwerdt des Geistes zu fechten. Also fieng Sich der Hader an, zwischen Lutherum \& Tetzel (Schuhmacher Chronik II: 425)

Nach einer alternativen Analyse wäre der untergeordnete $d a$-Satz mit afiniter Konstruktion (vgl. Kapitel 3.3.1) von Anno 1517 abhängig. Er wäre also ein nachgestellter Nebensatz, der mit dem nachfolgenden Hauptsatz nur semantisch-pragmatisch verknüpft ist. Nach dieser Analyse wäre $D a$ am Anfang des Hauptsatzes kein Korrelat, sondern ein Temporaladverbial. Die Interpunktion unterstützt zwar diese Analyse, solche nachgestellten $d a$-Nebensätze sind jedoch im Korpustext - im Gegensatz zu anschließenden da-Hauptsätzen wie in (140) - nicht belegt.

(140) und hat daß Feur gewehret biß Mitwoch morgens umb Fünff Uhr, da hat Es auffgehöret (Schuhmacher Chronik II: 401)

Auch beim Beleg (139) ist die Analyse als Apokoinu-Konstruktion (d.h., die Abhängigkeit des $d a$-Nebensatzes sowohl von der Wortgruppe Anno 1517 als auch vom nachfolgenden 
Hauptsatz) nicht auszuschließen. Die Analyse als Apokoinu-Konstruktion legt eine rein temporale Deutung des $d a$-Satzes nahe. Bis auf diesen Einzelbeleg wurde im Korpustext in den Belegen für korrelative Wiederaufnahme von Temporalsätzen das Korrelat so verwendet.

Zusammenfassend können die Temporalsätze in den Korpustexten aus dem 18. Jh. wie folgt charakterisiert werden: In beiden Korpustexten finden sich nur relativ wenige Temporalsätze, ihr Anteil ist jedoch im nähesprachlichen Text deutlich höher. Im distanzsprachlichen Korpustext Aichinger III finden sich nur drei Beispiele für vorangestellte Temporalsätze, diese werden jedoch ausnahmslos integrativ angebunden. Im nähesprachlichen Korpustext Schuhmacher Chronik II ist der Anteil des resumptiven Typs (5 Belege) etwas höher als der des integrativen Typs (4 Belege). In den beiden Korpustexten aus dem 18. Jh. findet sich kein einziger Beleg für die nicht-integrative Stellung von vorangestellten Temporalsätzen. Wie auch bei den Korpustexten aus dem 17. Jh., so ist auch hier ein Unterschied bei der Satzkomplexität in der Einheit vor dem Obersatz zu bemerken. Im nähesprachlichen Text wird die resumptive Variante, im distanzsprachlichen Text die integrative Variante verwendet. Da aber nur Einzelbelege zur Verfügung stehen, lassen sich daraus keine sicheren Schlüsse ziehen.

\subsubsection{Temporalsätze in den Korpustexten aus dem 19. Jh.}

Die Verwendung der Temporalsätze im 19. Jh. wird aufgrund der beiden Korpustexte Auswandererbriefe $\mathrm{V}$ und Burckhardt $\mathrm{V}$ untersucht. Im distanzsprachlichen Korpustext Burckhardt V sind nur 7 einschlägige Belege zu finden. Dabei liegt bis auf einen Beleg eine singuläre Verwendung der Temporalsätze vor. Der frequenteste Subjunktor ist als (5 Belege), der im folgenden Beleg Gleichzeitigkeit ausdrückt:

(141) Aber auch in kleinem Städten strengte man sich bisweilen sehr an. Als Herzog Borso 1453 zur Huldigung nach Reggio kam, empfing man ihn am Tor mit einer großen Maschine, auf welcher S. Prospero, der Stadtpatron, zu schweben schien, überschattet durch einen von Engeln gehaltenen Baldachin (Burckhardt V: 300)

Die semantische Relation zwischen den Teilsätzen ist jedoch nicht in jedem Fall eindeutig zu bestimmen. Im folgenden Beleg könnte beim als-Satz eine Vorzeitigkeit angenommen werden, wobei auch die kausale Deutung nicht auszuschließen ist, wie dies weiter oben am Beispiel der nachdem-Sätze gezeigt wurde (vgl. Kapitel 5.1.1).

(142) Als hierauf absolute Fürsten und Tyrannen emporkamen, hatte der Adel in den meisten Städten allen Anlaß und alle Muße, sich ein Privatleben zu schaffen, welches politisch gefahrlos und mit jeglichem feinern Lebensgenusse geschmückt, dabei 
übrigens von dem der reichen Bürger gewiß kaum zu unterscheiden war. (Burckhardt $\mathrm{V}: 262)$

Im Korpusvolltext ist auch die Verwendung des Subjunktors nachdem bei vorangestellten Temporalsätzen der Vorzeitigkeit belegt. Da der folgende Satz nicht dem Korpustext entnommen wurde, sondern im Korpusvolltext belegt ist, wird er bei der quantitativen Analyse nicht berücksichtigt:

(143) auf zwei hohen Säulen standen »der Götzendienst» und die »Fides«; nachdem letztere, ein schönes Mädchen, ihren Gruß hergesagt, stürzte die andere Säule samt ihrer Puppe zusammen (Burckhardt V: 301)

Die durch als eingeleiteten Temporalsätze werden bis auf einen Beleg integrativ angeschlossen. Im Beleg (144) wird dem Matrixsatz eine sehr komplexe Einheit mit vier Nebensätzen und einer Infinitivkonstruktion vorangestellt. Am Anfang des Obersatzes steht das Resumptivum $d a$, das bei temporalen Korrelatkonstruktionen im Gegenwartsdeutschen am häufigsten verwendet wird (vgl. Pasch et al. 2003: 263f.). Im nachfolgenden Beleg (145) wird der durch einen indirekten Fragesatz erweiterte Temporalsatz integrativ angeschlossen.

(144) Und als die neue Poesie und Literatur seit Dante Sache eines jeden wurde, als vollends die Bildung im Sinne des Altertums und das Interesse für den Menschen als solchen hinzutrat, während Condottieren Fürsten wurden und nicht nur die Ebenbürtigkeit, sondern auch die eheliche Geburt aufhörten, Requisite des Thrones zu sein, da konnte man glauben, ein Zeitalter der Gleichheit sei angebrochen, der Begriff des Adels völlig verflüchtigt. (Burckhardt V: 262)

(145) Auch am Hofe Alexanders VI. kamen Turniere vor. Als Kardinal Ascanio Sforza den Türkenprinzen Dschem fragte, wie ihm dies Schauspiel gefalle, antwortete derselbe sehr weise: in seiner Heimat lasse man dergleichen durch Sklaven aufführen, um welche es, wenn sie fielen, nicht schade sei. (Burckhardt V: 266)

Der einzige ,Frequenz spezifizierende‘ Temporalsatz im Sinne von Breindl et al. (2014: 255) wird durch wenn eingeleitet und mit dem resumptiven so wiederaufgenommen. Die generische Leseart einer sich wiederholenden Handlung wird nicht durch den Subjunktor, sondern durch den Kontext gesichert.

(146) Der Reichtum an Instrumenten sodann geht besonders daraus hervor, daß es sich lohnte, aus Kuriosität Sammlungen derselben anzulegen. In dem höchst musikalischen Venedig gab es mehrere dergleichen, und wenn eine Anzahl Virtuosen sich dazu einfand, so ergab sich gleich an Ort und Stelle ein Konzert. (Burckhardt V: 284)

Im folgenden Beleg wird der vorangestellte Temporalsatz durch den Subjunktor sowie eingeleitet, der aus Ausdrücken des Vergleichs entstanden ist und im Gegenwartsdeutschen 
als Zeitpunkt spezifizierender, grenzbezogener Konnektor verwendet wird (vgl. Breindl et al. 2014: 279, 1208).

(147) darauf folgten die Geschichten des Lancelot vom See, und die "von Athen«. Und sowie der König nur in Chieri anlangte, wartete man ihm wieder mit einer Pantomime auf, die ein Wochenbette mit vornehmem Besuch darstellte. (Burckhardt $\mathrm{V}: 298)$

Hier fällt die Endgrenze des im Temporalsatz ausgedrückten Sachverhalts mit der Anfangsgrenze des im Obersatz ausgedrückten Sachverhalts zusammen (vgl. Breindl et al. 2014: 292). ${ }^{189}$ Der Temporalsatz wird integrativ angebunden.

Der Anteil an vorangestellten Temporalsätzen ist im Nähetext aus dem 19. Jh. (Auswandererbriefe V) - wie im Korpustext Burckhardt V - relativ niedrig (11 Belege). Es fällt jedoch auf, dass die Temporalsätze in den Auswandererbriefen nur sehr selten resumptiv angebunden werden. Während der Anteil des resumptiven Typs in den Nähetexten Güntzer I und Schuhmacher Chronik II 40,6\% und 55,6\% ausmachten, beträgt ihr Anteil im Nähetext aus dem 19. Jh. lediglich 18,2\% (2 Belege). Im folgenden, durch den Zeitdauer spezifizierenden Subjunktor seitdem eingeleiteten Temporalsatz wird die integrative Stellung realisiert, obwohl eine recht komplexe Einheit vor dem Obersatzfinitum steht: Der Temporalsatz wird parenthetisch erweitert, wobei die beiden Untersätze insg. 31 Wörter umfassen.

(148) Seitdem die Radicalen (südliche Repuplicaner welche von der Vereinigten Staaten Regierung nach beendigung der Rebellion hierher geschickt wurden) von den Democraten im letzten Jahre bei der Wahl der Staatsregierung geschlagen wurden gehen die Geschäfte etwas besser aber nicht wie es erwartet wurde. (Auswandererbriefe V: 2)

Auch im anderen Beleg für die Zeitdauer spezifizierende Verwendung findet sich die integrative Stellung des Temporalsatzes. Der Subjunktor solange drückt Durativität aus, im Temporalsatz wird ein Zeitintervall festgelegt, „zu dem der im externen Konnekt bezeichnete Sachverhalt kontinuierlich der Fall ist.“ (Breindl et al. 2014: 132)

(149) Solange das Fieber anhält darf der Kranke nicht auf einen Augenblick allein gelassen werden. (Auswandererbriefe V: 3)

Obwohl dieser Beleg aufgrund einer konversationellen Implikatur auch eine konditionale Deutung zulässt (vgl. Zifonun et al. 1997: 1146), wird hier die Analyse als Temporalsatz bevorzugt. Aufgrund der geringen Belegzahl ist kein direkter Zusammenhang zwischen der

189 „In Sequenzrelationen mit Grenzbezug ist die Vorstellung eines Zusammenfalls der Endgrenze des einen Sachverhalts mit der Anfangsgrenze des anderen eine Abstraktion von den wirklichen physikalischen Verhältnissen und bedeutet nicht notwendig, dass keinerlei Zeitintervall zwischen den Sachverhalten liegen darf.“ (Breindl et al. 2014: 293). 
Zeitdauer spezifizierenden Verwendung und der integrativen Stellung der Temporalsätze nachzuweisen. ${ }^{190}$

Der Subjunktor als wird nur in einem Einzelbeleg verwendet. Die semantischen Beziehungen im Satzkomplex sind in mehreren Fällen nicht eindeutig markiert, sodass neben der temporalen Interpretation auch andere Lesearten möglich sind. Im folgenden Beleg ist eine konditionale Leseart nicht auszuschließen, da der im Temporalsatz bezeichnete Sachverhalt noch nicht eingetreten ist. Weil hier jedoch weniger die Frage der Wahrheit des Untersatzes als relevant dargestellt wird, ist in diesem Fall konditional eher nur eine Nebenbedeutung. Der Beleg wird als Temporalsatz mit vager semantischer Beziehung eingestuft. Der durch sobald eingeleitete Untersatz wird mit dem Resumptivum so wiederaufgenommen, während bei einschlägigen Korrelatkonstruktionen im Gegenwartsdeutschen eher die Korrelate $d a$, dann, sobald verwendet werden (s. Kapitel 3.4.2).

(150) Sobald ich einmal das Geld beisamen habe, so gehe ich aufs Land, u. ich kaufe eine Besitzung, wo ich reich zu werden hoffe, (Auswandererbriefe V: 1)

Beide einschlägigen Belege für den obigen Subjunktor sind in diesem Korpustext zusammengeschrieben. In den älteren Korpustexten aus dem 18. Jh. (vgl. 5.1.3 bzw. 5.1.4) traten so bald bzw. so offt (wie auch in den Quellentexten von Lefèvre 2012: 497) noch getrennt auf. Der folgende Beleg zeigt die generalisierende Verwendung des durch nachdem eingeleiteten Temporalsatzes. Auch in diesem Fall sind andere Lesearten nicht auszuschließen.

(151) Hier sind die Schulen gradirt. Nachdem die Schuler durch alle Klaßen der gewöhnlichen Stadtschulen durch // gegangen sind komen sie in die Hochschule, (Auswandererbriefe V: 14)

Im folgenden Beleg ist eine kausale Nebenbedeutung möglich, der Subjunktor markiert jedoch eher eine temporale Relation. In beiden Fällen werden die nachdem-Sätze integrativ angebunden.

(152) Nachdem ich lange $u$. vergeblich auf einen Brief von Euch gewartet will ich Euch nun wieder einmal schreiben. (Auswandererbriefe V: 2)

190 Es wäre jedoch aufschlussreich, aufgrund größerer Korpora des Neuhochdeutschen bzw. des Gegenwartsdeutschen zu untersuchen, welche Häufigkeit die bei Pasch et al. (2003: 263f.) aufgeführten Korrelate da (bei den Subjunktoren seit(dem) und solange), so lange/solange (beim Subjunktor solange) bzw. eventuell weitere Korrelate aufweisen. Breindl et al. (2014: 315) vermerken, dass die durativen temporalen Adverbialia „sich schlecht mit $d a$ wiederaufnehmen lassen.“ Genauere empirische Daten zur Variation und eine Erklärung des Phänomens bleiben das Desiderat weiterer Forschungen. 
Im nächsten Beleg ist keine scharfe Trennung von Temporalität bzw. Konditionalität möglich. Da die Wahrheit des Untersatzes nicht in Frage gestellt wird, kann eine generalisierende Verwendung im Sinne von ,jedes Mal wenn` angenommen werden.

(153) Wenn ich mit dem Todtenwagen eine Leiche nach dem Todtenhofe zu fahren habe, halte ich einen Mann, der 2 Pferde stellt, dem ich für jede Ausfahrt 5 Th. zu bezahlen habe. Für ärmere Leute od. Kinder nihmt man natürlich den Wagen nicht. (Auswandererbriefe V: 7)

Neben den Varianten wenn, weñ und wen ist auch wan belegt. Die semantische Beziehung ist auch diesmal nicht eindeutig, der Sachverhalt im Untersatz bezieht sich wohl nicht auf den Willen, Fotos machen zu lassen, sondern auf die Zeit des Fotografierens, wie dies der weitere Kontext (vielleicht dieses Spätjahr noch) vermuten lässt.

(154) wan̄ wir Zeit haben, wollen wir uns wieder abnehmen lassen vielleicht dieses Spätjahr noch und wollen dir sie schicken in einem Brief. (Auswandererbriefe V: 15)

In den meisten Fällen sind die vorangestellten Temporalsätze des Korpustextes syntaktisch weniger komplex. Im folgenden Beleg wird jedoch der wen-Satz mit einem kopulativ verknüpften Temporalsatz (mit Herstellung der syntaktischen Ruhelage) fortgesetzt, ${ }^{191}$ der mit einem indirekten Fragesatz erweitert wird. Diese komplexe Einheit wird am Anfang des Obersatzes resumptiv wiederaufgenommen.

(155) wen ich von der Arbeit nach Hauße kam, und frackte sie ob sie geschrieben hat, so war die Andwort nein. (Auswandererbriefe V: 20)

Im folgenden Beleg verweist auf den elliptisch realisierten Untersatz (vgl. Kapitel 3.1.1) mit fehlendem Subjektpronomen und Finitum das sog. nichtresumptive Korrelat (im Sinne von Ágel 2010: 914f., s. Kapitel 3.3.1) dā̄ im Mittelfeld des Obersatzes. Dieser Beleg wird in der quantitativen Bewertung aufgrund der Vorfeldstellung des wenn-Satztes als integrativ eingestuft.

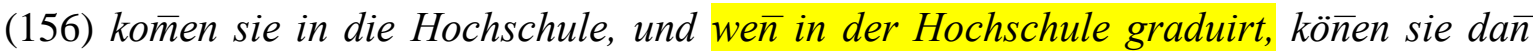
unentgeldlich die Staatsuniversität besuchen, Unsere Jugend unserer Stadt hat was Schulbildung anbelangt große Vorrechte, den sie haben die Gelegenheit in allen wisentschaftlichen Zweigen unterrichtet zu werden. (Auswandererbriefe V: 14)

Es kann festgehalten werden, dass die vorangestellten Temporalsätze in beiden Korpustexten nur selten resumptiv angebunden werden. Wegen der niedrigen Belegzahl und der relativ geringen Unterschiede im Stellungsverhalten der Temporalsätze darf man nur

191 In solchen Fällen werden die vorangestellten Teilsätze als ein Beleg verrechnet, die koordinierten Temporalsätze im obigen Beispiel können ja nur als eine komplexe Einheit in den nachfolgenden Obersatz eingefügt werden. 
vorsichtig mit Schlussfolgerungen umgehen. ${ }^{192}$ Die Daten zeigen keine größeren Unterschiede bei der Verteilung der Stellungstypen, im Distanztext scheinen jedoch die semantischen Beziehungen zwischen den Teilsätzen eindeutiger markiert zu sein. Es soll auch erwähnt werden, dass im Distanztext auch $d a$ als Resumptivum belegt ist, während in den Nähetexten des Gesamtkorpus das Korrelat so überwiegt. Auch dies zeigt eine Tendenz zur eindeutige(re)n Markierung der semantischen Beziehungen im Distanztext.

\subsubsection{Temporalsätze in den Korpustexten aus dem 20. Jh.}

Im Korpustext Lorenz VII sind lediglich 4 Belege für Temporalsätze zu finden. Diese werden, auch wenn sie zu komplexen Einheiten mit mehreren Teilsätzen erweitert werden, ausnahmslos integrativ angebunden. Im folgenden Beleg wird der vorangestellte Temporalsatz durch zwei koordinierte Infinitivkonstruktionen erweitert, bevor das Obersatzfinitum realisiert wird.

(157) Dieser alltägliche Vorgang ist ein gutes Beispiel für eine Leistung, die für unser Erkennen der außersubjektiven Wirklichkeit von grundlegender Bedeutung ist. Sie bringt unser Erkennen dem an sich Seienden dadurch näher, daß sie Vorgänge und Zustände innerhalb des erlebenden Subjekts mit ins Kalkül zieht. Sooft es uns gelingt, die Herkunft eines Teil-Phänomens in unserem Erleben auf innere, subjektive Vorgänge oder Zustände zurückzuführen und aus unserer Betrachtung der außersubjektiven Wirklichkeit auszuschalten, sind wir in unserer Erkenntnis einen kleinen Schritt näher an das unabhängig von unserem Erkennen Existierende herangekommen. (Lorenz VII: 13)

Die Aufnahmefähigkeit des Vorfeldes wird beim folgenden Beleg noch stärker in Anspruch genommen. Die jeweils mit wann eingeleiteten, koordinierten Temporalsätze werden mit einem Relativsatz bzw. mit einer Infinitivkonstruktion erweitert, wobei die im Vorfeld des Obersatz stehende komplexe Einheit 52 Wörter umfasst.

(158) Wann immer eine kleine Zunahme des Wissens über unseren Weltbildapparat eine neue kleine Korrektur des Bildes erheischt, das er von der außersubjektiven Realität entwirft, und wann immer umgekehrt ein kleiner Fortschritt unseres Wissens um das an sich Seiende uns in den Stand setzt, eine neue Kritik an unserem »perceiving apparatus« zu üben, wächst unsere Berechtigung, unsere Erkenntnistheorie für richtig zu halten, deren Natürlichkeit nicht mehr mit Naivität verwechselt werden darf. (Lorenz VII: 26)

Die beiden obigen Belege sind aus semantischer Sicht ähnlich, in beiden Fällen liegt eine Frequenzspezifikation (iterativ) mit der Bedeutung ,jedes Mal wenn` vor. Bezüglich der

192 Würde man den Einzelbeleg für das nichtresumptive Korrelat in den Auswandererbriefen nicht als integrativ, sondern resumptiv einstufen, wäre der Anteil des resumptiven Typs im Nähetext etwas höher als im Distanztext. 
Subjunktoren lässt sich jedoch ein wichtiger Unterschied beobachten: Bei dem (etymologisch auf den Ausdruck des Vergleichs zurückführbaren) sooft ist das temporale Adverbial oft nicht mehr als eigenständige Einheit aufzufassen, sondern ist als Teil des Subjunktors lexikalisiert (vgl. Breindl et al. 2014: 387). Das getrennt geschriebene wann immer ist dagegen nicht als Subjunktor grammatikalisiert. Für die vorliegende Untersuchung sind auch Temporalsätze einschlägig, denen unmittelbar ein temporales Adverb als Modifikator vorangestellt wird (vgl. Kapitel 4.2, s. auch die Belege (163) sowie (164)). Im folgenden Beleg wird eine komplexe Einheit von zwei koordinierten Temporalsätzen integrativ angebunden:

(96) Einen einzelnen Stern, der durch eine kleine Lücke in der Wolkendecke scheint, vermag man nicht zu benennen; erst wenn ein Stück klaren blauen Himmels größer wird und man auf ihm mehrere Sterne in ihrer räumlichen Beziehung zueinander sieht, ist man in der Lage, dieses Muster mit einem bestimmten Teil der bekannten Sternekarte zur Deckung zu bringen, mit ihm zu identifizieren. (Lorenz VII: 41)

Im Gegensatz zum Distanztext dominieren im Nähetext aus dem 20. Jh. (Jugendtagebuch VII) syntaktisch einfache Voranstellungen.

Im Korpustext Jugendtagebuch VII sind 19 Belege für Temporalsätze zu finden, davon weisen 4 Belege einen komplexen bzw. erweiterten Temporalsatz auf. Selbst beim längsten, parenthetisch erweiterten, vorangestellten Temporalsatz (159) wird die durchschnittliche Satzlänge der Temporalsätze aus dem Distanztext nicht erreicht (19 vs. 28 Wörter). Bezüglich des Stellungsverhaltens zeigen sich jedoch keine Unterschiede: Alle einschlägigen Belege aus dem Nähetext weisen - unabhängig von semantischen und syntaktischen Faktoren - eine integrative Stellung auf. Dies ist auch bei komplexen Voranstellungen der Fall:

(159) also M. war echt saumäßig süß. Er grinste einen so lieb an, tja und ich bin halt auch nur ein Mädchen (nein, nicht ein LIEBESBLITZ!!) L. ist MEIN PERFEKTER TRAUM) Als ich nun am so kurz vor der Abfahrt einen Sauerkraut schlabberte (ich saß gegenüber zur stets geöffneten Küchentür) stand dort der 3. Mr. X, na, ich nenn' ihn mal G., und grinste mich zwischen den Handtüchern. (Jugendtagebuch VII: 9f.)

Die meisten vorangestellten Temporalsätze werden durch als eingeleitet (11 Belege). Beim Ausdruck der Vorzeitigkeit wird neben dem Subjunktor als auch der Subjunktion nachdem verwendet, der die semantischen Beziehungen innerhalb des Satzkomplexes eindeutiger markiert (1 Beleg). Beim unspezifisch situierenden Subjunktor wenn kann neben Zeitpunktspezifikation bzw. Frequenzspezifikation auch eine konditionale Nebenbedeutung auftreten. Beim folgenden Beleg ist eine temporale Leseart anzunehmen, da sich keine Hinweise darauf finden, dass es in der aktuellen Gesprächssituation für die Textproduzentin denkbar ist, dass der im Temporalsatz bezeichnete Sachverhalt nicht zutrifft. 
(160) Ich liebe ihn sehr, und ich glaube auch nicht, daß es zu früh ist. Die einzige riesengroße klitzekleine Hürde, die leider noch nehmen muß ist das Gespräch mit Mama und der Frauenarzt, dann die Zeit, bis man die Pille hat, die zu einem paßt. Wenn das alles überstanden ist, ist sicher auch noch ein Monat vorbei. (Jugendtagebuch VII: 7)

Im folgenden Beleg ist eine konditionale Leseart nicht auszuschließen, da der im Temporalsatz bezeichnete Sachverhalt noch nicht eingetreten ist. Dabei ist jedoch weniger die Frage der Wahrheit des Untersatzes relevant, sondern die Zeit des geplanten Beischlafs.

(161) [Wenn die Umstände anders gewesen wären, P.K.] hätten miteinander geschlafen. Es blieb bei der Vorstufe und das ist ja auch gut so. Wenn wir miteinander schlafen brauchen wir absolute Ruhe! (Jugendtagebuch VII: 14)

Sowohl der obige als auch der folgende Beleg werden als Temporalsatz eingestuft. Eine konditionale Deutung ist auch beim folgenden Beleg denkbar, aufgrund des Frequenzadverbials im wenn-Satz ist jedoch eher eine iterative Lesart anzunehmen. Der vorangestellte Adverbialsatz referiert auf wiederkehrende Zeitintervalle, nicht auf die Gültigkeit des Antezedens.

(162) Er hat keine anderen Kumpels. Er soll doch mal was ohne mich unternehmen. Ich weiß, wenn ich So abends nichts mit ihm unternehme sitzt er gelangweilt herum. (Jugendtagebuch VII: 8)

Wie im entsprechenden Distanztext (s. Beleg (158) weiter oben), so ist auch im Jugendtagebuch VII eine Erweiterung durch einen restriktiven Modifikator belegt (vgl. Pasch et al. 2003: 411, Breindl et al. 2014: 295f., 757). Während im nächsten Beleg durch das Temporaladverb immer die Frequenzbezogenheit ausgedrückt wird, bezeichnet kurz im übernächsten Beleg ein Ausmaß (zu immer wenn s. auch Kapitel 3.4.2).

(163) Da wird ein Haus gebaut und immer wenn ein Arbeiter einem anderen einen Stein gibt geht es "bitteschön, dankeschön, bitteschön, dankeschön... “. (Jugendtagebuch VII: 17)

(164) Dabei: Papa, Mama, R., N., und L. Kurz bevor mein Zug kommen sollte ging er. Eigentlich wäre ich geschockt gewesen, aber ich wußte, daß er mit mir bis nach Stuttgart fahren würde (von Mama). (Jugendtagebuch VII: 16)

Im folgenden Beleg für eine elliptische Realisierung des Temporalsatzes fehlen neben dem Hauptsatzfinitum das Subjekts- und das Objektspronomen.

(165) So ist er (D.) vom Krankenhaus in die Nervenklinik verlegt worden. Kaum verlassen, war er auch wieder drin - erneutes Koma. Nun soll er nach Tübingen verlegt werden! (Jugendtagebuch VII: 1) 
Ob kaum - im Gegensatz zu kaum dass - als Subjunktor aufzufassen ist, ist strittig (vgl. Breindl et al. 2014: 361). Durch kaum eingeleitete, finite Nicht-Verbletztsätze werden im Modell der expliziten Junktion (Ágel 2010: 912f.) als Subjunktorersatz aufgefasst (vgl. auch Kapitel 3.3.1). Der obige Beleg stellt eine reduzierte Form des Subjunktorersatzes dar. Solche Strukturen können wie voll ausgebaute Nebensätze ins Vorfeld des Obersatzes integriert werden.

Zusammenfassend ist festzustellen, dass die vorangestellten Temporalsätze in beiden Korpustexten aus dem 20. Jh. nur integrativ angeschlossen werden. Relevante Unterschiede zwischen den beiden Texten zeigen sich nur bei der Aufnahmefähigkeit des Vorfeldes bzw. bei der Eindeutigkeit der semantischen Beziehung zwischen den Teilsätzen.

\subsubsection{Zusammenfassung}

Im Gesamtkorpus liegt kein eindeutiger Beleg für die nicht-integrative Stellung von vorangestellten Temporalsätzen vor. Dies steht sicherlich auch damit im Zusammenhang, dass sich Temporalsätze i.d.R. auf die propositionale Ebene beziehen. ${ }^{193}$

\begin{tabular}{|l|c|c|c|}
\hline & integrativ & resumptiv & nicht-integrativ \\
\hline \multirow{2}{*}{ Güntzer I (n=64) } & 38 & 26 & 0 \\
& $59,4 \%$ & $40,6 \%$ & $0 \%$ \\
\hline \multirow{2}{*}{ Harsdörffer I (n=4) } & 4 & 0 & 0 \\
& $100 \%$ & $0 \%$ & $0 \%$ \\
\hline \multirow{2}{*}{ Schuhmacher Chronik II (n=9) } & 4 & 5 & 0 \\
& $44,4 \%$ & $55,6 \%$ & $0 \%$ \\
\hline \multirow{2}{*}{ Aichinger III (n=3) } & 3 & 0 & 0 \\
& $100 \%$ & $0 \%$ & $0 \%$ \\
\hline \multirow{2}{*}{ Auswandererbriefe V (n=11) } & 9 & 2 & 0 \\
& $81,8 \%$ & $18,2 \%$ & $0 \%$ \\
\hline \multirow{2}{*}{ Burckhardt V (n=7) } & 5 & 2 & 0 \\
& $71,4 \%$ & $28,6 \%$ & $0 \%$ \\
\hline \multirow{2}{*}{ Jugendtagebuch VII (n=19) } & 19 & 0 & 0 \\
& $100 \%$ & $0 \%$ & $0 \%$ \\
\hline \multirow{2}{*}{ Lorenz VII (n=4) } & 4 & 0 & 0 \\
& $100 \%$ & $0 \%$ & $0 \%$ \\
\hline \multirow{2}{*}{ Gesamt $(n=121)$} & 86 & 35 & 0 \\
& $71,1 \%$ & $28,9 \%$ & $0 \%$ \\
\hline
\end{tabular}

Tabelle 3: Verteilung der Stellungstypen bei vorangestellten Temporalsätzen

193 Durch bevor eingeleitete Nebensätze können z.B. auch auf der Sprechaktebene verwendet werden. Es ist jedoch fraglich, ob in solchen Fällen tatsächlich eine temporale Leseart anzunehmen ist. Nach Breindl et al. (2014: 297f.) wird der Beispielsatz (i) mit nicht-integrativer Stellung konditional interpretiert:

(i) Bevor ich es vergesse: Hans hat angerufen. 
Der Anteil des resumptiven Typs hängt nicht nur vom zeitlichen Parameter, sondern auch vom Nähe-Distanz-Parameter ab. Der Zusammenhang zwischen den Zeitabschnitten und den Stellungstypen ,resumptiv ${ }^{\star}$ und ,integrativ ${ }^{`}$ ist statistisch signifikant (P-Wert $\left.=0,010635\right)$. Das Ergebnis, dass Temporalsätze noch im Neuhochdeutschen zur zunehmenden Integration tendieren, lässt sich auf in der bisherigen Forschung nicht beachtete Nähetexte mit einem hohen Anteil des resumptiven Typs zurückführen, bei diesem Teilkorpus ist die Signifikanz des Ergebnisses, dass mit der Zeit der integrative Typ häufiger wird, noch höher $(\mathrm{P}-\mathrm{Wert}=$ 0,007414). Bei Temporalsätzen im Distanzkorpus ist jedoch keine Tendenz zur zunehmenden Integration zu beobachten, sie werden in den einschlägigen Korpustexten bis auf zwei Belege (Burckhardt V) nur integrativ angebunden (insg. 18 Belege). Im Gegensatz dazu erscheint der ,resumptive“ Typ in den beiden Nähetexten aus dem 17. bzw. 18. Jh. noch mit einem Anteil von 40,6\% bzw. 55,6\%. Im Nähetext aus dem 19. Jh. ist der Anteil des resumptiven Typs etwa derselbe wie im entsprechenden Distanztext. In den beiden Korpustexten aus dem 20. Jh. werden Temporalsätze ausschließlich integrativ angebunden. Das Ergebnis, dass die Verteilung der Stellungstypen in Nähe- und Distanztexten unterschiedlich ist, ist auf der Grenze der statistischen Signifikanz (P-Wert = 0,070809). Die Irrtumswahrscheinlichkeit von ca. $7 \%$ ist v.a. auf die niedrige Belegzahl in den Distanztexten zurückzuführen. ${ }^{194}$ Zur prozentualen Verteilung der Stellungstypen bei vorangestellten Temporalsätzen in den Nähebzw. Distanztexten s. die Diagramme 1 und 2 im Anhang (8.2).

In den Nähetexten ist der Gebrauch des Korrelats so dominant (bis auf je eine Ausnahme vom resumptiven $d a$ nach einem $d a$-Satz in Schuhmacher Chronik II sowie einem als-Satz in Güntzer I). In den Distanztexten liegen insg. nur zwei Belege vor. Bei Burckhardt V werden je einmal so bzw. da als Korrelat verwendet. Bezüglich der Distanztexte können aus der Wahl der resumptiven Elemente aufgrund der geringen Belegzahl keine weiteren Schlüsse gezogen werden.

\subsection{Konditionalsätze}

Dieser Nebensatztyp ist im Korpus mit den meisten Belegen vertreten. Der Anteil an Konditionalsätzen ist jedoch in den einzelnen Teilkorpora unausgeglichen, vgl. die folgende Tabelle:

\footnotetext{
${ }^{194}$ Eine erneute Stichprobe aus Distanztexten könnte, wenn sie eine ähnliche Verteilung der Stellungstypen liefert, das Ergebnis bestätigen.
} 


\begin{tabular}{|l|l|l|l|}
\hline & Nähe & Distanz & insg. \\
\hline 17. Jh. & $\begin{array}{l}\text { Güntzer I } \\
12 \text { Belege }\end{array}$ & $\begin{array}{l}\text { Harsdörffer I } \\
\text { 42 Belege }\end{array}$ & 54 Belege \\
\hline 18. Jh. & $\begin{array}{l}\text { Schuhmacher Chronik II } \\
1 \text { Beleg }\end{array}$ & $\begin{array}{l}\text { Aichinger III } \\
\text { 21 Belege }\end{array}$ & 22 Belege \\
& $\begin{array}{l}\text { Auswandererbriefe V } \\
\text { 20 Belege }\end{array}$ & $\begin{array}{l}\text { Burckhardt V } \\
12 \text { Belege }\end{array}$ & 32 Belege \\
\hline 19. Jh. & $\begin{array}{l}\text { Jugendtagebuch VII } \\
7 \text { Belege }\end{array}$ & $\begin{array}{l}\text { Lorenz VII } \\
\text { 22 Belege }\end{array}$ & 29 Belege \\
\hline insg. & 40 Belege & 97 Belege & 137 Belege \\
\hline
\end{tabular}

Tabelle 4: Verteilung der Belege für vorangestellte Konditionalsätze in den Korpustexten bzw. in den Teilkorpora

Insgesamt finden sich häufig Belege in den untersuchten Distanztexten, in den Korpustexten aus dem 17. und 20. Jh. sogar mehr als dreimal so viele als in den entsprechenden Nähetexten. In Schuhmacher Chronik II ist nur ein Einzelbeleg zu finden. Nur die Korpustexte aus dem 19. Jh. verhalten sich diesbezüglich umgekehrt, hier fallen im Vergleich zu den anderen Korpustexten des jeweiligen Teilkorpus die relativ hohe Anzahl der einschlägigen Belege im Nähetext und die relativ niedrige Anzahl im Distanztext auf.

Es soll betont werden, dass ,Irrelevanzkonditionale“ (vgl. Kapitel 3.4.3) an dieser Stelle nicht berücksichtigt sind, sie werden im Kapitel 5.4.5 behandelt.

\subsubsection{Konditionalsätze in den Korpustexten aus dem 17. Jh.}

Die meisten Konditionalsätze finden sich in Harsdörffer I (insg. 42 Belege). Dabei überwiegen zwar die eingeleiteten Konditionalsätze (30 Belege), der Anteil an uneingeleiteten Konditionalsätzen (12 Belege) ist jedoch mit 28,6\% fast doppelt so hoch wie im Nähetext aus dem 17. Jh. (16,7\%, 2 von 12 Belegen). Der frequenteste konditionale Subjunktor in Harsdörffer I ist wann (26 Belege von 30 eingeleiteten Konditionalsätzen), der in hypothetischer Verwendung häufiger vorkommt als in kontrafaktischer. Die Offenheit bzw. Ungültigkeit des Antezedens wird in den folgenden Fällen durch Konjunktiv II (wäre) bzw. mit Hilfe eines Modalverbs (sollte) + Infinitiv Perfekt (gedruckt haben) markiert:

(166) Wann eine Furstin/ Gräfin/ oder Freyfrau ihres Herrn oder Gemahls Stammens wärel und nach Geburt gleichformige Ttul führel wuerden ihr gleich nach dem Tauffnamen alle Herrschafften und Ehrwort/ vermöge ihres Herrn und Gemahls Titul/ ebenmässig zugeschrieben/ mit Auslassung des Worts: Geborene R. von/ ... (Harsdörffer I: 30) 
(167) Wann man von Anfang der Welt sollte Bücher gedruckt haben/ so würden derselbigen noch viel eine grossere Anzahl zu finden seyn; ... (Harsdörffer I: 15)

In den folgenden Fällen ist die Trennung zwischen temporaler und konditionaler Verwendung nicht ganz eindeutig (vgl. Kapitel 5.1.1). Das Antezedens in Beleg (168) könnte eventuell im Sinne von, jedes Mal wenn' gedeutet werden und somit als generalisierende, temporale Verwendung identifiziert werden. Da aber die Gültigkeit des Antezedens in diesem Fall offen ist, wurde bei diesem Beleg für die Einstufung als Konditionalsatz entschieden.

(168) Wann ihr von Gottes wegen mit mir redet/ so solt ihr die eitlen Titul auslassen/... (Harsdörffer I: 27)

(169) ... daß mann mehrgemelde Schreib-Kunst mit dem Senffkorn vergleichen kann/ welches das kleineste ist unter allen Samen: wann es aber bekleibet und auffwächst/ so ist es das gröste unter allen Kohlkräutern; ... (Harsdörffer I: 16)

Der indikativisch realisierte Adverbialsatz aus dem Beleg (169) situiert den im Obersatz ausgedrückten Sachverhalt nicht zeitlich, da nicht aus jedem Samen eine Pflanze wird, bzw. da das Zeitintervall des Untersatzes nicht näher festgelegt wird. Deshalb wird an dieser Stelle eine konditionale Relation angenommen.

Bei wann-Sätzen ist der resumptive Stellungstyp häufiger anzutreffen als der integrative (14 vs. 11 Belege). Bei komplexeren Voranstellungen kommt der resumptive Typ in mehreren Fällen vor, aber auch der integrative Stellungstyp ist belegt (vgl. Beleg (166)). Als konditionaler Subjunktor ist auch wenn belegt. In beiden einschlägigen Belegen tritt ein resumptives so im Vorfeld des Obersatzes auf.

(170) Denn wenn ich sage Glük / so gibt es einen groben / langsamen Ausspruch / wie bey den Schlesiern und Voigtländern zu finden. (Harsdörffer I: 468)

(171) Wenn ich aber sage Glück / so gehet es hastig / und resch von der Zunge / und ist lieblicher anzuhoren. (Harsdörffer I: 468)

Die beiden konditionalen Subjunktoren wann und wenn scheinen Varianten ohne Bedeutungsunterschied zu sein (vgl. auch Behaghel 1928: 342), sie sind weitgehend austauschbar, was aus dem gleichen Verwendungskontext der Belege (170), (171) bzw. (172) leicht abzulesen ist.

(172) Wann ich sage ein Kartenspiel/ so weiß ich/ daß ein Spiel sey/ welchesman mit der Karten spielet: ... (Harsdörffer I: 565)

Auch bei diesem Vergleichsbeleg (172) ist die Vorfeldstellung des resumptiven Elements so zu beobachten. Die Wahl des resumptiven Stellungstyps könnte auch dadurch bedingt sein, dass in den obigen Konditionalsätzen ein Substantiv ((170), (171)) bzw. eine 
Substantivgruppe (172) ausgeklammert wird. Es liegen zwei weitere Belege für Ausklammerung vor, auch in diesen Fällen wird der resumptive Typ gewählt.

Neben wenn/wann treten weitere konditionale Subjunktoren im Korpustext nur vereinzelt vor. Im folgenden Beleg wird der vorangestellte Adverbialsatz durch in dem eingeleitet und integrativ angebunden. Eine temporale Verwendung, die bis zum Ende des 18. Jhs. üblich war (vgl. Behaghel 1928: 190), oder eine modal-instrumentale Bedeutungsrelation, die im Gegenwartsdeutschen beim Subjunktor indem ebenfalls möglich oder gar prototypisch ist (vgl. Breindl et al. 2014: 588, Duden-Grammatik 2016: 640, Fabricius-Hansen 2011: 21), kann bei diesem Beleg wohl nicht angenommen werden:

(173) In dem nun beyde Grundseul der Schreibrichtigkeit nicht ergreiffen/ muß man sich an die in Cantzleyen beliebten Gewohnheiten halten/ und lieber mit andern fehlen/ als allein klug seyn wollen/ und mit guten Ursachen verlachet werden. (Harsdörffer I: 467)

Ginge man davon aus, dass nun im vorangestellten Adverbialsatz auf die Gültigkeit des Antezedens schließen lässt, so wäre eine kausale Interpretation möglich. Aus dem Linkskontext geht es jedoch eindeutig hervor, dass an dieser Stelle eine gewisse Reihenfolge bei den Prinzipien der Rechtschreibung aufgestellt wird und der Inhalt des Satzes wie folgt zu paraphrasieren ist: Wenn weder die Etymologie noch die Aussprache (= die beiden „Grundseul“ der Rechtschreibung) ausschlaggebend ist, dann muss man sich an die in Kanzleien üblichen Gewohnheiten halten. Ein Konditionalsatz mit vergleichbarem Inhalt wird durch wann eingeleitet, was ebenfalls die konditionale Deutung des Belegs (173) nahelegt:

(174) Wann solcher Ursprung ermangelt/ so muß die Aussprache Richter seyn/.... (Harsdörffer I: 467)

Der Beleg (173) ist daher als faktisches Konditional zu deuten, das zwischen Kausalität und Konditionalität einzuordnen ist (vgl. die entsprechende Analyse begründender wenn-Sätze von Gohl 2002b).

In einem Einzelbeleg wird der Konditionalsatz nicht durch einen Subjunktor eingeleitet. Der Ausdruck im Fall wird zwar mit Verbendstellung realisiert, es handelt sich aber wohl um einen Vorläufer der komplexen konnektoralen Verknüpfung im Fall(e), dass bzw. im Falle als Verbzweitsatz-Einbetter (vgl. Breindl et al. 2014: 692, 782):

(175) ... und wird verhoffentlich in keinem verfehlet seyn; Im Fall aber solches aus ungleichem Bericht beschehen/ ist es ohne Willen und praejudicirlichen Nachtheil/ zu entschuldigen (Harsdörffer I: 31) 
Bei uneingeleiteten Konditionalsätzen taucht der integrative Stellungstyp viel seltener auf ( 2 vs. 10 Belege). In beiden Fällen handelt es sich um syntaktisch einfache Voranstellungen, vgl. die folgenden Belege:

(176) Solten auch andere ein anstädgeres Mittel vorschlagen konnen/ wollen wir denselben gerne beypflichten/ ... (Harsdörffer I: 169)

(177) Ist aber etwan ein freyes Wort in Behandlung unstrâfflichem Liebs-Hôflichkeit eingeschlichen / wird doch solches in folgendem Antwortschreiben genugsam hintertrieben / und beantwortet seyn / und sind wir zu solchem Schreiben an Frauenzimmer / veranlasst worden/durch das Exempel anderer / welche von diesem / von dergleichen/ wiewol gar gezwungen aus dem Frantzosischen/ oder gar zu unvernemlich und verdrießlich davon gehandelt haben. (Harsdörffer I: 368)

Die meisten uneingeleiteten Konditionalsätze stellen syntaktisch einfache Voranstellungen dar und werden resumptiv angebunden. Im Vorfeld des Obersatzes steht stets das Korrelat so, vgl. den folgenden Beleg:

(178) Schreibt man an seines gleichen/so pflegt man sie zu ehren wie wir von ihnen wollen geehret seyn/ und soll sie zum wenigsten geirzt und geherret oder mit ihnen in der dritten Person geredet werden. (Harsdörffer I: 30)

Der obige Beleg ist - wie die meisten einschlägigen Belege bei konditional-hypothetischer Verwendung - als ,generisch/generalisierend“ (vgl. Zifonun et al. 1997: 2282f.) einzustufen.

Im folgenden uneingeleiteten Konditionalsatz kommt zum Vorschein, dass finale und konditionale Verhältnisse verwandt sind. Das finale Verhältnis ist nämlich nach Zifonun et al. (1997: 2291, 2294f.) konditional fundiert.

(179) Soll unsre teutsche Sprache zu hochster Ubertrefflichkeit gelangen/ so wird auch diesem und vielen andern Stücken/von den Bücherschreibern/ und Drucksetzern ein mehrer Fleiß/ als bishero/ angewendet werden müssen. (Harsdörffer I: 564)

Das zugrundeliegende Konditionalschema lautet, wenn Bücherschreiber und Drucksetzer fleißiger daran arbeiten, dann wird unsere deutsche Sprache zu höchster Übertrefflichkeit gelangen` $\left(, p \rightarrow q^{`}\right)$. Dieses Argument könnte auch im Rahmen eines finalen Verhältnisses ausgedrückt werden: Bücherschreiber und Drucksetzer müssen fleißiger daran arbeiten, damit unsere deutsche Sprache zu höchster Übertrefflichkeit gelangt. Der obige Beleg könnte also durch einen Finalsatz umschrieben werden, in der vorliegenden Struktur liegt jedoch eine konditionale Beziehung vor: Wenn man den im vorangestellten Adverbialsatz ausgedrückten Sachverhalt erreichen möchte (und dieser Wunsch gilt als gegeben), dann sind die im Obersatz aufgeführten Handlungen notwendig. 
Der einzige, mit einem Untersatz erweiterte, uneingeleitete Konditionalsatz wird resumptiv angebunden:

(180) Betrachtet man den geringen Anfang des Schreibens/ welcher in geraden und krummen Strichlein bestehet/ so muß man sich billich verwundern/ daß eine geringe Sache so grosse Dinge leisten kann. (Harsdörffer I: 16)

Der Gebrauch des uneingeleiteten Konditionalsatzes steht der pragmatischen Verwendung nahe. Er könnte als ,diskursstrukturierenden“ Konditionalsatz eingestuft werden, denn er „hat vor allem die Funktion, die Aufmerksamkeit des Hörers in eine bestimmte Richtung zu lenken“ (Pittner 2000: o.S., vgl. auch Günthner 1999). Dieser Beleg kann jedoch auch als ,rein` konditional eingestuft werden, wenn man davon ausgeht, dass der Wahrheitswert des Konsequens von dem des Antezedens abhängt.

In Güntzer I sind 10 Belege für vorangestellte, eingeleitete Konditionalsätze zu finden. In den meisten Fällen werden sie durch den Subjunktor so eingeleitet und durch das Korrelat so wieder aufgenommen, sodass eine gewisse Parallelität zwischen Obersatz und Untersatz entsteht, vgl. den folgenden Beleg:

(181) Aldar wahr gleich ein Dorff. Schieß also meinen Busser lo $\beta$, daß die Kugel in eine Eichen ging, undt sprach zu den zweyen Merdtern: Saet ihr es, ihr Merdter, so ihr noch einmall an unß angesetz hetten, so wolt ich einem diße Kugel in Leib geschoßen haben. (Güntzer I: 43v)

In diesem Beleg ist die kontrafaktische Verwendung aufgrund der Konjunktivform hetten eindeutig zu erkennen. Im folgenden Beleg wird zwar sowohl im Obersatz als auch im Untersatz die finite Verbform hatten verwendet, aufgrund des Kontextes und des Modalwortes vileicht kann jedoch (trotz des Fehlens einer eindeutig erkennbaren Konjunktivform) eine kontrafaktische Verwendung angenommen werden.

(182) So sie mein Gelt gewußt hatten, so hatten sie mich vileicht ermordet, dan sie haben sich auch in dißem Grieg auffgehalten. (Güntzer I: 54r)

Bei der Identifizierung der hypothetischen Verwendungen kann auch die Verwendung von Modalverben ausschlaggebend sein. Im folgenden Beleg ist eine Abgrenzung von der temporalen Verwendung aufgrund der finiten Verbform solt möglich, da sie die Gültigkeit des Antezedens offen lässt (vgl. Kapitel 4.2 bzw. 5.1.1).

(104) So ein Waltforster solt zu eich komen, wirdt er eich gefenglich nacher Hagenau fieren. (Güntzer I: 41r) 
In (104) ist der vorangestellte Konditionalsatz ${ }^{195}$ in den übergeordneten Satz integriert. Die durch so eingeleiteten, vorangestellten Konditionalsätze werden bis auf diese Ausnahme resumptiv angebunden. Der nicht-integrative Stellungstyp ist bei propositionsbezogenen Konditionalsätzen nicht belegt (vgl. aber Kapitel 5.5).

Die vorangestellten Konditionalsätze sind in den meisten Fällen syntaktisch weniger komplex. Es finden sich nur zwei Voranstellungen mit koordinierender Verknüpfung, diese werden - wie auch die meisten syntaktisch einfachen Konditionalsätze - resumptiv angebunden. Der folgende Beleg weist eine aggregative Koordinationsellipse auf, da im zweiten, mit und verknüpften Untersatz das Subjektspronomen ich weggelassen wurde, obwohl es vorher im ersten Untersatz formal nicht realisiert ist. Das Subjekt des ersten Untersatzes ist nämlich der Babst.

(183) Habe aber die Wahrheitt gespartt, dan ich und die Grallen haben den Babst niemallen gesehen, dan so mich der Babst gesehen hatt und [ich] verratten wehr worden, so dirffte er mich im Rauch gen Himel geschickt haben. (Güntzer I: 63r)

Der folgende Beleg enthält keinen Subjunktor, der die Inhaltsrelation zwischen dem vorangestellten Verbletztsatz und seinem Obersatz anzeigen könnte. Da aber das Fehlen des Subjunktors nicht durch eine besondere Wortstellung kompensiert wird (,Subjunktorersatz', vgl. Ágel 2010: 912f.), sondern der vorangestellte Teilsatz mit Verbendstellung realisiert wird, scheint hier kein uneingeleiteter Konditionalsatz vorzuliegen. Vielmehr ist es möglich, dass bei der Häufung der einsilbigen Wörter der Subjunktor so (der sich formal dem Subjektspronomen sie ähnelt) unrealisiert bleibt.

(184) Die Bauren reittet[e]n unß nach, wolten un $\beta$ dodt schlagen, sagten, wihr hetten $i[h]$ nen Weißgezig von Leinwatt von der Pleich gestollen. Sie zogen un $\beta$ au $\beta$, suchet[e]n alenthalben bey un $\beta$, aber es hats sich nicht erfunden. Und sie es bey un $\beta$ finden werden, so wollen sie un $\beta$ gleich alsobalt ohn ale Genadt an die Beim auffhencken. (Güntzer I: 55v)

In Güntzer I sind zwei uneingeleitete Konditionalsätze mit Verberststellung belegt, beide werden resumptiv angebunden. Im folgenden Beispiel ist allerdings die Abgrenzung von der temporalen Verwendung fraglich:

(185) Von dißer Statt Prunßburck lag nicht weitt von der Portten eine Heidt und Walt. Dahin ging ich alen Abent, wan ich von meiner Arbeidt Fei[e]rabent hatt, darmit ich weitt von Leidten wahr und mich niemandt sahe. Ist es dan ein Fei[e]rdag, so wahr ich balt den gantzen Dag in dißem Walt oder auff andern Heidten, da mich niemand

\footnotetext{
195 Der durch so eingeleitete Vordersatz weist nur scheinbar eine Verbzweitstellung auf. Auch in anderen Nebensätzen mit zweigliedrigem Prädikat ist die Voranstellung des Finitums belegt (vgl. z.B. Beleg (115) weiter oben). Das Besondere im vorliegenden Beleg ist die Stellung der nicht-verbalen Einheiten als Erweiterung des Vollverbs zwischen den beiden Teilen des Verbalkomplexes, die im 17. Jh. noch belegt ist (vgl. Ágel 2000: 1878).
} 
sahe, und ruffete dan mit heller Stim zu Gott in der Heen um Trost, Hilff und Errettung. Ich wahr offt so miede, schwach und chrafftloß von dem Reden und Schreyen zu Gott, daß ich offt eine gantze Stunde auff der Erden lag. (Güntzer I: $101 \mathrm{v}-102 \mathrm{r}$ )

Am Anfang der Textstelle wird beschrieben, dass Güntzer jeden Abend, nach Dienstschluss, aus der Stadt in den umliegenden Wald ging. Obwohl der wan-Satz im Linkskontext syntaktisch als Attribut zu alen Abent aufzufassen ist, drückt er semantisch eine temporale Beziehung aus. Auch beim uneingeleiteten V1-Satz könnte angenommen werden, dass er angibt, in welchen Zeitintervallen die Aussage des Hauptsatzes gültig ist und daher temporal zu interpretieren ist. ${ }^{196}$ Er könnte somit wie folgt paraphrasiert werden: „An den Feiertagen war ich fast den ganzen Tag in diesem Wald (...)“. Auch die im weiteren Kontext auftretenden Ausdrücke wie offt lassen diese temporale Interpretation mit generalisierender Verwendung vermuten. Da jedoch uneingeleitete Adverbialsätze i.d.R. als Konditionalsätze (Verberstsatz als wenn-Subjunktorersatz) bzw. als Konzessivsätze (z.B. Nicht-Verbletzt mit mag/möge + Infinitiv als Subjunktorersatz) fungieren (Ágel 2010: 912, zum uneingeleiteten Konzessivsatz vgl. auch Kapitel 5.4.1), bzw. die konditionale Verwendung im Korpus bei uneingeleiteten Verberstsätzen mehrfach belegt ist, ist bei diesem Beleg die Annahme eines Konditionalsatzes naheliegend.

\subsubsection{Konditionalsätze in den Korpustexten aus dem 18. Jh.}

Im Korpustext Aichinger III stellen Konditionalsätze mit insg. 21 Belegen den frequentesten Nebensatztyp dar. 12 Konditionalsätze werden durch den Subjunktor wenn eingeleitet, während so viel seltener gewählt wird (3 Belege). Bei den beiden Subjunktoren findet sich je ein Einzelbeleg für den integrativen Stellungstyp:

(186) Ue, wenn es genennet wird, heißt ui; wenn es aber geschrieben wird, setzt man nach 27. §. ein e drüber oder dahinter. (Aichinger III: 25) ${ }^{197}$

(187) so sie aber zertrennet werden, muss man am Ende der Zeile gewiß ein kurzes machen, als: Aus-satz, das-selbige (Aichinger III: 14)

In diesen Fällen handelt es sich um syntaktisch einfache Voranstellungen. Beide Belege können als generalisierende, hypothetische Verwendung analysiert werden. Dies kann jedoch

\footnotetext{
196 Auch im Gegenwartsdeutschen sind V1-Sätze mit temporaler Leseart möglich, s. Breindl et al. (2014: 319f.). Der Vollständigkeit halber ist jedoch zu erwähnen, dass Temporalsätze im Gegenwartsdeutschen wohl nur seltener mit uneingeleiteten Nebensätzen mit Verberststellung ausgedrückt werden.

197 Der erste wenn-Satz (wenn es genennet wird) wird als Zwischensatz analysiert, denn das Subjekt des Obersatzes erscheint vor, der Rest des Obersatzes nach dem wenn-Satz.
} 
die Wahl des Stellungstyps nicht erklären, da es bei dieser Verwendung auch der resumptive Typ belegt ist.

Die meisten eingeleiteten Konditionalsätze werden mit so am Anfang des Obersatzes aufgegriffen (86,7\%, 13 von 15 Belegen). Bei syntaktisch komplexen Voranstellungen wird stets der resumptive Stellungstyp gewählt, vgl. den folgenden Beleg mit einem in den wennSatz eingeschobenen Relativsatz:

(188) Wenn man demnach die Wörter, so mit $\beta$ geschrieben werden, mit lauter grossen Buchstaben schreiben will, so muß man auch zwey S machen, als: GROSS, HEISST; (Aichinger III: 14)

In Aichinger III sind 6 Belege für uneingeleitete Konditionalsätze zu finden, diese werden stets mit dem Korrelat so resumptiv angebunden, vgl. den folgenden Beleg:

(189) $H$ wird nur im Anfang eines Wortes als ein Hauch ausgesprochen. Steht es hinter einem Selbstlauter, so dehnt es sich, wo ein Mitlauter darauf folgt, als: Zahn, wie Zaan, (Aichinger III: 9)

Der folgende Beleg für einen uneingeleiteten Konditionalsatz soll den Konjunktivgebrauch veranschaulichen, der bei Konditionalsätzen des Korpustextes insgesamt nur in 4 Fällen vorkommt.

(190) Man muß sich nicht irren in der Beurtheilung, welches das Stammwort sey. Wollte man schreiben flussen, fluere, in der Meinung, daß es von Fluß herkomme: so wäre es geirret: denn fliessen ist selbst das Stammwort, und Fluß nur das abgeleitete (Aichinger III: 30)

Im anderen Korpustext aus dem 18. Jh. sind keine uneingeleiteten Konditionalsätze belegt. ${ }^{198}$ Die Belegzahl der Konditionalsätze in Schuhmacher Chronik II ist im Vergleich zu den anderen Korpustexten mit Abstand am niedrigsten. Es findet sich bloß der folgende Einzelbeleg, in dem der vorangestellte ${ }^{199}$ Konditionalsatz mit dem Subjunktor wan eingeleitet wird. Der Subjunktor wird mit dem Subjektspronomen verbunden und zusammengeschrieben.

(191) So hat Er zu Ihm gesprochen, stehe auff Bruder, dein Hauß und deiner Freunden Hauß sollen verschont werden. So hat erstgemelter H. Clewer zur Antwort versetzt:

198 Im Korpustext können jedoch uneingeleitete Konditionalsätze mit Verberststellung nicht immer scharf von selbständigen Verberstsätzen getrennt werden. Der Beleg (i) wurde als selbständiger Verberst-Aussagesatz aussortiert:

(i) Siehet ein grosser Herr ein waidlichs Baüerlein im Hauffen, Männlein, spricht Er, welches Regiment gefelt dir am besten, der Bauren oder der Fürsten. Der Arm Mann spricht sehnlich, mit einem Hertzlichen Seuffzer: O mein lieber Herr, kein Messer schärffer schneit, denn wenn ein Baur deß andren Herr wird, wo bey Thomas Müntzer \& Pfeiffer und Storck über die klingen springen müssen, die Köpff in freyem Feld auff Pföl gestegt, womit die Bauren seind erschrögt. (Schuhmacher Chronik II: 426)

199 Es handelt sich nicht um eine Zwischenstellung des Konditionalsatzes, denn die Anredeform Bruder stellt eine Einheit dar, die aus der Satzstruktur in hohem Maße gelöst ist. Sie steht nicht im Vorfeld des Obersatzes, sondern im linken Außenfeld (vgl. Zifonun et al. 1997: 1577 bzw. Kapitel 3.2.1). 
Bruder wan $\beta$ soll auff daß ankom [m]en, So soll mein Hauß daß erste sein. So ist Es auff eine gnädige Capitulation ankom [m]en, (Schuhmacher Chronik II: 443f.)

Dieser Beleg zeigt, dass die strukturelle Trennung zwischen Haupt- und Nebensätzen im 18. Jh. noch nicht abgeschlossen ist. Das Finitum des Untersatzes steht nicht an der letzten, sondern direkt nach dem Subjektspronomen an der zweiten Stelle, sodass (scheinbar?) eine Hauptsatzwortstellung $\mathrm{zu}$ beobachten ist. Eine alternative Analyse besteht darin, die besondere Stellung des Finitums auf seine Voranstellung im Verbalkomplex zurückzuführen.

Es fällt jedoch auf, dass ein Standardkonditional in hypothetischer Verwendung mit dem Korrelat so resumptiv angeschlossen wird. Die Faktoren bei der Wahl des Stellungstyps können bei einem Einzelbeleg nicht näher analysiert werden.

\subsubsection{Konditionalsätze in den Korpustexten aus dem 19. Jh.}

In Burckhardt V kommen 12 (reine) Konditionalsätze vor, 10 davon werden durch einen Subjunktor eingeleitet. In diesen Fällen wird immer der Subjunktor wenn verwendet. Die vorangestellten Konditionalsätze werden im Korpustext ohne Ausnahme resumptiv angebunden, wobei stets das Korrelat so auftritt. Der Einfluss des strukturellen Faktors kann nicht näher untersucht werden, da sich einfache (192) und komplexe Voranstellungen (193) bezüglich des gewählten Stellungstyps nicht unterscheiden.

(192) Die Mäßigkeit und die freie, leichte Geselligkeit schwanden zu gleicher Zeit. Wenn wir die Verfasser von Dialogen beim Wort nehmen dürften, so hätten auch die höchsten Probleme des Daseins das Gespräch zwischen auserwählten Geistern ausgefüllt; (Burckhardt V: 276)

(193) Wenn sich dagegen schon im 15. Jahrhundert Vespasiano Fiorentino dahin ausspricht, daß die Reichen ihr ererbtes Vermögen nicht vermehren, sondern ihre ganze Einnahme ausgeben sollten, so kann dies im Munde eines Florentiners nur von den großen Grundbesitzern gelten. (Burckhardt V: 265)

Die Korrelation von konditionaler Relation und resumptiver Anbindung ist so stark, dass der strukturelle Faktor keinen Einfluss auf die Wahl des Stellungstyps hat.

Die kontrafaktische Verwendung ist nicht belegt. Bei (193) liegt ein faktisches Konditional vor. Auch der folgende Beleg ist als faktisches Konditional zu interpretieren, denn weder das Antezedens noch das Konsequens hypothetisch ist.

(194) Bistümer, Domherrnstellen und Abteien wurden oft nach den merkwürdigsten Rücksichten, aber doch nicht wesentlich nach Stammtafeln vergeben, und wenn die Bischöfe viel zahlreicher, ärmer und aller weltlichen Fürstenhoheit in der Regel bar und ledig waren, so blieben sie dafür in der Stadt wohnen, wo ihre Kathedrale stand, 
und bildeten samt ihrem Domkapitel ein Element der gebildeten Bevölkerung derselben. (Burckhardt V: 261f.)

Im Mittelfeld des Obersatzes erscheint der Ausdruck dafür, der im Gegenwartsdeutschen auch als nicht positionsbeschränkter adversativer Adverbkonnektor verwendet werden kann (Breindl et al. 2014: 1183). Die adversative und die konzessive Relation sind jedoch bei diesem Beleg auszuschließen, da kein Gegensatz zwischen dem Inhalt des Unter- und Obersatzes vorliegt. Aufgrund der Faktizität des Antezedens und des Konsequens könnte allerdings auch eine kausale Relation angenommen werden. Ob der wenn-Satz in diesem Beleg eine verwirklichte Bedingung oder einen Grund ausdrückt, lässt sich schwer entscheiden. An dieser Stelle gehen wir davon aus, dass auf der Ebene der sprachlichen Kodierung ein konditionaler Subjunktor verwendet wird und der wenn-Satz (trotz der durchaus möglichen kausalen Interpretation) eine Bedingung für die Aussage im Obersatz formuliert, die wie folgt zu paraphrasieren ist: ,Die Bischöfe blieben unter der Bedingung in der Stadt wohnen, dass sie ärmer waren und nicht dem Adel angehörten'. Daher wird dieser Beleg als konditional analysiert.

Bei der weit frequenteren hypothetischen Verwendung können Konjunktivgebrauch (192) oder Modalisierungsausdrücke wie sollte (195) dazu beitragen, dass die Gültigkeit des Untersatzes offen bleibt.

(195) Das Beste tat freilich Dante selber durch sein großes Gedicht. Der toscanische Dialekt wurde wesentlich die Basis der neuen Idealsprache. Wenn damit zu viel gesagt sein sollte, so darf der Ausländer um Nachsicht bitten, indem er schlechtweg in einer höchst bestrittenen Frage der vorherrschenden Meinung folgt. (Burckhardt $\mathrm{V}: 273)$

Der wenn-Satz im obigen Beleg ist auf einen ,singulären' Sachverhalt (vgl. Zifonun et al. 1997: 2282f.) bezogen.

Die beiden Belege für uneingeleitete Konditionalsätze mit Verberststellung weisen eine resumptive Stellung auf. Im folgenden Beleg wird der uneingeleitete Konditionalsatz nicht durch weitere Untersätze erweitert, er zeigt jedoch eine relativ lange Voranstellung mit insg. 45 Wortformen.

(196) Der Hof des Pietro Riario, der von Ferrara usw. ließen es dabei gewiß nicht an der ersinnlichsten Pracht fehlen. Vergegenwärtigt man sich das szenische Talent und die reichen Trachten der Schauspieler, die Darstellung der Örtlichkeiten durch ideale Dekorationen des damaligen Baustils, durch Laubwerk und Teppiche, endlich als Hintergrund die Prachtbauten der Piazza einer großen Stadt oder die lichten Säulenhallen eines Palasthofes, eines großen Klosterhofes, so ergibt sich ein überaus reiches Bild. (Burckhardt V: 296f.) 
Die Länge der Voranstellung könnte bei der Wahl des Stellungstyps eine Rolle gespielt haben, es soll jedoch bemerkt werden, dass auch der andere Beleg (197) mit deutlich kürzerer Voranstellung (bzw. die eindeutigen Belege für eingeleitete Konditionalsätze allesamt) eine resumptive Stellung aufweisen:

(197) Suchen wir nun die wesentlichsten Vorzüge des italienischen Festwesens gegenüber dem Auslande vorläufig auszumitteln, so steht in erster Linie der Sinn des entwickelten Individuums für Darstellung des Individuellen, d. h. die Fähigkeit, eine vollständige Maske zu erfinden, zu tragen und zu agieren. (Burckhardt V: 293)

In Auswandererbriefe V kommen insg. 20 propositionsbezogene Konditionalsätze vor. Konditionalsätze werden in den meisten Fällen durch den Subjunktor wenn bzw. wann (mit den Schreibvarianten wen, we $\bar{n}$ bzw. wañ) eingeleitet. Dabei kommt wenn viel häufiger als wann vor (16 vs. 3 Belege). Zudem findet sich ein Einzelbeleg für einen vorangestellten soSatz. Uneingeleitete Konditionalsätze kommen im Korpustext nicht vor.

Der Anteil des resumptiven Stellungstyps ist doppelt so hoch wie derjenige des integrativen Typs (13 vs. 7 Belege). In den meisten Fällen wird das Korrelat so verwendet (11 Belege). In den beiden folgenden Belegen erscheint $d a$ bzw. $d a \bar{n}$ im Vorfeld des Obersatzes:

(198) und bin froh das ich in dieses Land gekomen bin aber wē̄ ich Euch noch einmal sehen würde da meitte ich Ich hätte die ganze Welt Doch ich hoffe daß wir einander wieder sehen. (Auswandererbriefe V: 26)

(199) Die Mutter soll ganz außer Sorgen sein mir könte es nicht besser gehen wen Ihr liebste Mutter wistet wie lustig als es bei uns wär dā̄ würde sie keine Sorge mehr haben. (Auswandererbriefe V: 25)

Die Wahl zwischen den Stellungstypen kann auf mehrere Faktoren zurückgeführt werden. Beim Einzelbeleg für einen vorangestellten so-Satz ist die resumptive Stellung zu beobachten:

(200) Nun so es Gott so gefällt so sollte es uns auch gefallen und wir sollten mit dem fromen Dichter sagen könen was Gott thut das ist wohlgethan. (Auswandererbriefe $\mathrm{V}: 10)$

Der konditionale Gebrauch des Subjunktors so ist im Gegenwartsdeutschen veraltet (Zifonun et al. 1997: 2281). In älteren Korpustexten (Güntzer I und Aichinger III) sind konditionale soSätze belegt, sie werden bis auf je ein Gegenbeispiel resumptiv angeschlossen.

Der Einzelbeleg für einen kontrafaktischen Konditionalsatz mit Konjunktiv Plusquamperfekt im nachfolgenden Obersatz zeigt die integrative Stellung:

(201) Zur Zeit, da ich das Gold in Papier umwechselte, stuhnd es niedrig, nehmlich ein Gold Thaler entwarf 130 in Papier, die 377 Th. entwarfen 489. in Papier, u. einige Wochen später stieg das Gold auf 140, wē̄ ich es zu dieser Zeit hätte kön̄en umwechsele, hätte ich 37 Papier Th. gewon̄en. (Auswandererbriefe V: 6) 
Im folgenden Beleg für einen irrealen Konditionalsatz ist ebenfalls die integrative Stellung zu beobachten:

(202) Uebrigens, wenn, ihr liebe Eltern!, die Uebelstände des Gesellenlebens wissen u. im rechten Lichte erkennen könntet, wurdet ihr Gott danken, daß ich nun einmal frei $u$. nicht ein ganz verdorbenes Subject nach Leib $u$. Seele geworden bin; (Auswandererbriefe V: 7)

Der Einfluss des strukturellen Faktors ist nicht eindeutig nachzuweisen. Der resumptive Typ kommt auch bei sehr kurzen, syntaktisch einfachen Voranstellungen vor, wie im folgenden Beleg:

(203) Aber Gescheftsbriefe durch andere Schreiben zu lassen, ist hier nicht Mode, wenn einer das thut, so wird er nicht als Gescheftsmann angesehen, $u$. so behelfe ich mir selbst so gut ich kann. (Auswandererbriefe V: 7)

In den Belegen für vorangestellte Konditionalsätze, die (min.) einen Untersatz enthalten, kommen zweimal der resumptive und einmal die integrative Stellung vor, der Anteil des resumptiven Typs ist hier also nicht höher als bei den eingeleiteten Konditionalsätzen im Allgemeinen.

(204) Wē man sich theuschen thäte, daß sich der Papier Th. nicht nach $u$. nach verwerthen u. dem Gold Tha. gleich komen würde, so würde unser beschiedenes Erbtheil zuletzt sehr klein ausfallen, dē̄ bildet euch nicht ein, daß ein Papier Th. soviel Werth habe wie ein 5 Fr. Stück, (Auswandererbriefe V: 6)

(205) Liebe Geschwister Schwager und Schwägerin Gottes Segen und Frieden zum Gruß wē̄ Euch unser schreiben Gesund antrift wie es uns verläßt wird es uns herzlich freuen, wir sind Gottlob auch alle gesund der Vater hat viele Altersbeschwerlichkeiten [...] (Auswandererbriefe V: 15)

5.2.4 Konditionalsätze in den Korpustexten aus dem 20. Jh.

In Lorenz VII kommen 18 einschlägige Belege für eingeleitete Konditionalsätze vor. Bis auf eine Ausnahme (208) werden sie stets durch wenn eingeleitet, vgl. den folgenden Beleg (206):

(206) Daß lebende Systeme um so mehr Energie schlucken können, je mehr sie schon geschluckt haben, ist selbstverständlich: Wenn es einem Lebewesen gut geht, so wächst es, und es pflanzt sich fort. (Lorenz VII: 35)

In den meisten Fällen werden eingeleitete Konditionalsätze resumptiv angeschlossen (10 Belege). Dieser Stellungstyp tritt unabhängig davon auf, ob es sich um syntaktisch einfache (206) oder komplexe Voranstellungen (207) handelt. 
(207) Wenn wir z. B. fragen: »Wozu hat die Katze spitze, krumme Krallen?" - und darauf antworten: "zum Mäusefangen", so sind Frage und Antwort Kurzfassungen für das Stellen und die Lösung eines Problems. (Lorenz VII: 38)

Für den integrativen Stellungstyp finden sich 8 Belege. Auch hier halten sich einfache Voranstellungen, in denen der Konditionalsatz der einzige vorangestellte Untersatz des obersten Obersatzes ist (vgl. z.B. (208), bzw. komplexe Voranstellungen mit mehreren Untersätzen vor dem obersten Obersatzes (vgl. z.B. (209)) die Waage (4 vs. 4 Belege).

(208) Gerade angesichts der gewaltigen Verschiedenheit all dieser Weltbildapparaturen erscheint eine Tatsache von tiefster Bedeutung: Soweit sich ihre Meldungen auf dieselbe Umweltgegebenheit beziehen, widersprechen sie einander nie. (Lorenz VII: 24)

(209) Schlechterdings alle komplexen Strukturen sämtlicher Organismen sind unter dem Selektionsdruck bestimmter arterhaltender Leistungen entstanden. Wenn der Biologe auf eine Struktur stößt, deren Funktion er nicht kennt, ist es für ihn selbstverständliche Pflicht zu fragen, worin ihre Leistung bestehe. (Lorenz VII: 38)

Die Rolle der Komplexität der Voranstellung bei der Wahl zwischen den Stellungstypen kann jedoch nicht völlig ausgeschlossen werden. Bei der kontrafaktischen Verwendung kommt der resumptive Stellungstyp nur in den Korpustexten aus dem 17. Jh. vor. Dass im folgenden Beleg das Korrelat so im Vorfeld des Obersatzes erscheint, könnte daher auch damit im Zusammenhang stehen, dass hier eine längere Voranstellung mit zwei, kopulativ verknüpften wenn-Sätzen vorliegt.

(210) Das erste dieser Probleme ist die Geschwindigkeit der Evolution. Wenn diese auf die rein zufällige Ausmerzung des Ungeeigneten angewiesen wäre und wenn es die Rückspeisungsvorgänge von Kapital- und Informationsgewinn nicht gäbe, so würde die Zeit von wenigen Milliarden Jahren, die von den Physikern aus den Zerfallszeiten radioaktiver Substanzen als das Alter unserer Planeten errechnet wurden, sicherlich nicht ausreichen für die Entstehung des Menschen aus einfachsten Organismen. (Lorenz VII: 45)

Die Wahl zwischen den Stellungstypen könnte auch von weiteren Faktoren abhängen. Bei faktischen wenn-Sätzen scheint der resumptive Stellungstyp bevorzugt zu sein. Im folgenden Beleg wird in der komplexen Voranstellung die Tatsache beschrieben, dass wir ein Blatt Papier unabhängig von der Farbe des einfallenden Lichtes immer in derselben Farbe sehen. Auf diese Tatsache wird im Mittelfeld des Obersatzes auch mit dem anaphorischem dies referiert.

(211) Von besonderem Interesse für den nach Objektivität strebenden Forscher sind jene Leistungen unserer Wahrnehmung, die uns das Erlebnis jener Qualitäten vermitteln, die gewissen Umweltgegebenheiten konstant anhaften. Wenn wir einen bestimmten 
Gegenstand, etwa ein Blatt Papier, in den verschiedensten Beleuchtungen in derselben Farbe »weiß « sehen, wobei die von ihm reflektierten Wellenlängen je nach Farbe des einfallenden Lichtes recht verschieden sein können, so beruht dies auf der Funktion eines sehr komplizierten physiologischen Apparates, der aus Beleuchtungsfarbe und reflektierter Farbe eine dem Objekt konstant anhaftende Eigenschaft errechnet, die wir schlicht als die Farbe des Gegenstandes bezeichnen.. (Lorenz VII: 22)

Auch im nächsten Beleg liegt ein faktischer wenn-Satz vor. Es handelt sich um eine Situation, in der das Antezedens erwartungsgemäß wahr sein muss, denn keiner würde ernsthaft der Aussage widersprechen.

(212) Es scheint mir abstrus, eine andere [Erklärung der Übereinstimmung zwischen den Repräsentationen der Außenwelt verschiedener Lebewesen] finden zu wollen als die, daß sich alle diese so mannigfaltig abgewandelten Formen möglicher Erfahrung auf dieselbe außersubjektive Wirklichkeit beziehen. "Wenn die Teilnehmer an unserer Diskussion«, so sagte ich einst in später Nacht im Parkhotel in Königsberg bei einer "Nachtsitzung" der Kantgesellschaft, "darin übereinstimmen, daß jetzt und hier fünf Weingläser auf dem Tische stehen, so ist es mir unverständlich, wie irgend jemand eine andere Erklärung hierfür suchen kann als die, daß, was immer sich hinter der Erscheinung $>$ Weinglas $<$ verbergen mag, tatsächlich fünffach vorhanden ist.«. (Lorenz VII: 24f.)

Der faktische Gebrauch korreliert auch im nächsten Beleg mit dem resumptiven Stellungstyp.

Auf die Faktizität des Antezedens wird im Obersatz explizit verwiesen (Tatsache).

(213) Wenn man das eine Mal den Blick auf unseren Weltbildapparat richtet und das andere Mal auf die Dinge, die er schlecht und recht abbildet, und wenn man beide Male, trotz der Verschiedenheit der Blickrichtung, Ergebnisse erzielt, die Licht aufeinander werfen, so ist dies eine Tatsache, die nur aufgrund der Annahme des hypothetischen Realismus erklärt werden kann, der Annahme nämlich, daß alle Erkenntnis auf Wechselwirkung zwischen dem erkennenden Subjekt und dem erkannten Objekt beruht, die beide gleichermaßen wirklich sind. (Lorenz VII: 26)

Das Auftreten des resumptiven Typs setzt jedoch den faktischen Gebrauch keineswegs voraus. In (206) tritt die resumptive Variante bei hypothetischer Verwendung auf, auch in diesem Fall mit dem resumptiven so.

Im Korpustext finden sich 4 Belege für uneingeleitete Konditionalsätze. Diese werden stets mit dem Korrelat so resumptiv angeschlossen, vgl. z.B. (214):

(214) Nun kann man auch jenen zuerst gesehenen Stern benennen, wofern es ein Fixstern ist. Sollte es ein Planet sein, so bedarf man einer ganzen Menge weiteren Wissens über ein »Sternenmuster « höherer, zeitlicher Ordnung, um aus der »Konstellation" auf seine Identität schließen zu können. (Lorenz VII: 41) 
Die Wahl zwischen den Stellungstypen kann also auf den strukturellen Faktor zurückgeführt werden. Während die Anbindung von eingeleiteten Konditionalsätzen variiert, wird bei uneingeleiteten Konditionalsätzen der resumptive Typ bevorzugt.

In Jugendtagebuch VII sind 5 vorangestellte, durch wenn eingeleitete Konditionalsätze und zwei uneingeleitete Konditionalsätze zu finden. Drei eingeleitete Konditionalsätze werden integrativ angebunden, vgl. den folgenden Einzelbeleg für die kontrafaktische Verwendung:

(215) Alles mußte klappen, denn wenn wir in Dresden den direkten Zug nach Stgtt verpaßt hätten, wäre es schwierig geworden heim zu kommen. (Jugendtagebuch VII: 14)

In den beiden anderen Fällen wird das Korrelat dann im Vorfeld des Obersatzes realisiert. Während der wenn-Satz in (216) eindeutig eine Satzstruktur aufweist, ist in (217) eine elliptische Struktur zu finden.

(216) Wir haben zusammen viel Scheiß gemacht und echt Spaß gehabt. Leider kann man ja draußen nicht viel machen, aber wenn ihm das nicht gefallen hat, dann weiß ich nicht, was er für Vorstellungen hat (Jugendtagebuch VII: 3)

(217) J.: „Deutschland wird nie wieder eins. Wenn ja, dann nur weil ihr sozialistisch werden werdet! “ (Jugendtagebuch VII: 12)

Der Subjunktor leitet hier keinen finiten Nebensatz ein, es liegt eine elliptische Struktur vor: Sie bezieht sich eindeutig auf die ihr direkt vorangehende Aussage (Deutschland wird nie wieder eins), deshalb ist ein entsprechender, syntaktisch ausgebauter wenn-Satz zu rekonstruieren. Es liegt ein verkürzter Adverbialsatz vor, eine ,Verdichtung der Hypotaxe“ (vgl. Auer 2002, Ágel/Hennig 2006c: 396), dieser Beleg soll also nicht aussortiert werden (vgl. Kapitel 4.2). Auch das Konsequens im wenn-dann-Gefüge ist elliptisch realisiert und mit Bezug auf den vorangehenden Sachverhalt rekonstruierbar. ${ }^{200}$ Dieser Beleg wird als resumptiver Anschluss eingestuft.

Die beiden Belege für uneingeleitete Konditionalsätze mit den Verbformen im Konjunktiv Plusquamperfekt können der kontrafaktischen Verwendung zugeordnet werden. In (218) wird der vorangestellte Verberstsatz integrativ angebunden:

(218) Naja und irgendwann lagen wir dann beide nackt da. Hätte ich meine Zwischenblutung (wegen der Pille) nicht gehäbt, hätten wir wohl glatt miteinander geschlafen! Naja, aber haben wir nicht. (Jugendtagebuch VII: 15)

Durch die Verwendung der beiden konjunktivischen Verbformen am Anfang des uneingeleiteten Konditionalsatzes bzw. direkt danach im Obersatz entsteht eine formale

\footnotetext{
200 Die syntaktisch voll ausgebaute Paraphrase klingt wohl etwas umständlich: ,Deutschland wird nie wieder eins. Wenn Deutschland doch wieder eins wird, dann wird Deutschland nur deshalb wieder eins, weil ihr sozialistisch werden werdet!' Die elliptische Variante dient der sprachlichen Ökonomie.
} 
Parallelität, die uns an die paarbildenden Einleitungselemente wie so-so erinnern lässt (vgl. Kapitel 2.2.2).

Die nicht-integrative Stellung in (219) ist wohl darauf zurückzuführen, dass zwischen dem Konditionalsatz und seinem Bezugssatz ein Einschub (Das Gefühl war so stark) auftritt.

(219) Wir merkten es auch beide, daß der Frühling ausgebrochen ist. Wir waren echt „, $G$, Geils". (,,rallig“) und wären die Umstände nicht so beschissen gewesen $\rightarrow$ Das Gefühl war so stark wir hätten miteinander geschlafen. Es blieb bei der Vorstufe und das ist ja auch gut so (Jugendtagebuch VII: 14)

Ein weiterer Grund könnte sein, dass sich die starke emotionale Beteiligung auch in der Abweichung von frequenten Satzmustern abbildet. ${ }^{201}$

Im Korpustext sind keine Belege für komplexe Voranstellungen, daher ist hier die Rolle des strukturellen Faktors bei der Wahl zwischen den Stellungstypen nicht näher zu bestimmen. Der Einzelbeleg für einen pragmatischen Konditionalsatz wird im Kapitel 5.5 behandelt.

\subsubsection{Zusammenfassung}

Zunächst soll die Verteilung der Stellungstypen bei eingeleiteten Konditionalsätzen diskutiert werden, anschließend wird auf uneingeleitete Konditionalsätze eingegangen. ${ }^{202} \mathrm{Im}$ Korpus dominieren die eingeleiteten Konditionalsätze (109 von 137 Belegen). Die nicht-integrative Stellung ist nicht belegt. Der integrative Typ kommt, wie dies der folgenden Tabelle abzulesen ist, in den Korpustexten aus dem 17., 18. sowie 19. Jh. seltener vor: Der Anteil der integrativen Anbindung liegt - mit der Ausnahme des Distanztextes aus dem 17. - zwischen $0 \%$ und 35\%, während er in den Korpustexten aus dem 20. Jh. häufiger anzutreffen ist $(44,4 \%$ sowie 60\%). Er ist nur im Nähetext aus dem 20. Jh. der frequenteste Stellungstyp.

201 Die Wahl des nicht-integrativen Stellungstyps kann auch im Sturm-und-Drang-Drama Die Kindermörderinn mit emotionaler Beteiligung erklärt werden. Obwohl im Beleg (i) eine Verknüpfung auf der propositionalen Ebene zu finden ist, wird der wenn-Satz aufgrund expressiver Hervorhebung des Prädikativs fuchswild im Obersatz nicht-integrativ angeschlossen:

(i) Lauter Rätzel! - wenn dein Vater wieder fo eine Antwort hörte, fuchswild würd er darüber: -Du weißt, er kann das hinter dem Berg halten nicht ausftehn! ich auch nicht. (Wagner III: 64)

202 Zur prozentualen Verteilung der Stellungstypen bei allen (eingeleiteten und uneingeleiteten) Konditionalsätzen in den Nähe- bzw. Distanztexten s. auch die Diagramme 7 und $8 \mathrm{im}$ Anhang (8.2). Zur differenzierten Darstellung von eingeleiteten und uneingeleiteten Konditionalsätzen s. die Diagramme 3 und 4 bzw. 5 und 6 im Anhang (8.2). 


\begin{tabular}{|l|c|c|c|}
\hline & integrativ & resumptiv & nicht-integrativ \\
\hline \multirow{2}{*}{ Güntzer I (n=10) } & 1 & 9 & 0 \\
& $10,0 \%$ & $90,0 \%$ & $0 \%$ \\
\hline \multirow{2}{*}{ Harsdörffer I (n=30) } & 13 & 17 & 0 \\
& $43,3 \%$ & $56,7 \%$ & $0 \%$ \\
\hline \multirow{2}{*}{ Schuhmacher Chronik II (n=1) } & 0 & 1 & 0 \\
Aichinger III (n=15) & $0 \%$ & $100 \%$ & $0 \%$ \\
\hline \multirow{2}{*}{ Auswandererbriefe V (n=20) } & 2 & 13 & 0 \\
& $13,3 \%$ & $86,7 \%$ & $0 \%$ \\
\hline \multirow{2}{*}{ Burckhardt V (n=10) } & 7 & 13 & 0 \\
\hline \multirow{2}{*}{ Jugendtagebuch VII (n=5) } & 0 & $65,0 \%$ & $0 \%$ \\
\hline \multirow{2}{*}{ Lorenz VII (n=18) } & $0 \%$ & 10 & 0 \\
\hline \multirow{2}{*}{ Gesamt $(n=109)$} & 3 & $100 \%$ & $0 \%$ \\
\hline
\end{tabular}

Tabelle 5: Verteilung der Stellungstypen bei vorangestellten, eingeleiteten Konditionalsätzen

Die Annahme, dass bei eingeleiteten Konditionalsätzen die integrative Stellung bevorzugt wird (vgl. König/van der Auwera 1988: 117, s. dazu Kapitel 2.1.1), ist aufgrund dieser Ergebnisse zu relativieren. Der Anteil des integrativen Typs ist in den einzelnen Korpustexten nicht ausgewogen: In den Teilkorpora für das 17. und 18. Jh. ist er in den Distanztexten, in den Teilkorpora für das 19. und 20. Jh. aber in beiden Nähetexten deutlich höher als in den Vergleichstexten aus dem jeweiligen Zeitabschnitt.

Vorangestellte Konditionalsätze werden überwiegend durch die Subjunktoren wenn bzw. wann eingeleitet (92 von 109 Belegen, 84,4\%). Im Nähetext aus dem 17. Jh. wird jedoch stets so als Subjunktor verwendet, der in anderen Korpustexten nur marginale Verwendung findet (im Distanztext aus dem 18. Jh. 3 von 15 Belegen (20\%) bzw. im Nähetext aus dem 19. Jh. 1 von 20 Belegen (5\%)). Der Anteil des integrativen Typs liegt beim Subjunktor so bei 15,4\% (2 von 13 Belegen) und fällt damit deutlich geringer aus als bei wenn/wann (30 von 92, 32,6\%). Dies kann darauf zurückgeführt werden, dass bei so bis auf wenige Ausnahmen zugunsten einer Parallelstruktur so-so der resumptive Typ bevorzugt wird. Bei der Verwendung der resumptiven Elemente erscheint in den meisten Korpustexten fast ausschließlich so. Als Alternative erscheint nur selten da/dann. Die einzige Ausnahme stellt der Nähetext aus dem 20. Jh., der in beiden resumptiven Belegen dann aufweist. 
Was den Einfluss struktureller Faktoren betrifft, so ist aufgrund der niedrigen Anzahl des integrativen Typs Vorsicht geboten. Es fällt jedoch auf, dass in den früheren Quellentexten nur syntaktisch einfache Adverbialsätze integrativ angeschlossen werden. Dabei liegt jedoch keine eindeutige Korrelation vor. Im Nähetext aus dem 19. Jh. sowie im Distanztext aus dem 20. Jh. ist der Anteil des integrativen Typs in einfachen bzw. komplexen Voranstellungen sogar gleich hoch.

Uneingeleitete Konditionalsätze sind mit 20,6\% (28 von 136 Belegen) vertreten. Der Anteil der einschlägigen Belege ist im Distanz-Teilkorpus wesentlich höher (25,8\%, 24 von 93 Belegen). Im Näheteilkorpus finden sich insg. nur 4 Belege (von 41 Belegen, 9,8\%).

\begin{tabular}{|l|c|c|c|}
\hline & integrativ & resumptiv & nicht-integrativ \\
\hline \multirow{2}{*}{ Güntzer I (n=2) } & 0 & 2 & 0 \\
& $0 \%$ & $100 \%$ & $0 \%$ \\
\hline \multirow{2}{*}{ Harsdörffer I (n=12) } & 2 & 10 & 0 \\
& $16,7 \%$ & $83,3 \%$ & $0 \%$ \\
\hline \multirow{2}{*}{ Schuhmacher Chronik II (n=0) } & 0 & 0 & 0 \\
& $0 \%$ & $0 \%$ & $0 \%$ \\
\hline \multirow{2}{*}{ Aichinger III (n=6) } & 0 & 6 & 0 \\
& $0 \%$ & $100 \%$ & $0 \%$ \\
Auswandererbriefe V (n=0) & 0 & 0 & 0 \\
& $0 \%$ & $0 \%$ & $0 \%$ \\
\hline \multirow{2}{*}{ Burckhardt V (n=2) } & 0 & 2 & 0 \\
& $0 \%$ & $100 \%$ & 1 \\
\hline \multirow{2}{*}{ Jugendtagebuch VII (n=2) } & 1 & 0 & $50,0 \%$ \\
\hline \multirow{2}{*}{ Lorenz VII (n=4) } & $50,0 \%$ & 4 & 0 \\
& 0 & $100 \%$ & $0 \%$ \\
\hline \multirow{2}{*}{ Gesamt $(n=28)$} & $0 \%$ & 24 & 1 \\
& $10,7 \%$ & & $3,6 \%$ \\
\hline
\end{tabular}

Tabelle 6: Verteilung der Stellungstypen bei vorangestellten, uneingeleiteten Konditionalsätzen

Bei der überwiegenden Mehrheit der Belege (85,7\%, 24 von 28) ist die resumptive Wiederaufnahme mit so zu beobachten. Der resumptive Stellungstyp ist damit eindeutig die unmarkierte, bevorzugte Variante (vgl. Kapitel 2.1). Im Korpus finden sich lediglich 4 Gegenbeispiele. Der integrative Typ ist mit zwei Belegen aus dem Distanztext des 17. Jhs. und einem Einzelbeleg aus dem Nähetext des 20. Jhs. vertreten ((176), (177) sowie (218)). Im Einzelbeleg für den nicht-integrativen Typ aus dem Nähetext aus dem 20. Jh. (219) konnte eine starke emotionale Beteiligung als ein möglicher Grund für die Abweichung von frequenten Satzmustern ermittelt werden. Dabei ist Van den Nest (2010: 105) zuzustimmen, 
der den nicht-integrativen Typ bei nicht-kanonischen Konditionalen im Gegensatz zu König/van der Auwera (1988: 117) nicht als „unmarked, non-favoured“, sondern als markiert einstuft. Was aber die Präferenz der einzelnen Stellungstypen bei kanonischen bzw. nichtkanonischen Konditionalen betrifft, so konnten relevante Unterschiede nachgewiesen werden. Der Vergleich der Tabellen 5 und 6 zeigt einen eindeutigen Einfluss des strukturellen Kriteriums bei Konditionalsätzen: Eingeleitete Konditionalsätze werden integrativer angebunden als uneingeleitete. Dieser Zusammenhang erwies sich als statistisch signifikant $(\mathrm{P}-\mathrm{Wert}=0,035804)$.

Das Ergebnis, dass Konditionalsätze im Neuhochdeutschen zur zunehmenden Integration tendieren, ist auf der Grenze der statistischen Signifikanz (P-Wert = 0,051979). Für den Zusammenhang zwischen den Zeitabschnitten und den Stellungstypen, integrativ ${ }^{6}$ bzw. ,resumptiv ${ }^{6}$ wurde für das Distanzkorpus ein signifikantes Ergebnis berechnet $(\mathrm{P}-\mathrm{Wert}=$ 0,038576), im Bereich der Nähetexte (insg. 40 Belege) sind jedoch weitere Untersuchungen nötig (P-Wert $=0,081248)$. Im Bereich der Konditionalsätze besteht keine statistisch signifikante Korrelation zwischen den Stellungstypen und Nähe- bzw. Distanzsprachlichkeit $(\mathrm{P}-\mathrm{Wert}=0,612417)$.

\subsection{Kausalsätze}

Vorangestellte, uneingeleitete Kausalsätze kommen im Korpus nicht vor. Der Anteil an eingeleiteten Kausalsätzen ist in den einzelnen Zeitabschnitten jeweils unterschiedlich hoch, wobei zwischen den Korpustexten aus dem jeweiligen Zeitabschnitt keine großen Unterschiede zu beobachten sind:

\begin{tabular}{|l|l|l|l|}
\hline & Nähe & Distanz & insg. \\
\hline $17 . \mathrm{Jh}$. & $\begin{array}{l}\text { Güntzer I } \\
8 \text { Belege }\end{array}$ & $\begin{array}{l}\text { Harsdörffer I } \\
11 \text { Belege }\end{array}$ & 19 Belege \\
\hline $18 . \mathrm{Jh}$. & $\begin{array}{l}\text { Schuhmacher Chronik II } \\
12 \text { Belege }\end{array}$ & $\begin{array}{l}\text { Aichinger III } \\
15 \text { Belege }\end{array}$ & 27 Belege \\
\hline $19 . \mathrm{Jh}$. & $\begin{array}{l}\text { Auswandererbriefe V } \\
\text { 8 Belege }\end{array}$ & $\begin{array}{l}\text { Burckhardt V } \\
\text { 6 Belege }\end{array}$ & 14 Belege \\
\hline $20 . \mathrm{Jh}$. & $\begin{array}{l}\text { Jugendtagebuch VII } \\
\text { 3 Belege }\end{array}$ & $\begin{array}{l}\text { Lorenz VII } \\
\text { 3 Belege }\end{array}$ & 6 Belege \\
\hline insg. & 31 Belege & 35 Belege & 66 Belege \\
\hline
\end{tabular}

Tabelle 7: Verteilung der Belege für vorangestellte Kausalsätze in den Korpustexten bzw. in den Teilkorpora $(n=66)$ 
5.3.1 Kausalsätze in den Korpustexten aus dem 17. Jh.

In Harsdörffer I finden sich 11 einschlägige Belege, diese werden stets durch den Subjunktor weil eingeleitet. Vorangestellte weil-Sätze werden 8mal integrativ angebunden. Dieser Stellungstyp kommt in mehreren Fällen (6 Belege) vor, wenn der Kausalsatz syntaktisch nicht erweitert wird, vgl. folgenden Beleg:

(220) Weil aber noch keine solche Neurung eingeführet/lassen wir es hierinnen/ wie in vielen andern/ bey der alten Gewohnheit verbleiben: (Harsdörffer I: 566)

Der integrative Stellungstyp begegnet einem auch bei syntaktisch komplexen Voranstellungen, vgl. die folgenden beiden Belege:

(221) Weil nun unser Papyr/ welches aus alten Lumpen bereitet wird/ demselbigen gleichet/ hat es auch eben besagten Namen/ bis auff gegenwartige Stund. (Harsdörffer I: 11)

(222) Weil nun die Steine nicht leichtlich zu bewegen/ und sehr wenig/ sondern grosse Mühe gebraucht/ etwas darein zu bilden, zuweitlen auch gantze Stucke ausgesprungen/ haben sie Holtz und Metall zu ihren Schrifften gebraucht/ und sonderlich den Buchbaumen erwehlet/... (Harsdörffer I: 8)

In (221) wird der vorangestellte Kausalsatz durch einen Relativsatz erweitert. Viel komplexer ist die Voranstellung von vier Satzstrukturen in (222). Mit dem ersten (durch weil eingeleiteten) Teilsatz wird ein weiterer Teilsatz koordinierend verknüpft, dem zweiten Teilsatz werden eine Infinitivkonstruktion und ein weiterer Adverbialsatz untergeordnet. Erst nach dieser komplexen Voranstellung wird das Finitum des Obersatzes realisiert. In den vorangestellten Strukturen dieses komplexen Satzes werden die finiten Verbformen nicht realisiert, was auch bei weiteren Belegen aus dem Korpustext (vgl. z.B. (220)) zu beobachten ist.

Der resumptive Stellungstyp tritt dreimal auf, in diesen Fällen tritt das Korrelat als im Vorfeld des Obersatzes auf. Zwei Voranstellungen ((223) und (224)) sind auf der Satzebene, eine (225) auf der Phrasenebene (Attribuierung des Substantivs Holtz) komplex.

(223) Weil nun nicht zu erwarten/ daß sich die Mund-Arten in gleichstimmende Aussprache verienigen sollten: Als ist auch ihre Schreibrichtigkeit auff keine Weise endlich zu vergleichen/ und singet ein jeder Vogel/ wie ihm der Schnabel gewachsen ist. (Harsdörffer I: 17)

(224) Weil aber sonderlich an jetzo bedagtem Sprachstucke der Rechtschreibung viel gelegen/ und einem jeden frey stehet/ seine Gedancken mehrverstandiger Straffurtheil zu untergeben; als ist fur schicklich erachtet worden/ etwas weniges auff Verbesserung mehrvernüfftigen Gutachtens hierbey anzufügen; (Harsdörffer I: 560) 
(225) Weil aber solches zwar hartel doch endlich faulende Holtz/ nicht viel Jahr dauren konnen/als haben sie zu denckwürdigen Sachen/ das Metall/ Bley/ Kupffer und Messing gebraucht/ und ihre Schrifften darein gegraben/wie wir dann lesen in den Büchern der Maccaboer ... (Harsdörffer I: 8)

Der strukturelle Faktor der Komplexität scheint zwar eine Rolle bei der Wahl der Stellungstypen zu spielen, da das Auftreten des resumptiven Typs eine Komplexität auf Satzebene (oder zumindest auf der Phrasenebene) voraussetzt, im Korpustext ist aber der integrative Typ trotz Komplexität der Voranstellung möglich. Mit diesen Ergebnissen muss aufgrund der niedrigen Belegzahl vorsichtig umgegangen werden.

In Güntzer I sind 8 eingeleitete, vorangestellte Kausalsätze belegt. Die meisten einschlägigen Belege werden durch den Subjunktor dieweil eingeleitet (6 Belege). Zudem finden sich zwei eindeutig als Kausalsatz zu identifizierende Belege für den Subjunktor $d a$, der im Korpustext auch rein temporal (vgl. Kapitel 5.1.1) bzw. temporal-kausal (vgl. Kapitel 5.4.9) verwendet wird. ${ }^{203}$ Beim folgenden Beleg ist jedoch nur eine kausale Interpretation möglich.

(226) Da er nichts kunde außrichten bey mihr, so setzete er weitters an mich, ich solte mich entleiben mit Strek oder Waffen oder in ein Waßer springen und dergleichen mehrn Gestalt, so mihr auß dem Sin ist gevalen. (Güntzer I: 101r)

Im $d a$-Satz wird der Grund für den im Obersatz ausgedrückten Sachverhalt angegeben: Weil Güntzer der Versuchung widerstanden hat, wird er vom Teufel erneut in Versuchung geführt. Der $d a$-Satz wird, wie auch im folgenden Beleg, resumptiv angebunden. In beiden Fällen tritt das Korrelat so auf. Die Wahl des Stellungstyps kann nicht auf strukturelle Faktoren zurückgeführt werden, weil in beiden Fällen syntaktisch einfache Voranstellungen vorliegen, vgl. den folgenden Beleg:

(227) Ich dachte aber in meinem Sin, da die fihrneme Leidt in Seiden und Samet i[h]me die Fieß kißen, so mießte ich ihme den Arsch kißen, dieweil ich sehr unsauber, zerrißen Kleider trug, dieselbige voler Leiß und Fle. (Güntzer I: 61r)

Obwohl der komplexe Satz aus vier Teilsätzen (u.a. einem nachgestellten, durch dieweil eingeleiteten Kausalsatz) und einem weiteren Nicht-Satz (der elliptischen Struktur dieselbige voler Leiß und Fle) besteht, kann von einer syntaktisch einfachen Voranstellung ausgegangen werden, weil der $d a$-Satz durch keine Untersätze erweitert wird, ihm keine gleichrangigen

\footnotetext{
203 Man könnte allerdings annehmen, dass der $d a$-Satz in (227) weniger eine Ursache, sondern eine verwirklichte Bedingung ausdrückt. Dieser Beleg zeigt möglicherweise gewisse Ähnlichkeiten mit der konditionalen Verwendung, $d a$ kann jedoch - im Gegensatz zu wenn - nicht als rein konditional verwendet werden. Bei wenn-Sätzen kann zwischen faktischen und hypothetischen Verwendungen unterschieden werden (vgl. Zifonun et al. 1997: 2282f.). Dasselbe bei $d a$ anzunehmen, wäre aufgrund eines Einzelbelegs nicht angemessen.
} 
Satzstrukturen koordiniert werden und er auf Phrasenebene ebenfalls einfach gestaltet ist. Nur das Subjekt wird durch ein adjektivisches und ein präpositionales Attribut erweitert. Bei diesem Beleg stellt sich auch die Frage der semantischen Zuordnung, da die semantische Relation zwischen dem Unter- und Obersatz nicht mit der klassischen Ursache-WirkungFormel zu erfassen ist: Der wirkliche Grund für den im Obersatz ausgedrückten Sachverhalt (bzw. eigentlich dafür, dass Güntzer nicht die Füße des Papstes küssen wollte) wird durch den nachgestellten dieweil-Satz markiert. Der $d a$-Satz drückt einen im Linkskontext gegebenen, faktischen Grund aus, mit dem für eine Konsequenz argumentiert wird, die sich aus der Gegenüberstellung der gegebenen Handlung vornehmer Leute in schönen Kleidern und der notwendigen Handlung eines unsauberen Mannes in zerrissenen Kleidern ergibt.

Die durch dieweil eingeleiteten Kausalsätze werden in zwei Fällen integrativ angebunden. Beide Voranstellungen enthalten nur koordinierende Verknüpfungen, den dieweil-Sätzen wird kein Nebensatz untergeordnet.

(228) Dieweil wihr von nichts kondten essen und trincken, sparen wihr unßer Gelt. (Güntzer I: 62v)

(229) Dieweil der Kinig Christian der 4. kein hoffertiger Man wahr, sonder mit dem gemeinen Man also gern redet als mit großen Heren, hielte er selber dem Gemeinen als dem Ho[h]en Antientz. (Güntzer I: 95r)

Werden dieweil-Sätze durch Nebensätze erweitert, werden sie resumptiv angebunden. Der folgende Beleg enthält eine äußerst komplexe Voranstellung mit zwei dieweil-Sätzen, die wiederum weitere Untersätze haben. Dieser Beleg wird in der Auszählung nur einmal gewertet, weil nur ein Obersatz (so ferchte ich) vorliegt und der Bezug des ersten dieweilSatzes unklar ist, da er nicht den Grund für den im Obersatz ausgedrückten Sachverhalt (die Furcht vor Gottes Strafe) angibt:

(230) Mein Vatter und Geschwister sindt Tag und Nacht an mihr, ich solte mich verheiraten, dieweil ich aber gantz kein Lust darzu hatt und sahe beynoben, daz es mit mihr die Lengen kein Gudt wirdte thun, lenger in meim Vatterlandt zu verpleipen, dieweil ich daß gottloße Leben doglich vohr Augen sahe, so in meinem Vatte[r]landt fohrgehet mit Schweren und Fluchen, Freßen und Sauffen und alerley Sinden, und ich mich derer auch teilhafftig gemacht hab, so ferchte ich, Gott wirdt mich an Leib und Sele straffen. (Güntzer I: 66r)

Der resumptive Anschluss ist jedoch nicht nur auf komplexe Voranstellungen beschränkt. Er tritt auch bei einfachen Voranstellungen auf, vgl. den folgenden Beleg:

(231) Dieweil kein deischter Zingießersgesel alhie befirdtet wirdt, so bin ich alhie spatzieren gangen, ... (Güntzer I: 108r) 
Bei der resumptiven Anbindung vorangestellter Kausalsätze erscheint bis auf eine Ausnahme stets das Korrelat so. Im folgenden Beleg wird der vorangestellte dieweil-Satz mit dem Korrelat darum wieder aufgenommen. Obwohl der Obersatz selbst Verbendstellung aufweist (und daher ohne Korrelat wohl nicht mit dem Untersatz verknüpft werden könnte), wird er als übergeordneter Satz gedeutet. Es handelt sich um eine Grund-Folge-Beziehung, das Satzgefüge ließe sich wie folgt paraphrasieren: Dass das Schiff leer war, war der Grund für die Schwierigkeiten bei der Schifffahrt.

(232) Dieweil daß Schiff lehr wahr, dan nuhr den Balast, darum wihr bo̊ $\beta$ Soglen hatten. Wihr bekamen beßes Wette[r], hatten groß Sturm. [...] Der Sturmwindt spilet mit dem Schiff, wirffte daz Schiff hin und herr, gleich als wie eine Katzen mit einer Mau $\beta$ spilete. (Güntzer I: 95r-v)

An dieser Stelle soll auch der folgende Beleg diskutiert werden. Auf den ersten Blick könnte der markierte Teilsatz als ein integrativ angebundener, vorangestellter Kausalsatz gedeutet werden. Es liegt zwar in der Tat eine Grund-Folge-Beziehung vor. Der einschlägige, durch derohalber eingeleitete Teilsatz gibt jedoch nicht den Grund für den im nachfolgenden Teilsatz ausgedrückten Sachverhalt an, sondern markiert die Konsequenz der im Linkskontext geschilderten Situation. ${ }^{204}$

(233) Demnach ging der Kauff- und Schiffman in die Statt. Die Bö[t]ßköncht erwoehlet[e]n mich dieweil 8 Tag auff dem Schiff zu ihrem Koch. Sie hielten mich die 8 Wochen auff dem Schiff lieb und werdt, dan ich wahr under dienstbar mit Arbeiden, die Gietter halff ich auß- und einladen, Anck[e]r ziehen, daz Feir anzintten, Waßer ziehen, daß Bortt butzen. Derohalber sie von mihr kein Schifflon fordern, gaben mihr umsunst Essen und Trincken. (Güntzer I: 107v)

Der einschlägige Teilsatz ist also kein vorangestellter, sondern ein nachgestellter Adverbialsatz. Der Konnektor derohalber ist nicht kataphorisch, sondern anaphorisch. Aus gegenwartssprachlicher Sicht wäre bei einem Konjunktionaladverb eine adverbiale Verwendung mit Verbzweitstellung zu erwarten, im Korpustext ist jedoch derohalben in mehreren Verbendsätzen als Subjunktor belegt. Dieser Beleg wird im Sinne des Gesagten bei der quantitativen Auswertung nicht berücksichtigt.

5.3.2 Kausalsätze in den Korpustexten aus dem 18. Jh.

Im Korpustext Aichinger III finden sich ausschließlich resumptiv angebundene, eingeleitete Kausalsätze. Am Anfang des Obersatzes erscheint bei jedem Beleg das Korrelat so,

204 Der Konnektor derohalber scheint in diesem Beleg ,konsequensmarkierend‘ im Sinne von Breindl et al. (2014: 790) zu sein und kann als konsekutiv im engeren Sinne aufgefasst werden. 
unabhängig davon, ob es sich einfache oder komplexe Voranstellungen handelt, vgl. die folgenden Belege:

(234) Weil nun $\beta$ ein zweyfaches $s$ ist: so ists gefehlt, wenn man es den Wortern aufbringet, die nur ein einfaches haben, z.B. Muß, pulmentum, da man doch sagt Gemüe (Aichinger III: 14)

(235) Weil es aber doch angenommen ist, daß etlichen Wortern solche zweyfache Vocale gegeben werden, als: Aas, Heer, Schoos: so stehen sie, weil sie zu einer Syllbe gehoeren, billig unter den Doppellauten. (Aichinger III: 22)

Der frequenteste kausale Subjunktor des Korpustextes ist weil (13 von 15 Belegen). In zwei Fällen wird der Subjunktor $d a$ verwendet, vgl. den folgenden Beleg:

(236) Man muß für edel nicht schreiben ådel, als wenn es von Adel herkäme. Denn dieses stammt vielmehr von jenem, wie Satz von setzen, Strang von strengen, Da aber von einem hergeleiteten wieder ein anders kommen kann: so richtet sich das letztere nicht nach dem ersten Ursprung, sondern nach seinem eignen. (Aichinger III: 30)

In Schuhmacher Chronik II ist der Anteil an Kausalsätzen mit 12 Belegen höher als derjenige an Temporal- oder Konditionalsätzen. Es finden sich 12 Belege für vorangestellte weil-Sätze, die bis auf eine Ausnahme (243) resumptiv aufgenommen werden, vgl. z.B. (237) und (238) weiter unten.

Die Wahl des Stellungstyps scheint nicht von strukturellen Faktoren abhängig zu sein, da sowohl bei syntaktisch einfachen als auch bei komplexeren Voranstellungen der resumptive Typ bevorzugt wird. Die folgenden Belege sollen komplexe Voranstellungen veranschaulichen, die in diesem Korpustext dominieren. In (237) werden dem nachfolgenden Bezugssatz zwei koordinierte, kopulativ verknüpfte Kausalsätze untergeordnet. Nach dem Konjunktor und wird im zweiten Untersatz der Subjunktor weil nicht mehr wiederholt. Beide Untersätze werden als afinite Konstruktion realisiert. In (238) wird nach dem zweiten, ebenfalls kopulativ verknüpften Prädikat eine Infinitivkonstruktion eingefügt, erst dann wird der Obersatz realisiert.

(237) So hat Sie durch Teuffels Eingeben gemeint, der Sachen zu entrinnen und den Männern entlauffen. Weil man Sie aber fäst gehalten und wider zurück geführet so hat Sie Es auff dem Weg dem schon gemelten Statt-Diener Elias Klein bestanden, Sie habe Es auff der Büne in einem Wollen-Ständlein in Speier ligen, (Schuhmacher Chronik II: 379)

(238) Im Anfang dises Jahrs hat sich ein trauriges Spectacul gleichsam in der ersten viertel stundt zuge-Tragen. Weil daß sich die Vor-Stätter Söhn haben zusamen gefüegt $u$. geschlossen, daß Neue Jahr anzuschiessen, so hat sich auch der Beckh Johannes Lobmüller noch ein Junger Haußhälter auch darzu gemacht, welcher bey dem Eychhäusslein wohnhafft, und durch einen ungefähren unglückhafften Schutz 
seinen Schwager Joh. Georg Ruoffen, Lediger Gesell, hinde[n] In den Fuoß geschossen, daß Er sich so sehr verbluttet, daß Er dadurch wegen Überhandnehmung deß Brands sein Leben enden u. schliessen müssen, welches höchst zu-betrauren gewessen. (Schuhmacher Chronik II: 342f.)

In diesem Beleg wird der Subjunktor weil durch daß ergänzt. Dadurch wird der vorangestellte Teilsatz als untergeordnet markiert, obwohl er keine Verbendstellung aufweist (Voranstellung des Finitums). Die Belege, in denen zwei koordinierte Untersätze realisiert werden, werden bei der quantitativen Auswertung nur einmal mitgezählt, da sie sich auf den selben Obersatz beziehen und nicht getrennt in ihn integriert werden, sondern als ein (komplexes) Stellungsglied aufzufassen sind. Auch der folgende Beleg könnte als eine Voranstellung mit zwei koordinierten Kausalsätzen aufgefasst werden. Der Bezug des ersten weil-Satzes ist jedoch unklar.

(239) Gott der Vatter des Liechts wölle uns vor der Ewigen völlen Finsternus behüten und Bewahren c.\&. Weil dise Finsternus so erschröcklich ist zu sehen gewessen daß es bey uns Menschen hier auff Erden scheinet. Als wann Gott der Allgemeine Weltrichter kom $[\mathrm{m}]$ en werde, zu richten die böße Geister und gottlose Menschen, weil es gantz Nacht gewessen daß man die Stern am Himmel gesehen, so hat mann bald in allen Häußer Liechter angezindt wegen Forcht und Schrecken, der warthenden Dingen, die da kommen möchten auff Erden ... (Schuhmacher Chronik II: 328f.)

Der erste weil-Satz könnte auch als Nachsatz oder als Apokoinu-Konstruktion analysiert werden. Zudem ist auch die ausgeklammerte Präpositionalgruppe wegen Forcht und Schrecken als Kausaladverbial zu deuten. Es scheint hier also eine komplexe Begründung vorzuliegen. Der Bezug der weil-Sätze aus diesem Korpustext ist in mehreren Fällen schwer zu bestimmen, vgl. den folgenden Beleg (s. auch Kapitel 4.2):

(240) Hiemit ist merckwürdig zu beobachten, daß den 1. 2. 3. \& 5. mertzen daß vor Augen stehende Obere Thor Neu erbaut und auffgericht ist worden, welches durch den Total-Brand ist verzert worden. Dieser Monat stellet Sich recht winterisch Ein mit Schnee Nebel \& Sonnenschein, auch regen, weil Es scheinet noch Winter nicht Früeling zu werden, So hat daß Brodt den 15ten ein Kreutzer auffgeschlagen, So ist der Schnee schleinig abgangen, daß Es umb Palmarum erst auff dem Blachfeld Blatten geben, in der Caar-Wochen ist Es schön und warm Wetter gewessen, daß der Schnee fast alles weggangen (Schuhmacher Chronik II: 446)

Die Interpretation dieses Belegs als nachgestellter Kausalsatz ist zu verwerfen, da der Inhalt des weil-Satzes nicht als Grund für den im voraus realisierten Teilsatz aufzufassen ist (in beiden Fällen wird auf die winterlichen Temperaturen im März referiert). Analysiert man diesen Beleg als vorangestellten Kausalsatz, dann muss eine Verknüpfung auf der propositionalen Ebene angenommen werden: Die im März ungewohnte Kälte war der Grund 
für die Veränderung der Brotpreise. Bezüglich der Wortstellung im weil-Satz soll die Frage gestellt werden, ob hier eine Hauptsatzwortstellung/Verbzweitstellung angenommen werden kann. Für die Diskussion des Alters der weil-Verbzweitsätze würde dies bedeuten, dass die von Selting (1999: 191) konstatierte „Beschreibungslücke“ für den Zeitraum vom 16. bis zum 19. Jh. doch zu füllen ist. Der weil-Satz in (240) weist jedoch nur scheinbar bzw. an der Oberfläche eine Verbzweitstellung auf, der Beleg kann auch so analysiert werden, dass dem Vollverb am Ende des Kausalsatzes (scheinet) eine $z u+$ Infinitiv-Konstruktion nachgestellt wird. Hier konnte also kein sicherer Beleg für die Hauptsatzwortstellung im weil-Satz identifiziert werden.

In einem Beleg wird nicht das Resumptivum so, sondern also verwendet. Es handelt sich hier um eine syntaktisch komplexe Voranstellung. Der Kausalsatz wird durch einen $d a \beta-$ Satz und einen durch und verknüpften Teilsatz erweitert, der mit Verbzweitstellung realisiert wird. Dieser steht trotz Herstellung der syntaktischen Ruhelage auf derselben Ebene wie der daßSatz.

(241) Da hat Lutherus anheben darwider zu prodestieren und mit dem Schwerdt des Geistes zu fechten. Also fieng Sich der Hader an, zwischen Lutherum \& Tetzel. Da Tetzel Lutherum als ein Ertz ketzer thut verdam[m]en, wie solches bey Joh. Matheri, mit mehren Unständen zu lesen ist, weil aber daß Licht des Evangeli durch unterschidliche Päpst so verdunckelt worden, daß der Leichter deß waren GottesDinsts schier von seiner Stelle gestossen, und der Papst Johannes der XXIII hat selbsten keine Auffersteung der Todten nicht geglaubet. Also hat Lutherus daß Revormations Werck angefangen und hat geweret biß auff 30ig in werenter Zeit hat Sich viel Unheil gezeiget von allerhand Rotten \& Secten und theure Zeiten, (Schuhmacher Chronik II: 425)

In diesem Beleg tritt auch ein da-Satz auf (Da Tetzel Lutherum als ein Ertz ketzer thut verdam $[m] e n)$, der jedoch als Nachsatz zu interpretieren ist.

Weitere kausale Subjunktoren kommen nur in Belegen vor, deren Status nicht eindeutig ist. Im folgenden Beleg wird ein Nebensatz durch dieweil eingeleitet:

(242) So hat uns Gott einen köstlichen Tranck geschert, aber wenig wie in vorigen Jahren. So hat man auch diß Sprichwort gebraucht wenig $u$. Gutt, macht auch Ein Muth, und widrum Vinum est bonum Certissime. Er geschmeckt mir woll in Corpore. Dieweil Ich leb in Tempore, dafür sag Ich Gott Lob, Preiß u. Ehr, und geb daß künfftig Jahr noch mehr. An Obs hat Es nicht gar viel geben, Gott wöll unß fenner mehr bescheren. (Schuhmacher Chronik II: 357f.)

In diesem Beleg wird ein (möglicherweise etwas älterer) Spruch aufgeführt, der aus vier Teilsätzen besteht (Er geschmeckt mir woll in Corpore. Dieweil Ich leb in Tempore, dafür sag Ich Gott Lob, Preiß u. Ehr, und geb daß künfftig Jahr noch mehr). Er wurde als Zitat 
aussortiert und wird in der quantitativen Auswertung nicht berücksichtigt. Auch sein syntaktischer Status ist problematisch, da er sowohl als nachgestellter Kausalsatz als auch als vorangestellter Objektsatz analysiert werden könnte.

Der einzige Beleg für vorangestellte weil-Sätze, der keinen resumptiven Anschluss aufweist, wird nicht-integrativ angeschlossen:

(243) Es haben auch vier Haußhaltungen Gelt zusamen geschossen umb ein Emy Erbsen welches $30 \mathrm{xr}$. gekostet $u$. ist den 15 Brach-monath von Joh. Jacob Hum[m]el, Müller in der Kämermühlin gemahlen worden und seind 19 Personen mit abgespeiset worden. Weil wir den vor Augen stehende[n] reichen-Seegen den Som $[\mathrm{m}]$ er über angeschaut, mann vermeint, Es solte wohlfailer werden. So continuierte die Teurung biß auff den 11ten Augusti. (Schuhmacher Chronik II: 343f.)

Es handelt sich auch hier um eine Verknüpfung auf der propositionalen Ebene. Der Satzkomplex lässt sich wie folgt paraphrasieren: Während der großen Hungersnot im Jahre 1713 hat man wegen des guten Wetters damit gerechnet, dass man in absehbarer Zeit keinen Mangel an bezahlbaren Nahrungsmitteln erleiden muss.

Der folgende Beleg weist eine integrative Stellung auf. Er wird jedoch bei der quantitativen Auswertung nicht berücksichtigt. $\mathrm{Zu}$ überprüfen ist, ob hier ein Prädikatsausdruck wie ist der Grund dafür vorliegt, der als ,unifizierender Junktor' zwei Konnekte (mit der Reihenfolge Grund-Folge) regiert (vgl. Ágel 2010: 906f.). Solche ,unifizierenden Junktoren' sind im Korpustext belegt. ${ }^{205}$

(244) am Tage der unschuldige[n] Kindlein hat sich der Him[m]el Claar gemacht und cont. biß zum End deß Jahrs mit kalte Morgen und Sonnenschein, war daß Jahr beschlossen fein. Weil Ich daß Jahr nach allen 12 Monat beschriben hab war dises die Ursach weil vor 20ig Jahren Ein so kalter Winter gegesen und diser in disem Jahr gleich gehalten worden, in der kälte, weil Mir daß wasser in den feicht-Kössel auff der werckstatt gefrohren und ein sehr kalter Frühling gewessen, So hat man vermutet, Es werde widrum Ein böß Jahr darauff erfolgen. Aber dem nicht Also, wie Anno 9. (Schuhmacher Chronik II: 422f.)

Der Inhalt des ersten weil-Satzes kann weder als Ursache noch als Folge für den Inhalt der nachfolgenden Teilsätze verstanden werden. Die Tatsache, dass man das Wetter im aktuellen Jahr nach Monaten beschreibt, verursacht nicht, dass zur gegebenen Zeit oder vor 20 Jahren eine große Kälte herrschte bzw. dass man aufgrund der Ähnlichkeit mit den Wetterverhältnissen vor 20 Jahren ein $b \ddot{\partial} \beta J a h r$ erwartet hat. Der Ausdruck dises wird hier nicht anaphorisch mit Bezug auf den weil-Satz, sondern kataphorisch verwendet (vgl. auch

\footnotetext{
205 Vgl. das Beispiel (i), in dem Dises anaphorisch verwendet wird und der $d a \beta$-Satz die Folge ausdrückt. (i) Dises wahr Also die Ursach, daß Daß Jubel, Lob \& Danck-Fest ist gehalten worden,... (Schuhmacher Chronik II: 445).
} 
Beleg (139) weiter oben). Er drückt gemeinsam mit dem folgenden weil-Satz ${ }^{206}$ (und den nachfolgenden Teilsätzen) den Grund dafür aus, warum man für das Jahr 1729 mit einer schlechten Ernte gerechnet hat. Es kann also festgehalten werden, dass hier kein ,unifizierender Junktor` vorliegt und der erste, vorangestellte weil-Satz nicht den Grund für den Inhalt der nachfolgenden Teilsätze ausdrückt. Da der weil-Satz weder als Subjektsatz zum Obersatz war dises die Ursach noch als Kausalsatz zu einem der nachfolgenden Teilsätze analysiert werden kann, wird hier nur ein loses, assoziatives Verhältnis zwischen dem weilSatz und seinem Rechtskontext angenommen. Daher wird dieser Beleg bei der quantitativen Auswertung nicht berücksichtigt.

\subsubsection{Kausalsätze in den Korpustexten aus dem 19. Jh.}

In Burckhardt V finden sich insg. 6 vorangestellte, eingeleitete Kausalsätze. In fünf Belegen wird der Subjunktor $d a$ verwendet, bei ihnen wird stets - und unabhängig vom Grad der Komplexität der Voranstellungen - das Korrelat so im Vorfeld des Obersatzes realisiert, vgl. die folgenden Belege:

(245) Da nun die Sprache eine Angelegenheit der lebendigen Gesellschaft geworden war, so setzten die Archaisten und Puristen trotz aller Anstrengung ihre Sache im wesentlichen nicht durch. (Burckhardt V: 275)

(246) Die Idealfarbe aber, welche man in den eigenen, wie in den aufgesetzten Haaren zu erreichen strebte, war blond. Und da die Sonne im Rufe stand, das Haar blond machen zu können, so gab es Damen, welche bei gutem Wetter den ganzen Tag nicht aus der Sonne gingen, sonst braucht man auch Färbemittel und außerdem Mixturen für den Haarwuchs. (Burckhardt V: 269)

Weitere Subjunktoren kommen nur vereinzelt vor. Im folgenden Einzelbeleg für gerade weil ist jedoch der integrative Stellungstyp zu beobachten:

(247) Zum Verständnis der höhern Geselligkeit der Renaissance ist endlich wesentlich zu wissen, dass das Weib dem Manne gleich geachtet wurde. Man darf sich ja nicht irre machen lassen durch die spitzfindigen und zum Teil boshaften Untersuchungen über die vermutliche Inferiorität des schönen Geschlechtes, wie sie bei den Dialogenschreibern hin und wieder vorkommen, auch nicht durch eine Satire wie die dritte des Ariosto, welcher das Weib wie ein gefährliches grosses Kind betrachtet, das der Mann zu behandeln wissen müsse, während es durch eine Kluft von ihm geschieden bleibt. Letzteres ist allerdings in einem gewissen Sinne wahr; gerade weil das ausgebildete Weib dem Manne gleichstand, konnte in der Ehe das, was man

\footnotetext{
206 Da die Sätze weil vor 20ig Jahren Ein so kalter Winter gegesen bzw. weil Mir daß wasser in den feicht-Kössel auff der
} werckstatt gefrohren in (237) keine vorangestellten Nebensätze sind, werden sie im Weiteren nicht berücksichtigt. 
geistige und Seelengemeinschaft oder höhere Ergänzung nennt, nicht so zur Blüte gelangen wie später in der gesitteten Welt des Nordens. (Burckhardt V: 285)

Die Belegzahl der vorangestellten Kausalsätze in den beiden Korpustexten aus dem 19. Jh. unterscheidet sich kaum voneinander. In Auswandererbriefe $\mathrm{V}$ kommen insg. 8 vorangestellte, eingeleitete Kausalsätze vor. In diesem Korpustext tritt stets das resumptive so im Vorfeld des Obersatzes vor. Der Subjunktor $d a$ ist nur 3 mal belegt (vgl. z.B. (248), der frequenteste Subjunktor ist indem mit 5 Belegen (vgl. z.B. (249)), der heute standardsprachlich in kausaler Verwendung nur archaisch auftritt (vgl. Zifonun et al. 1997: 2278). Alle Belege für $d a$ bzw. indem stammen jedoch aus je einem einzigen Brief von zwei verschiedenen Schreibern, sodass bei der Wahl des Subjunktors soziolinguistische oder gar individuelle Faktoren vermutet werden können. ${ }^{207}$

(248) Da wir auf unserer Reiße das Unglück gehabt haben unsere Bildnisse zu erbrechen sogar das Gemälde zu verschmieren so würde es mich freuen wen Ihr sie uns schicken würdet (Auswandererbriefe V: 28)

(249) das größte Mädchen könnte wohl schon viel Hülf sein wenn sie dazu erzogen worden wäre, aber indem das eben nicht der Fall war, so ist inn anstrengender Arbeit nicht viel aus ihr herauszubringen,... (Auswandererbriefe V: 18)

In zwei Belegen ist die Abgrenzung der propositionsbezogenen Kausalsätze gegenüber ihrer pragmatischen Verwendung nicht unproblematisch. ${ }^{208}$

(250) Da wir auf unserer Reiße das Unglück gehabt haben unsere Bildnisse zu erbrechen sogar das Gemälde zu verschmieren so würde es mich freuen wen Ihr sie uns schicken würdet (Auswandererbriefe V: 28)

(251) Indem es nahe an Weinachten ist, so will ich euch auch wieder einige Zeil schreiben, um euch auf Weinachten durch einen Brief von mir, ein wenig zu erfreuen (Auswandererbriefe V: 18)

In (250) wird mit dem Obersatz und dem ihm untergeordneten wen-Satz ein indirekter Sprechakt durchgeführt, der als eine Bitte bzw. eine höflich formulierte Aufforderung zu interpretieren ist. Anders als bei Imperativsätzen (vgl. z.B. (39) weiter oben im theoretischen Teil, 3.1.3), in denen der Kommunikationspartner explizit aufgefordert wird, gilt hier aber auch die wörtliche Interpretation: Im $d a$-Satz wird der Grund dafür angeführt, warum sich der Schreiber über das Verschicken der Bilder freuen würde. Dass diese propositionale

207 Bei dem kausalen Gebrauch der Subjunktors indem sehen Zifonun et al. (1997: 2278) eine ,inzwischen standardsprachlich archaisierte[...]“ Verwendung: „Bis ins 19. Jahrhundert wurde indem kausal gebraucht im Sinne von weil, dadurch, daß.“

208 Pittner nimmt zu Recht an, dass manche „Adverbiale sowohl mit propositionalem Bezug wie auch mit Äußerungsbezug interpretiert werden [können]" (1999: 320). Da als distinktives Merkmal die Opposition integrative vs. nicht-integrative Stellung aufgeführt wird, bleibt beim resumptiven Typ die Frage offen, welche Interpretation(en) anzunehmen sind. 
Begründung implizit auf die Sprechaktebene übertragen werden kann und dem Leser einen Handlungszwang auferlegt, wird nicht grammatisch kodiert und ist nur unter Berücksichtigung konversationeller Regeln zu erklären. Daher wird bei diesem Beleg ein propositionaler Bezug angenommen. Bei Beleg (251) wird ebenfalls keine Verknüpfung auf der Sprechaktebene (im Sinne eines metakommunikativen Kommentars mit dem Wortlaut ,Weil es bald Weihnachten ist, teile ich euch mit: Ich will euch mit meinem Brief eine kleine Freude bereiten'), sondern eine Verknüpfung auf der propositionalen Ebene (,Weil es bald Weihnachten ist, habe ich die Absicht, euch mit meinem Brief eine kleine Freude zu bereiten') angenommen.

Die Wahl des resumptiven Stellungstyps ist unabhängig vom Grad der Komplexität der Voranstellungen, vgl. die einfache bzw. die komplexe Voranstellung in den obigen Belegen (249) und (251) bzw. (248) und (250).

5.3.4 Kausalsätze in den Korpustexten aus dem 20. Jh.

In Lorenz VII sind drei durch $d a$ eingeleitete Kausalsätze zu finden. Diese werden allesamt integrativ angebunden. Die folgenden Belege zeigen komplexe Voranstellungen: Dem $d a-$ Satz wird ein nachgestellter (252) bzw. ein zwischengestellter Relativsatz (253) untergeordnet.

(252) Da durch die Selektion Strukturen »herausgezüchtet" werden, die eine bestimmte arterhaltende Funktion besonders gut erfüllen, sehen sie im Enderfolg so aus, als wären sie von einem weise voraussehenden und klug planenden Geist zu eben diesem Zwecke erschaffen. (Lorenz VII: 38)

(253) sie [die Veränderungen durch Mutation und Neukombination von Erbanlagen, P.K.] wirken sich keineswegs immer zum Vorteil der betroffenen Individuen aus - im Gegenteil: Da alle diese kleinen und kleinsten Veränderungen, die durch Mutation und Neukombination von Erbanlagen verursacht werden, völlig ungerichtet vor sich gehen, haben sie in den allermeisten Fällen eine Verminderung der Aussichten zur Folge, die das betreffende Individuum auf Energiegewinn und Überleben hat. (Lorenz VII: 37)

Der dritte Beleg enthält zwar keinen Nebensatz zweiten Grades, aber aufgrund der Länge der Voranstellung könnte eine Gedächtnisbelastung beim Kodieren bzw. Dekodieren des komplexen Satzes angenommen werden:

(254) Da nun die kognitiven Vorgänge auf höchster Ebene ebenso wie die auf der denkbar tiefsten und ältesten auf dem gleichen Prinzip beruhen, könnte man meinen, es gäbe keine andere Art des Wissensgewinns. (Lorenz VII: 41) 
Diese Belege zeigen also eine erweiterte Vorfeldfähigkeit von Adverbialsätzen.

In Jugendtagebuch VII sind ein durch $d a$ und zwei durch weil eingeleitete Kausalsätze zu finden. Diese werden allesamt integrativ angebunden, unabhängig davon, ob eine syntaktisch einfache (255) oder komplexe (256) Voranstellung vorliegt.

(255) Wir haben heute telefoniert und da das so unpersönlich ist habe ich - und darauf bin ich mächtig stolz - mich spontan auf den Weg zu ihm gemacht. Er soll sehen, daß er mir nicht gleich ist, daß ich alles für ihn tun würde. (Jugendtagebuch VII: 4)

(256) Überall mußte man Schlange stehen, obwohl Plätze frei waren! (Das ist bei HOCafés so. Weil der Betrieb dem Staat gehört und jeder seinen festen Lohn erhält egal wie gut oder schlecht das Café geht - sorgt man eben dafür, daß man nie zu viel zu tun hat und u. U. in Stress kommt. (Jugendtagebuch VII: 13f.)

Im letzten Beleg wird der vorangestellte weil-Satz mit einem weiteren, durch den kopulativen Konnektor und verknüpften Kausalsatz koordiniert und durch einen parenthetischen Irrelevanzausdruck erweitert. Dies zeigt eine stark ausgeprägte Vorfeldfähigkeit.

\subsubsection{Zusammenfassung}

In Tabelle 8 ist eine historische Veränderung zu beobachten: Während in den Korpustexten aus dem 20. Jh. stets der integrative Typ anzutreffen ist (6 von 6 Belegen), dominiert in den älteren Korpustexten bis auf einen der resumptive Typ.

\begin{tabular}{|l|c|c|c|}
\hline & integrativ & resumptiv & nicht-integrativ \\
\hline \multirow{2}{*}{ Güntzer I (n=8) } & 2 & 6 & 0 \\
& $25,0 \%$ & $75,0 \%$ & $0 \%$ \\
\hline \multirow{2}{*}{ Harsdörffer I (n=11) } & 8 & 3 & 0 \\
& $72,7 \%$ & $27,3 \%$ & $0 \%$ \\
\hline \multirow{2}{*}{ Schuhmacher Chronik II (n=12) } & 0 & 11 & 1 \\
& $0 \%$ & $91,7 \%$ & $8,3 \%$ \\
\hline \multirow{2}{*}{ Aichinger III (n=15) } & 0 & 15 & 0 \\
& $0 \%$ & $100 \%$ & $0 \%$ \\
\hline \multirow{2}{*}{ Auswandererbriefe V (n=8) } & 0 & 8 & 0 \\
& $0 \%$ & $100 \%$ & $0 \%$ \\
\hline \multirow{2}{*}{ Burckhardt V (n=6) } & 1 & 5 & 0 \\
& $16,7 \%$ & $83,3 \%$ & $0 \%$ \\
\hline \multirow{2}{*}{ Jugendtagebuch VII (n=3) } & 3 & 0 & 0 \\
& $100 \%$ & $0 \%$ & $0 \%$ \\
\hline \multirow{2}{*}{ Lorenz VII (n=3) } & 3 & 0 & 0 \\
& $100 \%$ & $0 \%$ & $0 \%$ \\
\hline
\end{tabular}

Tabelle 8: Verteilung der Stellungstypen bei vorangestellten Kausalsätzen 
Die meisten vorangestellten Kausalsätze des Korpus sind resumptiv angebunden (48 von 66 Belegen, 72,7\%). Der integrative Typ ist in den beiden Korpustexten aus dem 18. Jh. sowie im Nähetext aus dem 19. Jh. gar nicht belegt. In drei weiteren Korpustexten ist eine Varianz zwischen integrativem und resumptivem Anschluss zu beobachten. In zwei Texten (Burckhardt V und Güntzer I) herrscht der resumptive Typ vor, der integrative Typ tritt nur vereinzelt auf (nur 1 von 6 bzw. 2 von 8 Belegen, 16,7\% bzw. 25\%). Im Distanztext aus dem 17. Jh. überwiegt jedoch der integrative Typ (72,7\%). Der nicht-integrative Typ ist im Korpus mit einem Einzelbeleg vertreten. ${ }^{209}$ Die Korrelation zwischen den Zeitabschnitten und den Stellungstypen, integrativ ${ }^{`}$ und, resumptiv ${ }^{`}$ erweist sich als statistisch hoch signifikant (PWert $=0,00001$ ). Sowohl in den Nähe- als auch in den Distanztexten ist die Tendenz, dass mit der Zeit die integrative Stellung häufiger wird, nachweisbar (P-Wert $=0,033052$ bzw. 0,000677). Im Distanzkorpus sind insgesamt mehr als doppelt so viele Belege für den integrativen Typ zu finden (12 von 35, 34,3\%) als im Nähekorpus (5 von 31, 16,1\%). ${ }^{210}$ Die Korrelation ist jedoch statistisch nicht signifikant $(\mathrm{P}-\mathrm{Wert}=0,107104)$. Im Bereich der Kausalsätze sind weitere Untersuchungen nötig.

Bei der Wahl des resumptiven Elements wird in den meisten Fällen so bevorzugt. Aus den insg. 48 einschlägigen Belegen wurde der Nachsatz 43mal mit so angeschlossen. Im Distanzkorpus sind nur drei Belege für resumptives als (alle aus Harsdörffer I), im Nähekorpus nur je einer für also bzw. daran (Schuhmacher Chronik II bzw. Güntzer I) zu finden. Aufgrund des Ergebnisses, dass das Resumptivum so nicht nur bei einer semantischen Relation verwendet wird, sondern bei mehreren Adverbialsatztypen (z.B. auch bei Temporalsowie Kausalsätzen), kann angenommen werden, dass die „Tendenz zur semantischen Reduzierung“ von so als Korrelat im Gegensatz zu den Daten von Lefèvre (2012: 501) vor Ende des 19. Jh. noch nicht abgeschlossen ist. Bezüglich der Subjunktionen kann zumindest im Nähekorpus eine größere Varianz festgestellt werden: Während im Distanzkorpus weil bzw. $d a$ benutzt werden, kommen im Nähekorpus außer weil und $d a$ auch die Subjunktoren dieweil und indem mit ziemlich hoher Frequenz ( 6 bzw. 5 Belege) vor.

Die Relevanz struktureller Faktoren bei der Wahl zwischen den Stellungstypen ist nicht eindeutig zu bestimmen. Es ist allerdings hervorzuheben, dass der integrative Typ im Nähekorpus nur einmal gewählt wird, wenn dem vorangestellten Adverbialsatz ein weiterer

\footnotetext{
209 Aus diesem Grund wird er in den folgenden statistischen Berechnungen nicht berücksichtigt.

210 Zur prozentualen Verteilung der Stellungstypen bei Kausalsätzen in den Nähe- bzw. Distanztexten s. die Diagramme 9 und 10 im Anhang (8.2).
} 
Nebensatz untergeordnet ist. Dieser Beleg stammt aus dem Nähetext aus dem 20. Jh. und kann als ein Ausbau der Vorfeldfähigkeit gedeutet werden. Im Nähekorpus wird bis auf diesen Einzelbeleg in solchen Fällen der resumptive Typ gewählt. Im Distanzkorpus gibt es jedoch nicht nur im Quellentext aus dem 20. Jh. einschlägige Belege, sondern auch im Quellentext aus dem 17. Jh.

\subsection{Weitere Typen von Adverbialsätzen im engeren Sinne}

Zunächst werden Adverbialsatztypen aus dem Kernkanon behandelt, für die im Korpus nur relativ wenige Belege zu finden sind: Final- sowie Konsekutivsätze. Anschließend werden Adversative und spezifizierende Adverbialsätze mit den Untertypen Komparativ- und Proportionalsätze (Duden-Grammatik 2016: 1117, vgl. auch Kapitel 3.4.1), sowie Irrelevanzkonditionale untersucht. Zuletzt werden die Mischtypen beschrieben, bei denen eine eindeutige Zuordnung zu einem reinen Adverbialsatztyp nicht angemessen wäre (temporalkausal, lokal-konditional). Auf eine tabellarische Darstellung der Ergebnisse wird verzichtet, wenn die Belegzahl zu niedrig ist, um Schlussfolgerungen bezüglich historischer Veränderungen oder Nähe-Distanz-Unterschiede zu ziehen.

\subsubsection{Finalsätze}

Im Korpus sind nur zwei Belege für vorangestellte Finalsätze zu finden. Beide stammen aus dem 17. Jh. und werden integrativ angebunden.

(257) Darmit ich aber auch von ihrem Gotzenwerck etwaß konde sagen, ging ich in der Maria Capel, hohrete darinen $2 \mathrm{Me} \beta$ an, aber wenig Trost und Krafft darbey gesehen. (Güntzer I: 57v)

(258) ... damit sie nun unterschieden würden/ hat ein jeder/ benebenst dem Tauffnamen/ auch sein Leibgut anmelden/ und sich also in die Reichsbücher einschreiben lassen/ .... (Harsdörffer I: 28)

Die Verwendung der Subjunktoren darmit $^{211}$ bzw. damit entspricht der Beobachtung von Babenko (1988: 108): Vorangestellte Finalsätze werden im 17. Jh. „fast ausnahmslos durch damit eingeleitet“. In ihrem Aufsatz belegt sie jedoch auch den resumptiven Stellungstyp nach vorangestelltem Finalsatz (1988: 108). Welche Faktoren bei der Wahl des integrativen

211 Die Form darmit zeigt die Herkunft des Subjunktors, denn damit ist aus dem Pronominaladverb darmit (gebildet aus dem Demonstrativum dar und der Präposition mit) entstanden (vgl. Babenko 1988: 104). 
Stellungstyps in den obigen Belegen eine Rolle spielen, lässt sich aufgrund der geringen Belegzahl nicht feststellen.

\subsubsection{Konsekutivsätze}

In den Korpustexten kommt ein Einzelbeleg für vorangestellte Konsekutivsätze vor. Der daßSatz, der die Folge der sommerlichen Temperaturen ausdrückt, wird vorangestellt. Die Reihenfolge der Teilsätze weicht vom gegenwartssprachlichen Gebrauch und vom Prinzip der Ikonizität ab. Am Anfang des Obersatzes wird so als Teil einer Adjektivphrase (so warm) realisiert (vgl. Zifonun et al. 1997: 2308).

(259) da gab Es einen Stürmischen Wind, gleich darauff einen lieblichen Som[m]ertag, daß daß weibervolck hat Spitz auff der Gaß würcken können, So warm war Es, den 20. \& 21igsten wider Sturmwind und geregnet, (Schuhmacher Chronik II: 422)

Bei der Identifizierung des Bezugssatzes ist jedoch festzustellen, dass sowohl vor als auch nach dem daß-Satz ein Teilsatz steht, der auf die oben erwähnten sommerlichen Temperaturen referiert. Somit könnte hier eine Apokoinu-Konstruktion angenommen werden. Wie im Kapitel 3.4.5.3 bereits erwähnt, wird in Gegenwartsgrammatiken die Möglichkeit der Voranstellung von Konsekutivsätzen nicht behandelt. In einer gegenwartsdeutschen Umschreibung des obigen Belegs wäre der dass-Satz wohl in Nachstellung realisiert (Es war so warm, dass ...). Im einschlägigen Korpustext aus dem 18. Jh. ist auch die Nachstellung von Konsekutivsätzen belegt:

(260) Anno 1705, den 25igsten Mayen Montags vor Pfüngsten, Hab Ich mit meiner Sponsa Agnes Schauppin Hochzeit gehalten, und an meiner Nah-Hochzeit ist ein Schnee gefallen, als den 26igste [n] dito. Daß Er auff der Alb eines Schuß tieff gewessen, Es hat in den weinbergen die Zwickling gebogen und ist Gott sey Danck ohne Schaden abgangen. (Schuhmacher Chronik II: 326)

Dass hier trotz der Interpunktion und Großschreibung des Subjunktors $D a \beta$ eine Nachstellung angenommen wird, ergibt sich daraus, dass sich der $d a \beta$-Satz auf den vorangestellten Teilsatz bezieht, der die Intensität des Schneefalls schildert.

\subsubsection{Adversative Adverbialsätze}

Für adversative bzw. konfrontative während-Sätze in Voranstellung finden sich lediglich zwei Belege, beide werden integrativ angebunden. Der Einzelbeleg aus Burckhardt V enthält zwar 
keine weiteren Teilsätze vor dem Obersatz, zeigt aber aufgrund der Länge des vorangestellten Nebensatzes (34 Wortformen) eine erweiterte Vorfeldfähigkeit:

(261) Während im Norden der Adel und die Fürsten ihre Muße entweder einsam oder mit Kampf, Jagd, Gelagen und Zeremonien, die Bürger die ihrige mit Spielen und Leibesübungen, allenfalls auch mit Verskünsten und Festlichkeiten hinbrachten, gab es in Italien zu all diesem noch eine neutrale Sphäre, wo Leute jeder Herkunft, sobald sie das Talent und die Bildung dazu hatten, der Unterredung und dem Austausch von Ernst und Scherz in veredelter Form oblagen. (Burckhardt V: 276)

Adversative Adverbialsätze sind zwar im Korpus bereits seit dem 19. Jh. mehrfach belegt, werden jedoch i.d.R. ihrem Bezugssatz nachgestellt, vgl. die folgenden Belege.

(262) Außerdem beklagen sie den bereits äußerst raschen Wechsel der Moden und (wenn wir die Worte richtig deuten) die törichte Verehrung alles dessen, was aus Frankreich kommt, während es doch oft ursprünglich italienische Moden seien, die man nur von den Franzosen zurückerhalte. (Burckhardt V: 268)

(263) In diesen beiden Sätzen sind, wie man sich klarmachen muß, zwei Postulate aufgestellt, deren eines den Gegenstand der Forschung betrifft, während das zweite an den Forschenden gerichtet ist. (Lorenz VII: 11)

Nachgestellte während-Sätze werden nicht näher untersucht. Der folgende Beleg enthält einen vorangestellten, adversativen Adverbialsatz, der jedoch nicht einen Gegensatz, sondern den „Ersatz eines Umstandes“ (vgl. Duden-Grammatik 2016: 640) ausdrückt.

(264) Doch statt man jetzt denken müßte also fahre ich jetzt aus der Fremde heim ist es wieder total anders! (Jugendtagebuch VII: 20)

Dieser Typ ist nach Musan (2013: 63) als Substitutivadverbial einzustufen. Die Besonderheit des obigen Belegs besteht aus syntaktischer Sicht darin, dass statt als Subjunktor verwendet wird. Dieser Gebrauch von statt wird dem Substandard zugewiesen, er ist jedoch nach Breindl et al. (2014: 497) nicht auf den oberdeutschen Sprachraum (dem der obige Beleg entstammt), sondern ,,auf den niederdeutschen und mitteldeutschen Sprachraum [beschränkt]“.

\subsubsection{Konzessivsätze}

Die Verteilung der Konzessivsätze in den Korpustexten bzw. in den Teilkorpora wird in Tabelle 9 angegeben. Dabei soll allerdings bemerkt werden, dass ,Irrelevanzkonditionale‘, die von Baschewa (1983) als Konzessivsätze aufgefasst werden, hier nicht berücksichtigt, sondern an anderer Stelle behandelt werden (vgl. Kapitel 5.4.5). 


\begin{tabular}{|l|l|l|l|}
\hline & Nähe & Distanz & insg. \\
\hline 17. Jh. & $\begin{array}{l}\text { Güntzer I } \\
\text { 0 Belege }\end{array}$ & $\begin{array}{l}\text { Harsdörffer I } \\
5 \text { Beleg }\end{array}$ & 5 Belege \\
\hline 18. Jh. & $\begin{array}{l}\text { Schuhmacher Chronik II } \\
1 \text { Beleg }\end{array}$ & $\begin{array}{l}\text { Aichinger III } \\
3 \text { Belege }\end{array}$ & 4 Belege \\
\hline 19. Jh. & $\begin{array}{l}\text { Auswandererbriefe V } \\
5 \text { Belege }\end{array}$ & $\begin{array}{l}\text { Burckhardt V } \\
1 \text { Beleg }\end{array}$ & 6 Belege \\
\hline 20. Jh. & $\begin{array}{l}\text { Jugendtagebuch VII } \\
0 \text { Belege }\end{array}$ & $\begin{array}{l}\text { Lorenz VII } \\
1 \text { Beleg }\end{array}$ & 1 Beleg \\
\hline insg. & 6 Belege & 10 Belege & 16 Belege \\
\hline
\end{tabular}

Tabelle 9: Verteilung der Belege für vorangestellte Konzessivsätze in den Korpustexten bzw. in den Teilkorpora $(n=16)$

Im Korpus sind 13 Belege für eingeleitete Konzessivsätze zu finden. Sie werden vorrangig resumptiv angeschlossen $(84,6 \%, 11$ von 13 Belegen). Die nicht-integrative Stellung von vorangestellten Konzessivsätzen ist nicht belegt, der integrative Typ kommt in zwei Korpustexten mit je einem Einzelbeleg vor.

Bei der Verwendung der Verknüpfungsmittel ist eine Tendenz zur Grammatikalisierung zu beobachten. Bei den Subjunktoren der ob-Gruppe dominiert ab dem 18. Jh. die Kontaktstellung ( $o b$ wohl) bzw. die Zusammenschreibung (obgleich, obwohl), während in den Korpustexten aus dem 17. Jh. die Zusammenschreibung nur in einem Beleg (266) auftritt. Im folgenden Beleg für $o b$... wol in Distanzstellung tritt zwischen $o b$ und wol die Partikel nun auf, die möglicherweise dazu beiträgt, dass das Antezedens als gültig dargestellt wird.

(265) Ob nun wol viel aus Ehr- und Gewinnsucht solche Bedienung deß gemeinen Nutzens/ angetretten/so sind wir doch von solchen Absehen dieses Orts weit entfernet/ und ... (Harsdörffer I: 3)

In (266) ist die Zusammenrückung von Subjunktor $o b$ und Partikel wol belegt. In (267) erscheint der Subjunktor $o b$ in Zusammenschreibung mit der ursprünglich geltungsbekräftigenden Partikel gleich. Die Kontaktstellung der beiden Elemente und ihre Zusammenschreibung kann als ein Zeichen der Grammatikalisierung der konzessiven Verknüpfungsmittel angesehen werden (vgl. Di Meola 1997: 164).

(266) Dann obwol etliche Thiere auch Häde zu haben scheinen/ so sind doch solche nur den Unsrigen der gestalt nach gleich/ und können wie allein unsren Willen durch Bezeichnung der Buchstaben/zu verstehen geben (Harsdörffer I: 15f.)

(267) Dem Valentin und Anton Rast aus Pfaffenhofen geht es recht gut dē̄ sie sind jetzt auch in Springba: und zwar schon seit Ende August wo sie einige Zeit bei uns gearbeitet haben und dā haben sie sich ein Haus gemiethet und betreiben seiddem die Metzgerei wobei sie recht guten Verdienst haben. Obgleich schon ein Metzger 
hier ist, so verkaufen sie deñoch viel Fleisch und Wurst sie führen auch viel Fleisch nach Peoria wo sie auch viel verdienen. (Auswandererbriefe V: 28)

Bis auf eine Ausnahme (268) steht in den Belegen vom 17. bis zum 19. Jh. die Partikel doch (266) bzw. deñoch (267) im Mittelfeld des Obersatzes, die beim Ausdruck der konzessiven Relation konstitutiv sein dürfte. Der folgende Beleg aus der wenn-Gruppe enthält keine Partikel im Mittelfeld des Obersatzes:

(268) Wenngleich, als ich Europa verließ, ich nicht recht wuste was ich that, u. mein Geist u. Gemüthe mit Dunkelheit umhüllet waren, so ist es jetzt mein unerschütterlicher glaube, daß ich durch Gottes weise Rathschluss hier bin, u. ich kann sagen der Herr hat alles wohl gemacht (Auswandererbriefe V: 7)

Für die wenn-Gruppe liegen ingesamt nur 3 Belege aus dem 19. Jh. vor, die beiden anderen Belege mit diskontinuierlichem wenn ... auch bzw. we $\bar{n}$... schon werden im Gegensatz zu (268) mit doch im Mittelfeld des Obersatzes realisiert, vgl. z.B. den folgenden Einzelbeleg aus dem Distanztext des 19. Jhs.: ${ }^{212}$

(269) Wenn aber auch die Allegorien der italienischen Dichtungen, Kunstwerke und Feste an Geschmack und Zusammenhang im ganzen höher stehen, so bilden sie doch nicht die starke Seite. (Burckhardt V: 295)

Die Realisierung der Partikel im Mittelfeld des Obersatzes geht mit der Zeit zurück. Im Einzelbeleg aus dem 20. Jh. wird die semantische Relation nur durch die Verwendung des semantisch eindeutigen Subjunktors obwohl explizit bezeichnet:

(270) Obwohl ich also schon früh zu einer durchdachten erkenntnistheoretischen Einstellung gekommen war und obwohl ich mir darüber im klaren war, daß auch der Mensch angeborene Normen des Verhaltens hat, die den Fragestellungen und der Methodik der Naturforschung zugänglich sind, machte mein Erkenntnisstreben scharf von jenen spezifisch menschlichen Eigenschaften und Leistungen halt, die sich auf der Ebene des Kulturellen aufbauen. (Lorenz VII: 32)

Diese Veränderungen betreffen den Grad der semantischen Integration der Konzessivsätze. Was die topologische Integration betrifft, so ist eine historische Entwicklung im Sinne der Verbreitung des integrativen Stellungstyps nicht eindeutig abzulesen, obwohl in den Korpustexten aus dem 19. Jh. nur der resumptive Stellungstyp vorkommt, während im Einzelbeleg aus dem 20. Jh. (270) der integrative Stellungstyp zu beobachten ist. Es soll auch angemerkt werden, dass der komplexe Satz in (270) zwei koordinierte, durch den kopulativen

${ }^{212}$ Im Linkskontext wird beschrieben, dass es in der italienischen Kultur oft „,mit lauter verfehlten Attributen [überladene]“, aber „auch besser gelungene Allegorien“ gibt (Burckhardt V: 294) und diese insgesamt wertvoller sind als jene vom burgundischen Hofe. Da der Nebensatz keine Bedingung, auch keine irrelevante oder „blockierte Bedingung“ (Breindl et al. 2014: 263, 983), ausdrückt, wird er nicht als konditional oder irrelevanzkonditional gedeutet. Vielmehr ist die Analyse als Konzessivsatz sinnvoll. Der wenn ... auch-Satz drückt einen faktischen Sachverhalt aus, der eigentlich zum Gegenteil dessen führen sollte, was im Obersatz versprachlicht wird. 
Konnektor und verknüpfte obwohl-Sätze enthält, wobei der zweite durch einen Nebensatz zweiten Grades (daß auch der Mensch angeborene Normen des Verhaltens hat) und einen Nebensatz dritten Grades (die den Fragestellungen und der Methodik der Naturforschung zugänglich sind) erweitert wird. Dass diese komplexe Voranstellung mit dem integrativen Stellungstyp realisiert wird, zeigt die erweiterte Aufnahmefähigkeit des Vorfeldes in diesem Distanztext. In (268) aus dem Nähetext aus dem 19. Jh. liegt ebenfalls eine extreme Komplexität mit zwei koordinierten, mit kopulativem und verknüpften Konzessivsätzen und zwei weiteren Untersätzen (ein Temporal- und ein Objektsatz) vor, der Konzessivsatz wird jedoch mit resumptiver Stellung realisiert. Die Rolle des strukturellen Faktors kann hier aber nicht näher bestimmt werden, da die eingeleiteten Konzessivsätze vom 17. bis zum 19. Jh. mit Ausnahme des folgenden Belegs - stets resumptiv angeschlossen werden, unabhängig davon, ob sie eine einfache oder komplexe Struktur aufweisen. Für das 18. Jh. sind jedoch nur eingeleitete Konzessivsätze belegt, die weder mit weiteren Konzessivsätzen koordiniert noch mit weiteren Untersätzen erweitert sind.

(271) ist der gewalt des Eyses $u$. Wassers so schleunig angeloffen daß Es die Brücke eingerissen wovon 24 Menschen ertruncken obgleich viel hundert menschen am Uffer gestanden, konten sie doch disen Armen Leuthen nicht helffen. (Schuhmacher Chronik II: 331)

Der Konzessivsatz könnte sich auch auf den Inhalt des ihm vorangehenden Teilsatz beziehen. Der Beleg könnte als Apokoinu gedeutet werden, wenn man eine Abhängigkeit des obgleichNebensatzes sowohl vom ihm vorangehenden als auch vom ihm anschließenden Teilsatz annehmen würde. ${ }^{213}$

Für uneingeleitete Konzessivsätze liegen im Korpustext nur insgesamt drei eindeutige Belege vor. Ob der folgende Einzelbeleg aus Güntzer I als uneingeleiteter Konzessivsatz einzuordnen ist, ist fraglich:

(272) kam zu mihr ein Wolkemer von Etingen. Mit i[h]m reißete ich einen weidten Wog in Niderlandt. Mihr wahren 2 gudte Reißbrieder, hette[n] beide wol Gelt, hielten beide darmit zuruck, fordern den Zinß bey den Bauren ein. Unßer Fornemen wahr, weidt zu reißen, und kondten nicht alen Tag bey unßern Eltern Gelt und Gleider abhollen. (Güntzer I: 80r)

${ }^{213}$ Im folgenden Beleg wird - trotz des Interpunktionszeichens ,.,“ vor dem Subjunktor - eine Nachstellung des obwohlSatzes angenommen: Er relativiert die Gültigkeit der voranstehenden Aussage über die Länge der Freundschaft. Angesichts der Nachstellung wird dieser Beleg in der quantitativen Auswertung nicht berücksichtigt.

(i) Nun sind wir 4 Monate zusammen, das ist für die erste Freundschaft aber gar nicht schlecht. Obwohl es in letzter Zeit so aussah, als ob es zu Ende gehen würde, aber gestern waren wir auf einem Säet-Faschingsfeschd. (Jugendtagebuch VII: 6) 
Stünde im uneingeleiteten Nebensatz eine Verbform im Konjunktiv I oder mögen mit einem Beliebigkeitselement, würde dies eindeutig für die Annahme eines Konzessivsatzes sprechen (vgl. Baschewa 1983: 98ff.). Auch die bei Konzessivsätzen übliche Partikel doch fehlt aus dem Mittelfeld des Obersatzes. Diese formalen Elemente sind im obigen Beleg nicht zu identifizieren, nur die Partikel wol im Mittelfeld des vorangestellten Satzes könnte zum Ausdruck der konzessiven Relation beitragen. Die konzessive Relation ist also nur unter Einbeziehung des Kontextes nachvollziehbar. Der Beleg ist daher nicht eindeutig von Aussagesätzen mit Verberststellung abgrenzbar (Kappel 2004: 71f.), die in diesem Korpustext relativ frequent sind. Dieser Beleg wird deshalb in der quantitativen Analyse nicht berücksichtigt.

Im Einzelbeleg aus dem 17. Jh. ist die Wahl des integrativen Stellungstyps insofern überraschend, als in anderen Konzessivsätzen des Korpustextes stets der resumptive Typ gewählt wird.

(273) Ist meine Sprache schwartz!/ sind doch die Worter weiß. (Harsdörffer I: 12)

Im Korpus von Wiktorowicz (2013: 396) werden Konzessivsätze aus dem 17. Jh. ausschließlich resumptiv angebunden. Die Wahl des Stellungstyps könnte bei diesem Beleg durch rhythmische Faktoren bzw. durch die festgelegte Silbenzahl in einem Reim bedingt sein. Sowohl der Unter- als auch der Obersatz bestehen aus 6 Silben, die Verwendung eines Resumptivums würde den Rhythmus zerstören. Des Weiteren fällt auf, dass im VerberstKonzessivsatz die im Gegenwartsdeutschen obligatorische Partikel auch (Zifonun et al. 1997: 2313, von Polenz 1985: 271) nicht realisiert wird. Der nachgestellte Obersatz enthält die Partikel doch, die beim Ausdruck der konzessiven Relation konstitutiv sein dürfte.

Im Korpustext Aichinger III sind zwei Belege für uneingeleitete Konzessivsätze mit Verberststellung zu finden, beide werden mit dem resumptiven so angeschlossen. Eine weitere Gemeinsamkeit ist, dass im Mittelfeld des Obersatzes stets die Partikel doch realisiert wird, vgl. folgenden Beleg:

(274) Wird gleich der Laut, den es hat, meistens mit chs umschrieben, als in Wachs, wech//seln, Ochs, Fuchs, so hat sich doch das $x$ in etlichen erhalten, als in Axt, Eidexe, Hexe (Aichinger III: 17f.)

Im Untersatz erscheint die Partikel gleich, die im Korpustext bei konzessiven Verknüpfungen auch beim diskontinuierlichen $o b$... gleich belegt ist. ${ }^{214}$ Aufgrund der geringen Belegzahl

\footnotetext{
${ }^{214}$ Vgl. den folgenden Beleg für einen nachgestellten Konzessivsatz:

(i) so thut es beym erstern eben diese Wirkung, ob es gleich in der Absetzung zur folgenden Syllbe gezogen wird, als: gehen, wie gee en. (Aichinger III: 9).
} 
sind der Interpretation der Stellung von uneingeleiteten Konzessivsätzen in der vorliegenden Untersuchung Grenzen gesetzt. Dass ab dem 19. Jh. keine uneingeleiteten Konzessivsätze mehr belegt sind, entspricht jedoch der Tendenz der Zunahme eingeleiteter Konzessivsätze im Neuhochdeutschen (vgl. Ágel 2000: 1885, Baschewa 1983: 86f.).

Tabelle 10 zeigt zusammenfassend die Ergebnisse für eingeleitete und uneingeleitete Konzessivsätze. Zusammenfassend ist festzuhalten, dass in den analysierten Korpustexten die nicht-integrative Stellung von vorangestellten Konzessivsätzen nicht belegt ist. Der resumptive Anschluss mit so dominiert mit 81,3\% (13 von 16 Belegen) deutlich, die integrative Stellung ist in 3 Korpustexten mit je einem Einzelbeleg nachzuweisen.

\begin{tabular}{|l|c|c|c|}
\hline & integrativ & resumptiv & nicht-integrativ \\
\hline \multirow{2}{*}{ Güntzer I (n=0) } & 0 & 0 & 0 \\
& $0 \%$ & $0 \%$ & $0 \%$ \\
\hline \multirow{2}{*}{ Harsdörffer I (n=5) } & 1 & 4 & 0 \\
& $20,0 \%$ & $80,0 \%$ & $0 \%$ \\
\hline \multirow{2}{*}{ Schuhmacher Chronik II (n=1) } & 1 & 0 & 0 \\
& $100 \%$ & $0 \%$ & $0 \%$ \\
\hline \multirow{2}{*}{ Aichinger III (n=3) } & 0 & 3 & 0 \\
& $0 \%$ & $100 \%$ & $0 \%$ \\
\hline \multirow{2}{*}{ Auswandererbriefe V (n=5) } & 0 & 5 & 0 \\
& $0 \%$ & $100 \%$ & $0 \%$ \\
\hline \multirow{2}{*}{ Jugendtagebuch VII (n=0) } & 0 & 1 & $0 \%$ \\
\hline \multirow{2}{*}{ Lorenz VII $(\mathrm{n}=1)$} & $0 \%$ & 0 & $0 \%$ \\
\hline \multirow{2}{*}{ Gesamt $(n=16)$} & $0 \%$ & $0 \%$ & 0 \\
& 1 & 0 & $0 \%$ \\
\hline
\end{tabular}

Tabelle 10: Verteilung der Stellungstypen bei vorangestellten Konzessivsätzen

Aus der Beobachtung, dass der prozentuale Anteil des integrativen Typs im Teilkorpus für Distanzsprachlichkeit etwas höher ist als im Teilkorpus für Nähesprachlichkeit (1 von 6 vs. 2 von 10 Belegen), dürfen aufgrund der geringen Belegzahl keine Schlussfolgerungen gezogen werden. $^{215}$

\footnotetext{
215 Zur prozentualen Verteilung der Stellungstypen bei allen (eingeleiteten und uneingeleiteten) Konzessivsätzen in den Nähe- bzw. Distanztexten s. die Diagramme 13 und 14, zu eingeleiteten Konzessivsätzen s. die Diagramme 11 und $12 \mathrm{im}$ Anhang (8.2). Zu uneingeleiteten Konzessivsätzen wurde wegen der niedrigen Belegzahl kein Diagramm erstellt.
} 


\subsubsection{Irrelevanzkonditionale}

Im folgenden Beleg wird - wie auch bei eingeleiteten Konzessivsätzen (vgl. Kapitel 5.4.4) ein resumptives so im Vorfeld bzw. die Partikel doch im Mittelfeld des Obersatzes realisiert. Im vorangestellten Adverbialsatz erscheint die Partikel gleich, die im Text auch als Teil der zusammengerückten Form obgleich als konzessiver Subjunktor belegt ist (Harsdörffer I: 22).

(275) Lernen wir gleich Hebraeisch und Griechisch/Lateinisch/Frantzosisch/Italianisch und andere ausländische Sprachen/ so können wir doch in allen Städen/ unsrem geehrten Vatterland mit keiner mehr als unsrer angeborenen teutschen Sprache dienen/ und müssen wir Teutsche uns mit dem Teutschen behelffen / ja fast in allen Standen unsre Nahrung damit erwerben. (Harsdörffer I: 4)

Dieser Beleg ist jedoch nicht als konzessiv einzustufen, denn es „kann nicht auf die Gültigkeit des Antezedens geschlossen werden“ (Zifonun et al. 1997: 2319). Es bleibt allerdings zu bemerken, dass das Konsequens unabhängig vom Inhalt des Antezedens als gültig angenommen werden kann. Damit teilt dieser Beleg eine wichtige Eigenschaft des ,Irrelevanzkonditionals', wobei sich wiederum der Inhalt des Verberstsatzes nicht unbedingt als „Menge von Antezedensbedingungen, die ein ganzes Spektrum von Möglichkeiten mehr oder weniger erschöpfend erfassen“ (Zifonun et al. 1997: 2319), beschreiben lässt.

Der folgende Beleg für Irrelevanzkonditionale tritt mit dem integrativen Stellungstyp auf:

(276) Es ist so miserabel und Greilich zugangen, daß nicht gnugsam zu beschreiben, man hat einander beraubt und bestolen, daß Es ein Greyl gewessen. Es ist ärger zugangen als zur Zerstörung Jerusalem wangleich der Feind mit höresmacht wehre über unser Statt gezogen und unß rein außgeblindert, hät Es nicht miserabler können daher gehen, der eine hat Bett verlohren, der andere hat Schreinwerck verlohren, der dritte hat Leinwand verlohren, der vierte hat Faß verlohren, und so Fort, und ist ärger und leichtförtiger Zugangen als im grösten Krieg. (Schuhmacher Chronik II: 402)

Es handelt sich hier um den Subtyp ,skalares Irrelevanzkonditional' (vgl. Kapitel 3.4.3), in dem beim Inhalt des Antezedens ein extrem hoher Wert aus einer skalar geordneten Menge von Alternativen genannt wird, der aber eine irrelevante Bedingung für die Gültigkeit des Konsequens darstellt (,irreales Konzessivgefüge“ nach der Duden-Grammatik 2016). Für den Ausdruck des Extremwerts ist die Partikel gleich verantwortlich (Breindl et al. 2014: 983), die hier mit dem Subjunktor wan zusammengeschrieben wird. Irrelevanzkonditionale kommen im Gegenwartsdeutschen zwar häufig desintegriert vor, ,skalare Irrelevanzkonditionale‘ können jedoch auch integrativ oder (seltener) resumptiv angeschlossen werden (vgl. Breindl et al. 2014: 983 bzw. Kapitel 3.4.3). Zu bemerken ist, dass hier der integrative Stellungstyp gewählt 
wurde, obwohl die Gültigkeit der Proposition im Hauptsatz nicht durch das Antezedens eingeschränkt wird. In diesem Sinne ist dieser Beleg als eine Verletzung des Prinzips der Ikonizität zu interpretieren (vgl. Kapitel 2.1).

Die beiden Irrelevanzkonditionale (277) sowie (278) enthalten den Ausdruck so + Adjektiv, in beiden Fällen werden sie durch resumptives so wieder aufgenommen:

(277) Und so ungewohnt es am Ende aussieht, so man schreibet: ei, bei, frei, so nimmt doch diese Schreibart jetziger Zeit selbst überhand. (Aichinger III: 18)

(278) So gut die angegebenen Regeln sind: so wird man doch aus denselben nicht so leicht, als aus einem Verzeichnusse der Worter, deren Schreibart zweifelhafft ist, Rath holen konnen, wie dieses oder jenes auszudrücken sey. (Aichinger III: 39)

Im vorangestellten Adverbialsatz kommt im Gegensatz zum Gegenwartsdeutschen kein auch oder immer vor (vgl. Breindl et al. 2014: 1206). Bemerkenswert ist jedoch das Auftreten der bei konzessiver Verwendung frequenten Partikel doch im Mittelfeld des Obersatzes. ${ }^{216}$ Die Unterscheidung zwischen konzessiv bzw. irrelevanzkonditional ist nicht einfach. Da jedoch weniger von der Faktizität des Untersatzes (vgl. Breindl et al. 2014: 950f.) ausgegangen werden kann, sondern eher davon, dass die Gültigkeit des Antezedens offen bleibt, wurde bei (278) die Entscheidung für irrelevanzkonditional getroffen.

Auch in Burckhardt V sind Irrelevanzkonditionalsätze belegt. Im folgenden, durch w-auch immer eingeleiteten Nebensatz trägt auch die Modalisierung durch mögen dazu bei, dass das Antezedens einer offenen Menge von Propositionen entspricht, deren Wahrheit als irrelevant für die Wahrheitsbedingungen des Konsequens betrachtet wird. Es handelt sich um ein universales Irrelevanzkonditional (vgl. Kapitel 3.4.3).

(279) Abgesehen von diesem nicht unwesentlichen Anhalt der Ritterwürde gab es auch bereits, z. B. in Ferrara wahre Hoforden, welche den Titel Kavaliere mit sich führten. Welches aber auch die einzelnen Ansprüche und die Eitelkeiten der Adligen und Kavaliere sein mochten, immerhin nahm der italienische Adel seine Stellung in der Mitte des Lebens und nicht an einem äußern Rande desselben. (Burckhardt V: 266f.)

Das Vorfeld des Obersatzes ist durch immerhin besetzt, das von Breindl et al. (2014: 1008) als irrelevanzkonditionaler Adverbkonnektor beschrieben wird. Bei dieser Kategorie wird eine „mehr oder weniger ausgeprägte Vorfeldfeindlichkeit“ (Breindl et al. 2014: 998) angenommen. Die Besonderheit des obigen Belegs besteht aber auch darin, dass die

${ }^{216}$ Die folgende Beschreibung, die von Breindl et al. (2014: 948) für die konzessive Verwendung von wenn auch aufgeführt wird, könnte auch für die einschlägigen Belege mit so+Adjektiv zutreffen: „Die typische Verwendung ist die im Rahmen des kompensatorischen Vorteil-Nachteil-Schemas. Dabei bezeichnet das interne Argument von wenn auch einen Sachverhalt, aus dem eigentlich auf das Gegenteil dessen zu schließen wäre, was aus dem mit dem externen Konnekt bezeichneten folgt." 
irrelevanzkonditionale Relation sowohl durch eine w-auch-immer-Phrase im Untersatz als auch durch einen Adverbkonnektor im Obersatz markiert wird. Der Ausdruck immerhin wird hier nicht als Korrelat analysiert, weil es syntaktisch gesehen keine Entsprechung des Adverbialsatzes im Obersatz darstellt (vgl. Kapitel 3.3.2 bzw. Zitterbart 2013: 602-606). Daher wird hier eine nicht-integrative Stellung des Nebensatzes angenommen. Auch im folgenden Beleg wird der vorangestellte Nebensatz nicht-integrativ angeschlossen. Der vor dem Nebensatz ,wie dem auch sei“ realisierte Ausdruck allein ist ein adversativer (Adverb-)Konnektor, der zwei komplexe Sätze miteinander verbindet (vgl. Duden-Grammatik 2016: 635, Breindl et al. 2014: 561). An der Position von allein könnte die adversative Konjunktion aber stehen. Diese Position vor dem Vorfeld wird ,Nullstelle' genannt (Pasch et al. 2003: 487), sie befindet sich außerhalb der Felderstruktur des Satzes. Da allein nicht der Satzstruktur im engeren Sinne zugerechnet werden kann, wird der einschlägige Satz nicht als Parenthese, sondern als Voranstellung eingestuft. Im Vorfeld des Obersatzes steht hier eine Präpositionalgruppe.

(280) Und andere, die der Sprache im höchsten Sinne mächtig waren, verließen sich hinwiederum auf den prachtvoll wogenden Gang und Wohllaut derselben als auf einen vom Inhalt unabhängigen Vorzug. Auch eine geringe Melodie kann nämlich, von solch einem Instrument getragen, herrlich klingen. Allein, wie dem auch sei, in gesellschaftlicher Beziehung hatte diese Sprache einen hohen Wert. (Burckhardt V: 273)

Der vorangestellte Nebensatz ist ein formelhafter Ausdruck, der die im Linkskontext aufgeführten und alle denkbaren Inhalte als irrelevant für die Gültigkeit des Konsequens darstellt. Das Verb im Konjunktiv Präsens (sei) dient zum Ausdruck von Möglichkeiten aus einer offenen Menge (,universales Irrelevanzkonditional', vgl. Kapitel 3.4.3). Der formelhafte Ausdruck wie dem auch sei ist auch im neuhochdeutschen Korpus von Baschewa (1983: 97) belegt, ebenfalls in nicht-integrativer Stellung.

Neben den obigen Belegen für eingeleitete Irrelevanzkonditionalsätze tritt im Korpustext auch ein uneingeleiteter Irrelevanzkonditionalsatz auf. In der Textstelle werden die Benutzung von Gesichtspflegeprodukten und ihre einstige Kritik beschrieben.

(281) Es ist möglich, dass die häufigen und prachtvollen Aufführungen von Mysterien, wobei Hunderte von Menschen bemalt und geputzt wurden, den Missbrauch im täglichen Leben fördern halfen; jedenfalls war er ein allgemeiner, und die Landmädchen hielten dabei nach Kräften mit. Man konnte lange predigen, dass dergleichen ein Abzeichen von Buhlerinnen sei; gerade die ehrbarsten Hausfrauen, die sonst das ganze Jahr keine Schminke anrührten, schminkten sich doch an Festtagen, wo sie sich öffentlich zeigten. - Möge man nun diese ganze Unsitte betrachten als einen Zug von Barbarei, wofür sich das Schminken der Wilden als 
Parallele anführen läßt, oder als eine Konsequenz des Verlangens nach normaler jugendlicher Schönheit in Zügen und Farbe, wofür die grosse Sorgfalt und Vielseitigkeit dieser Toilette spräche - jedenfalls haben es die Männer an Abmahnungen nicht fehlen lassen. (Burckhardt V: 269f.)

Im uneingeleiteten Verberstsatz werden zwei Alternativen formuliert, die in disjunktiver Relation stehen. Sie werden nicht als gültig dargestellt, vielmehr wird ihre Gültigkeit offen gelassen und als irrelevant für die Gültigkeit des Konsequens dargestellt. Im Vorfeld des Obersatzes steht jedenfalls, das im Gegenwartsdeutschen nach Breindl et al. (2014: 1198) ein nicht positionsbeschränkter Adverbkonnektor ist. Wie auch beim obigen Beleg (276), so wird auch hier eine nicht-integrative Stellung des Nebensatzes angenommen.

In Auswandererbriefe $\mathrm{V}$ sind zwei Belege für Irrelevanzkonditionalsätze $\mathrm{zu}$ finden. Sowohl der durch wie ... noch eingeleitete (282) als auch der uneingeleitete Irrelevanzkonditionalsatz (283) werden resumptiv angeschlossen:

(282) wie sehr es noch Eltern ueber Verlust kleiner Kinder schmerz so haben doch solche Kinder ein großes Vorrecht den sie sind des Gefahr und mühevollen Erdenlebens enthoben und können Anstat deßen da sein wo kein leid und Geschrei mer ist sondern lauter Seeligkeit. (Auswandererbriefe V: 11)

(283) Jedoch könen wir es angreifen wie wir wollen, so wird unser beschiedenes Theil, das uns wird zugemessen werden, noch bedeutend verschmällert ehe es die Küste Amerika erreichen kan̄. (Auswandererbriefe V: 6)

Die Partikel doch erscheint nur in (282) im Mittelfeld des Obersatzes. Der uneingeleitete Konzessivsatz mit Verbzweitstellung (jedoch ist als Konnektor im Vorfeld zu analysieren) hat nicht die strukturellen Merkmale, die für das Gegenwartsdeutsche angenommen werden. Leuschner (2005: 289) nennt jedoch ein Beispiel für die asyndetische Formvariante mit der Protasis sie können machen, was sie wollen, die als universales Irrelevanzkonditional zu interpretieren ist, obwohl es „,einen grammatisch völlig selbständigen Satz“ enthält. In der Tat ist hier die Abgrenzung von hypotaktischen und parataktischen Verknüpfungen nicht unproblematisch.

Der Korpustext Lorenz VII enthält zwei vorangestellte Irrelevanzkonditionale. In (284) liegt ein ,universales Irrelevanzkonditional' vor, das nicht die kanonische Form so ADJ auch (immer), sondern nur so+ADJ enthält. Im Mittelfeld des Obersatzes wird jedoch die Partikel doch realisiert, die zur Herstellung der semantischen Relation zwischen den Konnekten beiträgt.

(284) So himmelhoch sich diese Systemeinheit über alle anderen uns bekannten erhebt, teilt sie doch mit ihnen ein unvermeidliches Schicksal: Wie alle lebenden Systeme, so 
ist auch der menschliche Geist und mit ihm die menschliche Kultur Störungen unterworfen. (Lorenz VII: 16)

Im folgenden Beleg wird nur die Wahrheit des Konsequens impliziert, die nicht davon abhängt, ob der Sachverhalt im vorangestellten Adverbialsatz wahr ist. Der Adverbialsatz wird durch auch wenn eingeleitet und enthält ,eine Kombination aus noch so + Adjektiv/Adverb (...), durch die die freie Wahl unter Alternativen aufgezeigt wird“" (Zifonun et al. 1997: 2321). Im Mittelfeld des Obersatzes tritt die Partikel doch nicht auf. Es handelt sich um ein ,skalares Irrelevanzkonditional', denn das Antezedens enthält einen Extremwert und ,impliziert dadurch, dass die Konditionalbeziehung auch für weitere, weniger auffällige Werte gilt" (Leuschner 2005: 286).

(285) Wir können nichts erfahren, nichts anschauen und nichts denken, ohne dies aufgrund von Voraussetzungen, von Unterstellungen zu tun, in denen solche angeborene Hypothesen stecken: Sie sind in unserer »Weltbildapparat" eingebaut! Auch wenn wir noch so sehr danach trachten, in freier Tat neue Hypothesen zu erfinden, können wir nicht verhindern, daß in ihnen diese uralten durch Mutation und Neukombination von Genen entstandenen und durch äonenlanges "pattern matching « erprobten Hypothesen des Apriorischen stecken, die nie ganz dumm, aber immer starr und nie restlos zutreffend sind. (Lorenz VII: 44)

Beide vorangestellten Irrelevanzkonditionale aus dem 20. Jh. werden integrativ angeschlossen. Die folgende Tabelle zeigt die Verteilung der Stellungstypen:

\begin{tabular}{|l|c|c|c|}
\hline & integrativ & resumptiv & nicht-integrativ \\
\hline \multirow{2}{*}{ Güntzer I (n=0) } & 0 & 0 & 0 \\
& $0 \%$ & $0 \%$ & $0 \%$ \\
\hline \multirow{2}{*}{ Harsdörffer I (n=1) } & 0 & 1 & 0 \\
& $0 \%$ & $100 \%$ & $0 \%$ \\
\hline \multirow{2}{*}{ Schuhmacher Chronik II (n=1) } & 1 & 0 & 0 \\
& $100 \%$ & $0 \%$ & $0 \%$ \\
\hline \multirow{2}{*}{ Aichinger III (n=2) } & 0 & 2 & 0 \\
& $0 \%$ & $100 \%$ & $0 \%$ \\
\hline \multirow{2}{*}{ Buswandererbriefe V (n=2) } & 0 & 2 & 0 \\
& $0 \%$ & $100 \%$ & 3 \\
\hline \multirow{2}{*}{ Jugendtagebuch VII (n=0) } & 0 & 0 & $100 \%$ \\
\hline \multirow{2}{*}{ Lorenz VII (n=2) } & 0 & $0 \%$ & $0 \%$ \\
\hline \multirow{2}{*}{ Gesamt $(n=11)$} & $0 \%$ & $0 \%$ & 0 \\
& 2 & 0 & 3 \\
\hline
\end{tabular}

Tabelle 11: Verteilung der Stellungstypen bei vorangestellten Irrelevanzkonditionalen 
Zusammenfassend ist jedoch festzustellen, dass trotz der beiden Belege aus dem 20. Jh. keine klare Tendenz zur Verbreitung des integrativen Typs zu beobachten ist. Der Anteil der integrativen Anbindung (3 von 11 Belegen, 27,3\%) könnte nämlich auch mit den Untertypen der Irrelevanzkonditionale im Zusammenhang stehen. Die integrative Stellung, die im Gegenwartsdeutschen nach Breindl et al. (2014: 983) nur beim Untertyp ,skalare Irrelevanzkonditionale' möglich ist, ist bei diesem Untertyp zweimal belegt ((279) und (285)). Leuschner (2005: 288) nennt jedoch auch einen Beleg für ,universales Irrelevanzkonditional` mit integrativer Anbindung aus der Gegenwartssprache. Der dritte Beleg (284) für integrative Anbindung entspricht diesem Untertyp. Zu bemerken ist, dass auch der nicht-integrative Typ mit drei Belegen (alle aus dem Distanztext aus dem 19. Jh.) vertreten ist. Im Näheteilkorpus finden sich insg. nur 3 Irrelevanzkonditionale, daher scheint eine quantitative Auswertung des Materials bezüglich der Nähe-Distanz-Dimension nicht aussagekräftig zu sein. ${ }^{217}$

\subsubsection{Spezifizierende Adverbialsätze (Explikativ- und Restriktivsätze)}

Im Korpus sind lediglich drei einschlägige Belege zu finden. Der Subjunktor insofern leitet sowohl einen Beleg für Explikativsätze als auch den Einzelbeleg für Restriktivsätze ein, zudem findet sich ein durch indem eingeleiteter Adverbialsatz. Alle einschlägigen Adverbialsätze sind integrativ angebunden. Eine weitere Gemeinsamkeit ist, dass alle Belege aus dem Distanztext aus dem 19. Jh. stammen. Beim folgenden Beleg ist eine explikative Relation anzunehmen, durch den vorangestellten Adverbialsatz wird die Aussage des Obersatzes erklärt:

(286) Außerdem aber ist der Galateo eine schön und geistvoll geschriebene Unterweisung in der guten Lebensart, in Delikatesse und Takt überhaupt. Noch heute können ihn Leute jedes Standes mit großem Nutzen lesen, und die Höflichkeit des alten Europas wird wohl schwerlich mehr über seine Vorschriften hinauskommen. Insofern der Takt Herzenssache ist, wird er von Anfang aller Kultur an bei allen Völkern gewissen Menschen angeboren gewesen sein, und einige werden ihn auch durch Willenskraft erworben haben, allein als allgemeine gesellige Pflicht und als Kennzeichen von Bildung und Erziehung haben ihn erst die Italiener erkannt. (Burckhardt V: 271)

Auch der folgende Beleg ist explikativ zu deuten:

(287) wir erfahren auch, er sei ein großer Neuerer gewesen, allein ob er oder andere den entscheidenden Schritt in die Tonsprache der modernen Welt hinein getan haben, wird nicht so erörtert, dass der Laie sich einen Begriff von dem Tatbestand machen könnte. Indem wir daher die Geschichte der musikalischen Komposition gänzlich auf

217 Zur prozentualen Verteilung der Stellungstypen in den Nähe- bzw. Distanztexten s. die Diagramme 15 und 16 im Anhang (8.2). 
sich beruhen lassen, suchen wir die Stellung der Musik zur damaligen Gesellschaft auszumitteln. (Burckhardt V: 283)

Der Subjunktor insofern ist auch im Gegenwartsdeutschen sowohl als explikative als auch als restriktive Subjunktion bekannt (vgl. Duden-Grammatik 2016: 1114f.). Im folgenden Beleg schränkt der Adverbialsatz die Gültigkeit der nachstehenden Aussage ein:

(288) Außerdem beklagen sie den bereits äußerst raschen Wechsel der Moden und (wenn wir die Worte richtig deuten) die törichte Verehrung alles dessen, was aus Frankreich kommt, während es doch oft ursprünglich italienische Moden seien, die man nur von den Franzosen zurückerhalte. Insofern nun der häufige Wechsel der Kleiderformen und die Annahme französischer und spanischer Moden der gewöhnlichen Putzsucht diente, haben wir uns damit nicht weiter zu beschäftigen; allein es liegt darin außerdem ein kulturgeschichtlicher Beleg für das rasche Leben Italiens überhaupt in den Jahrzehnten um 1500. (Burckhardt V: 268)

Der Adverbialsatz bezieht sich auf einen Aspekt des Kleiderwechsels, der für Burckhardts Anliegen nicht einschlägig ist. Der komplexe Satz ist paraphrasierbar mit einem Obersatz, der die semantische Beziehung, restriktiv“ explizit markiert (vgl. von Polenz 1985: 237): „Dass wir uns damit nicht weiter zu beschäftigen haben, gilt nur insofern, als der häufige Wechsel der Kleiderformen und die Annahme französischer und spanischer Moden der gewöhnlichen Putzsucht diente“. Diese Interpretation wird auch dadurch unterstützt, dass das einschlägige Thema im Text unter kulturgeschichtlicher Perspektive weiter behandelt wird. Diese Gebrauchsweise wird von Zifonun et al. (1997: 2326) wie folgt beschrieben: „Mit dem Untersatz wird derjenige Aspekt genannt, auf den die Gültigkeit der Aussage eingeschränkt werden soll.“ Der Untersatz könnte im Gegenwartsdeutschen mit soweit paraphrasiert werden (vgl. Zifonun et al. 1997: 2326, Engel 2004: 152).

\subsubsection{Komparativsätze}

Das Vorkommen von vorangestellten Komparativsätzen ist in den einzelnen Korpustexten unausgeglichen: Während in den meisten Korpustexten vorangestellte Komparativsätze nur vereinzelt nachzuweisen (Güntzer I, Schuhmacher Chronik II, Aichinger III, Buckhardt V, Lorenz VII) oder gar nicht belegt sind (Auswandererbriefe V, Jugendtagebuch VII), kommen in Harsdörffer I 10 Belege (50\% aller Belege) vor. 


\begin{tabular}{|l|l|l|l|}
\hline & Nähe & Distanz & insg. \\
\hline 17. Jh. & $\begin{array}{l}\text { Güntzer I } \\
1 \text { Beleg }\end{array}$ & $\begin{array}{l}\text { Harsdörffer I } \\
10 \text { Belege }\end{array}$ & 11 Belege \\
\hline 18. Jh. & $\begin{array}{l}\text { Schuhmacher Chronik II } \\
1 \text { Belege }\end{array}$ & $\begin{array}{l}\text { Aichinger III } \\
\text { 4 Belege }\end{array}$ & 5 Belege \\
\hline 19. Jh. & $\begin{array}{l}\text { Auswandererbriefe V } \\
\text { 0 Belege }\end{array}$ & $\begin{array}{l}\text { Burckhardt V } \\
1 \text { Beleg }\end{array}$ & 1 Beleg \\
\hline 20. Jh. & $\begin{array}{l}\text { Jugendtagebuch VII } \\
\text { 0 Belege }\end{array}$ & $\begin{array}{l}\text { Lorenz VII } \\
\text { 3 Belege }\end{array}$ & 3 Belege \\
\hline insg. & 2 Belege & 18 Belege & 20 Belege \\
\hline
\end{tabular}

Tabelle 12: Verteilung der Belege für vorangestellte Komparativsätze in den Korpustexten bzw. in den Teilkorpora $(n=20)$

Im Teilkorpus des 17. Jhs. sind insg. 11 Belege für vorangestellte Komparativsätze zu finden, diese werden stets resumptiv wieder aufgenommen. In beiden Korpustexten aus dem 17. Jh. sind vorangestellte Komparativsätze belegt, die durch gleichwie bzw. gleich wie eingeleitet werden. Diese Belege werden resumptiv mit also bzw. so angebunden. Der Einzelbeleg (289) in Güntzer I weist dabei eine aus syntaktischer Sicht einfache Voranstellung auf, der Beleg (290) aus Harsdörffer I ist jedoch syntaktisch komplex, der Komparativsatz steht in kopulativer Verknüpfung mit einem weiteren Komparativsatz, der durch einen Relativsatz erweitert wird.

(289) Gleich wie diße Merdter auff den Reder ligen, so werdtet ihr auch darauff gelegt werden, darum nemet ein Exenpel an dißen Merder, welche dahier geredert sindt worden. (Güntzer I: 43v)

(290) Gleichwie nun ein jeder Christ fur sich in der Noht ein Gebetlein zu Gott soll verabfassen konnen/ un er zu solchem Ende die H. Schrifft und unterschiedene Betbucher lieset/ daraus er sein Wort/ bey dem Herrn Himmels und der Erden furbringen lernet: Also soll ein jeder seine Gedancken/ nach erheischter Begebenheit nach hier begriffenel oder andre Weise ein Gruß-oder Handels-Brieflein verfassen lernen/ ... (Harsdörffer I: 17)

In Harsdörffer I kommen neben den beiden durch gleichwie eingeleiteten Komparativsätzen acht wie-Sätze vor, auch diese werden stets resumptiv wieder aufgenommen (mit den Korrelaten als bzw. also), unabhängig davon, ob sie syntaktisch einfach oder komplex sind:

(291) Was wåre der Streit zwischen Fleisch und Geist vonnơhten/wann wir alle lüstrenden Begierden ablegen oder derselben mit leichter Hand obsiegen könten? Nein/ wie die Seele der Freude und des Leibs fähig ist/ also ist der Leibe der Wollust und dem Schmerzen zugethan (Harsdörffer I: 367) 
(292) ... und Wie es einem Schuster keine Schande ist/ daß er kein Kleid machen kann/ also ist es auch einem Ungelehrten nicht zu verargen/ wann er nicht weiß/ was er nicht gelernet hat. (Harsdörffer I: 169)

Im Teilkorpus aus dem 18. Jh. sind insg. 5 vorangestellte Komparativsätze belegt, sie werden - wie auch die entsprechenden Sätze aus dem 17. Jh. - resumptiv angeschlossen. In Aichinger III werden drei Belege durch wie und ein Beleg durch gleichwie eingeleitet, wobei alle durch also wieder aufgenommen werden, vgl. (293):

(293) Wie es nun zu viel ist, so man in der Verdopplung 2. z machet, als: Hizze: also ists zu wenig, überall nur eins brauchen (Aichinger III: 19)

Bei diesem Beleg wird dem Ausdruck zu viel (im Vergleichssatz) der Ausdruck zu wenig (im Obersatz) gegenüber gestellt. Im Einzelbeleg aus Schuhmacher Chronik II wird der wie-Satz durch das Korrelat $s o$ wieder aufgenommen. ${ }^{218}$

(294) hat Es Zwey Nächt geregnet und ein groß Gewässer geben, daß Es zwey Weer zurüssen, daß erste bey der Graben-Mühle und daß andre bey der undren Mühle, folget gelinde Wütterung zu deß Monats Ende. Wie der Monat sich geendet, So nimbt der Feb. seinen Anfang, in der wütterung den 5ten dito wider kalt, und cont. biß auff den 12ten dito, da hat Es wider geschnien und kalt darauff und cont. biß auff den 16. \& 17ten dito, Es hat 31ig Stund an Einander geschnien, (Schuhmacher Chronik II: 417f.)

Trotz der geringen Belegzahl ist eine klare Tendenz beim Gebrauch der Resumptiva zu beobachten: Während bei den Distanztexten aus dem 17. und 18. Jh. durchgehend also/als verwendet wird (insg. 14 von 14 Komparativsätzen), tritt in den beiden Nähetexten so auf (2 von 2 Belegen).

Im Distanztext aus dem 19. Jh. ist ein Einzelbeleg für Komparativsätze zu finden, er wird resumptiv mit dem Korrelat so wieder aufgenommen:

(295) Wie aber das weltliche Drama eben durch eine solche Ausstattung zu Schaden kam, so ist auch wohl die höhere poetische Entwicklung des Mysteriums selber durch dieses unmäßige Vordrängen der Schaulust gehemmt worden. (Burckhardt V: 297)

Weitere Komparativsätze sind im Teilkorpus für das 19. Jh. nicht belegt.

Während im Nähetext aus dem 20. Jh. keine Komparativsätze auftreten, sind in Lorenz VII durch wie eingeleitete vorangestellte Komparativsätze belegt. In (296) wird der durch wie eingeleitete Komparativsatz mit resumptivem so wieder aufgenommen.

${ }^{218}$ Beim folgenden Beleg ist der Bezugssatz des wie-Satzes schwer zu bestimmen. Beim Komparativsatz wird hier eine Nachstellung angenommen, da hier die Geschehnisse am dritten Tag des Empfanges des Grafen mit denen am ersten Tag verglichen werden:

(i) Am dritten Tag hat sich widerum eine löbliche Burgerschafft, sich schön in ein Proceß gemacht, und dem Grafen Eben daß Gelayt hinaus gegeben, wie es den erste[n] Tag ist geschehen mit losung der Stuck und Doppelhocken ist alles volendet worden. (Schuhmacher Chronik II: 327) 
(296) All die physiologischen Leistungen, auf denen diese sog. Konstanzphänomene beruhen, sind erkenntnis-theoretisch deshalb von so großem Interesse, weil sie der schon besprochenen Leistung der bewußten, verstandesmäßigen Objektivierung streng analog sind. Wie der Mensch in meinem Beispiel die Temperatur der wahrnehmenden Hand in Rechnung stellt und so die "subjektive" Wahrnehmung "fieberheiß" auf ein "objektiveres" Maß reduziert, so sieht auch die »konstantmachende" Wahrnehmung der Gegenstandsfarbe von der augenblicklichen Beleuchtungsfarbe ab, um eine dem Objekt eigene Reflexionseigenschaft zu ermitteln. (Lorenz VII: 23)

In (297) wird jedoch so direkt vor dem Komparativsatz realisiert, sodass so und der wie-Satz gemeinsam das Vorfeld des Obersatzes besetzen (vgl. Thurmair 2001: 76).

(297) Es gibt aber auch Leistungen des Wissensgewinns, die gänzlich anderer Art sind. So wie die Anpassung körperliche Strukturen schuf, die dem Erwerb und der Verwertung von Energie dienen, hat sie auch solche hervorgebracht, deren Funktion im Gewinnen und Auswerten von Information, von Wissen besteht, und zwar von Wissen über augenblicklich in der Welt des Organismus obwaltende Umstände, denen sofort Rechnung getragen werden muß. (Lorenz VII: 42)

So wird hier also nicht resumptiv verwendet: „In diesen Fällen löst so den Vergleich aus, indem es auf eine allgemeine, nicht näher spezifizierte Modalität verweist"“. (Thurmair 2001: 76) Da Voranstellungen mit so + wie-Satz auch mit resumptivem so aufgegriffen werden können, sollte dieser Beleg nicht als Zwischenstellung aussortiert werden, es ist vielmehr eine Tendenz zur Entstehung des komplexen Subjunktors so wie zu vermuten. ${ }^{219}$ Dies ist der einzige Beleg in den untersuchten Korpustexten, der eindeutig den integrativen Stellungstyp aufweist. Zudem wird ein weiterer Beleg dem integrativen Typ zugerechnet. Er zeigt einen elliptisch realisierten wie-Satz:

(298) Wie die große Mehrzahl der Dinge in unserem Universum ist aber der Hund durchaus nicht von menschlicher Planung entworfen. (Lorenz VII: 29)

Bei diesem Beleg stellt sich die Frage, ob eine wie-Phrase (Präpositionalphrase) oder ein wieSatz ins Vorfeld integriert ist. Im ersten Falle müsste der Beleg aussortiert werden, da er dem Satzkriterium nicht erfüllt. An dieser Stelle wird für die zweite Alternative argumentiert: Da die elliptische Konstruktion in Bezug auf die Satzstruktur im Obersatz durchaus rekonstruierbar ist (Wie die große Mehrzahl der Dinge in unserem Universum nicht von menschlicher Planung entworfen ist), liegt hier ein (elliptisch realisierter) wie-Satz vor.

${ }^{219}$ In Thurmair (2001: 77) findet sich Beleg (i) für die Voranstellung von so + wie-Satz mit resumptivem so im Vorfeld des Obersatzes:

(i) So wie uns die Löwen neben unserem Bett tief beeindrucken, so mußte Myles umgekehrt erst einmal mit den vielen Menschen auf den Straßen fertig werden. 
In der folgenden Tabelle wird zusammenfassend der Anteil der Stellungstypen bei vorangestellten Komparativsätzen gezeigt:

\begin{tabular}{|l|c|c|c|}
\hline & integrativ & resumptiv & nicht-integrativ \\
\hline \multirow{2}{*}{ Güntzer I (n=1) } & 0 & 1 & 0 \\
& $0 \%$ & $100 \%$ & $0 \%$ \\
\hline \multirow{2}{*}{ Harsdörffer I (n=10) } & 0 & 10 & 0 \\
& $0 \%$ & $100 \%$ & $0 \%$ \\
\hline \multirow{2}{*}{ Schuhmacher Chronik II (n=1) } & 0 & 1 & 0 \\
& $0 \%$ & $100 \%$ & $0 \%$ \\
\hline \multirow{2}{*}{ Aichinger III (n=4) } & 0 & 4 & 0 \\
& $0 \%$ & $100 \%$ & $0 \%$ \\
\hline \multirow{2}{*}{ Auswandererbriefe V (n=0) } & 0 & 0 & 0 \\
& $0 \%$ & $0 \%$ & $0 \%$ \\
\hline \multirow{2}{*}{ Burckhardt V (n=1) } & 0 & 1 & 0 \\
\hline \multirow{2}{*}{ Jugendtagebuch VII (n=0) } & $0 \%$ & $100 \%$ & 0 \\
\hline \multirow{2}{*}{ Lorenz VII (n=3) } & $0 \%$ & $0 \%$ & $0 \%$ \\
\hline \multirow{2}{*}{ Gesamt $(n=20)$} & 2 & 1 & 0 \\
& $66,7 \%$ & $33,3 \%$ & $0 \%$ \\
\hline
\end{tabular}

Tabelle 13: Verteilung der Stellungstypen bei vorangestellten Komparativsätzen

Bei Komparativsätzen dominiert also der resumptive Stellungstyp (90\%, 18 von 20 Belegen). In den Korpustexten vom 17. bis zum 19. Jh. sind keine Unterschiede nachzuweisen, die auf die Nähe-Distanz-Dimension zurückzuführen wären. ${ }^{220}$ Allerdings ist auffällig, dass die beiden Belege für die integrative Stellung von Komparativsätzen aus dem Distanztext aus dem 20. Jh. stammen. Vergleichsbelege aus dem Nähetext aus dem 20. Jh. liegen nicht vor.

\subsubsection{Proportionalsätze}

In den Teilkorpora für das 18. und 20. Jh. sind keine Proportionalsätze zu finden. Für die Nähetexte aus dem 17. und 19. Jh. liegen je ein Einzelbeleg vor. Die beiden Distanztexte aus dem 17. und 19. Jh. enthalten insgesamt 6 Belege. Die Verteilung der Proportionalsätze in den Korpustexten bzw. in den Teilkorpora wird in der folgenden Tabelle angegeben:

${ }^{220}$ Zur prozentualen Verteilung der Stellungstypen in den Nähe- bzw. Distanztexten s. die Diagramme 17 und 18 im Anhang (8.2). 


\begin{tabular}{|l|l|l|l|}
\hline & Nähe & Distanz & insg. \\
\hline $17 . \mathrm{Jh}$. & $\begin{array}{l}\text { Güntzer I } \\
1 \text { Beleg }\end{array}$ & $\begin{array}{l}\text { Harsdörffer I } \\
\text { 4 Belege }\end{array}$ & 5 Belege \\
\hline $18 . \mathrm{Jh}$. & $\begin{array}{l}\text { Schuhmacher Chronik II } \\
\text { 0 Belege }\end{array}$ & $\begin{array}{l}\text { Aichinger III } \\
\text { 0 Belege }\end{array}$ & 0 Belege \\
\hline $19 . \mathrm{Jh}$. & $\begin{array}{l}\text { Auswandererbriefe V } \\
\text { 1 Beleg }\end{array}$ & $\begin{array}{l}\text { Burckhardt V } \\
\text { 2 Belege }\end{array}$ & 3 Belege \\
\hline $20 . \mathrm{Jh}$. & $\begin{array}{l}\text { Jugendtagebuch VII } \\
\text { 0 Belege }\end{array}$ & $\begin{array}{l}\text { Lorenz VII } \\
\text { 0 Belege }\end{array}$ & 0 Belege \\
\hline insg. & 2 Belege & 6 Belege & 8 Belege \\
\hline
\end{tabular}

Tabelle 14: Verteilung der Belege für vorangestellte Proportionalsätze in den Korpustexten bzw. in den Teilkorpora $(\mathrm{n}=8)$

In den beiden Korpustexten aus dem 17. Jh. kommen insgesamt 5 Proportionalsätze vor. Einem Einzelbeleg aus Güntzer I stehen 4 Belege aus Harsdörffer I gegenüber. Bei Proportionalsätzen besteht keine Möglichkeit zur Wahl zwischen den Stellungstypen. Sie werden stets resumptiv angeschlossen, wobei das Anschlusselement im Vorfeld des Obersatzes nicht als resumptives Korrelat im Sinne von König/van der Auwera (1988) zu betrachten ist. Bei den entsprechenden komplexen Sätzen tragen ,paarige Junktoren“ am Anfang des Ober- und Untersatzes zur weitgehenden Parallelität der Konstruktion bei (vgl. Kapitel 3.1.4).

Bei der Steigerungsstufe Positiv wird der Proportionalsatz durch so + ein Adjektiv/Adverb eingeleitet, am Anfang des Obersatzes steht ebenfalls so + ein Adjektiv/Adverb:

(299) So viel der Innhalt/ den Worten nach/ an ihrer Reinlichkeit abnimmet/ so viel nimmet hingegen die Zierlichkeit der Schrifft zu/ dergestalt/ daß nirgend keine trefflichere Schreiber- und Buchdruckereyen/ als in Teutschland zu finden sind. (Harsdörffer I: 10)

(300) So wenig man das Hohelied aus der Schrifft mustern kann / so wenig kann man auch die Reden und Meinung von der Liebe entfernen / und wird solche Liebesneigung nicht als Laster / sondern als eine Tugend betrachtet. (Harsdörffer I: 368)

Diese Struktur, in der so + Adjektiv zur Bildung einer ,sowohl syntaktisch als auch semantisch relativ komplexe[n] Struktur“ beiträgt, ist zwar auch im Gegenwartsdeutschen bekannt, aber selten anzutreffen und „einer hohen Stilebene zuzurechnen“ (Thurmair 2001: 34). Die Untersuchungsergebnisse stehen im Einklang mit der obligatorischen resumptiven Stellung von durch so+Adjektiv eingeleiteten Proportionalsätzen im Gegenwartsdeutschen (vgl. Zifonun et al. 1997: 2351f.). 
Bei der Steigerungsstufe Komparativ wird der Vergleichssatz mit dem Ausdruck je + Adjektiv im Komparativ eingeleitet, am Anfang des Obersatzes stehen jedoch nicht die im Gegenwartsdeutschen üblichen Formen desto bzw. umso, sondern ebenfalls je + Adjektiv im Komparativ (vgl. Zitterbart 2002: 160):

(301) Je kürtzer er seine Meinung schliessen kann/ je lieblicher/ und leichter ist das Lied/ und das Gedicht. (Harsdörffer I: 567f.)

Auch im Bereich der Proportionalsätze können jedoch integrativere und aggregativere Techniken unterschieden werden. In den Belegen aus dem 17. Jh. ist eine formale Parallelität (je ..., je ...) zu beobachten, der in zwei Belegen auch die Verbendstellung im Ober- und Untersatz entspricht. In den folgenden Belegen werden sowohl der vorangestellte Untersatz als auch der Obersatz mit Verbendstellung realisiert.

(302) Aber diße Statt wahr gantz der babistischer Religion, derohalben ich keinen evanielischen gelerdten Heren kundt haben, mich zu trosten. Ja, je mehr ich Gott anruffete und bitte um sinen heiligen Geist, Hilff und Beystandt, je mehr sich der Teiffel an mich setzete zu plagen mit schwohrmietigen Gedancken. So ich die schöne Sprich und Verheißungen Gottes Wordts nicht wußtete und deß Jobs gedachte, so wehre ich in meinem Leiden vergangen (Güntzer I: 102r)

(303) Je họher einer geadelt/ je gnådiger er seyn soll! (Harsdörffer I: 27)

Die Verbstellung im Obersatz ist insofern nicht verwunderlich, da die Endstellung im Nachsatz bei Verknüpfungen mit je ... je/desto/umso im 17. Jh. noch den Normalfall darstellt und sie bis zum 18. Jh. zugunsten der Verbzweitstellung im Nachsatz verdrängt wird (vgl. Ágel 2000: 1876, Behaghel 1932: 27f.; Dal/Eroms 2014: 210f.). Da im 17. Jh. noch keine klare formale Trennung zwischen Haupt- und Nebensatz erfolgt ist, kann bei den obigen Nachsätzen nicht von Hauptsätzen nach dem formalen Kriterium, sondern nur von Obersätzen die Rede sein. Die Technik mit der Endstellung des Verbs im Obersatz des Proportionalsatzes ist weniger integrativ.

In den Belegen aus dem Distanztext des 19. Jhs. steht desto+Adjektiv im Vorfeld des Obersatzes. Mit dem Junktionsausdruck des Typs je+Adjektiv, desto+Adjektiv werden die grammatische und semantische Relation expliziter ausgedrückt, vgl. den folgenden Beleg:

(304) Je konsequenter hierauf der Humanismus sich die Anschauungsweise der Italiener dienstbar machte, desto fester überzeugte man sich auch, daß die Abstammung über den Wert des Menschen nicht entscheide. (Burckhardt V: 262) 
In Auswandererbriefe V hat der einzige Beleg für Proportionalsatz die Struktur wie ..., desto ... . Auf den vorangestellten Proportionalsatz folgt keine satzwertige Einheit, sondern eine elliptische Struktur, die mit einem nachfolgenden Kopulaverb zu ergänzen ist:

(305) ich mußte nehmen was ich bekomen konnte, und nicht was ich hätte sollen haben und wie bälder dieselben verkauft wurden desto besser indem sie // täglich im Werthe fielen, ... (Auswandererbriefe V: 18)

Auch bei dieser elliptischen Konstruktion wird desto+Adjektiv im Nachsatz realisiert. Dies zeigt wiederum die enge Zusammengehörigkeit der Bestandteile von Parallelstrukturen. Die formale Parallelität ist in diesen Fällen nicht mehr so offensichtlich, die semantische Relation wird jedoch expliziter ausgedrückt.

\subsubsection{Temporal-kausale Adverbialsätze}

In diesem Kapitel werden Belege diskutiert, bei denen die Trennung der temporalen Bedeutung von anderen Lesarten schwierig ist (vgl. die Erläuterungen im Kapitel 3.4.5.7 bzw. 4.2). Im folgenden Beleg liegt ein Mischtyp temporal-kausal vor:

(306) Da es Tage wirdt, danckete ich ihme um die Herberg und ginge meine Straß weidters fordt durch den Walt. (Güntzer I: 91r-v)

Hier ist die Temporalität kaum von der Kausalität zu trennen: Der Subjunktor $d a$ könnte sowohl im Sinne von nachdem (Vorzeitigkeit) als auch im Sinne von während (Gleichzeitigkeit) verwendet werden (vgl. Kapitel 5.1.1): Der Tagesanbruch im obigen Kontext ist im engeren, wirklichkeitsbezogenen Sinne sicherlich als vorzeitig aufzufassen, in der sprachlichen Realisierung kann es jedoch auch als die Zeit des Danksagens und Fortgehens bestimmt werden. Im Gegensatz zur eindeutig temporalen Relation zwischen den Sachverhalten Tagesanbruch und Güntzers Dank beim Gastgeber bei den durch als eingeleiteten Adverbialsätzen (121) sowie (122) wird der obige Adverbialsatz mit dem Konnektor $d a$ eingeleitet, der in diesem Korpustext auch bei rein kausalen Verwendungen auftritt (vgl. Kapitel 5.3.1). Die Trennung der temporalen bzw. kausalen Bedeutung ist beim Subjunktor $d a$ oft schwierig, vgl. den folgenden Beleg:

(307) Da er sahe, daß ich hardt schlieff, wolte er seine Schelmenstuck und Unkischheidt an mihr volpringen, mitt Refferentz, am After. (Güntzer I: 56v)

Der Inhalt des internen Konnekts (= der Räuber sah, dass Güntzer tief schlief) kann nicht nur den Grund, sondern auch die Zeit dafür bezeichnen, dass der Räuber versuchte, sein Verbrechen zu begehen. Es stellt sich die Frage, seit wann die Kausalität in der lexikalischen 
Bedeutung von $d a$ anzunehmen ist bzw. welche semantische(n) Relation(en) es im frühen Neuhochdeutschen markiert. Die obigen Belege für $d a$-Sätze erwecken den Eindruck, dass im Nähetext aus dem 17. Jh. die semantische Relation zwischen den Konnekten nicht immer ganz explizit markiert wird. Die Belegzahl für diesen Typ ist so gering, dass eine Auszählung bezüglich der Stellungstypen nicht sinnvoll erscheint.

\subsubsection{Lokalsätze (inkl. lokal-konditional)}

Lokalsätze werden durch wo eingeleitet, das kein Subjunktor, sondern ein „W-Adverb“ (Zifonun et al. 1997: 2280) ist. Im Kapitel 3.4.5.6 wurde bereits auf die Tendenz hingewiesen, dass neben der rein lokalen Bedeutung auch andere Inhaltsbeziehungen auftreten. Diese Tendenz führt Eisenberg darauf zurück, „dass das Lokale besonders häufig metaphorisiert wird und Grundstrukturen für viele andere Inhaltsbereiche abgibt.“ (2013: 277) Behaghel (1928: 350) zeigt anhand einiger Belege aus dem älteren Deutsch, dass bei wo eingeleiteten Adverbialsätzen ,aus der greifbaren Örtlichkeit (...) die geistige hervorgehen [kann]“: Der wo-Satz kommt in diesen Fällen ,der allgemeinen Bedingung nahe“. Die meisten einschlägigen Sätze aus den untersuchten Korpustexten zeigen einen Übergang zwischen der rein lokalen und der konditionalen Inhaltsrelation, die rein lokale Verwendung ist nur in einem Einzelbeleg zu beobachten. Daher werden die einschlägigen Belege für wo-Sätze nicht in getrennten Kapiteln behandelt.

Die ,rein' lokale Verwendung ist nur in Burckhardt V belegt. Im folgenden Einzelbeleg wird durch den Nachtrag wie z. B. in Neapel verdeutlicht, dass hier keine geistige Örtlichkeit, sondern Örtlichkeit im engeren Sinne gemeint ist:

(308) In Venedig und Florenz gab es zur Zeit der Renaissance für die Männer vorgeschriebene Trachten und für die Frauen Luxusgesetze. Wo die Trachten frei waren, wie z. B. in Neapel, da konstatieren die Moralisten, sogar nicht ohne Schmerz, daß kein Unterschied mehr zwischen Adel und Bürger zu bemerken sei. (Burckhardt V: 268)

Auf den wo-Satz wird im Vorfeld des Obersatzes mit dem Korrelat $d a$ verwiesen, es liegt also ein resumptiver Anschluss vor. Im Folgenden werden Belege für den Mischtyp lokalkonditional behandelt.

Im Teilkorpus des 17. Jhs. sind drei einschlägige Belege zu finden. Diese stammen aus Harsdörffer I. Die folgenden Belege veranschaulichen den Übergang zum temporalen bzw. konditionalen Gebrauch: 
(309) Wo die Ursachen auffhoren/ fanget die Gewonheit an: (Harsdörffer I: 560)

(310) wo man keine Ursach geben kan/ folget man bildlich der gebrächlichen Mundart/ und lassset die blinde Gewohnheit des unverståndigen Póbelvolcks an seinem Ort verbleiben. (Harsdörffer I: 560)

Hier werden weniger ,(Charakteristiken von) Orte(n) [...], an denen Ereignisse stattfinden“ (Zifonun et al. 1997: 2280), beschrieben. Im Beleg (309) könnte der wo-Satz noch als Verweis auf eine ,geistige Örtlichkeit‘ (vgl. Behaghel 1928: 350) aufgefasst werden. Der Beleg (310) ist jedoch eher konditional zu interpretieren, er ist wie folgt zu paraphrasieren: ,Falls man keine Ursache geben kann, folgt man der gebräuchlichen Mundart ... ‘. Beide Belege weisen eine integrative Anbindung der wo-Sätze auf. In Harsdörffer I findet sich jedoch auch ein Einzelbeleg für den resumptiven Anschluss mit $d a$ :

(311) Wo man treibt keine Tugend mehr/ da steigert man der Titul Ehr. (Harsdörffer I: 28)

In den Nähetexten finden sich keine einschlägigen Belege für Lokalsätze oder für den Mischtyp lokal-konditional. Die Distanztexte Aichinger III, Burckhardt V sowie Lorenz VII enthalten je ein Einzelbeleg für den Mischtyp lokal-konditional:

(312) wo aber ein Mitlauter in eben der Syllbe folgt, klinget es hell, wie das e ouvért, als: wer, denket. (Aichinger III: 8)

(313) Offenbar ist dieser Dilettantismus, sowohl der vornehmern als der mittlern Stände, in Italien verbreiteter und zugleich der eigentlichen Kunst näher verwandt gewesen als in irgend einem andern Lande. Wo irgend Geselligkeit geschildert wird, ist auch immer und mit Nachdruck Gesang und Saitenspiel erwähnt; (Burckhardt V: 284)

(314) Wo sie [die Kausalität, P.K.] experimentell erarbeitete Tatsachen nicht mehr einzuordnen vermochte, stellte er sie einfach beiseite und ersetzte sie durch Wahrscheinlichkeitsrechnung. (Lorenz VII: 31)

Diese Belege weisen eine integrative Stellung des vorangestellten Adverbialsatzes auf.

Es finden sich also insg. 7 einschlägige wo-Sätze in den untersuchten Korpustexten, wovon sechs als konditional zu interpretieren sind. Den resumptiven Anschluss findet man beim Einzelbeleg für die rein lokale Verwendung und bei einem konditional zu interpretierenden Beleg. Der integrative Stellungstyp dominiert mit 5 Belegen.

\subsection{Pragmatische Adverbialsätze}

In diesem Unterkapitel werden ,periphere Adverbialsätze‘ (vgl. Kapitel 3.1.2), d.h. alle nichtprototypischen, nicht-propositionsbezogenen Satzadverbialsätze behandelt. Auf die Schwierigkeiten, die bei der Abgrenzung auftauchen, wurde bereits oben eingegangen (s. die 
Erläuterungen z.B. beim Beleg (180). Die nicht-propositionsbezogenen Satzadverbialsätze sind inhaltlich nur locker angefügt (vgl. Zifonun et al. 1997: 2242ff.), sodass die Frage nach der Desintegration ,peripherer Adverbialsätze“ besonders relevant ist. Die untersuchten Quellentexte enthalten insg. 57 einschlägige Belege, u.a. sprechaktbezogene Kausal- und Konditionalsätze. Es handelt sich also im Vergleich $\mathrm{zu}$ den propositionsbezogenen Adverbialsätzen um eine relativ kleine Gruppe von Belegen. Pragmatische Adverbialsätze werden in folgende Untergruppen eingeteilt (vgl. Kapitel 3.4.6): ,moduskommentierend' und ,modusmodifizierend“ (s. Kapitel 5.5.1 bzw. 5.5.2), ,diktums- bzw. einstellungskommentierend ${ }^{6}$ (s. Kapitel 5.5.3), ,formulierungskommentierend " sowie ,diskursstrukturierend“ (s. Kapitel 5.5.4 bzw. 5.5.5) und letztlich ,thematisierende Adverbialsätze‘ (s. Kapitel 5.5.6).

Bevor auf die Diskussion der Belege eingegangen wird, sei vermerkt, dass die Verteilung der Belege in den Teilkorpora gar nicht einheitlich ist.

\begin{tabular}{|l|l|l|l|}
\hline & Nähe & Distanz & insg. \\
\hline $17 . \mathrm{Jh}$. & $\begin{array}{l}\text { Güntzer I } \\
\text { 2 Belege }\end{array}$ & $\begin{array}{l}\text { Harsdörffer I } \\
\text { 3 Belege }\end{array}$ & 5 Belege \\
\hline $18 . \mathrm{Jh}$. & $\begin{array}{l}\text { Schuhmacher Chronik II } \\
\text { 27 Belege }\end{array}$ & $\begin{array}{l}\text { Aichinger III } \\
\text { 0 Belege }\end{array}$ & 27 Belege \\
\hline $19 . \mathrm{Jh}$. & $\begin{array}{l}\text { Auswandererbriefe V } \\
\text { 16 Belege }\end{array}$ & $\begin{array}{l}\text { Burckhardt V } \\
\text { 0 Belege }\end{array}$ & 16 Belege \\
\hline $20 . \mathrm{Jh}$. & $\begin{array}{l}\text { Jugendtagebuch VII } \\
\text { 3 Belege }\end{array}$ & $\begin{array}{l}\text { Lorenz VII } \\
\text { 6 Belege }\end{array}$ & 9 Belege \\
\hline insg. & 48 Belege & 9 Belege & 57 Belege \\
\hline
\end{tabular}

Tabelle 15: Verteilung der Belege für pragmatische Adverbialsätze in den Korpustexten bzw. in den Teilkorpora $(n=57)$

Die überwiegende Mehrheit der Belege stammt aus den Nähetexten des 18. und 19. Jhs $(47,4 \%$ sowie $28,1 \%)$. In den beiden Distanztexten aus dem 18 . sowie 19 . Jh. treten dagegen keine einschlägigen Belege auf. In den Quellentexten aus dem 17. Jh. und 20. Jh. sind lediglich vereinzelt pragmatische Adverbialsätze nachzuweisen. Daher können historische Veränderungen kaum nachgezeichnet werden. Die Belege sollen deshalb im Folgenden nach Typen geordnet beschrieben werden. 
In diesem Abschnitt werden sprechaktbezogene Adverbialsätze behandelt, bei denen nur in wenigen Fällen eine Wahl zwischen den Stellungstypen besteht. Im folgenden Beleg wird der bereits Anfang Dezember realisierte Sprechakt des Neujahrwunsches damit begründet, dass der Brief wahrscheinlich erst „,am Neuenjahre“ beim Empfänger eintreffen wird:

(315) Da, wie ich hoffe Euch dieser Brief am Neuenjahre antreffen wird, so wünsche ich Euch hiermit Glück u. Freude zum Neuen-Jahre, Gesundheit und noch recht viele glücklichen frohen Jahre nach diesem. (Auswandererbriefe V: 2)

Es liegt hier also ein Kausalsatz mit Sprechaktbezug vor, der mit resumptivem so angeschlossen wird. Auch die folgenden Belege enthalten vorangestellte Kausalsätze, die den Vollzug einer Aufforderung begründen.

(316) Es hat uns sehr gefreut daß dein Fritz auch einen Gruß an uns geschrieben hat, und weil du geschrieben hast ich soll deinem Johañes ein paar Hosen kaufen so kom $\overline{d u}$ nur mit ihm heraus so kauf ich ihm gleich ein paar. (Auswandererbriefe V: 16)

(317) Wihr kunden vohr Miedigkeitt deß Schroeckens und Ruffen zu Gott balt kein Atem mehr recht erholen und sprachen: Herr, Gott, unßer Vatter im Himel, dieweil du unß um der Sinden wilen also wilt straffen, daz wihr zugrundt sollen gon, so nime unßere Sele in deine Hende und laße es balt geschehen, dan wihr sindt schwache Menschen, kunden balt in Ungedult komen. (Güntzer I: 66r)

Der wesentliche Unterschied zum Beleg (315) liegt darin, dass das Korrelat so am Anfang des Obersatzes nicht als Vorfeldelement betrachtet werden kann, da der Obersatz sowohl in (316) als auch in (317) ein Aufforderungssatz mit Verberststellung ist. ${ }^{221}$ Weder der vorangestellte, durch einen Untersatz erweiterte Kausalsatz noch das Resumptivum so sind topologisch in den nachfolgenden Aufforderungssatz integrierbar, sie stehen im linken Außenfeld. In solchen Fällen liegt eine nicht-integrative Anbindung mit einem resumptiven Element im linken Außenfeld des Obersatzes vor (vgl. Kapitel 3.1.3).

In den Nähetexten aus dem 17. und 19. Jh. sind auch Konditionalsätze mit Sprechaktbezug belegt, die als Relevanzkonditional aufzufassen sind. Beim folgenden Beleg tritt das Korrelat so am Anfang des Obersatzes auf. ${ }^{222}$ Da er nicht als Imperativsatz mit Verberststellung realisiert wird, wird hier der resumptive Stellungstyp angenommen.

\footnotetext{
${ }^{221}$ Zifonun et al. (1997: 654f.) gehen bei Aufforderungssätzen wie Also/Und/Aber arbeite nicht so viel. von Verberstsätzen aus, da bestimmte Konjunktoren bzw. Konnektive nicht das Vorfeld besetzen, sondern im linken Außenfeld stehen (vgl. Kapitel 3.2.1).

222 Die Frage nach dem Obersatz bzw. Obersätzen im Beleg (318) ist ebenfalls zu klären. Es wird zwar ein kopulativer Konnektor (und) verwendet, aus syntaktischer Sicht stehen der Obersatz so möchtet ihr so gut s sein und der nachfolgende Ausdruck mir die Atresse schicken also auf derselben Ebene. Obwohl hier keine syntaktische Subordination vorliegt, kann nach Ágel (2007: 44) eine semantische Subordination aufgrund der Adjektivvalenz angenommen werden.
} 
(318) Deshalb erßuche ich euch, wē̄ sie euch auch schton geschrieben hat, so möchtet ihr so gut s sein und mir die Atresse schicken. (Auswandererbriefe V: 20)

In mehreren Belegen steht nach dem vorangestellten Konditionalsatz ein Aufforderungssatz, in diesen Fällen kann der vorangestellte Adverbialsatz topologisch nicht in den Aufforderungssatz integriert sein. In den folgenden Belegen gibt der Konditionalsatz an, unter welchen Bedingungen die Äußerung der koordinierten Aufforderungssätze relevant ist. Auch hier tritt vor dem Aufforderungssatz so auf.

(319) So er es nicht glauben wolt, so no $\stackrel{e}{\text { mpt }}$ mich alhie gefangen und fieret mich morgen in die Statt, so werdt ihr sehen, wer ich bin. (Güntzer I: 89r)

(320) Liebste Eltern wen sich Euer Geschäft nicht rendirt oder wē̄ diese Krankheiten nicht aufhören so verkauft wen Ihr könt und kō̄t zu uns ... (Auswandererbriefe V: 26)

Im Gegenwartsdeutschen ist die korrelative Anbindung von Relevanzkonditionalsätzen nach Pittner (1999: 345, 2000: o.S.) „unmöglich“. Im Korpus ist nur ein einschlägiger Beleg zu finden, in dem kein resumptives Element realisiert wird:

(321) ich glaube daß es für dich besser wäre hier als in Deutschland. wē $d u$ Lust hast hierher zu kommen, und es dir sollte an Reisegeld fehlen, laß mich es wissen ich werd dir sogleich das Reisegeld, oder einen Reisebas schicken, (Auswandererbriefe $\mathrm{V}: 21)$

Dieser Beleg wird trotz der Verberststellung im Obersatz nicht dem integrativen Typ zugeordnet, da der Obersatz ein Imperativsatz ist (vgl. Kapitel 3.1.3).

Im folgenden Beleg steht ein $d$ am Anfang des Obersatzes, das durchgestrichen ist. Dies könnte als ein Zeichen dafür interpretiert werden, dass die (nicht zu Ende geschriebene) Wortform $d a(\bar{n})$ durch den frequenteren Ausdruck so ersetzt wurde bzw. dass die Wahl zwischen den Wortformen $d a(\bar{n}) /$ so dem Schreiber durchaus bewusst war. Eine andere Interpretation wäre, dass hier das (nicht zu Ende geschriebene) Subjektspronomen getilgt wurde und somit ein eigenständiges Stellungsglied durch das resumptive Element ersetzt wurde.

(322) weñ $d u$ er das es erhalten habst $d$ so solst $d u$ auch eine Quittung schicken. (Auswandererbriefe V: 15)

Der komplexe Satz im folgenden Beleg weist eine stark aggregative Form auf, seine Analyse als ,nicht-integrativ ‘ ist jedoch angesichts seines Einzellfallcharakters strittig. Es stellt sich dabei die Frage, ob hier ein reguläres Satzgefüge vorliegt, weil bezüglich der linearen Anordnung der Teilsätze mehrere Besonderheiten zu beobachten sind. Der Inhalt des ersten wenn-Satzes (wēn sie ausbricht) wird durch den zweiten wenn-Satz (wē̄ ... in Eurem Hauße 
diese Seiche nochmal anbrechen wirde) präzisiert, der einen parenthetischen Charakter hat. Zudem wird der Obersatz diskontinuierlich realisiert (dieses Rezept schickt ... in die Apotheke). Der erste Teil des übergeordneten Imperativsatzes wird zwischen den beiden wenn-Sätzen realisiert, der zweite Teil ist erst am Ende des Satzkomplexes zu finden. Der erste Teil kann als Anakoluth analysiert werden, er scheint aber durch die nachgetragene Direktivergänzung nach dem zweiten Konditionalsatz zu einer vollständigen Satzstruktur ergänzt zu werden.

(323) Der Onkel schreibt Euch ein Rezept und sendet es Euch es ist für die [Halsbrume] dem hier darf Kein Mensch sterben man denkt wē̄ sie ausbricht dieses Rezept schickt wen was ich nicht hoffe in Eurem Hauße diese Seiche nochmal anbrechen wirde in die Apotheke und laßt Euch dieses geben. (Auswandererbriefe V: 27)

Es ist fraglich, ob hier eine Analyse als Verbzweitsatz, mit einem topikalisierten Stellungsglied (das Objekt dieses Rezept) im Vorfeld des Imperativsatzes, sinnvoll erscheint (zu vergleichbaren Strukturen im Gegenwartsdeutschen s. Zifonun et al. 1997: 655). Wegen der Störung in der Projektionsstruktur (s. Kapitel 4.2) wird dieser Beleg bei der quantitativen Untersuchung nicht berücksichtigt.

In den folgenden Belegen aus den Distanztexten aus dem 17. und 20. Jh. werden sprechaktbezogene Konditionalsätze nicht-integrativ angebunden, wobei die nicht-integrative Stellung obligatorisch ist, weil der Obersatz eine Ergänzungsfrage darstellt. In den Belegen (324) sowie (325) steht das Frageadverb wie im Vorfeld des Obersatzes:

(324) Wann der Mensch seine Stimme und Aussprache verandert/ in der Kindheit klar/ in dem Alter groeber zu sprechen pfleget; wie sollte dann nicht auch die Art zu reden sich verä èdern/ und zugleich mit der; in den nechsten hundert Jahren viel zierlicher Schrifft/ gewandelt worden seyn: ... (Harsdörffer I: 170)

In diesem Beleg tritt im Mittelfeld des Obersatzes das nicht-resumptive (da nicht linksversetzende, vgl. Ágel 2010: 914f.) Korrelat dann auf, das bei Konditionalsätzen sonst nicht auftritt (zum Gebrauch des resumptiven so bei Konditionalsätzen s. 5.2.1). In einem Einzelbeleg wird ein uneingeleitetes Konditional nicht-integrativ angeschlossen.

(325) Ist dieses gantze Weltwesen so wandelbar/ wie sollte man dann in einem so flüchtigen Wortlaut einige Beständigkeit erhärten oder bejahen können? (Harsdörffer I: 559)

Der einzige Beleg aus dem 20. enthält eine syntaktisch komplexe Voranstellung, daher ist das Fehlen des (nicht-resumptiven) Korrelats dann im Mittelfeld des Obersatzes auffallend. Der Obersatz wird als Ergänzungsfrage realisiert, die Stellung des Fragewortes wer ist aus diesem 
Grunde strukturell determiniert. Die Desintegration wird auch durch die Einfügung von ja vor dem Obersatz markiert.

(326) Wenn der Blick nach innen unmittelbar tiefste Wahrheiten erschauen konnte, während der nach außen gerichtete bestenfalls die Gesetzlichkeiten einer Chimäre, eines Traumes, aufzuzeigen vermochte, ja, wer wollte sich da mit der langwierigen und mühsamen Erforschung der Außenwelt befassen, die noch dazu in manchen ihrer Aspekte wenig anziehend? (Lorenz VII: 28f.)

\subsubsection{Modusmodifizierende Adverbialsätze}

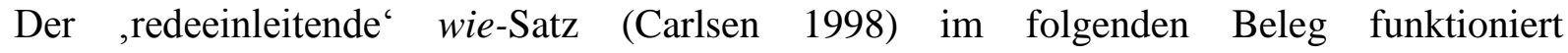
referatsanzeigend, in dem die Quelle der Information genannt wird.

(327) So seind Sie deßwegen zum Schwert verurtheilt worden und die Köpff auff die Spitz zu stecken, und der Mutter die rechte Hand abhauen, welches abhauen der Scharffrüchter mit Namen Johannes Volmar erbetten, Sie möchte Ihm zu Schwach werden, daß Er Sie nicht könnte rüchten. Welches rüchten ist geschehen den 29. Augs. an Johanny Enthauptung Freitags und wie Ich von den Alten gehört hab, So solle Es Just 45ig Jahr sein, daß man der alten Ihren Vatter u. Bruder auch mit dem Schwerdt solle hingerücht haben, und seind die bayde Mutter $u$. Tochter wider in Ihr Grab unter dem Haupt-Gericht oder Galgen gelegt worden, und seind die Köpff auff zwey Spitz gesteckt worden, welche man lange Zeit zum Spectacul hat söhen können, hernach ist der Vatter Johannes Ziegler umb 40ig Florin gestrafft worden, und der Joh. Jacob Spohn mit 80ig Florin Straff belegt worden und ist also die Sach zum Ende gebracht. (Schuhmacher Chronik II: 380)

Neben diesem Verweis auf den Indirektheitskontext funktioniert auch das Modalverb sollen im Obersatz als ein Mittel zur Einschränkung der Gültigkeit der Aussage. Da der Adverbialsatz die Wissensgrundlage des Schreibers thematisiert, kann er als epistemisch betrachtet werden.

Der modusmodifizierende Gebrauch ist in zwei Belegen aus Auswandererbriefe V zu beobachten. Mit den vorangestellten so-Sätzen wird die Gültigkeit der nachfolgenden Aussage einschränkt (vgl. Zifonun et al. 1997: 2327 bzw. Kapitel 3.4.6):

(328) Unsre Bilder wirst du noch nicht erhalten haben, so viel wir gehört haben ist der Mā̄ noch da er habe den Prozeß verspie[lt.] (Auswandererbriefe V: 15)

(329) ich werde gleich nach Neujahr wieder hinmüßen, um wahrscheinlich mehrere Wochen dort zu bleiben es wäre mir allerdings lieb wenn ich könnte die Geschäft mehr durch andere Leute verrichten lassen ich werde so viel ich kann, aber so viel ich bis jetzt wahrgenomen habe, wird unsers Bruders hinterlassenschaft als eine fette Gans betrachtet, an welcher jeder gern rupfen möcht ich halte es deßwegen für meine Pflicht, selbst so viel wie möglich danach zu sehen, ... (Auswandererbriefe V: 18) 
Im Gegensatz zum einschlägigen Beleg (327) aus Schuhmacher Chronik II wird in den beiden obigen Belegen die integrative Stellung realisiert.

Der folgende Beleg zeigt einen durch wie eingeleiteten pragmatischen Nebensatz. Die Floskel wie man sieht wird - wie in den meisten Belegen (89\%) einer Stichprobe von 100 einschlägigen Voranstellungen aus dem DeReKo (Institut für Deutsche Sprache 2016) integrativ angeschlossen:

(330) Wie man sieht, eignet sich die Ausdrucksweise der Informationstheorie nur wenig für die Beschreibung von Lebensvorgängen. (Lorenz VII: 39)

Die Besonderheit dieses Belegs besteht darin, dass hier die Illokutionskraft des Obersatzes nicht abgeschwächt, sondern verstärkt wird (vgl. Pittner 2000: o.S.).

5.5.3 Diktums- bzw. einstellungskommentierende Adverbialsätze

Im Korpus sind lediglich 3 einschlägige Nebensätze belegt. Der durch wiewol eingeleitete Nebensatz in (331) ist als ,einstellungskommentierend` ${ }^{`} \mathrm{zu}$ analysieren: ${ }^{223}$

(331) Etliche sagen c sey kein teutscher Buchstab: sie irren aber weit / weil es in den allerältesten Schrifften befindlich; wiewol ich gerne zugebe er sey neuer als das $k$ / und eben der Lieblichkeit halber erfunden worden: (Harsdörffer I: 468)

Es liegt hier sicherlich kein prototypischer Adverbialsatz vor, der Nebensatz ist weder semantisch noch topologisch integriert. Aufgrund der ,Nebensatzgestalt' (Zifonun et al. 1997: 2250) kann er trotzdem als subordinierter Satz analysiert werden (vgl. Kapitel 3.3). Das Verhältnis zwischen Obersatz und Untersatz ist jedoch kompliziert, weil der subordinierte Satz selbst als Redeeinleitung fungiert: Der Untersatz wird dank des Vollverbs zugebe mit dem nachgestellten Konjunktivsatz in Beziehung gesetzt. ${ }^{224}$ Im Gegenwartsdeutschen wäre eine verkürzte Formulierung möglich (etwa mit dem Wortlaut wobei: er ist neuer als das K, vgl. Günthner 2000). In diesem Sinne lässt sich der obige Beleg als Zwischenstufe bei der Grammatikalisierung, als Diskursmarker auffassen.

Der nächste Beleg ist als Diktumskommentar (vgl. Zifonun et al. 1997: 2334) zu analysieren:

\footnotetext{
${ }^{223}$ Nach einer alternativen Analyse enthält der wiewol-Satz eine Korrektur, eine nachgetragene Einschränkung der Gültigkeit des voranstehenden Obersatzes (vgl. Kapitel 3.4.5.3). Demnach wäre der Konzessivsatz seinem Bezugssatz nachgestellt und sollte in der quantitativen Auswertung nicht berücksichtigt werden.

224 Vergleichbare Konstruktionen mit subordiniertem redeeinleitenden Satz und anschließendem Hauptsatz als Redewiedergabe sind auch im Gegenwartsdeutschen bekannt (vgl. Carlsen 1998: 64).
} 
(332) Heute ist ein total mieser Tag gewesen! In der Schule hab' ich Stunde gehabt, siehe Zettel, aber - was am schlimmsten ist, es kriselt gerade sehr ernsthaft. (Jugendtagebuch VII: 4)

Dieser Satztyp steht hinsichtlich seiner Form den Pseudoclefts nahe (vgl. Kapitel 3.4.6), hier wird jedoch kein Satzglied links herausgestellt. Die Funktion des durch was eingeleiteten Nebensatzes besteht vielmehr darin, eine Bewertung der Schreiberin zum im nachfolgenden Hauptsatz bezeichneten Sachverhalt auszudrücken, er ist demnach als , bewertend-assertives Satzadverbial` einzuordnen (vgl. Zifonun et al. 1997: 1128). ${ }^{225}$ Der vorangestellte Nebensatz weist eine nicht-integrative Stellung auf, die integrative Anbindung ist hier wohl keine Option. Der Ausdruck könnte jedoch durch ein Satzadverbial wie bedauerlicherweise ersetzt und im Vorfeld realisiert werden und hätte nach diesem Kriterium deshalb einen Satzgliedwert (vgl. Zifonun et al. 1997: 2331).

Der folgende Beleg enthält einen elliptischen Satz. Er ist nicht als Vergleichssatz zu analysieren im Sinne von ,Ich ging so (schnell/leise) runter, wie es vereinbart worden ist", sondern eher als Diktumskommentar (vgl. Zifonun et al. 1997: 2334), der Adverbialsatz ist paraphrasierbar mit ,Wie wir untereinander vereinbart haben“.

(333) Um 3 Uhr schaute ich aus dem Fenster, da kam L. gerade. Wie ausgemacht, ging ich runter. Im Schlafzi. schlug sich Papa unruhig von einer Seite auf die andere, sein Atem ging unruhig und ich hatte Angst mein lauter, starker Herzschlag könnte ihn wecken. (Jugendtagebuch VII: 21)

\subsubsection{Formulierungskommentierende Adverbialsätze}

Der folgende Beleg kommentiert die im Obersatz durchgeführte Äußerung bzw. eine bestimmte Formulierung und ist paraphrasierbar durch, Weil ich das Jahr nach den Monaten beschreibe, beginne ich die Beschreibung mit dem ersten Monat: ... . Der Adverbialsatz funktioniert als ,metakommunikativ‘ (Günthner 1999).

(334) 17 A N N O 31 // So Ich daß Jahr nach den Monat beschreibe So hat sich der Janw. mit Sonnenschein angelassen, den 4ten mit regen, den 9ten \& 11ten geschnien, darauff Sonnenschein und kalt den gantzen Monat durch, auch zuweilen trieb, den 30igsten daß wetter gebrochen, den 31igsten geschnien. (Schuhmacher Chronik II: 445)

Da der Adverbialsatz zur thematischen Orientierung dient, könnte er auch als ,diskursstrukturierend‘ gedeutet werden: Durch den so-Satz wird die Beziehung der folgenden

\footnotetext{
225 Als alternative Analyse kommt eine Umkehrung des Obersatz-Untersatz-Verhältnisses in Frage, der Satz „,es kriselt gerade sehr ernsthaft" wäre demnach Subjekt des was-Satzes. Dagegen spricht jedoch, dass der was-Satz selbst angesichts der Nebensatzgestalt als ein subordinierter Satz aufzufassen ist (vgl. Kapitel 3.3).
} 
Äußerung zum Gesprächskontext verdeutlicht. Dieser Adverbialsatztyp wird v.a. am Beispiel der Konditionalsätze ausführlicher beschrieben, vgl. Günthner (1999) und Pittner (2000), eine ähnliche Verwendung findet sich jedoch auch bei sprechaktbezogenen Kausalsätzen (vgl. Thim-Mabrey 1988: 55).

Im folgenden Beleg liegt eine metakommunikative Verwendung des Konditionalsatzes vor. Der wenn-Satz nimmt auf unterschiedliche Möglichkeiten der Abgrenzung der Kindheit gegenüber dem Erwachsensein Bezug und schwächt dadurch auch die Gültigkeit des im Hauptsatz bezeichneten Sachverhalts ab: Die Kindheit der Schreiberin ist nur dann vorbei, wenn man eine bestimmte Definition von Kindheit anwendet.

(335) Also wenn man's genau nimmt, ist meine Kindheit jetzt vorbei. (Jugendtagebuch VII: 20)

Diese Verwendung ist von modusmodifizierenden bzw. restriktiven Adverbialsätzen schwer abzugrenzen.

Der folgende Beleg wird in der quantitativen Auszählung dem nicht-integrativen Typ zugerechnet, zumal er (trotz Verberststellung im nachstehenden Teilsatz) eine lose Verknüpfung mit seinem Bezugssatz zeigt. Der wie-Satz ist nicht in das Konditionalgefüge mit zwei Verberstsätzen integriert: Im Vorfeld des Obersatzes steht der uneingeleitete Konditionalsatz Gibt es nicht ein Ding an sich. Der wie-Satz, in dem die Quelle der nachstehenden Originaläußerung angegeben wird, steht im linken Außenfeld.

(336) Wie der Freiburger Philosoph Szilasi in seiner durch geringe Beherrschung der deutschen Sprache zu lapidarer Größe gezwungenen Ausdrucksweise zu sagen pflegte: "Gibt es nicht ein Ding an sich, gibt es viele Ding-an-siche." (Lorenz VII: 23)

Der Bezugssatz stellt eine Redewiedergabe dar, daher wird seine lineare Anordnung nicht durch die Stellung des wie-Satzes beeinflusst. Auch das graphische Desintegrationssignal Doppelpunkt weist darauf hin, dass der vorangestellte wie-Satz keinesfalls in den nachfolgenden Obersatz integriert ist.

\subsubsection{Diskursstrukturierende Adverbialsätze}

Der vorangestellte $w a \beta$-Satz steht im folgenden Beleg in einer losen Beziehung zum nachstehenden übergeordneten Satz. Er kann als, diskursstrukturierend' analysiert werden, denn er nimmt auf den Textaufbau Bezug. In diesem Beleg wird der vorangestellte Nebensatz ebenfalls mit dem Resumptivum so aufgegriffen: 
(337) Es folget sehr wenig Buß, man verfolgt einander, welches Ich selbsten hab fühlen müssen und grosse Verfolgung außstehen. Waß noch weiters passiert. So hat es den 9 ten Novemb. einen grossen Sturmwind geben und den 11ten dito als an Martini hat Es einen Schnee herunter geworffen und kalt darauff worden. (Schuhmacher Chronik II: 411)

In Auswandererbriefe V finden sich redekommentierende Nebensätze (vgl. Eggs 2006: 495), die der intertextuellen Vernetzung von Sachverhalten dienen. Sie funktionieren wie vergleichbare Fälle der diskursstrukturierenden Konditionalsätze (vgl. Pittner 2000, Günthner 1999a): Die SchreiberInnen möchten den Briefpartnern etwas in Erinnerung rufen, das sie schon wissen. Bloß hier liegen keine textinternen Verweise vor, es wird auf die Äußerungen der Briefpartner Bezug genommen. Diese Nebensätze werden mit wie eingeleitet: ${ }^{226}$

(338) Und wie du geschrieben hast unsere Kinder nehmen nun schon den Platz ein den wir noch ohnlängst besasen und wir werden alt ohne daß wir es wissen. (Auswandererbriefe V: 12)

(339) Wir wir mit Bedauern vernomen haben hat sich bei Euch so maches Schicksal zugetragen und Ihr habt freilich wenig Freude gehabt. (Auswandererbriefe V: 26)

(340) Wie ich in dem Briefe vernōmen habe ist der Grünwald nach euch da ich denk er wird sich recht Gelde ersparen und dā̄ schreiben ein Gruß an seine Frau. (Auswandererbriefe V: 26)

Die Belege (338) und (340) könnten eventuell auch als ,formulierungskommentierend“ eingestuft werden, denn sie könnten sich nicht nur auf den Inhalt, sondern auch auf die sprachliche Formulierung des Bezugssatzes beziehen (vgl. Eggs 2006: 511). Der Beleg (338) drückt zudem eine negative Bewertung des Sachverhalts im Obersatz aus (vgl. Zifonun et al. 1997: 1128). In mehreren Fällen wird also der jeweilige Aspekt metakommunikativer Kommentare nicht explizit bezeichnet. Der Beleg (338), der eventuell auch als formulierungskommentierend eingestuft werden könnte, weist eine nicht-integrative Stellung auf, die Belege (339) und (340) werden jedoch integrativ angebunden. Da diese metakommunikativen Sätze ,,auf das Thema der folgenden Äußerung [verweisen] und [...] dann im Grunde als eine Form des ,Freien Themas' aufzufassen [sind]“, wäre nach Pittner (2000: o.S.) - zumindest bei diskursstrukturierenden Konditionalsätzen - eine Stellung an der linken Peripherie zu erwarten. Ein ,freies Thema‘ liegt auch im folgenden Beleg vor, in dem jedoch nur eine sehr lose Beziehung zwischen den Teilsätzen besteht. Der erste Teilsatz ist hier kein untergeordneter, sondern ein selbständiger Satz, daher wird dieser Beleg bei der quantitativen Auswertung nicht berücksichtigt:

\footnotetext{
${ }^{226}$ Im Beleg (339) wird das erste Vorkommen der Wortform wir als Schreibvariante von wie analysiert.
} 
(341) Und wie leicht sind wir von den Lebendigen weggeblasen letzten Somer mußte ich hälfen ein Grab machen und zuschaufeln der Verstorbene war ein junger starcker Man von 35 Jahren. (Auswandererbriefe V: 12)

In Lorenz VII sind zwei Belege für diskursstrukturierende wie-Sätze zu finden, die jedoch - im Gegensatz zu den oben diskutierten Beispielen aus Auswandererbriefe V - der textinternen Vernetzung von Sachverhalten dienen. Sie haben die Funktion, etwas in Erinnerung zu rufen, das im Text schon behandelt wurde. Eggs (2006: 506) bezeichnet diesen Typ ,intratextuelle ,wie 6 -Kommentare“. In (342) liegt eine besondere Form der Textdeixis vor, indem rückbezüglich und explizit auf den Ort der betroffenen Textstelle verwiesen wird.

(342) Wie schon erwähnt, besteht ein solches Bildverhältnis zwischen Organismus und Wirklichkeit schon auf der Ebene des Körperbaus, der "Morphogenese«, man denke an die Sonnenhaftigkeit des Auges oder an die Wellenbewegung der Fischflosse (S. 17). (Lorenz VII: 40)

(343) Wie schon gesagt, ist das Leben gleichzeitig Informationserwerb, $d$. h. ein kognitiver Vorgang, und ein ökonomisches, man ist versucht zu sagen, ein kommerzielles Unternehmen. (Lorenz VII: 45f.)

In beiden Fällen wird der integrative Stellungstyp realisiert. Die oben behandelte intertextuelle Vernetzung mit einem diskursstrukturierenden Adverbialsatz ist im Korpustext ebenfalls belegt:

(344) Wie schon in den Prolegomena auseinandergesetzt, gründet sich meine Betrachtungsweise der Erkenntnisfunktionen und aller Lebensvorgänge überhaupt auf eine erkenntnistheoretische Einstellung, die ich mit Donald Campbell als die des hypothetischen Realismus bezeichnet habe. (Lorenz VII: 303)

Auch dieser Beleg aus dem Distanztext aus dem 20. Jh. weist integrative Stellung auf.

5.5.6 Thematisierende Adverbialsätze

,Formelhafte Thematisierungsausdrücke‘ (Zifonun et al. 1997: 522-526) sind lediglich in zwei Quellentexten nachzuweisen. In Schuhmacher Chronik II kommen die meisten einschlägigen Belege (24 von 29) vor, die die Form eines durch waß eingeleiteten Nebensatzes mit dem Prädikatsausdruck betreffen/anbetreffen/anbelangen haben (vgl. Kapitel 3.2.1).

(345) Es hat daß Obs bey unß zimlich verzogen, aber unsre benachtbarte Örter nämlich im Uracher-Thal, im Hausamer-Thal und Gininger-Thal, daß sie es zum theil reichlich bekom[m]en und genossen. Waß der Som [m]er anbetrifft, So ist Er Iederzeit warm gewessen, daß alles ist wol ist einkommen von allerhand Frucht und Futter (Schuhmacher Chronik II: 410) 
Es handelt sich um „besondere grammatische Strukturen, welche ermöglichen, ein Thema grammatisch zu isolieren und in einer durchsichtigen Weise an den Satzanfang zu stellen“ (Lötscher 2006: 43). Diese sind den sog. ,Sperrsätzen“ oder ,unechten Spaltsätzen“ (auch ,Pseudo-Clefts‘ genannt) ähnlich (vgl. Kapitel 3.4.6). In einigen Fällen (wie beim obigen Beleg (345)) wird die herausgestellte Substantivgruppe (der Som[m]er) im Bezugssatz anaphorisch (er) fortgeführt. An der Position des Pronomens könnte auch die herausgestellte Substantivgruppe stehen. In anderen Fällen kann jedoch das herausgestellte Element des waßSatzes nicht (oder nicht ohne Modifizierung) in den Bezugssatz integriert werden, vgl. die folgenden Belege (346) und (347).

(346) Waß der Herbst betreffend So hat uns Gott einen köstlichen Tranck geschert, aber wenig wie in vorigen Jahren. (Schuhmacher Chronik II: 399)

(347) Es war umb Georgy der Rebstock noch gantz blind, daß kein Traub nicht ist gesehen worden. So hat Es eine mittelmeßige Ernd an Frucht geben, aber sehr wol und gut heimkom[m]en, waß daß Obs anbelangt, So hat Es wenig geben, theil Orten gar keinß, die zweschgen seind an theil Orten auch gerathen. (Schuhmacher Chronik II: 423)

In diesen Fällen sind die Ähnlichkeiten mit Pseudoclefts geringer. Es finden sich fließende Übergänge, wenn etwa die herausgestellte Substantivgruppe und die entsprechenden Ausdrücke des Bezugssatzes nur in einer loseren Beziehung zueinander stehen, wie im folgenden Beleg:

(348) Der Septemb. gehet Ein mit reiffen, den 1. \& 2. hat Es Eys geben, fast alle Tag reiffen, doch ohne Schaden biß auff den 7ten dito, hernach den gantzen Monat durch gutes wetter mit regen \& Sonnenschein, zum Ende warmes Wetter, als den 28. 29. \& 30igsten Sonnenschein, starcke reiffen \& kiele morgen. Waß der Authumnus betrifft, So hat Sich der October wider warm angelassen mit Sonnenschein, hernach den 7ten ein starcken reiffen \& gefrohren und cont. biß auff den 12. 13. 14. \& 15ten dito, daß die Weinberg ohne unterscheid erfrohren wegen grosser kälte. (Schuhmacher Chronik II: 442)

Bezüglich der Form der waß-Sätze sind drei Besonderheiten festzustellen. Die Variable X in der was X VERB \{(an)betrifft/angeht/an(be)langt $\}$ Konstruktion wird - im Gegensatz zum heutigen Gebrauch - stets durch eine Substantivgruppe im Nominativ bezeichnet (vgl. (346) sowie (348)). ${ }^{227}$ Das Verb kann - wie auch im Gegenwartsdeutschen, vgl. Birkner (2008:

\footnotetext{
${ }^{227}$ In einer Stichprobe von 87.989 einschlägigen Treffern aus dem DeReKo (Institut für Deutsche Sprache 2016) konnte ein einziger Beleg mit eindeutiger Nominativform (Maskulinum Singular) identifiziert werden. Der was-Satz tritt in (i) allerdings nachgestellt auf:

(i) Der Hauswart gehört zum Schulhaus. Er ist Techniker, Raumpfleger, Aufpasser. Die gute Seele des Hauses, Tag und Nacht zur Stelle. Und er wohnt auf dem Schulareal. Dieses Bild ist in vielen Köpfen verankert. Doch die Realität sieht anders aus, zumindest was der Wohnort des Hauswarts betrifft. (A09/OKT.09000 St. Galler Tagblatt, 31.10.2009, S. 51; «Hauswart kann abschrecken»)
} 
63f.) - sowohl im Singular als auch im Plural stehen. Des Weiteren sind Belege (wie etwa (346)) zu finden, die mit Partizipialkonstruktionen wie betreffend + Akkusativ (Zifonun et al. 1997: 2227f.) verwandt sind. Da hier jedoch trotz der Partizipform betreffend sowohl das Subjekt als auch das Pronomen waß realisiert werden, werden die einschlägigen Belege nicht als Partizipialkonstruktionen aus dem Korpus eliminiert (vgl. Kapitel 3.1.1).

Die ,formelhaften Thematisierungsausdrücke“ des Typs was X VERB \{(an)betrifft/ anbelangt $\}$ werden in Schuhmacher Chronik II bis auf eine Ausnahme immer mit einem Korrelat angeschlossen (vgl. die obigen Belege). Dabei ist ausschließlich das Korrelat so belegt, $d a$ kommt hingegen nicht vor. Hier sei das einzige Gegenbeispiel aufgeführt:

(349) Am Auffarths Tag Ist abermahl der König in Franckreich im Würtemberger Land eingefallen und überzogen, daß daß Land 10 Tonnen Golds Rantzion geben, auch die Vöstung Schondorff eingenom [m]en und hat daß Land viel hundert Centner Meel geben müssen. Bald darauff ist er widerum Abgewichen $u$. daß meel stehen bliben wegen unserer Völcker die Ihm den Paß haben wollen Abschneiden u. also daß Land widerumb befreyhet. Waß die reichstätt anbelangen, ist Ihnen ebenmässig auch ergangen, wir haben es auch gefühlet In unser geliebten Vatterstatt Reuttlingen. (Schuhmacher Chronik II: 329)

Da das Korrelat so in den obigen Fällen keine wiederaufnehmende (resumptive) Funktion hat, ist es fraglich, ob hier von Resumption im Sinne von König/van der Auwera (1988) gesprochen werden kann (vgl. 3.1.4). Das Korrelat ist eher als struktureller Anschluss aufzufassen. Bei diesen musterhaften Konstruktionen in Schuhmacher Chronik II scheint auch das Korrelat so Teil der Konstruktion zu sein. Das obige Beispiel (349) für integrative Stellung sowie die lexikalische und syntaktische Variation in den obigen Belegen legen jedoch den Schluss nahe, dass thematisierende Adverbialsätze keinen stark formalisierten Charakter aufweisen.

In Auswandererbriefe $\mathrm{V}$ sind 5 Belege für ,formelhafte Thematisierungsausdrücke‘ des Typs was X VERB \{(an)betrifft/anbelangt $\} \mathrm{zu}$ finden. Sie weisen jedoch mehrere Unterschiede gegenüber den Belegen aus Schuhmacher Chronik II. Die Ausdrücke für die Formel X im was-Syntagma stehen in Auswandererbriefe V im Akkusativ (vgl. die Personalpronomina mich bzw. uns in (350) und (351)). Das Verb anbetrifft ist in diesem Korpustext nicht belegt. Im Gegensatz zu Schuhmacher Chronik II ist hier keine Dominanz des resumptiven Typs nachzuweisen, die Belege für integrative und resumptive Stellungen halten sich fast die Waage (2 vs. 3 Belege). Zum prozentualen Verhältnis der Stellungstypen in den beiden Nähetexten s. Diagramm 24 im Anhang (8.2). Die beiden Belege für integrative Stellungen haben die Gemeinsamkeit, dass das Subjektspronomen des Folgesatzes (ich bzw. 
wir) als eine Art ,Cleft-Ausdruck“ im was-Syntagma „abgespalten“ wird und sie somit den freien Relativsätzen nahestehen (vgl. 3.4.6).

(350) Was mich betrifft bin ich gesund und munter und es geht mir und dem Carl recht gut es gefällt uns hier jeden Tag besser. (Auswandererbriefe V: 26)

(351) Was uns betrifft sind wir alle gesund und munter und es gent uns recht gut. (Auswandererbriefe V: 28)

In den Belegen für resumptiven Anschluss sind zwar auch pronominale Formen im Folgesatz $\mathrm{zu}$ finden, diese sind jedoch nicht referenzidentisch mit den Subjektspronomina des Folgesatzes.

(352) Was die Arbeit betrift so konte sie dieselbe wohl aushalten sie hatte viele halbe Tage wo sie fir sich arbeiten Nähen oder Stüken kante. (Auswandererbriefe V: 23)

(353) was das Essen betraft so kan ich darüber keine Ke Klage haben und ich denke sie konte es bei ihrer Arbeit auch dabei aushalten. (Auswandererbriefe V: 23)

(354) Was uns anbelangt so kan ich dir schreiben das es uns gut geht. (Auswandererbriefe $\mathrm{V}: 12)$

Auch in diesem Korpustext ist ausschließlich das Resumptivum so belegt, da kommt nicht vor. Während im Gegenwartsdeutschen auch die nicht-integrative Stellung von thematisierenden Adverbialsätzen möglich ist (vgl. Kapitel 3.4.6), sind im Korpus nur die Stellungstypen ,resumptiv ‘ und, integrativ` belegt, wobei ,resumptiv` die bevorzugte Variante ist.

\subsubsection{Zusammenfassung}

Aufgrund der Forschungsliteratur zum Gegenwartsdeutschen war ein hoher Anteil der nichtintegrativen Stellung von pragmatischen Adverbialsätzen im Neuhochdeutschen anzunehmen. Diese Annahme konnte durch die vorliegende Untersuchung bestätigt werden. Während der Anteil des nicht-integrativen Typ in den propositionsbezogenen Adverbialsätzen nur 1,3\% beträgt (5 Belege von 396, 3 davon aus dem Typ ,irrelevanzkonditional'), ist die nichtintegrative Stellung bei pragmatischen Adverbialsätzen deutlich häufiger (21,1\%, 12 von 57 Belegen). Die Korrelation zwischen propositionsbezogenen bzw. pragmatischen Adverbialsätzen und den Stellungstypen ist statistisch hoch signifikant (P-Wert <0,00001).

Bei den einzelnen Typen konnten unterschiedliche Stellungsmöglichkeiten nachgewiesen werden. Bei ,formelhaften Thematisierungsausdrücken', die mehr als die Hälfte der einschlägigen Belege ausmachen (insg. 29 Belege, 50,9\%), kam der nicht-integrative Тур nicht vor. Angesichts ihres musterhaften Charakters ist es umstritten, ob sie bei der 
Bewertung der Stellungsvariation zu berücksichtigen sind. Im Bereich der traditionell beachteten pragmatischen Adverbialsätze (ohne den Untertyp ,thematisierend') ist dagegen die nicht-integrative Stellung sogar die bevorzugte Variante mit 42,9\% (12 von 28 Belegen). Die Daten für diesen Untertyp werden in der folgenden Tabelle bzw. im Anhang (8.2, in den Diagrammen 21 und 22) getrennt aufgeführt.

\begin{tabular}{|l|c|c|c|}
\hline & integrativ & resumptiv & nicht-integrativ \\
\hline $\begin{array}{l}\text { Pragmatische Adverbialsätze ohne } \\
\text { thematisierende AS }(\mathrm{n}=28)\end{array}$ & 10 & 6 & 12 \\
\hline $\begin{array}{l}\text { Thematisierende Adverbialsätze } \\
(\mathrm{n}=29)\end{array}$ & $35,7 \%$ & $21,4 \%$ & $42,9 \%$ \\
\hline Gesamt $(n=57)$ & $10,3 \%$ & 26 & 0 \\
& 13 & $89,7 \%$ & $0 \%$ \\
\hline
\end{tabular}

Tabelle 16: Verteilung der Stellungstypen bei pragmatischen Adverbialsätzen

Wenn man solche Fälle, die nur einen Stellungstyp zulassen, nicht berücksichtigt, dann ergeben sich z.T. andere Frequenzwerte für die einzelnen Stellungstypen. Im Bereich der traditionellen pragmatischen Adverbialsätze konnten 8 Belege identifiziert werden, bei denen der Obersatz ein Imperativ- oder Ergänzungsfragesatz war. In diesen Fällen besteht keine Wahl zwischen den Stellungstypen. Im Bereich der propositionsbezogenen Adverbialsätze können die Proportionalsätze aus demselben Grund aussortiert werden. Auch ohne diese Belege ergibt sich für den nicht-integrativen Typ ein bedeutender Frequenzunterschied zwischen propositionsbezogenen (1,3\%, 5 von 388 Belegen) und pragmatischen (20\%, 4 von 20 Belegen) Adverbialsätzen. Die Korrelation zwischen propositionsbezogenen bzw. pragmatischen Adverbialsätzen und den Stellungstypen ist auch nach dieser Berechnung statistisch hoch signifikant (P-Wert $<0,00001)$.

Das Ergebnis, dass im Bereich der pragmatischen Adverbialsätze der resumptive und der integrative Stellungstyp möglich sind, steht im Einklang mit den Ergebnissen zum Gegenwartsdeutschen (vgl. z.B. Pittner 2013a: 521, s. dazu Kapitel 2.1.2). Propositionsbezogene Adverbialsätze sind tendenziell stärker integriert als nichtpropositionsbezogene, es besteht jedoch keine Eins-zu-eins-Entsprechung zwischen dem Parameter (Des-)Integration und der Verknüpfungsebenen. Die topologische Desintegration ist im Neuhochdeutschen erwartungsgemäß nicht voll funktionalisiert. Dabei konnten sogar bei den einzelnen Typen unterschiedliche Stellungsmöglichkeiten nachgewiesen werden. Während bei modusmodifizierenden Adverbialsätzen der nicht-integrative Typ nicht belegt ist, lassen bestimmte diktums- bzw. einstellungskommentierende Adverbialsätze nur die 
nicht-integrative Stellung zu. Die Erforschung der einschlägigen Unterschiede ist Gegenstand weiterer Analysen.

Eine Untersuchung historischer Veränderungen ist aufgrund der vorliegenden Daten kaum möglich. Aus der folgenden Tabelle geht aber hervor, dass der integrative Typ nur in den beiden Korpustexten aus dem 20. Jh. und dem Nähetext aus dem 19. Jh. eine relativ frequente Variante darstellt.

\begin{tabular}{|l|c|c|c|}
\hline & integrativ & resumptiv & nicht-integrativ \\
\hline \multirow{2}{*}{ Güntzer I (n=2) } & 0 & 0 & 2 \\
& $0 \%$ & $0 \%$ & $100 \%$ \\
\hline \multirow{2}{*}{ Harsdörffer I (n=3) } & 0 & 0 & 3 \\
& $0 \%$ & $0 \%$ & $100 \%$ \\
\hline \multirow{2}{*}{ Schuhmacher Chronik II (n=27) } & 1 & 26 & 0 \\
& $3,7 \%$ & $96,3 \%$ & $0 \%$ \\
\hline \multirow{2}{*}{ Aichinger III (n=0) } & 0 & 0 & 0 \\
\multirow{2}{*}{ Auswandererbriefe V (n=16) } & $0 \%$ & $0 \%$ & $0 \%$ \\
\hline \multirow{2}{*}{ Burckhardt V (n=0) } & $67,5 \%$ & 6 & 4 \\
\multirow{2}{*}{ Jugendtagebuch VII (n=3) } & 0 & $0 \%, 5 \%$ & $25,0 \%$ \\
\hline \multirow{2}{*}{ Lorenz VII (n=6) } & 2 & $0 \%$ & $0 \%$ \\
\hline \multirow{2}{*}{ Gesamt $(n=57)$} & $66,7 \%$ & $0 \%$ & 1 \\
& 4 & 0 & $33,3 \%$ \\
\hline
\end{tabular}

Tabelle 17: Verteilung der Stellungstypen bei pragmatischen Adverbialsätzen

Für den integrativen Typ ist im Teilkorpus für das 18. Jh. ein Einzelbeleg enthalten (3,7\%). Im 19. und im 20. Jh. ist er bereits ein frequenter Stellungstyp (37,5\% bzw. 66,7\%). Am Gesamtbild ändert sich nicht viel, wenn man thematisierende Adverbialsätze und Belege mit Imperativ/Ergänzungsfrage im Obersatz nicht berücksichtigt. In diesem Fall sind vom 17. bis zum 19. Jh. nur in drei Korpustexten einschlägige Belege zu finden. In Harsdörffer I und in Schuhmacher Chronik II ist nur je ein Stellungstyp belegt (1mal nicht-integrativ bzw. 3mal resumptiv). In Auswandererbriefe V entfällt die Hälfte der Belege auf diese Varianten (1mal nicht-integrativ bzw. 3mal resumptiv von 8 Belegen), die integrative Stellung ist jedoch mit 50\% (4 Belege) die dominierende Variante. Die Verteilung der Stellungstypen in den Texten aus dem 20. Jh. ändert sich bei dieser Berechnung nicht.

Eine vergleichende Analyse des Integrationsgrades bei Untertypen, die in mehreren Quellentexten belegt sind, wäre sicherlich wünschenswert, dies ist jedoch auf der Basis der 
vorliegenden Daten nur in den folgenden Fällen und nur beschränkt möglich. Im Distanztext aus dem 20. Jh. werden Adverbialsätze der intertextuellen/textinternen Vernetzung stets integrativ angeschlossen (3 von 3 Belegen), während im Nähetext aus dem 19. Jh. auch der nicht-integrative Typ mit einem Einzelbeleg vertreten ist (von 3 Belegen) und der einzige Beleg aus dem Nähetext aus dem 18. Jh. resumptiven Anschluss zeigt. Beim modusmodifizierenden Gebrauch ist im Nähetext aus dem 18. Jh. ein Einzelbeleg mit resumptiver Stellung zu finden, im Nähetext aus dem 19. Jh. werden dagegen beide einschlägigen Belege integrativ angebunden; der Distanztext aus dem 20. Jh. enthält auch einen einzigen modusmodifizierenden Adverbialsatz mit integrativer Stellung.

Was das Verhältnis der Stellungstypen und Nähe- bzw. Distanz betrifft, so sind im Bereich der pragmatischen Adverbialsätze (bei Berücksichtigung aller Typen) trotz der geringen Belegzahl deutliche Unterschiede $\mathrm{zu}$ erkennen. Es ist hervorzuheben, dass sich im Distanzkorpus kein Beleg für die resumptive Anbindung von pragmatischen Adverbialsätzen findet, während der resumptive Typ im Nähekorpus dominiert. Zum prozentualen Verhältnis der Stellungstypen in den Nähe- bzw. Distanztexten s. die Diagramme 19 und 20 im Anhang (8.2). Die Korrelation zwischen Stellungstypen und Nähe-Distanz erweist sich als statistisch signifikant (P-Wert $=0,003421)$. Zählt man Belege für die Typen ,thematisierend' bzw. Fragesatz/Imperativ-Obersatz nicht mit, ist das Ergebnis jedoch statistisch nicht signifikant.

\subsection{Diskussion der Ergebnisse}

\subsubsection{Historische Aspekte}

In diesem Kapitel soll geprüft werden, welche Schlussfolgerungen aus den Ergebnissen zu den einzelnen Adverbialsatztypen $\mathrm{zu}$ ziehen sind. Im Allgemeinen wird die integrative Stellung als die dominierende Variante des Gegenwartsdeutschen angesehen (vgl. Zifonun et al. 1997: 2349 sowie die im Kapitel 2.1.1 angeführten Korpusergebnisse von Axel 2002: 9). Aufgrund der einschlägigen Daten aus den Quellentexten aus dem 17. Jh. soll im Folgenden die Frage diskutiert werden, ob die wesentlichen Veränderungen im Bereich der Satzintegration zu Beginn des Neuhochdeutschen bereits abgeschlossen sind und der integrative Typ bereits im 17. Jh. die dominierende Variante ist. In der folgenden Tabelle finden sich zusammenfassende Daten zur Verteilung der Stellungstypen bei verschiedenen Adverbialsatztypen im 17. Jh. Diese lassen den Schluss zu, dass sich die integrative Stellung nicht bereits vor Beginn der neuhochdeutschen Periode durchgesetzt hat. 


\begin{tabular}{|l|c|c|c|}
\hline & integrativ & resumptiv & nicht-integrativ \\
\hline \multirow{2}{*}{ Temporalsätze (n=68) } & 42 & 26 & 0 \\
& $61,8 \%$ & $38,2 \%$ & $0 \%$ \\
\hline \multirow{2}{*}{ Konditionalsätze (n=54) } & 16 & 38 & 0 \\
& $29,6 \%$ & $70,4 \%$ & $0 \%$ \\
\hline Kausalsätze (n=19) & 10 & 9 & 0 \\
& $52,6 \%$ & $47,4 \%$ & $0 \%$ \\
\hline Finalsätze (n=2) & 2 & 0 & 0 \\
Konzessivsätze (n=5) & $100 \%$ & $0 \%$ & $0 \%$ \\
\hline Irrelevanzkonditionale (n=1) & 1 & 4 & 0 \\
\hline \multirow{2}{*}{ Komparativsätze (n=11) } & $0,0 \%$ & $80,0 \%$ & $0 \%$ \\
\hline \multirow{2}{*}{ Proportionalsätze (n=5) } & $0 \%$ & 1 & $0 \%$ \\
\hline \multirow{2}{*}{ Temporal-kausale AS (n=2) } & 0 & $100 \%$ & 0 \\
& $0 \%$ & 11 & $0 \%$ \\
\hline Lokal(-konditional) (n=3) & 0 & 5 & $0 \%$ \\
\hline Pragmatische Adverbialsätze (n=5) & $0 \%$ & $100 \%$ & 0 \\
\hline \multirow{2}{*}{ Gesamt $(n=175)$} & $0 \%$ & 0 & $0 \%$ \\
\hline
\end{tabular}

Tabelle 18: Verteilung der Stellungstypen bei verschiedenen Adverbialsatztypen im 17. Jh.

Bei den einzelnen Adverbialsatztypen konnte nur bei den Typen ,final` und ,temporal-kausal` der Anteil von 100\% des integrativen Typs ermittelt werden, die Korpustexte enthalten allerdings insgesamt nur je zwei einschlägige Belege. Im Gegensatz dazu treten bei Vergleichssätzen (Komparativ- und Proportionalsätzen) und Irrelevanzkonditionalen der resumptive Typ bzw. bei pragmatischen Adverbialsätzen der nicht-integrative Typ mit einem Anteil von $100 \%$ auf. Bei den weiteren Satztypen ist eine Varianz zu beobachten, wobei der resumptive Typ stets einen Anteil zwischen 33,3\% und $80 \%$ hat. Er ist bei Konditional- und Konzessivsätzen sogar die bevorzugte Variante (70,4\% bzw. 80\%). Der integrative Typ dominiert bei Temporal- und Kausalsätzen, wobei der Frequenzunterschied zwischen ,resumptiv‘ und ,integrativ‘ im kausalen Bereich gering ist (9 vs. 10 von insgesamt 19 Belegen). Bei Temporalsätzen konnte im Gegensatz zu früheren Forschungsergebnissen (z.B. Wiktorowitz 2013: 394f.) ein bedeutender Anteil des Resumptivums nachgewiesen werden (38,2\%). Der hohe Anteil des Resumptivums bei diesem Satztyp lässt sich auf in der bisherigen Forschung nicht beachtete Nähe-Distanz-Unterschiede zurückführen. Durch die 
Untersuchung des Nähekorpus konnten also frühere Forschungsergebnisse relativiert werden, was die Relevanz der Unterscheidung von Nähe und Distanz bei der Integration (und der Berücksichtigung von Nähetexten in sprachgeschichtlichen Untersuchungen im Allgemeinen) untermauert.

Die Verteilung der Stellungstypen ist abhängig von den verschiedenen Adverbialsatztypen. Der nicht-integrative Typ ist nur bei nicht-propositionsbezogenen, pragmatischen Adverbialsätzen belegt. Im Bereich der propositionsbezogenen Adverbialsätze ist die folgende Korrelation zu entdecken (Proportionalsätze, bei denen es keine Wahlmöglichkeit zwischen den Stellungstypen gibt, werden in der folgenden Tabelle nicht berücksichtigt): Im temporal-kausalen Bereich dominiert der integrative Typ, während bei den restlichen Adverbialsätzen der resumptive Typ bevorzugt wird, vgl. die folgende Tabelle:

\begin{tabular}{|l|c|c|}
\hline & integrativ & resumptiv \\
\hline Temporal und kausal gleichläufig (kausal i.e.S., final), & 56 & 35 \\
temporal-kausal (n=91) & $61,5 \%$ & $38,5 \%$ \\
\hline Rest (konditional, konzessiv, komparativ, lokal- & 19 & 55 \\
konditional (n=74) & $25,7 \%$ & $74,3 \%$ \\
\hline \multirow{2}{*}{ Gesamt $(n=165)$} & 75 & 90 \\
& $45,5 \%$ & $54,5 \%$ \\
\hline
\end{tabular}

Tabelle 19: Stellungstypen und semantische Bereiche im 17. Jh.

Die Korrelation zwischen Stellungstypen und den semantischen Bereichen ist statistisch hoch signifikant $(\mathrm{P}-\mathrm{Wert}=0,000004)$. Im Kapitel 2.4 wurde folgende Hypothese formuliert:

Hypothese 1: In den Korpustexten aus dem 17. Jahrhundert dominiert bereits der integrative Typ. Die Durchsetzung des integrativen Typs ist vor dem Beginn der neuhochdeutschen Periode bereits abgeschlossen.

Im Lichte der oben angeführten Ergebnisse muss diese Hypothese verworfen werden. Der integrative Typ scheint sich nur im temporal-kausalen Bereich durchgesetzt zu haben. Die vorliegende Untersuchung relativiert damit die Daten von Axel (2004: 40), die eine frühere Dominanz des integrativen Typs im Bonner Frühneuhochdeutsch-Korpus zeigten (vgl. Kapitel 2.2.2.2).

Die nächste, zu klärende Frage ist, ob sich der integrative Typ in der neuhochdeutschen Periode als die bevorzugte Variante erweist. Die unten stehende Tabelle 20, in der die Gesamtergebnisse für die einzelnen Adverbialsatztypen zusammengefasst sind, lässt erkennen, dass bezüglich des Neuhochdeutschen von einer Dominanz des integrativen Typs nicht die Rede sein kann. 


\begin{tabular}{|c|c|c|c|}
\hline & integrativ & resumptiv & nicht-integrativ \\
\hline Temporalsätze $(\mathrm{n}=121)$ & $\begin{array}{c}86 \\
71,1 \%\end{array}$ & $\begin{array}{c}35 \\
28,9 \%\end{array}$ & $\begin{array}{c}0 \\
0 \%\end{array}$ \\
\hline Konditionalsätze (n=137) & $\begin{array}{c}37 \\
27,0 \%\end{array}$ & $\begin{array}{c}99 \\
72,3 \%\end{array}$ & $\begin{array}{c}1 \\
0,7 \%\end{array}$ \\
\hline Kausalsätze (n=66) & $\begin{array}{c}17 \\
25,8 \%\end{array}$ & $\begin{array}{c}48 \\
72,7 \%\end{array}$ & $\begin{array}{c}1 \\
1,5 \%\end{array}$ \\
\hline Finalsätze $(n=2)$ & $\begin{array}{c}2 \\
100 \%\end{array}$ & $\begin{array}{c}0 \\
0 \% \\
\end{array}$ & $\begin{array}{c}0 \\
0 \%\end{array}$ \\
\hline Konsekutivsätze (n=1) & $\begin{array}{c}0 \\
0 \%\end{array}$ & $\begin{array}{c}1 \\
100 \%\end{array}$ & $\begin{array}{c}0 \\
0 \%\end{array}$ \\
\hline Adversativsätze (n=2) & $\begin{array}{c}2 \\
100 \%\end{array}$ & $\begin{array}{c}0 \\
0 \%\end{array}$ & $\begin{array}{c}0 \\
0 \%\end{array}$ \\
\hline Konzessivsätze (n=16) & $\begin{array}{c}3 \\
18,6 \%\end{array}$ & $\begin{array}{c}13 \\
81,3 \%\end{array}$ & $\begin{array}{c}0 \\
0 \%\end{array}$ \\
\hline Irrelevanzkonditionale $(\mathrm{n}=11)$ & $\begin{array}{c}3 \\
27,3 \%\end{array}$ & $\begin{array}{c}5 \\
45,5 \%\end{array}$ & $\begin{array}{c}3 \\
27,3 \% \\
\end{array}$ \\
\hline Spezifizierende AS $(n=3)$ & $\begin{array}{c}3 \\
100 \%\end{array}$ & $\begin{array}{c}0 \\
0 \%\end{array}$ & $\begin{array}{c}0 \\
0 \%\end{array}$ \\
\hline Komparativsätze (n=20) & $\begin{array}{c}2 \\
10,0 \%\end{array}$ & $\begin{array}{c}18 \\
90,0 \%\end{array}$ & $\begin{array}{c}0 \\
0 \%\end{array}$ \\
\hline Proportionalsätze $(\mathrm{n}=8)$ & $\begin{array}{c}0 \\
0 \% \\
\end{array}$ & $\begin{array}{c}8 \\
100 \% \\
\end{array}$ & $\begin{array}{c}0 \\
0 \% \\
\end{array}$ \\
\hline Temporal-kausale AS $(n=2)$ & $\begin{array}{c}2 \\
100 \%\end{array}$ & $\begin{array}{c}0 \\
0 \%\end{array}$ & $\begin{array}{c}0 \\
0 \%\end{array}$ \\
\hline Lokal(-konditional) $(\mathrm{n}=7)$ & $\begin{array}{c}5 \\
71,4 \%\end{array}$ & $\begin{array}{c}2 \\
28,6 \%\end{array}$ & $\begin{array}{c}0 \\
0 \%\end{array}$ \\
\hline $\begin{array}{l}\text { Pragmatische Adverbialsätze ohne } \\
\text { thematisierende AS }(n=28)\end{array}$ & $\begin{array}{c}10 \\
35,7 \%\end{array}$ & $\begin{array}{c}6 \\
21,4 \%\end{array}$ & $\begin{array}{c}12 \\
42,9 \%\end{array}$ \\
\hline $\begin{array}{l}\text { Thematisierende Adverbialsätze } \\
(\mathrm{n}=29)\end{array}$ & $\begin{array}{c}3 \\
10,3 \%\end{array}$ & $\begin{array}{c}26 \\
89,7 \%\end{array}$ & $\begin{array}{c}0 \\
0 \%\end{array}$ \\
\hline Gesamt $(n=453)$ & $\begin{array}{c}175 \\
38,6 \%\end{array}$ & $\begin{array}{c}261 \\
57,6 \%\end{array}$ & $\begin{array}{c}17 \\
3,8 \%\end{array}$ \\
\hline
\end{tabular}

Tabelle 20: Verteilung der Stellungstypen bei vorangestellten Adverbialsätzen im Gesamtkorpus

Diese Ergebnisse belegen, dass der integrative Typ insgesamt nicht den frequentesten Stellungstyp darstellt (38,6\%). Der integrative Typ dominiert zwar im Bereich der Temporalsätze und bei einigen weniger frequenten Satztypen (final, adversativ, spezifizierend, temporal-kausal, lokal-konditional). Der resumptive Typ kommt aber mit dem höchsten Anteil im Bereich der Kausal-, Konditional-, Irrelevanzkonditional-, Konzessiv-, Komparativsätzen sowie bei Proportionalsätzen und thematisierenden Adverbialsätzen vor. In den letzten beiden Typen ist die Wahl der integrativen Variante nicht möglich bzw. nur selten anzutreffen. Würde man nur die Gesamtwerte für propositionsbezogene Adverbialsätze ohne 
Proportionalsätze berücksichtigen, käme es beim integrativen Typ auf 41,6\% (162 von 388 Belegen), während sich der Anteil des resumptiven Typs auch in diesem Fall nicht relevant verändern würde (57,0\%). Die im Kapitel 2.4 formulierte Hypothese lautete wie folgt:

Hypothese 2: Der integrative Typ ist die bevorzugte Variante im Neuhochdeutschen.

Durch die obigen Ergebnisse kann diese Hypothese nicht bestätigt werden. Während die integrative Stellung die dominierende Variante des Gegenwartsdeutschen darstellt (vgl. Zifonun et al. 1997: 2349, Axel 2002: 9, s. auch Kapitel 2.1.1), konnte im neuhochdeutschen Korpus nur im Bereich der Temporalsätze eine deutliche Dominanz des integrativen Typs nachgewiesen werden. Bei einigen Adverbialsätzen lässt die Datengrundlage keine weitreichenden Schlüsse zu, bei mehreren Adverbialsatztypen wurde jedoch die Dominanz des resumptiven Typs ermittelt. In mehreren Fällen konnte die Wahl des resumptiven Typs auch darauf zurückgeführt werden, dass dadurch eine paarige Struktur entsteht. Während den konditionalen und irrelevanzkonditionalen so-so-Strukturen auch integrativ angeschlossene Gegenbeispiele (s. (104) bzw. (284)) gegenüberstehen, wurden Proportionalsätze als paarige Strukturen analysiert, in denen $s o+$ Adj. konstitutiver Bestandteil des ,paarigen Junktionsausdrucks ist (s. Kapitel 3.1.4).

Was die nicht-integrative Stellung betrifft, so ist festzustellen, dass er im Bereich der propositionsbezogenen Adverbialsätze nur eine marginale Rolle spielt (1,3\%, 5 von 388 Belegen, Proportionalsätze nicht mitgerechnet). Sein Anteil ist erwartungsgemäß bei Irrelevanzkonditionalen am höchsten (27,3\%), da hier die topologische Desintegration den geringen semantischen Zusammenhang zwischen den Teilsätzen widerspiegelt (vgl. z.B. Küper 1993: 42, s. dazu Kapitel 2.1.3). Der hohe Anteil des nicht-integrativen Typs im Bereich der pragmatischen Adverbialsätze (42,9\%, ohne den Untertyp ,thematisierend“) ist z.T. darauf zurückzuführen, dass in 8 Belegen, in denen der Obersatz ein Imperativ- oder Fragesatz ist, nur die Desintegration möglich ist. Eine Wahlmöglichkeit zwischen den Stellungstypen besteht nur bei Aussagesätzen, bei denen der nicht-integrative Typ nicht die häufigste Variante darstellt (20\%, 4 von 20 Belegen). Die Stellungstypen ,integrativ` und ,resumptiv‘ einerseits bzw. ,nicht-integrativ` andererseits haben also keine klare Verteilung auf propositionsbezogene und nicht-propositionsbezogene Adverbialsätze.

Die oben angeführten Daten bestätigen jedoch die bisherigen Forschungsergebnisse (s. Kapitel 2.1.3) insofern, dass sie statistisch hoch signifikante Unterschiede zwischen den einzelnen Adverbialsatztypen bezüglich ihrer Stellung belegen. Temporalsätze sind stärker integriert als Konzessivsätze (P-Wert $=0,000037)$, Konditionalsätze stärker als 
Irrelevanzkonditionale (P-Wert < 0,00001). Beim Vergleich konditional-irrelevanzkonditional ist zwar der Anteil des integrativen Typs gleich (27,0\% vs. 27,3\%), bei Irrelevanzkonditionalen werden aber insgesamt aggregativere Techniken verwendet, weil hier der Anteil des nicht-integrativen Typs höher ist als bei Konditonalen. Zudem konnte nachgewiesen werden, dass eingeleitete Konditionalsätze integrativer angebunden werden als uneingeleitete $(\mathrm{P}-\mathrm{Wert}=0,035804$, vgl. 5.2.5) bzw. dass propositionsbezogene Adverbialsätze integrativer angebunden werden als nicht-propositionsbezogene (P-Wert < 0,00001, vgl. 5.5.7). Die folgende Hypothese konnte somit bestätigt werden:

Hypothese 3: Der Integrationsgrad ist bei den einzelnen semantischen Adverbialsatztypen z.T. unterschiedlich bzw. bei ihnen werden jeweils verschiedene Stellungstypen bevorzugt.

Diese Ergebnisse zeigen m.E. eindeutig, dass eine grobe, pauschale Korpusauswertung nach der Frequenz der Stellungstypen ohne Berücksichtigung der obigen Unterschiede nicht befriedigend sein kann. Würde man verschiedene Adverbialsatztypen undifferenziert behandeln, hätte die uneinheitliche Verteilung der Adverbialsatztypen in den Korpustexten bzw. in den Teilkorpora einen wesentlichen Einfluss auf die Frequenz der einzelnen Stellungstypen in den Teilkorpora. Dies könnte wiederum dazu führen, dass Frequenzunterschiede bei den semantischen Typen fälschlicherweise als Unterschiede im Integrationsgrad interpretiert werden. Die Auszählungen zu den einzelnen Typen sollen daher getrennt behandelt und erst dann zueinander in Beziehung gesetzt werden.

Nun wenden wir uns der Frage zu, ob bzw. welche Unterschiede zwischen den untersuchten Zeitabschnitten festzustellen sind. Zunächst sollen die Gesamtergebnisse für die einzelnen Teilkorpora präsentiert werden:

\begin{tabular}{|l|c|c|c|}
\hline & integrativ & resumptiv & nicht-integrativ \\
\hline \multirow{2}{*}{ 17. Jh. $(\mathrm{n}=175)$} & 75 & 95 & 5 \\
& $42,9 \%$ & $54,3 \%$ & $2,9 \%$ \\
\hline \multirow{2}{*}{ 18. Jh. $(\mathrm{n}=102)$} & 13 & 88 & 1 \\
& $12,7 \%$ & $86,3 \%$ & $1,0 \%$ \\
\hline \multirow{2}{*}{ 19. Jh. $(\mathrm{n}=101)$} & 33 & 61 & 7 \\
& $32,7 \%$ & $60,4 \%$ & $6,9 \%$ \\
\hline \multirow{2}{*}{ 20. Jh. $(\mathrm{n}=75)$} & 54 & 17 & 4 \\
& $72,0 \%$ & $22,7 \%$ & $5,3 \%$ \\
\hline \multirow{2}{*}{ Gesamt $(n=453)$} & 175 & 261 & 17 \\
& $38,6 \%$ & $57,6 \%$ & $3,8 \%$ \\
\hline
\end{tabular}

Tabelle 21: Verteilung der Stellungstypen nach Zeitabschnitten 
Die in der obigen Tabelle angeführten Ergebnisse müssen unter Vorbehalt betrachtet werden, da - wie oben gezeigt - die Daten zu verschiedenen Adverbialsätzen nicht unreflektiert miteinander verglichen werden dürfen. Sie zeigen jedoch, dass in den Korpustexten aus dem 17. bis zum 19. Jh. im Allgemeinen der resumptive Typ dominiert. ${ }^{228}$ In den Korpustexten aus dem 18. Jh. nimmt der Anteil des resumptiven Typs im Vergleich zu den entsprechenden Daten aus dem 17. Jh. sogar deutlich zu, was im Widerspruch zur Annahme einer unidirektionalen Entwicklung von Desintegration zur Integration im Sinne von König/van der Auwera (1988) steht. Erst im Teilkorpus aus dem 20. Jh. nimmt der Gebrauch des resumptiven Typs stark ab (22,7\%), er ist nur im konditionalen Bereich und bei Vergleichssätzen belegt. ${ }^{229}$

Im Folgenden wird auf historische Veränderungen bei den einzelnen Untertypen eingegangen. Im Bereich der Temporalsätze sind die wesentlichen Veränderungen auf die Nähetexte beschränkt, zumal im Teilkorpus der Distanztexte bis auf zwei Gegenbeispiele stets der integrative Stellungstyp auftritt. Die Dynamik bei der Integration der Temporalsätze im Nähebereich kann damit beschrieben werden, dass der ,resumptive" Typ in den beiden Quellentexten aus dem 17. bzw. 18. Jh. noch mit einem Anteil von 40,6\% bzw. 55,6\% vorkommt, dann im 19. Jh. unter $20 \%$ fällt und im 20. Jh. gänzlich außer Gebrauch kommt. Das Ergebnis, dass im temporalen Bereich die integrative Stellung häufiger wird und der resumptive Typ seltener, ist statistisch signifikant (P-Wert $=0,010635)$.

Bei Konditionalsätzen ist insgesamt eine Verbreitung des integrativen Typs zu beobachten, wobei dieses Ergebnis auf der Grenze der statistischen Signifikanz ist (P-Wert = 0,051979). Während der Anteil des integrativen Typs bei eingeleiteten Konditionalsätzen in den Korpustexten aus dem 17., 18. sowie 19. Jh. - mit der Ausnahme des Distanztextes aus dem 17. - zwischen $0 \%$ und $35 \%$ liegt, ist er in den Korpustexten aus dem 20. Jh. bereits häufiger anzutreffen $(44,4 \%$ sowie $60 \%)$. Er ist jedoch nur im Nähetext aus dem 20. Jh. der frequenteste Stellungstyp. Uneingeleitete Konditionalsätze sind von der Integrationstendenz weniger betroffen: Der resumptive Stellungstyp ist eindeutig die unmarkierte, bevorzugte Variante (vgl. Kapitel 2.1), bei der überwiegenden Mehrheit der einschlägigen Belege $(85,7 \%, 24$ von 28$)$ ist die resumptive Wiederaufnahme mit so zu beobachten. Insgesamt konnte für den Zusammenhang zwischen den Zeitabschnitten und den Stellungstypen ,integrativ‘ bzw. ,resumptiv‘ nur für das Distanzkorpus ein signifikantes Ergebnis berechnet

\footnotetext{
${ }^{228}$ Die Gründe für die Dominanz des integrativen Typs in einigen Texten des 16. Jhs. (vgl. z.B. Hammarström 1923, Axel 2004: 40, s. dazu Kapitel 2.2.2) können an dieser Stelle nicht diskutiert werden.

${ }^{229}$ Dass der Gebrauch des resumptiven Typs im 20. Jh. bereits eingeschränkt ist, zeigt sich auch darin, dass im ProtoNähetext Daniel Domian VII die resumptive Stellung nur bei wenn-Sätzen belegt ist.
} 
$(\mathrm{P}-\mathrm{Wert}=0,038576)$ werden, im Bereich der Nähetexte (insg. 40 Belege) sind jedoch weitere Untersuchungen nötig $(\mathrm{P}-\mathrm{Wert}=0,081248)$.

Die Daten aus dem kausalen Bereich zeigen auch eine historische Veränderung: Während in den älteren Korpustexten der resumptive Typ bis auf einen Korpustext dominiert, tritt in den Korpustexten aus dem 20. Jh. stets der integrative Typ auf (6 von 6 Belegen). Die Tendenz, dass mit der Zeit die integrative Stellung häufiger wird, konnte sowohl in den Näheals auch in den Distanztexten nachgewiesen werden (P-Wert $=0,033052$ bzw. 0,000677). Die Korrelation zwischen den Zeitabschnitten und den Stellungstypen ,integrativ ${ }^{`}$ und ,resumptiv erweist sich im kausalen Bereich als statistisch hoch signifikant $(\mathrm{P}-\mathrm{Wert}=0,00001)$.

Bei den weiteren Untertypen von Adverbialsätzen im engeren Sinne (Kapitel 5.4) ermöglicht die Belegzahl keine weiteren Schlüsse. Es soll jedoch angemerkt werden, dass die Ergebnisse zu den folgenden Untertypen die Annahme einer Integrationstendenz bestätigen. Bei Konzessivsätzen steht der absoluten Dominanz des resumptiven Stellungstyps in den Korpustexten aus dem 19. Jh. ein Einzelbeleg für den integrativen Stellungstyp aus den Korpustexten des 20. Jhs. entgegen. Auch in Komparativsätzen ist der resumptive Stellungstyp dominant (18 von 20 Belegen, 90\%), die beiden Gegenbeispiele mit integrativer Stellung stammen jedoch aus dem 20. Jh. Bei Irrelevanzkonditionalen wird in den Belegen aus dem 17. bis zum 19. Jh. bis auf einen Einzelbeleg entweder der resumptive (5 Belege) oder der nicht-integrative Typ (3 Belege, alle aus einem einzigen Korpustext) verwendet, während die beiden Belege aus dem 20. Jh. eine integrative Stellung zeigen. Die folgende Tabelle enthält die Gesamtergebnisse für die weiteren Untertypen von Adverbialsätzen im engeren Sinne (Kapitel 5.4). Proportionalsätze, bei denen keine Wahl zwischen den Stellungstypen besteht, werden hier nicht berücksichtigt.

\begin{tabular}{|l|c|c|c|}
\hline & integrativ & resumptiv & nicht-integrativ \\
\hline \multirow{2}{*}{ 17. Jh. $(\mathrm{n}=24)$} & 7 & 17 & 0 \\
& $29,2 \%$ & $70,8 \%$ & $0 \%$ \\
\hline \multirow{2}{*}{ 18. Jh. $(\mathrm{n}=14)$} & 3 & 11 & 0 \\
& $21,4 \%$ & $78,6 \%$ & $0 \%$ \\
\hline \multirow{2}{*}{ 19. Jh. $(\mathrm{n}=18)$} & 5 & 10 & 3 \\
& $27,8 \%$ & $55,6 \%$ & $16,7 \%$ \\
\hline \multirow{2}{*}{ 20. Jh. $(\mathrm{n}=8)$} & 7 & 1 & 0 \\
\hline \multirow{2}{*}{ Gesamt $(n=64)$} & $87,5 \%$ & $12,5 \%$ & $0 \%$ \\
\hline
\end{tabular}

Tabelle 22: Verteilung der Stellungstypen nach Zeitabschnitten bei weiteren Untertypen von Adverbialsätzen im engeren Sinne 
Aufgrund dieser Gesamtergebnisse ist die Korrelation zwischen Stellungstypen und Zeitabschnitten auch bei weiteren Untertypen von Adverbialsätzen im engeren Sinne statistisch signifikant (P-Wert=0,041863).

Die obigen Ergebnisse bestätigen m.E. eindrucksvoll, dass in der neuhochdeutschen Periode relevante Veränderungen im Bereich der Integration vorangestellter Adverbialsätze vorgehen. Die im Kapitel 2.4 aufgestellte Hypothese wurde in den einzelnen Adverbialsatztypen durch empirische Daten belegt.

Hypothese 4: Im Neuhochdeutschen sind relevante syntaktische Veränderungen im Bereich der Satzintegration nachzuweisen, die sich in deutlichen Häufigkeitsunterschieden bei den Stellungstypen ,nicht-integrativ', ,resumptiv` und ,integrativ` erfassen lassen.

Die Daten zu den einzelnen Adverbialsatztypen zeigen einstimmig die folgende Tendenz der Veränderung: Die resumptive Anbindung von Adverbialsätzen wird durch die integrative verdrängt bzw. überlagert, aber im Falle der verschiedenen Adverbialsatztypen mit unterschiedlichem Tempo. Die Analyse zeigte auch entgegengesetzte Tendenzen: Angesichts der Zunahme des Anteils der resumptiven Anbindung im 18. Jh. scheint der Integrationsprozess nicht eindeutig unidirektional zu verlaufen.

\subsubsection{Nähe-Distanz-Unterschiede}

Auf einzelne Nähe-Distanz-Unterschiede wurde oben bereits verwiesen, im Folgenden ist jedoch auf Unterschiede, die auf die Variable (konzeptionelle) Mündlichkeit/Schriftlichkeit zurückzuführen sind, näher einzugehen. An dieser Stelle soll die im Kapitel 2.4 formulierte Hypothese wiederholt werden:

Hypothese 5: Bei der Verteilung der Stellungstypen ,nicht-integrativ`, ,resumptiv ${ }^{`}$ und ,integrativ ${ }^{6}$ sind relevante Unterschiede zwischen konzeptionell mündlichen bzw. konzeptionell schriftlichen Texten nachzuweisen.

Die folgende Tabelle enthält die empirischen Ergebnisse zur Verteilung der Stellungstypen in den beiden Teilkorpora der Nähe- bzw. Distanztexte. In dieser Überblickstabelle werden der Vollständigkeit halber die Ergebnisse für alle Adverbialsatztypen angegeben. 


\begin{tabular}{|c|c|c|c|c|c|c|c|}
\hline & \multicolumn{3}{|c|}{ Nähetexte } & \multicolumn{3}{|c|}{ Distanztexte } & \\
\hline & int & res & $n-i$ & int & res & $n-i$ & \\
\hline $\begin{array}{l}\text { Temporal } \\
(\mathrm{n}=103)\end{array}$ & $\begin{array}{c}70 \\
68,0 \% \\
\end{array}$ & $\begin{array}{c}33 \\
32,0 \% \\
\end{array}$ & $\begin{array}{c}0 \\
0 \%\end{array}$ & $\begin{array}{c}16 \\
88,9 \%\end{array}$ & $\begin{array}{c}2 \\
11,1 \% \\
\end{array}$ & $\begin{array}{c}0 \\
0 \%\end{array}$ & $\begin{array}{l}\text { Temporal } \\
(\mathrm{n}=18)\end{array}$ \\
\hline $\begin{array}{l}\text { Konditional, eing. } \\
(\mathrm{n}=36)\end{array}$ & $\begin{array}{c}11 \\
30,6 \%\end{array}$ & $\begin{array}{c}25 \\
69,4 \%\end{array}$ & $\begin{array}{c}0 \\
0 \%\end{array}$ & $\begin{array}{c}23 \\
31,5 \%\end{array}$ & $\begin{array}{c}50 \\
68,5 \%\end{array}$ & $\begin{array}{c}0 \\
0 \%\end{array}$ & $\begin{array}{l}\text { Konditional, eing. } \\
(\mathrm{n}=73)\end{array}$ \\
\hline $\begin{array}{l}\text { Konditional, } \\
\text { uneing. }(n=4)\end{array}$ & $\begin{array}{c}1 \\
25 \% \\
\end{array}$ & $\begin{array}{c}2 \\
50 \%\end{array}$ & $\begin{array}{c}1 \\
25 \%\end{array}$ & $\begin{array}{c}2 \\
8,3 \%\end{array}$ & $\begin{array}{c}22 \\
91,7 \%\end{array}$ & $\begin{array}{c}0 \\
0 \%\end{array}$ & $\begin{array}{l}\text { Konditional, } \\
\text { uneing. }(n=24)\end{array}$ \\
\hline $\begin{array}{l}\text { Kausal } \\
(\mathrm{n}=31)\end{array}$ & $\begin{array}{c}5 \\
16,1 \% \\
\end{array}$ & $\begin{array}{c}25 \\
80,6 \%\end{array}$ & $\begin{array}{c}1 \\
3,2 \% \\
\end{array}$ & $\begin{array}{c}12 \\
34,3 \% \\
\end{array}$ & $\begin{array}{c}23 \\
65,7 \% \\
\end{array}$ & $\begin{array}{c}0 \\
0 \% \\
\end{array}$ & $\begin{array}{l}\text { Kausal } \\
(\mathrm{n}=35)\end{array}$ \\
\hline $\begin{array}{l}\text { Final } \\
(n=1)\end{array}$ & $\begin{array}{c}1 \\
100 \%\end{array}$ & $\begin{array}{c}0 \\
0 \%\end{array}$ & $\begin{array}{c}0 \\
0 \%\end{array}$ & $\begin{array}{c}1 \\
100 \%\end{array}$ & $\begin{array}{c}0 \\
0 \%\end{array}$ & $\begin{array}{c}0 \\
0 \%\end{array}$ & $\begin{array}{l}\text { Final } \\
(\mathrm{n}=1)\end{array}$ \\
\hline $\begin{array}{l}\text { Konsekutiv } \\
(\mathrm{n}=1)\end{array}$ & $\begin{array}{c}0 \\
0 \%\end{array}$ & $\begin{array}{c}1 \\
100 \%\end{array}$ & $\begin{array}{c}0 \\
0 \%\end{array}$ & - & - & - & $\begin{array}{l}\text { Konsekutiv } \\
(\mathrm{n}=0)\end{array}$ \\
\hline $\begin{array}{l}\text { Adversativ } \\
(\mathrm{n}=1)\end{array}$ & $\begin{array}{c}1 \\
100 \%\end{array}$ & $\begin{array}{c}0 \\
0 \%\end{array}$ & $\begin{array}{c}0 \\
0 \%\end{array}$ & $\begin{array}{c}1 \\
100 \%\end{array}$ & $\begin{array}{c}0 \\
0 \%\end{array}$ & $\begin{array}{c}0 \\
0 \%\end{array}$ & $\begin{array}{l}\text { Adversativ } \\
(\mathrm{n}=1)\end{array}$ \\
\hline $\begin{array}{l}\text { Konzessiv } \\
(\mathrm{n}=6)\end{array}$ & $\begin{array}{c}1 \\
16,7 \%\end{array}$ & $\begin{array}{c}5 \\
83,3 \%\end{array}$ & $\begin{array}{c}0 \\
0 \%\end{array}$ & $\begin{array}{c}2 \\
20 \%\end{array}$ & $\begin{array}{c}8 \\
80 \%\end{array}$ & $\begin{array}{c}0 \\
0 \%\end{array}$ & $\begin{array}{l}\text { Konzessiv } \\
(\mathrm{n}=10)\end{array}$ \\
\hline $\begin{array}{l}\text { Irrelevanz- } \\
\text { konditional }(n=3)\end{array}$ & $\begin{array}{c}1 \\
33,3 \% \\
\end{array}$ & $\begin{array}{c}2 \\
66,7 \% \\
\end{array}$ & $\begin{array}{c}0 \\
0 \% \\
\end{array}$ & $\begin{array}{c}2 \\
25 \%\end{array}$ & $\begin{array}{c}3 \\
37,5 \%\end{array}$ & $\begin{array}{c}3 \\
37,5 \%\end{array}$ & $\begin{array}{l}\text { Irrelevanz- } \\
\text { konditional }(n=8)\end{array}$ \\
\hline $\begin{array}{l}\text { Spezifizierend } \\
(\mathrm{n}=0)\end{array}$ & - & - & - & $\begin{array}{c}3 \\
100 \% \\
\end{array}$ & $\begin{array}{c}0 \\
0 \% \\
\end{array}$ & $\begin{array}{c}0 \\
0 \% \\
\end{array}$ & $\begin{array}{l}\text { Spezifizierend } \\
(\mathrm{n}=3)\end{array}$ \\
\hline $\begin{array}{l}\text { Komparativsätze } \\
(\mathrm{n}=2)\end{array}$ & $\begin{array}{c}0 \\
0 \%\end{array}$ & $\begin{array}{c}2 \\
100 \%\end{array}$ & $\begin{array}{c}0 \\
0 \%\end{array}$ & $\begin{array}{c}2 \\
11,1 \%\end{array}$ & $\begin{array}{c}16 \\
88,9 \%\end{array}$ & $\begin{array}{c}0 \\
0 \%\end{array}$ & $\begin{array}{l}\text { Komparativsätze } \\
(\mathrm{n}=18)\end{array}$ \\
\hline $\begin{array}{l}\text { Proportionalsätze } \\
(\mathrm{n}=2)\end{array}$ & $\begin{array}{c}0 \\
0 \% \\
\end{array}$ & $\begin{array}{c}2 \\
100 \% \\
\end{array}$ & $\begin{array}{c}0 \\
0 \% \\
\end{array}$ & $\begin{array}{c}0 \\
0 \%\end{array}$ & $\begin{array}{c}6 \\
100 \%\end{array}$ & $\begin{array}{c}0 \\
0 \%\end{array}$ & $\begin{array}{l}\text { Proportionalsätze } \\
(\mathrm{n}=6)\end{array}$ \\
\hline $\begin{array}{l}\text { Temporal-kausal } \\
(\mathrm{n}=2)\end{array}$ & $\begin{array}{c}2 \\
100 \%\end{array}$ & $\begin{array}{c}0 \\
0 \%\end{array}$ & $\begin{array}{c}0 \\
0 \%\end{array}$ & - & - & - & $\begin{array}{l}\text { Temporal-kausal } \\
(\mathrm{n}=0)\end{array}$ \\
\hline $\begin{array}{l}\text { Lokal(-konditional) } \\
(\mathrm{n}=0)\end{array}$ & - & - & - & $\begin{array}{c}5 \\
71,4 \% \\
\end{array}$ & $\begin{array}{c}2 \\
28,6 \% \\
\end{array}$ & $\begin{array}{c}0 \\
0 \% \\
\end{array}$ & $\begin{array}{l}\text { Lokal(-konditional) } \\
(\mathrm{n}=7)\end{array}$ \\
\hline $\begin{array}{l}\text { Pragmatisch - ohne } \\
\text { thematisierend } \\
(n=19)\end{array}$ & $\begin{array}{c}6 \\
31,6 \%\end{array}$ & $\begin{array}{c}6 \\
31,6 \%\end{array}$ & $\begin{array}{c}7 \\
36,8 \%\end{array}$ & $\begin{array}{c}4 \\
44,4 \%\end{array}$ & $\begin{array}{c}0 \\
0 \%\end{array}$ & $\begin{array}{c}5 \\
55,6 \%\end{array}$ & $\begin{array}{l}\text { Pragmatisch - ohne } \\
\text { thematisierend } \\
(\mathrm{n}=9)\end{array}$ \\
\hline $\begin{array}{l}\text { Thematisierend } \\
(\mathrm{n}=29)\end{array}$ & $\begin{array}{c}3 \\
10,3 \% \\
\end{array}$ & $\begin{array}{c}26 \\
89,7 \%\end{array}$ & $\begin{array}{c}0 \\
0 \%\end{array}$ & - & - & - & $\begin{array}{l}\text { Thematisierend } \\
(\mathrm{n}=0)\end{array}$ \\
\hline $\begin{array}{l}\text { Gesamt } \\
(n=240)\end{array}$ & $\begin{array}{c}102 \\
42,5 \% \\
\end{array}$ & $\begin{array}{c}129 \\
53,8 \% \\
\end{array}$ & $\begin{array}{c}9 \\
3,8 \% \\
\end{array}$ & $\begin{array}{c}73 \\
34,3 \% \\
\end{array}$ & $\begin{array}{l}132 \\
62 \% \\
\end{array}$ & $\begin{array}{c}8 \\
3,8 \% \\
\end{array}$ & $\begin{array}{l}\text { Gesamt } \\
(n=213)\end{array}$ \\
\hline
\end{tabular}

Tabelle 23: Verteilung der Stellungstypen in den Nähe- bzw. Distanztexten

Betrachtet man die letzte, zusammenfassende Zeile, so sind auf den ersten Blick keine relevanten Unterschiede hinsichtlich der Verteilung der Stellungstypen zu finden. Der Anteil des nicht-integrativen Typs ist in den beiden Teilkorpora im Grunde genommen gleich, bei den beiden anderen Stellungstypen sind die prozentualen Unterschiede unter $10 \%$. Überraschenderweise ist der Anteil des integrativen Typs im Näheteilkorpus sogar etwas höher als im Distanzteilkorpus. Dies bedeutet m.E. jedoch nicht, dass die obige Hypothese 
vorschnell verworfen werden sollte. Es wurde bereits mehrfach betont, dass die Belegzahlen für unterschiedliche Adverbialsatztypen in den beiden Teilkorpora z.T. große Unterschiede aufweisen. Im Nähekorpus ist eine größere Anzahl an Temporalsätzen zu finden. Weil Temporalsätze im Allgemeinen integrativer angebunden werden als z.B. Konditionalsätze, führt der hohe Anteil der Temporalsätze im Nähekorpus (103 von 240 Belegen) dazu, dass der Anteil des integrativen Typs hier überraschend hoch ist. Mit anderen Worten: Der vergleichsweise hohe Anteil des integrativen Typs im Nähekorpus kann darauf zurückgeführt werden, dass der temporale Typ im Nähekorpus stark überrepräsentiert ist. Dies zeigt aber m.E. keinesfalls, dass im Nähekorpus die Adverbialsätze an sich integrativer angebunden werden. Wenn man Temporalsätze und die anderen Adverbialsätze jeweils getrennt auswertet, zeigt sich sogar, dass der integrative Anschluss in beiden Fällen im Distanzkorpus häufiger vorkommt (68,0\% vs. $88,9 \%$ sowie $23,4 \%$ vs. $29,2 \%)$.

Im konditionalen Bereich zeigt sich in beiden Teilkorpora eine starke Korrelation von eingeleiteten Konditionalsätzen und dem resumptiven Typ (69,4\% bzw. 65,5\%). Es besteht keine statistisch signifikante Korrelation zwischen den Stellungstypen und Nähe- bzw. Distanzsprachlichkeit $(\mathrm{P}-\mathrm{Wert}=0,612417)$, der relativ hohe Anteil der eingeleiteten Konditionalsätze in den Distanztexten (73 vs. 36 Belege) erhöht jedoch in diesem Teilkorpus die Frequenz des resumptiven Typs in der Gesamtbewertung. Dasselbe gilt für Komparativsätze, die ebenfalls stark mit dem resumptiven Typ korrelieren. Dass nur im Distanzteilkorpus integrativ angeschlosse Komparativsätze zu finden sind, kann jedoch als relevanter Unterschied betrachtet werden, wenn auch die Belegzahlen keine eindeutigen Schlussfolgerungen erlauben.

Bei allen oben behandelten Untertypen ist der Anteil des integrativen Typs im Distanzkorpus höher: Temporalsätze (88,9\% vs. 68\%), eingeleitete Konditionalsätze (31,5\% vs. $30,6 \%)$, Komparativsätze $(11,1 \%$ vs. $0 \%)$. Angesichts ihrer Korrelation mit den Integrationstypen (temporal: ,integrativ ${ }^{\star}$ vs. konditional und komparativ: ,resumptiv') und ihrer Frequenz in den beiden Teilkorpora kann eine undifferenzierte Analyse des Integrationsgrades nicht hinreichen. Es soll auch erwähnt werden, dass die obige Überblickstabelle Belege enthält, bei denen keine Wahl zwischen den Stellungstypen besteht (Proportionalsätze sowie pragmatische Adverbialsätze vor Nicht-Aussagesätzen). Wertet man nur die restlichen Adverbialsätze (also alle außer den Typen ,temporal', ,konditional', ,vergleichend‘ sowie ,pragmatisch“ vor Nicht-Aussagesätzen) aus, dann erweist sich die Korrelation zwischen den Stellungstypen und Nähe-Distanz als statistisch signifikant (P-Wert $=0,010497)$. 
Im Folgenden soll auf einige Untertypen ausführlicher eingegangen werden. Bei Temporalsätzen ist der Anteil des integrativen Typs in den Distanztexten deutlich höher als in den Nähetexten. Das Ergebnis, dass die Verteilung der Stellungstypen in Nähe- und Distanztexten unterschiedlich ist, ist - v.a. wegen der niedrigen Belegzahl in den Distanztexten - auf der Grenze der statistischen Signifikanz (P-Wert = 0,070809). Die Daten sind jedoch mit vergleichbaren Untersuchungsergebnissen (s. Wiktorowitz 2013: 394f., AxelTober 2012: 349) zu stützen, die eine Dominanz des integrativen Typs im frühen Neuhochdeutschen zeigen. Angesichts des hohen Anteils des resumptiven Typs in den Nähetexten vom 17. bis zum 19. Jh. (insg. 39,3\%) scheinen im temporalen Bereich relevante Nähe-Distanz-Unterschiede vorzuliegen, die durch weitere Analysen von Distanztexten aus diesem Zeitraum bestätigt werden könnten. Es soll angemerkt werden, dass im temporalen Bereich die wesentlichen Veränderungen auf die Nähetexte beschränkt sind: Die in den Distanztexten bereits im 17. Jh. zu beobachtende Dominanz des integrativen Typs wird im Laufe der neuhochdeutschen Periode auch im Nähebereich erreicht. Dies steht im Einklang mit Ágels Untersuchungsergebnissen zum Junktionsprofil von Distanz- und Nähetexten, die wie folgt erklärt werden (2012: 202): „Infolge der medialen und strukturellen Umschichtung nähern sich die tendenziell aggregativen Nähetexte, wenn auch zeitversetzt, den tendenziell integrativen Distanztexten strukturell an.“ Die obigen Ergebnisse liefern unabhängige Evidenz für diese Schlussfolgerung.

Auch die Ergebnisse im kausalen Bereich sprechen für die obige Hypothese. In den Distanztexten ist der Anteil des integrativen Typs mehr als doppelt so hoch wie im Nähekorpus (34,3\% vs. 16,1\%). Die Belegzahl ist jedoch in beiden Teilkorpora gering (35 bzw. 31 Belege), die Korrelation zwischen Stellungstypen und Nähe-Distanz ist daher statistisch nicht signifikant $(\mathrm{P}-$ Wert $=0,107104)$. Im kausalen Bereich sind weitere Untersuchungen zum Integrationsverhalten von Adverbialsätzen in Nähe- und Distanztexten nötig.

Die obige Hypothese unterstützen auch der vergleichsweise hohe Anteil des integrativen Typs bzw. das Fehlen der resumptiven Anbindung bei pragmatischen Adverbialsätzen im Distanzteilkorpus. Dabei soll der Anteil des nicht-integrativen Typs ausführlicher diskutiert werden. Im Distanzteilkorpus war die nicht-integrative Stellung propositionsbezogener Adverbialsätze nicht belegt, dafür ist sie jedoch bei Irrelevanzkonditionalen und bei pragmatischen Adverbialsätzen häufiger anzutreffen als im Näheteilkorpus. Dies zeigt eine ausgeprägte Funktionalisierung der nicht-integrativen bzw. integrativen Stellungen als ikonisches Zeichen der semantischen (Des-)Integration im Distanzbereich (vgl. z.B. Küper 
1993: 43), die jedoch - wie die integrativen Belege zeigen - noch keineswegs abgeschlossen ist. Im Näheteilkorpus sind auch nicht-integrativ angeschlossene propositionsbezogene Adverbialsätze zu finden (wenn auch mit einer geringen Frequenz), dieser Stellungstyp tritt bei Irrelevanzkonditionalen gar nicht bzw. bei pragmatischen Adverbialsätzen mit einem relativ niedrigen Anteil auf. Diese Ergebnisse zeigen m.E., dass der Grad der Funktionalisierung der Wortstellungsopposition in den beiden Teilkorpora unterschiedlich ist. Soweit die wichtigsten Ergebnisse, die für die obige Hypothese sprechen.

Als Gegenargument sind nur Frequenzunterschiede aufzuführen, die aufgrund der niedrigen Belegzahl unter Vorbehalt betrachtet werden müssen. So fällt bei uneingeleiteten Konditionalsätzen der vergleichsweise hohe Anteil des integrativen Typs im Näheteilkorpus auf. Es findet sich jedoch nur ein Einzelbeleg (aus 4 Belegen) mit integrativer Stellung im Korpustext aus dem 20. Jh. Aus demselben Grund erlaubt der unterschiedliche Anteil des integrativen Typs bei Irrelevanzkonditionalen keine weiteren Schlüsse. Aufgrund des Fehlens einschlägiger Belege in einem Teilkorpus können Nähe-Distanz-Unterschiede in mehreren Bereichen nicht untersucht werden (konsekutiv, spezifizierend, temporal-kausal, lokalkonditional, thematisierend). In anderen Bereichen sind keine oder nur kleinere Unterschiede $\mathrm{zu}$ beobachten (Final- und Adversativsätze bzw. eingeleitete Konditionalsätze, Konzessivsätze). Es kann festgehalten werden, dass keine gewichtigen Gegenargumente angeführt werden konnten und daher die obige Hypothese nicht verworfen werden soll. Im Gegensatz dazu wurden mehrere stichhaltige Argumente für die Richtigkeit der Hypothese vorgebracht. Zwischen konzeptionell mündlichen bzw. konzeptionell schriftlichen Texten bestehen relevante Unterschiede bezüglich der Anbindung vorangestellter Adverbialsätze. Dieses Ergebnis steht im Einklang mit den Untersuchungsergebnissen, die eine Korrelation von Nähe und Aggregation bzw. Distanz und Integration zeigen (vgl. z.B. Ágel 2012: 202, Ágel 2015: 127f., Czicza/Hennig 2013: 28, Hennig 2010a: 98).

Nicht nur die Ergebnisse zur topologischen Integration stützen die obige Hypothese. In mehreren Fällen konnte der Unterschied nachgewiesen werden, dass die semantischen Beziehungen zwischen den Teilsätzen in den Distanztexten eindeutiger markiert sind als in den Nähetexten. Zudem ist auch ein wichtiger Unterschied bezüglich der Vorfeldfähigkeit vorangestellter Adverbialsätze und der mit ihnen (koordinierend, subordinierend oder parenthetisch) verknüpften weiteren Untersätze zu beobachten: In Distanztexten treten komplexere Voranstellungen öfter mit dem integrativen Typ vor, in solchen Fällen wird dagegen in den Nähetexten häufig der resumptive Typ verwendet. Dieses Ergebnis bestätigt die Auffassung Lötschers (2010: 128), nach der das sprachpsychologische Kriterium des 
Verarbeitungsaufwandes mit dem Kriterium der grammatischen Kohärenz konkurriert und letzteres in den Distanztexten immer mehr zur Geltung kommt, während in Nähetexten lange das gedächtnisentlastende resumptive Element gesetzt wird. Angesichts der niedrigen Frequenz komplexer Voranstellungen in den Nähetexten ist jedoch dieses Ergebnis durch weitere Analysen zu untermauern. 


\section{Zusammenfassung und Ausblick}

Als Zielstellung der vorliegenden Arbeit wurde ein Beitrag zur historischen Syntax angegeben. Obwohl zum Prozess der Integration der vorangestellten Adverbialsätze in den nachfolgenden Hauptsatz bereits mehrere empirische Untersuchungen vorliegen (vgl. z.B. Baschewa 1983, König/van der Auwera 1988, Axel 2002, Lötscher 2005), konnten vom Stand der Forschung mehrere Desiderate abgeleitet werden. Es wurde für die Notwendigkeit einer systematischen Untersuchung des Integrationsgrades vorangestellter Adverbialsätze aus dem Zeitraum 1650-2000 argumentiert, die alle Adverbialsatztypen berücksichtigt. Demnach wurden in der vorliegenden Arbeit nicht nur ,,prototypische Gliedsätze“ (Pittner 2013: 521) erfasst, sondern auch Adverbialsätze im weiteren Sinne, bei denen die Satzgliedeigenschaft fehlt und die daher von den prototypischen, propositionsbezogenen Adverbialsätzen getrennt analyisiert wurden, z.B. modusmodifizierende, moduskommentierende, diktumskommentierende Adverbialsätze (vgl. Zifonun et al. 1997: 2242ff.) bzw. als ,thematisierende Satzadverbialsätze` bezeichnete was-X-betrifft/angeht-Konstruktionen.

$\mathrm{Da}$ in der Literatur kontroverse Auffassungen zur Erklärung des Integrationsprozesses vertreten werden, wurde eine Analyse durchgeführt, die sich neben historischen Veränderungen auf außersprachliche Faktoren, genauer auf das Spannungsfeld zwischen konzeptioneller Mündlichkeit und konzeptioneller Schriftlichkeit (im Sinne von Koch/Oesterreicher 1985 und Ágel/Hennig 2006) fokussierte. Somit konnte überprüft werden, ob die neu erhobenen empirischen Daten rein syntaktische oder soziopragmatische Erklärungsansätze untermauern. Im Folgenden wird der wissenschaftliche Ertrag der Arbeit zusammengefasst.

Die früheren Untersuchungen zur Integration vorangestellter Adverbialsätze zeigten eine Tendenz zur Verbreitung integrativerer Techniken (vgl. z.B. König/van der Auwera 1988, Axel 2002, Baschewa 1983). Dies wurde auch durch die vorliegende Untersuchung zum Neuhochdeutschen bestätigt. Für die Annahme eines Grammatikalisierungspfades mit drei Stufen (nicht-integrativ $>$ resumptiv $>$ integrativ) im Sinne von König/van der Auwera (1988: 127) fehlt es jedoch sowohl an empirischer Evidenz als auch an einem angemessenen theoretischen Rahmen, der mit verschiedenen Stellungsvarianten vor der Entwicklung des Vorfelds im Deutschen umgehen kann. Die Möglichkeit der topologischen Integration 
vorangestellter Adverbialsätze (,integrativer Typ`) setzt nämlich eine dem Gegenwartsdeutschen ähnliche Felderstruktur voraus, insbesondere ein Vorfeld, „das von genau einem, beliebigen vollen Satzglied besetzt werden kann“ (Lötscher 2005: 355). Diese Voraussetzung war im Frühalthochdeutschen noch keineswegs erfüllt (vgl. Lötscher 2005: 355, Ágel 2015). Mit der Entwicklung der Felderstruktur und der Vorfeldfähigkeit vorangestellter Adverbialsätze (Axel 2002) entsteht eine neue Möglichkeit, die enge semantische und pragmatische Relation zwischen den verknüpften Sätzen formal zu markieren. Als theoretischer Rahmen zur Erfassung der verschiedenen Satzverknüpfungstechniken wurde ein Junktionsmodell mit einem Kontinuum zwischen ,Aggregation“ und ,Integration“ vorgeschlagen (vgl. Raible 1992 und Ágel 2010). Ferner wurden die Subordination in Anlehnung an Fabricius-Hansen (1992) und Zifonun et al. (1997: 2250-2253) als vieldimensionales Verfahren dargestellt und die Zusammenhänge zwischen den formalen und funktionalen Aspekten der Subordination diskutiert (Kapitel 3.3.2). Es stellte sich heraus, dass sich das Modell von König/van der Auwera (1988) im Bereich der Aussagesätze als hinreichende Grundlage für die Diskussion des Integrationsverhaltens von vorangestellten Adverbialsätzen im Neuhochdeutschen erwiesen hat. Das Dreiermodell von König/van der Auwera (1988: 102f.) kann jedoch nicht auf komplexe Sätze angewandt werden, in denen der Adverbialsatz einem Imperativ- oder Fragesatz vorangestellt wird. Auch in diesen Fällen sind jedoch mehr oder weniger aggregative Verknüpfungstechniken zu unterscheiden.

Wenn der untergeordnete Satz als Satzglied in den übergeordneten Satz eingebettet ist, entsteht eine kohärente syntaktische Einheit. Dadurch wird eine monozentrische Organisationsweise ermöglicht (Ágel 2007: 53). Die einschlägigen syntaktischen Veränderungen sind jedoch nicht von außersprachlichen Faktoren zu trennen. Dabei scheint es angebracht, auf die Konzepte der Verschriftlichung (Koch/Oesterreicher 1994: 587 sowie Hennig 2009: 9) und der Vertikalisierung (Reichmann 1988, 2003) zu verweisen, die soziopragmatisch bedingte Veränderungen des Integrationsgrades nahelegen. Die beiden Stellungsvarianten ,resumptiv ${ }^{‘}$ und ,integrativ ${ }^{‘}$ folgen zeitlich nicht einfach aufeinander, sondern existieren zu Beginn des Neuchochdeutschen im Sinne einer vertikalen Umschichtung nebeneinander bzw. über-/untereinander.

Die vorliegende Untersuchung liefert wertvolle Ergebnisse zu den Satztypen, die bislang kaum Beachtung fanden (z.B. Vergleichssätze, bestimmte Untertypen pragmatischer Adverbialsätze), und zum Bereich der konzeptionellen Mündlichkeit, der in den einschlägigen Untersuchungen bisher weitgehend vernachlässigt wurde. Die Analyseergebnisse zeigen 
relevante Unterschiede zwischen Nähe- und Distanztexten. Letztere scheinen eine Vorreiterrolle bei der Verbreitung integrativerer Techniken zu spielen. Der resumptive Typ wird bei mehreren Adverbialsatztypen auch in Nähetexten zugunsten des integrativen Stellungstyps tendenziell (und zeitversetzt) abgebaut. Überraschend ist die Zeit des Durchbruchs des integrativen Typs. In den Korpustexten aus dem 20. Jh. überwiegt diese Variante mit insgesamt 72\%, nur in Konditionalsätzen und Vergleichssätzen konkurriert sie mit dem resumptiven Typ. Die Korpustexte vom 17. bis zum 19. Jh. zeigen jedoch einen bedeutenden Anteil des resumptiven Typs (im 18. Jh. sogar 86,3\%). Diese Ergebnisse legen nahe, dass im Bereich der Integration vorangestellter Adverbialsätze auch in neuhochdeutscher Zeit wesentliche Veränderungen stattfinden und zur syntaktischen Variation bei ihrer Anbindung an den Obersatz weitere Analysen lohnenswert wären.

Die vorliegende Arbeit konnte nicht alle möglichen Aspekte der Integration systematisch behandeln, die Forschungsergebnisse zeigen jedoch, in welchen Bereichen weitere Forschungen nötig sind. Aufgrund der niedrigen Frequenz komplexer Voranstellungen in den Nähetexten ist der eventuelle Einfluss des Komplexitätsprinzips auf die Wahl der Stellungstypen durch weitere Analysen zu untermauern. Zur Untersuchung des Durchbruchs des integrativen Typs bei mehreren Adverbialsatztypen wären Analysen zum jüngeren Neuhochdeutsch auf der Basis eines größeren Korpus wünschenswert. Zudem könnten gezielte Analysen zu weniger frequenten Satztypen und v.a. zu nicht-propositionsbezogenen Adverbialsätzen die hier ermittelten Ergebnisse ergänzen, präzisieren oder relativieren. Für die vorliegende Untersuchung konnte kein repräsentatives Korpus des Neuhochdeutschen herangezogen werden. Die Ergebnisse können jedoch aufgrund der weitgehenden Beachtung außersprachlicher Faktoren in Zukunft durch die Analyse weiterer Korpustexte (z.B. aus den hier nicht behandelten Dialektgebieten) ergänzt werden. Die Unterschiede zu den hier vorgelegten Forschungsergebnissen könnten die Aufmerksamkeit auf Aspekte lenken, die uns $\mathrm{zu}$ einem besseren Verständnis der syntaktischen Variation bei der Integration vorangestellter Adverbialsätze verhelfen können. 


\section{Literaturverzeichnis}

\subsection{Primärliteratur}

Korpustexte:

Aichinger III = Aichinger, Carl Friedrich (1754/1972): Versuch einer teutschen Sprachlehre, anfănglich nur zu eignem Gebrauche unternommen, endlich aber, um den Gelehrten zu fernerer Untersuchung Anlaß zu geben, ans Liecht gestellt von Carl Friedrich Aichinger, d. Z. Stadtprediger zu Sulzbach. Hildesheim/New York: Olms [Reprographischer Nachdruck der Ausgabe Frankfurt/Leipzig 1754]. (= Documenta Linguistica, Reihe V).

Auswandererbriefe V = Briefkorpus zur Monographie von Stephan Elspaß (2005): Sprachgeschichte von unten. Untersuchungen zum geschriebenen Alltagsdeutsch im 19. Jahrhundert. Tübingen: Niemeyer (Reihe Germanistische Linguistik 263).

Burckhardt V = Burckhardt, Jacob (1860/1988): Die Kultur der Renaissance in Italien. Ein Versuch. 11. Aufl. Stuttgart: Kröner 1988 (Kröners Taschenbuchausgabe Bd. 53).

Güntzer I = Güntzer, Augustin (1657/2002): Kleines Biechlin von meinem gantzen Leben. Die Autobiographie eines Elsässer Kannengießers aus dem 17. Jahrhundert. Hg. v. Sebastian Brändle. Köln/Weimar: Böhlau (Selbstzeugnisse der Neuzeit 8).

Harsdörffer I = Harsdörffer, Georg Philipp (1656/1971): Der Teutsche Secretarius. Das ist: Allen Cantzleyen/ Studir- und Schreibstuben nutzliches/fast nohtwendiges und zum drittenmal vermehrtes Titular- und Formularbuch I. Hildesheim/New York: Olms. [Reprografischer Nachdruck der Ausgabe Nürnberg 1656]

Jugendtagebuch VII = Tagebuch Nr. 4 von Lilly A. Deutsches Tagebucharchiv Emmendingen, Signatur-Nr. 936 / I, 4.

Lorenz VII = Lorenz, Konrad (1973/1977): Die Rückseite des Spiegels: Versuch einer Naturgeschichte menschlichen Erkennens. München: Deutscher Taschenbuch Verlag.

Schuhmacher Chronik II = Die Chronik von Johann Georg Launer. Stadtarchiv Reutlingen, Handschriftenabteilung Hs 51. Transkription Stadtarchiv Reutlingen (unveröffentlicht).

Weitere Quellentexte ${ }^{230}$

Daniel Domian VII = Günthner, Susanne: Radio-phone-in (Domian-Daniel) 2002. Abgedruckt in: Hennig, Mathilde (2006): Grammatik der gesprochenen Sprache in Theorie und Praxis. Kassel: University Press, 298-304.

\footnotetext{
230 An dieser Stelle werden Quellentexte aufgeführt, die keinen Bestandteil des Korpus bildeten, aus denen jedoch einzelne Belege besprochen wurden. Zusätzlich wurden einzelne Belege der Sekundärliteratur entnommen, zu den bibliographischen Daten dieser Quellen vgl. die Angaben in den jeweiligen Arbeiten.
} 
Wagner III = Wagner, Heinrich Leopold (1776): Die Kindermörderinn. Ein Trauerspiel. Leipzig: Schwickertsche Verlag.

\section{Digitale Korpora}

Das Bonner Frühneuhochdeutsch-Korpus. http://www.korpora.org/Fnhd/ [zuletzt abgerufen am 30.08.2015]

DeReKo = Institut für Deutsche Sprache (2004-2016): Deutsches Referenzkorpus - DeReKo. Archiv der Korpora geschriebener Gegenwartssprache. (Releases von 2004-I bis 2016-I). Mannheim: Institut für Deutsche Sprache. http://www.idsmannheim.de/kl/projekte/korpora/ [zuletzt abgerufen am 13.06.2016]

\subsection{Sekundärliteratur}

Admoni, Wladimir G. (1980): Zur Ausbildung der Norm der deutschen Literatursprache im Bereich des neuhochdeutschen Satzgefüges (1470-1730). Ein Beitrag zur Geschichte des Gestaltungssystems der deutschen Sprache. Berlin: Akademie (Bausteine zur Sprachgeschichte des Neuhochdeutschen 56/IV).

Admoni, Wladimir (1990): Historische Syntax des Deutschen. Tübingen: Niemeyer.

Ágel, Vilmos (2000): Syntax des Neuhochdeutschen bis zur Mitte des 20. Jahrhunderts. In: Besch, Werner/Betten, Anne/Reichmann, Oskar/Sonderegger, Stefan (Hrsg.): Sprachgeschichte. Ein Handbuch zur Geschichte der deutschen Sprache und ihrer Erforschung. Bd. 2. 2. Aufl. Berlin/New York: de Gruyter (HSK 2.2), 1855-1903.

Ágel, Vilmos (2003): Prinzipien der Grammatik. In: Lobenstein-Reichmann, Anja/Reichmann, Oskar (Hrsg.): Neue historische Grammatiken. Zum Stand der Grammatikschreibung historischer Sprachstufen des Deutschen und anderer Sprachen. Tübingen: Niemeyer, 1-46.

Ágel, Vilmos (2007): Was ist „grammatische Aufklärung“ in einer Schriftkultur? Die Parameter ,Aggregation' und ,Integration'. In: Feilke, Helmuth/Knobloch, Clemens/Völnitz, Paul-Ludwig (Hrsg.): Was heißt linguistische Aufklärung? Sprachauffassungen zwischen Systemvertrauen und Benutzerfürsorge. Heidelberg: Synchron, 39-57.

Ágel, Vilmos (2010): Explizite Junktion. Theorie und Operationalisierung. In: Ziegler, Arne/Braun, Christian (Hrsg.): Historische Textgrammatik und Historische Syntax des Deutschen. Traditionen, Innovationen, Perspektiven. Bd. 2: Frühneuhochdeutsch, Neuhochdeutsch. Berlin: de Gruyter, 897-936.

Ágel, Vilmos (2012): Junktionsprofile aus Nähe und Distanz. Ein Beitrag zur Vertikalisierung der neuhochdeutschen Grammatik. In: Bär, Jochen A./Müller, Marcus (Hrsg.): Geschichte der Sprache - Sprache der Geschichte. Probleme und Perspektiven der historischen Sprachwissenschaft des Deutschen. Oskar Reichmann zum 75. Geburtstag. Berlin: Akademie (Lingua Historica Germanica 3), 181-206.

Ágel, Vilmos (2015): Die Umparametrisierung der Grammatik durch Literalisierung. Onlineund Offlinesyntax in Gegenwart und Geschichte. In: Eichinger, Ludwig M. (Hrsg.): 
Sprachwissenschaft im Fokus. Positionsbestimmungen und Perspektiven. Berlin/Boston: de Gruyter (Jahrbuch des Instituts für Deutsche Sprache, 2014), 121-155.

Ágel, Vilmos (2016): Obwohl (.) fährt der eigentlich auch am Sonntag? Der VerbzweitMythos. In: Handwerker, Brigitte/ Bäuerle, Rainer/ Sieberg, Bernd (Hrsg.): Gesprochene Fremdsprache Deutsch. Baltmannsweiler: Schneider Verlag Hohengehren, 75-100.

Ágel, Vilmos (o.J.): Projektskizze Neuhochdeutsche Grammatik. Abrufbar unter: http://www.uni-kassel.de/ agel/ProjektNhdGramm.pdf (zuletzt gesehen am 30.07.2015).

Ágel, Vilmos/Diegelmann, Carmen (2010): Theorie und Praxis der expliziten Junktion. In: Ágel, Vilmos/Hennig, Mathilde (Hrsg.): Nähe und Distanz im Kontext variationslinguistischer Forschung. Berlin: de Gruyter (Linguistik - Impulse \& Tendenzen; 35), 347-396.

Ágel, Vilmos/Hennig, Mathilde (Hrsg.) (2006): Grammatik aus Nähe und Distanz. Theorie und Praxis am Beispiel von Nähetexten 1650-2000. Tübingen: Niemeyer.

Ágel, Vilmos/Hennig, Mathilde (2006a): Theorie des Nähe- und Distanzsprechens. In: Ágel, Vilmos/Hennig, Mathilde (Hrsg.): Grammatik aus Nähe und Distanz. Theorie und Praxis am Beispiel von Nähetexten 1650-2000. Tübingen: Niemeyer, 3-31.

Ágel, Vilmos/Hennig, Mathilde (2006b): Praxis des Nähe- und Distanzsprechens. In: Ágel, Vilmos/Hennig, Mathilde (Hrsg.): Grammatik aus Nähe und Distanz. Theorie und Praxis am Beispiel von Nähetexten 1650-2000. Tübingen: Niemeyer, 33-74.

Ágel, Vilmos/Hennig, Mathilde (2006c): Modelglossar. In: Ágel, Vilmos/Hennig, Mathilde (Hrsg.): Grammatik aus Nähe und Distanz. Theorie und Praxis am Beispiel von Nähetexten 1650-2000. Tübingen: Niemeyer, 387-396.

Ágel, Vilmos/Hennig, Mathilde (2007): DFG-Projekt „Explizite und elliptische Junktion in der Syntax des Neuhochdeutschen“. In: Zeitschrift für germanistische Linguistik 35, 185189.

Ágel, Vilmos/Hennig, Mathilde (2010): Einleitung. In: Ágel, Vilmos/Hennig, Mathilde (Hrsg.): Nähe und Distanz im Kontext variationslinguistischer Forschung. Berlin: de Gruyter (Linguistik - Impulse \& Tendenzen; 35), 1-22.

Altmann, Hans (1981): Formen der „Herausstellung“ im Deutschen. Rechtsversetzung, Linksversetzung, Freies Thema und verwandte Konstruktionen. Tübingen: Niemeyer (Linguistische Arbeiten 106).

Altmann, Hans/Hofmann, Ute (2004): Topologie fürs Examen. Verbstellung, Klammerstruktur, Stellungsfelder, Satzglied- und Wortstellung. Wiesbaden: VS Verlag für Sozialwissenschaften (Linguistik fürs Examen; 4).

Altmann, Hans (2009): Cleft- und Pseudocleft-Sätze (Spalt- und Sperrsätze) im Deutschen. In: Brdar-Szabó, Rita/Knipf-Komlósi, Elisabeth/Péteri, Attila (Hrsg.): An der Grenze zwischen Grammatik und Pragmatik. Frankfurt am Main u. a.: Peter Lang (Deutsche Sprachwissenschaft international, 3), 13-34.

Arndt, Erwin (1956): Die begründenden Sätze im Neuhochdeutschen und ihre wichtigsten Konjunktionen. Phil. Diss. Berlin (DDR).

Auer, Peter (1997): Formen und Funktionen der Vor-Vorfeldbesetzung im gesprochenen Deutsch. In: Schlobinski, Peter (Hrsg.): Syntax des gesprochenen Deutsch. Opladen: Westdeutscher Verlag, 55-91. 
Auer, Peter (1998): Zwischen Parataxe und Hypotaxe: ,abhängige Hauptsätze ‘ im Gesprochenen und Geschriebenen Deutsch. In: Zeitschrift für germanistische Linguistik 26/3, 284-307.

Auer, Peter (2000): Pre- and post-positioning of wenn-clauses in spoken and written German. In: Couper-Kuhlen, Elizabeth/Kortmann, Bernd (eds.): Clause Condition Concession Contrast. Berlin: de Gruyter, 173-204.

Auer, Peter (2002): Die Verdichtung der konditionalen Hypotaxe im gesprochenen Deutsch. In: Orosz, Magdolna/Herzog, Andreas (Hrsg.): Jahrbuch der ungarischen Germanistik. Budapest: DAAD, 189-204.

Axel, Katrin (2002): Zur diachronen Entwicklung der syntaktischen Integration linksperipherer Adverbialsätze im Deutschen: Ein Beispiel für syntaktischen Wandel? In: Beiträge zur Geschichte der deutschen Sprache und Literatur 124, 1-43.

Axel, Katrin (2004): The syntactic integration of preposed adverbial clauses on the German left periphery: A diachronic perspective. In: Lohnstein, Horst/Trissler, Susanne (Hrsg.): The Syntax and Semantics of the Left Periphery. Berlin, New York: de Gruyter, 23-58.

Axel, Katrin (2007): Studies on Old High German Syntax. Left Sentence Periphery, Verb Placement and Verb Second. Amsterdam/Philadelphia: John Benjamins (Linguistik Aktuell 112).

Axel, Katrin (2009): Die Entstehung des dass-Satzes - ein neues Szenario. In: Ehrich, Veronika et al. (Hrsg.): Koordination und Subordination im Deutschen. Hamburg: Buske (Linguistische Berichte Sonderheft 16.), 21-41.

Axel-Tober, Katrin (2012): (Nicht-)kanonische Nebensätze im Deutschen. Synchrone und diachrone Aspekte. Berlin/Boston: de Gruyter (Linguistische Arbeiten 542).

Axel, Katrin/Wöllstein, Angelika (2009): German verb-first conditionals as unintegrated clauses. A case study in converging synchronic and diachronic evidence. In: Winkler, Susanne/Featherston, Sam (eds.): The Fruits of Empirical Linguistics. Band II. Berlin/New York: Mouton de Gruyter, 1-36.

Babenko, Natalja Sergejevna (1988): Einige Entwicklungstendenzen im Bereich des Satzgefüges in der deutschen Sprache des 16. und 17. Jahrhunderts (am Material finaler Unterordnung). In: Beiträge zur Erforschung der deutschen Sprache 8, 95-129.

Bartsch, Renate (1972): Adverbialsemantik. Frankfurt/M.: Athenäum.

Baschewa, Emilia (1983): Untersuchungen zur Diachronie des Konzessivsatzes im Neuhochdeutschen. In: Beiträge zur Erforschung der deutschen Sprache 3, 77-107.

Behaghel, Otto (1903): Herstellung der syntaktischen Ruhelage im Deutschen. In: Indogermanische Forschungen 14, 438-459.

Behaghel, Otto (1923, 1924, 1928, 1932): Deutsche Syntax. Eine geschichtliche Darstellung. 4 Bde. Heidelberg: Winter (Sammlung germanischer Elementar- und Handbücher, Reihe Grammatiken 10).

Behaghel, Otto (1929): Der Nachsatz. In: Beiträge zur Geschichte der deutschen Sprache 53, 401-418.

Bergerová, Hana (1998): Zu Problemen der Nebensatzbeschreibung am Beispiel der Vergleichssätze. In: Deutsch als Fremdsprache. Zeitschrift zur Theorie und Praxis des Faches Deutsch als Fremdsprache, 35/3, 148-153. 
Betten, Anne (1987): Grundzüge der Prosasyntax. Stilprägende Entwicklungen vom Althochdeutschen zum Neuhochdeutschen. Tübingen: Niemeyer (Reihe Germanistische Linguistik; 82).

Betten, Anne (2000): Zum Verhältnis von geschriebener und gesprochener Sprache im Frühneuhochdeutschen. Besch, Werner/Betten, Anne/Reichmann, Oskar/Sonderegger, Stefan (Hrsg.): Sprachgeschichte. Ein Handbuch zur Geschichte der deutschen Sprache und ihrer Erforschung. Bd. 2. 2. Aufl. Berlin/New York: de Gruyter (HSK 2.2), 16461664.

Biener, Clemens (1920/22): Wie ist die nhd. Regel über die Stellung des Verbums entstanden? In: Zeitschrift für deutsches Altertum 59, 165-179.

Birkner, Karin (2008): Was X betrifft: Textsortenspezifische Aspekte einer Redewendung. In: In: Stefanowitsch, Anatol/Fischer, Kerstin (Hrsg.): Konstruktionsgrammatik II. Von der Konstruktion zur Grammatik. Tübingen: Stauffenburg (Stauffenburg Linguistik), 59-80.

Blatz, Friedrich (1880): Neuhochdeutsche Grammatik mit Berücksichtigung der historischen Entwickelung der deutschen Sprache. 2., theilw. verb. und verm. Aufl.; Tauberbischofsheim: Lang. (zugänglich über http://reader.digitalesammlungen.de/de/fs1/object/display/bsb11023177_00799.html, zuletzt gesehen am 21 . 08. 2015)

Blatz, Friedrich (1896): Neuhochdeutsche Grammatik mit Berücksichtigung der historischen Entwickelung der Deutschen Sprache. Dritte, völlig neu bearbeitete Auflage. Zweiter Band. Satzlehre (Syntax). Karlsruhe: Lang (Nachdruck aus dem Jahre 1900).

Blühdorn, Hardarik (2004): Temporalkonnektoren. Einleitung. In: Blühdorn, Hardarik/Breindl, Eva/Waßner, Ulrich Hermann (Hrsg.): Brücken schlagen. Grundlagen der Konnektorensemantik. Berlin/New York: de Gruyter (Linguistik: Impulse \& Tendenzen 5), 125-136.

Blühdorn, Hardarik (2006): Kausale Satzverknüpfungen im Deutschen. In: Pandaemonium Germanicum. Revista de Estudos Germanísticos 10, 253-282.

Blühdorn, Hardarik (2012a): Zur Syntax adverbialer Satzverknüpfungen: Deutsch Italienisch - Portugiesisch. In: Gunkel, Lutz / Gisela Zifonun (Hrsg.): Deutsch im Sprachvergleich. Grammatische Kontraste und Konvergenzen. Berlin: de Gruyter, 301332.

Blühdorn, Hardarik (2012b): Verknüpfungseigenschaften von Satzkonnektoren im Deutschen. Am Beispiel der Kausal- und Konsekutivkonnektoren. In: Deutsche Sprache 40, 193-220.

Blühdorn, Hardarik (2013): Syntaktische Nebensatzklassen im Deutschen. In: Pandaemonium Germanicum. Revista de Estudos Germanísticos 21, 149-189.

Boettcher, Wolfgang (2009): Grammatik verstehen. II - Einfacher Satz. Tübingen: Niemeyer (Niemeyer Studienbuch).

Boszák, Gizella (2009): Realisierung der valenzbestimmten Korrelate des Deutschen. Frankfurt am Main: Peter Lang.

Brandt, Margareta (1989): Zur Fokus-Hintergrund-Gliederung in komplexen Sätzen. In: Sprache und Pragmatik 13, 43-53.

Brandt, Margareta (1990): Weiterführende Nebensätze. Stockholm: Almqvist \& Wiksell International. (Lunder germanistische Forschungen 57). 
Breindl, Eva/Waßner, Ulrich H. (2006): Syndese vs. Asyndese. Konnektoren und andere Wegweiser für die Interpretation semantischer Relationen in Texten. In: Blühdorn, Hardarik/Breindl, Eva/Waßner, Ulrich H. (Hrsg.): Text - Verstehen. Grammatik und darüber hinaus. (Jahrbuch des Instituts für Deutsche Sprache, 2005). Berlin/New York: de Gruyter, 46-70.

Breindl, Eva et al. (2014): Handbuch der deutschen Konnektoren. Band 2: Semantik. Berlin/New York: de Gruyter (Schriften des Instituts für Deutsche Sprache 13.1-13.2).

Breul, Carsten (1998): On Adverbial Clauses and Their Status within Concepts of Hypotaxis, Subordination and Clause Embedding. In: Studia Neophilologica 70(2), 129-137.

Brooks, Thomas (2006): Untersuchungen zur Syntax in oberdeutschen Drucken des 16.-18. Jahrhunderts. Frankfurt am Main u. a.: Peter Lang (Schriften zur deutschen Sprache in Österreich 36).

Bußmann, Hadumod (2008): Lexikon der Sprachwissenschaft. 4., durchges. und bibliogr. erg. Auflage. Stuttgart: Kröner.

Carlsen, Laila (1998): Redewiedergabe mit redeeinleitendem wie-Satz. In: Deutsche Sprache 26(1), 63-89.

Cortès, Colette (2003): Subjunktoren und Korrelate im Deutschen. In: Baudot, Daniel/Behr, Irmtraud (Hgg.): Funktion und Bedeutung. Modelle einer syntaktischen Semantik des Deutschen. Festschrift für François Schanen. Tübingen: Stauffenburg, 141-160.

Czicza, Dániel (2014): Das es-Gesamtsystem im Neuhochdeutschen. Ein Beitrag zu Valenztheorie und Konstruktionsgrammatik. Berlin/Boston: de Gruyter (Studia Linguistica Germanica 120).

Czicza, Dániel et al. (2012): Zur Verortung von Texten zwischen den Polen maximaler und minimaler Wissenschaftlichkeit. Ein Operationalisierungsvorschlag. In: Fachsprache 34(12), 2-44.

Czicza, Dániel/Hennig, Mathilde (2013): Aggregation, Integration und Sprachwandel. In: Vogel, Petra M. (Hrsg.): Sprachwandel im Neuhochdeutschen. Berlin/Boston: de Gruyter (Jahrbuch für Germanistische Sprachgeschichte 4), 1-33.

Dal, Ingerid/Eroms, Hans-Werner (2014): Kurze deutsche Syntax auf historischer Grundlage. 4. Auflage. Neu bearbeitet von Hans-Werner Eroms. Berlin/Boston: de Gruyter.

Dittmer, Arne/Dittmer, Ernst (1998): Studien zur Wortstellung - Satzgliedstellung in der althochdeutschen Tatianübersetzung. Für den Druck bearbeitet von Michael Flöer und Juliane Klempt. Göttingen: Vandenhoeck \& Ruprecht (Studien zum Althochdeutschen 31).

Demske, Ulrike (2009): Zur Markierung von Konsekutivität im Deutschen: Diachrone Aspekte. In: Ehrich, Veronika et al. (Hgg.): Koordination und Subordination im Deutschen. Sonderheft der Linguistischen Berichte 16, 43-66.

Demske, Ulrike (2011): Subordinationsmarker im Deutschen. Zur Geschichte hypothetischer Vergleichssätze. In: Simmler, Franz/Wich-Reif, Claudia (Hrsg.): Geschichte der Gesamtsatzstrukturen vom Althochdeutschen bis zum Frühneuhochdeutschen. Bern: Peter Lang (= Jahrbuch für Internationale Germanistik 104), 9-31.

Di Meola, Claudio (1997): Der Ausdruck der Konzessivität in der deutschen Gegenwartssprache: Theorie und Beschreibung anhand eines Vergleichs mit dem Italienischen. Tübingen: Niemeyer (Linguistische Arbeiten; 372). 
Donaldson, Bryan (2012): Initial subordinate clauses in Old French: Syntactic variation and the clausal left periphery. In: Lingua 122: 1021-1046.

Dorchenas, Ingeborg (2005): Etymologie und Syntax der Konjunktion ,dass ' in der deutschen Grammatik von ihren Anfängen bis 1800 vor dem Hintergrund antiker und moderner daßForschung. Berlin: Rhombos.

Duden-Grammatik (2016) = Wöllstein, Angelika/Dudenredaktion (Hrsg.) (2016): Duden. Die Grammatik. Unentbehrlich für richtiges Deutsch. 9., vollständig überarbeitete und aktualisierte Auflage. Berlin: Dudenverlag (Der Duden in 12 Bänden, Band 4).

Dürscheid, Christa (2012): Syntax. Grundlagen und Theorien. 6., aktualisierte Aufl. Göttingen: Vandenhoeck \& Ruprecht.

DWb $=$ Grimm, Jakob/Grimm, Wilhelm (1854-1961): Deutsches Wörterbuch. 16 Bände in 32 Teilbänden. Leipzig: S. Hirzel. (im Internet unter http://dwb.bbaw.de/)

Ebert, Robert Peter (1998): Verbstellungswandel bei Jugendlichen, Frauen und Männern im 16. Jahrhundert. Tübingen: Niemeyer (Reihe Germanistische Linguistik)

Eggs, Frederike (2006): Die Grammatik von als und wie. Tübingen: Gunter Narr (Tübinger Beiträge zur Linguistik 496).

Eichinger, Ludwig M. (1995): Syntaktischer Wandel und Verständlichkeit. Zur Serialisierung von Sätzen und Nominalgruppen im frühen Neuhochdeutschen. In: Kretzenbacher, Heinz L./Weinrich, Harald (Hrsg.): Linguistik der Wissenschaftssprache. Berlin/New York: de Gruyter (Akademie der Wissenschaften zu Berlin. Forschungsbericht 10), 303-324.

Eisenberg, Peter (2013): Grundriss der deutschen Grammatik. Band 2: Der Satz. 4., aktualisierte und überarbeitete Auflage. Stuttgart/Weimar: Metzler.

Elspaß, Stephan (2005): Sprachgeschichte von unten. Untersuchungen zum geschriebenen Alltagsdeutsch im 19. Jahrhundert. Tübingen: Niemeyer (Reihe Germanistische Linguistik 263).

Elspaß, Stephan (2007a): ,Neue Sprachgeschichte(n)`. Einführung in das Themenheft. In: Neue Sprachgeschichte(n). Themenheft der Zeitschrift Der Deutschunterricht, 59.3, 2-6.

Elspaß, Stephan (2007b): Briefe und Tagebücher als Quellen der Alltags- und Alltagssprachgeschichte. In: Neue Sprachgeschichte(n). Themenheft der Zeitschrift Der Deutschunterricht, 59.3, 42-51.

Elspaß, Stephan (2008): Vom Mittelneuhochdeutschen (bis ca. 1950) zum Gegenwartsdeutsch. In: Zeitschrift für Dialektologie und Linguistik (ZDL) 75, 1-20.

Elspaß, Stephan (2010): Zum Verhältnis von ,Nähegrammatik‘ und Regionalsprachlichkeit in historischen Texten. In: Ágel, Vilmos/Hennig, Mathilde (Hrsg.): Nähe und Distanz im Kontext variationslinguistischer Forschung. Berlin: de Gruyter (Linguistik - Impulse \& Tendenzen; 35), 65-83.

Elspaß, Stephan (2015): Grammatischer Wandel im (Mittel-) Neuhochdeutschen - von oben und von unten. Perspektiven einer Historischen Soziolinguistik des Deutschen. In: Zeitschrift für germanistische Linguistik 43/3, 387-420.

Engel, Ulrich (1988): Deutsche Grammatik. Heidelberg: Groos.

Engel, Ulrich (2004): Deutsche Grammatik. Neubearbeitung. München: Iudicium.

Erben, Johannes (2000): Syntax des Frühneuhochdeutschen. In: Besch, Werner/Betten, Anne/Reichmann, Oskar/Sonderegger, Stefan (Hrsg.): Sprachgeschichte. Ein Handbuch 
zur Geschichte der deutschen Sprache und ihrer Erforschung. 2. Aufl. Berlin/New York: de Gruyter (HSK 2.2), 1584-1593.

Eroms, Hans-Werner (2000): Syntax der deutschen Sprache. Berlin/New York: de Gruyter (De Gruyter Studienbuch).

Fabricius-Hansen, Cathrine (1992): Subordination. In: Hoffmann, Ludger (Hrsg.): Deutsche Syntax. Ansichten und Aussichten. Berlin/New York: de Gruyter (Institut für deutsche Sprache, Jb. 1991), 458-483.

Fabricius-Hansen, Cathrine (2007): Subjunktor. In: Hoffmann, Ludger (Hrsg.): Handbuch der deutschen Wortarten. Berlin/New York: de Gruyter (de Gruyter Lexikon), 759-790.

Fabricius-Hansen, Cathrine (2011): Was wird verknüpft, mit welchen Mitteln - und wozu? Zur Mehrdimensionalität der Satzverknüpfung. In: Breindl, Eva/Ferraresi, Gisella/Volodina, Anna (Hrsg.): Satzverknüpfungen. Zur Interaktion von Form, Bedeutung und Diskursfunktion. Berlin: de Gruyter, 15-40.

Faucher, Eugène (2000): Wozu Subordination gut ist. In: Lefèvre, Michel (Hrsg.): Subordination in Syntax, Semantik und Textlinguistik. Tübingen: Stauffenburg (Eurogermanistik 15), 1-12.

Feilke, Helmuth/Hennig, Mathilde (2016): Zur Karriere von ,Nähe und Distanz'. Rezeption und Diskussion des Koch-Oesterreicher-Modells. Berlin/Boston: de Gruyter (Reihe Germanistische Linguistik).

Fiehler, Reinhard (2000): Über zwei Probleme bei der Untersuchung gesprochener Sprache. In: Sprache und Literatur 31, Heft 85, 23-42.

Flämig, Walter (1964): Untersuchungen zum Finalsatz im Deutschen (Synchronie und Diachronie). Berlin: Akademie (Sitzungsberichte der deutschen Akademie der Wissenschaften zu Berlin, Klasse für Sprachen, Literatur und Kunst 1964/5).

Fleischer, Jürg (2006): Zur Methodologie althochdeutscher Syntaxforschung. In: Beiträge zur Geschichte der deutschen Sprache und Literatur 128, 25-69.

Fleischer, Jürg (2015): Perspektiven der historischen Syntax des Deutschen: Einführung. In: Zeitschrift für germanistische Linguistik 43/3, 377-386.

Fleischmann, Klaus (1973): Verbstellung und Relieftheorie. Ein Versuch zur Geschichte des deutschen Nebensatzes. München: Hueber (Münchener Germanistische Beiträge 6).

Frey, Werner (2011): Peripheral adverbial clauses, their licensing and the prefield in German. In: Breindl, Eva/Ferraresi, Gisella/Volodina, Anna (Hrsg.): Satzverknüpfungen. Zur Interaktion von Form, Bedeutung und Diskursfunktion. Berlin: de Gruyter, 41-77.

Freywald, Ulrike (2013): Uneingeleiteter V1- und V2-Satz. In: Meibauer, Jörg/Steinbach, Markus/Altmann, Hans (Hrsg.): Satztypen des Deutschen. Berlin/New York: de Gruyter, 317-337.

Gardt, Andreas/Mattheier, Klaus J./Reichmann, Oskar (Hrsg.) (1995): Sprachgeschichte des Neuhochdeutschen. Gegenstände, Methoden, Theorien. Tübingen: Niemeyer (Reihe Germanistische Linguistik; 156).

Gohl, Christine (2002a): Retrospektive Markierung von Begründungen. In: InLiSt Interaction and Linguistic Structures 30. [Abrufbar unter: http://www.inlist.unibayreuth.de/issues/30/Inlist30.pdf, zuletzt abgerufen am 11. August 2016) 
Gohl, Christine (2002b): Zwischen Kausalität und Konditionalität: Begründende wennKonstruktionen. In: Deutsche Sprache 30, 193-219.

Gohl, Christine (2006): Bedeutungskonstitution im Gespräch: Zur Interpretation und Präsentation konnektiver Einheiten. In: Ágel, Vilmos/Hennig, Mathilde (Hrsg.): Zugänge zur Grammatik der gesprochenen Sprache. Tübingen: Niemeyer (Reihe Germanistische Linguistik; 269), 35-71.

Glück, Helmut/Rödel, Michael (Hrsg.) (2016): Metzler Lexikon Sprache. 5., aktualisierte und überarbeitete Auflage. Stuttgart/Weimar: Metzler.

Günthner, Susanne (1999a): Wenn-Sätze im Vor-Vorfeld: Ihre Formen und Funktionen in der gesprochenen Sprache. In: Deutsche Sprache 3, 209-235.

Günthner, Susanne (1999b): Entwickelt sich der Konzessivkonnektor obwohl zum Diskursmarker? Grammatikalisierungstendenzen im gesprochenen Deutsch. In: Linguistische Berichte 180, 409-446.

Günthner, Susanne (2000): 'wobei (.) es hat alles immer zwei seiten'. Zur Verwendung von wobei im gesprochenen Deutsch. In: Deutsche Sprache 28/4, 313-341.

Günthner, Susanne (2002): Zum kausalen und konzessiven Gebrauch des Konnektors wo im gesprochenen Umgangsdeutsch. In: Zeitschrift für germanistische Linguistik 30/3: 310341.

Günthner, Susanne (2006): 'Was ihn trieb, war vor allem Wanderlust' (Hesse: Narziss und Goldmund). Pseudocleft-Konstruktionen im Deutschen. In: Günthner, Susanne/Imo, Wolfgang (Hrsg.): Konstruktionen in der Interaktion. Berlin: de Gruyter, 59-90.

Haegeman, Liliane (2006): Conditionals, factives and the left periphery. In: Lingua 116, 1651-1669.

Hammarström, Emil (1923): Zur Stellung des Verbums in der deutschen Sprache. Studien in volkstümlicher Literatur und Urkundensprache der Übergangszeit vom Mittelhochdeutschen zum Neuhochdeutschen. Lund: Håkan Ohlssons Buchdruckerei.

Härd, John Evert (1981): Studien zur Struktur mehrgliedriger deutscher Nebensatzprädikate. Diachronie und Synchronie. Göteborg: Acta Univerversitatis Gothoburgensis (Göteborger Germanistische Forschungen, 21).

Helbig, Gerhard (2003): Koordination vs. Subordination von Sätzen. Hauptsatz vs. Nebensatz. In: Dimova, Ana/Wiegand, Herbert Ernst (Hrsg.): Wort und Grammatik. Hildesheim u.a.: Olms (Germanistische Linguistik 171-172), 1-10.

Hennig, Mathilde (2006): Grammatik der gesprochenen Sprache in Theorie und Praxis. Kassel: University Press.

Hennig, Mathilde (2007): Gesprochene Sprache gestern und heute. In: Neue Sprachgeschichte(n). Themenheft der Zeitschrift Der Deutschunterricht, 59.3, 17-27.

Hennig, Mathilde (2009): Nähe und Distanzierung. Verschriftlichung und Reorganisation des Nähebereichs. Kassel: University Press.

Hennig, Mathilde (2010a): Elliptische Junktion in der Syntax des Neuhochdeutschen. In: Schmid, Hans Ulrich (Hrsg.): Perspektiven der germanistischen Sprachgeschichtsforschung. Berlin/New York (Jahrbuch der Gesellschaft für germanistische Sprachgeschichte 1), 76-103. 
Hennig, Mathilde (2010b): Aggregative Koordinationsellipsen im Neuhochdeutschen. In: Ziegler, Arne (Hrsg.): Historische Textgrammatik und Historische Syntax des Deutschen. Traditionen, Innovationen, Perspektiven. Bd. 2: Frühneuhochdeutsch, Neuhochdeutsch. Berlin/New York: de Gruyter, 937-963.

Hennig, Mathilde/Buchwald-Wargenau, Isabel (2010): Ausdrucksarten - ein neuer Zugang zur Wortschatzvermittlung im DaF-Unterricht? In: Kresic, Marijana/Ferraresi, Gisela (Hrsg.): Neue Perspektiven auf das Verhältnis zwischen linguistischer und didaktischer Grammatik. Linguistik online 41/2010. [Abrufbar unter: http://www.linguistikonline.com/41_10/hennigBuchwald.html]

Hentschel, Elke (Hrsg.) (2010): Deutsche Grammatik. Berlin: de Gruyter (de Gruyter Lexikon).

Hentschel, Elke/Weydt, Harald (2013): Handbuch der Deutschen Grammatik. 4. vollständig überarbeitete Auflage. Berlin/New York: de Gruyter Mouton.

Hilpert, Martin (2010): What can synchronic gradience tell us about reanalysis? Verb-first conditionals in written German and Swedish. In: Traugott, Elizabeth Closs; Trousdale, Graeme (Hrsg.): Gradience, Gradualness and Grammaticalization. Amsterdam: John Benjamins (Typological Studies in Language; No. 90), 181-201.

Hoffmann, Ludger (2013): Deutsche Grammatik: Grundlagen für Lehrerausbildung, Schule, Deutsch als Zweitsprache und Deutsch als Fremdsprache. Berlin: Schmidt.

Holler, Anke (2013): $d$ - und w-Relativsätze. In: Meibauer, Jörg/Steinbach, Markus/Altmann, Hans (Hrsg.): Satztypen des Deutschen. Berlin/New York: de Gruyter, 266-300.

Horacek, Blanka (1957): Zur Verbindung von Vorder- und Nachsatz im Deutschen. In: Beiträge zur Geschichte der deutsche Sprache und Literatur (Halle) 79, Sonderband, 415439.

Imo, Wolfgang (2007): Construction Grammar und Gesprochene-Sprache-Forschung: Konstruktionen mit zehn matrixsatzfähigen Verben im gesprochenen Deutsch. Tübingen: Niemeyer.

Kappel, Péter (2004): Zur Verberststellung im Neuhochdeutschen. Am Beispiel eines nähesprachlichen Textes aus dem 17. Jahrhundert. In: Czicza, Dániel/Hegedüs, Ildikó/Kappel, Péter/Németh, Attila (Hrsg.): Wertigkeiten, Geschichten und Kontraste. Festschrift für Péter Bassola zum 60. Geburtstag. Szeged: Grimm, 67-80.

Kappel, Péter (2006): Näheanalyse des Textes "Augustin Güntzer: Kleines Biechlin von meinem gantzen Leben. Die Autobiographie eines Elsässer Kannengießers aus dem 17. Jahrhundert. [= Güntzer I.]". In: Ágel, Vilmos/Hennig, Mathilde (Hrsg.): Grammatik aus Nähe und Distanz. Theorie und Praxis am Beispiel von Nähetexten 1650-2000. Tübingen: Niemeyer, 101-120.

Kappel, Péter (2007): Überlegungen zur diatopischen Variation in der gesprochenen Sprache. In: Ágel, Vilmos/Hennig, Mathilde (Hrsg.): Zugänge zur Grammatik der gesprochenen Sprache. Tübingen: Niemeyer (Reihe Germanistische Linguistik; 269), 215-244.

Kappel, Péter (2008): Integrationsgrad vorangestellter Adverbialsätze im Neuhochdeutschen. Anmerkungen zum Forschungsstand und Umriss eines Neuansatzes. In: Lovász, Ágnes/Molnár, Krisztina (Hrsg.): Linguistische Beiträge ungarischer Nachwuchsgermanisten. Referate der IV. linguistischen Tagung ungarischer Nachwuchsgermanisten an der Universität Pécs am 29.-30. März 2007. Pécs: Universitätsverlag 2008 (Studien zur Germanistik; 9.), 32-44. 
Kappel, Péter (2014): Satzintegration in neuhochdeutschen Texten. Zum Schnittstellencharakter der Integration vorangestellter Adverbialsätze. In: Bassola, Péter/Drewnowska-Vargáné, Ewa/Scheibl, György (Hrsg.): Zugänge zum Text. Frankfurt am Main: Lang (Szegediner Schriften zur germanistischen Linguistik; Bd. 3.), 193-215.

Kappel, Péter (i.V.): Vorangestellte Temporalsätze und ihre Integration in den Hauptsatz. Eine empirische Analyse von Nähe- und Distanztexten (1650-2000). In: Nubert, Roxana (Hrsg.): Temeswarer Beiträge zur Germanistik, Heft 13.

Kappel, Péter/Németh, Attila (2003): Monosemierung von Satzkonnektoren als Ergebnis der Vertikalisierung des Varietätenspektrums. Vergleichende Analyse zweier Varietäten der Gegenwartssprache. In: Németh, Attila (Hrsg.): Linguistische Beiträge ungarischer Nachwuchsgermanisten. Referate der I. Linguistischen Tagung ungarischer Nachwuchsgermanisten an der Universität Veszprém vom 28.-29. März 2003. Veszprém: Universitätsverlag/Wien: Edition Praesens (Studia Germanica Universitatis Vesprimiensis, Supplement Bd. 3), 91-115.

Karhiaho, Izabela (2003): Der Doppelpunkt im Deutschen: Kontextbedingungen und Funktionen. Göteborg: Acta Universitatis Gothoburgensis (Göteborger germanistische Forschungen; 42).

Koch, Peter/Oesterreicher, Wulf (1985): Sprache der Nähe - Sprache der Distanz. Mündlichkeit und Schriftlichkeit im Spannungsfeld von Sprachtheorie und Sprachgeschichte. In: Romanisches Jahrbuch 36, 15-43.

Koch, Peter/Oesterreicher, Wulf (1990): Gesprochene Sprache in der Romania: Französisch, Italienisch, Spanisch. Tübingen: Niemeyer.

Koch, Peter/Oesterreicher, Wulf (1994): Schriftlichkeit und Sprache. In: Günther, Hartmut/Ludwig, Otto (Hrsg.): Schrift und Schriftlichkeit/Writing and Its Use. Ein interdisziplinäres Handbuch internationaler Forschung Halbbd.1. Berlin/New York: de Gruyter (HSK 10.1), 587-604.

Konopka, Marek (2006): Topologie komplexer Sätze und Textverstehen. Zur Stellung von Verbletztsätzen mit weil. In: Blühdorn, Hardarik/Breindl, Eva/Waßner, Ulrich Hermann (Hrsg.): Text - Verstehen. Grammatik und darüber hinaus. Berlin/New York: de Gruyter (Jahrbuch des Instituts für Deutsche Sprache, 2005), 108-124.

Kotin, Michail L. (2013): Die wenn-dann-Hypotaxe aus synchroner, diachroner und typologischer Sicht. In: Zeitschrift für germanistische Linguistik, 41/3, 413-442.

Köller, Wilhelm (1993): Perspektivität in Bildern und Sprachsystemen. In: Eisenberg, Peter / Klotz, Peter (Hrsg.): Deutsch im Gespräch. Stuttgart: Klett, 15-34.

Köller, Wilhelm (2004): Perspektivität und Sprache. Zur Struktur von Objektivierungsformen in Bildern, im Denken und in der Sprache. Berlin/New York: de Gruyter.

König, Ekkehard/Eisenberg, Peter (1984): Zur Pragmatik von Konzessivsätzen. In: Stickel, Gerhard (Hrsg.): Pragmatik in der Grammatik. Jahrbuch 1983 des Instituts für deutsche Sprache. Düsseldorf: Schwann. (= Sprache der Gegenwart 60), 313-332.

König, Ekkehard/Auwera, Johan van der (1988): Clause integration in German and Dutch conditionals, concessive conditionals, and concessives. In: Haiman, John/Thompson, Sandra A. (eds.): Clause combining in grammar and discourse. Amsterdam/Philadelphia: Benjamins (Typological Studies in Language 18), 101-133. 
Köpcke, Klaus-Michael/Panther, Klaus-Uwe (1989): On correlations between word order and pragmatic function of conditional sentences in German. In: Journal of Pragmatics 13, 685711.

Kronberger, Gerald (2008): Weinpreis, Mordtat, Jubelfeier: Die Reutlinger "Cronica" 16871738 des Johann Georg Launer. In: Stadtarchiv Reutlingen und Reutlinger Geschichtsverein e. V. (Hrsg.): Reutlinger Geschichtsblätter 2007. Neue Folge Nr. 46. Reutlingen: Eigenverlag Stadtarchiv, 115-159.

Küper, Christoph (1991): Geht die Nebensatzstellung im Deutschen verloren? In: Deutsche Sprache 19, 133-158.

Küper, Christoph (1993): Pragmatische Motiviertheit in der Syntax. Haupt- und Nebensätze im Deutschen. In: Küper, Christoph (Hrsg.): Von der Sprache zur Literatur. Motiviertheit im sprachlichen und im poetischen Kode. Tübingen: Stauffenburg (Probleme der Semiotik 14), 37-49.

Lefèvre, Michel (2012): Von der Periode zum Satz in Prosaschriften um 1730. Untersuchung an Hand der Korrelation mit so. In: Lefèvre, Michel (Hrsg.): Syntaktischer Wandel in Gegenwart und Geschichte. Akten des Kolloquiums in Montpellier vom 9. bis 11. Juni 2011. Berlin: Weidler (Berliner Sprachwissenschaftliche Studien 28), 483-508.

Lefèvre, Michel (2013): Textgestaltung, Äußerungsstruktur und Syntax in deutschen Zeitungen des 17. Jahrhunderts. Zwischen barocker Polyphonie und solistischem Journalismus. Berlin: Weidler (Berliner Sprachwissenschaftliche Studien 29).

Lehmann, Christian (1984): Der Relativsatz. Typologie seiner Strukturen, Theorie seiner Funktionen, Kompendium seiner Grammatik. Tübingen. (Language Universals Series 3).

Lehmann, Christian (1988): Towards a typologie of clause linkage. In: Haiman, John/Thompson, Sandra A. (Hrsg.): Clause Combining in Grammar und Discourse. Amsterdam/Philadelphia: Benjamins (Typological Studies in Language 18), 181-225.

Lehmann, Christian (1995): Relativsätze. In: Jacobs, Joachim et al. (Hrsg.): Syntax: Ein internationales Handbuch zeitgenössischer Forschung. Berlin, New York: de Gruyter (HSK 9.1), 1199-1216.

Lehmann, Christian (2015): Thoughts on grammaticalization. $3^{\text {rd }}$ edition. Berlin: Language Science Press (Classics in Linguistics 1).

Lemnitzer, Lothar/Zinsmeister, Heike (2010): Korpuslinguistik. Eine Einführung. 2., durchgesehene und aktualisierte Auflage. Tübingen: Gunter Narr (narr studienbücher).

Leuschner, Torsten (2005): Ob blond, ob braun, ich liebe alle Frau'n. Irrelevanzkonditionalen als grammatikalisierter Diskurs. In: Leuschner, Torsten/Mortelmans, Tanja/De Groodt, Sarah (Hrsg.): Grammatikalisierung im Deutschen. Berlin/New York: de Gruyter, 279-307.

Leuschner, Torsten (2007): Irrelevanzausdruck und Feldergrammatik. Linguistische Beschreibung - didaktische Folgerungen. In: Deutsch als Fremdsprache 44, 219-226.

Lötscher, Andreas (1995): Herausstellung nach links in diachroner Sicht. In: Sprachwissenschaft 20, 32-63.

Lötscher, Andreas (2000): Verbendstellung im Hauptsatz in der deutschen Prosa des 15. und 16. Jahrhunderts. In: Sprachwissenschaft 25.2, 153-191. 
Lötscher, Andreas (2005): Linksperiphere Adverbialsätze in der Geschichte des Deutschen. Pragmatische Aspekte eines grammatischen Wandels. In: Beiträge zur Geschichte der deutschen Sprache und Literatur (PBB) 127, 347-376.

Lötscher, Andreas (2006): Die Formen der Sprache und die Prozesse des Verstehens. Textverstehen aus grammatischer Sicht. In: Blühdorn, Hardarik/Breindl, Eva/Waßner, Ulrich (Hrsg.): Text - Verstehen. Berlin/New York: de Gruyter (Jahrbuch des Instituts für Deutsche Sprache, 2005), 46-70.

Lötscher, Andreas (2010): Auf der Suche nach syntaktischen „Nähe-Distanz“-Signalen in frühneuhochdeutschen Texten. In: Ágel, Vilmos/Hennig, Mathilde (Hrsg.): Nähe und Distanz im Kontext variationslinguistischer Forschung. Berlin: de Gruyter (Linguistik Impulse \& Tendenzen; 35), 111-134.

Lühr, Rosemarie (2004): Universale Konzessivsätze. In: Kozianka, Maria et al. (Hrsg.): Indogermanistik - Germanistik - Linguistik. Akten der Arbeitstagung der Indogermanischen Gesellschaft, Jena 18.-20.09.2002. Hamburg: Kovac (Philologia 63), $345-359$.

Lühr, Rosemarie (2010): Bedingungsstrukturen im älteren Deutsch. In: Ziegler Arne (Hrsg.): Historische Textgrammatik und historische Syntax des Deutschen. Traditionen, Innovationen, Perspektiven. Bd. 1.: Diachronie, Althochdeutsch, Mittelhochdeutsch. Berlin/New York: de Gruyter, 157-172.

Marschall, Matthias (1994): Satzklammer und Textverstehen. Zur Funktion der Verbendstellung im Deutschen. Deutsche Sprache 22, 310-330.

Mattheier, Klaus J. (1981): Wege und Umwege zur neuhochdeutschen Schriftsprache. In: Zeitschrift für germanistische Linguistik 9/3, 274-307.

Mattheier, Klaus J. (1995): Sprachgeschichte des Deutschen. Desiderate und Perspektiven. In: Gardt, Andreas/Mattheier, Klaus J./Reichmann, Oskar (Hrsg.): Sprachgeschichte des Neuhochdeutschen. Gegenstände, Methoden, Theorien. Tübingen: Niemeyer (Reihe Germanistische Linguistik 156), 1-18.

Maurer, Friedrich (1926): Untersuchungen über die deutsche Verbstellung in ihrer geschichtlichen Entwicklung. Heidelberg: Winter.

Molnár, Valéria (2012): Zur Relevanz der linken Peripherie für die Strukturierung der Information: kontrastive und typologische Überlegungen. In: Gunkel, Lutz/Zifonun, Gisela (Hrsg.): Deutsch im Sprachvergleich. Grammatische Kontraste und Konvergenzen. Berlin/Boston: de Gruyter (Jahrbuch des Instituts für Deutsche Sprache, 2011), 383-416.

Musan Renate (2013): Satzgliedanalyse. 3., aktualisierte Auflage. Heidelberg: Winter (Kurze Einführungen in die germanistische Linguistik; 6).

Neef, Martin (2014): Satzgliedfunktionen im Deutschen: eine realistische Weiterentwicklung. Zeitschrift für germanistische Linguistik 42/3, 420-455.

Nübling, Damaris et al. (2013): Historische Sprachwissenschaft des Deutschen. Eine Einführung in die Prinzipien des Sprachwandels. 4., komplett überarb. u. erw. Auflage. Tübingen: Narr (Narr-Studienbücher).

Oppenrieder, Wilhelm (2006): Subjekt- und Objektsätze. In: Ágel, Vilmos et al. (Hrsg.): Dependenz und Valenz. Ein internationales Handbuch zeitgenössischer Forschung, 2. Halbbd. Berlin/New York: de Gruyter (HSK 25.2), 900-914. 
Panofsky, Erwin (1927): Die Perspektive als 'symbolische Form'. In: Saxl, Fritz (Hrsg.): Vorträge der Bibliothek Warburg 1924/25. Leipzig/Berlin: Teubner, 258-330.

Pasch, Renate et al. (2003): Handbuch der deutschen Konnektoren. Linguistische Grundlagen der Beschreibung und syntaktische Merkmale der deutschen Satzverknüpfer (Konjunktionen, Satzadverbien und Partikeln). Berlin/New York: de Gruyter (Schriften des Instituts für Deutsche Sprache 9).

Pasch, Renate/Zifonun, Gisela (2006): Adverbial- und Relativsätze. In: Ágel, Vilmos/Eichinger, Ludwig M./Eroms, Hans-Werner/Hellwig, Peter/Heringer, Hans Jürgen/Lobin, Henning (Hrsg.): Dependenz und Valenz. Ein internationales Handbuch der zeitgenössischen Forschung. 2. Halbband. Berlin/New York: de Gruyter (HSK 25.2), 921936.

Paul, Hermann (1920): Deutsche Grammatik. Bd. IV.: Syntax. Halle: Niemeyer.

Peyer, Ann (1997): Satzverknüpfung: syntaktische und textpragmatische Aspekte. Tübingen: Niemeyer (Reihe Germanistische Linguistik; 178).

Pittner, Karin (1999): Adverbiale im Deutschen. Untersuchungen zu ihrer Stellung und Interpretation. Tübingen: Stauffenburg (Studien zur deutschen Grammatik 60).

Pittner, Karin (2000): Sprechaktbedingungen und bedingte Sprechakte: Pragmatische Konditionalsätze im Deutschen. In: Fetzer, Anita/Pittner, Karin (Hrsg.): Gesprächsforschung: Neue Entwicklungen. Linguistik online 5/2000. [Abrufbar unter: http://www.linguistik-online.org/1_00/PITTNER.HTM]

Pittner, Karin (2004): Wo in Relativsätzen - eine korpusbasierte Untersuchung. In: Zeitschrift für Germanistische Linguistik 32/2004, 357-375.

Pittner, Karin (2011): Anmerkungen zur (Un-)Integriertheit von Konditionalsätzen mit Verberststellung. In: Zeitschrift für Sprachwissenschaft 30/1, 75-105.

Pittner, Karin (2013a): Adverbialsätze. In: Meibauer, Jörg/Steinbach, Markus/Altmann, Hans (Hrsg.): Satztypen des Deutschen. Berlin/New York: de Gruyter, 501-525.

Pittner, Karin (2013b): Artikel "Adverbial". In: Dürscheid, Christa/Schierholz, Stefan J. (Hrsg.): WSK-Online. Grammatik: Syntax. Ein Lern- und Konsultationswörterbuch. Mit einer Systematischen Einführung und englischen Übersetzungen (Wörterbücher zur Sprach- und Kommunikationswissenschaft, 1.2). Berlin/New York: de Gruyter [InternetDatenbank, http://www.degruyter.com/view/db/wsk].

von Polenz, Peter (1985): Deutsche Satzsemantik. Grundbegriffe des Zwischen-den-ZeilenLesens. Berlin/New York: de Gruyter (Sammlung Göschen 2226).

von Polenz, Peter (1994): Deutsche Sprachgeschichte vom Spätmittelalter bis zur Gegenwart. Bd. 2: 17. und 18. Jahrhundert. Berlin/New York: de Gruyter (de Gruyter Studienbuch).

von Polenz, Peter (1995): Sprachsystemwandel und soziopragmatische Sprachgeschichte in der Sprachkultivierungsepoche. In: Gardt, Andreas/Mattheier, Klaus J./Reichmann, Oskar (Hrsg.): Sprachgeschichte des Neuhochdeutschen. Gegenstände, Methoden, Theorien. Tübingen: Niemeyer (Reihe Germanistische Linguistik 156), 39-67.

von Polenz, Peter (1998): Deutsche Sprache und Gesellschaft in historischer Sicht. In: In: Besch, Werner/Betten, Anne/Reichmann, Oskar/Sonderegger, Stefan (Hrsg.): Sprachgeschichte. Ein Handbuch zur Geschichte der deutschen Sprache und ihrer Erforschung. Bd. 1. 2. Aufl. Berlin/New York: de Gruyter (HSK 2.1), 41-54. 
von Polenz, Peter (2000): Deutsche Sprachgeschichte vom Spätmittelalter bis zur Gegenwart. Bd. 2: 17. und 18. Jahrhundert. Berlin/New York: de Gruyter (de Gruyter Studienbuch).

Raible, Wolfgang (1992): Junktion. Eine Dimension der Sprache und ihre Realisierungsformen zwischen Aggregation und Integration. Heidelberg: Winter (Sitzungsberichte der Heidelberger Akademie der Wissenschaften, Philosophischhistorische Klasse 1992/2).

Ramers, Karl Heinz (2005): Verbstellung im Althochdeutschen. In: Zeitschrift für germanistische Linguistik 33/1, 78-91.

Redder, Angelika (1987): Wenn...so. Zur Korrelatfunktion von so. In: Rosengren, Inger (Hrsg.): Sprache und Pragmatik; Lunder Symposium 1986. Stockholm: Almqvist \& Wiksell. (Lunder Germanistische Forschungen 55), 315-326.

Reich, Ingo/Reis, Marga (2013): Koordination und Subordination. In: Meibauer, Jörg/Steinbach, Markus/Altmann, Hans (Hrsg.): Satztypen des Deutschen. Berlin/New York: de Gruyter, 536-569.

Reichmann, Oskar (1988): Zur Vertikalisierung des Varietätenspektrums in der jüngeren Sprachgeschichte des Deutschen. In: Munske, Horst Haider et al. (Hrsg.): Deutscher Wortschatz. Lexikologische Studien. Festschrift für Ludwig Erich Schmitt von seinen Marburger Schülern. Berlin/New York: de Gruyter, 151-180.

Reichmann, Oskar (2003): Die Entstehung der neuhochdeutschen Schriftsprache: Wo bleiben die Regionen? In: Berthele, Raphael et al. (Hgg.): Die deutsche Schriftsprache und die Regionen. Entstehungsgeschichtliche Fragen in neuer Sicht. Berlin/New York: de Gruyter (Studia Linguistica Germanica 65), 29-56.

Reis, Marga (1997): Zum syntaktischen Status unselbständiger Verbzweit-Sätze. In: Dürscheid, Christa et al. (Hrsg.): Sprache im Fokus: Festschrift für Heinz Vater zum 65. Geburtstag. Tübingen: Niemeyer, 121-144.

Reis, Marga/Wöllstein, Angelika (2010): Zur Grammatik (vor allem) konditionaler V1Gefüge im Deutschen. In: Zeitschrift für Sprachwissenschaft 29, 111-179.

Rieck, Susanne (1977): Untersuchungen zu Bestand und Varianz der Konjunktionen im Frühneuhochdeutschen unter Berücksichtigung der Systementwicklung zur heutigen Norm. Heidelberg: Winter (Studien zum Frühneuhochdeutschen 2).

Robinson, Orrin W. (1997): Clause Subordination and Verb Placement in the Old High German ,Isidor‘ Translation. Heidelberg: Winter.

Roelcke, Thorsten (1995): Periodisierung der deutschen Sprachgeschichte. Analysen und Tabellen. Berlin/New York: de Gruyter (Studia Linguistica Germanica, Bd. 40).

Schildt, Joachim (1976): Zur Ausbildung des Satzrahmens. In: Kettmann, Gerhard/Schildt, Joachim (Hrsg.): Zur Ausbildung der Norm der deutschen Literatursprache auf der syntaktischen Ebene (1470-1730). Der Einfachsatz. Berlin: Akademie (Bausteine zur Sprachgeschichte des Neuhochdeutschen 56/I), 235-284.

Schrodt, Richard (2004): Althochdeutsche Grammatik II: Syntax. Tübingen: Niemeyer (Sammlung kurzer Grammatiken germanischer Dialekte A. Hauptreihe 5/2)

Selting, Margret (1993): Voranstellungen vor den Satz. Zur grammatischen Form und interaktiven Funktion von Linksversetzung und Freiem Thema im Deutschen. In: Zeitschrift für germanistische Linguistik 21/3, 291-319. 
Selting, Margaret (1999): Kontinuität und Wandel der Verbstellung von ahd. wanta bis gwd. weil. Zur historischen und vergleichenden Syntax der weil-Konstruktionen. In: Zeitschrift für germanistische Linguistik 27/2, 167-204.

Sinner, Carsten (2014): Varietätenlinguistik. Eine Einführung. Tübingen: Narr (narr studienbücher).

Spiekermann, Helmut (2010): Variation in der deutschen Sprache. In: Krumm, Hans-Jürgen et al. (Hrsg.): Deutsch als Fremd- und Zweitsprache. Ein internationales Handbuch. 2. Aufl. Berlin/New York: de Gruyter (HSK 35.1), 343-359.

Starker, Josef (1883): Die Wortstellung der Nachsätze in den ahd. Übersetzungen des Matthäus-Evangeliums, des Isidor und des Tatian. Beuthen: Wiss. Beilage des 16. Jahresberichts des Städt. Kathol. Gymnasiums zu Beuthen, O.-S. (Abrufbar unter: http://dbooks.bodleian.ox.ac.uk/books/PDFs/600048075.pdf, zuletzt abgerufen am 11.8.2015)

Sweetser, Eve E. (1990): From Etymology to Pragmatics. Metaphorical and cultural aspects of semantic structure. Cambridge: Cambridge University Press (Cambridge studies in linguistics 54).

Takada, Hiroyuki (1998): Grammatik und Sprachwirklichkeit von 1640-1700: zur Rolle deutscher Grammatiker im schriftsprachlichen Ausgleichsprozeß. Tübingen: Niemeyer (Reihe Germanistische Linguistik; 203).

Thim-Mabrey, Christiane (1987): Adverbiale + so: Distribution und Funktion des Korrelats so im Frühneuhochdeutschen. In: Sprachwissenschaft 12/2, 180-219.

Thim-Mabrey, Christiane (1988): Satzadverbialia und andere Ausdrücke im Vorvorfeld. In: Deutsche Sprache 16, 52-67.

Thurmair, Maria (1991): Warten auf das Verb. Die Gedächtnisrelevanz der Verbklammer im Deutschen. Jahrbuch Deutsch als Fremdsprache 17, 174-202.

Thurmair, Maria (2001): Vergleiche und Vergleichen: Eine Studie zu Form und Funktion der Vergleichsstrukturen im Deutschen. Tübingen: Niemeyer (Linguistische Arbeiten 433).

Van den Nest, Daan (2005): Asyndetische Subordination diachronisch. Konditionalen zwischen Diskurs und Syntax. Germanistische Mitteilungen 62, 29-43

Van den Nest, Daan (2010): Should conditionals be emergent ...: Asyndetic subordination in German and English as a challenge to grammaticalization research. In: Van linden, An/Verstraete, Jean-Christophe/Davidse, Kristin (Hrsg.): Formal Evidence in Grammaticalization Research (Typological Studies in Language, 94), 93-136.

Vandenberghe, Roxane (2003): Het syntactische gedrag van het Middelnederlandse zinsinleidende doe ('toen'). In: Dialectsyntaxis in bloei. Taal en Tongval themanummer 15-16, 251-276.

Vandergriff, Ilona (2009): Conditionals and Mental Space Set-up: Evidence from German Word Order. In: Bergs, Alexander/Diewald, Gabriele (Hrsg.): Contexts and Constructions Amsterdam: John Benjamins (Constructional Approaches to Language CAL), 193-212.

Viti, Carlotta (2015): Variation und Wandel in der Syntax der alten indogermanischen Sprachen. Tübingen: Narr.

Volodina, Anna (2006): wenn-Relationen: Schnittstelle zwischen Syntax, Semantik und Pragmatik. In: Breindl, Eva/Gunkel, Lutz/Strecker, Bruno (Hrsg.): Grammatische 
Untersuchungen. Analysen und Reflexionen. Tübingen: Narr (Studien zur deutschen Sprache 36), 359-379.

Volodina, Anna (2011a): Sweetsers Drei-Ebenen-Theorie: Theoretische Überlegungen vor dem Hintergrund einer korpuslinguistischen Studie über konditionale und kausale Relationen. In: Gisella Ferraresi (Hrsg.): Konnektoren im Deutschen und im Sprachvergleich: Beschreibung und grammatische Analyse. Tübingen: Narr (Studien zur deutschen Sprache 53), 127-155.

Volodina, Anna (2011b): Konditionalität und Kausalität im Diskurs. Eine korpuslinguistische Studie zum Einfluss von Syntax und Prosodie auf die Interpretation komplexer Äußerungen. Tübingen: Narr (Studien zur deutschen Sprache 54).

Waßner, Ulrich Hermann (2006): Zur Relevanz von und zur Irrelevanz bei Irrelevanzkonditionalen. In: Breindl, Eva/Gunkel, Lutz/Strecker, Bruno (Hrsg.): Grammatische Untersuchungen, Analysen und Reflexionen. Festschrift für Gisela Zifonun. Tübingen: Narr (Studien zur Deutschen Sprache 36), 381-399.

Wegener, Heide (2000): Koordination und Subordination - semantische und pragmatische Unterschiede. In: Lefèvre, Michel (Hrsg.): Subordination in Syntax, Semantik und Textlinguistik. Tübingen: Stauffenburg (Eurogermanistik 15), 33-44.

Wegener, Heide (2001): Integration und Nichtintegration von Satzkonjunkten im Deutschen und im Französischen. In: Haßler, Gerda (Hrsg.): Sprachkontakt und Sprachvergleich. Münster: Nodus Publikationen (Studium Sprachwissenschaft: Beiheft 34), 89-105.

Wegera, Klaus-Peter/Waldenberger, Sandra (2012): Deutsch diachron. Eine Einführung in den Sprachwandel des Deutschen. Berlin: Erich Schmidt (Grundlagen der Germanistik 52).

Weinrich, Harald (2005): Textgrammatik der deutschen Sprache. Unter Mitarb. von Maria Thurmair. 3., rev. Aufl. Darmstadt: Wissenschaftliche Buchgesellschaft.

Welke, Klaus (2007): Einführung in die Satzanalyse. Die Bestimmung der Satzglieder im Deutschen. Berlin/New York: de Gruyter (de Gruyter Studienbuch).

Wich-Reif, Claudia (2008): Zur Relevanz des Vorvorfeldbegriffs in Gegenwart und Geschichte. In: Sprachwissenschaft 33.2, 173-209.

Wiktorowicz, Józef (2013): Die vorangestellten Adverbialsätze und ihre Integration mit dem Hauptsatz im 17. Jahrhundert. In: Wiktorowicz, Józef/Just, Anna/Gaworski, Ireneusz (Hrsg.): Satz und Text. Zur Relevanz syntaktischer Strukturen zur Textkonstitution. Frankfurt am Main: Lang Ed. (Schriften zur diachronen und synchronen Linguistik, Bd. 8.), 393-398.

Wolf, Georg (2001): Zur Pragmatik der konjunktionalen Nebensätze des Deutschen. In: Liedtke, Frank/Hundsnurscher, Franz (Hrsg.): Pragmatische Syntax. Tübingen: Niemeyer (Beiträge zur Dialogforschung 23), 141-164.

Wolf, Norbert Richard (1998): Metakommunikative Nebensätze im Vorvorfeld. In: Donhauser, Karin/Eichinger, Ludwig M. (Hrsg.): Deutsche Grammatik: Thema in Variationen. Festschrift für Hans-Werner Eroms zum 60. Geburtstag. Heidelberg: Universitätsverlag C. Winter, 93-99.

Zifonun, Gisela et al. (1997): Grammatik der deutschen Sprache. 3 Bde. Berlin/New York: de Gruyter (Schriften des Instituts für deutsche Sprache 7). 
Zifonun, Gisela (2015): Satz - oberflächennah. In: Dürscheid, Christa/Schneider, Jan Georg (Hrsg.): Handbuch Satz, Äußerung, Schema. Berlin: de Gruyter (Handbücher Sprachwissen; 4), 155-179.

Zitterbart, Jussura Paranhos (2002): Zur korrelativen Subordination im Deutschen. Tübingen: Niemeyer (Linguistische Arbeiten 464).

Zitterbart, Jussura Paranhos (2013): Satztyp und Korrelat/Platzhalter/Bezugsausdruck. In: Meibauer, Jörg/Steinbach, Markus/Altmann, Hans (Hrsg.): Satztypen des Deutschen. Berlin/New York: de Gruyter, 602-626. 


\section{Anhang}

\subsection{Belegliste}

Die Belegliste enthält nur die einschlägigen Belege für vorangestellte Adverbialsätze aus dem für die vorliegende Untersuchung zusammengestellten Korpus. Die Belege werden nach semantischen Typen geordnet. Vorangestellte Adverbialsätze und weitere Teilsätze, die vor ihrem Obersatz realisiert werden, sind durch Unterstreichung markiert. Die Subjunktoren der einschlägigen Adverbialsätze werden durch Fettdruck hervorgehoben. Alle weiteren Markierungen stammen - wenn nicht gesondert markiert - aus den Originaltexten. ${ }^{231}$

Temporalsätze (Kapitel 5.1)

1. So offt wir aus der gemeinen Lufft Odem schöpffen/ bekennen wir stillschweigend die allgemeine Wolthat: (Harsdörffer I: 1)

2. So offt wir Speis und Getränck zu uns nehmen/ sollen wir uns erinnern/ daß wir von unsrem Vatterland genehret und erhalten werden: (Harsdörffer I: 1)

3. So offt wir in das freye Feld spatziren/ und desselben zuwachsende Fruchtbarkeit betrachten sollen wir unvergessen seyn/ daß wir von solchem unsren Vatterland/ nechst GOtt/ das Leben haben: (Harsdörffer I: 1)

4. Als aber der Jăgermeister geantwortet: Er wisse nicht/ was ein Extract seye? hat der Kåiser versetzt: Mein Knoll/ so laß mir auch meine Schreiber und Secretarien zu frieden. (Harsdörffer I: 13)

5. Nachdem ich nun im Jahrr ano 1615 auff Barttolimey außzog zu wandtern, ließ mihr mein Vatter ein ney Kleidt machen von Leder, gab mihr zugeho̊hrige Nodtdurfft wie auch 12 fl. in Gelt (Güntzer I: 40v)

6. Als wihr ein Meill Wex in den Hagenauer Forst kamen, so kame auß einem Busch ein Merdter, kurtz von Person, sprach zu unß: Wie weidt wolt ihr? (Güntzer I: 41r)

7. So offt als er hinder mich ging, spring ich von den gelegten Beimen herunder undt ging widerumb dahinden. (Güntzer I: 42v)

8. Als wihr mitten in den Walt kamen, ging er widerumb hinder mihr, zog ein Meßer auß dem Hossensack, stick mihr nach dem Halß, aber Gott behiedtet mich fihr einem solchen Dodt, der Stich ging mihr no̊ben dem Kragen hinauß. (Güntzer I: 43r)

9. Als ich nacher Trieß[t] kam, erqwickete mich widerumb, lag 5 Tag im Wirtzhauß. (Güntzer I: 55r)

\footnotetext{
${ }^{231}$ Die Graphie $\int$ wird durchgehend als $s$ wiedergegeben.
} 
10. Als ich von Batuwa auß ging, kam zu mihr ein Bandit, ging daher als wie ein Freyher oder Graff, also statlich in der Gleidung. (Güntzer I: 55r)

11. Als ich nun solches hordte auff der italienischer Sprach, daß diße 3 Gesellen Banditen und Merder wahren, da wahr mihr angst und bange. (Güntzer I: 56 r)

12. Als ich nun nacher Farara kam, ließ ich meinen Degen bey einem deischten Laudtenmacher ligen, zoge nacher Lohreta, alda die groste Walfardt ist bey den Babisten. (Güntzer I: 56v)

13. Als ich aber hinkam gon Loreta, flohe ich deß Minchen auff den Gaßen. (Güntzer I: 57v)

14. Als ich zur Portten hinaußkam, fandt ich meine 2 Spießgesellen von Ambsterdam in eines Bauren Hauß auff dem Mist im Stro ligen, zu welchen ich mich schlaffen auff den Mist legte. (Güntzer I: 58v)

15. Als ich auffstundt, fiel ich widerumb nider, ging auff alen Fieren gleich wie Dierr. (Güntzer I: 59v)

16. Als ich auffstundt, wirdt ich alererst solches gewahr, sae aber dem Dieb nicht mer. (Güntzer I: 59v)

17. Als wihr miteinander gon Rom kamen, so versteckte ich mich vohr ihme, dieweil er jedezeitt an mich begerdte, daz ich beichten solt. (Güntzer I: 60r)

18. Wan mihr etwaß wol ist, so beschaue ich die Statt, ihr Gebey und abgôttische Kirchen. In dem ging ich in S. Petterskirch. (Güntzer I: 60v)

19. Nachdem ich nun auß Italiam in daß deischt Landt kam, arbeidet ich zu Botzen in Eschtlandt 14 Tag, mehr 4 Wochen zu Schootz in Tirol, alhie wahr ich ubel staffierdt. (Güntzer I: 63r)

20. Als ich von ihme den wolte den Abscheidt nemen, so wolte er mihr die Handt nicht bieden, dan es wahr i[h]me leidt, daß ich widerumb fordtzog. (Güntzer I: 65v)

21. Als ich nun den 3ten May 1620 von Hauß zog zum ander Mal, so nam ich meinen Wóg in daß Westreich. (Güntzer I: 78r)

22. Als ich mich bey ihnen durch Gottes Hilff beschultigt hab und außgerett, ich seye kein Soldadt, sonder ein Handtwercksgesel, so kam ich fihr die Oberkeitt und die Bauren verglagt. (Güntzer I: 79r)

23. Als es Tage wirdt, so danckete ich ihren um die Herberg, gab ihr eine Verehr. (Güntzer I: $79 \mathrm{v})$

24. Wan wihr dan in ein Scheiren oder Stall im Abent kamen, so zogen wihr unß auß biß auff daß Hemet und legten den Sack auff daß Stro, (Güntzer I: 80v)

25. Nachdem wihr auß Lickerlandt kemen sindt, so zogen wihr ins Landt zu Gleffen. (Güntzer I: 82r)

26. Als ich nacher Ambsterdam zu reißete in der Nacht, auff der Wel bey Harlem, kamen 2 Soldaten zu mihr, setzet[e]n mit Gewalt an mich, wolten mich plindern. (Güntzer I: 83v)

27. So man die Dore zuschließt, so ging ich hinauß. (Güntzer I: 84r)

28. Als ich nacher Ambsterdam kam, ließe ich eine Dockaten wôxlen, darmit die Statt besichtigen. (Güntzer I: 84r)

29. Als daß Fich daß Fuder auffgetzet hatt, fingen sie an, auff mich zu beißen und nagen, als wolten sie mich auch freßen. (Güntzer I: 84r) 
30. Als ich die feindliche Biß am Kopff und Fießen empfinde, so erwachete ich, erschr[e]cke uber dißer Geschicht und wunderlicher Gest, die freßen wolten. (Güntzer I: 84r-v)

31. Als es Tag wirdte, reißete ich mein[e]r Straßen und dancket dem Wirdt um die Herberg. (Güntzer I: 84v)

32. Als ich in daß Grining[e]r Landt kam in der Schantz zu Delffsil, wahren ankomen 6 Heren von den Erenstaden. (Güntzer I: 84v)

33. Als die Mustgatierer Salfey goben, stund er fohr den Stuck, den Ladtstock heraußzuziehen. (Güntzer I: 84v)

34. Als ich nun auff der Pricken zusahe, so fier mihr der Ladtstock neben die Seidten hinweck, mich zwar gerierdt, aber nichts am Leib verletzet worden. (Güntzer I: 85r)

35. Als ich nun deß Nachts vohr der Statt Altenburck von den Waßerfóglin erleßet bin worden, so kam ich deß Morgens frie in die Statt zu einem Zingießer. (Güntzer I: 85v)

36. Als ich nach der Statt Krompen reißete, wirdte ich verspediget, der Statt Portten wirdten zugemocht. (Güntzer I: 86v)

37. Als nun der Tag anpricht, machte ich mich auß dem Milerwagen auff in die Statt, danckete Gott, daz er mich von dißem Windtmiller errettet hatt. (Güntzer I: 89v)

38. Als ich nacher Etzoho kam in dißem Landte, hielten die Burger ein Fogelschießen. (Güntzer I: 89v)

39. Als er vernam, daß ich ein Hochdeischter wahr, so fragt er mich alerhandt, wie es im deischt Landt stunde, aber wußt es beßer, als ich es i[h]m sagen kondt. (Güntzer I: 90r)

40. Als ich 2 Meil im Walt gangen bin, kame ich zu einem Wirtzhauß und Meyerhoff. (Güntzer I: 90r)

41. Als ich hiezu kam, saßen ein gantzer Disch vol reiberischer Gesellen. (Güntzer I: 90v)

42. Als ich nun 5 Meil Wơgx in dißem Walt gangen wahr, so kame ich in ein klines Dorff, sprich den Bauren um die Herberg an, im Stal zu ligen, dan es wahr kein Wirdt alda. (Güntzer I: 91r)

43. Als ich geßen hatt, hungerte mich vester dan zuvor. (Güntzer I: 91r)

44. Als ich nun deß Waltes ein Ende kam, sahe ich ein großes Ackerfelt und Stettin vohr mihr ligen. (Güntzer I: 91v)

45. Als ich i[h]n erkandte, das es ein Man wahr, erschracke ich zim Teil, dieweil er so lang auff mich warttete, er dirfft mich dodt schlagen. (Güntzer I: 91v)

46. Als ich nun nahe zu i[h]m kam, grießete ich ihnen. (Güntzer I: 92r)

47. Als ich ungefahr 200 Schritt fordtging, wolte ich den Meder nachmallen anschauen, aber er wartt mihr entgangen. (Güntzer I: 93r)

48. Als ich nacher Lauenburck kam in Caßsuben, so triff ich ein Dantzig[er] Fuhrman an mit einem leren Wagen, der fieret mich vergebens 18 Meil Wögx durch den langen Walt und Gestriß. (Güntzer I: 93v)

49. Als ich nach[e]r Dantzig kam, wolten mihr die Zingießer kein Arbeidt geben von wògen meiner schlechten Gleidung. (Güntzer I: 93v)

50. Als aber der Zingießer von andern seinen Gesellen erfuhr, wehr ich sey, so gab er mihr den andern Tag Arbeidt. (Güntzer I: 94r) 
51. Als wihr bey der Inßel Bornuhol vihriber schiff[e]n, so warff der Schiffman Ancker. (Güntzer I: 94v)

52. Als wihr nun in die rigische Einfardt köhmen, hatten wihr abermal ein Gefohr, dieweil wihr etwaß auff den Sandt furen. (Güntzer I: 96v)

53. Als wihr nun nacher Riga kamen, so bekamen wihr beide Zingießerarbeidt bey einem Zingieß[er]. (Güntzer I: 96v)

54. Als ich nun mit den Darttern uber daz Eiß der Duna reißete, wahr ein auffprochen Eiß am Landt. (Güntzer I: 97r)

55. Indem wihr in Churlandt thun reißen, durchzogen wihr vil großer langer Welt. (Güntzer I: $97 \mathrm{v})$

56. Als wihr in Litan kamen, wahr die Kelten und Schne noch gròßer. (Güntzer I: 98r)

57. Als wihr nahe zu der Wiltau kamen, hette ich nichts mehr zu essen, ku[n]dt auch nichts um daz Gelt bekomen. (Güntzer I: 98v)

58. So mihr dan die Schenkel an daß Geschire wolten frieren, so name ich ein Hamer, schlug daz Eiß mit auff. (Güntzer I: 98v)

59. Als ich aber mich mit einem Zingießergesel von hieruß zog nacher Gintzburck in Preißen, so sindt mihr die Schenckel widerum erfrohren. (Güntzer I: 99r)

60. Als wihr miteinander in die Statt Kauen eingingen, zoge ich meine Belckkapen von den Gesicht, dan sie wahr gemacht wie eine Larffen. (Güntzer I: 99v)

61. Wan ich dan deß Abens spadt nacher Нauß kam, so fragt man mich, waß mihr an dem Augen wahr, daz sie also rodt wahren. (Güntzer I: 101v-102 r)

62. Als es nun geyen dem Friejahr ging und ich die Schiff auß fernen Landen sach komen, so name ich von meim Meister Uhrlaub und begabe mich auff ein englisches Schiff, mit in Engelandt zu fahren. (Güntzer I: 102v)

63. Als wihr nacher Gintzburck kamen, so bitete ich den Kauffman und Schiffman gantz frindlich, [mich] mit mitzunömen. (Güntzer I: 103r)

64. Als es Tage wirdt, triff ich diße 3 Schelmen widerum an. (Güntzer I: 104v)

65. Da wihr gutten Sudtwindt bekamen und fordtseglet[e]n dem Sundt zu, empfinde ich eine Lifferung meiner schwöhrmiedtigen Gedancken und sehe, daß Gott nahe ist, mihr zu helffen in meiner großer Nodt und Traurigkeitt, die mich umpfangen hatt in dem Landt zu Preißen. (Güntzer I: 105v)

66. Als wihr auff diße Ostse in Danenmarck dem Sondt zu segelt[e]n, wirdte ich zwar etwaß sekranck. (Güntzer I: 106r)

67. Als wihr gudten Windt bekamen, so seglet[e]n wihr durch den Sundt dißes Baß in die Nordtse geyen Engelandt. (Güntzer I: 106v)

68. Als nun der Kauffman und Schiffman sahen die große Nodt, ristet[e]n sie sich auch zu sterben. (Güntzer I: 107r)

69. Z.B. wenn ich das Wort Baum aussprechen soll: so muß ich erstlich die Lippen zusammen schliessen, und, so bald ich sie aufthu, muß jener Schall heraus fahren, der die weitere Erôffnung des Mundes erfordert. (Aichinger III: 20) 
70. Ehe die lateinische Sprache zu ihrer Schónheit gelangte, hatte sie auch allerley diphthongos, welche in alten römischen Schriften, als, den Gesetzten der Könige, den 12. Tafeln, der Duillischen und Sripionischen Tafel gefunden werden, als: $e i$ für $e$ oder $i$, (Aichinger III: 26)

71. Denn als der König in Frankreich dem Kaiser im Jahr 1444. etliche 1000. Mann wider die Schweizer zu Hůlffe schickte, deren Oberhaubt war der Marschall Graf von Armagnac, diese Franzosen aber gegen das teutsche Reich selbst nichts gutes im Sinne hatten, und daher etliche Mahle geschlagen wurden, wurde ihnen von den Teutschen nach ihrem Feldherrn, und zugleich zu Schimpfe der Name der armen Jàcken beygeleget. (Aichinger III: 44)

72. Nachmittag hat man daß Gastmal gehalten, mit einer schönen Auffwartung der zwelff Falckenäten, wie auch dero Schiltwachten, die Herren haben auch gespeiset mit dem Graffen Eben, dem Keyser auch zu Ehren des Keysers Gesundheit truncken, hat man auch Loß gebrunnen 12 Falckeneten Hunden es waren auch besonnen die Herren nun von Stunden, der Keyserin Gesundheit zu trincken mit Bescheidt so hat man auch zu Ehren 10 Falckenäten gehen, wie es an graffen kom[m]en, hat man 2 weniger genom[m]en und sie so gelöset, wie es zu Ehren gneset. (Schuhmacher Chronik II: 327)

73. Nehmlich vierzig Personen seind auff die Donau Brücken gangen dem Eiß durch zu helffen, so bald sie aber angefangen zu schaffen ist der gewalt des Eyses u. Wassers so schleunig angeloffen daß Es die Brücke eingerissen wovon 24 Menschen ertruncken (Schuhmacher Chronik II: 331)

74. So seind die zwey Gerber sampt anderen auß dem WirtsHauß gegangen u. auff die zwey Metzger gewartet, wie sie aber herau $\beta \operatorname{kom}[\mathrm{m}] \mathrm{en}$, so hat der eine Gerber N. Steffen Grieninger den Metzger Michael Werenwag angriffen $u$. in den Bach zu boden geschmissen (Schuhmacher Chronik II: 336)

75. So hat mann sie Gefänglich eingezogen. (So ist der pacient den 17 Novemb. gestorben) Biß sie von einem Ehrsamen rath durch Zeugen u. Kundschafft seindt verhört worden So haben die Medici u. Barbierer in Eröffnung gefunden, daß ein Stich so gefährlich gewessen, der Ihm daß Leben verkürtzt. (Schuhmacher Chronik II: 336)

76. und ist von den Weh-Müetteren besichtiget worden. So haben Sie bey Ihren gefunden, $\mathrm{Da} ß$ Sie Milch gegeben, welches schon ein anzeigen eines Kinds gewessen, darauff ist Sie widerumb von der Obrigkeit Examiniert worden, Ob Sie ein Kind gebohren oder nicht, Sie es aber durchauß gelaugnet, wie man Ihren hat vorgehalten, warumb Sie dann Milch geben, So hat Sie Sich mit disen Worten vernem[m]en lassen, Sie habe vor einem Jahr schon Milch gegeben, (Schuhmacher Chronik II: 378)

77. hat Sie Es auff dem Weg dem schon gemelten Statt-Diener Elias Klein bestanden, Sie habe Es auff der Büne in einem Wollen-Ständlein in Speier ligen, man solle nur daß Schlisselein bey ihrem Vatter holen, wie mans von Ihm begert, So ist Ihm hart unmächtig worden, daß man vermeint Er werde gleich deß Todes sein. (Schuhmacher Chronik II: 379)

78. den 5. Janw. deß Nachts, So daß die Sheur auff der Alten-Burg ist in die Asche gelegt worden und ist deß Nachts umb 1 uhr der Lerm angangen, biß man aber ist hinauff kom[m]en, ist Sie schon fast verbrunnen gewessen, daß keine Errettung mehr zu hoffen und ist durch einen Gott und ruchlossen Menschen angezindt worden \& also sampt Frucht und Futter Jäm[m]erlich verbrunnen. (Schuhmacher Chronik II: 398f.) 
79. Dises war die Ursach weil der Papst Leo der Zehende den Johann Tetzel ein Dominicaner Münch ins Teuschland außgesant Ablaß umbs Gelt zu verkauffen, damit Er Alle Schärtz in Eropa zusamen gebracht welches ist geschehen Anno 1517 Da Tetzeleus seinen verdampten Ablaß-Kram zu Gütterbach ausgeleget. Da hat Lutherus anheben darwider zu prodestieren und mit dem Schwerdt des Geistes zu fechten. Also fieng Sich der Hader an, zwischen Lutherum \& Tetzel. (Schuhmacher Chronik II: 425)

80. Also hat Lutherus daß Revormations Werck angefangen und hat geweret biß auff 30ig in werenter Zeit hat Sich viel Unheil gezeiget von allerhand Rotten \& Secten und theure Zeiten, auch Anno 1524 der Bauren-Krieg sich erhoben, da Sich die Bauren mit ihren Müstgablen \& Pfleglen und Bauersfedren verfast, pfiff Pfeiffer dem gemeinen Mann zum Tantz und bließ Lerma. in dieser Auffrur sind viel Tausend Bauren umkom[m]en, wie die gefangne Auffrierer erbärmlich zusamen koppet. (Schuhmacher Chronik II: 425f.)

81. Als hierauf absolute Fürsten und Tyrannen emporkamen, hatte der Adel in den meisten Städten allen Anlaß und alle Muße, sich ein Privatleben zu schaffen, welches politisch gefahrlos und mit jeglichem feinern Lebensgenusse geschmückt, dabei übrigens von dem der reichen Bürger gewiß kaum zu unterscheiden war. (Burckhardt V: 262)

82. Und als die neue Poesie und Literatur seit Dante Sache eines jeden wurde, als vollends die Bildung im Sinne des Altertums und das Interesse für den Menschen als solchen hinzutrat, während Condottieren Fürsten wurden und nicht nur die Ebenbürtigkeit, sondern auch die eheliche Geburt aufhörten, Requisite des Thrones zu sein, da konnte man glauben, ein Zeitalter der Gleichheit sei angebrochen, der Begriff des Adels völlig verflüchtigt. (Burckhardt V: 262)

83. Auch am Hofe Alexanders VI. kamen Turniere vor. Als Kardinal Ascanio Sforza den Türkenprinzen Dschem fragte, wie ihm dies Schauspiel gefalle, antwortete derselbe sehr weise: in seiner Heimat lasse man dergleichen durch Sklaven aufführen, um welche es, wenn sie fielen, nicht schade sei. (Burckhardt V: 266)

84. Der Reichtum an Instrumenten sodann geht besonders daraus hervor, daß es sich lohnte, aus Kuriosität Sammlungen derselben anzulegen. In dem höchst musikalischen Venedig gab es mehrere dergleichen, und wenn eine Anzahl Virtuosen sich dazu einfand, so ergab sich gleich an Ort und Stelle ein Konzert. (Burckhardt V: 284)

85. Als z. B. S. Bernardino von Siena durch den Papst heilig gesprochen wurde (1450), gab es, wahrscheinlich auf dem grossen Platz seiner Vaterstadt, eine Art von dramatischer Nachahmung (rappresentazione) seiner Kanonisation, nebst Speise und Trank für jedermann. (Burckhardt V: 297)

86. darauf folgten die Geschichten des Lancelot vom See, und die »von Athen«. Und sowie der König nur in Chieri anlangte, wartete man ihm wieder mit einer Pantomime auf, die ein Wochenbette mit vornehmem Besuch darstellte. (Burckhardt V: 298)

87. Aber auch in kleinem Städten strengte man sich bisweilen sehr an. Als Herzog Borso 1453 zur Huldigung nach Reggio kam, empfing man ihn am Tor mit einer großen Maschine, auf welcher S. Prospero, der Stadtpatron, zu schweben schien, überschattet durch einen von Engeln gehaltenen Baldachin, (Burckhardt V: 300)

88. Sobald ich einmal das Geld beisamen habe, so gehe ich aufs Land, u. ich kaufe eine Besitzung, wo ich reich zu werden hoffe, // (Auswandererbriefe V: 1)

89. Nachdem ich lange u. vergeblich auf einen Brief von Euch gewartet will ich Euch nun wieder einmal schreiben. (Auswandererbriefe V: 2) 
90. Seitdem die Radicalen (südliche Repuplicaner welche von der Vereinigten Staaten Regierung nach beendigung der Rebellion hierher geschickt wurden) von den Democraten im letzten Jahre bei der Wahl der Staatsregierung geschlagen wurden gehen die Geschäfte etwas besser aber nicht wie es erwartet wurde. (Auswandererbriefe V: 2)

91. Solange das Fieber anhält darf der Kranke nicht auf einen Augenblick allein gelassen werden. (Auswandererbriefe V: 3 )

92. Wenn ich mit dem Todtenwagen eine Leiche nach dem Todtenhofe zu fahren habe, halte ich einen Mann, der 2 Pferde stellt, dem ich für jede Ausfahrt 5 Th. zu bezahlen habe. Für ärmere Leute od. Kinder nihmt man natürlich den Wagen nicht. (Auswandererbriefe V: 7)

93. Hier sind die Schulen gradirt. Nachdem die Schuler durch alle Klaßen der gewöhnlichen Stadtschulen durch // gegangen sind komen sie in die Hochschule, (Auswandererbriefe V: 14)

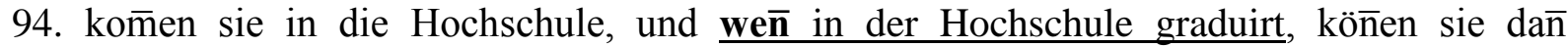
unentgeldlich die Staatsuniversität besuchen, Unsere Jugend unserer Stadt hat was Schulbildung anbelangt große Vorrechte, de $\bar{n}$ sie haben die Gelegenheit in allen wisentschaftlichen Zweigen unterrichtet zu werden. (Auswandererbriefe V: 14)

95. wā̄ wir Zeit haben, wollen wir uns wieder abnehmen lassen vielleicht dieses Spätjahr noch und wollen dir sie schicken in einem Brief. (Auswandererbriefe V: 15)

96. Wir geniesen jetzt Gottlob wieder Frieden Sobald Östreich mit den Süddeutschen geschlagen war schloß es unter vermittlung Napoleons Frieden ohne an seine Verbündeten zu denken. (Auswandererbriefe V: 15)

97. Als ich gestern in der Stadt Perry war habe ich Johanes Kempf Bruder von Pfeifle und Schulkamerad von Schwester Magdalena auch gesehen [...] (Auswandererbriefe V: 19)

98. wen ich von der Arbeit nach Hauße kam, und frackte sie ob sie geschrieben hat, so war die Andwort nein. (Auswandererbriefe V: 20)

99. Dieser alltägliche Vorgang ist ein gutes Beispiel für eine Leistung, die für unser Erkennen der außersubjektiven Wirklichkeit von grundlegender Bedeutung ist. Sie bringt unser Erkennen dem an sich Seienden dadurch näher, daß sie Vorgänge und Zustände innerhalb des erlebenden Subjekts mit ins Kalkül zieht. Sooft es uns gelingt, die Herkunft eines Teil-Phänomens in unserem Erleben auf innere, subjektive Vorgänge oder Zustände zurückzuführen und aus unserer Betrachtung der außersubjektiven Wirklichkeit auszuschalten, sind wir in unserer Erkenntnis einen kleinen Schritt näher an das unabhängig von unserem Erkennen Existierende herangekommen. (Lorenz VII: 13)

100.Ohne den physiologischen Mechanismus, der das quantitative Kontinuum verschiedener Wellenlängen in Bänder verschieden erlebter Qualitäten einteilt, hätten die Physiker nie bemerkt, daß ein Zusammenhang zwischen der Wellenlänge und dem Winkel besteht, in dem das Licht vom Prisma gebrochen wird. Als der Physiker, der Erforscher des Außersubjektiven, das $\mathrm{zu}$ verstehen gelernt hatte, war wieder der Erforscher des erkennenden Subjektes an der Reihe: (Lorenz VII: 25) 
101.Wann immer eine kleine Zunahme des Wissens über unseren Weltbildapparat eine neue kleine Korrektur des Bildes erheischt, das er von der außersubjektiven Realität entwirft, und wann immer umgekehrt ein kleiner Fortschritt unseres Wissens um das an sich Seiende uns in den Stand setzt, eine neue Kritik an unserem »perceiving apparatus « zu üben, wächst unsere Berechtigung, unsere Erkenntnistheorie für richtig zu halten, deren Natürlichkeit nicht mehr mit Naivität verwechselt werden darf. (Lorenz VII: 26)

102.Einen einzelnen Stern, der durch eine kleine Lücke in der Wolkendecke scheint, vermag man nicht zu benennen; erst wenn ein Stück klaren blauen Himmels größer wird und man auf ihm mehrere Sterne in ihrer räumlichen Beziehung zueinander sieht, ist man in der Lage, dieses Muster mit einem bestimmten Teil der bekannten Sternekarte zur Deckung zu bringen, mit ihm zu identifizieren. (Lorenz VII: 41)

103.Dieser Schlaf sollte nicht lang' dauern, da kam N. Als sie uns durchgekitzelt hatte und wir fällig (?!?) wach waren, servierte ich Eis um die Gemüter zu kühlen. Da kamen auch meine Eltern aus Brühl zurück. (Jugendtagebuch VII: 1)

104. So ist er (D.) vom Krankenhaus in die Nervenklinik verlegt worden. Kaum verlassen, war er auch wieder drin - erneutes Koma. Nun soll er nach Tübingen verlegt werden! (Jugendtagebuch VII: 1)

105.Am Nachmittag zeigte uns I. noch Dias von Jersey und danach wurde rumgealbert und als wir alleine waren hörten wir unsere traditionelle G. Geils Band. (Freeze-Frame/Angel in Blue...) $\rightarrow$ MAMA findet die a Songs gut. (Jugendtagebuch VII: 5)

106.Ich liebe ihn sehr, und ich glaube auch nicht, daß es zu früh ist. Die einzige riesengroße klitzekleine Hürde, die leider noch nehmen muß ist das Gespräch mit Mama und der Frauenarzt, dann die Zeit, bis man die Pille hat, die zu einem paßt. Wenn das alles überstanden ist, ist sicher auch noch ein Monat vorbei. (Jugendtagebuch VII: 7)

107.Um 8 Uhr abends waren L. und ich bei N., zum Lasagne kochen! Wir standen da zu viert (+ Frau M.) in der Küche und wurschdelten so vor uns hin. Besonders gierig wurde von der „Beschamellsoße“ genascht und als wir die 1. Lasagneform (für 4 Pers) voll hatten war noch ein/4 vom Topf voll mit der Hackfleischfüllung. Also löffelten wir sie so aus, und machten uns an die 2. Lasagneform. Denn wir wollten Lasagne für 8 Personen machen, damit auch ja jeder genug hatte! (Jugendtagebuch VII: 7)

108.Er hat keine anderen Kumpels. Er soll doch mal was ohne mich unternehmen. Ich weiß, wenn ich So abends nichts mit ihm unternehme sitzt er gelangweilt herum. (Jugendtagebuch VII: 8)

109.Mit L. zusammen sind wir rumgefahren, der fährt schon echt gut. (Man glaubt kaum, daß er in seinem Leben insgesamt 7 Tage skigefahren ist!) Es hat wahnsinnig Spaß gemacht. Wir haben laut gesungen, und als wir zum „Mittagessen“ zum BUS runterfuhren kugelten wir in 1-m-hohen SPulverschnee! den Berg runter. (Jugendtagebuch VII: 9)

110.Da war auch schon der Bus, der 60 Nägianer in die rallige Schnee - und Skifans sind in's Allgäu zu transportieren. Als endlich! ALLES verstaut war, hatten auch L. und ich unseren Platz. Los ging's! (Jugendtagebuch VII: 9)

111.also M. war echt saumäßig süß. Er grinste einen so lieb an, tja und ich bin halt auch nur ein Mädchen (nein, nicht ein LIEBESBLITZ!!) L. ist MEIN PERFEKTER TRAUM) Als ich nun am so kurz vor der Abfahrt einen Sauerkraut schlabberte (ich saß gegenüber zur stets geöffneten Küchentür) stand dort der 3. Mr. X, na, ich nenn' ihn mal G., und grinste mich zwischen den Handtüchern. (Jugendtagebuch VII: 9f.) 
112. Oder starb er schon beim Dauerlauf die $5 \mathrm{~km}$ bis nach oben, wenn er hinfiel, fiel der Schuß, oder er wurde zu Tode getrampelt. (Jugendtagebuch VII: 12)

113.Nach der Stadtrundfahrt ging's zurück zum Bahnhof. 1/2 Std. Fahrt ins nächste Kaff von dort noch 1 Std Fahrt mit dem Bus nach Hohenstein. Nachdem wir die „U-HA-Stelle“ passiert hatten (es ging kurz, steil ab und der Magen hopste) war es nicht mehr weit bis zur Herberge. (Jugendtagebuch VII: 13)

114.Nach dem Essen hatten wir erst noch das Bettzeug verteilt und als wir dann endlich fertig waren gingen wir: Ich, B., C., S., D., L. und ich, S., und K., I., A., E., K. und K. zur Dischgo. (Jugendtagebuch VII: 13)

115.Es blieb bei der Vorstufe und das ist ja auch gut so. Wenn wir miteinander schlafen brauchen wir absolute Ruhe! (Jugendtagebuch VII: 14)

116.Als dann plötzlich jemand reinkam lagen wir auf dem Bett auf dem Bauch und lasen „Hägar“. (+ Licht) Da kam noch die ganze Meute herauf und es kam die größte Kissenschlacht in Gange, die man sich je vorstellen kann. (Jugendtagebuch VII: 14)

117.Dabei: Papa, Mama, R., N., und L. Kurz bevor mein Zug kommen sollte ging er. Eigentlich wäre ich geschockt gewesen, aber ich wußte, daß er mit mir bis nach Stuttgart fahren würde (von Mama) (Jugendtagebuch VII: 16)

118. Als es dammrig[?] wurde, wurden auch mal Pausen gemacht, wo gesungen wurde und christliches vorgelesen wurde. (Jugendtagebuch VII: 17)

119.Ch. versuchte mir einen Witz auf Deutsch zu erklären. Als ich ihm Französisch nacherzählte, wurde er mir klar: (Jugendtagebuch VII: 17)

120.Da wird ein Haus gebaut und immer wenn ein Arbeiter einem anderen einen Stein gibt geht es „bitteschön, dankeschön, bitteschön, dankeschön...“ (Jugendtagebuch VII: 17)

121.Es ist komisch. Als ich aus Stuttgart losfuhr, fuhr ich in's Unbekannte. (von daheim in's Fremde) (Jugendtagebuch VII: 20) können Stănden müssen

Konditionalsätze (Kapitel 5.2)

\section{Eingeleitete Konditionalsätze}

1. ... wann aber die Daurung eine Sache schătzbar machet/ so ist das geschriebene Wort beståndiger/ und deßwegen wehrter zu achten/ als die in leichter Lufft verlohrne und bald vergessne Rede. (Harsdörffer I: 6)

2. ... und wann man solcher eingeschnittenen Schrifft-Zeichen viel/ auff etliche zusammengehefften Bretlein gesamlet/ darzu gemeider Baum sehr bequemlich/ist es ein Buch genennet worden: ... (Harsdörffer I: 8)

3. Hiervon saget die Ràthsel also: ... Die Gans (Federn) beflecket mich: wann ich besudelt bin/ erhellt der Menschen Kunst und hält der Warheit Sinn. (Harsdörffer I: 11)

4. Wann meine stumme Red urkundet di $\beta$ und das/ so bring ich manchem Gunst/ und manchem Neid und Haß. (Harsdörffer I: 12)

5. Wann sie aber die Feder auff den Hut stecken/ so müssen sie selbsten bekennen/ die Feder seye das oberste in der Welt/ ... (Harsdörffer I: 13) 
6. Denn wann alle Kundigung in dem mundlichen Unterricht von Anfang der Welt bestanden wăre/ sollte/ besorglich/ so wenig als nichts/ von der alten nutzlichen und nỏhtigen Beobachtungen/ auff uns geerbet seyn/ und wir von vielen Wissenschafften nicht mehr wissen/ als von der Barder Heldenliedern. (Harsdörffer I: 15)

7. Wann man von Anfang der Welt sollte Bücher gedruckt haben/ so würden derselbigen noch viel eine grossere Anzahl zu finden seyn; ... (Harsdörffer I: 15)

8. ... ein jeder aber/ der recht schreiben und rechnen kann/ findet seine Stelle noch wol in dieser Welt/ und wann er solches treiben mag/ wird er nicht Hungers sterben. (Harsdörffer I: 16)

9. ... daß mann mehrgemelde Schreib-Kunst mit dem Senffkorn vergleichen kann/ welches das kleineste ist unter allen Samen: wann es aber bekleibet und auffwăchst/ so ist es das gröste unter allen Kohlkråutern; ... (Harsdörffer I: 16)

10. Wann ihr von Gottes wegen mit mir redet/ so solt ihr die eitlen Titul auslassen/ und weiß ich wol/ was Christus spricht: ... (Harsdörffer I: 27)

11.... und wann derselben Beambten geschrieben wird/ setzet man darzu Landgråfisch/ Hessisch oder Leuchtenbergisch. (Harsdörffer I: 30)

12. Wann eine Fưrstin/ Gräfin/ oder Freyfrau ihres Herrn oder Gemahls Stammens wăre/ und nach Geburt gleichformige Ttul führe/ wuerden ihr gleich nach dem Tauffnamen alle Herrschafften und Ehrwort/ vermòge ihres Herrn und Gemahls Titul/ ebenmåssig zugeschrieben/ mit Auslassung des Worts: Geborene R. von/ ... (Harsdörffer I: 30)

13. So auch eines Kơnigs/ der Fưrsten Gemahl/ an Geburt und Stammen/ viel nidriger/ dann ihr Herr und Gemahl gradiert/ als: Wann sie eine geborne Grăfin/ Freyin/ 2c. wăre/ wird ihr Geburt Stamm nicht/ aber ihr sonst alle Ehrenwort und Herrschafften/ wie ihrem Gemahl/ zugeschrieben. (Harsdörffer I: 30f.)

14. ... und wird verhoffentlich in keinem verfehlet seyn; $\underline{\text { Im Fall aber solches aus ungleichem }}$ Bericht beschehen/ ist es ohne Willen und praejudicirlichen Nachtheil/ zu entschuldigen. (Harsdörffer I: 31)

15. Wann wir uns nun zu der guten Gewonheit halten sollen/ (massen die bòse Gewonheit/ wie etwan das gebrächliche Vollsauffen/ Spielen/ und Müssiggehen/ niemand rechtfertigen wird/) ist ferners zu wissen/ daß solche ungezweiffelt und richtig/ oder zweiffelhafftig und streittig ist. (Harsdörffer I: 167)

16. Wann sich nun ansehliche Leute fănden/ welche erstbesagte und dergleichen Worter in gebrauch brächten/ so wurde ihnen ein jeder billich folgen/ und nachahmen: (Harsdörffer I: 168)

17. Wann solcher Ursprung ermangelt/ so muß die Aussprache Richter seyn/ ... (Harsdörffer I: 467)

18. In dem nun beyde Grundseul der Schreibrichtigkeit nicht ergreiffen/ muß man sich an die in Cantzleyen beliebten Gewohnheiten halten/ und lieber mit andern fehlen/ als allein klug seyn wollen/ und mit guten Ursachen verlachet werden. (Harsdörffer I: 467)

19. Denn wenn ich sage Glük / so gibt es einen groben / langsamen Ausspruch / wie bey den Schlesiern und Voigtlăndern zu finden. (Harsdörffer I: 468) 
20. Wenn ich aber sage Glück / so gehet es hastig / und resch von der Zunge / und ist lieblicher anzuhờren. (Harsdörffer I: 468)

21. Dann im Anfang gehen die Krăfften hervor auff ihre Vollkommenheit zu / wann selbe erlangt / nehmen sie ab / und erleschen: (Harsdörffer I: 468)

22. Wann man unwidersprechlich recht schreibet: Jahr/sagen/ jenen/ und Laut/ Haus/ euch: so müssen die unterschiedlichen Buchstaben einen unterschiedlichen Gebrauch haben ... (Harsdörffer I: 561)

23. Wann man die Gebietungsweise für ein Stammwort setzet/ kann man aus den Vor- und Nachsyllben alles leichtlich beurtheilen/ da man sonsten schwerlich eine durchgeheude Richtigkeit wird finden kỏnnen. (Harsdörffer I: 562)

24. Wann aber ein Wort aus erstbesagten Ursachen nicht kann beurtheilet werden/ so sihet man auff die gewóhnliche Schreibung/ deren Grund ist die wolausgesprochene Rede/ oder Mundart/ ... (Harsdörffer I: 563)

25. Wann ich sage ein Kartenspiel/ so weiß ich/ daß ein Spiel sey/ welchesman mit der Karten spielet: (Harsdörffer I: 565)

26. Wann man die Sache genau nehmen wolte/ so müßte man einen Unterscheid machen/ zwischen dem Zwergstrichlein/ comma genannt/ (/) und das Beystrichlein / semicomma (,) genannt/ ... (Harsdörffer I: 566)

27. Wann etwas darzwischen gesetzet wird/ daß zwar zu der gantzen Meinung gehört/ aber dieselbe zweiflig/ oder zu verstehen schwer machet/ so könte man wol das besagte Beystrichlein gebrauchen/ ... (Harsdörffer I: 567)

28. Wann die Meinung durch etliche Doppelpunct gesondert wird/ und selbe widerumb zertheilet werden/ so hat das Strichpünctlein statt/ ... (Harsdörffer I: 567)

29. Dann wann sie glauben könten/ das Glück und Unglück von GOTT komme/ (Sirach. II.) und daß der Wille GOTTES die hochste Gerechtigkeit seyel wie Petr. Martyr redet/ so würden sie solchen nicht ungeduldig widerstreben/ sondern der Gnaden-Hülffe des himmlischen Vatters in der Stille werwarten. (Harsdörffer I: 665)

30. Wann nun wir Menschen dieses einfältig glaubten/ und behertzigen wollten/ ist keines Weges zu zweifflen/ sie wừrden Gott ůber alles vertrauen/ ihn fürchten/ und auch ob der Straffe willen kindlich lieben; (Harsdörffer I: 666)

31. So ein Waltforster solt zu eich komen, wirdt er eich gefenglich nacher Hagenau fieren. (Güntzer I: 41r)

32. Aldar wahr gleich ein Dorff. Schieß also meinen Busser loß, daß die Kugel in eine Eichen ging, undt sprach zu den zweyen Merdtern: Saet ihr es, ihr Merdter, so ihr noch einmall an unß angesetz hetten, so wolt ich einem diße Kugel in Leib geschoßen haben. (Güntzer I: $43 \mathrm{v})$

33. So sie mein Gelt gewußt hatten, so hatten sie mich vileicht ermordet, dan sie haben sich auch in dißem Grieg auffgehalten. (Güntzer I: 54r)

34. So ich nuhr ein Trunck Waßers kondt haben auß einer Murrlachen, so wirdte ich erfreyet. (Güntzer I: 54v) 
35. Die Bauren reittet[e]n unß nach, wolten unß dodt schlagen, sagten, wihr hetten $\mathrm{i}[\mathrm{h}] \mathrm{nen}$ Weißgezig von Leinwatt von der Pleich gestollen. Sie zogen un $\beta$ auß, suchet[e]n alenthalben bey un $\beta$, aber es hats sich nicht erfunden. Und sie es bey unß finden werden, so wollen sie unß gleich alsobalt ohn ale Genadt an die Beim auffhencken. (Güntzer I: $55 \mathrm{v})$

36. So ich verradten wirdt, so dirfften sie mich ins Feihr setzen. (Güntzer I: 58r)

37. Habe aber die Wahrheitt gespartt, dan ich und die Grallen haben den Babst niemallen gesehen, dan so mich der Babst gesehen hatt und [ich] verratten wehr worden, so dirffte er mich im Rauch gen Himel geschickt haben. (Güntzer I: 63r)

38. So ich alenthalben um ein Gelt solte zeren, so hete ich 5 oder 6 Wochen Zerung gehapt, hette doch entlich die Bauren mießen anspróechen. (Güntzer I: 83r)

39. So mihr einer auß dißem Landt zuhanden [kommt], so wil ich solche Gudtdaten widerunb vergelten und wettmachen. (Güntzer I: 92v-93r)

40. So ich die schöhne Sprich und Verheißungen Gottes Wordts nicht wußtete und deß Jobs gedachte, so wehre ich in meinem Leiden vergangen und hette nicht könen außharen in dißem schwôhren Streitt und Kampff. (Güntzer I: 102r)

41. Wenn dann aber die andre Gattung der gemeinen fremden Wörter das $C$, auch nach Herrn Gottscheds Meinung, behalten sollen: so werden, wie ich meine, noch vielmehr die eignen griechischen und lateinischen Namen das $C$ behaubten: (Aichinger III: 7)

42. Diesen spricht zwar Herr Gottsched, so sie griechisch sind, das $K$ zu, ausser, wo die Aussprache das $C$ erfordert, wie in Cimon: doch, so wir den Griechen recht folgen wollten, so müssten wir auch in solchen ja selbst in lateinischen das $c$ verwerffen, indem ja Cimon ganz griechisch ist, und nicht Simon, sondern Kimon heißt; (Aichinger III: 7)

43. so wir aber in einem Stuck der Gewohnheit nicht ungehorsam sind, so müssen wir auch in andern dabey bleiben, damit wir nicht unbeständig seyen. (Aichinger III: 7)

44. Wenn aber $n$ vorhergehet, so wird der Laut dieser beiden also vermischet, daß man ihn nicht anderst als mit lebendiger Rede ausdrücken kann, als Gang, Jüngling, singen. (Aichinger III: 9)

45. Wenn nun einer sonst im lateinischen das $u$ und $v$ im Schreiben unterscheidet, so thut er unrecht, wenn er dem $q$ das $u$, nicht $v$, beysetzet. (Aichinger III: 13)

46. so sie aber zertrennet werden, muss man am Ende der Zeile gewiß ein kurzes machen, als: Aus-satz, das-selbige. (Aichinger III: 14)

47. Wenn man demnach die Wörter, so mit $\beta$ geschrieben werden, mit lauter grossen Buchstaben schreiben will, so muß man auch zwey $S$ machen, als: GROSS, HEISST; (Aichinger III: 14)

48. Wenn das $h$ dem $t$ seinen Hauch mittheilete, so müsste th schärffer lauten, als $t$. (Aichinger III: 16)

49. Z.B. wenn ich das Wort Baum aussprechen soll: so muß ich erstlich die Lippen zusammen schliessen, und, so bald ich sie aufthu, muß jener Schall heraus fahren, der die weitere Eröffnung des Mundes erfordert. (Aichinger III: 20) 
50. Wenn ich Herrn Prof. Gottsched folgen wollte: so müsste ich nun die Selbstlauter in lange und kurze eintheilen. Ich thue es aber nicht. (Aichinger III: 21)

51. Wenn aber $A, O, U$, Versale sind, so wird das $e$ dahinter gesetzt, als: Aerger, Oehr, Uebel. (Aichinger III: 23)

52. Ue, wenn es genennet wird, heißt $u i$; wenn es aber geschrieben wird, setzt man nach 27 . §. ein $e$ drüber oder dahinter. (Aichinger III: 25)

53. Doch wenn dreyerley Mitlauter zusammen kommen, wie in Kampf, Schimpf u.d.g. so mag die Verdopplung der letzten wohl für überflüssig gehalten werden. (Aichinger III: 38)

54. Sonderlich, wenn kernicht so viel heißt, als kräffrig, nachdrücklich: so kommt es von Kern, nicht von Korn. (Aichinger III: 49)

55. Wenn gedachter Name vor dem 17ten Jahrhunderte nicht gefunden wird: so ist es glaubwürdig genug, daß Obentraut am ersten der teutsche Michel genennet worden sey. (Aichinger III: 52)

56. So hat Sich der wol Meridierte Herr B. M. Samuel Clewer einen Fußfahl vor Ihro Generalitet gethan, weil Sie tautzbrüder gewessen. So hat Er zu Ihm gesprochen, stehe auff Bruder, dein Hauß und deiner Freunden Hauß sollen verschont werden. So hat erstgemelter H. Clewer zur Antwort versetzt: Bruder wan $\beta$ soll auff daß ankom[m]en, So soll mein Hauß daß erste sein. So ist Es auff eine gnädige Capitulation ankom[m]en, (Schuhmacher Chronik II: 443f.)

57. Bistümer, Domherrnstellen und Abteien wurden oft nach den merkwürdigsten Rücksichten, aber doch nicht wesentlich nach Stammtafeln vergeben, und wenn die Bischöfe viel zahlreicher, ärmer und aller weltlichen Fürstenhoheit in der Regel bar und ledig waren, so blieben sie dafür in der Stadt wohnen, wo ihre Kathedrale stand, und bildeten samt ihrem Domkapitel ein Element der gebildeten Bevölkerung derselben. (Burckhardt V: 261f.)

58. Wenn sich dagegen schon im 15. Jahrhundert Vespasiano Fiorentino dahin ausspricht, daß die Reichen ihr ererbtes Vermögen nicht vermehren, sondern ihre ganze Einnahme ausgeben sollten, so kann dies im Munde eines Florentiners nur von den großen Grundbesitzern gelten. (Burckhardt V: 265)

59. Der sonstige Aufenthalt von Nichtadligen in der Nähe des Fürsten ist damit vollends nicht ausgeschlossen; es handelt sich nur darum, daß dem vollkommenen Menschen, dem Cortigiano, kein irgend denkbarer Vorzug fehle. Wenn ihm dann eine gewisse Zurückhaltung in allen Dingen zum Gesetze gemacht wird, so geschieht dies, nicht weil er von edlerm Geblüte stammt, sondern weil seine zarte individuelle Vollendung es so verlangt. (Burckhardt V: 267)

60. Ein Beweis wird sich jedoch natürlich niemals leisten lassen, und wenn es sich um die Priorität von Reinlichkeitsvorschriften handelt, so möchte die Ritterpoesie des Mittelalters deren ältere aufweisen können. (Burckhardt V: 270)

61. Das Beste tat freilich Dante selber durch sein großes Gedicht. Der toscanische Dialekt wurde wesentlich die Basis der neuen Idealsprache. Wenn damit zu viel gesagt sein sollte, so darf der Ausländer um Nachsicht bitten, indem er schlechtweg in einer höchst bestrittenen Frage der vorherrschenden Meinung folgt. (Burckhardt V: 273) 
62. Die Mäßigkeit und die freie, leichte Geselligkeit schwanden zu gleicher Zeit. Wenn wir die Verfasser von Dialogen beim Wort nehmen dürften, so hätten auch die höchsten Probleme des Daseins das Gespräch zwischen auserwählten Geistern ausgefüllt; (Burckhardt V: 276)

63. Wenn irgend etwas unsere obige Behauptung beweist, so ist es diese Frauenpoesie mit ihrem völlig männlichen Ton. (Burckhardt V: 286)

64. Aktive literarische Tätigkeit verlangt man nicht von ihr, und wenn sie Dichterin ist, so erwartet man wohl irgendeinen mächtigen Klang der Seele, aber keine speziellen Intimitäten in Form von Tagebüchern und Romanen. (Burckhardt V: 287)

65. Wenn aber irgendein Kirchenfest einen allgemeinen Anspruch auf die höchste Anstrengung hatte, so war es Fronleichnam, an dessen Feier sich ja in Spanien jene besondere Gattung von Poesie anschloß. (Burckhardt V: 298)

66. Wenn bei allen Festen lebende Figuren als Statuen in Nischen, auf und an Pfeilern und Triumphbogen vorkamen und sich dann doch mit Gesang und Deklamation als lebend erwiesen, so waren sie dazu durch natürliche Farbe und Gewandung berechtigt; (Burckhardt V: 299)

67. Wen es gut geht kan man gewöhnlich am 10 Tag das Bett u. am 12 Tage das Haus verlassen; Man muß sich aber dan noch sehr in acht nehmen im Essen $u$. Trinken, den die kleinste Unregelmäßigkeit führt sehr oft den Tod herbei. (Auswandererbriefe V: 3)

68. Zur Zeit, da ich das Gold in Papier umwechselte, stuhnd es niedrig, nehmlich ein Gold Thaler entwarf 130 in Papier, die 377 Th. entwarfen 489. in Papier, u. einige Wochen später stieg das Gold auf 140, wē̄ ich es zu dieser Zeit hätte könen umwechsele, hätte ich 37 Papier Th. gewōnen. (Auswandererbriefe V: 6)

69. Wēn man sich theuschen thäte, daß sich der Papier Th. nicht nach $u$. nach verwerthen $u$. dem Gold Tha. gleich komen würde, so würde unser beschiedenes Erbtheil zuletzt sehr klein ausfallen, den bildet euch nicht ein, daß ein Papier Th. soviel Werth habe wie ein 5 Fr. Stück, (Auswandererbriefe V: 6)

70. bildet euch nicht ein, daß ein Papier Th. soviel Werth habe wie ein 5 Fr. Stück, nein, wē̄ wir ihn im Magasin für Kleider oder d. gl. ausgeben, so hat er bei uns nicht mehr werth, als bei euch ein 2 Fr. Stück. (Auswandererbriefe V: 6)

71. den ich bin so seelenallein in meinem neuen Geschefte, welches meine Gedanken, Kräfte, kurze Wiß[en]schaft $u$. besonders die Zeit sehr in Anspruch nihmt, auch denke ich, wen̄ ihr den Botschafter gut durchmustert, könt ihr manches daraus entnehmen, das ich euch schonst gerne schreiben möchte. (Auswandererbriefe V: 6)

72. Aber Gescheftsbriefe durch andere Schreiben zu lassen, ist hier nicht Mode, wenn einer das thut, so wird er nicht als Gescheftsmann angesehen, u. so behelfe ich mir selbst so gut ich kann. (Auswandererbriefe V: 7)

73. Ich meine aber, wenn einer seit 12 Jahren in der Fremde, wie ich, unter anderer Autorität gestanden ist, so soll es Pflicht u. Aufgabe sein selbständig zu werden, oder müssten wir nicht von Wilhelm Tells Gefühlen besselt sein. (Auswandererbriefe V: 7) 
74. Uebrigens, wenn, ihr liebe Eltern!, die Uebelstände des Gesellenlebens wissen u. im rechten Lichte erkennen könntet, wurdet ihr Gott danken, daß ich nun einmal frei u. nicht ein ganz verdorbenes Subject nach Leib u. Seele geworden bin; (Auswandererbriefe V: 7)

75. Mithin geht's mir auch wie andere Europäere, wenn man hier einmal klimanisirt ist, so ist man nicht leicht zu bewegen in's alte Land, wie man's hier nennt, zurück zu kehren. (Auswandererbriefe V: 8)

76. Nun so es Gott so gefällt so sollte es uns auch gefallen und wir sollten mit dem fromen Dichter sagen könen was Gott thut das ist wohlgethan. (Auswandererbriefe V: 10)

77. Und wenn erst einmal die projecktirte // Eisenbahn nach Saanen wird vollendet sein, und ich und Br. Jakob noch leben, werden wir Euch so nebenbei einem Besuch abstatten. (Auswandererbriefe V: 14)

78. Liebe Geschwister Schwager und Schwägerin Gottes Segen und Frieden zum Gruß wē̄ Euch unser schreiben Gesund antrift wie es uns verläßt wird es uns herzlich freuen, wir sind Gottlob auch alle gesund der Vater hat viele Altersbeschwerlichkeiten [...] (Auswandererbriefe V: 15)

79. wir sind beide bei der Landwehr bis ins 32. Jahr. wan̄ etwas losbricht so müßen wir beide fort. (Auswandererbriefe V: 15)

80. wān ihr es 4 Wochen lang so machet so sei sie Ka Kurirt er habe schon ziemlich der Art Kurirt täglich 1 mal soll sie baden. // (Auswandererbriefe V: 15)

81. Wā̄ es ihm diesmal mit seinem Weinhandel gelingt so kō̄t er in einigen Jahr[en] wieder, die Reise sagte er fürchte er nicht. (Auswandererbriefe V: 16)

82. Du bist ietzt nicht mer zu iung, und ich denke es wird dich auch manchmal in den Elbogen

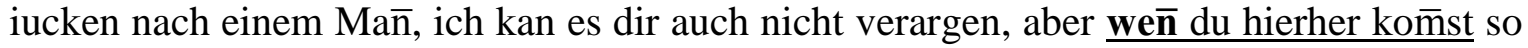
will ich thun was ich $k a \bar{n}$ daß du einen bekomst ich habe einige die für mich Arbeiten welch gute Men̄er machen würden // (Auswandererbriefe V: 21)

83. Die Mutter soll ganz außer Sorgen sein mir kön̄te es nicht besser gehen wen Ihr liebste Mutter wistet wie lustig als es bei uns wär dañ würde sie keine Sorge mehr haben. (Auswandererbriefe V: 25)

84. kauft Euch und das Geld Lebensmittel oder eine Farm // wē̄ ihr nicht mehr so viel Geld habt so giebt auch der Onkel oder ihr pachtet Euch Land und seid fleißig ich geh dan auch wieder zu Euch dan könt Ihr Euch inn 2 Jahr selbst eine Farm kaufen. (Auswandererbriefe $\mathrm{V}: 26)$

85. und bin froh das ich in dieses Land gekomen bin aber wē ich Euch noch einmal sehen würde da meitte ich Ich hätte die ganze Welt Doch ich hoffe daß wir einander wieder sehen. (Auswandererbriefe V: 26)

86. Liebste Eltern seid außer Sorgen ich kā̄ in der neuen Welt gut durchkōmen und das Heimweh plagt mich nicht wē es mich quelen sollte denke ich jedesmal an Eure Dienstplätze doch ist keine Stunde im Tage wo ich nicht an Euch Ihr Lieben denke. (Auswandererbriefe V: 28)

87. Als Historiker glaubte er [Spengler, P.K], daß eine unentrinnbare »Logik der Zeit« und ein unaufhaltsamer Prozeß des Alterns an der Auflösung jeder Hochkultur, einschließlich der unseren, die Schuld trüge. Wenn man aber als vergleichender Verhaltensforscher und 
Arzt die heute viel deutlicher gewordenen Verfallserscheinungen unserer eigenen Kultur betrachtet, sieht man selbst bei dem geringen Stande unseres gegenwärtigen Wissens eine Reihe von Störungen, deren pathologische Natur offensichtlich ist. (Lorenz VII: 16)

88. Von besonderem Interesse für den nach Objektivität strebenden Forscher sind jene Leistungen unserer Wahrnehmung, die uns das Erlebnis jener Qualitäten vermitteln, die gewissen Umweltgegebenheiten konstant anhaften. Wenn wir einen bestimmten Gegenstand, etwa ein Blatt Papier, in den verschiedensten Beleuchtungen in derselben Farbe »weiß« sehen, wobei die von ihm reflektierten Wellenlängen je nach Farbe des einfallenden Lichtes recht verschieden sein können, so beruht dies auf der Funktion eines sehr komplizierten physiologischen Apparates, der aus Beleuchtungsfarbe und reflektierter Farbe eine dem Objekt konstant anhaftende Eigenschaft errechnet, die wir schlicht als die Farbe des Gegenstandes bezeichnen. (Lorenz VII: 22)

89. Gerade angesichts der gewaltigen Verschiedenheit all dieser Weltbildapparaturen erscheint eine Tatsache von tiefster Bedeutung: Soweit sich ihre Meldungen auf dieselbe Umweltgegebenheit beziehen, widersprechen sie einander nie. (Lorenz VII: 24)

90. Es scheint mir abstrus, eine andere [Erklärung der Übereinstimmung zwischen den Repräsentationen der Außenwelt verschiedener Lebewesen, P.K.] finden zu wollen als die, daß sich alle diese so mannigfaltig abgewandelten Formen möglicher Erfahrung auf dieselbe außersubjektive Wirklichkeit beziehen. "Wenn die Teilnehmer an unserer Diskussion«, so sagte ich einst in später Nacht im Parkhotel in Königsberg bei einer »Nachtsitzung« der Kantgesellschaft, »darin übereinstimmen, daß jetzt und hier fünf Weingläser auf dem Tische stehen, so ist es mir unverständlich, wie irgend jemand eine andere Erklärung hierfür suchen kann als die, daß, was immer sich hinter der Erscheinung $>$ Weinglas $<$ verbergen mag, tatsächlich fünffach vorhanden ist. «(Lorenz VII: 24f.)

91. Durch eine vereinfachte didaktische Darstellung mag der Eindruck entstehen, daß der erste Schritt des naturwissenschaftlichen Vorgehens im Voraussetzen der physikalischen Realität bestehe. Wenn man etwa die Leistungen des Farbsehens zu erklären versucht, fängt man meist mit der physikalischen Natur des Lichtes und dem Kontinuum der Wellenlängen an, um dann erst zu den physiologischen Vorgängen überzugehen, die aus diesem ein Diskontinuum von Qualitäten machen. (Lorenz VII: 25)

92. Wenn man das eine Mal den Blick auf unseren Weltbildapparat richtet und das andere Mal auf die Dinge, die er schlecht und recht abbildet, und wenn man beide Male, trotz der Verschiedenheit der Blickrichtung, Ergebnisse erzielt, die Licht aufeinander werfen, so ist dies eine Tatsache, die nur aufgrund der Annahme des hypothetischen Realismus erklärt werden kann, der Annahme nämlich, daß alle Erkenntnis auf Wechselwirkung zwischen dem erkennenden Subjekt und dem erkannten Objekt beruht, die beide gleichermaßen wirklich sind. (Lorenz VII: 26)

93. Wenn etwa ein Tischler einen Tisch schafft, so ist tatsächlich die Idee des Tisches vorhanden, ehe sie ihre Verwirklichung in einen realen Tisch findet, sie ist vollkommener als dieser, denn sie hat z. B. keine Astlöcher, die dem planenden Handwerker sicher ebensowenig »vorgeschwebt« waren wie ein gelegentliches Ausrutschen des Hobels. (Lorenz VII: 29)

94. Die Idee ist auch unvergänglicher als das reale Möbel, denn wenn dieses von Holzwürmern zerfressen oder von Kindern zertrümmert wird, kann sie durch die Reparatur des alten oder die Herstellung eines neuen Tisches eine neue Verwirklichung finden. (Lorenz VII: 29) 
95. Daß lebende Systeme um so mehr Energie schlucken können, je mehr sie schon geschluckt haben, ist selbstverständlich: Wenn es einem Lebewesen gut geht, so wächst es, und es pflanzt sich fort. (Lorenz VII: 35)

96. Schlechterdings alle komplexen Strukturen sämtlicher Organismen sind unter dem Selektionsdruck bestimmter arterhaltender Leistungen entstanden. Wenn der Biologe auf eine Struktur stößt, deren Funktion er nicht kennt, ist es für ihn selbstverständliche Pflicht zu fragen, worin ihre Leistung bestehe. (Lorenz VII: 38)

97. Wenn wir z. B. fragen: „Wozu hat die Katze spitze, krumme Krallen?« - und darauf antworten: »zum Mäusefangen«, so sind Frage und Antwort Kurzfassungen für das Stellen und die Lösung eines Problems. (Lorenz VII: 38)

98. Wenn ich nun dieses Wort [den Begriff Information, P.K.] im Sinne der Umgangssprache gebrauche, könnte dies deshalb $\mathrm{zu}$ Mißverständnissen führen, weil die Informationstheoretiker mit ihm einen anderen, um sehr viel weiteren Begriff verbinden. (Lorenz VII: 38)

99. Wenn ich im folgenden von der aller Anpassung zugrunde liegenden Information rede, so meine ich damit immer den in der Umgangssprache üblichen Begriff, eine Information also, die für ihren Empfänger oder Besitzer Sinn und Zweck hat. (Lorenz VII: 39)

100. Wenn durch eine Mutation oder durch eine Neukombination von Erbanlagen die Wahrscheinlichkeit des Energiegewinnes so wesentlich vergrößert wird, daß Selektion zugunsten des mit dieser Verbesserung begabten Wesens wirksam wird, vergrößert sich auch die Zahl seiner Nachkommen. (Lorenz VII: 44)

101.Das erste dieser Probleme ist die Geschwindigkeit der Evolution. Wenn diese auf die rein zufällige Ausmerzung des Ungeeigneten angewiesen wäre und wenn es die Rückspeisungsvorgänge von Kapital- und Informationsgewinn nicht gäbe, so würde die Zeit von wenigen Milliarden Jahren, die von den Physikern aus den Zerfallszeiten radioaktiver Substanzen als das Alter unserer Planeten errechnet wurden, sicherlich nicht ausreichen für die Entstehung des Menschen aus einfachsten Organismen. (Lorenz VII: 45)

102.Wenn wir die Attribute »niedriger« und »höher" in erstaunlich gleichem Sinne auf Lebewesen wie auf Kulturen anwenden, so bezieht sich diese berechtigte Wertung unmittelbar auf den Gehalt an unbewußtem oder bewußtem Wissen, der diesen lebenden Systemen eignet, gleichgültig, ob er durch Selektion, Lernen oder explorative Forschung erworben wurde und ob er im Genom, im Gedächtnis des Einzelwesens oder in der Tradition einer Kultur aufbewahrt ist. (Lorenz VII: 46)

103.Die Aussage, daß auch im sozialen Verhalten des Menschen Instinkthaftes enthalten sie, das durch kulturelle Einwirkungen nicht verändert werden kann, wird uns Ethologen häufig als ein Bekenntnis zum extremen Kulturpessimismus ausgelegt. Dies ist völlig ungerechtfertigt. Wenn jemand auf drohende Gefahren hinweist, so zeigt dies, daß er kein Fatalist ist, der das drohende Unheil für unabwendbar hält. (Lorenz VII: 303)

104.Ich glaube sichere Anzeichen dafür wahrzunehmen, daß eine auf naturwissenschaftlichen Erkenntnissen sich aufbauende Selbsterkenntnis der Kulturmenschheit aufzuleuchten beginnt. Wenn diese - was durchaus im Bereich des Möglichen liegt - zur Blüte und zum Tragen kommen sollte, würde damit das kulturelle geistige Streben der Menschheit ebenso auf eine höhere Stufe gehoben werden, wie in grauer Vorzeit durch das 
Fulgurieren der Reflexion die Erkenntnisfähigkeit des Einzelmenschen auf eine neue und höhere Stufe gehoben wurde. (Lorenz VII: 303f.)

105. Wir haben zusammen viel Scheiß gemacht und echt Spaß gehabt. Leider kann man ja draußen nicht viel machen, aber wenn ihm das nicht gefallen hat, dann weiß ich nicht, was er für Vorstellungen hat (Jugendtagebuch VII: 3)

106.J. wollte Offizier werden und war ein ganz überzeugter Sozialist und riet uns Lenin zu lesen, daß wir wissen ,wie schlecht es euch im Westen geht“. Arbeitslose gibt es nicht nur Assoziale, wenn die nicht wären würde unsere Wirtschaft funktionieren. (Jugendtagebuch VII: 11)

107.E.: „Wenn Du nicht in der FDJ bist, hast du keine Aufstiegs - geschweige denn Berufschancen!“ (Jugendtagebuch VII: 12)

108.J.: „Deutschland wird nie wieder eins. Wenn ja, dann nur weil ihr sozialistisch werden werdet!“"(Jugendtagebuch VII: 12)

109.Es wurde noch Abschied von M. gefeiert. Sie wurde beschenkt und dann gingen wir in

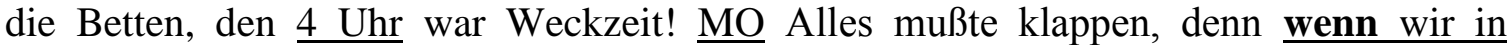
Dresden den direkten Zug nach Stgtt verpaßt hätten, wäre es schwierig geworden heim zu kommen. (Jugendtagebuch VII: 14)

\section{Uneingeleitete Konditionalsätze}

1. ... nimbt man das Haubt (das F.[Feder, P.K.] dahin/ so hab ich nichts gelitten: ... (Harsdörffer I: 12)

2. Ist es nach dem Käiserlichen Ausspruch Iustiniani, eine Gôttliche Sache alles in dem Gedåchtniß behalten; so muß auch in dem Schreiben/ welches benebens der edlen Druckerkunst alles unser Wissen/ gleichsam in einem Schrein beschrancket, etwas Göttliches enthalten seyn/ und ist nicht ohne sonderbare Vorsehung des Höchsten / berüht Druckerkunst / in den letzten Zeiten hervor und empor genommen / in welche sich / nach des Propheten Weissagung die Künste vermehren sollten / darzu nichts füglichers in Gebrauch gelangen können. (Harsdörffer I: 15)

3. Betrachtet man den geringen Anfang des Schreibens/ welcher in geraden und krummen Strichlein bestehet/ so muß man sich billich verwundern/ daß eine geringe Sache so grosse Dinge leisten kann. (Harsdörffer I: 16)

4. ... unn schreibet also nach Belieben alle Buchstaben doppelt. Will man/ so kann man eine Såiten aufspannen/ so viel geråder zu schreiben. (Harsdörffer I: 21)

5. Schreibt man an geringere/ als Unterthanen/Knechte/ Kinder/ 2c. so pflegt man sie zu dutzen. (Harsdörffer I: 30)

6. Schreibt man an seines gleichen/ so pflegt man sie zu ehren wie wir von ihnen wollen geehret seyn/ und soll sie zum wenigsten geirzt und geherret oder mit ihnen in der dritten Person geredet werden. (Harsdörffer I: 30)

7. Schreibet man an höhere/ so muß man ihnen ihren angebornen Titul beylegen/ nach ihren Stand und Ambtsdiensten. (Harsdörffer I: 30) 
8. Folge ich der Gewohnheit/ so wird mich ein jeder verstehen: (Harsdörffer I: 168)

9. folge ich der verhassten Neurung/ so werden mich nicht wenig verspotten/ ... (Harsdörffer I: 168)

10. Solten auch andere ein anståndgerers Mittel vorschlagen kònnen/ wollen wir denselben gerne beypflichten/ und beharren in der Meinung/ daß es nicht an dem Vermògen unserer Sprache / sondern an den teutschen Liebhabern derselben ermangle/ daß sie nicht åndern allen vorgezogenen und zu höchster Reinlichkeit erheben werde / und gehet es den Worten wie den Menschen / daß / wann sie sich gemein machen/ in Verachtung und Abbruch ihrer Wethaltung zu fallen pflegen. (Harsdörffer I: 169)

11. Ist aber etwan ein freyes Wort in Behandlung unstråfflichem Liebs-Hôflichkeit eingeschlichen / wird doch solches in folgendem Antwortschreiben genugsam hintertrieben / und beantwortet seyn / und sind wir zu solchem Schreiben an Frauenzimmer / veranlasst worden/ durch das Exempel anderer / welche von diesem / von dergleichen/ wiewol gar gezwungen aus dem Frantzósischen/ oder gar zu unvernemlich und verdrießlich davon gehandelt haben. (Harsdörffer I: 368)

12. Soll unsre teutsche Sprache zu höchster Ubertrefflichkeit gelangen/ so wird auch diesem und vielen andern Stücken/ von den Bücherschreibern/ und Drucksetzern ein mehrer Fleiß/ als bishero/ angewendet werden müssen. (Harsdörffer I: 564)

13. Es vertrieß die jung Gesellen, daß ich nicht wolte mitmachen, sie machten mihr Sprichwordt und singen mihr erdichte grobianische Lieder. Wolte ich von dißen groben Geselen Ruhe haben, so mieß ich mich ihrer Grobheitt undergeben, mit ihnen Dag und Nacht zechen und grob, lustig sien. (Güntzer I: 64v)

14. Von dißer Statt Prunßburck lag nicht weitt von der Portten eine Heidt und Walt. Dahin ging ich alen Abent, wan ich von meiner Arbeidt Fei[e]rabent hatt, darmit ich weitt von Leidten wahr und mich niemandt sahe. Ist es dan ein Fei[e]rdag, so wahr ich balt den gantzen Dag in dißem Walt oder auff andern Heidten, da mich niemand sahe, und ruffete dan mit heller Stim zu Gott in der Heen um Trost, Hilff und Errettung. Ich wahr offt so miede, schwach und chrafftloß von dem Reden und Schreyen zu Gott, daß ich offt eine gantze Stunde auff der Erden lag. (Güntzer I: 101v-102r)

15. $H$ wird nur im Anfang eines Wortes als ein Hauch ausgesprochen. Steht es hinter einem

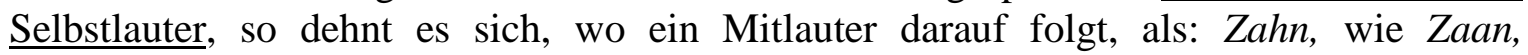
(Aichinger III: 9)

16. Steht es zwischen zween Selbstlautern, so thut es beym erstern eben diese Wirkung, ob es gleich in der Absetzung zur folgenden Syllbe gezogen wird, als: ge-hen, wie gee en. (Aichinger III: 9)

17. Man muß sich nicht irren in der Beurtheilung, welches das Stammwort sey. Wollte man schreiben flüssen, fluere, in der Meinung, daß es von Flu $\beta$ herkomme: so wäre es geirret: denn fliessen ist selbst das Stammwort, und Flu $\beta$ nur das abgeleitete. (Aichinger III: 30)

18. Ist jemand anders Sinnes, sonderlich in Ansehung derer, die ein $c h$ haben: so werde ich mit ihm keinen Streit anheben. (Aichinger III: 32)

19. Will man mir nicht zu Willen seyn: so steht es dahin, was man für Ehre davon habe, wenn ... (Aichinger III: 38) 
20. Michel, teutscher Michel. Soll dieser einen starken Kerl bedeuten: so möchte man lieber Müchel schreiben, von mögen. (Aichinger III: 51)

21. Suchen wir nun die wesentlichsten Vorzüge des italienischen Festwesens gegenüber dem Auslande vorläufig auszumitteln, so steht in erster Linie der Sinn des entwickelten Individuums für Darstellung des Individuellen, d. h. die Fähigkeit, eine vollständige Maske zu erfinden, zu tragen und zu agieren. (Burckhardt V: 293)

22. Der Hof des Pietro Riario, der von Ferrara usw. ließen es dabei gewiß nicht an der ersinnlichsten Pracht fehlen. Vergegenwärtigt man sich das szenische Talent und die reichen Trachten der Schauspieler, die Darstellung der Örtlichkeiten durch ideale Dekorationen des damaligen Baustils, durch Laubwerk und Teppiche, endlich als Hintergrund die Prachtbauten der Piazza einer großen Stadt oder die lichten Säulenhallen eines Palasthofes, eines großen Klosterhofes, so ergibt sich ein überaus reiches Bild. (Burckhardt V: 296f.)

23. Erstens muß selbstverständlich, wenn unsere Forschung überhaupt einen Sinn haben soll, die reale Existenz dessen vorausgesetzt werden, was wir zu erforschen trachten. Zweitens aber muß an den Forschenden eine Forderung gestellt werden, die keineswegs leicht zu formulieren ist. Wäre sie es, so brauchte ich dieses Buch nicht zu schreiben. (Lorenz VII: 11)

24. Uns allen, vor allem uns Deutschen, ist von Kindheit auf mit jedem Wort unserer Lehrer und unserer großen Dichter eine platonisch-idealistische Grundeinstellung so radikal eingebleut worden, daß sie uns selbstverständlich ist. Wundern wir uns aber einmal gründlich darüber, so drängt sich uns die Frage auf, welche Gründe es wohl gewesen sein mögen, die eine Vielzahl ernst nach Erkenntnis ringender Menschen veranlassen konnten, das Verhältnis zwischen phänomenaler und realer Welt genau verkehrt zu sehen. (Lorenz VII: 28)

25. Nun kann man auch jenen zuerst gesehenen Stern benennen, wofern es ein Fixstern ist.

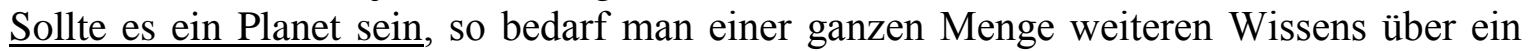
»Sternenmuster« höherer, zeitlicher Ordnung, um aus der »Konstellation« auf seine Identität schließen zu können. (Lorenz VII: 41)

26. Ihr eingebauter Verrechnungsapparat enthält notwendigerweise »Hypothesen«, an denen er in geradezu doktrinärer Weise festhält. Treten Umstände ein, die von dem Anpassungsvorgang, der sie erzeugte, nicht »vorgesehen" sind, so können sie Fehlmeldungen erstatten, an denen sie dann in unbelehrbarer Weise festhalten. (Lorenz VII: 43f.)

27. Naja und irgendwann lagen wir dann beide nackt da. Hätte ich meine Zwischenblutung (wegen der Pille) nicht gehäbt, hätten wir wohl glatt miteinander geschlafen! Naja, aber haben wir nicht. (Jugendtagebuch VII: 15)

28. Wir merkten es auch beide, daß der Frühling ausgebrochen ist. Wir waren echt „G, Geils“. (,rallig“) und wären die Umstände nicht so beschissen gewesen $\rightarrow$ Das Gefühl war so stark wir hätten miteinander geschlafen. Es blieb bei der Vorstufe und das ist ja auch gut so (Jugendtagebuch VII: 14) 
1. Weil nun die Steine nicht leichtlich zu bewegen/ und sehr wenig/ sondern grosse Mưhe gebraucht/ etwas darein zu bilden, zuweitlen auch gantze Stücke ausgesprungen/ haben sie Holtz und Metall zu ihren Schrifften gebraucht/ und sonderlich den Buchbaumen erwehlet/... (Harsdörffer I: 8)

2. Weil aber solches zwar harte/ doch endlich faulende Holtz/ nicht viel Jahr dauren können/ als haben sie zu denckwürdigen Sachen/ das Metall/ Bley/ Kupffer und Messing gebraucht/ und ihre Schrifften darein gegraben/ wie wir dann lesen in den Büchern der Maccaboer ... (Harsdörffer I: 8)

3. Weil nun unser Papyr/ welches aus alten Lumpen bereitet wird/ demselbigen gleichet/ hat es auch eben besagten Namen/ bis auff gegenwärtige Stund. (Harsdörffer I: 11)

4. Weil nun nicht zu erwarten/ daß sich die Mund-Arten in gleichstimmende Aussprache verienigen sollten: Als ist auch ihre Schreibrichtigkeit auff keine Weise endlich zu vergleichen/ und singet ein jeder Vogel/ wie ihm der Schnabel gewachsen ist. (Harsdörffer I: 17)

5. Der Gebrauch welcher hierinnen die Meisterschafft fŭhret/ ist zweyerley: gut und bò / und weil dieser ein König/ jener ein Tyrann genennet wird/ wollen wir beeder Gebot und Gebiet in dieser Vorrede betrachten/ und solches auff den Innhalt folgender Briefe ziehen. (Harsdörffer I: 165)

6. Weil nun Reden und Schreiben nur eine zufällige Unterscheidung hat/ setzet man beedes billich zusammen/ und ist nicht genug/ daß man andrer flügenden Worten nachspreche; ... (Harsdörffer I: 166)

7. Weil man sich aber wegen der Aussprache nicht wird vergleichen/ ist zu besorgen/ man treffe auch in der Wortschreibung keine durchgehende Vereinigung. (Harsdörffer I: 467)

8. Weil aber etliche Wôrter eine Gleichheit in der Ausrede haben / sind solche in der Schrifft deutlich zu unterscheiden / wie folgt. (Harsdörffer I: 468)

9. Weil aber sonderlich an jetzo bedagtem Sprachstucke der Rechtschreibung viel gelegen/ und einem jeden frey stehet/ seine Gedancken mehrverståndiger Straffurtheil zu untergeben; als ist fur schicklich erachtet worden/ etwas weniges auff Verbesserung mehrvernünfftigen Gutachtens hierbey anzufügen; (Harsdörffer I: 560)

10. Weil man aber das Teutsche vor Alters mit lateinischen Buchstaben geschrieben/ ist das c für das k gebraucht worden. (Harsdörffer I: 561)

11. Weil aber noch keine solche Neurung eingefŭhret/ lassen wir es hierinnen/ wie in vielen andern/ bey der alten Gewohnheit verbleiben: (Harsdörffer I: 566)

12. Ich dachte aber in meinem Sin, da die fihrneme Leidt in Seiden und Samet i[h]me die Fieß kißen, so mießte ich ihme den Arsch kißen, dieweil ich sehr unsauber, zerrißen Kleider trug, dieselbige voler Leiß und Fle. (Güntzer I: 61r)

13. Dieweil wihr von nichts kondten essen und trincken, sparen wihr unßer Gelt. (Güntzer I: $62 \mathrm{v})$ 
14. Mein Vatter und Geschwister sindt Tag und Nacht an mihr, ich solte mich verheiraten, dieweil ich aber gantz kein Lust darzu hatt und sahe beynóben, daz es mit mihr die Lengen kein Gudt wirdte thun, lenger in meim Vatterlandt zu verpleipen, dieweil ich daß gottloße Leben dogglich vohr Augen sahe, so in meinem Vatte[r]landt fohrgehet mit Schweren und Fluchen, Freßen und Sauffen und alerley Sinden, und ich mich derer auch teilhafftig gemacht hab, so ferchte ich, Gott wirdt mich an Leib und Sele straffen. (Güntzer I: 66r)

15. Dieweil wihr der Se nicht also gewonet sindt, so wirdten wihr alzumal kranck, kundten nicht eßen noch trincken 4 Tag, 60 Meil, biß nacher Kopenhagen. (Güntzer I: 94v)

16. Dieweil der Kinig Christian der 4. kein hoffertiger Man wahr, sonder mit dem gemeinen Man also gern redet als mit großen Heren, hielte er selber dem Gemeinen als dem Ho[h]en Antientz. (Güntzer I: 95r)

17. Dieweil daß Schiff lehr wahr, dan nuhr den Balast, darum wihr bo̊ß So̊glen hatten. Wihr bekamen beßes Wette[r], hatten groß Sturm. [...] Der Sturmwindt spilet mit dem Schiff, wirffte daz Schiff hin und herr, gleich als wie eine Katzen mit einer Mauß spilete. (Güntzer I: 95r-v)

18. Da er nichts kunde außrichten bey mihr, so setzete er weitters an mich, ich solte mich entleiben mit Strek oder Waffen oder in ein Waßer springen und dergleichen mehrn Gestalt, so mihr auß dem Sin ist gevalen. (Güntzer I: 101r)

19. Dieweil kein deischter Zingießersgesel alhie befirdtet wirdt, so bin ich alhie spatzieren gangen, 12 Tag die Statt sampt der kiniglicher Begróbniß, daz alte Schloß besichtiget, daz neye Barlamendthauß, daz Gewelb, wadurch Engelandt hatt solen verraten werden. (Güntzer I: 108r)

20. Weil nun die Art, die Syllben nach ihrer Grösse auszusprechen, in allen Sprachen in ungebundener Rede anderst ist, als in der gebundenen: so wird billig in der Grammatik einer ieden Sprache eine Unterweisung vom Syllbenmaß, so viel zur Aussprache nöthig ist, nicht bis in die Prosodie gesparet. (Aichinger III: 2)

21. Und weil bey den Teutschen die Aussprache der Worter in Versen sehr wenig von ihrer Aussprache in ungebundener Rede abweichet oder abweichen soll: so muß die teutsche Grammatik die Lehre // von der Grôsse der Syllben so vollståndig vortragen, daß der Prosodie hievon nichts ůbrig bleibet, als ihre gebührende Freyheiten. (Aichinger III: 2)

22. Doch weil im Schreiben das $k$ fast der mühsamste Buchstab ist, so kann man, ihn zu verdoppeln, wohl das $c$ gehalten, welches zu diesem Ende ohnfehlbar der Bequemlichkeit wegen angenommen worden ist. (Aichinger III: 11)

23. Weil nun $\beta$ ein zweyfaches $s$ ist: so ists gefehlt, wenn man es den Wortern aufbringet, die nur ein einfaches haben, z.B. Muß, pulmentum, da man doch sagt Gemuse (Aichinger III: 14)

24. Weil er aber nicht nach einem ganzen zweyfachen Laut verdoppelt wird, da er sonst klingen müsste, wie $t s$ ts; sondern nur in Anlehnung des erstern ursprünglichen Buchstaben $t$ : so drücken die Teutschen solche Verdoppelung am beßten mit $t z$ aus. (Aichinger III: 19)

25. Weil ein einiges Wort so viererley Bewegungen im Munde erfordert, und doch in einem Augenblick fertig ist: so hat man sich ůber die geschickte Has//tigkeit zu wundern, die der grosse Schôpffer dem menschlichen Munde verliehen hat. (Aichinger III: 20f.) 
26. Weil es aber doch angenommen ist, daß etlichen Wortern solche zweyfache Vocale gegeben werden, als: Aas, Heer, Schoos: so stehen sie, weil sie zu einer Syllbe gehören, billig unter den Doppellauten. (Aichinger III: 22)

27. Doch weil die diphthongi ae und oe schon lang genug sind: so darf man diese Unkosten ersparen; und dem $a a$ und $o o$ wird nicht zu kurz geschehen, wenn sie in Doppellaute verwandelt werden. Man schreibe nur: Såle, loffeln. (Aichinger III: 25)

28. Weil nun das Stammwort nicht fadem sondern faden heisset, so folgt, daß einfädeln besser teutsch sey, als einfämen. (Aichinger III: 30)

29. Man muß für edel nicht schreiben $\stackrel{e}{a} d e l$, als wenn es von Adel herkåme. Denn dieses stammt vielmehr von jenem, wie Satz von setzen, Strang von strengen, Da aber von einem hergeleiteten wieder ein anders kommen kann: so richtet sich das letztere nicht nach dem ersten Ursprung, sondern nach seinem eignen. (Aichinger III: 30)

30. weil dergleichen Art zu schreiben nie gewo̊hnlich gewesen ist, so mag es nach §. 39. bey den einfachen Mitlautern bleiben. (Aichinger III: 36)

31. Also, weil die weiblichen Namen, die auf in ausgehen, in der mehrern Zahl innen haben, als: Koniginnen, so můssen sie in den einfachen auch ein gedoppelt $n$ haben, als: Koniginn. (Aichinger III: 37)

32. Ja weil Frau und frey, liber, der Bedeutung nach miteinander verwandt sind: so kann auch dieses aus eben derselben Quelle kommen. (Aichinger III: 43)

33. Leugnen von lìgen. Weil man aber laugnen sagt: so kann lägnen auch so unrecht nicht seyn. (Aichinger III: 50)

34. Leugnen von lügen. Weil man aber laugnen sagt: so kann lägnen auch so unrecht nicht seyn. Doch da laugnen auch heisset mit Leuge begiessen, so behält man zum leugnen, negare lieber das blosse eu. (Aichinger III: 50)

35. Gott der Vatter des Liechts wölle uns vor der Ewigen völlen Finsternus behüten und Bewahren c.\&. Weil dise Finsternus so erschröcklich ist zu sehen gewessen daß es bey uns Menschen hier auff Erden scheinet. Als wann Gott der Allgemeine Weltrichter kom[m]en werde, zu richten die böße Geister und gottlose Menschen, weil es gantz Nacht gewessen daß man die Stern am Himmel gesehen, so hat mann bald in allen Häußer Liechter angezindt wegen Forcht und Schrecken, der warthenden Dingen, die da kommen möchten auff Erden auff diese Finsternus hat sich daß Jahr angelassen daß alles ist genug gewachsen, (Schuhmacher Chronik II: 328f.)

36. Im Anfang dises Jahrs hat sich ein trauriges Spectacul gleichsam in der ersten viertel stundt zuge-Tragen. Weil daß sich die Vor-Stätter Söhn haben zusamen gefüegt $u$. geschlossen, daß Neue Jahr anzuschiessen, so hat sich auch der Beckh Johannes Lobmüller noch ein Junger Haußhälter auch darzu gemacht, welcher bey dem Eychhäusslein wohnhafft, und durch einen ungefähren unglückhafften Schutz seinen Schwager Joh. Georg Ruoffen, Lediger Gesell, hinde[n] In den Fuoß geschossen, daß Er sich so sehr verbluttet, daß Er dadurch wegen Überhandnehmung deß Brands sein Leben enden u. schliessen müssen, welches höchst zu-betrauren gewessen. (Schuhmacher Chronik II: 342f.) 
37. Es haben auch vier Haußhaltungen Gelt zusamen geschossen umb ein Emy Erbsen welches 30 xr. gekostet $u$. ist den 15 Brach-monath von Joh. Jacob Hum[m]el, Müller in der Kämermühlin gemahlen worden und seind 19 Personen mit abgespeiset worden. Weil wir den vor Augen stehende[n] reichen-Seegen den Som[m]er über angeschaut, mann vermeint, Es solte wohlfailer werden. So continuierte die Teurung biß auff den 11ten Augusti. (Schuhmacher Chronik II: 343f.)

38. Merckwürdig-Keit: Weil der Allweise Gott unser Geliebte Vatter-Statt bey der reinen Lehr deß H. Evangely schon in die zweyhundert Jahr vätterlich Erhalten, und den Leichter deß wahren Gottes-Dinsts nicht von seiner Stätte gestossen, So ist des wegen ein drey Tägiges Jubel-Fest angestelt worden auff den 30ig und 31igsten Octb. und 1. Novemb. welches Ich hernach umbständlich beschreibe (Schuhmacher Chronik II: 358)

39. So hat Sie durch Teuffels Eingeben gemeint, der Sachen zu entrinnen und den Männern entlauffen. Weil man Sie aber fäst gehalten und wider zurück geführet so hat Sie Es auff dem Weg dem schon gemelten Statt-Diener Elias Klein bestanden, Sie habe Es auff der Büne in einem Wollen-Ständlein in Speier ligen, (Schuhmacher Chronik II: 379)

40. In disem Jahr hat sich eine traurige Mordthat zugetragen mit zwey Schwäger der Täter mit Namen Heinrich Lamparter Beckh Der andre Christopff Gebel, so entleibet, dises hat sich zugetragen den 13. Juny zwischen acht \& neun Uhr, weil seine Schwester mit ihrem Mann dem H. L. etwaß Händel gehabt, daß Er Sie geschlagen, so ist der Bruder Ihren Zuhülffe gestanden, nachgehens seind Sie beede auff der Gassen mit rauffen und Schlagen aneinander gerathen, daß der Lamparter seinen Schwager mit Fäusten braff erschlagen und grüttlingen auff Ihm troben gesessen, daß Er so verbüttert und Ihm vor daß Hauß geloffen, geflucht \& geschworen, Er Ihn aber vom Hauß haissen weg gehen oder Er schieß Ihn Tod, darauff sprach Er der Gebel: Hund Schiess zu, worauff der Knall gegeschehen, darauff Jesus geschriehen und nach etliche Schritt vor sich geloffen und zu Boden gefallen und kein Wort Zaichen mehr geben und kein Stundt mehr gelebet und also seinen Geist auffgeben. (Schuhmacher Chronik II: 397f.)

41. am Tage der unschuldige[n] Kindlein hat sich der Him[m]el Claar gemacht und cont. biß zum End deß Jahrs mit kalte Morgen und Sonnenschein, war daß Jahr beschlossen fein. Weil Ich daß Jahr nach allen 12 Monat beschriben hab war dises die Ursach weil vor 20ig Jahren Ein so kalter Winter gegesen und diser in disem Jahr gleich gehalten worden, in der kälte, weil Mir daß wasser in den feicht-Kössel auff der werckstatt gefrohren und ein sehr kalter Frühling gewessen, So hat man vermutet, Es werde widrum Ein böß Jahr darauff erfolgen. Aber dem nicht Also, wie Anno 9. (Schuhmacher Chronik II: 422f.)

42. Da hat Lutherus anheben darwider zu prodestieren und mit dem Schwerdt des Geistes zu fechten. Also fieng Sich der Hader an, zwischen Lutherum \& Tetzel. Da Tetzel Lutherum als ein Ertz ketzer thut verdam[m]en, wie solches bey Joh. Matheri, mit mehren Unständen zu lesen ist, weil aber daß Licht des Evangeli durch unterschidliche Päpst so verdunckelt worden, daß der Leichter de $\beta$ waren Gottes-Dinsts schier von seiner Stelle gestossen, und der Papst Johannes der XXIII hat selbsten keine Auffersteung der Todten nicht geglaubet. Also hat Lutherus daß Revormations Werck angefangen und hat geweret biß auff 30ig in werenter Zeit hat Sich viel Unheil gezeiget von allerhand Rotten \& Secten und theure Zeiten, (Schuhmacher Chronik II: 425)

43. 17 A N N O 31 // Jubel und Danck Fest, Waß massen \& Gestalt Es gehalten worden. Ist nämlich dises die Ursach, weil Just vor hundert Jahren an S. Petri \& Pauli die Statt in Ruin verderbt und ausgeplindert werden. Weil der Graff E G O N von Fürsten-berg, So 
auß Italia herauß kommen und in Ober Schwaben viel Stätt in Ruin verderbet und außgeplündert, und solches Unheyl unser Statt auch sollen widerfahren, So hat Sich der General mit vierzig Tausend Mann auff der Winge gegen Eningen zu gelagert und einen schröck Capitin in die Statt geschickt, den Untergang anzutrohen, (Schuhmacher Chronik II: 444f.)

44. Hiemit ist merckwürdig zu beobachten, daß den 1. 2. 3. \& 5. mertzen daß vor Augen stehende Obere Thor Neu erbaut und auffgericht ist worden, welches durch den TotalBrand ist verzert worden. Dieser Monat stellet Sich recht winterisch Ein mit Schnee Nebel \& Sonnenschein, auch regen, weil Es scheinet noch Winter nicht Früeling zu werden, So hat daß Brodt den 15ten ein Kreutzer auffgeschlagen, So ist der Schnee schleinig abgangen, daß Es umb Palmarum erst auff dem Blachfeld Blatten geben, in der CaarWochen ist Es schön und warm Wetter gewessen, daß der Schnee fast alles weggangen, (Schuhmacher Chronik II: 446)

45. Weil Ich daß Jahr nach allen 12 Monaten beschriben hab mit der Wütterung, so ist gut darauß prognostizieren, waß Es für ein Jahr gewessen, nämlich ein Cron-Jahr, daß alles genug gewachsen an Frucht, Obs und Wein, und ist alles köstlich und gut Einkom[m]en. (Schuhmacher Chronik II: 449)

46. Es haben auch theil Som[m]er-Früchten Noth gelütten wegen der Dürre, daß es etwaß weniger geben und seind am Stroh gar kurtz bliben, weil wir so einen reichen Seegen an Obß bekom[m]en haben, So hat man daß Simry Püren umb 4 \& 5 Kreutzer kauffen können, der Apffel gleichfahls, mann hat umb Weynachten noch 20ig biß 24ig auch 30ig Apffel umb ein Kreutzer kauffen können. (Schuhmacher Chronik II: 449)

47. Die Idealfarbe aber, welche man in den eigenen, wie in den aufgesetzten Haaren zu erreichen strebte, war blond. Und da die Sonne im Rufe stand, das Haar blond machen zu können, so gab es Damen, welche bei gutem Wetter den ganzen Tag nicht aus der Sonne gingen, sonst braucht man auch Färbemittel und außerdem Mixturen für den Haarwuchs. (Burckhardt V: 269)

48. Da nun die Sprache eine Angelegenheit der lebendigen Gesellschaft geworden war, so setzten die Archaisten und Puristen trotz aller Anstrengung ihre Sache im wesentlichen nicht durch. (Burckhardt V: 275)

49. Da die Bewirtung dabei Nebensache war, so konnte man stumpfe und gefräßige Individuen ohne Schwierigkeit fernhalten. (Burckhardt V: 276)

50. Mit andern Worten, da der Gesang trotz aller konventionellen Bescheidenheit eine Exhibition des einzelnen Gesellschaftsmenschen bleibt, so ist es besser, man höre (und sehe) jeden besonders. (Burckhardt V: 284)

51. Zum Verständnis der höhern Geselligkeit der Renaissance ist endlich wesentlich zu wissen, dass das Weib dem Manne gleich geachtet wurde. Man darf sich ja nicht irre machen lassen durch die spitzfindigen und zum Teil boshaften Untersuchungen über die vermutliche Inferiorität des schönen Geschlechtes, wie sie bei den Dialogenschreibern hin und wieder vorkommen, auch nicht durch eine Satire wie die dritte des Ariosto, welcher das Weib wie ein gefährliches grosses Kind betrachtet, das der Mann zu behandeln wissen müsse, während es durch eine Kluft von ihm geschieden bleibt. Letzteres ist allerdings in einem gewissen Sinne wahr; gerade weil das ausgebildete Weib dem Manne gleichstand, konnte in der Ehe das, was man geistige und Seelengemeinschaft oder höhere Ergänzung nennt, nicht so zur Blüte gelangen wie später in der gesitteten Welt des Nordens. (Burckhardt V: 285) 
52. Es erregt den Italienern der Renaissance nicht das geringste Bedenken, den literarischen und selbst den philologischen Unterricht auf Töchter und Söhne gleichmäßig wirken zu lassen; da man ja in dieser neuantiken Kultur den höchsten Besitz des Lebens erblickte, so gönnte man sie gerne auch den Mädchen. (Burckhardt V: 285f.)

53. Indem es nahe an Weinachten ist, so will ich euch auch wieder einige Zeil schreiben, um euch auf Weinachten durch einen Brief von mir, ein wenig $\mathrm{zu}$ erfreuen (Auswandererbriefe V: 18)

54. das größte Mädchen könnte wohl schon viel Hülf sein wenn sie dazu erzogen worden wäre, aber indem das eben nicht der Fall war, so ist inn anstrengender Arbeit nicht viel aus ihr herauszubringen, (Auswandererbriefe V: 18)

55. und an Arbeit fehlt es auch nicht, denn bei uns bewahrheitet sich der Spruch, im Schweiß deines Angesichts sollst du dein Brod essen, indem wir aber beide von etwas besserem nichts wissen so können wir uns ziemlich gut inn unsere Lage schicken, denn unser Feierabend wird auch einmahl komenen, (Auswandererbriefe V: 19)

56. Indem ihr meinen letztjährigen Brief nicht bekomen habt inn welchem ich euch eine beschreibung von unserer Scheuer gab so will ich dasselbe jetzt noch einmahl inn kürze thun, (Auswandererbriefe V: 19)

57. Obst haben wir dießes Jahr etwas mehr bekomen als wie jemals zuvor, indem wir verschiedene Sorten hatten so hatten wir seid Juni seitdem die frühen Kirschen reif wurden imer ein wenig etwas für die Kinder sowie auf den Tisch wir haben jetzt noch etwas Äpfel im Keller sowie eingemachte Beeren. (Auswandererbriefe V: 19)

58. Da es bei uns sehr viel wilde Enten gibt, welche so groß wie die zahme sind auch Gänse Hühner und dergleichen welche eine Masse über unsere Stadt komen und dem Karl viele Freude machen so wollte ich auch den Vater als Jagdliebhaber bei uns haben. (Auswandererbriefe V: 28)

59. Da wir auf unserer Reiße das Unglück gehabt haben unsere Bildnisse zu erbrechen sogar das Gemälde zu verschmieren so würde es mich freuen wen Ihr sie uns schicken würdet (Auswandererbriefe V: 28)

60. da es aber bei Euch so theuer kō̄t so will ich in stetter Hoffnung und Geduld warten bis Ihr sie mir nebst deai Ohrenringe einmal selbst überbringt. // (Auswandererbriefe V: 28)

61. sie [die Veränderungen durch Mutation und Neukombination von Erbanlagen, P.K.] wirken sich keineswegs immer zum Vorteil der betroffenen Individuen aus - im Gegenteil: Da alle diese kleinen und kleinsten Veränderungen, die durch Mutation und Neukombination von Erbanlagen verursacht werden, völlig ungerichtet vor sich gehen, haben sie in den allermeisten Fällen eine Verminderung der Aussichten zur Folge, die das betreffende Individuum auf Energiegewinn und Überleben hat. (Lorenz VII: 37)

62. Da durch die Selektion Strukturen »herausgezüchtet« werden, die eine bestimmte arterhaltende Funktion besonders gut erfüllen, sehen sie im Enderfolg so aus, als wären sie von einem weise voraussehenden und klug planenden Geist zu eben diesem Zwecke erschaffen. (Lorenz VII: 38)

63. Da nun die kognitiven Vorgänge auf höchster Ebene ebenso wie die auf der denkbar tiefsten und ältesten auf dem gleichen Prinzip beruhen, könnte man meinen, es gäbe keine andere Art des Wissensgewinns. (Lorenz VII: 41) 
64. Gegen 15 Uhr kam er wieder und wir hörten Kinderschallplatten (z. B. Pumuchl und die kleine Hexe etc. $p+p)$ Weil wir so müde waren schliefen wir ein. (Jugendtagebuch VII: 1)

65. Wir haben heute telefoniert und da das so unpersönlich ist habe ich - und darauf bin ich mächtig stolz - mich spontan auf den Weg zu ihm gemacht. Er soll sehen, daß er mir nicht gleich ist, daß ich alles für ihn tun würde. (Jugendtagebuch VII: 4)

66. Überall mußte man Schlange stehen, obwohl Plätze frei waren! (Das ist bei HO-Cafés so. Weil der Betrieb dem Staat gehört und jeder seinen festen Lohn erhält - egal wie gut oder schlecht das Café geht - sorgt man eben dafür, daß man nie zu viel zu tun hat und $u$. $U$. in Stress kommt. (Jugendtagebuch VII: 13f.)

\section{Weitere Typen von Adverbialsätzen im engeren Sinne (Kapitel 5.4)}

Finalsätze (Kapitel 5.4.1)

1. Darmit ich aber auch von ihrem Gótzenwerck etwaß konde sagen, ging ich in der Maria Capel, hò hrete darinen $2 \mathrm{Me} ß$ an, aber wenig Trost und Krafft darbey gesehen. (Güntzer I: $57 \mathrm{v})$

2. ... damit sie nun unterschieden würden/ hat ein jeder/ benebenst dem Tauffnamen/ auch sein Leibgut anmelden/ und sich also in die Reichsbücher einschreiben lassen/ .... (Harsdörffer I: 28)

Konsekutivsätze (Kapitel 5.4.2)

1. da gab Es einen Stürmischen Wind, gleich darauff einen lieblichen Som[m]ertag, daß daß weibervolck hat Spitz auff der Gaß würcken können, So warm war Es, den 20. \& 21igsten wider Sturmwind und geregnet, (Schuhmacher Chronik II: 422)

Adversative Adverbialsätze (Kapitel 5.4.3)

1. Während im Norden der Adel und die Fürsten ihre Muße entweder einsam oder mit Kampf, Jagd, Gelagen und Zeremonien, die Bürger die ihrige mit Spielen und Leibesübungen, allenfalls auch mit Verskünsten und Festlichkeiten hinbrachten, gab es in Italien zu all diesem noch eine neutrale Sphäre, wo Leute jeder Herkunft, sobald sie das Talent und die Bildung dazu hatten, der Unterredung und dem Austausch von Ernst und Scherz in veredelter Form oblagen. (Burckhardt V: 276)

2. Doch statt man jetzt denken müßte also fahre ich jetzt aus der Fremde heim ist es wieder total anders! (Jugendtagebuch VII: 20) 
1. Ob nun wol viel aus Ehr- und Gewinnsucht solche Bedienung deß gemeinen Nutzens/ angetretten/ so sind wir doch von solchen Absehen dieses Orts weit entfernet/ und ... (Harsdörffer I: 3)

2. Ob nun etliche an der Warheit solcher Erzehlung zweiffeln; so ist doch gewiß/ daß bald nach der Sündflut/ von den Mitternåchtigen Vôlkern/ die Steine zu Bezeichnung ihrer Heldengeschichte gebrauchet worden/ ... (Harsdörffer I: 8)

3. Ist meine Sprache schwartz!/ sind doch die Worter weiß. (Harsdörffer I: 12)

4. Dann obwol etliche Thiere auch Hănde zu haben scheinen/ so sind doch solche nur den Unsrigen der gestalt nach gleich/ und kônnen wie allein unsren Willen durch Bezeichnung der Buchstaben/ zu verstehen geben (Harsdörffer I: 15f.)

5. Ob nun wol GOTT des Jobs Meinung recht gesprochen/ so ist doch solches von den Worten zu verstehen/ welches er von der schweren Anfechtung hòren lassen/ oder Gott hat ihm seinen Fehler wegen ůberhăffter Schmertzen verziehen. (Harsdörffer I: 665)

6. Haben gleich die Griechen diesen Buchstaben nicht gehabt, und die lateinischen Namen anderst ausgedruckt, als: [...], Quartus: so haben doch unsre Voreltern, welche ihre Schrift von den Ro̊mern gelernet haben, von diesen entlehnen kỏnnen, was sie fưr nồthig befanden. (Aichinger III: 13)

7. Wird gleich der Laut, den es hat, meistens mit chs umschrieben, als in Wachs, wech//seln, Ochs, Fuchs, so hat sich doch das $x$ in etlichen erhalten, als in Axt, Eidexe, Hexe. (Aichinger III: 17f.)

8. Graf besser als Grav. Denn ob wohl vor alters meistens Grav oder Graue geschrieben worden: so ist doch dieses $v$ am Ende, wie in Hov, Hoven, u.d.g. schon lăngstens geåndert worden. (Aichinger III: 45)

9. ist der gewalt des Eyses u. Wassers so schleunig angeloffen daß Es die Brücke eingerissen wovon 24 Menschen ertruncken obgleich viel hundert menschen am Uffer gestanden, konten sie doch disen Armen Leuthen nicht helffen. (Schuhmacher Chronik II: 331)

10. Wenn aber auch die Allegorien der italienischen Dichtungen, Kunstwerke und Feste an Geschmack und Zusammenhang im ganzen höher stehen, so bilden sie doch nicht die starke Seite. (Burckhardt V: 295)

11. Weñ ich schon den Kopf so voll von Gedanken habe, u. ich euch gerne über vieles, über natürliches, pollitisches u. kirchliches Gebiet dieses Landes schreiben möchte, so will ich mich doch auf['s] kürzeste für dieses mal beschrenken, (Auswandererbriefe V: 6)

12. Obgleich ich im englisch Schreiben noch schwach bin, so hatte ich doch bis jetzt noch keinen Anstand. (Auswandererbriefe V: 7)

13. Wenngleich, als ich Europa verlie $\beta$, ich nicht recht wuste was ich that, $u$. mein Geist $u$. Gemüthe mit Dunkelheit umhüllet waren, so ist es jetzt mein unerschütterlicher glaube, daß ich durch Gottes weise Rathschluss hier bin, u. ich kann sagen der Herr hat alles wohl gemacht (Auswandererbriefe V: 7) 
14. Es sind jedoch nach den Vorlagen des neuen Milletärgesezes einige härten ausgelaßen und obgleich alles in den Waffen eingeübt wird so soll doch noch die Landwehr die auch noch ausgelost wird mit einigen Monaten wiederwegkomen. (Auswandererbriefe V: 15)

15. Dem Valentin und Anton Rast aus Pfaffenhofen geht es recht gut den sie sind jetzt auch in Springba: und zwar schon seit Ende August wo sie einige Zeit bei uns gearbeitet haben und dā haben sie sich ein Haus gemiethet und betreiben seiddem die Metzgerei wobei sie recht guten Verdienst haben. Obgleich schon ein Metzger hier ist, so verkaufen sie den̄och viel Fleisch und Wurst sie führen auch viel Fleisch nach Peoria wo sie auch viel verdienen. (Auswandererbriefe V: 28)

16. Obwohl ich also schon früh zu einer durchdachten erkenntnistheoretischen Einstellung gekommen war und obwohl ich mir darüber im klaren war, daß auch der Mensch angeborene Normen des Verhaltens hat, die den Fragestellungen und der Methodik der Naturforschung zugänglich sind, machte mein Erkenntnisstreben scharf von jenen spezifisch menschlichen Eigenschaften und Leistungen halt, die sich auf der Ebene des Kulturellen aufbauen. (Lorenz VII: 32)

\section{Irrelevanzkonditionale (Kapitel 5.4.5)}

1. Lernen wir gleich Hebræisch und Griechisch/ Lateinisch/ Frantzo̊sisch/ Italiănisch und andere auslăndische Sprachen/ so kônnen wir doch in allen Ständen/ unsrem geehrten Vatterland mit keiner mehr als unsrer angeborenen teutschen Sprache dienen/ und müssen wir Teutsche uns mit dem Teutschen behelffen / ja fast in allen Ständen unsre Nahrung damit erwerben. (Harsdörffer I: 4)

2. Es ist so miserabel und Greilich zugangen, daß nicht gnugsam zu beschreiben, man hat einander beraubt und bestolen, daß Es ein Greyl gewessen. Es ist ärger zugangen als zur Zerstörung Jerusalem wangleich der Feind mit höresmacht wehre über unser Statt gezogen und unß rein außgeblindert, hät Es nicht miserabler können daher gehen, der eine hat Bett verlohren, der andere hat Schreinwerck verlohren, der dritte hat Leinwand verlohren, der vierte hat $\mathrm{Faß}$ verlohren, und so Fort, und ist ärger und leichtförtiger Zugangen als im grösten Krieg. (Schuhmacher Chronik II: 402)

3. Und so ungewohnt es am Ende aussieht, so man schreibet: ei, bei, frei, so nimmt doch diese Schreibart jetziger Zeit selbst ůberhand. (Aichinger III: 18)

4. So gut die angegebenen Regeln sind: so wird man doch aus denselben nicht so leicht, als aus einem Verzeichnusse der Wörter, deren Schreibart zweifelhafft ist, Rath holen kỏnnen, wie dieses oder jenes auszudrücken sey. (Aichinger III: 39)

5. Abgesehen von diesem nicht unwesentlichen Anhalt der Ritterwürde gab es auch bereits, z. B. in Ferrara wahre Hoforden, welche den Titel Kavaliere mit sich führten. Welches aber auch die einzelnen Ansprüche und die Eitelkeiten der Adligen und Kavaliere sein

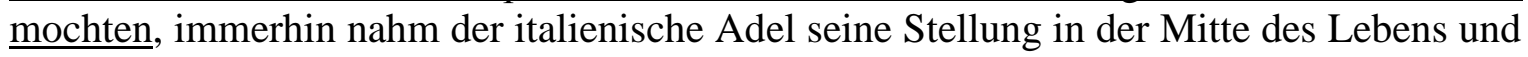
nicht an einem äußern Rande desselben. (Burckhardt V: 266f.)

6. Und andere, die der Sprache im höchsten Sinne mächtig waren, verließen sich hinwiederum auf den prachtvoll wogenden Gang und Wohllaut derselben als auf einen vom Inhalt unabhängigen Vorzug. Auch eine geringe Melodie kann nämlich, von solch einem Instrument getragen, herrlich klingen. Allein, wie dem auch sei, in gesellschaftlicher Beziehung hatte diese Sprache einen hohen Wert. (Burckhardt V: 273) 
7. Es ist möglich, dass die häufigen und prachtvollen Aufführungen von Mysterien, wobei Hunderte von Menschen bemalt und geputzt wurden, den Missbrauch im täglichen Leben fördern halfen; jedenfalls war er ein allgemeiner, und die Landmädchen hielten dabei nach Kräften mit. Man konnte lange predigen, dass dergleichen ein Abzeichen von Buhlerinnen sei; gerade die ehrbarsten Hausfrauen, die sonst das ganze Jahr keine Schminke anrührten, schminkten sich doch an Festtagen, wo sie sich öffentlich zeigten. - Möge man nun diese ganze Unsitte betrachten als einen Zug von Barbarei, wofür sich das Schminken der Wilden als Parallele anführen läßt, oder als eine Konsequenz des Verlangens nach normaler jugendlicher Schönheit in Zügen und Farbe, wofür die grosse Sorgfalt und Vielseitigkeit dieser Toilette spräche - jedenfalls haben es die Männer an Abmahnungen nicht fehlen lassen. (Burckhardt V: 269f.)

8. wie sehr es noch Eltern ueber Verlust kleiner Kinder schmerz so haben doch solche Kinder ein großes Vorrecht den sie sind des Gefahr und mühevollen Erdenlebens enthoben und können Anstat deßen da sein wo kein leid und Geschrei mer ist sondern lauter Seeligkeit. (Auswandererbriefe V: 11)

9. Jedoch könen wir es angreifen wie wir wollen, so wird unser beschiedenes Theil, das uns wird zugemessen werden, noch bedeutend verschmällert ehe es die Küste Amerika erreichen kā̄. (Auswandererbriefe V: 6)

10. So himmelhoch sich diese Systemeinheit über alle anderen uns bekannten erhebt, teilt sie doch mit ihnen ein unvermeidliches Schicksal: Wie alle lebenden Systeme, so ist auch der menschliche Geist und mit ihm die menschliche Kultur Störungen unterworfen. (Lorenz VII: 16)

11. Wir können nichts erfahren, nichts anschauen und nichts denken, ohne dies aufgrund von Voraussetzungen, von Unterstellungen zu tun, in denen solche angeborene Hypothesen stecken: Sie sind in unserer »Weltbildapparat» eingebaut! Auch wenn wir noch so sehr danach trachten, in freier Tat neue Hypothesen zu erfinden, können wir nicht verhindern, daß in ihnen diese uralten durch Mutation und Neukombination von Genen entstandenen und durch äonenlanges "pattern matching" erprobten Hypothesen des Apriorischen stecken, die nie ganz dumm, aber immer starr und nie restlos zutreffend sind. (Lorenz VII: 44)

\section{Spezifizierende Adverbialsätze (Explikativ- und Restriktivsätze) (Kapitel 5.4.6)}

1. Außerdem aber ist der Galateo eine schön und geistvoll geschriebene Unterweisung in der guten Lebensart, in Delikatesse und Takt überhaupt. Noch heute können ihn Leute jedes Standes mit großem Nutzen lesen, und die Höflichkeit des alten Europas wird wohl schwerlich mehr über seine Vorschriften hinauskommen. Insofern der Takt Herzenssache $\underline{\text { ist, }}$ wird er von Anfang aller Kultur an bei allen Völkern gewissen Menschen angeboren gewesen sein, und einige werden ihn auch durch Willenskraft erworben haben, allein als allgemeine gesellige Pflicht und als Kennzeichen von Bildung und Erziehung haben ihn erst die Italiener erkannt. (Burckhardt V: 271)

2. wir erfahren auch, er sei ein großer Neuerer gewesen, allein ob er oder andere den entscheidenden Schritt in die Tonsprache der modernen Welt hinein getan haben, wird nicht so erörtert, dass der Laie sich einen Begriff von dem Tatbestand machen könnte. Indem wir daher die Geschichte der musikalischen Komposition gänzlich auf sich beruhen lassen, suchen wir die Stellung der Musik zur damaligen Gesellschaft auszumitteln. (Burckhardt V: 283) 
3. Außerdem beklagen sie den bereits äußerst raschen Wechsel der Moden und (wenn wir die Worte richtig deuten) die törichte Verehrung alles dessen, was aus Frankreich kommt, während es doch oft ursprünglich italienische Moden seien, die man nur von den Franzosen zurückerhalte. Insofern nun der häufige Wechsel der Kleiderformen und die Annahme französischer und spanischer Moden der gewöhnlichen Putzsucht diente, haben wir uns damit nicht weiter $\mathrm{zu}$ beschäftigen; allein es liegt darin außerdem ein kulturgeschichtlicher Beleg für das rasche Leben Italiens überhaupt in den Jahrzehnten um 1500. (Burckhardt V: 268)

Komparativsätze (Kapitel 5.4.7)

1. Wie zu einem Bau nicht nur Stein und Holtz/ sondern auch Kalg und Sand erfordert wird; als achten wir diese Briefe (so das freundliche Vertrauen gleichsam zusammen fügen und halten/) in erstbesagter Vergleichung/ nie weniger nutzlich/ als sie zu Erhöhung deß Verstandes/ mit verantwortlicher Liebs-Hóflichkeit luftreitzend und erfreulich seyn werden. (Harsdörffer I: 5)

2. Gleichwie nun ein jeder Christ für sich in der Noht ein Gebetlein zu Gott soll verabfassen konnen/ un er zu solchem Ende die H. Schrifft und unterschiedene Betbücher lieset/ daraus er sein Wort/ bey dem Herrn Himmels und der Erden fürbringen lernet: Also soll ein jeder seine Gedancken/ nach erheischter Begebenheit nach hier begriffene/ oder andre Weise ein Gruß- oder Handels-Brieflein verfassen lernen/ ... (Harsdörffer I: 17)

3. Wie kein Buch allein/ viel andre Bücher auffheben kann: Also kann eines Gelehrten Meinung/ und mit angemasster Neurung wolgemeint zu rucke stellen. (Harsdörffer I: 165)

4. Gleichwie der Bildhauer seine arbeit nicht nach einem andern Bild/ sondern nach dem natuerlichen Menschen/ oder Thiere gestaltet: Also soll auch der Schreiber nach dem besten Exempel/ deß rechtgesprochnen Gebrauchs/ sich zu schreiben angewöhnen/ und nicht dem groben und unstudirten Pổbel blöde und blinde Folge leisten. (Harsdörffer I: 166)

5. Oder: wie Apelles von allen schỏnen Jungfrauen das Schönste abgeseyen/ als von einer die lieblichen Augen/ von der andern die rosenfarben Wangen/ von der dritten den rubinrohten Munde/ 2c. Also soll der teutsche Secretarius viel gute Bücher und viel wolverfasste Briefe lesen/ daraus seiner Feder alle Zierlichkeit einzugeben/ ... (Harsdörffer I: 166)

6. Wie aber in allen Sachen die menschliche Schwachheit erhellet/ in dem man sich keiner einstimmigen/ durchgehenden und kunstgrundigen Richtigkeit vergleichen mag: Als ist sich nicht zu verwundern/ wann man so wol in dem Reden als Hören/ so wol in dem Schreiben als Lesen// irren/ und sich wegen keiner allgemeinen Gleichheit nicht vereinigen kann. (Harsdörffer I: 166)

7. ... und Wie es einem Schuster keine Schande ist/ daß er kein Kleid machen kann/ also ist es auch einem Ungelehrten nicht zu verargen/ wann er nicht weiß/ was er nicht gelernet hat. (Harsdörffer I: 169)

8. ... und wie der Krieg alles Unheil mit sich bringet/ und die rechmåssigen Besitzer aus ihren Gütern vertreibet: Also muß man sich nicht verwundern/ wann unter den kriegenden Partheyen ... (Harsdörffer I: 170) 
9. Was wăre der Streit zwischen Fleisch und Geist vonno̊̀ten/ wann wir alle lüstrenden Begierden ablegen oder derselben mit leichter Hand obsiegen könten? Nein/ wie die Seele der Freude und des Leibs fahig ist/ also ist der Leibe der Wollust und dem Schmerzen zugethan (Harsdörffer I: 367)

10. Wie nun alles/ was wir Menschen besitzen/ in stetsschwebender Unbeståndigkeit verharret: als ist sich nicht zu verwundern/ wann auch mit Zerruttung der Völcker/ durch ihre/ Theils benohtsachte/ Theils freywillige Wanderschafft durchgafften/ Kriegen/ Siegen/ und Unterligen die Sprachen in mancherley Veränderung/ Vermischung/ und Irrung mißgerahten und veråndert werden. (Harsdörffer I: 559)

11. Gleich wie diße Merdter auff den Reder ligen, so werdtet ihr auch darauff gelegt werden, darum nemet ein Exenpel an dißen Merder, welche dahier geredert sindt worden. (Güntzer I: $43 \mathrm{v}$ )

12. Gleichwie man aber långst angefangen hat, an Statt leyen, weyen, gedeyen, u. d.g. zu schreiben leihen, weihen, gedeihen: also kònnte man auch an Statt der übrigen schreiben: Die Leiher, (Aichinger III: 18)

13. Wie es nun zu viel ist, so man in der Verdopplung 2. $z$ machet, als: Hizze: also ists zu wenig, ùberall nur eins brauchen (Aichinger III: 19)

14. Denn, wie das $u$ in den Doppellaut eu nicht verwandelt wird, indem man nicht schreibet, neu, neuer: also wird es auch im au nicht verwandelt werden, sondern die vorkommende Verwandlung dem $a$ zuerkannt werden müssen. (Aichinger III: 25)

15. Wie nun dergleichen Art zu reden und zu schreiben nachmals gebessert worden ist, also důrffen diejenigen Teutschen, die solcherley Doppellaute in ihren Provinzen haben, sich derselben auch nicht růhmen, noch sich auf das Alterthum beruffen. (Aichinger III: 26f.)

16. hat Es Zwey Nächt geregnet und ein groß Gewässer geben, daß Es zwey Weer zurüssen, daß erste bey der Graben-Mühle und daß andre bey der undren Mühle, folget gelinde Wütterung zu deß Monats Ende. Wie der Monat sich geendet, So nimbt der Feb. seinen Anfang, in der wütterung den 5ten dito wider kalt, und cont. biß auff den 12 ten dito, da hat Es wider geschnien und kalt darauff und cont. biß auff den 16. \& 17ten dito, Es hat 31ig Stund an Einander geschnien, (Schuhmacher Chronik II: 417f.)

17. Wie aber das weltliche Drama eben durch eine solche Ausstattung zu Schaden kam, so ist auch wohl die höhere poetische Entwicklung des Mysteriums selber durch dieses unmäßige Vordrängen der Schaulust gehemmt worden. (Burckhardt V: 297)

18. All die physiologischen Leistungen, auf denen diese sog. Konstanzphänomene beruhen, sind erkenntnis-theoretisch deshalb von so großem Interesse, weil sie der schon besprochenen Leistung der bewußten, verstandesmäßigen Objektivierung streng analog sind. Wie der Mensch in meinem Beispiel die Temperatur der wahrnehmenden Hand in Rechnung stellt und so die »subjektive« Wahrnehmung »fieberheiß« auf ein »objektiveres« Maß reduziert, so sieht auch die »konstantmachende « Wahrnehmung der Gegenstandsfarbe von der augenblicklichen Beleuchtungsfarbe ab, um eine dem Objekt eigene Reflexionseigenschaft zu ermitteln. (Lorenz VII: 23)

19. Wie die große Mehrzahl der Dinge in unserem Universum ist aber der Hund durchaus nicht von menschlicher Planung entworfen. (Lorenz VII: 29) 
20. Es gibt aber auch Leistungen des Wissensgewinns, die gänzlich anderer Art sind. So wie die Anpassung körperliche Strukturen schuf, die dem Erwerb und der Verwertung von Energie dienen, hat sie auch solche hervorgebracht, deren Funktion im Gewinnen und Auswerten von Information, von Wissen besteht, und zwar von Wissen über augenblicklich in der Welt des Organismus obwaltende Umstände, denen sofort Rechnung getragen werden muß. (Lorenz VII: 42)

Proportionalsätze (Kapitel 5.4.8)

1. So viel der Innhalt/ den Worten nach/ an ihrer Reinlichkeit abnimmet/ so viel nimmet hingegen die Zierlichkeit der Schrifft $\mathrm{zu} /$ dergestalt/ daß nirgend keine trefflichere Schreiber- und Buchdruckereyen/ als in Teutschland zu finden sind. (Harsdörffer I: 10)

2. Je hôher einer geadelt/ je gnådiger er seyn soll! (Harsdörffer I: 27)

3. So wenig man das Hohelied aus der Schrifft mustren kann / so wenig kann man auch die Reden und Meigung von der Liebe entfernen / und wird solche Liebesneigung nicht als Laster / sondern als eine Tugend betrachtet (Harsdörffer I: 368)

4. Je kürtzer er seine Meinung schliessen kann/ je lieblicher/ und leichter ist das Lied/ und das Gedicht. (Harsdörffer I: 567f.)

5. Aber diße Statt wahr gantz der babistischer Religion, derohalben ich keinen evanielischen gelerdten Heren kundt haben, mich zu trôsten. Ja, je mehr ich Gott anruffete und bitte um sinen heiligen Geist, Hilff und Beystandt, je mehr sich der Teiffel an mich setzete zu plagen mit schwỏhrmietigen Gedancken. So ich die schỏhne Sprich und Verheißungen Gottes Wordts nicht wußtete und deß Jobs gedachte, so wehre ich in meinem Leiden vergangen (Güntzer I: 102r)

6. Je konsequenter hierauf der Humanismus sich die Anschauungsweise der Italiener dienstbar machte, desto fester überzeugte man sich auch, daß die Abstammung über den Wert des Menschen nicht entscheide. (Burckhardt V: 262)

7. Je weniger nun die Unterschiede der Geburt einen bestimmten Vorzug verliehen, desto mehr war das Individuum als solches aufgefordert, all seine Vorteile geltend zu machen; desto mehr musste auch die Geselligkeit sich aus eigener Kraft beschränken und veredeln. (Burckhardt V: 267)

8. ich mußte nehmen was ich bekomen konnte, und nicht was ich hätte sollen haben und wie bälder dieselben verkauft wurden desto besser indem sie // täglich i $\bar{m}$ Werthe fielen, ... (Auswandererbriefe V: 18)

Temporal-kausale Adverbialsätze (Kapitel 5.4.9)

1. Da es Tage wirdt, danckete ich ihme um die Herberg und ginge meine Straß weidters fordt durch den Walt. (Güntzer I: 91r-v)

2. Da er sahe, daß ich hardt schlieff, wolte er seine Schelmenstuck und Unkischheidt an mihr volpringen, mitt Refferentz am After. (Güntzer I: 56v) 
Lokalsätze (inkl. lokal-konditional) (Kapitel 5.4.10)

1. Wo man treibt keine Tugend mehr/ da steigert man der Titul Ehr. (Harsdörffer I: 28)

2. Wo die Ursachen auffhòren/ fănget die Gewonheit an: (Harsdörffer I: 560)

3. wo man keine Ursach geben kan/ folget man bildlich der gebrăchlichen Mundart/ und lăsset die blinde Gewohnheit des unverståndigen Pôbelvolcks an seinem Ort verbleiben. (Harsdörffer I: 560)

4. wo aber ein Mitlauter in eben der Syllbe folgt, klinget es hell, wie das e ouvért, als: wer, denket. (Aichinger III: 8)

5. In Venedig und Florenz gab es zur Zeit der Renaissance für die Männer vorgeschriebene Trachten und für die Frauen Luxusgesetze. Wo die Trachten frei waren, wie z. B. in Neapel, da konstatieren die Moralisten, sogar nicht ohne Schmerz, daß kein Unterschied mehr zwischen Adel und Bürger zu bemerken sei. (Burckhardt V: 268)

6. Offenbar ist dieser Dilettantismus, sowohl der vornehmern als der mittlern Stände, in Italien verbreiteter und zugleich der eigentlichen Kunst näher verwandt gewesen als in irgend einem andern Lande. Wo irgend Geselligkeit geschildert wird, ist auch immer und mit Nachdruck Gesang und Saitenspiel erwähnt; (Burckhardt V: 284)

7. Wo sie [die Kausalität, P.K.] experimentell erarbeitete Tatsachen nicht mehr einzuordnen vermochte, stellte er sie einfach beiseite und ersetzte sie durch Wahrscheinlichkeitsrechnung. (Lorenz VII: 31)

Pragmatische Adverbialsätze (Kapitel 5.5)

Moduskommentierende Adverbialsätze (Kapitel 5.5.1)

1. Wann der Mensch seine Stimme und Aussprache verandert/ in der Kindheit klar/ in dem Alter groeber zu sprechen pfleget; wie sollte dann nicht auch die Art zu reden sich veråndern/ und zugleich mit der; in den nechsten hundert Jahren viel zierlicher Schrifft/ gewandelt worden seyn: ... (Harsdörffer I: 170)

2. Ist dieses gantze Weltwesen so wandelbar/ wie sollte man dann in einem so flüchtigen Wortlaut einige Beståndigkeit erhărten oder bejahen ko̊nnen? (Harsdörffer I: 559)

3. Wihr kunden vohr Miedigkeitt deß Schröckens und Ruffen zu Gott balt kein Atem mehr recht erholen und sprachen: Herr, Gott, unßer Vatter im Himel, dieweil du unß um der Sinden wilen also wilt straffen, daz wihr zugrundt sollen gon, so nime unßere Sele in deine Hende und laße es balt geschehen, dan wihr sindt schwache Menschen, kunden balt in Ungedult komen. (Güntzer I: 66r)

4. So er es nicht glauben wolt, so nömpt mich alhie gefangen und fieret mich morgen in die Statt, so werdt ihr sehen, wer ich bin. (Güntzer I: 89r)

5. Da, wie ich hoffe Euch dieser Brief am Neuenjahre antreffen wird, so wünsche ich Euch hiermit Glück u. Freude zum Neuen-Jahre, Gesundheit und noch recht viele glücklichen frohen Jahre nach diesem. (Auswandererbriefe V: 2) 
6. wē̄ du er das es erhalten habst $\mathrm{a}$ so solst du auch eine Quittung schicken. (Auswandererbriefe $\mathrm{V}: 15$ )

7. Es hat uns sehr gefreut daß dein Fritz auch einen Gruß an uns geschrieben hat, und weil du geschrieben hast ich soll deinem Johan̄es ein paar Hosen kaufen so kom du nur mit ihm heraus so kauf ich ihm gleich ein paar. (Auswandererbriefe V: 16)

8. Deshalb erßuche ich euch, wē̄ sie euch auch schłon geschrieben hat, so möchtet ihr so gut s sein und mir die Atresse schicken. (Auswandererbriefe V: 20)

9. ich glaube daß es für dich besser wäre hier als in Deutschland. wen du Lust hast hierher zu kommen, und es dir sollte an Reisegeld fehlen, laß mich es wissen ich werd dir sogleich das Reisegeld, oder einen Reisebas schicken, (Auswandererbriefe V: 21)

10. Liebste Eltern wen sich Euer Geschäft nicht rendirt oder wen diese Krankheiten nicht aufhören so verkauft wen Ihr könt und kō̄t zu uns ... (Auswandererbriefe V: 26)

11. Wenn der Blick nach innen unmittelbar tiefste Wahrheiten erschauen konnte, während der nach außen gerichtete bestenfalls die Gesetzlichkeiten einer Chimäre, eines Traumes, aufzuzeigen vermochte, ja, wer wollte sich da mit der langwierigen und mühsamen Erforschung der Außenwelt befassen, die noch dazu in manchen ihrer Aspekte wenig anziehend erschien? (Lorenz VII: 28f.)

Modusmodifizierende Adverbialsätze (Kapitel 5.5.2)

1. So seind Sie deßwegen zum Schwert verurtheilt worden und die Köpff auff die Spitz zu stecken, und der Mutter die rechte Hand abhauen, welches abhauen der Scharff-rüchter mit Namen Johannes Volmar erbetten, Sie möchte Ihm zu Schwach werden, daß Er Sie nicht könnte rüchten. Welches rüchten ist geschehen den 29. Augs. an Johanny Enthauptung Freitags und wie Ich von den Alten gehört hab, So solle Es Just 45ig Jahr sein, daß man der alten Ihren Vatter u. Bruder auch mit dem Schwerdt solle hingerücht haben, und seind die bayde Mutter $u$. Tochter wider in Ihr Grab unter dem Haupt-Gericht oder Galgen gelegt worden, und seind die Köpff auff zwey Spitz gesteckt worden, welche man lange Zeit zum Spectacul hat söhen können, hernach ist der Vatter Johannes Ziegler umb 40ig Florin gestrafft worden, und der Joh. Jacob Spohn mit 80ig Florin Straff belegt worden und ist also die Sach zum Ende gebracht. (Schuhmacher Chronik II: 380)

2. Unsre Bilder wirst du noch nicht erhalten haben, so viel wir gehört haben ist der Mā̄ noch da er habe den Prozeß verspie[1t.] (Auswandererbriefe V: 15)

3. ich werde gleich nach Neujahr wieder hinmüßen, um wahrscheinlich mehrere Wochen dort zu bleiben es wäre mir allerdings lieb wenn ich könnte die Geschäft mehr durch andere Leute verrichten lassen ich werde so viel ich kann, aber so viel ich bis jetzt wahrgenomen habe, wird unsers Bruders hinterlassenschaft als eine fette Gans betrachtet, an welcher jeder gern rupfen möcht ich halte es deßwegen für meine Pflicht, selbst so viel wie möglich danach zu sehen, ... (Auswandererbriefe V: 18)

4. Wie man sieht, eignet sich die Ausdrucksweise der Informationstheorie nur wenig für die Beschreibung von Lebensvorgängen. (Lorenz VII: 39) 
Diktums- bzw. einstellungskommentierende Adverbialsätze (Kapitel 5.5.3)

1. Etliche sagen c sey kein teutscher Buchstab: sie irren aber weit / weil es in den allerăltesten Schrifften befindlich; wiewol ich gerne zugebe er sey neuer als das k / und eben der Lieblichkeit halber erfunden worden: (Harsdörffer I: 468)

2. Heute ist ein total mieser Tag gewesen! In der Schule hab' ich Stunde gehabt, siehe Zettel, aber - was am schlimmsten ist, es kriselt gerade sehr ernsthaft. (Jugendtagebuch VII: 4)

3. Um 3 Uhr schaute ich aus dem Fenster, da kam L. gerade. Wie ausgemacht, ging ich runter. Im Schlafzi. schlug sich Papa unruhig von einer Seite auf die andere, sein Atem ging unruhig und ich hatte Angst mein lauter, starker Herzschlag könnte ihn wecken. (Jugendtagebuch VII: 21)

Formulierungskommentierende Adverbialsätze (Kapitel 5.5.4)

1. 17 A N N O $31 / /$ So Ich daß Jahr nach den Monat beschreibe So hat sich der Janw. mit Sonnenschein angelassen, den 4ten mit regen, den 9ten \& 11ten geschnien, darauff Sonnenschein und kalt den gantzen Monat durch, auch zuweilen trieb, den 30igsten daß wetter gebrochen, den 31 igsten geschnien. (Schuhmacher Chronik II: 445)

2. Also wenn man's genau nimmt, ist meine Kindheit jetzt vorbei. (Jugendtagebuch VII: 20)

3. Wie der Freiburger Philosoph Szilasi in seiner durch geringe Beherrschung der deutschen Sprache zu lapidarer Größe gezwungenen Ausdrucksweise zu sagen pflegte: »Gibt es nicht ein Ding an sich, gibt es viele Ding-an-siche.« (Lorenz VII: 23)

Diskursstrukturierende Adverbialsätze (Kapitel 5.5.5)

1. Es folget sehr wenig Buß, man verfolgt einander, welches Ich selbsten hab fühlen müssen und grosse Verfolgung außstehen. Waß noch weiters passiert. So hat es den 9ten Novemb. einen grossen Sturmwind geben und den 11ten dito als an Martini hat Es einen Schnee herunter geworffen und kalt darauff worden. (Schuhmacher Chronik II: 411)

2. Und wie du geschrieben hast unsere Kinder nehmen nun schon den Platz ein den wir noch ohnlängst besasen und wir werden alt ohne daß wir es wissen. (Auswandererbriefe V: 12)

3. Wir wir mit Bedauern vernomen haben hat sich bei Euch so maches Schicksal zugetragen und Ihr habt freilich wenig Freude gehabt. (Auswandererbriefe V: 26)

4. Wie ich in dem Briefe vernomen habe ist der Grünwald nach euch da ich denk er wird sich recht Gelde ersparen und dañ schreiben ein Gruß an seine Frau. (Auswandererbriefe $\mathrm{V}: 26)$

5. Wie schon erwähnt, besteht ein solches Bildverhältnis zwischen Organismus und Wirklichkeit schon auf der Ebene des Körperbaus, der »Morphogenese«, man denke an die Sonnenhaftigkeit des Auges oder an die Wellenbewegung der Fischflosse (S. 17). (Lorenz VII: 40) 
6. Wie schon gesagt, ist das Leben gleichzeitig Informationserwerb, d. h. ein kognitiver Vorgang, und ein ökonomisches, man ist versucht $\mathrm{zu}$ sagen, ein kommerzielles Unternehmen. (Lorenz VII: 45f.)

7. Wie schon in den Prolegomena auseinandergesetzt, gründet sich meine Betrachtungsweise der Erkenntnisfunktionen und aller Lebensvorgänge überhaupt auf eine erkenntnistheoretische Einstellung, die ich mit Donald Campbell als die des hypothetischen Realismus bezeichnet habe. (Lorenz VII: 303)

\section{Thematisierende Adverbialsätze (Kapitel 5.5.6)}

1. Am Auffarths Tag Ist abermahl der König in Franckreich im Würtemberger Land eingefallen und überzogen, daß daß Land 10 Tonnen Golds Rantzion geben, auch die Vöstung Schondorff eingenom[m]en und hat daß Land viel hundert Centner Meel geben müssen. Bald darauff ist er widerum Abgewichen $u$. daß meel stehen bliben wegen unserer Völcker die Ihm den Paß haben wollen Abschneiden u. also daß Land widerumb befreyhet. Waß die reichstätt anbelangen, ist Ihnen ebenmässig auch ergangen, wir haben es auch gefühlet In unser geliebten Vatterstatt Reuttlingen. (Schuhmacher Chronik II: 329)

2. Waß sich [mehres] hat zugetrage[n] würdt Es die Feder erst zeigen. Waß der Früeling betreffend, So hat es gleich im Anfang deß Jahrs auffgeschlagen. So galt der Laib Brodt umb Liechtmes 20 x. (Schuhmacher Chronik II: 343)

3. Waß der Weinwachs betreffent, so hat derselbige frühzeitigen Anstoß gelitten wegen rauen wetters u. kalten Winden. (Schuhmacher Chronik II: 345)

4. Waß der Herbst betreffend So hat uns Gott einen köstlichen Tranck geschert, aber wenig wie in vorigen Jahren. (Schuhmacher Chronik II: 357)

5. Waß daß letztere Quartl. anbelangt, So hat sich der winter mit gutem Wetter angelassen, wie man Es gerne wünschet, mit Sonnenschein auch regen $u$. Schnee, ausgenom[m]en der Decemb. gehet Ein mit continuierlichem regenwetter, (Schuhmacher Chronik II: 358)

6. 17 A N N O 26 // Waß dises Jahr anbelangt, So hat sich ein unglick zugetragen, den 5. Janw. deß Nachts, So daß die Sheur auff der Alten-Burg ist in die Asche gelegt worden (Schuhmacher Chronik II: 398)

7. also sampt Frucht und Futter Jäm[m]erlich verbrunnen. // Fenner waß die drey erste Monat anbetreffen, So hat Sich der Janw. mit kaltem Wetter und Schnee hindurch praesentiert, der Feb. hat sein Art auch nicht verlassen sondern recht winterisch eingestelt mit Schnee und Sturmwind biß zum End. (Schuhmacher Chronik II: 399)

8. Waß der Mertz anbelangt, So hat Er sich auch noch recht winterisch im Anfang eingestelt mit vilem Schneien und kälte, mit reiffen und Kitzbonen zuletzt aber mit $\mathrm{O}$ schein. (Schuhmacher Chronik II: 399)

9. Waß der Herbst betreffend So hat uns Gott einen köstlichen Tranck geschert, aber wenig wie in vorigen Jahren. (Schuhmacher Chronik II: 399)

10. Waß der Augustus anbetrifft, So hat Er sich mit gutem wetter praes. in mittelst deß Monats Sturmwind, und cont. gut Wetter hindurch, daß also köstliche Frucht ist einge[...]net worden, aber wenig doch gut und seind die Trauben umb Bartholomey fast alle zeitig gewessen. (Schuhmacher Chronik II: 401) 
11. Waß fenner daß Wachstum anbelangt, So hat unß Gott theils Orten zimlich Obs bescheret, der Apffel mehr als der püren und sind merentheils durch den Brand verzert worden. (Schuhmacher Chronik II: 402)

12. Es hat daß Obs bey unß zimlich verzogen, aber unsre benachtbarte Örter nämlich im Uracher-Thal, im Hausamer-Thal und Gininger-Thal, daß sie es zum theil reichlich bekom[m]en und genossen. Waß der Som[m]er anbetrifft, So ist Er Iederzeit warm gewessen, daß alles ist wol ist einkommen von allerhand Frucht und Futter (Schuhmacher Chronik II: 410)

13. So daß der Laib Brodt nach der Ernd ist auff $6 \mathrm{x}$ komen und cont. durch daß gantze Jahr. Waß der Autumnus anbelangt, So hat unß Gott einnen reichen Seegen geben in den Weinbergen, welches man nicht verhofft wegen der gefrüre so sich im Frieling ereignet wie Oben gemelt. (Schuhmacher Chronik II: 410f.)

14. 17 A N N O 28 // Waß daß 28igste Jahr anbelangt, und Ich Es Quartaliter beschreibe, So ist es ein gutter Winter gewessen. So hat es im Janw. Sturmwind geben und offtmals geregnet, \& nicht viel kalt, doch hat sich der Winter im Feb. mit kälte mehr Spüren lassen. (Schuhmacher Chronik II: 412f.)

15. daß man auch hat können wider ein zeichen geben, in die Kürch zu gehen. Welches ist daß erste mal geschehen am Sontag Reminiscere. Waß der Fer anbelangt, so hat er Sich mit vermüschter Witterung praes. Im Anfang deß Mertzen etwan kalt, auch regen, hernach warmmer Son[n]enschein den gantzen Monat durch. (Schuhmacher Chronik II: 413)

16. Auch ist den 27igsten dito die erste Copulation \& Hochzeit gehalten worden. Waß der Aestas anbelangt, So hat Er Sich mit warmem Wetter praes. Daß der Traubenblüeet absonderlich in Juny föllig vorüber gewessen vor Johanne sein $\beta$ wenig mehr zu finden gewessen die noch nicht verblüet haben. (Schuhmacher Chronik II: 413f.)

17. Es hat auch viel Apffel geben, man hat 20ig auch 22 ig um ein Kreutzer geben. Waß der Wein betreffend, so hat Es dessen die Minge gegeben, und hät noch mehr geben wan[n] Er nicht hät dreyfachen Schaden gelütten, wegen deß Hagels und der Feule, die Trauben seind theils Orten so verfaulet, daß man schier kein reinen hat haben können. (Schuhmacher Chronik II: 415)

18. Waß der Wein-Preiß anbetrifft, will Ich fenner beschreiben. Waß die zwey letztere Monat betreffend, zu deß Herbsts und Jahrs Ende, So hat der Novemb. Sich mit kaltem Wetter vermercken lassen, um St. Catharina hat Es geschnien, vorher grosse Sturmwind um Andreae grosse Winterkälte, deßgleichen im Decemb. (Schuhmacher Chronik II: 415f.)

19. Es war umb Georgy der Rebstock noch gantz blind, daß kein Traub nicht ist gesehen worden. So hat Es eine mittelmeßige Ernd an Frucht geben, aber sehr wol und gut heimkom[m]en, waß daß Obs anbelangt, So hat Es wenig geben, theil Orten gar keinß, die zweschgen seind an theil Orten auch gerathen. (Schuhmacher Chronik II: 423)

20. 17 A N N O 30 // Waß daß Jahr anbetreffend, so hat sich der Janw. Feb. \& Martzy mit recht winterischem wetter, mit Schnee, Kützbonen und kälte eingestelt, den 23. \& 24igsten Martzy hat Es so viel geregnet, und ein gewässer geben, daß der Neccar übergeloffen war also ein kalter Hyem. (Schuhmacher Chronik II: 441)

21. daß der Neccar übergeloffen war also ein kalter Hyem. Waß der Fer anbelangt, So hat sich der Appril noch winterisch erzeigt, daß Es den 10. und 15ten reiffen geben wie auch den 22. \& 24igsten reiffen, auch viel regen, war also ein nasser Appril, wegen rauen wetters war der Rebstock umb Georgy noch gantz blind, daß erst im Mayen seind Trauben gesehen worden. (Schuhmacher Chronik II: 441) 
22. wegen rauen wetters war der Rebstock umb Georgy noch gantz blind, daß erst im Mayen seind Trauben gesehen worden. Waß der Aestas anbelangt, So hat sich der Julius gut angelassen, den 12. \& 13ten unfreundlich wetter, hernach wider guttes wetter, rege[n] und Sonnenschein biß auff den 30igsten hat es ein reiffen gehabt, darauff Oschein. (Schuhmacher Chronik II: 441f.)

23. Der Septemb. gehet Ein mit reiffen, den 1. \& 2. hat Es Eys geben, fast alle Tag reiffen, doch ohne Schaden biß auff den 7 ten dito, hernach den gantzen Monat durch gutes wetter mit regen \& Sonnenschein, zum Ende warmes Wetter, als den 28. 29. \& 30igsten Sonnenschein, starcke reiffen \& kiele morgen. Waß der Authumnus betrifft, So hat Sich der October wider warm angelassen mit Sonnenschein, hernach den 7 ten ein starcken reiffen \& gefrohren und cont. biß auff den 12.13. 14. \& 15ten dito, daß die Weinberg ohne unterscheid erfrohren wegen grosser kälte. (Schuhmacher Chronik II: 442)

24. waß für Trauben so nicht gar zeitig gewesen, hat man müssen stehen lassen, dan die unzeitige Beer seind gantz roth worden, absonderlich Butscheren, Feldleyner, rothwelsch, theil weisse und andre Arten mehr. Waß der November \& December betreffend, So hat sich der November trieb angelassen, mit warme regen, Sonnenschein \& Sturmwind, daß Es gut bezüen gewessen, cont. regenwetter biß zu deß Monats End. Schuhmacher Chronik II: (443)

25. Was uns anbelangt so kan ich dir schreiben das es uns gut geht. (Auswandererbriefe V: 12)

26. Was die Arbeit betrift so konte sie dieselbe wohl aushalten sie hatte viele halbe Tage wo sie fir sich arbeiten Nähen oder Stüken kante. (Auswandererbriefe V: 23)

27. was das Essen betraft so kan ich darüber keine Ke Klage haben und ich denke sie konte es bei ihrer Arbeit auch dabei aushalten. (Auswandererbriefe V: 23)

28. Was mich betrifft bin ich gesund und munter und es geht mir und dem Carl recht gut es gefällt uns hier jeden Tag besser. (Auswandererbriefe V: 26)

29. Was uns betrifft sind wir alle gesund und munter und es gent uns recht gut. (Auswandererbriefe V: 28)

\subsection{Diagramme}

Die folgenden Diagramme zeigen die prozentuale Verteilung der Stellungstypen bei verschiedenen Typen von Adverbialsätzen. Auf die Angabe der absoluten Werte kann an dieser Stelle, der besseren Übersichtlichkeit halber, verzichtet werden, da sie in den entsprechenden Tabellen im empirischen Teil (Kapitel 5) zu finden sind. Die Daten für das Nähe- bzw. Distanzteilkorpus werden getrennt angegeben. 


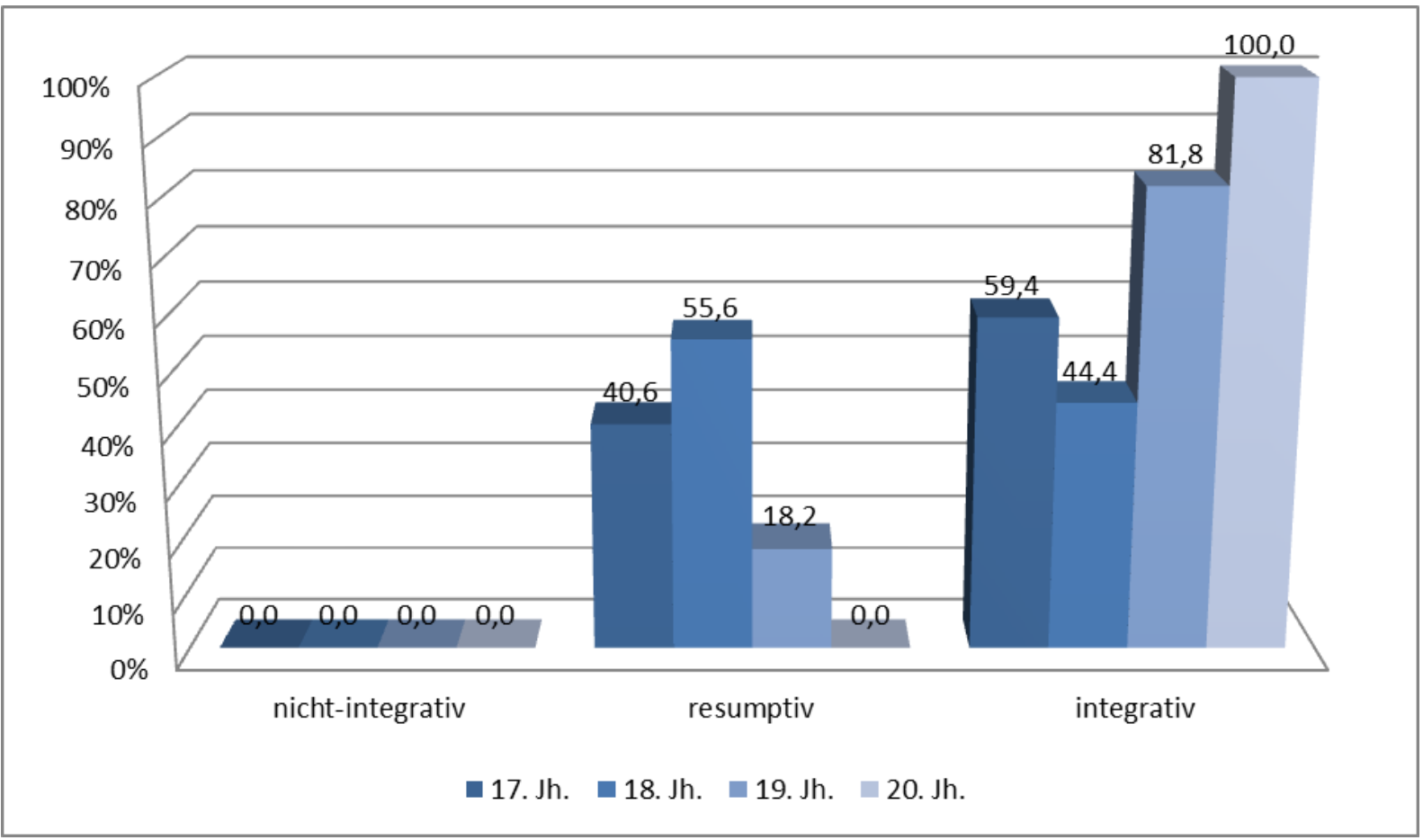

Diagramm 1: Prozentuale Verteilung der Stellungstypen bei vorangestellten Temporalsätzen im Näheteilkorpus [17. Jh.: Güntzer I ( $n=64)$, 18. Jh.: Schuhmacher Chronik II ( $n=9)$, 19. Jh.: Auswandererbriefe V ( $\mathrm{n}=11)$, 20. Jh.: Jugendtagebuch VII $(\mathrm{n}=19)]$

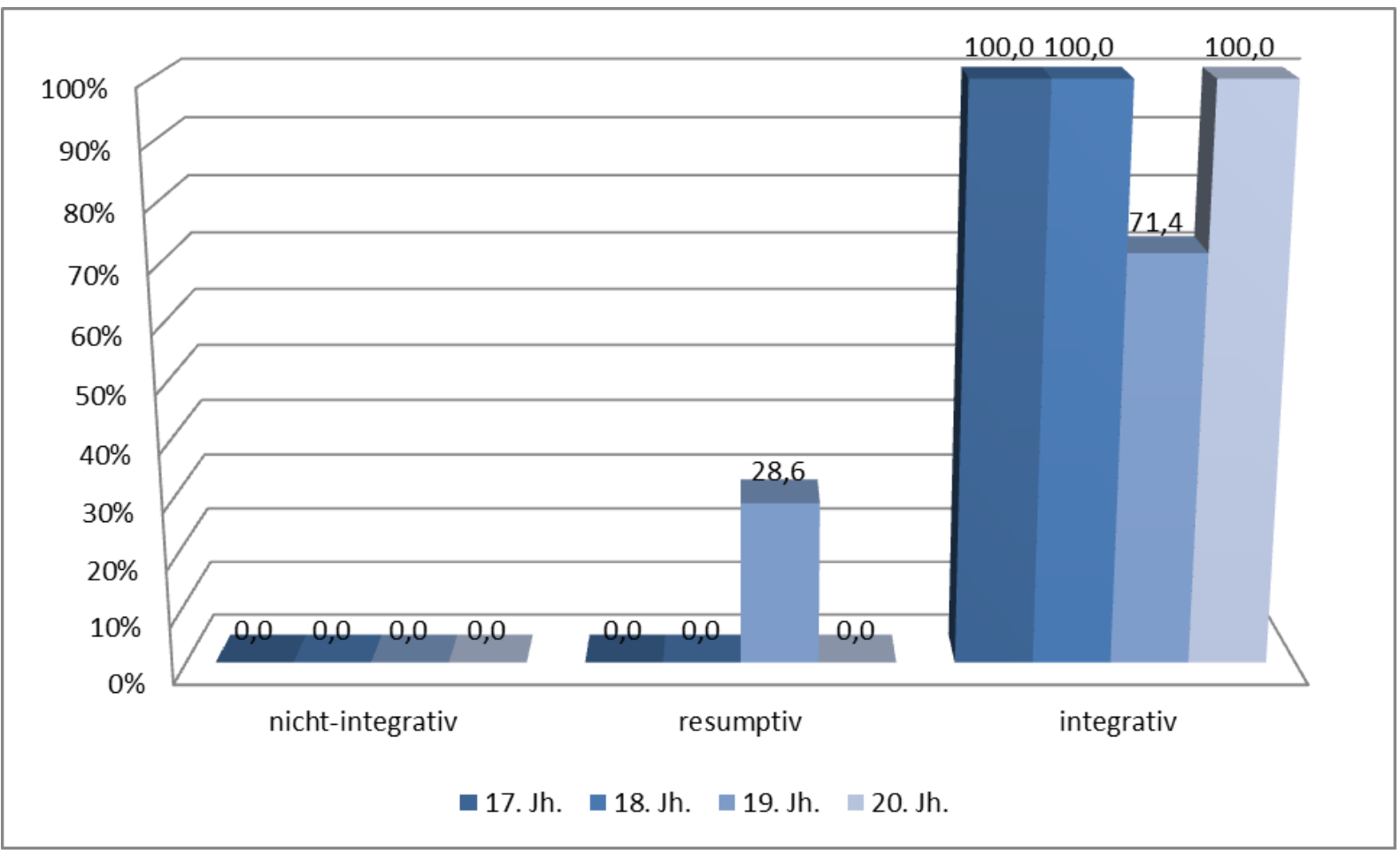

Diagramm 2: Prozentuale Verteilung der Stellungstypen bei vorangestellten Temporalsätzen im Distanzteilkorpus [17. Jh.: Harsdörffer I $(n=4)$, 18. Jh.: Aichinger III $(n=3)$, 19. Jh.:

Burckhardt V (n=7), 20. Jh.: Lorenz VII ( $n=4)]$ 


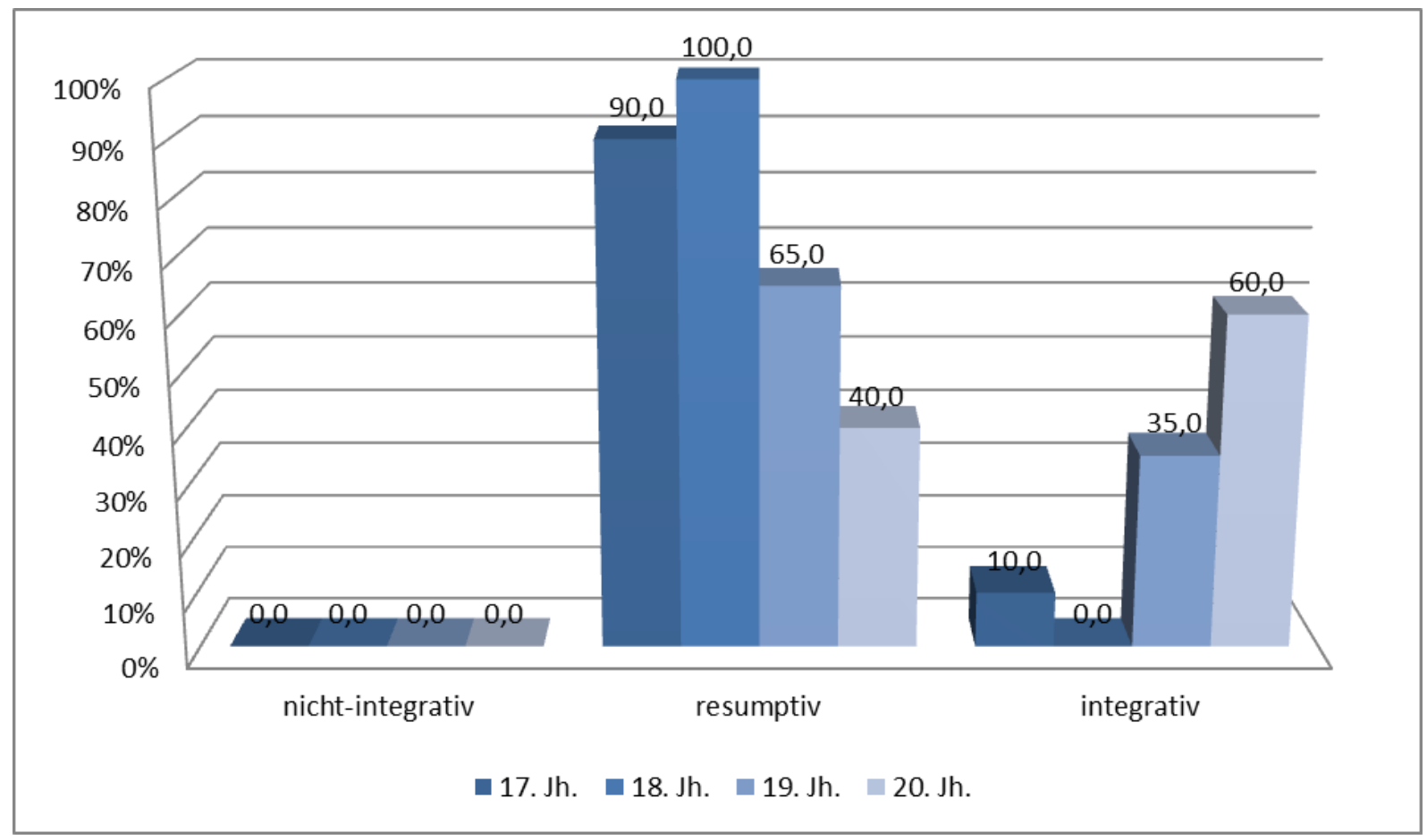

Diagramm 3: Prozentuale Verteilung der Stellungstypen bei vorangestellten, eingeleiteten Konditionalsätzen im Näheteilkorpus [17. Jh.: Güntzer I $(n=10)$, 18. Jh.: Schuhmacher Chronik II ( $n=1)$, 19. Jh.: Auswandererbriefe V (n=20), 20. Jh.: Jugendtagebuch VII ( $n=5)]$

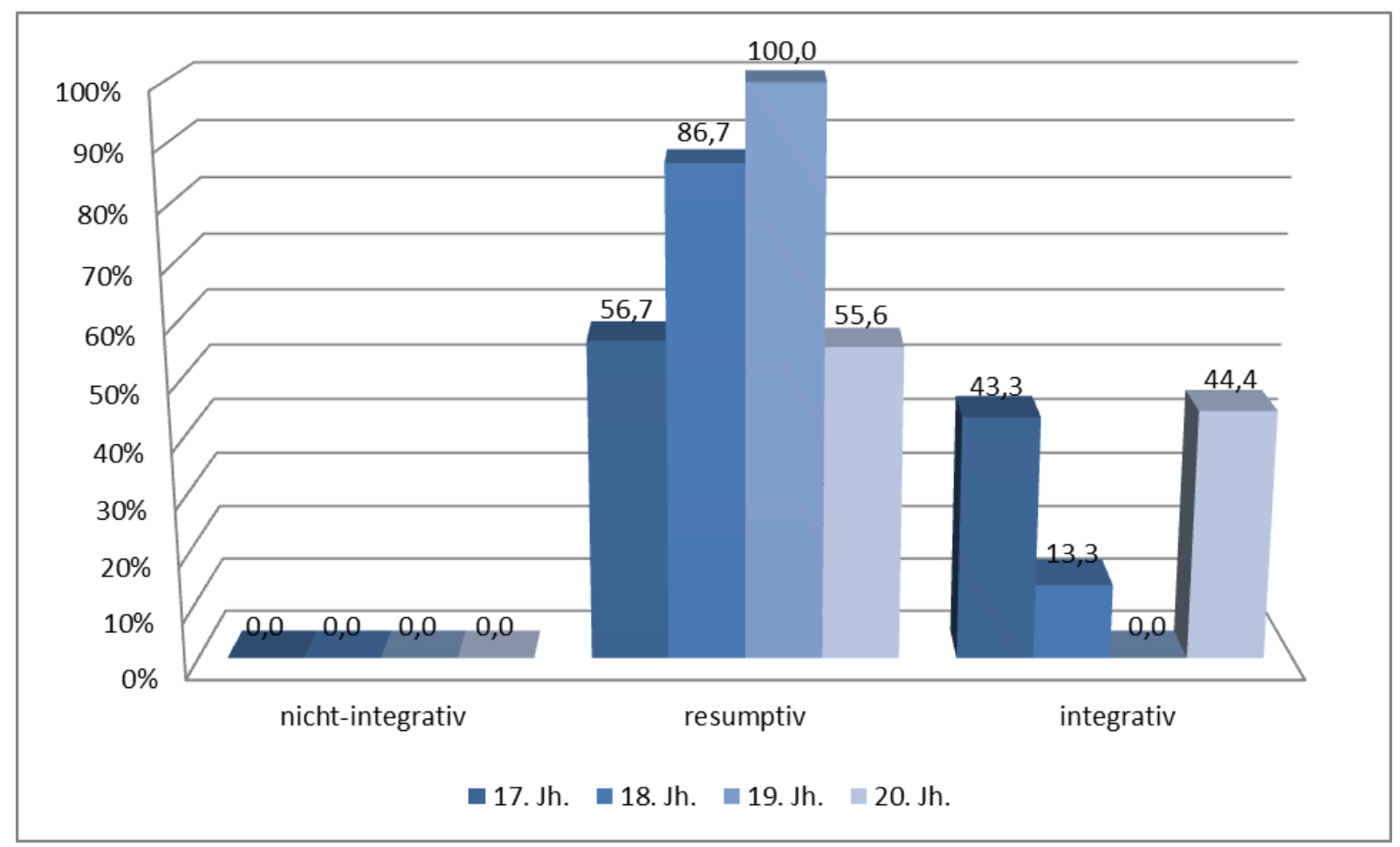

Diagramm 4: Prozentuale Verteilung der Stellungstypen bei vorangestellten, eingeleiteten Konditionalsätzen im Distanzteilkorpus [17. Jh.: Harsdörffer I $(n=30)$, 18. Jh.: Aichinger III $(n=15)$, 19. Jh.: Burckhardt V $(n=10)$, 20. Jh.: Lorenz VII $(n=18)]$ 


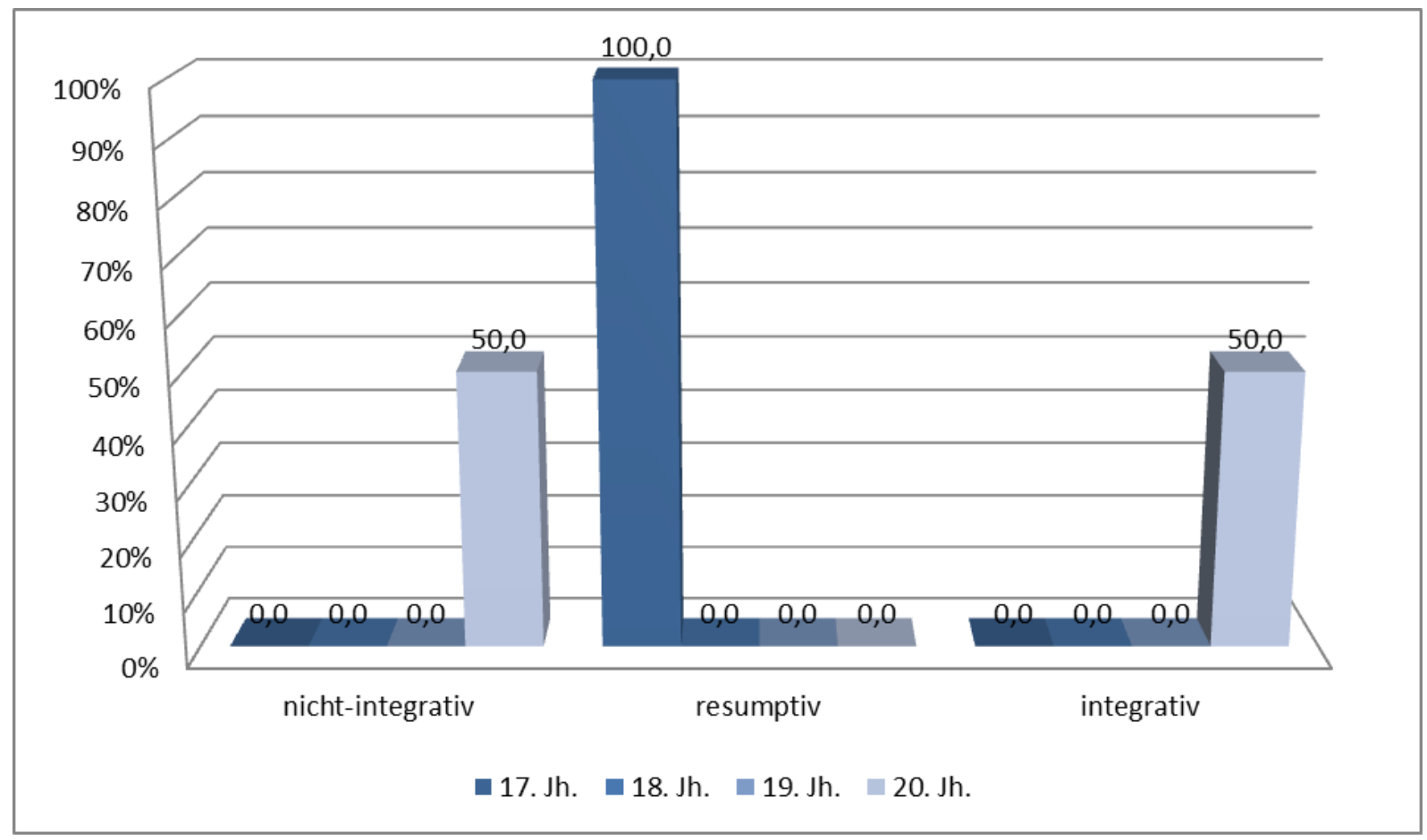

Diagramm 5: Prozentuale Verteilung der Stellungstypen bei vorangestellten, uneingeleiteten Konditionalsätzen im Näheteilkorpus [17. Jh.: Güntzer I ( $n=2)$, 18. Jh.: Schuhmacher Chronik II $(n=0)$, 19. Jh.: Auswandererbriefe V $(n=0)$, 20. Jh.: Jugendtagebuch VII $(n=2)]$

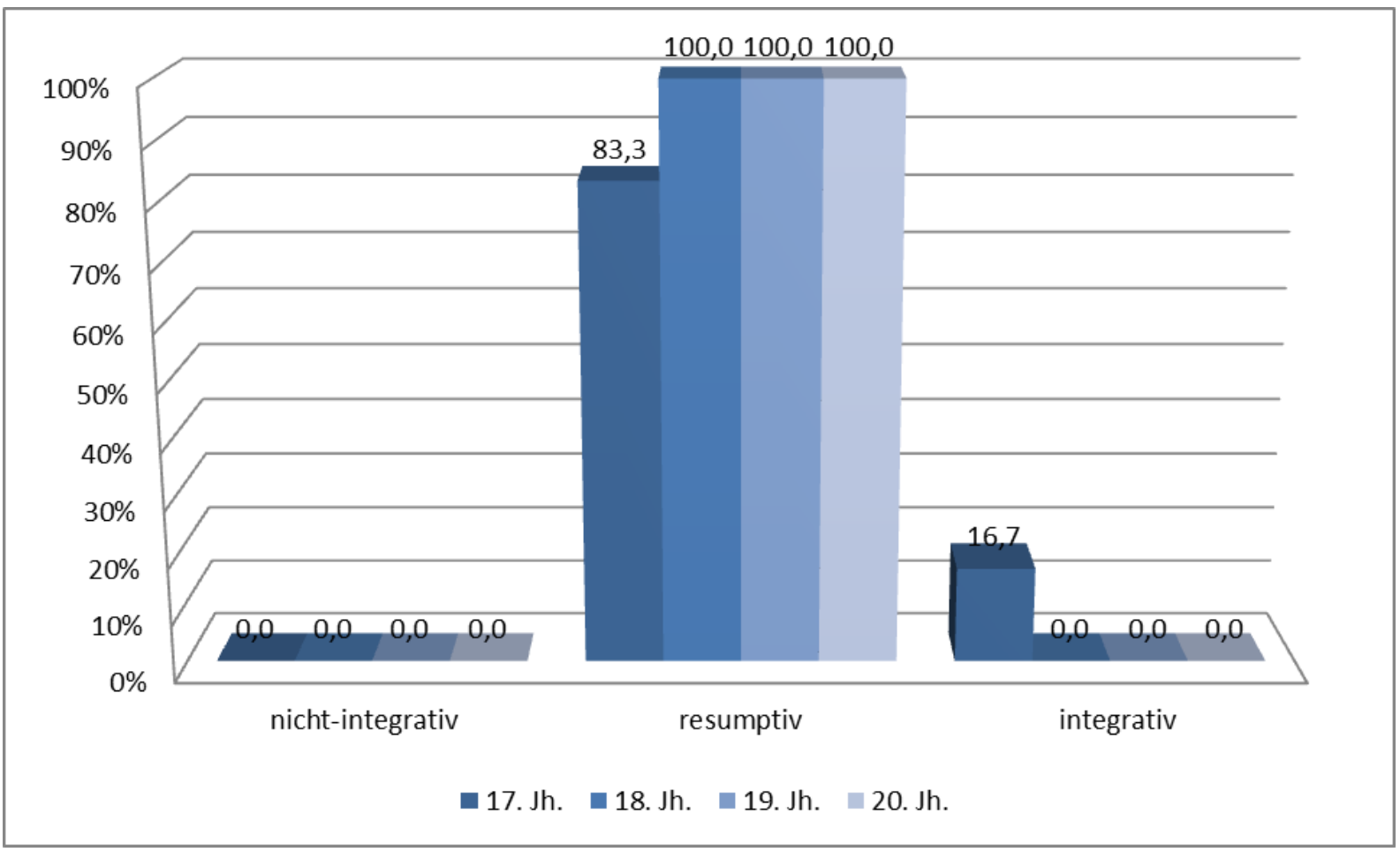

Diagramm 6: Prozentuale Verteilung der Stellungstypen bei vorangestellten, uneingeleiteten Konditionalsätzen im Distanzteilkorpus [17. Jh.: Harsdörffer I ( $\mathrm{n}=12)$, 18. Jh.: Aichinger III $(n=6)$, 19. Jh.: Burckhardt V (n=2), 20. Jh.: Lorenz VII $(n=4)]$ 


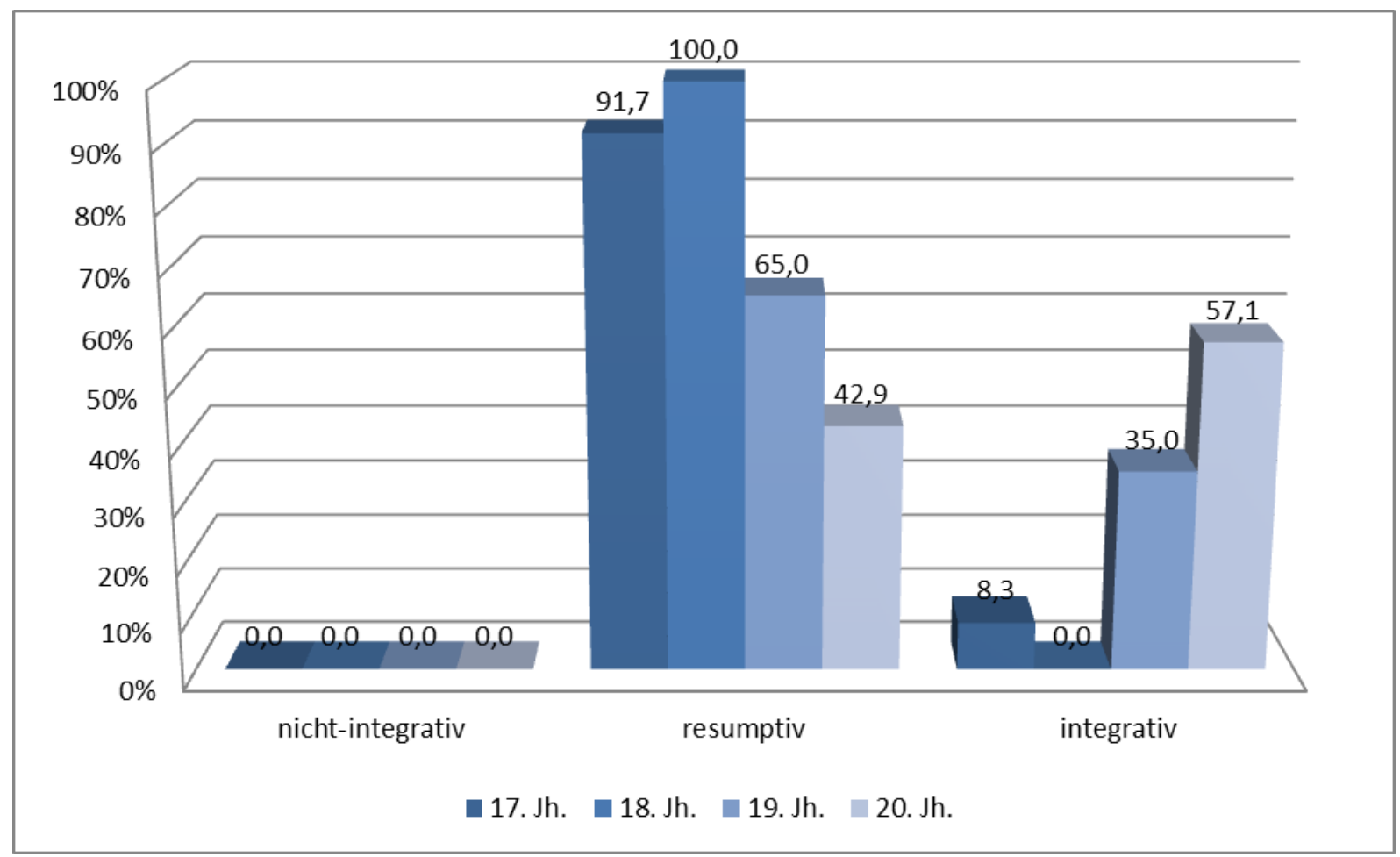

Diagramm 7: Prozentuale Verteilung der Stellungstypen bei vorangestellten Konditionalsätzen im Näheteilkorpus [17. Jh.: Güntzer I ( $n=12)$, 18. Jh.: Schuhmacher Chronik II $(n=1)$, 19. Jh.: Auswandererbriefe V $(n=20)$, 20. Jh.: Jugendtagebuch VII $(n=7)]$

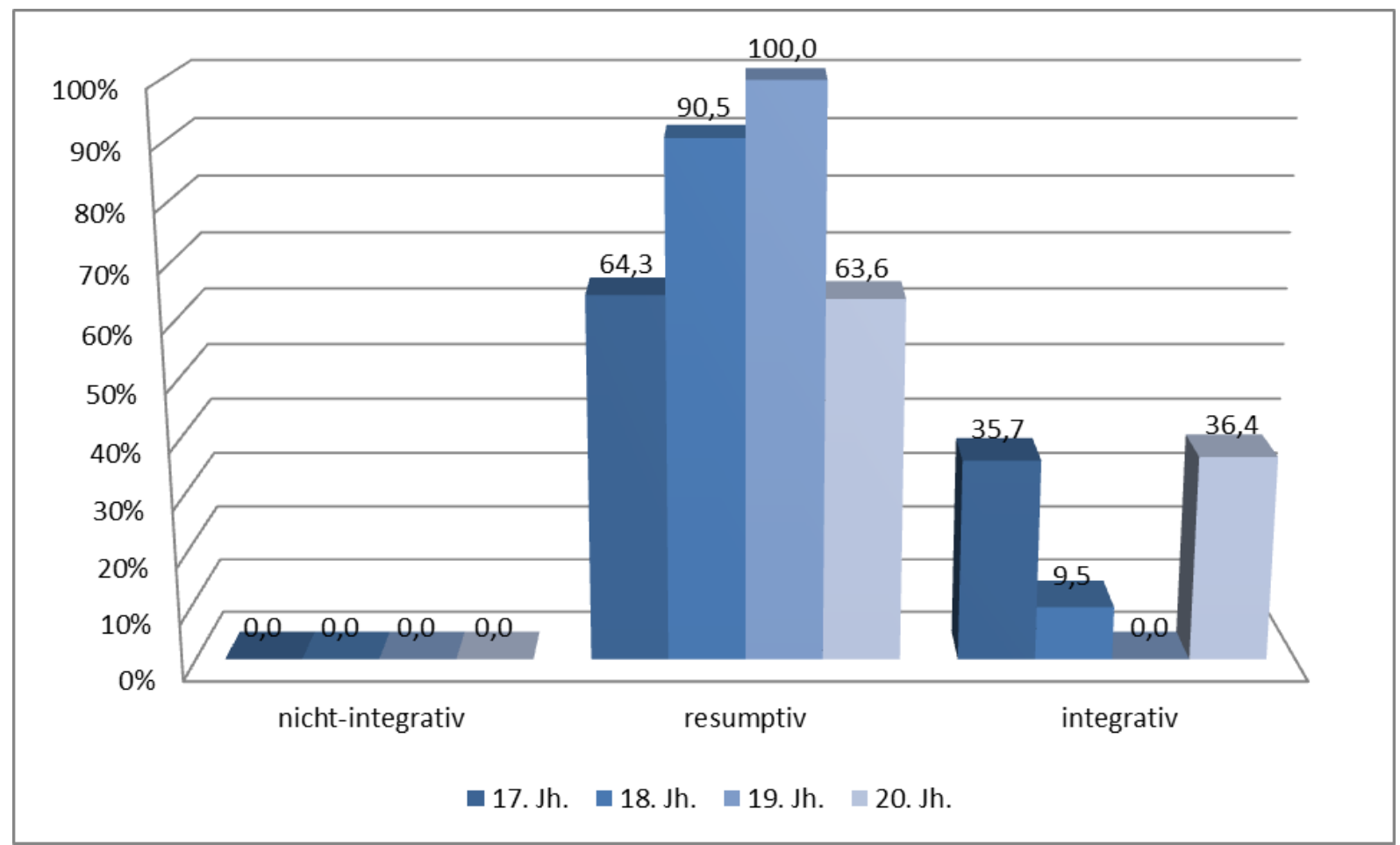

Diagramm 8: Prozentuale Verteilung der Stellungstypen bei vorangestellten Konditionalsätzen im Distanzteilkorpus [17. Jh.: Harsdörffer I ( $\mathrm{n}=42)$, 18. Jh.: Aichinger III $(n=21)$, 19. Jh.: Burckhardt V $(n=12)$, 20. Jh.: Lorenz VII $(n=22)]$ 


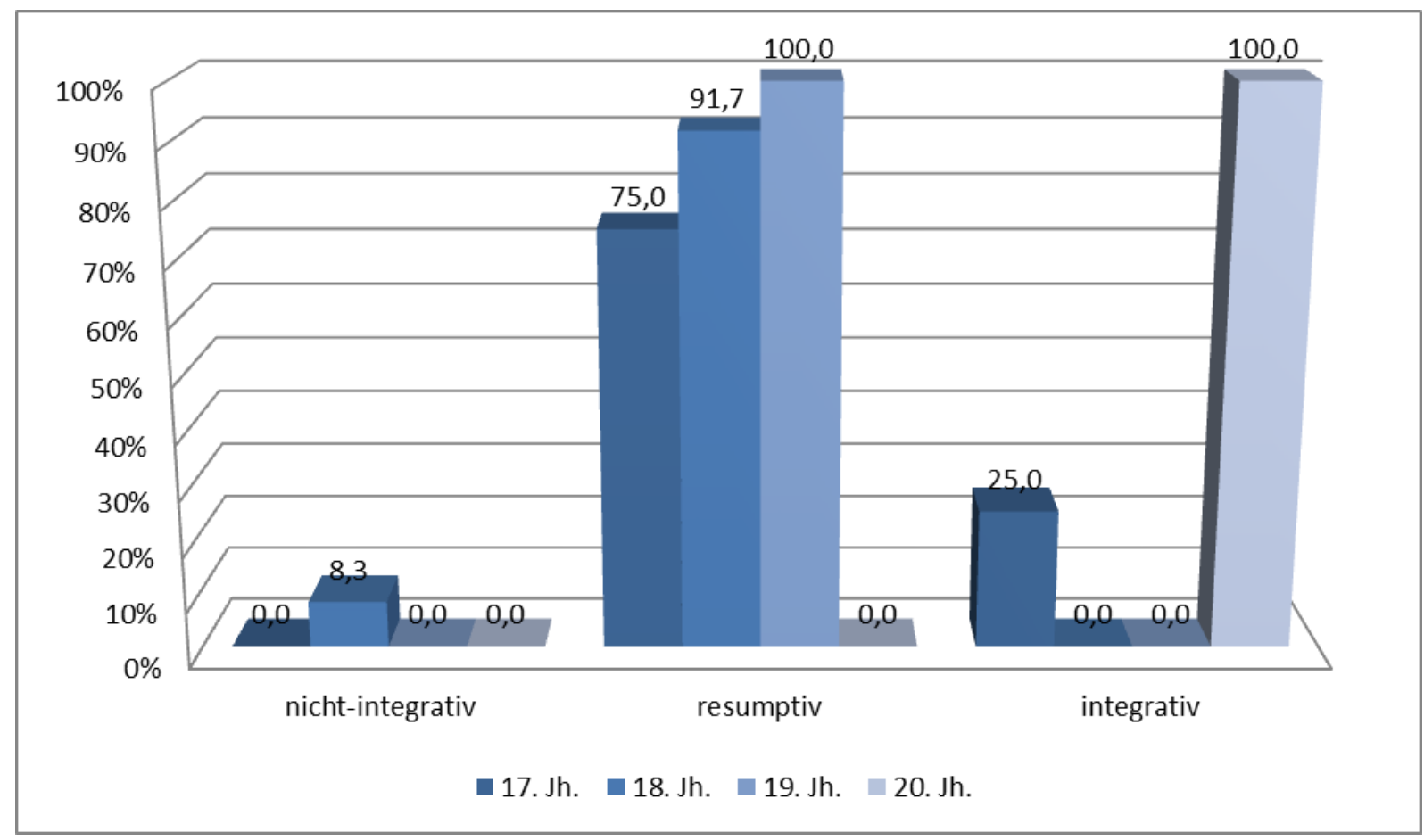

Diagramm 9: Prozentuale Verteilung der Stellungstypen bei vorangestellten Kausalsätzen im Näheteilkorpus [17. Jh.: Güntzer I ( $\mathrm{n}=8)$, 18. Jh.: Schuhmacher Chronik II ( $\mathrm{n}=12)$, 19. Jh.: Auswandererbriefe V (n=8), 20. Jh.: Jugendtagebuch VII ( $n=3)$ ]

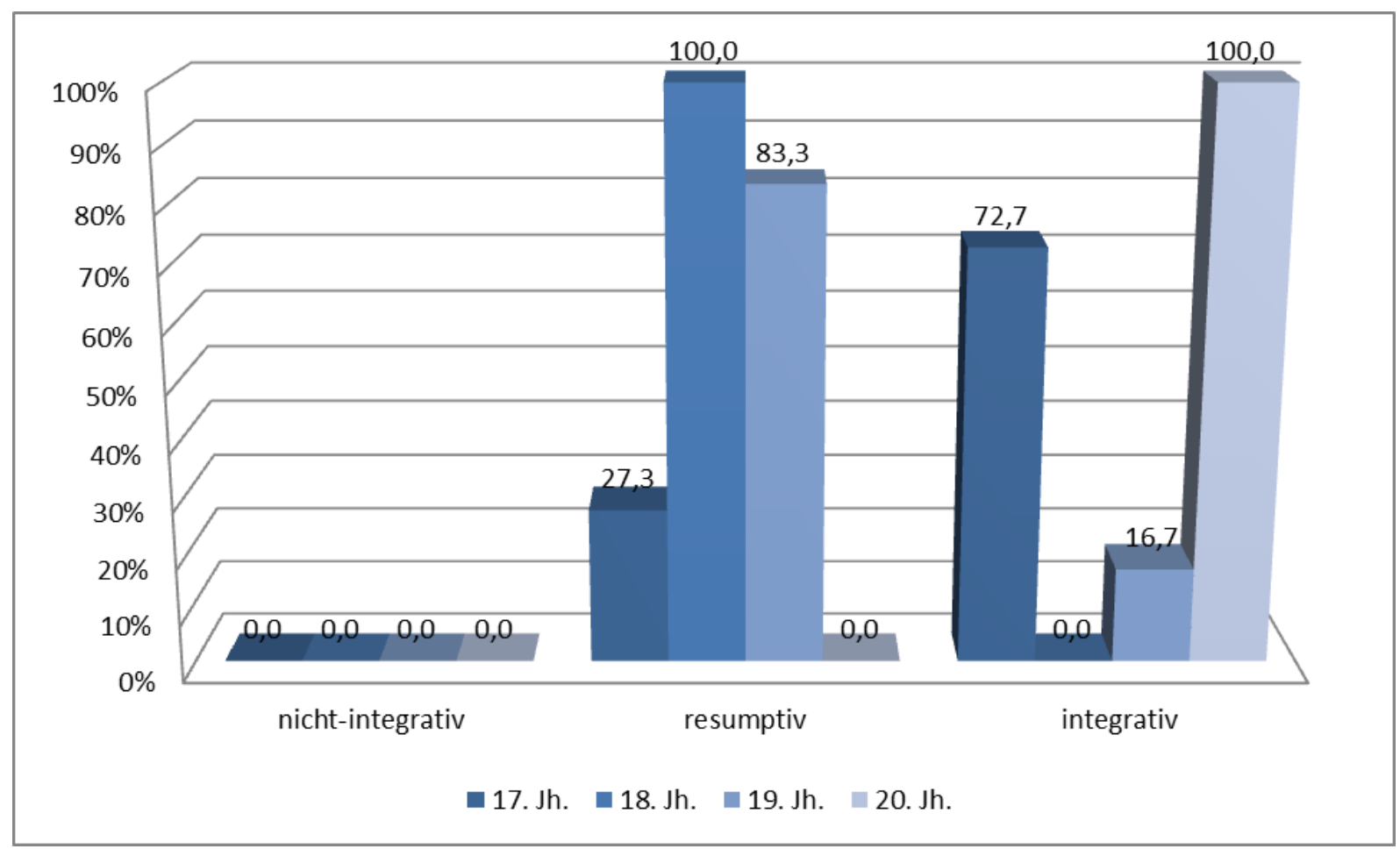

Diagramm 10: Prozentuale Verteilung der Stellungstypen bei vorangestellten Kausalsätzen im Distanzteilkorpus [17. Jh.: Harsdörffer I $(n=11)$, 18. Jh.: Aichinger III $(n=15), 19$. Jh.: Burckhardt V (n=6), 20. Jh.: Lorenz VII $(n=3)]$ 


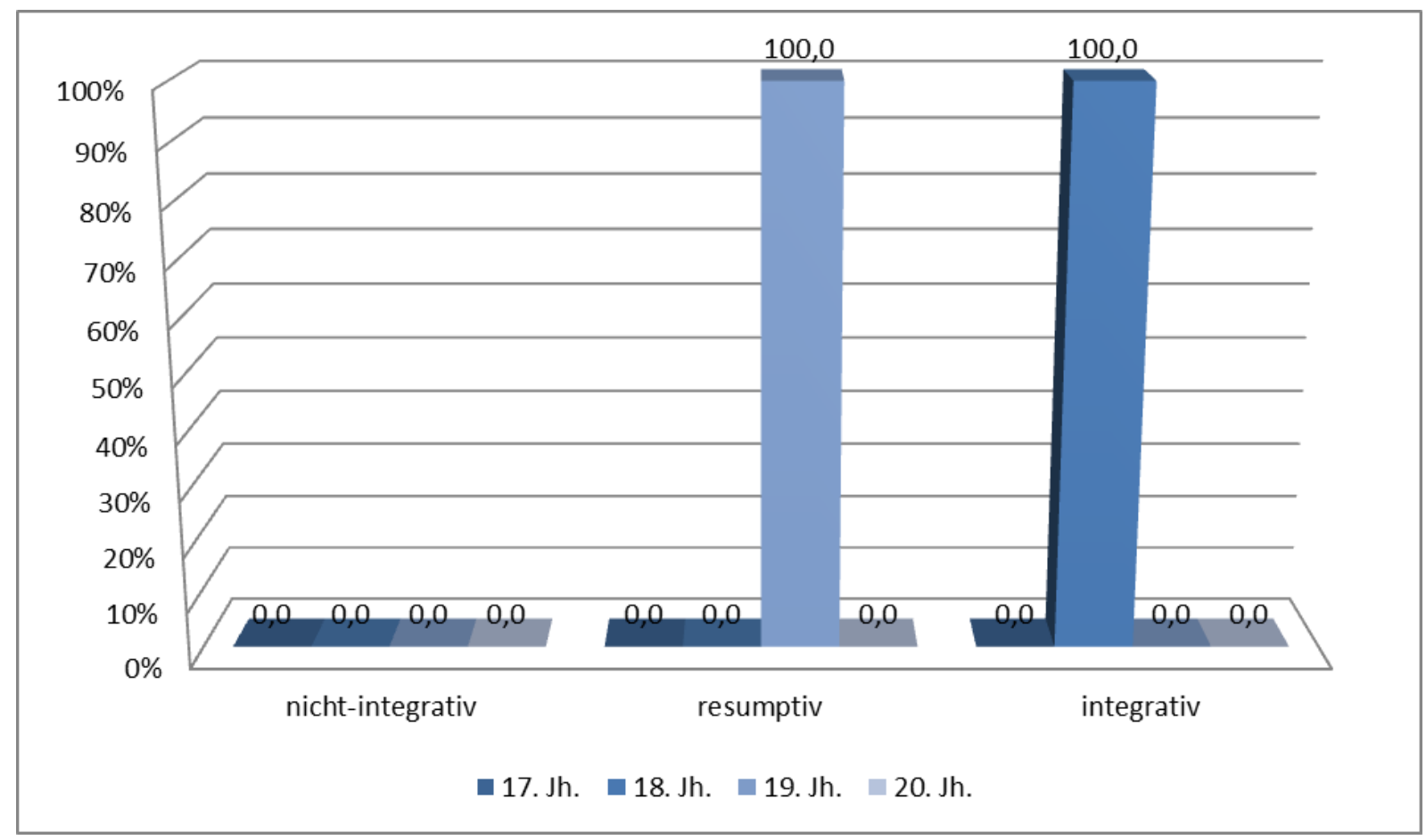

Diagramm 11: Prozentuale Verteilung der Stellungstypen bei vorangestellten, eingeleiteten Konzessivsätzen im Näheteilkorpus [17. Jh.: Güntzer I $(n=0)$, 18. Jh.: Schuhmacher Chronik II $(n=1)$, 19. Jh.: Auswandererbriefe V $(n=5)$, 20. Jh.: Jugendtagebuch VII $(n=0)]$

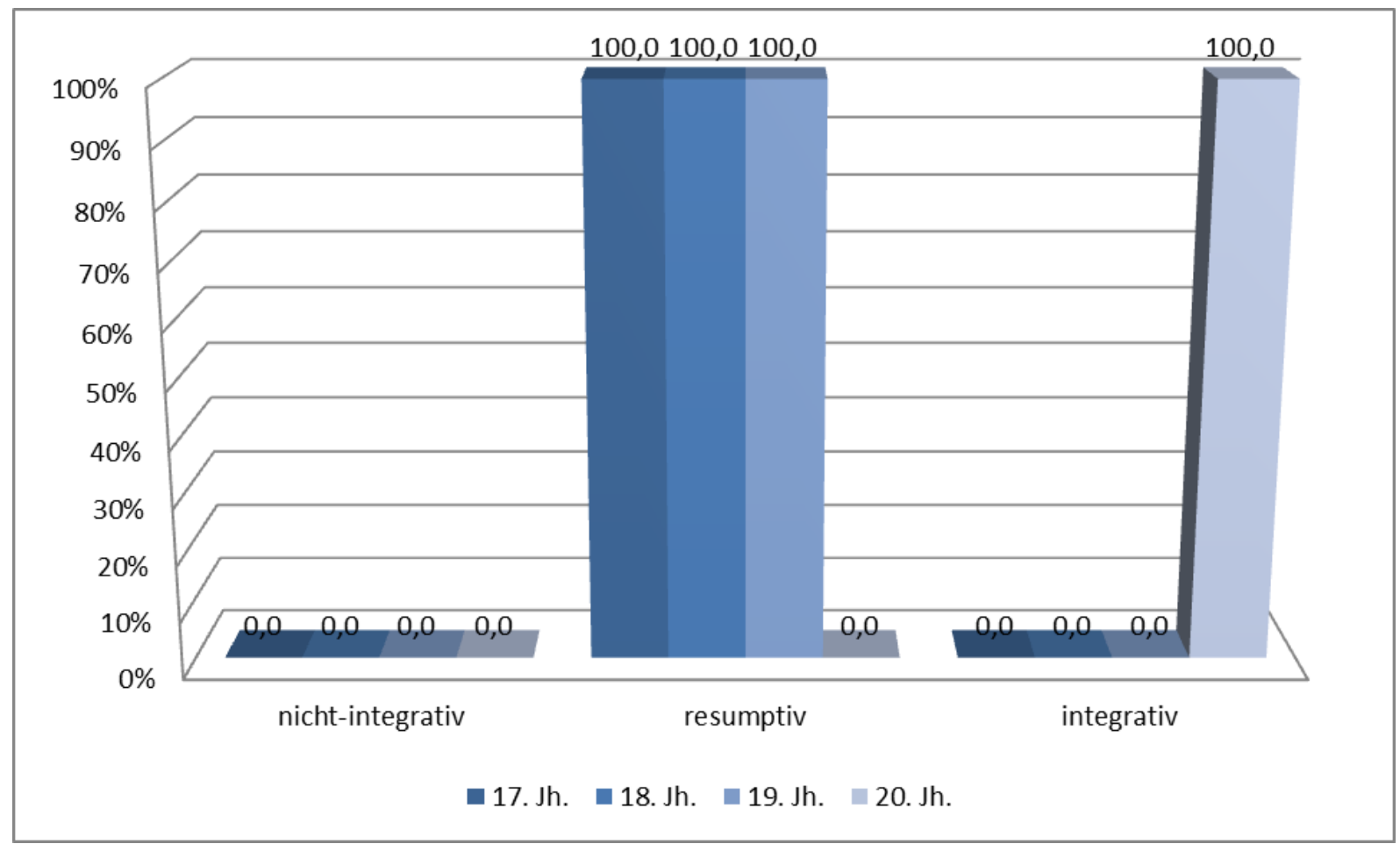

Diagramm 12: Prozentuale Verteilung der Stellungstypen bei vorangestellten, eingeleiteten Konzessivsätzen im Distanzteilkorpus [17. Jh.: Harsdörffer I $(n=4)$, 18. Jh.: Aichinger III $(\mathrm{n}=1)$, 19. Jh.: Burckhardt V ( $\mathrm{n}=1)$, 20. Jh.: Lorenz VII $(\mathrm{n}=1)]$ 


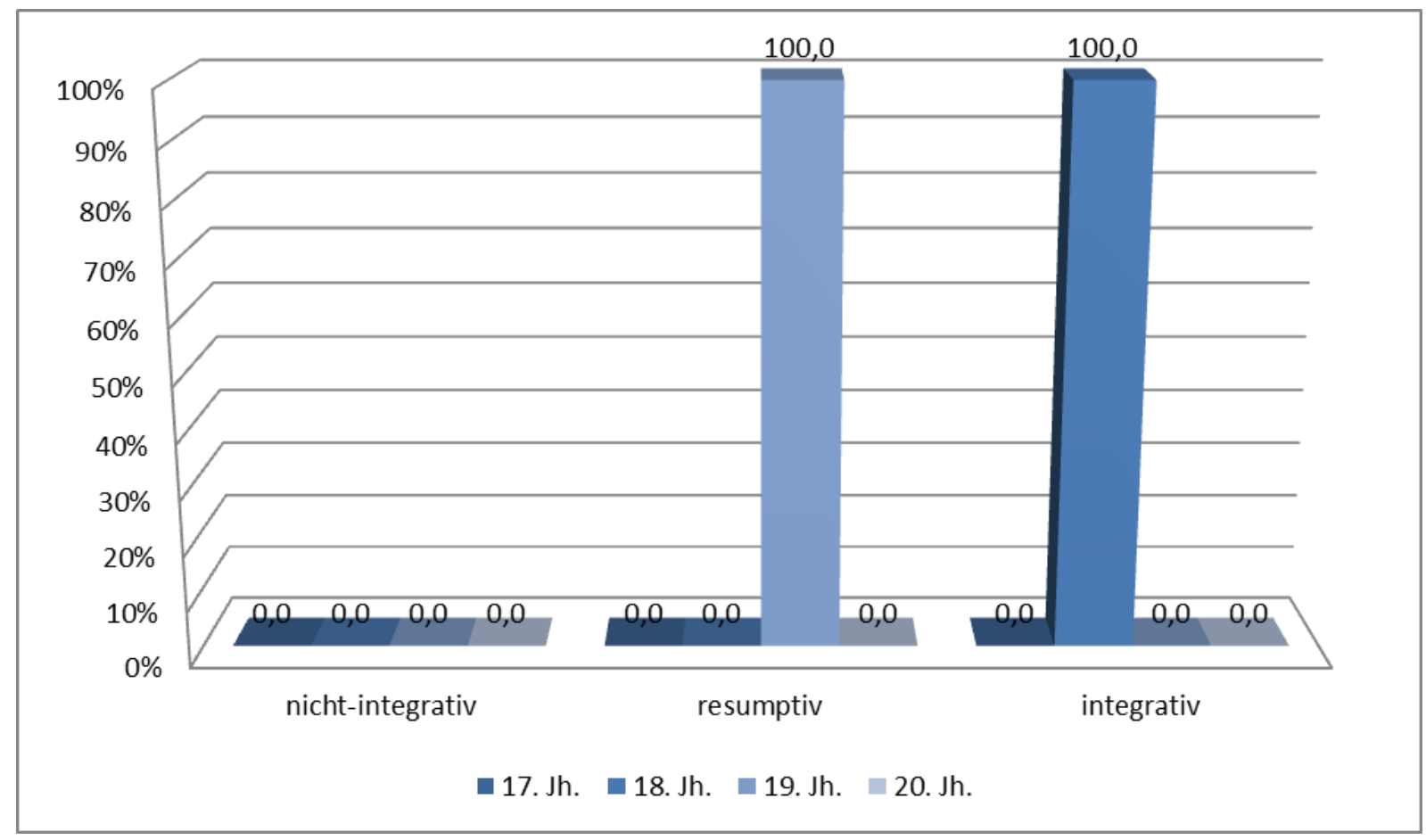

Diagramm 13: Prozentuale Verteilung der Stellungstypen bei vorangestellten Konzessivsätzen im Näheteilkorpus [17. Jh.: Güntzer I $(n=0)$, 18. Jh.: Schuhmacher Chronik II ( $n=1), 19$. Jh.: Auswandererbriefe V (n=5), 20. Jh.: Jugendtagebuch VII $(n=0)]$

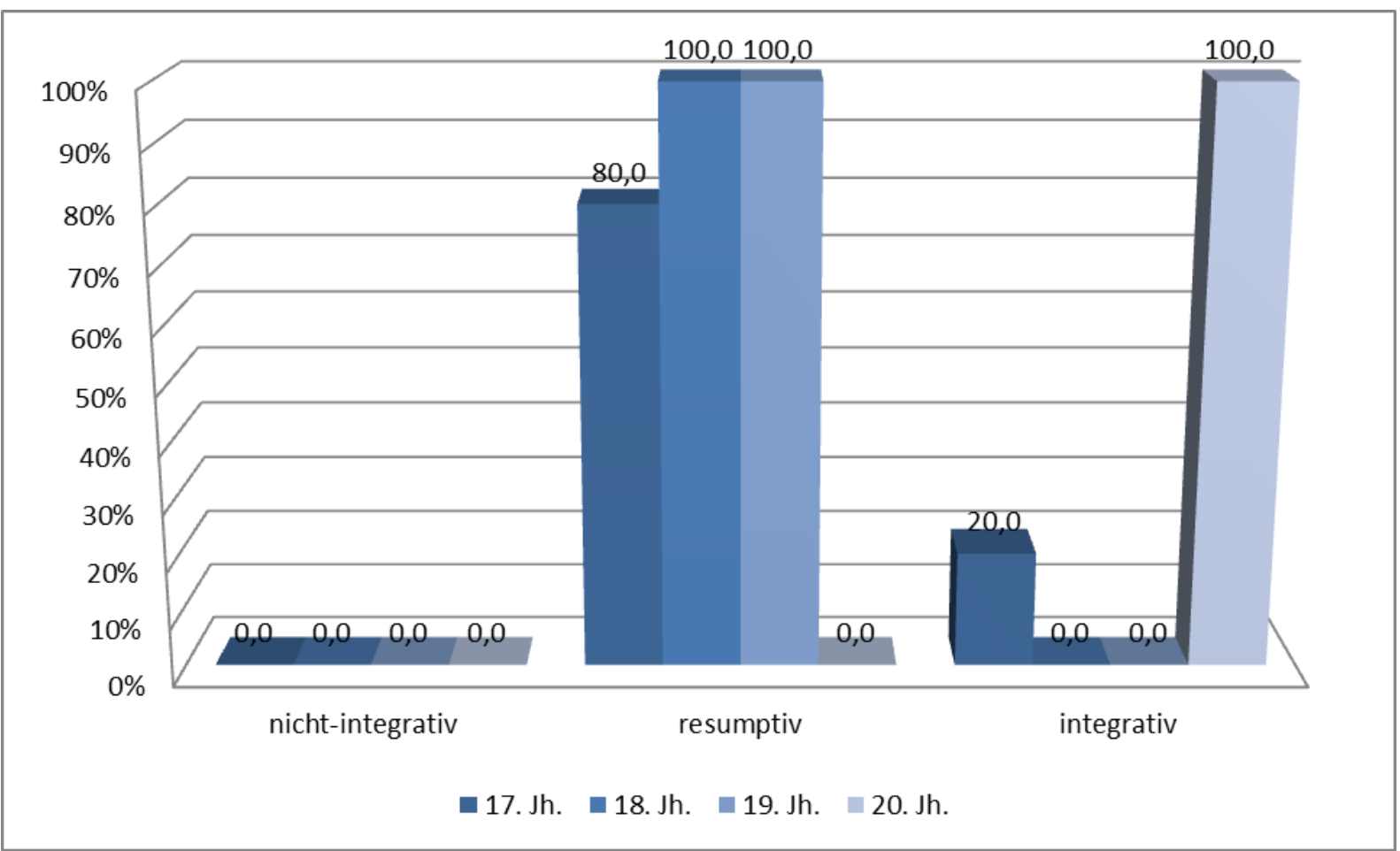

Diagramm 14: Prozentuale Verteilung der Stellungstypen bei vorangestellten Konzessivsätzen im Distanzteilkorpus [17. Jh.: Harsdörffer I $(n=5)$, 18. Jh.: Aichinger III $(n=3), 19$. Jh.:

Burckhardt V $(n=1)$, 20. Jh.: Lorenz VII $(n=1)]$ 


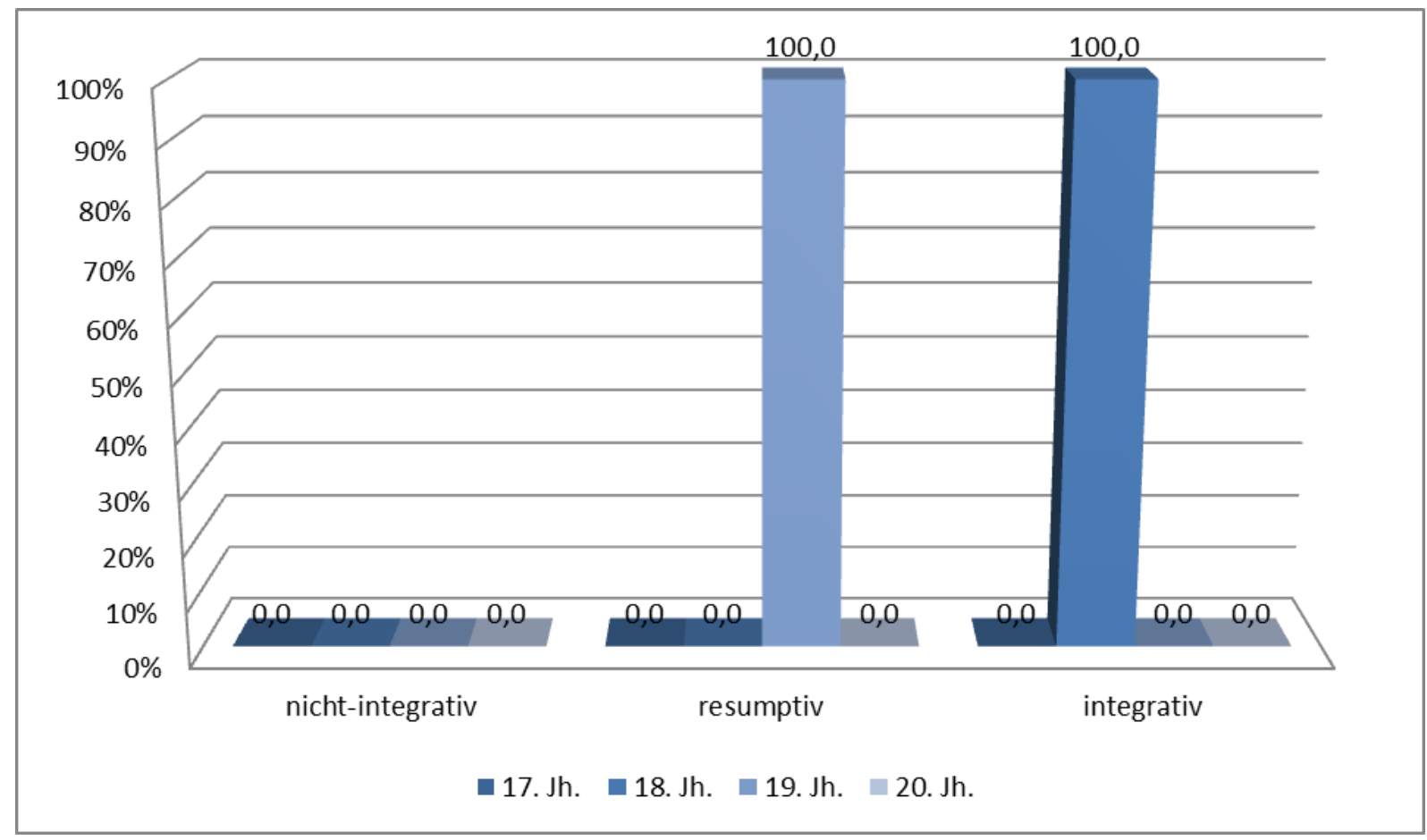

Diagramm 15: Prozentuale Verteilung der Stellungstypen bei vorangestellten Irrelevanzkonditionalen im Näheteilkorpus [17. Jh.: Güntzer I $(n=0)$, 18. Jh.: Schuhmacher Chronik II ( $n=1)$, 19. Jh.: Auswandererbriefe V $(n=2)$, 20. Jh.: Jugendtagebuch VII $(n=0)]$

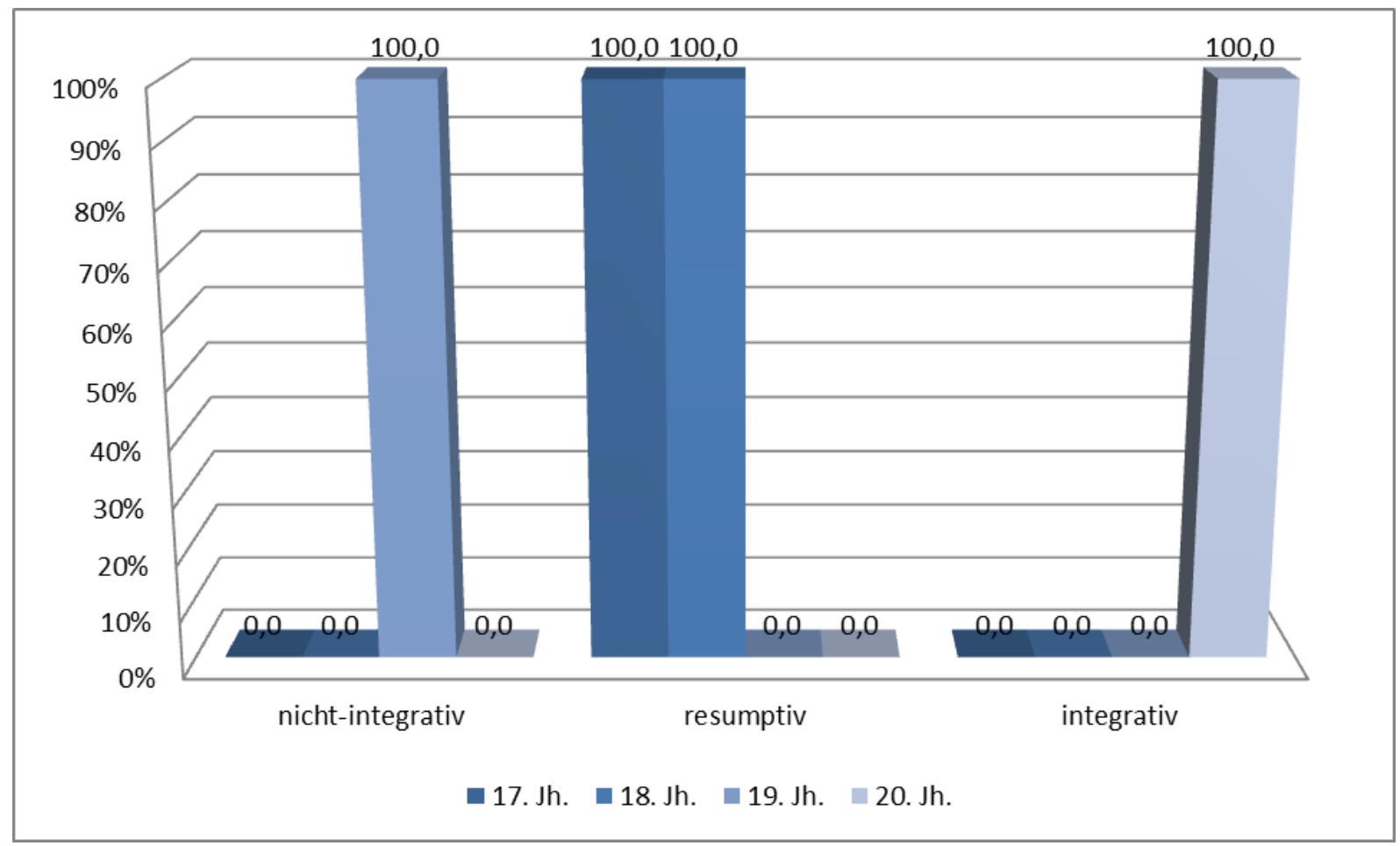

Diagramm 16: Prozentuale Verteilung der Stellungstypen bei vorangestellten Irrelevanzkonditionalen im Distanzteilkorpus [17. Jh.: Harsdörffer I $(n=1)$, 18. Jh.: Aichinger III $(n=2)$, 19. Jh.: Burckhardt V ( $n=3)$, 20. Jh.: Lorenz VII $(n=2)]$ 


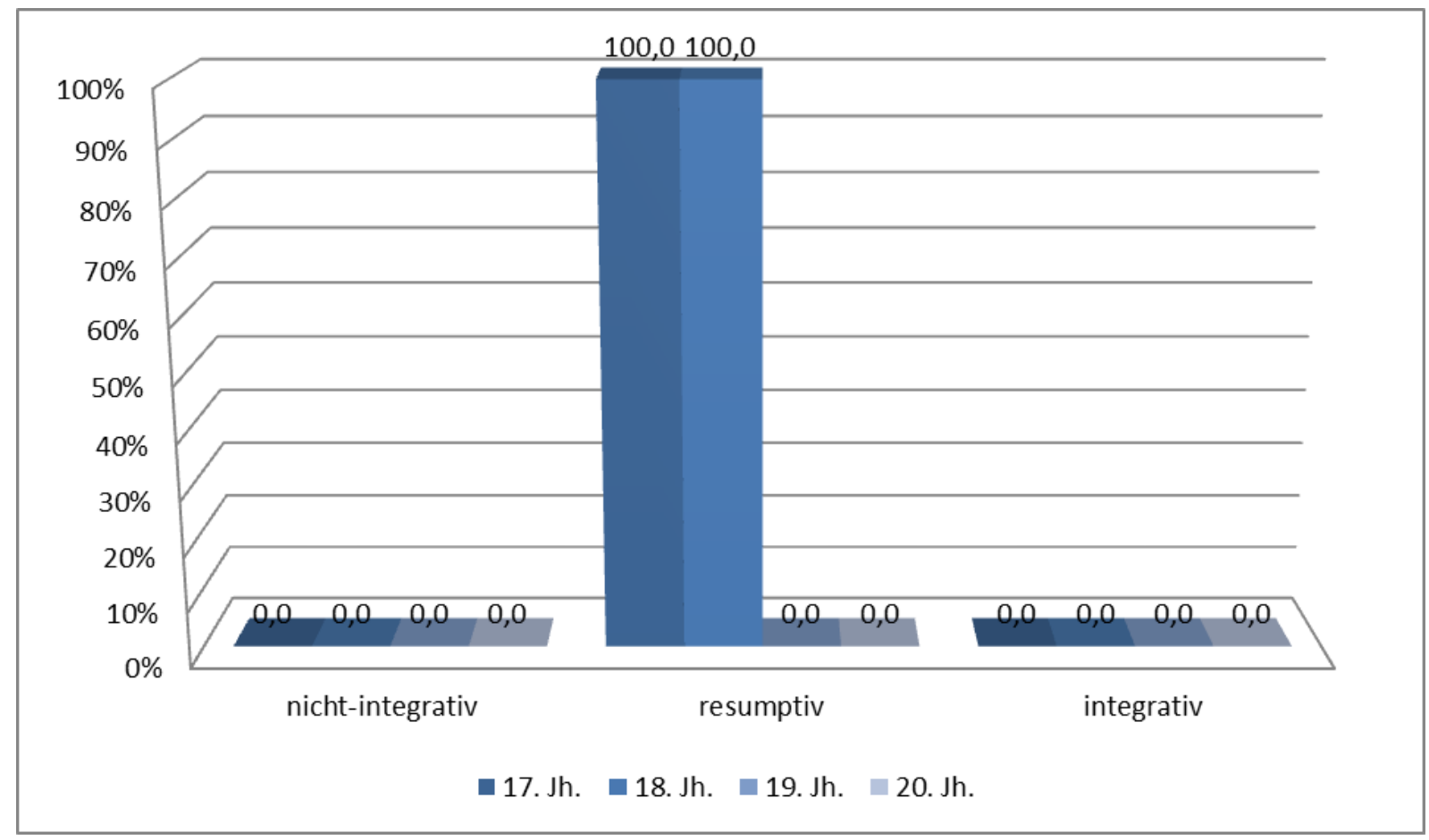

Diagramm 17: Prozentuale Verteilung der Stellungstypen bei vorangestellten Komparativsätzen im Näheteilkorpus [17. Jh.: Güntzer I (n=1), 18. Jh.: Schuhmacher Chronik II $(n=1)$, 19. Jh.: Auswandererbriefe V $(n=0)$, 20. Jh.: Jugendtagebuch VII $(n=0)$ ]

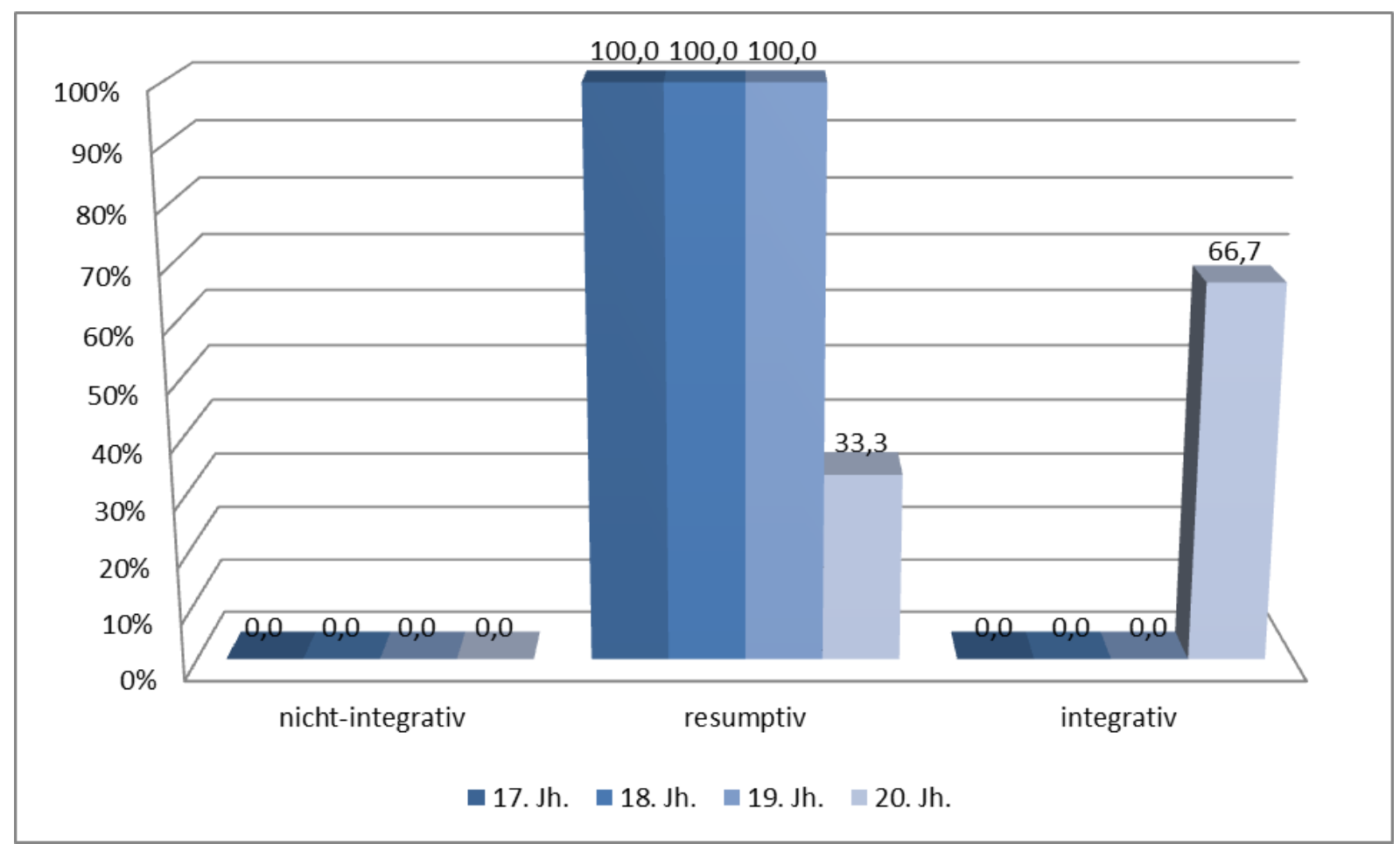

Diagramm 18: Prozentuale Verteilung der Stellungstypen bei vorangestellten Komparativsätzen im Distanzteilkorpus [17. Jh.: Harsdörffer I ( $\mathrm{n}=10)$, 18. Jh.: Aichinger III $(n=4)$, 19. Jh.: Burckhardt V ( $n=1)$, 20. Jh.: Lorenz VII $(n=3)]$ 


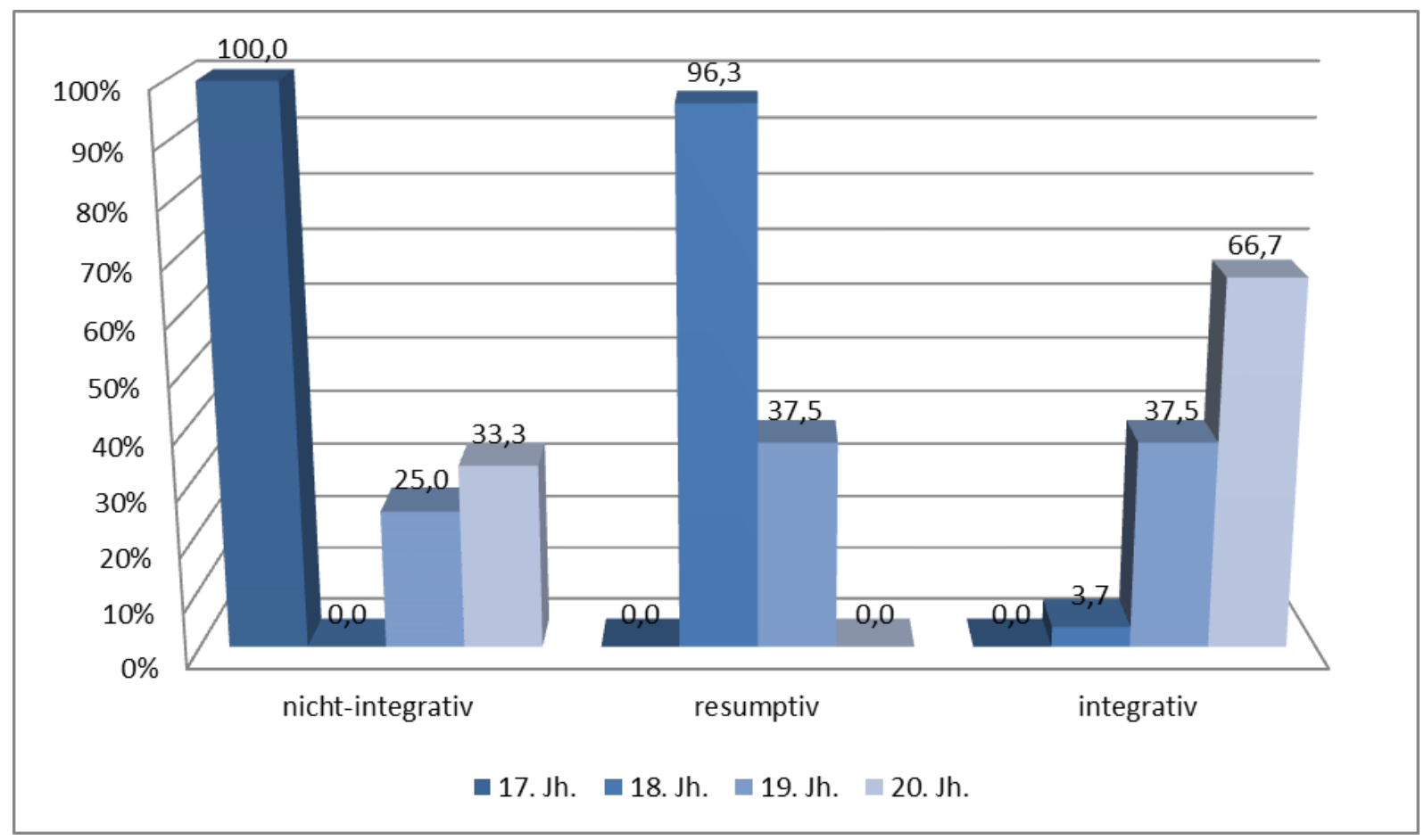

Diagramm 19: Prozentuale Verteilung der Stellungstypen bei vorangestellten pragmatischen Adverbialsätzen im Näheteilkorpus [17. Jh.: Güntzer I ( $n=2)$, 18. Jh.: Schuhmacher Chronik II $(n=27)$, 19. Jh.: Auswandererbriefe V $(n=16)$, 20. Jh.: Jugendtagebuch VII $(n=3)]$

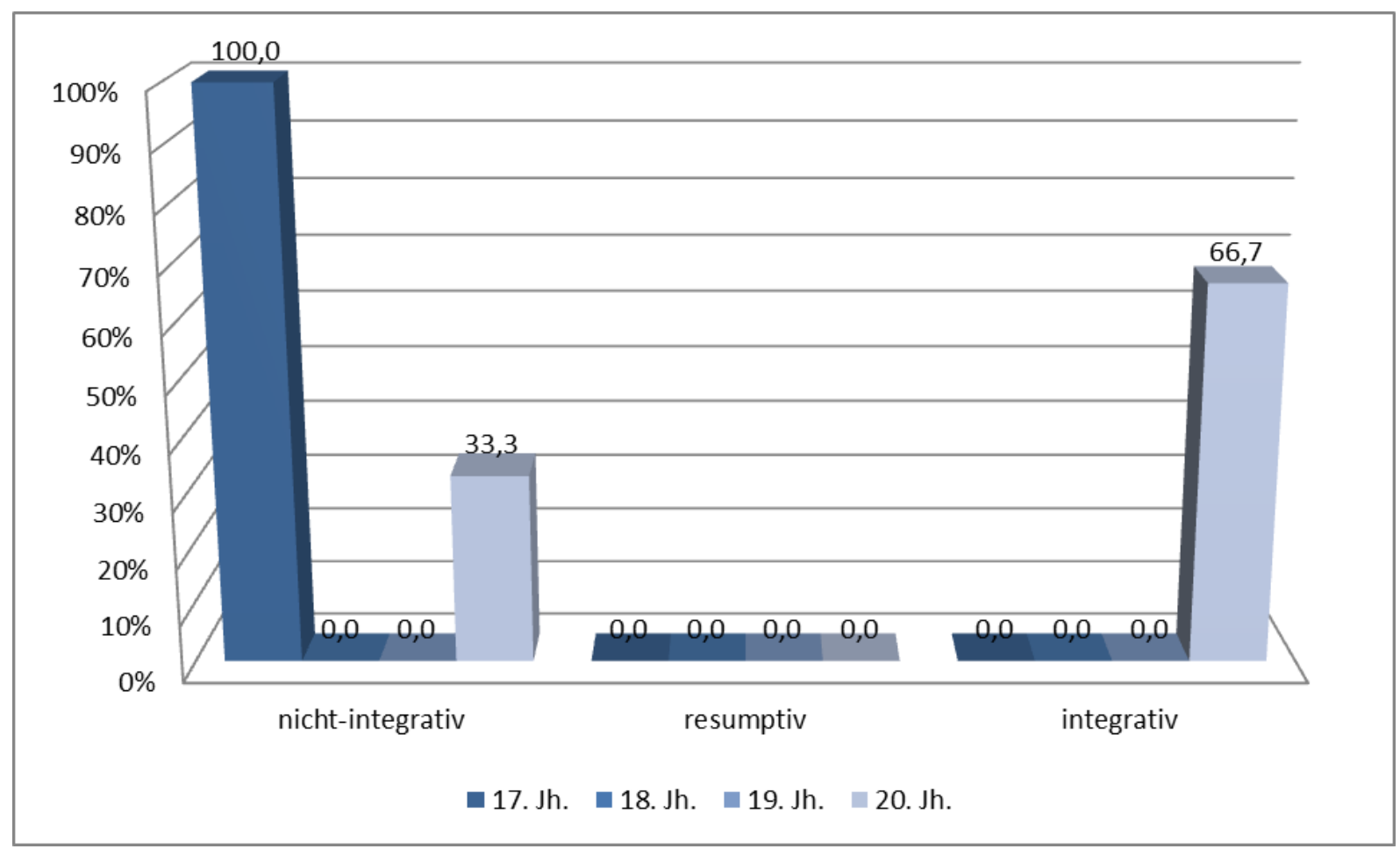

Diagramm 20: Prozentuale Verteilung der Stellungstypen bei vorangestellten pragmatischen Adverbialsätzen im Distanzteilkorpus [17. Jh.: Harsdörffer I $(n=3)$, 18. Jh.: Aichinger III $(\mathrm{n}=0)$, 19. Jh.: Burckhardt V $(\mathrm{n}=0)$, 20. Jh.: Lorenz VII $(\mathrm{n}=6)]$ 


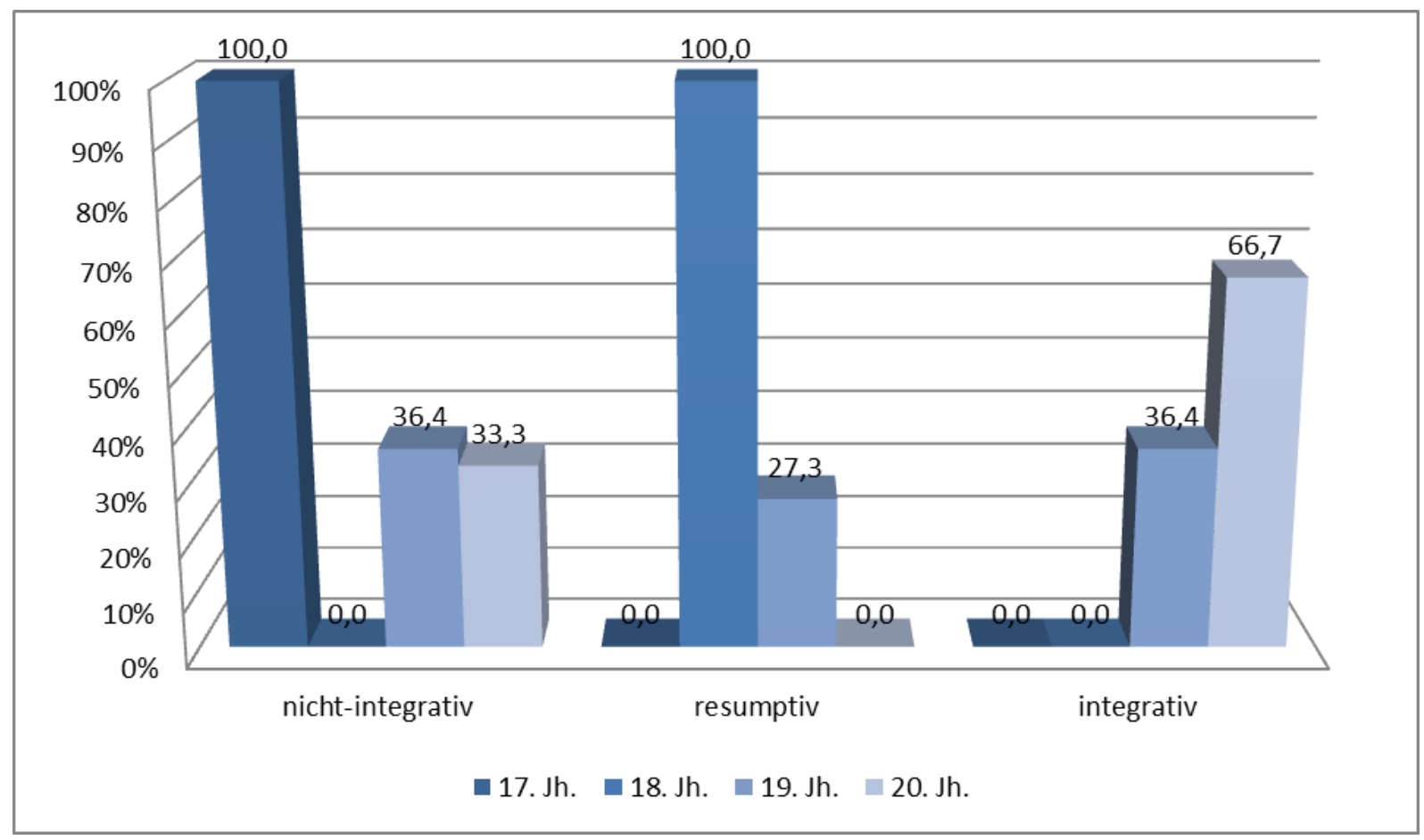

Diagramm 21: Prozentuale Verteilung der Stellungstypen bei vorangestellten pragmatischen Adverbialsätzen im Näheteilkorpus - Untertypen moduskommentierend und -modifizierend, diktums- bzw. einstellungskommentierend, formulierungskommentierend und diskursstrukturierend [17. Jh.: Güntzer I ( $n=2)$, 18. Jh.: Schuhmacher Chronik II ( $n=3), 19$. Jh.: Auswandererbriefe V ( $\mathrm{n}=11)$, 20. Jh.: Jugendtagebuch VII $(\mathrm{n}=3)]$

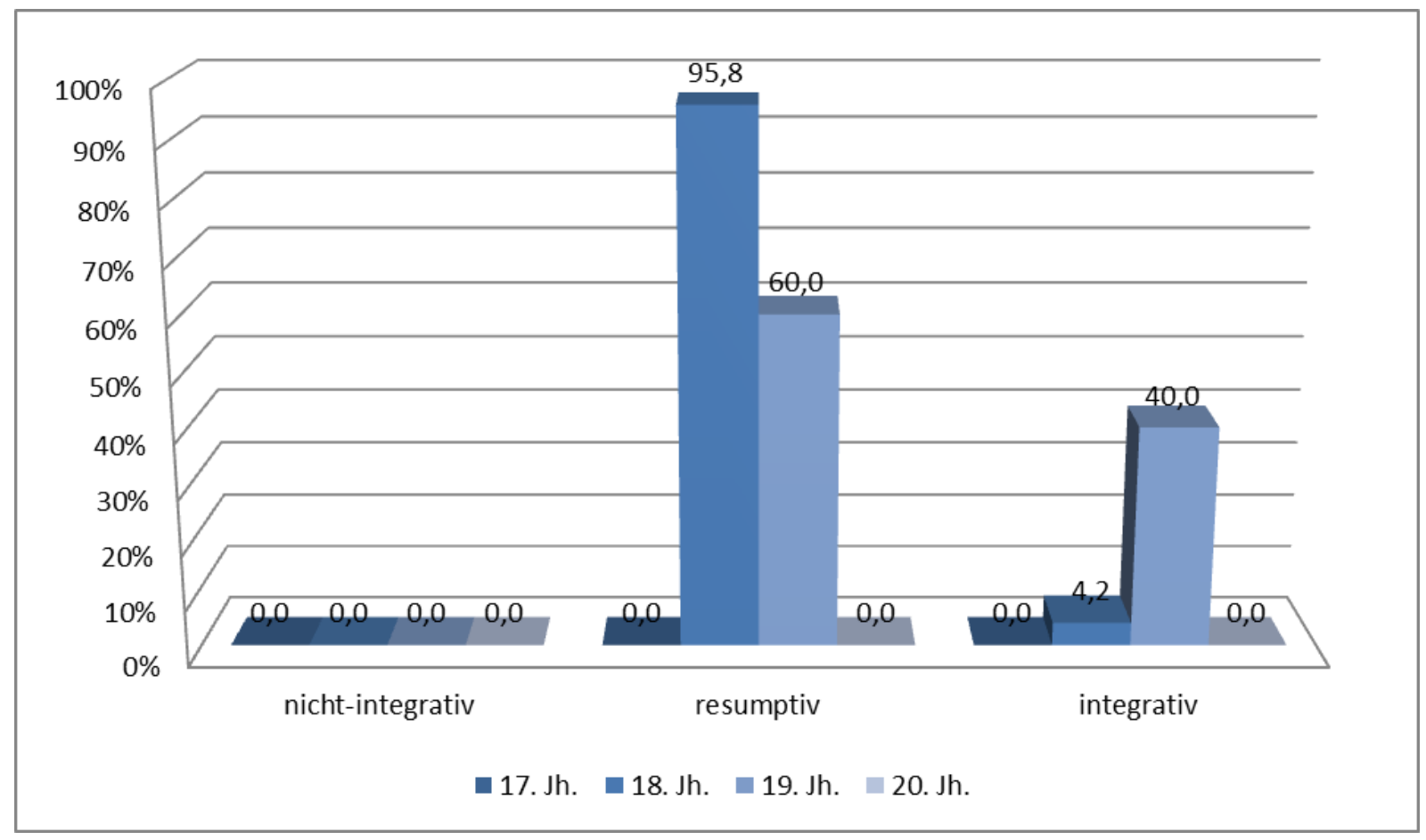

Diagramm 22: Prozentuale Verteilung der Stellungstypen bei vorangestellten thematisierenden Adverbialsätzen im Näheteilkorpus [17. Jh.: Güntzer I ( $n=0)$, 18. Jh.: Schuhmacher Chronik II ( $n=24)$, 19. Jh.: Auswandererbriefe V ( $n=5)$, 20. Jh.: Jugendtagebuch VII $(\mathrm{n}=0)]$ 